\title{
Udo Göttlich
}

Lothar Mikos

Rainer Winter (Hg.)

D i e

Werkze u g is te de $r$ Cultura I Studies Perspektiven, Anschlüsse und

ค

ш

$\overline{-}$

ᄀ

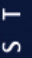

$-$

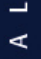

$\simeq$

$\supset$

$\vdash$

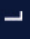

$=$

$u$

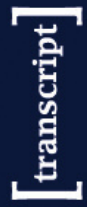


Udo Göttlich • Lothar Mikos • Rainer Winter (Hg.)

Die Werkzeugkiste der Cultural Studies 

Udo Göttlich • Lothar Mikos • Rainer Winter (Hg.)

Die Werkzeugkiste der Cultural Studies

Perspertiven, Anschlüsse und Interventionen

[transcript] CULTURALSTUDIES 


\section{(c) $(9)$ This work is licensed under a Creative Commons BY NC ND Attribution-NonCommercial-NoDerivatives 3.0 License.}

Die deutsche Bibliothek - CIP-Einheitsaufnahme

Die Werkzeugkiste der cultural studies : Perspektiven, Anschlüsse und Interventionen / Udo Göttlich ... (Hg.). - Bielefeld : Transcript, 200I (Cultural studies ; 2) ISBN 3-933I27-66-I

(C) 200I transcript Verlag, Bielefeld Lektorat: Karin Dirks Umschlaggestaltung \& Innenlayout: Kordula Röckenhaus, Bielefeld Satz: digitron $\mathrm{GmbH}$, Bielefeld Druck: Majuskel Medienproduktion GmbH, Wetzlar ISBN 3-933I27-66-I 
Inhalt

Vorbemerkung der Herausgeber | 7

Vorwort

Lawrence Grossberg | 9

Zur Epistemologie der Cultural Studies in kulturwissenschaftlicher Absicht: Cultural Studies zwischen kritischer Sozialforschung und Kulturwissenschaft

Udo Göttlich | 15

Ethnographie, Interpretation und Kritik: Aspekte der Methodologie der Cultural Studies

Rainer Winter | 43

Baustellen in Wien: Ein kulturwissenschaftlicher Werkstattbericht Christina Lutter | 63

Wozu Cultural Studies? Lektüreversuch aus einer «widerspenstigen« Perspektive

Manuela Ribeiro Sanches | 85

Sport und Cultural Studies: Zur ungleichzeitigen Formierung eines Forschungsfeldes

Roman Horak und Otto Penz | 105

Cultural Studies und Ethnologie: Zu einem schwierigen Verhältnis Sibylle Niekisch | 131

Das Neue im Zusammenspiel bildungs- und club-kultureller Prozesse Olaf Sanders | 159

Zur Rezeption der Cultural Studies in «SPEX - Magazin für Pop-Kulturu

Richard Gebhardt | 175 
Die Politik des Kulturellen: Cultural Studies in Wien und in Birmingham

Günther Sandner | 201

Denken (und Schreiben) in Netzwerken: Antonio Gramsci, Walter Benjamin und Antonio Machado

Birgit Wagner | 223

Medienkultur als »Nationalkultur» im Wandel: Cultural Studies und die Perspektive einer transkulturellen Medienforschung im deutschsprachigen Raum

Andreas Hepp | 243

Kulturimperialismus und Kulturindustrie ade? Zur Notwendigkeit einer Neuorientierung der Erforschung und Kritik von Medienkultur in den Cultural Studies

Carsten Winter | 283

Cultural Studies, Medienanalyse und Rezeptionsästhetik Lothar Mikos | 323

Die Autorinnen und Autoren | 343 


\section{Vorbemerkung der Herausgeber}

Cultural Studies sind nicht nur ein theoretischer Ansatz, sondern auch ein intellektuelles Projekt mit einem politischen Anspruch. Sie sind transdisziplinär, manchmal auch gegendisziplinär, transnational orientiert. Außerdem sind sie interpretativen und kritischen Methodologien verpflichtet. Ihre Geschichte zeigt, dass die Wahl der jeweiligen Forschungspraxis aus pragmatischen, strategischen und selbstreflexiven Gesichtspunkten heraus erfolgt. Zentral sind die Fragen, Konflikte und Probleme, die sich in einem spezifischen sozialen Kontext stellen. Es geht um die sozialen Auseinandersetzungen, wie sie sich im Alltag der Menschen zeigen - und es geht darum, die Strukturen und die Dynamik des Alltags im Rahmen ihres historischen, kulturellen, politischen und ökonomischen Kontextes zu betrachten. Aus dem Pool der vorhandenen theoretischen Konzepte, Methoden und Auswertungsverfahren werden diejenigen »Werkzeuge« ausgewählt, die für die jeweilige Aufgabe als geeignet erscheinen. Theorie wird in diesem Sinn als strategische Ressource benutzt. Werden neue Werkzeuge gebraucht, so werden diese hergestellt.

Cultural Studies sind notwendigerweise offen für unerwartete und ungebetene Möglichkeiten. Der Forschungsprozess lässt sich als Bricolage begreifen. Leitend für die Theorieentwicklung sind die kulturellen Praktiken und Erfahrungen der Menschen in ihrem Alltagsleben. Michel Foucaults Analysen folgend, wird der Zusammenhang von Wissenschaft und Macht kritisch reflektiert. Wissenschaftliche Forschung ist kein Selbstzweck, sondern Teil des Bemühens, eine demokratischere und gerechtere Gesellschaft zu schaffen. Cultural Studies geht es um die »Theoretisierung von Politik« und die »Politisierung 
von Theorie« (Lawrence Grossberg). Sie sind bestrebt, politisch brauchbares Wissen zu erzeugen. In diesem Sinn geht es ihnen vor allem um eine Politik der Anerkennung und der Ermöglichung von Handlungsfähigkeit.

Es gibt (bisher) nicht die Disziplin der Cultural Studies, sondern es gibt Wissenschaftler, die sich dem Projekt der Cultural Studies verpflichtet fühlen - jenseits disziplinärer Grenzen. Sie operieren an der Schnittstelle von Disziplinen, Kulturen, Wissenschaft und Politik. Das heißt auch, dass es zahlreiche Anknüpfungspunkte zu traditionellen Wissenschaftsdisziplinen gibt. In dem vorliegenden Buch versuchen Wissenschaftler und Wissenschaftlerinnen aus Deutschland, Österreich und Portugal das Projekt der Cultural Studies weiterzuführen und diskutieren insbesondere die Schnittstellen mit Disziplinen wie Soziologie, Philosophie, Ethnologie, Medien- und Kommunikationswissenschaft, Germanistik, Sportwissenschaft, Erziehungswissenschaft, Kritischer Theorie und Kulturwissenschaft. Sie benutzen die Werkzeugkiste der Cultural Studies, um sie für ihre »Mutterdisziplinen « fruchtbar zu machen. Sie diskutieren ihre Theorien, Methoden und Geschichte. Gleichzeitig arbeiten sie heraus, welche Anschlusspunkte, Parallelen und Unterschiede es in der deutschen Diskussion gibt. Ihr Hauptaugenmerk gilt einem produktiven Einbringen und einer kreativen Fortführung von Cultural Studies. Deren gefüllte, allerdings nicht komplette »Werkzeugkiste« lädt hierzu ein.

Die Herausgeber und AutorInnen hoffen, dass sich die Gebrauchsweisen, Fortsetzungen und Weiterentwicklungen vervielfachen und auf diese Weise vielfältige Verbindungen entstehen werden. Denn das Projekt der Cultural Studies lebt davon, permanent in unterschiedlichen Kontexten fortgeschrieben zu werden.

Starnberger See, im August 2001

Die Herausgeber 


\section{Vorwort}

LAWRENCE GROSSBERG

Vor einigen Jahren stellte ein eng befreundeter Kollege eine beiläufige Bemerkung (eigentlich eine Fußnote) in Frage, mit der ich sinngemäß gemeint hatte, es gäbe in Frankreich keine Cultural Studies, obwohl die französische Kulturtheorie einen enormen Einfluss auf die Cultural Studies gehabt hätte. Mein Freund, Charley Stivale, brachte einige aufschlussreiche Argumente an. Vor allem wies er darauf hin, dass ich mit vielen der Arbeiten aus Frankreich einfach nicht vertraut wäre, und zwar, weil sie entweder nicht übersetzt waren oder aber keine an Cultural Studies Interessierte außerhalb Frankreichs anzusprechen schienen (und auch nicht entsprechend vermarktet worden waren). Wichtiger aber ist, dass er den in meiner Beurteilung enthaltenen Widerspruch in Frage stellte. Denn ich hatte ja schließlich schon seit mehreren Jahren behauptet, dass die Cultural Studies eine radikal kontextuelle Herangehensweise an den Stellenwert von diskursiven Praktiken bei der Bildung und Umbildung sozialer Machtzusammenhänge darstellen. Und daher müssen die Cultural Studies an sich auch kontextuell verstanden werden. Das Erscheinungsbild der Cultural Studies, die spezifischen theoretischen, analytischen und methodologischen Umrisse und Praktiken, die sie in jedem Fall bestimmen, sind sowohl vom Kontext ihrer eigenen Produktion als auch von jenem Kontext abhängig, auf den sie sich beziehen. Und wenn dem so ist, wie kann man sich dann bereits im Vornherein sicher sein, woran die Cultural Studies zu erkennen sind? Wie weiß man, ob es sich bei einer bestimmten Studie um Cultural Studies handelt, um ein in einer 
angemessenen Weise kontextuell bestimmtes politisch-intellektuelles Projekt? Nichtsdestotrotz gibt es eine imaginäre Geografie der Cultural Studies - oder vielleicht auch mehrere solcher Geografien. Karten dieser Art ermöglichen uns angeblich ein Wissen darüber, wo und wann Cultural Studies stattgefunden haben, wo und wann sie stattfinden, stattfinden könnten und vielleicht auch betrieben werden sollten. Gleichzeitig gibt es zumindest einige Mythen über die Ursprünge der Cultural Studies und ihre Verbreitung, und wahrscheinlich noch viele andere Mythen, die erst noch erzählt werden müssen. Bedeutsam sind nicht die Mythen selbst, sondern jene Auswirkungen, die sie auf die Praktiken der Cultural Studies haben. Denn welche Absichten auch immer im Spiel sein mögen, so werden Mythen doch letztendlich häufig als Erklärungen für »angemessene« Formen, Engagements, Paradigmen, Maßnahmen etc. betrachtet. Wichtiger aber ist, dass die gebotenen strategischen Mittel - Exemplare im Sinne Thomas Kuhns - zur Konstruktion oder Kommunikation spezifischer Ausformungen und Zusammenhänge der Cultural Studies schließlich zu einer Bestimmung der Grenzen unseres Vermögens führen können, Cultural Studies in neuen und unterschiedlichen Formen zu erkennen. Obwohl derartige Mythen für die imaginierte Geografie der Cultural Studies nicht bestimmend sind, können sie doch in letzter Instanz die Breiten- und Längengrade, die zentrifugalen und zentripetalen Vektoren, der Cultural Studies-Karten definieren. Diese Grade und Vektoren ordnen die vielfältigen Stellen, Gemeinschaften, Traditionen und Historien der Cultural Studies. Und leider verlief die Bildung dieser Grade und Bahnen bislang auch häufig gemäß vorhersehbarer Dimensionen, die an den Linien der imperialen und ökonomischen Macht des zwanzigsten Jahrhunderts ausgerichtet sind. Und die mythische Bedeutung der britischen Cultural Studies sowie der mehr allgemeine Einfluss der Cultural Studies aus den Vereinigten Staaten beruht sicherlich zu einem großen Teil auf der Hegemonie der englischsprachigen akademischen Welt und dem schieren Ausmaß ihres potenziellen Marktes. Natürlich sind eine bedeutsame Sprachgemeinschaft (und ein potenzieller Markt) allein noch nicht alles. Zwei weitere Beispiele mögen genügen, um dies zu veranschaulichen. In der letzten Zeit wurden sich diejenigen von uns, die nicht in der spanischsprachigen Welt leben, der Mannigfaltigkeit jener kraftvollen und produktiven Cultural Studies-Traditionen bewusst, die bereits seit einigen Jahrzehnten in Lateinamerika gedeihen. Wie andere Orte und 
Traditionen auch sind diese Arbeiten als Antwort auf einzigartige historische und geografische Verhältnisse aus indigenen theoretischen und politischen Praktiken hervorgegangen. Daniel Mato hat jedoch behauptet, die Einheit der »lateinamerikanischen Cultural Studies« sei größtenteils ein von außerhalb aufgezwungenes Konstrukt, wenn auch die Aussichten auf Kooperation und Dialog bereits ansatzweise verwirklicht werden. Interessanterweise werden die bedeutsamsten Fachzeitschriften, die angeblich die englischsprachigen lateinamerikanischen Cultural Studies repräsentieren, nicht auf dem Kontinent selbst produziert. Andererseits musste sich Asien einer äußerst komplexen sprachlichen Vielfalt stellen, als es sich im Laufe des vergangenen Jahrzehnts seine eigene Bedeutung von »inter-asiatischen« Cultural Studies erarbeitete. Ungeachtet des starken Einflusses von britischen und amerikanischen Bildungseinrichtungen und Cultural Studies-Traditionen haben die asiatischen Cultural Studies durchwegs ein Engagement erkennen lassen, diese Einflüsse sowohl auf indigene intellektuelle Traditionen als auch auf spezifische politische Verhältnisse neu abzustimmen. Verglichen mit Lateinamerika werden die führenden Fachzeitschriften, die die englischsprachigen asiatischen Cultural Studies repräsentieren, großteils in Asien selbst hergestellt, wobei sich dies teilweise jedoch aufgrund der Tatsache erklären lässt, dass Englisch die Sprache ist, vermittels derer auch die verschiedenen Sprachgemeinschaften in Asien miteinander kommunizieren. All dies führt mich zur wohl am schwierigsten zu entwirrenden und zu interpretierenden regionalen Spielart der Cultural Studies: Europa. Obwohl die gegenwärtige politische Kritik dazu tendiert, die unterschiedlichen Nationalkulturen in einen Topf zu werfen (mit Begriffen wie »der Westen « und »Eurozentrismus«), ist es eine Tatsache, dass »Europa « ebenso sehr eine Abstraktion ist wie Asien oder Lateinamerika. Und will man die Anfänge der Cultural Studies in Europa begreifen, so darf man weder über die Problematik der Nationalsprachen noch über die verschiedenartigen Philosophie- und Kulturgeschichten und auch nicht über die einzigartigen politischen Probleme hinwegsehen, die den Kontext der Rezeption und der Produktion der Cultural Studies in jedem einzelnen Land Europas ausmachen. Es ist allgemein bekannt, dass beispielsweise die Anfänge der Cultural Studies in Großbritannien bis zu einem gewissen Grad eine Reaktion (großteils im Rahmen der Denkkategorien der eben erst entstandenen New Left) auf jenen Wandel sind, der für die Nachkriegsgesellschaft bezeichnend ist, in- 
klusive einer »drohenden Amerikanisierung « (was die anhaltende zentrale Rolle der Kunst und der Literatur in vielen der frühen Arbeiten zum Teil erklären könnte). (In den Vereinigten Staaten gingen andererseits verschiedene Artikulationen von Cultural Studies teilweise mit unterschiedlichen Versuchen einher, den »amerikanischen Exzeptionalismus « $\mathrm{zu}$ theoretisieren und $\mathrm{zu}$ politisieren.) Viele der nicht in Europa lebenden Vertreter der Cultural Studies sind sich wenigstens der starken Präsenz der Cultural Studies in den nordischen Ländern bewusst (obwohl sie vielleicht nicht im gleichen Maße von den sehr unterschiedlichen Formen wissen, die diese beispielsweise in Finnland und Schweden angenommen haben); weniger sind sie sich jedoch wohl der in Italien geleisteten Arbeiten und noch weniger der Situation auf der Iberischen Halbinsel bewusst. Erst neulich habe ich von Intellektuellengruppen gehört, die über Osteuropa verstreut das Banner der Cultural Studies ergriffen haben. Ich habe das Gefühl, dass die Cultural Studies in diesen Nationen im Gegensatz zu Frankreich (einmal angenommen, Charley Stivale hat Recht) stark von den angloamerikanischen Cultural Studies beeinflusst worden sind (wie auch von anderen englischsprachigen Arbeiten sowie von der französischen Theorie). Das heißt nicht, dass diese Kreise einfach nur die Modelle der britischen oder amerikanischen Cultural Studies kopiert haben, denn sie alle haben diese Arbeit auf ihre eigenen institutionellen und politischen Anforderungen abgestimmt. Es gibt eine merkliche Lücke in dieser allzu kurzen Würdigung der Cultural Studies in Europa: Deutschland und Österreich. Ich maße mir nicht an, etwas über die Entwicklung der Cultural Studies im deutschsprachigen Europa zu schreiben, und gestehe, dass ich dieses Vorwort mit einem bestimmten Maß an Beklommenheit verfasse. Ich kann Ihnen nur etwas von meiner Einschätzung geben, die auf meiner eigenen Erfahrung und Teilhabe basiert, die ich während des letzten Jahrzehnts hauptsächlich in Österreich machte. Natürlich tauchten die Cultural Studies im vergangenen Vierteljahrhundert immer wieder kurzzeitig in den deutschsprachigen Nationen auf, aber ganz egal, ob nun die spürbaren Folgen der Nazizeit, die alten Traditionen des kulturellen Elitedenkens in der deutschen akademischen Welt, die enorme Macht und das Prestige anderweitiger politisch-intellektueller Traditionen oder andere Faktoren (vielleicht auch eine Kombination dieser Elemente) im Spiel waren, so schienen diese Gelegenheiten bereits vertan, noch bevor die Cultural Studies in welcher Form auch 
immer Fuß fassen konnten. Obgleich einige wenige Cultural StudiesNester überleben konnten (beispielsweise Rolf Lindner in der Ethnologie, Roman Horak in der Populärkultur und Peter Wicke in der Populärmusik), blieben sie doch größtenteils isoliert. Aber diese Situation wandelt sich sehr rasch, was auch im vorliegenden Buch dokumentiert und beschrieben wird. Es ist nun beinahe ein Jahrzehnt her, dass ich zu meinem ersten von zahlreichen Besuchen nach Wien eingeladen wurde und auf einen lebhaften Kreis politisch engagierter, zum Großteil junger Intellektueller stieß. In den meisten Fällen bildeten die angloamerikanischen Cultural Studies nicht den Ausgangspunkt ihrer Arbeit. Viele von ihnen besaßen eigene wissenschaftliche und intellektuelle Traditionen (marxistische Arbeitergeschichte, Sozial- und feministische Historie, Kultursoziologie, Kunst etc.) und doch begeisterten sie manche jener grundlegenden Positionen, die sie in den besten Arbeiten der britischen und amerikanischen Cultural Studies erkannten, unter anderem die Kontextualität und Politisierung der Wissensproduktion, das anti-elitäre Engagement für das Populäre und Alltägliche sowie eine optimistischere (weil weniger gesicherte) Sicht auf historische Zukunftsaussichten. Deshalb halfen die Cultural Studies (in Form ihrer englischsprachigen Spielarten) dabei, unterschiedliche politische Entwicklungen und intellektuelle Arbeiten zusammenzuführen und für alle einen Diskurs, ein Bezugssystem und ein Projekt bereitzustellen. Seither sind die angloamerikanischen Cultural Studies in einer wahrlich dialektischen Weise nicht nur deutlich einflussreicher, sondern auch weniger bestimmend geworden, während die einzigartigen Kräfte, Historien und politischen Herausforderungen Österreichs ihren Einfluss auf das Erscheinungsbild der deutschen Cultural Studies ausgeübt haben. Zum Teil beruht diese Einzigartigkeit auf der Tatsache, dass sich die Cultural Studies in Österreich außerhalb der Universität entwickelt haben (was durch eine Reihe von mit Regierungsgeldern unterstützte Institutionen und Initiativen gefördert wurde) und gerade erst - nach einem Jahrzehnt - in die akademische Welt einzudringen beginnen. Zur selben Zeit hatte sich in einigen wenigen deutschen Universitäten eine parallele Entwicklung abgespielt. Auch in Deutschland entstand eine kleine aber eindrucksvolle Gemeinschaft von Cultural Studies-Vertretern, die im Wesentlichen die politischen Bedeutungen und Möglichkeiten der Massenmedien und der Populärkultur ausloten wollten. Es überrascht nicht, dass die britischen Cultural Studies (und ihre an anderen Orten 
entstandenen Spielarten) einen starken Einfluss auf diese Arbeiten hatten, aber auch hier werden die Cultural Studies - wie aus den verschiedenen, von dieser Gruppe produzierten Sammelbänden aus der letzten Zeit ersichtlich wird - durch eigenständige intellektuelle, wissenschaftliche und politische Traditionen, Projekte und Vektoren abgewandelt. Schließlich begannen sich diese beiden Entwicklungslinien - die deutsche und die österreichische - erst in den letzten Jahren zu vereinen, und hier liegt auch das bedeutsamste Ziel des vorliegenden Buches, das von ihm dokumentierte Ereignis. Dieses Zusammenlaufen bedeutet, dass die Zukunft der deutschen Cultural Studies so wie eh und je offen ist und neue Gelegenheiten eröffnet, die sich auch noch vor wenigen Jahren niemand hat vorstellen können. Sowohl Deutschland als auch Österreich sind bedeutsame Schauplätze für Cultural Studies-Arbeiten, wenn auch aus unterschiedlichen Gründen. Deutschland ist offensichtlich ein historischer Hauptschauplatz, und zwar sowohl in politischer als auch in philosophischer Hinsicht, aber auch in der Zukunft, die Europa ist, wird dieses Land ein Hauptschauplatz sein. Österreich bringt seine eigenen Traditionen und Zukunftsaussichten mit ein, aber auch seine traditionelle Position an der Schnittstelle von West- und Osteuropa, was nicht minder bedeutsam ist. Und weil eben dieser Unterschied besteht, ist der Dialog zwischen den in diesen beiden Nationen entstehenden Arbeiten - die Anfänge der deutschsprachigen Cultural Studies - wichtig und vielversprechend, mit all jenen Ambiguitäten, die die widersprüchliche Position des Westens kennzeichnen. Noch bedeutsamer jedoch ist es, dass sich, während diese emergente und andersartige Cultural Studies-Formation in Entwicklung begriffen ist, ihre intellektuellen und politischen Stimmen an der bereits rasch wachsenden Reihe von Gesprächen beteiligen werden, die Cultural Studies ausmachen. Und nur auf diesem Weg können die Cultural Studies ihre eigene Zukunft haben - eine Zukunft, die sich der wandelnden Gestalt der Welt wird stellen können, so wie der Position des Lokalen in den Wirrnissen des Globalen. 


\title{
Zur Epistemologie der Cultural Studies
}

\section{in kulturwissenschaftlicher Absicht: Cultural Studies}

\author{
zwischen kritischer Sozialforschung und
}

\section{Kulturwissenschaft}

Udo GÖTTLICH

\section{Vorbemerkung}

Eine Epistemologie der Cultural Studies ist noch nicht geschrieben. Dennoch findet sich kaum ein Text der theoretischen Häupter, der nicht die spezifische Ausrichtung gerade auch im Vergleich zu sozialund kulturwissenschaftlichen Zugängen herausstreicht. Auch liefern die einschlägigen Handbücher zur qualitativen Forschung im Umfeld der Cultural Studies eine umfassende Übersicht zu den Methoden und den erkenntniskritischen Positionen (vgl. Denzin/Lincoln I994, I998). Ethnographie, so zeigt sich, stellt nur eine ihrer Methoden dar, die vor allem im Rahmen der Rezeptions- und Zuschauerforschung Bedeutung erlangte (vgl. Ang I996). Weniger aufgearbeitet im transdisziplinären Dialog scheint jedoch der postmarxistische und poststrukturalistische Zuschnitt, der gerade auch die erkenntniskritische Perspektive gegenüber sozial- und kulturwissenschaftlichen $\mathrm{Zu}$ gangsweisen und Methoden konserviert (vgl. Morley/Chen I996). Der poststrukturalistische Strang dieser Kritik entspringt vor allem der 
literatur- und textwissenschaftlichen Herkunft der Cultural Studies, die ihre Methoden gerade auch aus der Diskursanalyse beziehen. Der postmarxistische Strang der Kritik hat über die Arbeiten verschiedener Cultural Studies-Vertreter, angefangen bei Raymond Williams über Stuart Hall bis hin zu Lawrence Grossberg, seine zentrale Bedeutung in sozial- und kulturwissenschaftlichen Feldern erfahren, nicht ohne Berührungen mit der (post-)strukturalistischen Richtung einzugehen. Bereits angesichts dieser Ausdifferenzierung muss gefragt werden, ob es ausreichend ist, Cultural Studies vordergründig - wie oftmals beobachtbar - als kontextualistisch, interventionistisch, inter- bzw. transdisziplinär und schließlich selbstreflexiv zusammenzufassen, ohne aber die kultur- und sozialtheoretische Frage- und Problemstellung als Ausgangspunkt $\mathrm{zu}$ benennen, in der die genannten theoretischen Richtungen ihre analytische Bedeutung entfalten und ihren kritischen Stellenwert erfahren.

Die Herausforderung durch die Cultural Studies besteht nämlich keineswegs in deren radikaler Kontextualität, sondern diese ist mit ihrem spezifischen Erkenntnisinteresse gegeben, kulturelle Praktiken in ihrer Beziehung und Begrenzung durch soziale Strukturen und Prozesse zu begreifen, wobei dieses Verhältnis insbesondere anhand von kulturellen Repräsentationen analysiert und thematisiert wird, wodurch das bearbeitete Problem in Abhängigkeit eines spezifischen theoretischen und damit perspektivischen Zuschnitt konzeptualisiert wird. Dieser Perspektivierung wird durch das Konzept der Artikulation entsprochen, was nicht nur zu jeweils eigenständigen Thematisierungen und Problemzugriffen führt, sondern jeweils auch methodische Entscheidungen verlangt. Die Frage nach einer Epistemologie der Cultural Studies in kulturwissenschaftlicher Absicht erscheint somit als notwendig, um die Besonderheit des Projekts im Spannungsfeld von kritischer Sozialforschung und Kulturwissenschaft zu erfassen.

Vorweg sei daher bereits hier und entgegen den ebenfalls oftmals anzutreffenden Festlegungen gesagt, dass der Gegenstand der Cultural Studies gewiss nicht Kultur per se ist, egal ob im anthropologischen oder semiotisch-strukturalistischen Sinn, oder gar Kultur in ihrer Beziehung zur Macht. Mit einer solchen Ausrichtung eignen sich Cultural Studies bestenfalls zu einer neuen Form der Kulturkritik, aber nicht zu einem (wissenschafts-)politischen Projekt, als das sie sich in Fortentwicklung ihrer marxistischen, genauer materialistischen Wur- 
zeln zu verstehen geben. Das Interesse gilt den am Aufbau einer kulturellen und sozialen Ordnung spezifischer Gruppen beteiligten Rahmenbedingungen, die sich in ihren Repräsentationen ausdrücken. Gegenüber dieser Ausrichtung ist die in der deutschsprachigen Cultural Studies-Rezeption anzutreffende Konzentration auf die ethnographische Medienforschung durchweg als Vereinseitigung zu betrachten, als deren Folge eine umfassende Bewertung der Cultural Studies zusehends schwer fällt. Denn die forschungspolitische Frage der Cultural Studies handelt davon, wie die »Leute von den besonderen Strukturen ihres Alltagslebens und den verschiedenen Widerständen und Mächten, denen sie dabei begegnen - sowohl ökonomischer als auch politischer Provenienz -, entmündigt oder ermächtigt werden und ferner wie sie selbst ihre Situation auslegen, darstellen, begreifen und zum Ausdruck bringen. Diese Perspektive, die auf den Kreislaufprozess kultureller Produktion und Reproduktion gerichtet ist (vgl. du Gay/Hall et al. 1997), verspricht den hierzulande geführten kulturwissenschaftlichen Diskurs mit einer Konzentration auf die Analyse gegenwärtiger sozialer und politischer Prozesse zu erweitern. Erwartbar wird auch eine Kritik der Differenzlosigkeit des Individualisierungstheorems, in dessen aktueller Anwendung Macht- und Herrschaftsprozesse kaum mehr behandelt werden.

Im vorliegenden Text wird es darum gehen, den theoretischen und methodischen Hintergrund dieses als Fortentwicklung der materialistischen Theoriebildung sich verstehenden Strangs der Kulturund Gesellschaftsanalyse auszuleuchten und seine Bedeutung im kulturwissenschaftlichen Diskurs zu ergründen. Dazu sollen in einem ersten Schritt die zentralen, in der internationalen Diskussion anzutreffenden Auffassungen der kultur- und sozialwissenschaftlichen Ausrichtung der Cultural Studies vorgestellt werden. Ausgehend von Positionsbestimmungen einzelner Cultural Studies-Vertreter im internationalen Kontext, werde ich das Spezifische anhand von Selbstbeschreibungen herausstellen (Kap. 2). Anschließend (Kap. 3) gehe ich auf die Ausdifferenzierung der marxistischen und strukturalistischen Theorierichtung ein, wobei es um die Genese des für die Cultural Studies kennzeichnenden Erkenntnisinteresses geht. Abschließend (Kap. 4) werde ich auf die Rolle und Bedeutung der Cultural Studies für die Theoretisierung von Fragen des gesellschaftlichen und kulturellen Zusammenhangs eingehen und diskutieren, wie sie eine Erweiterung der kulturwissenschaftlichen Perspektive bieten. ${ }^{\mathrm{I}}$ 


\section{Cultural Studies: Ein Kampf um Positionen?}

Es gehört zu den Eigenheiten kritisch-theoretischen Denkens, dass die Theoriegeschichte nicht von einer spezifischen theoriepolitischen Ausrichtung oder Denkstrategie, die über Leitideen verfügt, zu trennen ist. ${ }^{2}$ Diskussionswürdig und oftmals sogar strittig sind jedoch die Zuschreibungen und Einteilungen, die bei der Nachzeichnung oder Kritik eines wirkungsvollen Ansatzes gerade auch in der Rezeption erfolgen. In der anglo-amerikanischen Cultural Studies-Debatte trifft man wegen der Vielzahl an Strömungen, die sich in den letzten vier Jahrzehnten herausgebildet haben, auf teilweise sich widersprechende Klärungsversuche, die die Rezeption mitunter erschweren. John Hartley hat die aus dieser Uneinheitlichkeit erwachsende Situation - von der beobachtbare »Definitionsversuche« der Cultural Studies betroffen sind - folgendermaßen zugespitzt:

"Cultural Studies fallen wegen der Empfindlichkeit ihrer Anhänger gegenüber jeder Orthodoxie auf, die sich in einer bewussten interdisziplinären Haltung und einer ebenso beabsichtigten Vermeidung von Autorität ausdrückt; es gibt keine einheitliche Theorie, keinen Kanon an Grundlagentexten, keine ausschließliche Disziplin, keine gemeinsame Methode, keine gemeinsame Begrifflichkeit, keinen abfragbaren Inhalt und keine geschlossene Lehrmeinung, eigentlich überhaupt keine Geschlossenheitu (Hartley 1992: 17).

Der aus dieser Offenheit notwendigerweise erwachsenden Problemstellung für die Rezeption ist von unterschiedlicher Seite durch die Bezugnahme auf offensichtlich dennoch bestehende, aber anscheinend verdeckte theoriepolitische Leitlinien begegnet worden. Eine der deutlichsten Positionsbestimmungen findet sich in der Aufforderung Tony Bennetts, die er in seinem Aufsatz »Putting Policy into Cultural Studies« (I992) umrissen hat. Die bereits an dem Aufsatztitel erkennbare Forderung, die politischen Dimensionen kultureller Praxen und ihre offensichtliche Einbindung in Machtbeziehungen $z u$ thematisieren (Bennett I992: 23), wäre bis Mitte der I980er Jahre nicht nur ungewöhnlich, sondern von den meisten Cultural Studies-Vertretern auch mit Unverständnis aufgenommen worden. Schließlich war man nicht nur dem eigenen Selbstverständnis nach per se politisch, sondern stand in einer (Ahnen-)Reihe von (theorie-)politischen Auseinandersetzungen. Als eine Folge theoretischer Reorientierungen - vor allem als Folge der internationalen Ausweitung der Cultural Studies und 
ihrer akademischen Institutionalisierung in den Vereinigten Staaten sieht aber nicht nur Bennett die Gefahr, die aus der damit entstandenen »Elastizität des Gebrauchs « der Cultural Studies erwachsen ist (Bennett 1992: 23). Angela McRobbie betont fast gleichlautend das Problem, »dass wir unter Billigung des Pluralismus alle unsere eigenen Wege gehen und dass schlechterdings jeder Untersuchungsgegenstand als legitim angesehen wird, der nachweislich kulturellen Gehalt besitzt« (McRobbie 1995: I09).

Die in den aufgeführten Einwänden verstärkt laut werdende Kritik an der Fortentwicklung der Cultural Studies in den I990er Jahren scheint von einem bestimmten theoriepolitischen Verständnis auszugehen, das mit der aktuell dominanten poststrukturalistischen Schwerpunktsetzung anscheinend verfehlt wird. Lässt sich aber ein theoriepolitischer Orientierungsrahmen für die Cultural Studies einkreisen, der dieser Schwerpunktsetzung entgegensteht, und wie wäre durch eine wie auch immer geartete (Wieder-)Verankerung an einen solchen Rahmen das aktuelle Problem zu lösen? Oder anders gefragt: Zeigt nicht die Geschichte der Cultural Studies als eine Geschichte wechselvoller theoretischer Verschiebungen, dass es bei diesen Einwänden um Grundsätzlicheres geht, als es der Hinweis auf die Gefahr des Relativismus und Pluralismus anzudeuten vermag (vgl. Göttlich I999a).

Einen Schritt näher kommt man der Beantwortung dieser Frage mit Blick auf die von vielen Vertretern geteilte Vorstellung der Cultural Studies als einem Projekt mit unterschiedlichen Formationen (vgl. insb. Williams 1989b: 15I). Als Formationen gelten nicht die Fächer, Studienangebote und Studiengänge, die international unter dem Label »Cultural Studies« eingerichtet wurden. Formationen sind vielmehr Netzwerke, die aus Personen und der Verbindung von Texten und Personen zu bestimmten Zeitpunkten und über Fachgrenzen hinweg zu bestimmten Forschungsthemen bestehen (vgl. Göttlich/C. Winter I999: 25). Die Herausgeber des für die Cultural Studies-Debatte in den I990er Jahren maßgeblichen Vortrags- und Diskussionsbandes Cultural Studies (Grossberg et al. I992) entfalten daraus folgende Perspektive:

"Eine Reihe der (bisherigen) Anstrengungen, das Cultural Studies-Projekt zu definieren oder herzuleiten, ist hilfreich, die Diversität von Positionen und Traditionen, die diesen Namen beanspruchen, zu kartieren. Vor dem Hintergrund dieser Anstrengungen lässt sich 
zusammenfassend sagen, dass Cultural Studies ein interdisziplinäres, transdisziplinäres und manchmal auch gegendisziplinäres Gebiet bezeichnen, das im Spannungsverhältnis seiner beiderseitigen Anstrengungen, einen breiteren anthropologischen als auch einen engeren humanistischen Kulturbegriff zu umfassen, operiert. [...] [Im] Gegensatz zum traditionellen Humanismus weisen sie die Gleichsetzung von Kultur mit Elite- bzw. Hochkultur zurück und betonen, dass alle Formen kultureller Produktion in ihrem Verhältnis zu kulturellen Praxen wie zu sozialen und historischen Strukturen untersucht werden müssen. Cultural Studies befassen sich mit der Analyse des gesamten Spektrums von Kunst und Ideengebäuden (Glaubensvorstellungen) einer Gesellschaft, ihren Institutionen und ihren kommunikativen Praxen« (Nelson et al. 1992: 4).

Diese Perspektive verweist bereits auf den Spielraum, den die einzelnen Formationen bei der Analyse kultureller Phänomene beanspruchen und beansprucht haben, um sich in dem Projekt zu verorten, ohne bereits im Vorhinein auf bestimmte Methoden und Theorien festgelegt zu sein. Zugleich wird deutlich, dass eine Definition in direktem Widerspruch zum Selbstverständnis dieser Forschungsorientierung stünde, weil eine solche an den in den einzelnen Formationen gewählten Forschungsgegenständen vorbeizielte. Die Reichweite und Rolle des für die Cultural Studies maßgeblichen anthropologischen, auf die ganze Lebensweise (whole way of life) zielenden Kulturbegriffs, der den spezifischen Ausgangspunkt der materialen Kulturanalyse in den I95oer Jahren mitbegründete, bestimmt Stuart Hall dahin gehend, dass die Analyse damit dennoch nicht auf Kultur per se orientiert ist: Es geht in diesem Kulturbegriff darum, die »Kultur eher im Hinblick auf ihre Beziehung zwischen einer sozialen Gruppe und den Dingen, die deren Lebensweise ausdrücken« (Hall I977: 55), zu betrachten, »als im Hinblick auf die Dinge selbst - also nicht das Bild, der Roman, das Gedicht, die Oper, sondern die Beziehung zu der sozialen Gruppe, deren Leben sich in diesen Objekten widerspiegelt« (ebd.).

Mit dieser Unterscheidung ist nicht nur das Schlaglicht auf das prozessuale Kulturverständnis geworfen, von dem aus Gesellschaft als Resultat von Lebensprozessen begriffen wird, sondern ebenfalls die Besonderheit des Artikulationskonzepts vorgezeichnet, das den theoretischen Hintergrund des vor allem methodisch nicht unproblematischen Konzepts der radikalen Kontextualität bildet (vgl. Grossberg I994: 2I). Diese und weitere dann unmittelbar damit verknüpften Kritikkonzepte, wie der Anti-Reduktionismus, der Anti-Essenzialismus sowie die Selbstreflexivität (ebd.: 22 f.), haben im Zuge der theo- 
riepolitischen Entwicklungen oftmals eine unterschiedliche Gewichtung und auch externe Einschätzung erfahren, die das eigentliche theoretische Problem, die Frage nach der Reichweite des Artikulationskonzepts zur Analyse kultureller und sozialer Prozesse, überlagert haben. Vor dem Hintergrund dieser Überlagerung der theoretischen und methodischen Besonderheiten durch den Kulturbegriff sowie die besagten Schlagworte des »Anti-...« hat Morley darauf hingewiesen, dass für Außenstehende eine alleinige Annäherung über den spezifischen und unzweifelhaft wichtigen Kulturbegriff unbestimmt bleiben muss, wenn nicht seine theoretische Einbindung berücksichtigt wird, die darauf verweist, dass

wzu unterschiedlichen Zeiten und an unterschiedlichen Orten die gleichen Dinge nicht auch die gleiche Bedeutung (den gleichen Stellenwert) haben, und das ist die Gefahr, der jede unangebrachte universalisierende Anstrengung innerhalb der Cultural Studies zum Opfer fallen wirdı (Morley 1992: 3).

Unter das Dach einer Formation gelangt man anscheinend nur, wenn man die spezifische Genese ihrer Theoriebildung, die zusammen mit dem Kulturbegriff die Klammer des Projekts ausmacht, berücksichtigt. Aber reicht es dazu aus zu sagen, dass der Kulturbegriff oder das spezifische der Kulturauffassung der Cultural Studies historisch herzuleiten und theoretisch zu gewichten ist, d.h., dass die Modelle kontextuell adaptiert und (re-)formuliert werden müssen? Was aber heißt das vor dem Hintergrund des bislang gewonnenen Eindrucks, dass die Cultural Studies kein abgeschlossenes oder endgültiges methodisches und theoretisches Konzept der Kulturanalyse beherbergen? Können sie mit ihren Zugängen auf eine Theorie von Kultur und Gesellschaft hinarbeiten, oder weisen sie durch ihren Zuschnitt vielmehr auf die Grenzen einer interdisziplinär angelegten kritischen Gesellschaftstheorie in der Spätmoderne hin? Reagieren sie also gleichsam mit ihrem theoretischen und methodischen Gefüge auf die aktuellen Probleme kulturwissenschaftlicher Theoriebildung und worin besteht die durch sie eingebrachte Erweiterung?

Wer ihren Zuschnitt allzu schnell aus der Postmodernediskussion oder den besagten oberflächlichen Zugriff auf die die Cultural Studies angeblich charakterisierenden Schlagworte ableitet, braucht sich diese Fragen kaum zu stellen bzw. wird eine schnelle Antwort bereit halten. Die hier aufgeführten Positionsbestimmungen zeichnen sich an 
vielen Stellen aber gerade in ihrer kritischen Haltung gegenüber der Postmoderne und ihren theoretischen Konsequenzen aus, was in der poststrukturalistischen Hausse gerne auch übersehen wird. Im Grunde geht es um das Verhältnis der Theoriestränge, die entweder näher zur Birminghamer Tradition stehen, und jenen Strängen, die sich in der amerikanischen Rezeption herausgebildet haben.

Gegenüber dieser erst in Ansätzen stattfindenden Auseinandersetzungen (vgl. Grossberg et al. 1992) führt eine weitere Frage überraschenderweise unmittelbarer zum Verständnis der Cultural StudiesPerspektive und ihrer gesellschaftskritischen Richtung. Eine Einschätzung des Stellenwerts der Cultural Studies und ihrer aktuellen theoriepolitischen Auseinandersetzung ist möglich, wenn man sich das Erkenntnisinteresse in Form einer Fragestellung verdeutlicht. Diese lautet: »Wessen Cultural Studies? «3 (vgl. Göttlich/C. Winter 1999). Die Genese dieser Fragestellung ist im Zusammenhang mit dem von Stuart Hall reflektierten Verhältnis von akademischer und intellektueller Arbeit im Zuge seiner Auseinandersetzung mit Gramscis Diskussion des organischen Intellektuellen zu sehen. Konkret geht es um das Begründungsproblem, »intellektuelle und theoretische Arbeit als politische Praxis zu entwickeln « (Hall I992: 28I). Hierzu hebt Hall auf den prinzipiellen Unterschied zwischen intellektueller und akademischer Arbeit ab, woraus sich ein für die Sozialkritik der Cultural Studies unerlässliches Spannungsverhältnis ergibt, in dem sich die Kritik begründet. Dazu hält Hall fest:

"Wenn man diese Spannung verliert, lässt sich durchaus intellektuell anspruchsvoll arbeiten, aber um den Preis des Verlustes politischer intellektueller Praxis. [...] Ich denke, es ist genau das, was die Cultural Studies als Projekt ausmachen. [...] Sie halten theoretische und politische Fragen in einer unauflösbaren, aber andauernden Spannung. Sie erlauben der einen Seite, die andere Seite ständig zu irritieren, zu belästigen und zu stören, ohne auf einer endgültigen theoretischen Schließung zu bestehen« (Hall 1992: 284).

In dieser Positionierung begründet sich nicht nur das Motiv der Einbeziehung verschiedener theoretischer Richtungen, aus deren wechselseitiger Kritik der Erkenntnisgewinn über soziale und kulturelle Veränderungen und Prozesse erfolgen soll. Vielmehr versuchen die Cultural Studies aus diesem Spannungsverhältnis heraus den Rahmen für eine (Wieder-)Entdeckung und Darstellung nicht nur unterdrückter und marginalisierter Stimmen im Kulturprozess als auch im 
Wissenschaftsdiskurs abzustecken, sondern die Erarbeitung neuer Perspektiven auf bislang bekannte Gegenstände zu befördern. Das scheint insbesondere den kulturwissenschaftlichen Diskurs zu irritieren, wenn aus ihm heraus die hier dargestellte Perspektive als »interessegeleitet« kritisiert wird (Böhme et al. 2000: I3). Für den wechselseitigen Diskurs scheint man daher unbedingt an dieser Stelle ansetzen zu müssen, was wiederum ein anderer kulturwissenschaftlich inspirierter Sammelband erkannt hat, der den Faden an dieser Stelle aufnimmt (vgl. BMWV/IFK I999).

Von diesem Zuschnitt ausgehend wird auch die Positionierung Grossbergs deutlich, der die Aufgabenstellung der Cultural Studies darin festmacht, die »Rolle kultureller Praktiken bei der Konstruktion der Kontexte menschlichen Lebens als Machtmilieus « zu analysieren (Grossberg 1994: 23). Unverkennbar können hierin die marxistischen Wurzeln der Cultural Studies festgemacht werden, die in den Diskussionen und Bewegungen der I970er Jahre - durch die Intervention des Feminismus - und die Postmodernediskussion in den I980er Jahren eine Reihe unterschiedlicher Anschlusspunkte mit neuen theoretischen Konzepten gefunden haben. Die im Laufe der weiteren Entwicklung entstandenen theoretischen Öffnungen werden in den I990er Jahren nun mit den eingangs zitierten Positionsbestimmungen von Bennett, McRobbie, Grossberg sowie Hall u.a. zu bewerten und mit einer theoretischen Schließung an das Projekt der Cultural Studies (wieder) anzubinden gesucht.

Vor diesem Entwicklungshintergrund wird ersichtlich, dass die beiden in der internationalen Rezeption oftmals überwiegenden Bilder der Cultural Studies als Alltags- bzw. Populärkulturforschung sowie als Medien- bzw. Rezeptionsforschung nur mögliche Anwendungsgebiete bezeichnen. Im Zuge der theoriepolitischen Verschiebungen in den letzten zwanzig Jahren haben sich die Themen und die behandelten Probleme gesellschaftlicher Gruppen innerhalb der Cultural Studies beständig gewandelt. Mit unterschiedlicher Schwerpunktsetzung stellten und stellen die Kultur der Arbeiterklasse, jugendliche Subkulturen, das Erziehungs- und Schulbildungssystem, die staatliche Macht, die populären Medien und in einem immer stärkeren Maße die Medienkultur sowie Fragen der kulturellen Identität den Gegenstand der Cultural Studies dar. Das Interesse der durch die Arbeiten von Fiske angeregten Forschung galt dabei insbesondere der Frage, wie die politische und kulturelle Hegemonie z.B. über die Medien errichtet und 
aufrechterhalten wird und welche Strategien die »Leute« in Reaktion darauf entwickelt haben.

Die Vielzahl der Themen, denen sich die Cultural Studies vor allem in den letzen Jahren angenommen haben, machen deutlich, dass das Projekt, wie es etwa bei Hall oder Johnson formuliert wurde, mit der Analyse des Verhältnisses von Kultur und Politik, Ökonomie, Macht und Herrschaft »prinzipiell unabgeschlossen ist und einer grundsätzlichen Offenheit für neue Fragen und Probleme« bedarf (Bromley et al. I999: 24). Was das für die forschungspraktische Ausrichtung in kulturwissenschaftlicher Hinsicht bedeutet, werde ich in Kap. 4 nochmals aufgreifen, um ein abschließendes Verständnis der Cultural Studies gerade auch im Verhältnis zur kulturwissenschaftlichen Forschung aufzuweisen. Zur weiteren Einordnung ist jedoch ein Blick auf die Ausdifferenzierung der Cultural Studies notwendig, die das hier beschriebene aktuelle Bild aus der Perspektive der Theoriegenese erklärt.

\section{Cultural Studies in historischer Perspektive}

Als ein verlässlicher Klärungsversuch der Besonderheiten der Cultural Studies kann unzweifelhaft ein historischer Zugang angesehen werden. Zur Annäherung an den historischen Ausgangspunkt bietet sich in besonderer Weise das Werk von Raymond Williams an, da sich mit ihm nicht nur die entscheidenden Grundlagen in der Kulturanalyse verbinden (vgl. Williams I977a, I989, I998). An Williams lassen sich zudem eine Reihe von Aspekten - etwa seine kritische Auseinandersetzung mit dem Marxismus - aufzeigen, die in ähnlicher Form auch für andere Vertreter zutreffen, vor allem für Stuart Hall, der rückblickend zur Rolle des Marxismus herausstreicht, dass es

ınie einen Zeitpunkt gegeben [hat], an dem Cultural Studies und Marxismus eine völlige theoretische Übereinstimmung hatten. Von Anfang an [...] überwogen die Fragen nach den Unzulänglichkeiten, sowohl theoretisch wie politisch als auch, was das mächtige Schweigen zu den Auslassungen des Marxismus anbelangt [...] Die Begegnung zwischen britischen Cultural Studies und Marxismus hat also zunächst mit der Beschäftigung mit einem Problem zu tun [...] Sie beginnt und entwickelt sich über die Kritik an dem bestimmten Reduktionismus und Ökonomismus॥ (Hall 1992: 279). 
Die Besonderheit von Williams' Werk ergibt sich - wie an anderer Stelle von mir weiter ausgeführt - aus einer Art Stellvertreterfunktion, anhand der die von unterschiedlichen Personen zu unterschiedlichen Zeiten geleistete theoretische Arbeit in den Cultural Studies verdeutlicht werden kann (vgl. dazu ausführlich Göttlich I996). Gegenüber den zahlreichen und für ein deutsches Publikum überwiegend nur verstreut vorliegenden Beiträgen der unterschiedlichen Vertreter des Cultural Studies Approach zu kulturtheoretischen Problemen erlaubt es Williams' Werk zudem, die verschiedenen Punkte der früheren Theoriebildung exemplarisch im Zusammenhang zu verfolgen. Das gilt auch für die spätere Verarbeitung der kontinentalen marxistischen Einflüsse, die in die zweite Werkphase ab den I970er Jahren gehört. Wesentlich ab dieser Phase datiert die Differenzierung in unterschiedliche Strömungen des British Cultural Studies Approach und der beginnenden amerikanischen Entwicklung, die für die poststrukturalistische Ausweitung des Projekts steht. ${ }^{4}$

\section{I Die Rolle des linken Kulturalismus}

Die von Raymond Williams ab Mitte der 5oer Jahre mit seinen beiden Büchern Culture and Society (I958) und The Long Revolution (I96I) eingeleitete und mitgetragene kulturtheoretische Neuorientierung, die zu den Cultural Studies geführt hat, wurde wesentlich durch die in der unmittelbaren Nachkriegszeit aufkommenden Probleme, die sich mit dem wachsenden Einfluss der Massenmedien wie der neuen Rolle und Funktion massendemokratischer Entwicklungen und Organisationen ergaben, angestoßen. Es handelt sich dabei um Entwicklungen der britischen Nachkriegsgesellschaft, für die die konservativen, aus der englischen Tradition der Kulturdebatte herrührenden und fortwirkenden Theorieansätze für die Generation junger, zunehmend aus der Arbeiterklasse stammenden »Jungakademiker « keine Erklärungskraft mehr hatten.

Ganz entscheidend gehört zu den Cultural Studies der Emanzipationskampf der Arbeiterklasse, der sich in einer Ablehnung des Elitekulturbegriffs und in der selbstbewussten Betonung der eigenen gelebten Kultur ausdrückt, die auch dem als negativ bewerteten Einfluss der Massenkultur - man kann auch sagen der Kulturindustrie - entgegengesetzt wurde. Der diese Auseinandersetzung prägende Kulturbegriff findet sich am deutlichsten in Williams Werk wieder. Denn es 
war seine, für die folgende Entwicklung maßgebliche begriffliche Leistung, ${ }^{5}$ Kultur nicht allein auf Literatur, Theater oder Malerei zu beziehen, sondern als eine ganze Lebensweise, als a whole way of life im anthropologischen Sinne - einzuführen. Dieser Schritt erklärt sich vor allem aus seiner Kritik an dem konservativen Kulturverständnis, das die Populär- bzw. Massenkultur aus der Perspektive der minority culture kritisierte, Kultur aber nicht als Lebensweise einer Gruppe verstand. Stuart Hall fasst die zentrale Bedeutung und Rolle von Raymond Williams' The Long Revolution (I96r) für die Kulturdebatte im England der 5oer und Goer Jahre sowie für die Entwicklung des Centre for Contemporary Cultural Studies (CCCS) in Birmingham folgendermaßen zusammen:

"[The Long Revolution] [...] verschob die gesamte Auseinandersetzung von einer literarisch-moralischen zu einer anthropologischen Kulturauffassung. Diese wurde unter Einbeziehung der Art und Weise, in der Bedeutungen und Definitionen sozial und historisch konstruiert sind, als igesamter Prozessi definiert. Kunst und Literatur bildeten nurmehr eine privilegierte Form gesellschaftlicher Kommunikation« (Hall 1980: 19).

Williams' Stärke in dieser Zeit beruht unzweifelhaft darauf, in dieser Auseinandersetzung auf die Durchsetzung einer eigenständigen Perspektive auf die kulturelle Entwicklung hingearbeitet zu haben. Einer Perspektive, die Kultur mit einer ganz bestimmten politischen Zielrichtung als zusammengehörigen Prozess begreifbar machen sollte und so die Erforschung der Alltags- und Populärkultur einleitete. Eine Erforschung der Alltagskultur, die sich nicht gegenüber einem Elitekulturbegriff zu verteidigen haben sollte oder aus einer solchen Perspektive eine Kritik der Populärkultur forcierte, sondern eine Analyse von Macht- und Herrschaftsprozessen, die sich in der Populärkultur beobachten lassen, anstrebte, um die Tendenzen und Kräfte zu stärken, die auf eine Emanzipation und Selbstbestimmung der unteren Schichten mit ihrer Kultur zielen.

Die Bedeutung und Rolle der begrifflich und sozialtheoretisch zwar nicht unproblematischen, für Williams' Kritik jedoch zentralen Formel der culture as a whole way of life, zu der weitere Begriffe wie der von Paul Willis weiter verwandte der common culture (vgl. Williams I958a) oder culture is ordinary (vgl. Williams I958b) gehören, ist $\mathrm{zu}$ einem Großteil allein aus der Reaktion auf diese Zeitumstände und die konservative Kulturkritik erklärbar, mit der die New Left in Ausein- 
andersetzung stand. Diese Reaktion erfolgte im Kontext der kulturkritischen Auseinandersetzung im England der i950er und i96oer Jahre, auf deren einen Seite die Vertreter der sich formierenden New Left ${ }^{6}$ den auf der anderen Seite operierenden Vertretern der kulturkonservativen Richtung - mit F.R. Leavis an deren Spitze - gegenüberstanden (vgl. Leavis I930; Leavis/Thompson I933).

Konsequent hat Williams dieser elitären Position mit seinem Kulturbegriff der »culture as a whole way of life« und dem dazugehörigen Konzept der »Gemeinschaft einer Kultur« die Idee einer demokratisch partizipativen Kommunikationsgemeinschaft entgegengehalten. Williams' Idee der Gemeinschaft einer Kultur erscheint hier als Demonstration der Möglichkeit vollkommen neuer kultureller und gesellschaftlicher Beziehungen, vor allem wenn er an die Möglichkeiten des damals noch nicht so alten Mediums Kino oder gar an die zukünftigen Möglichkeiten des Fernsehens dachte. An dieses Konzept nun schloss eine Fülle materialer Kulturanalysen an, die bis heute unter »Cultural Studies« firmieren. Für die späten ı96oer und die I970er Jahre prägend sind schließlich die Arbeiten, die am und im Umfeld des CCCS Birmingham entstanden sind - woher sich der bedeutende britische Einfluss in den internationalen Cultural Studies bis heute erklärt. Für die Bedeutung des von Williams geebneten kulturbegrifflichen Weges ist noch ein weiterer Aspekt zentral, der unmittelbar zu den kommunikations- und medienwissenschaftlichen Analysen als einem Zweig der Cultural Studies hinüberweist.

Die von verschiedenen Vertretern des Cultural Studies Approach herausgestellte anthropologische Bedeutung des Kulturbegriffs beruht neben der Konzentration auf den gesamten Kulturprozess zusätzlich auf der von Williams herausgearbeiteten Rolle der schöpferischen Aktivität und der Kommunikation für die Reproduktion menschlicher Gemeinschaft(en). Im letzten Abschnitt des Kapitels über das »Schöpferische « in The Long Revolution gibt Williams einen zusammenfassenden Überblick über die aus der Beziehung von Kunst, Kommunikation und Gemeinschaft als zentrale Elemente seines Kulturverständnisses ableitbaren Bedingungen für die weitere Theoriebildung:

"Die menschliche Gemeinschaft wächst durch die Entdeckung gemeinsamer Bedeutungen und gemeinsamer Kommunikationsmittel. [...] Kommunikation ist in der Tat ein Gemeinschaftsprozess: das Teilen gemeinsamer Bedeutungen sowie gemeinsamer Handlungen und Ziele; die Bereithaltung, Entgegennahme und der Vergleich neuer Bedeutungen führt zu 
Spannungen und leitet so Wachstum und Wandel ein. Es ist ungeheuer wichtig, Kommunikation als umfassenden Gesellschaftsprozess (as a whole social process) zu verstehen« (Williams 1961: $38 \mathrm{f}$.).

Erst im Zusammenhang mit dieser für die Theoriebildung grundlegenden Erkenntnis wird die Frage der Vermittlung des Gesellschaftsprozesses mit den einzelnen kulturellen Produkten, Kunstwerken und weiteren kulturellen Artefakten als Ausdruck einer ganzen Lebensweise wichtig. Kultur beinhaltet also nicht nur kulturelle Objekte oder bezeichnet das akkumulierte Wissen, sondern ist soziale Praxis einschließlich der Sinn und Erfahrungsebene, die symbolisch repräsentiert sind. Im direkten Anschluss an diese theoretische Ausrichtung versteht sich Williams' späterer Ansatz des kulturellen Materialismus (I977a) als eine Theorie der Besonderheiten kultureller Produktion, die zur Analyse und Beschreibung gesellschaftlicher und kultureller Prozesse einen spezifischen, in der materialistischen Theoriebildung nach Williams' Auffassung vor allem in kritischer Abgrenzung zum Basis-Überbautheorem bis dahin nicht formulierbaren Beitrag leisten will.

Die Zielsetzung des kulturellen Materialismus ist es, der kulturellen Produktion eine mit der materiellen Basis, also der Produktion vergleichbare Rolle im Prozess der gesellschaftlichen Reproduktion zukommen zu lassen. Die damit verfolgte Thematisierung von Fragen der symbolischen kulturellen Ordnung findet sich dann ebenfalls in der für die Cultural Studies maßgeblich werdenden Strukturalismusrezeption, die zu neuen theoretischen Modellen führte und bis heute in der Verbindung von Postmarxismus und Poststrukturalismus nachwirkt. Die Einbindung sprachtheoretischer Überlegungen in die Theoriebildung erfolgte bei Williams jedoch schon einige Zeit früher (Hall I992: 283). Das Hauptinteresse der Kultur- und Gesellschaftsanalyse verlagerte sich schließlich mit dem Strukturalismus auf die die kulturellen Erfahrungen leitenden bzw. determinierenden semiotischen, linguistischen, textuellen (ideologischen) Strukturen. Diese Rezeption war zwar noch einen längeren Zeitraum marxistisch und materialistisch geprägt, ebenso wie die kulturalistische Phase eine Kritik am Marxismus leistete. Die Rezeption Althussers und LeviStrauss' wurde aber durch die Rezeption Foucaults und Derridas abgelöst, womit die materialistische An- und Rückbindung des Struktura- 
lismus im Zeichen des Postmarxismus (vor allem im US-amerikanischen Raum) weitestgehend obsolet wurde.

\subsection{Cultural Studies und die strukturalistische Ausrichtung}

Ohne die von dem neuen Paradigma ausgehende Entwicklung der Cultural Studies in ihrem vollen Umfang vorausahnen zu können, hatte Stuart Hall bereits 198I in dem Aufsatz »Cultural Studies: Two Paradigms « (vgl. auch Hall 1999) eine kritische Bestandsaufnahme der theoretischen und methodischen Möglichkeiten mit Bezug auf das kulturalistische und strukturalistische Paradigma geliefert.

Den kulturalistischen und strukturalistischen Strängen kommt nach Hall für die materiale Kulturanalyse eine gleichgewichtige Bedeutung bei. Wichtig war zunächst, dass beiden Strängen der radikale Bruch mit der Basis-Überbau-Konzeption gemeinsam ist, die bis weit in die I970er Jahre hinein noch als Orientierungspunkt für eine materiale Kulturanalyse galt. Stattdessen schrieben Kulturalisten und Strukturalisten - Letztere vor allem mit Bezugnahme auf Althusser den bislang dem Überbau zugeordneten Bereichen eine der Basis vergleichbare Wirksamkeit und ein ebensolches konstitutives Primat bei, wodurch die gesellschaftliche Vermittlung auf neue Art theoretisierbar wurde. Durch den Strukturalismus wurden nicht nur sprachwissenschaftliche Fragen in der Kulturanalyse relevant, sondern auch Aspekte der Textanalyse und das Verständnis der Vielgestaltigkeit von Bedeutungspraxen.

Maßgeblicher als die Gemeinsamkeiten wurden schließlich aber die aus dieser theoretischen und methodischen Erweiterung sich entwickelnden Hauptunterscheidungspunkte, die auch für die heutigen Cultural Studies-Projekte fortbestehen, aus denen sich bestimmte Folgerungen für die weitere theoretische Ausrichtung und die Aneignung neuer Theorieelemente herleiten. Um es mit den Worten von Richard Johnson zu sagen:

„Mitten durch die Cultural Studies verläuft eine große theoretische und methodologische Teilungslinie. Auf der einen Seite dieser Linie befinden sich diejenigen, die darauf bestehen, dass «ulturens als ganze Erscheinungen und in situ, vor Ort, in ihren materiellen Zusammenhängen untersucht werden müssen. [...] Auf der anderen Seite der Trennungslinie finden wir diejenigen Autoren, welche die relative Eigenständigkeit oder die tatsächli- 
che Autonomie subjektiver Zeichensysteme betonen. Hier ist der Ansatz normalerweise strukturalistisch orientiert, aber in einer Form, die der diskursiven Konstruktion von Situationen und Subjekten den Vorrang einräumt. [...] Die bevorzugte Methode besteht in der abstrakten, bisweilen ganz formalistischen Behandlung von Formen, bei der die Mechanismen der Bedeutungserzeugung in sprachlichen, narrativen oder anderen Zeichensystemen aufgedeckt werden« (Johnson 1999: 153 f.).

Die aus dieser »Trennung« resultierenden Hauptunterschiede, aber auch die Schwierigkeiten in der Analyse des Kulturprozesses lassen sich exemplarisch an der Gramsci-Rezeption und der Einbindung des Hegemoniekonzepts verdeutlichen. Im strukturalistischen Paradigma erscheint Hegemonie vornehmlich als ideologisches oder diskurstheoretisches Problemfeld. Im kulturalistischen Ansatz - nun vermittelt über Gramsci - ist Hegemonie materiell, d.h. als Prozess unterschiedlicher und in Beziehung miteinander stehender kultureller Praxen konzipiert, angefangen bei der kulturellen Produktion über die Distribution bis hin zur Rezeption, also ein Kreislaufmodell im Sinne Johnsons (Johnson I999: I48). Die daraus resultierenden Unterschiede in der Analyse kultureller Prozesse lassen sich daher mit Milner bündig folgendermaßen zusammenfassen: »Hegemonie als Kultur ist eine Frage materieller Produktion, Reproduktion und Konsumption, Hegemonie als Struktur ist Fall für textuelle Analyse« (Milner I993: 8I).

Während die kulturalistischen Ansätze also die Erfahrungskategorie und die materiellen kulturellen Praxen in den Vordergrund stellen, stehen in der strukturalistischen Perspektive ideologische, semiotische oder linguistische, d.h. überwiegend textanalytisch zu erschließende Gegenstände im Vordergrund. Dieser scheinbare Gegensatz ist für Halls Frage nach den Folgen für die theoriepolitische Ausrichtung der Cultural Studies in den I980er Jahren von zentraler Bedeutung. Die von ihm eingebrachte theoretische Lösung basiert nicht auf einer harschen Trennung materialistischer und strukturalistischer Perspektiven. Vielmehr geht es ihm um die aus den Unterschieden und Gemeinsamkeiten ableitbaren Stärken und Schwächen, die sich zur Fusion beider Perspektiven, sozusagen aus deren Komplementarität erst ergeben. ${ }^{7}$ Dabei war die gefundene Lösung für die Verbindung beider historischen Stränge theoriearchitektonisch nicht nur höchst riskant - besonders in ihrer Anfangszeit -, sondern auch höchst anspruchsvoll, da »weder der Kulturalismus noch der Strukturalismus« in ihrer gegenwärtigen Gestalt alleine dazu ausreichen, »die Kultur- 
analyse als ein theoretisch durchkonzipiertes Analysefeld auf den Weg zu bringen « (Hall I98r: 30). Die seitdem innerhalb der Cultural Studies zu beobachtende beständige Aufnahme und Integration neuer theoretischer Komponenten - vor allem in den letzten fünfzehn Jahren - wird von dieser Theoriearchitektur angestoßen, die angesichts kulturellen und gesellschaftlichen Wandels darauf zielt, jeweils neue Fragen zu stellen. Nach außen erscheint das als Eklektizismus, der aber eine komplexe theoriegeschichtliche Wurzel hat und jeweils seine theoretische und methodische Einlösung findet. Das geschieht vor allem durch die zu bestimmten Momenten bewusst vorgenommenen theoretischen Schließungen, auf die auch die eingangs zitierten Positionen in der aktuellen Situation der Cultural Studies hinarbeiten.

Wie Halls eigene Arbeiten verdeutlichen, bedarf es zu bestimmten historischen Phasen unbedingt dieser theoretischen Schließungen, damit die »theoretical fluency« (Hall I992: 286) - die theoretische Gewandtheit und Vielstimmigkeit der Cultural Studies -, aktuell vor allem angesichts der postmodernen Differenzierungen, nicht in Beliebigkeit mündet. Hall war es so beispielsweise mit Policing the Crisis (1978) gelungen, eine neue Grundlage für die politische Verortung von Themen wie Kriminalität, Jugend und die Kämpfe der people of colour in den Vorstädten angesichts der sich abzeichnenden gesellschaftspolitischen Krisen zu schaffen. Darüber hinaus markierte diese Arbeit zugleich den Einstiegspunkt in die aktuellen Themen der Cultural Studies im Zusammenhang mit Fragen kultureller Identitäten.

Neben den Leistungen, die sich unzweifelhaft mit den (post-)strukturalistischen Positionen in den letzten Jahren verbinden, ist besonders Halls Hinweis auf einige Leerstellen für die aktuelle Wahrnehmung der Cultural Studies wichtig. Kultur, so stellt er fest, wird uns in diesen Ansätzen in jedem Moment nur durch ihre besondere Textualität gewahr, und doch wissen wir, dass Textualität nicht alles ist (Hall I992: 284). Hall kritisiert an dieser Stelle ein Verständnis von Cultural Studies, das darauf hinausläuft, die Verbindung von Macht und Kultur in jedem Fall nur textuell zu analysieren. Halls Bedenken gegen eine rein textuelle Machtanalyse gründen dabei in den materialistischen Wurzeln der Cultural Studies. Für ihn gibt es genügend Beispiele, in denen die Artikulation von Macht und Kultur unausgesprochen wirkt und es keine Repräsentation dieser Struktur gibt. Diese aus der Theoriearchitektur resultierende Spannung mitzudenken und auszuhalten erachtet er als entscheidende Bedingung, das Projekt der Cultural 
Studies weiter zu verfolgen. Interpretiert man die Problemstellung der theoretischen Schließung und die damit einhergehenden Konsequenzen für die Theoriearchitektur unter erkenntniskritischen Gesichtspunkten, so erkennt man in ihnen auch die Wurzel für den oftmals spezifischen Problemzugriff, der in den unterschiedlichen Entwicklungsphasen des Projekts der Cultural Studies aus der Anwendung und Kombination von zum Teil konträren theoretischen Ansätzen resultiert.

Gerade da die Cultural Studies wegen dieser Verortung und der daraus gleichfalls resultierenden methodischen Uneinheitlichkeit die Kritik auf sich ziehen, sie korrumpierten mit ihrem Anspruch einen für sich bestehenden Erkenntnisapparat, dem Objektivität zu verdanken ist, gilt es an dieser Stelle für die Einschätzung ihrer Rolle im kulturwissenschaftlichen Diskurs die Frage nach Erkenntnis und Interesse erneut zu stellen. Dies soll im Folgenden über eine Annäherung an das Konzept der Artikulation geschehen, mit dem sich diese Frage an der herausragenden erkenntnisleitenden Perspektive der Cultural Studies behandeln lässt.

\section{Eine Epistemologie der Cultural Studies?}

Denkt man an die politische und intellektuelle Bewegung der I950er und Goer Jahre, auf die die Formierung der Cultural Studies zurückgeht, so erklärt sich ihre kulturanalytische Perspektive, die auf die jeweiligen historischen Situationen und Möglichkeiten der Veränderungen des Alltagslebens bezogen ist. Die These der abschließenden Ausführungen ist, dass bereits dieses für die Cultural Studies grundlegende Erkenntnisinteresse einen Unterschied zu kulturwissenschaftlichen Forschungsproblemen begründet - nicht zuletzt auch, weil es sich in die Tradition kritischer Sozialforschung einschreibt -, von dem zentrale Impulse zur Analyse der gegenwärtigen kulturellen und gesellschaftlichen Entwicklung zu erwarten sind.

Zentral für das in aktuellen Cultural Studies-Analysen zur Anwendung kommende theoretische Konzept der Artikulation ist, dass es von einem materialistischen Verständnis der Rolle und Funktion von Produktionsweisen (modes of production) ausgeht, diese aber nicht in einem orthodox-materialistischen Sinne wirksam sieht. Es geht 
nicht um die Ableitung von Folgen, sondern um ein Verständnis von Kontexten und Formationen, in denen eine bestimmte Verbindung (conjuncture) materieller und ideologischer Gegebenheiten die (strukturalen) Bedingungen für gesellschaftliche und kultureller Praxen bilden. »Anders ausgedrückt, die Formation selbst stellt den unterschiedlichen Gruppen mit ihrem jeweils anderen Verhältnis zur Formation eine Reihe unterschiedlicher Positionen zur Verfügung « (Grossberg I999: 2I8), die es jeweils in ihrer eigenen Ausprägung und in ihrem Verhältnis zueinander zu bestimmen gilt.

Wie John Hartley (O'Sullivan et al. I994: I7 f.) ausführt, geht es um die Analyse besonderer historischer Konfigurationen oder Formationen, die die strukturalen Bedingungen sozialer Praxen, Ereignisse oder kultureller Zeugnisse, wie z. B. Texte, hervorbringen, was angesichts globaler Differenzierungsprozesse ein Erkenntnisanspruch ist, den die geisteswissenschaftlich orientierte Kulturwissenschaft mit ihrem Anspruch auf Kanonisierung (Böhme et al. 2000: I3) - der hier als gegen die Cultural Studies gerichtet in Anschlag gebracht wird - so nicht einlösen kann. Daher stellt sich auch hier erneut die Frage, ob anstelle einer solchen Grenzlinie, die von kulturwissenschaftlicher Seite nicht zuletzt auch wegen der Gewinnung wissenschaftsdisziplinärer Eigenständigkeit vollzogen wird, nicht eine künstliche Abgrenzung vorgenommen wird, die das Spannungsverhältnis vertieft.

In Halls Kritik kommt dem Konzept der »Artikulation« nicht von ungefähr eine zentrale - epistemologische, theoriepolitische und strategische - Rolle zu. Beschreibt und umschließt es doch jenes Korrespondenz- bzw. Vermittlungsverhältnis im (angelsächsischen) Doppelsinn von speaking einerseits und jointing oder connecting andererseits und bildet sozusagen das theoretische Äquivalent für die Realisierung der radikalen Kontextualität, die auch jeweils methodische Entscheidungen fordert, wenn, wie u. a. Hall formuliert hat, »die Form einer Verbindung, die aus differenten Elementen - unter bestimmten Bedingungen - eine Einheit macht« (Grossberg 1996: I4I) aufgeschlossen werden soll. Zumal es sich um Verknüpfungen handelt, »die nicht notwendig, determiniert, absolut oder essenziell für alle Zeiten [sind]《 (ebd.). Daher gilt es zu fragen, »unter welchen Umständen [...] eine Verbindung geschmiedet oder gemacht werden [kann] « (ebd.) und wie man ihr in der Empirie nachkommt. In anderen Worten ausgedrückt, bedeutet das: 
"Was in den Cultural Studies artikuliert werden kann, sind [...] groß angelegte soziale Kräfte (besonders Produktionsweisen) in ihrer zu einer bestimmten Zeit gegebenen besonderen Konfiguriertheit oder Formation, also eine Zusammensetzung, die die strukturellen Determinanten einer bestimmten Praxis, eines Textes oder eines Ereignisses darstelltı (0'Sullivan et al. 1994: 17).

Wendet man dieses Konzept, zum Beispiel wie Grossberg (I999: 233), auf die Analyse der zeitgenössischen (Populär-)Kultur an, dann erscheint diese als Artikulation von Beziehungen, die das Populäre zunächst einmal historisch in sehr unterschiedlichen Formen und Gebieten konstituiert haben. Dazu gehören Beziehungen zu so unterschiedlichen Feldern wie Arbeit, Religion, Moralität und Politik, die zunächst einmal artikuliert werden müssen, um zu belegen, dass die Kategorie des Populären nicht auf die gleiche Art und Weise in jeder historischen Situation zu finden ist oder dass es unwandelbare Formen populären Vergnügens oder gefühlsmäßiger Einstellungen gibt. Das Populäre kann nur dann historisch verstanden werden, wenn es als Artikulation bestimmter Haltungen oder (politischer) Einstellungen, die sich u.a. in Stilen (Repräsentationen) ausdrücken und auf einer bestimmten Mittelwahl beruhen, gedacht wird. Dabei überlagern sich in einer Kultur neue Artikulationen des Populären mit älteren. Zusammen bilden sie den Kontext der Populärkultur. Es gibt jedoch keinen notwendigen Grund, dass z. B. die affektiven Haltungen beim populären Vergnügen primär auf dem Terrain der kommerziellen Populärkultur anzutreffen sind. Aber es ist natürlich so, dass hier für die breite Mehrheit der in entwickelten kapitalistischen Gesellschaften lebenden Menschen der Raum liegt, in dem Vergnügen erlebt wird und erlebt werden kann und in Analysen durch die Forschung auch entsprechend verfolgt wird. Die Machtbeziehungen, die in einem solchen Raum bzw. Kontext vorliegen und auf die es in der Analyse ebenfalls ankommt, lassen sich von den sozialen und kulturellen Praxen aus artikulieren, was aber jeweils einen spezifischen perspektivischen $\mathrm{Zu}$ schnitt bedeutet.

An dieser Stelle muss daher weiter danach gefragt werden, was das für manche Kulturwissenschaftler Problematische an den Cultural Studies ausmacht, wenn doch das Erkenntnisinteresse in der Bestimmung jener konkreten Verknüpfung mit gesellschaftlichen und kulturellen Strukturen nicht nur jenseits geschichtsphilosophischer Absich- 
ten liegt, sondern die Konstruiertheit der Perspektive im Forschungsprozess sogar mit thematisiert wird? Liegt die mögliche Irritation etwa in dem Hinweis auf die Unabschließbarkeit von Paradigmen und theoretischen Positionen, der auch die in diesem Sammelband verfolgte Überlegung der »Werkzeugkiste« mitbegründet?

Wie dem auch sei, gegenüber der quantitativ-empirischen Forschung, in der Daten als kontextlose Ausprägungen genommen und interpretiert werden, ist damit unter epistemologischen Gesichtspunkten eine grundlegend andere Ausrichtung der Forschung eingeschlagen, deren Stellung und Positionierung dem kulturwissenschaftlichen Interesse entsprechen müsste. Anscheinend aber sorgt die beschriebene Ausrichtung der Cultural Studies einstweilen für Missverständnisse, da ihre im Forschungsprozess mitgeleistete Erkenntniskritik als Kritik am probabilistischen Verfahren einerseits und der hermeneutischen Position andererseits gewertet wird. Ein Teil der Kritik ist mit Blick auf die methodischen Schwächen und Probleme, die einige Cultural Studies-Arbeiten offenbaren, durchaus verständlich und vertretbar. Aber gerade mit der impliziten erkenntniskritischen Position scheinen mir auf dem transdisziplinären Feld der »Kulturwissenschaft« entscheidende Einsichten zu gewinnen zu sein. Denn für die Cultural Studies wird der Kontext und damit auch das konkrete wissenschaftliche Problem - sozusagen von zwei Seiten - erst mit dem Verfahren der Artikulation hergestellt, was zugleich auf die rekonstruktive Haltung in der Kulturanalyse und Kulturkritik verweist. Artikulation ist ein Modell, das eine »nicht lineare expansive Praxis der Herstellung von Verbindungen« (Grossberg I994: 26) beschreibt. Es deutet auf die Möglichkeit hin, wie im Spiel der Differenzen, Bedeutungen oder Entsprechungen erst Relationen geknüpft werden müssen, die in die Interpretation eingehen. Dieser Denkmodus gibt eine Ahnung davon, wie Einheit und Differenz, Einheit in Differenz zu denken wären. Hier greift wieder die Frage »Wessen Cultural Studies? «als Motiv der kritischen Ausrichtung, wenn nach Grossberg die Praxis der Cultural Studies die »Leute in die Lage zu versetzen sucht, »bei ihren Bemühungen, ihre Kontexte zum Besseren zu verändern, strategischer vorgehen zu können« (Grossberg I994: 27).

Das Konzept der Artikulation muss somit als ein pars pro toto für die Praxis der Cultural Studies genommen werden, weil es einerseits auf die Wichtigkeit von Bedeutungsunterschieden verweist, die sich in 
spezifischen gesellschaftlichen bzw. medialen Situationen ergeben, und andererseits eine Selbstverpflichtung dafür enthält, wie auf sich verändernde gesellschaftliche und politische Bedingungen mit fortgesetzten theoriepolitischen Verschiebungen zu reagieren ist (Göttlich I999a: 63). Oder anders formuliert: Als das Charakteristikum der Cultural Studies und ihrer unterschiedlichen Formationen kann die Analyse kultureller Kontexte als die Erforschung und Kritik der Bedingungen der Möglichkeiten kultureller Selbstvergewisserung von Einzelpersonen sowie von gesellschaftlichen Gruppen und Schichten in ihrem Alltag und ihrer kulturellen Praxis unter sich wandelnden Machtkonstellationen gesehen werden (Göttlich/C. Winter I999: 26), die vorwiegend anhand von kulturellen Repräsentationen erfolgt.

Für die Auseinandersetzung und den Diskurs mit der Kulturwissenschaft sind daher an aktuellen gesellschaftlichen Problemen orientierte konkrete Fragen erst herauszuarbeiten, die es erlauben, weitere Gemeinsamkeiten und Unterschiede zu bestimmen. Diese sollten zum einen die unterschiedlichen Arten und Weisen der Themenbehandlung betonen. Aus Sicht der Cultural Studies handelt es sich um Erkenntnisse zu drängenden Gegenwartsthemen

॥wie Rasse, Geschlecht und Sexualität, nationale Souveränität und Nationalität, Kulturpolitik, aber auch neue Informationstechnologien und Cyberkulturen, die Informationsstadt und ihre räumlichen Formationen, interkulturelle Beziehungen, Körperdiskurse, kulturelle Institutionen, neue Ethnizitäten und Identitäten, IKartographienı der Diaspora, Kolonialismus, Postkolonialismus und die Auswirkungen der Globalisierung auf politische, wirtschaftliche und mediale Systeme» (Bromley 1999 et al.: 22),

auf die auch kulturwissenschaftliche Antworten zu erwarten sind.

Zum anderen sollten sie theoretisch gestellt sein, um die politische wie soziale Verankerung der Forschungsansätze und -perspektiven thematisieren zu können. Gerade darin sehe ich eine notwendige Ergänzung kulturwissenschaftlicher Fragestellungen um sozialwissenschaftliche Perspektiven, die vor dem Hintergrund der geisteswissenschaftlichen Grundlegung der Kulturwissenschaft(en) drohen, ausgeblendet oder sogar verfehlt zu werden. In diesem Beitrag konnte zu diesem möglichen Schritt nur die Richtung angegeben werden.

Wenn sich Cultural Studies als kritische Wissenschaft begreifen lassen, dann wäre es für die deutschsprachige Rezeption auch lohnend 
- um den Diskurs mit den Kulturwissenschaften ebenfalls in diese Richtung anzustoßen -, gemeinsamen Anschlusspunkten mit den aktuellen Diskussionen um die Perspektiven der Kritischen Theorie intensiver nachzugehen, womit die Rezeption der Cultural Studies über die bislang dominante einzelwissenschaftliche Wahrnehmung und Anwendung hinausgeführt werden könnte. In einer solchen Auseinandersetzung müssten die »normativen « Bezugspunkte der Kritik vertiefend behandelt werden. Ein interessantes Diskussionsfeld eröffnet sich in einem Vergleich des kritischen Motivs der Cultural Studies mit der Problemstellung innerhalb der Kritischen Theorie und den dort vorliegenden Konzepten einer normativen Gesellschaftskritik. Relevant ist das sowohl in historischer Dimension, z. B. anhand des von Horkheimer (1937) entwickelten Programms einer Kritischen Theorie als auch aktuell, z.B. anhand des von Honneth formulierten Ansatzes einer Kritik der Anerkennungsverhältnisse (Honneth I992: I994). Das Kritikkonzept der Cultural Studies gilt es aber auch mit den Kritikkonzepten in der Postmodernedebatte zu vergleichen. Eine weitere Diskussion wäre im Hinblick auf die handlungstheoretischen Überlegungen bei Joas (1992) an den Punkten zu eröffnen, wo es um die Klärung der Rolle der Medien für die Identitätsbildung geht. Damit könnte die Vielfalt der Aneignungsweisen an eine theoretische Tradition rückgebunden werden, die eine handlungstheoretische Aufklärung der Kreativität des Mediengebrauchs verspricht.

Es war hier nicht der Ort, detaillierter auf die inhaltlichen und thematischen Differenzierungen, die die Cultural Studies im Laufe ihrer Entwicklung bei unterschiedlichen Vertretern aufweisen, einzugehen und das Verhältnis zu den Kulturwissenschaften aus diesen Perspektiven zu rekonstruieren. Ohnehin zeigen die meisten Beispiele aus der internationalen Rezeptionsgeschichte, dass die Cultural Studies bislang überwiegend im Rahmen von Einzeldisziplinen rezipiert wurden, womit eine transdisziplinäre Einordnung erst an ihrem Anfang steht. Hierzu sollte an dieser Stelle eine erste Orientierung anhand von Fragestellungen und möglichen Vergleichspunkten gegeben werden. 


\section{Anmeruungen}

I Der Beitrag geht in Teilen zurück auf Göttlich r999b.

2 Darauf verwies bereits Lazarsfeld (I94I) in seinem grundlegenden Text zum Verhältnis von empirischer und kritischer Kommunikationsforschung.

3 Diese Frage bietet eine deutlichere Konkretisierung der Motive der Cultural Studies-Kritik als die von Grossberg in diesem Zusammenhang formulierte Frage »What's going on?«.

4 Es gibt eine ganze Reihe von Positions- und Ortsbestimmungen der Cultural Studies, die ihren Ausgangspunkt bei einer historischen Genealogie finden und die von den British Cultural Studies aus ansetzen - was in der internationalen Ausbreitung nicht unwidersprochen blieb. Vgl. Clarke I99I; Johnson I999; Grossberg I993; Hall ı980, I98I, I990. Vgl. bei Ang/Stratton die Kritik am Verständnis der Internationalität, die eine europäisch-amerikanische ist und dadurch lokale und regionale Varianten der Cultural Studies in Asien und Australien ausblendet.

5 Die Bedeutung dieser beiden Bücher wird bis heute in zahlreichen Kommentaren immer wieder herausgestellt. Nach Perry Anderson etwa war Culture and Society das »most influential socialist work of the past decade « und The Long Revolution »the one serious work of socialist theory«, hier zit. n. Milner ı989: I20.

6 Vgl. zur Entwicklung der New Left u.a. Blackburn I988; Milner I989.

7 Das verdeutlicht besonders gut seine Anwendung poststrukturalistischer Ansätze zur Analyse kultureller Identitäten.

\section{Literatur}

Ang, Ien (I996): Living Room Wars. Rethinking Media Audiences for a Postmodern World, London: Routledge.

Ang, Ien/Stratton, Jon (I996): »On the Impossibility of a Global Cultural Studies. >British< Cultural Studies in an >International< Frame«. In: David Morley/Kuan-Hsing Chen (Hg.), Stuart Hall. Critical Dialogues in Cultural Studies, London: Routledge, S. 36I391.

Bennett, Tony (I992): »Putting Policy into Cultural Studies«. In: Law- 
rence Grossberg et al. (Hg.), Cultural Studies, London/New York: Routledge, S. 23-34.

BMWV/IFK (Bundesministerium für Wissenschaft und Verkehr/Internationales Forschungszentrum für Kulturwissenschaften) (Hg.) (1999): The Contemporary Study of Culture, Wien: Turia + Kant.

Blackburn, Robin (I988): »Raymond Williams and the Politics of a New Left«. NLR I68, S. I2-22.

Böhme, Hartmut/Matussek, Peter/Müller, Lothar (2000): Orientierung Kulturwissenschaft. Was sie kann, was sie will, Reinbek bei Hamburg: rowohlts enzyklopädie.

Bromley, Roger/Göttlich, Udo/Winter, Carsten (Hg.) (I999): Cultural Studies. Grundlagentexte zur Einführung, Lüneburg: zu Klampen.

Clarke, John (1991): New Times and Old Enemies: Essays on Cultural Studies and America, London: HarperCollins.

Denzin, Norman K/Lincoln, Yvonna S. (Hg.) (I994): Handbook of Qualitative Research, Thousand Oaks/London: Sage Publications.

Denzin, Norman K./Lincoln, Yvonna S. (Hg.) (1998): The Landscape of Qualitative Research. Theories and Issues, Thousand Oaks/London: Sage Publications.

du Gay, Paul/Hall, Stuart et al. (1997): Doing Cultural Studies, London: Sage Publications.

Engelmann, Jan (Hg.) (1999): Die kleinen Unterschiede. Der Cultural Studies Reader, Frankfurt a.M.: Campus Verlag.

Fiske, John (1989a): Understanding Popular Culture, Boston et al.: Unwin Hyman.

Fiske, John (1989b): Reading the Popular, Boston et al.: Unwin Hyman. Göttlich, Udo (1996): Kritik der Medien. Reflexionsstufen kritisch-materialistischer Medientheorien am Beispiel von Leo Löwenthal und Raymond Williams, Opladen: Westdeutscher Verlag.

Göttlich, Udo (1999a): »Unterschiede durch Verschieben. Zur Theoriepolitik der Cultural Studies«. In: Jan Engelmann (Hg.), Die kleinen Unterschiede. Der Cultural Studies Reader, Frankfurt a.M.: Campus Verlag, S. 49-63.

Göttlich, Udo (I999b): »Die Wahrnehmung der Cultural Studies. Cultural Studies zwischen hilfswissenschaftlicher Vereinnahmung und radikaler Kontextualität«. Sociologia Internationalis, 37/2, S. I89-219.

Göttlich, Udo/Winter, Carsten (I999): »Wessen Cultural Studies?«. 
In: Roger Bromley/Udo Göttlich/Carsten Winter (Hg.), Cultural Studies. Grundlagentexte zur Einführung Lüneburg: zu Klampen, S. $25-39$.

Grossberg, Lawrence (I993): »The Formations of Cultural Studies: An American in Birmingham«. In: Valda Blundell/John Shepherd/ Ian Taylor (Hg.), Relocating Cultural Studies. Developments in Theory and Research, London: Routledge, S. 2I-66.

Grossberg, Lawrence (I994): »Cultural Studies. Was besagt ein Name?«. IKUS Lectures 3/I7-I8, S. II-40.

Grossberg, Lawrence (I996): »On Postmodernism and Articulation: an Interview with Stuart Hall«. In: David Morley/Kuan-Hsing Chen (Hg.), Stuart Hall. Critical Dialogues in Cultural Studies, London, NewYork: Routledge, S. I3I-I5O.

Grossberg, Lawrence (I999): »Zur Verortung der Populärkultur«. In: Roger Bromley/Udo Göttlich/Carsten Winter (Hg.), Cultural Studies. Grundlagentexte zur Einführung, Lüneburg: zu Klampen, S. $215-236$.

Grossberg, Lawrence/Nelson, Cary/Treichler, Paula A. (Hg.) (I992), Cultural Studies, London/New York: Routledge.

Hall, Stuart (I977): »Über die Arbeit des Centre for Contemporary Cultural Studies (Birmingham). Ein Gespräch mit H. Gustav Klaus«. Gulliver 2, S. 54-57.

Hall, Stuart (I980): »Cultural Studies and the Centre. Some Problematics and Problems«. In: Ders. et al. (Hg.), Culture, Media, Language, London: Open University Press, S. I5-47.

Hall, Stuart (198I): »Cultural Studies: Two Paradigms«. In: Tony Bennett et al. (Hg.), Culture, Ideology and the Social Process, London: Open University Press, S. I9-37.

Hall, Stuart (I990): »The Emergence of Cultural Studies and the Crisis of the Humanities «. October 53, S. II-23.

Hall, Stuart (I992): »Cultural Studies and its Theoretical Legacies«. In: Lawrence Grossberg/Cary Nelson/Paula A. Treichler (Hg.), Cultural Studies, London/New York: Routledge, S. 277-286.

Hall, Stuart (I999): »Cultural Studies. Zwei Paradigmen«. In: Roger Bromley/Udo Göttlich/Carsten Winter (Hg.), Cultural Studies. Grundlagentexte zur Einführung, Lüneburg: zu Klampen, S. II3I38.

Hall, Stuart et al. (1978): Policing the Crisis: »Mugging«, the State, and Law and Order, Basingstoke: Macmillan. 
Hartley, John (1992): The Politics of Pictures, London/New York: Routledge.

Horkheimer, Max (1937): »Traditionelle und kritische Theorie«. In: Ders. (I988), Gesammelte Schriften, Bd. 4, Frankfurt a.M.: Fischer Verlag, S. 162-216.

Joas, Hans (1992): Die Kreativität des Handelns, Frankfurt a.M.: Suhrkamp Verlag.

Johnson, Richard (I999): »Was sind eigentlich Cultural Studies?«. In: Roger Bromley/Udo Göttlich/Carsten Winter (Hg.), Cultural Studies. Grundlagentexte zur Einführung, Lüneburg: zu Klampen, S. I39-I88.

Lazarsfeld, Paul F. (I94I): »Remarks on Administrative and Critical Communications Research «. Studies in Philosophy and Social Science, Bd. 9, S. 2-16.

Leavis, F.R. (1930): Mass Civilization and Minority Culture, Cambridge. Leavis, F.R./Thompson, Denys (1933): Culture and Environment. The Trainig of Critical Awareness, London.

McRobbie, Angela (1995): »What is Happening in Cultural Studies? Kulturanalyse im Postmarxismus«. In: Wolfgang Kaschuba (Hg.), Kulturen - Identitäten - Diskurse, Berlin: Akademie Verlag, S. IOO-II3.

Milner, Andrew (1989): »Williams and the New Left«. Southern Review 22/2, S. II9-128.

Morley, David (1992): Television Audiences and Cultural Studies, London/New York: Routledge.

Morley, David/Chen, Kuan-Hsing (Hg.) (1996): Stuart Hall. Critical Dialogues in Cultural Studies, London/New York: Routledge.

Nelson, Cary/Treichler, Paula A./Grossberg, Lawrence (I992): »Cultural Studies: An Introduction«. In: Dies. (Hg.), Cultural Studies, London/New York: Routledge, S. I-I6.

O'Sullivan, Tim/Hartley, John/Saunders, Danny et al. (1994): Key Concepts in Communication an Cultural Studies, London/New York: Routledge.

Williams, Raymond (1958a): Culture and Society 1780-1950, London: Chatto \& Windus (hier zit.n. der Ausgabe von 1993).

Williams, Raymond (1958b): »Culture is Ordinary«. In: Norman McKenzie (Hg.), Conviction, London: MacGibbon and Kee, S. 74-92. Williams, Raymond (196r): The Long Revolution, Harmondsworth: Penguin. 
Williams, Raymond (1977a): Marxism and Literature, London: Oxford University Press.

Williams, Raymond (1977b): Innovationen. Über den Prozeßcharakter von Literatur und Kultur, hg. v. H. Gustav Klaus, Frankfurt a.M: Suhrkamp Verlag.

Williams, Raymond (1989a): Raymond Williams on Television. Selected Writings, hg. v. Alan O'Connor, London: Routledge.

Williams, Raymond (1989b): »The Future of Cultural Studies«. In: Ders., The Politics of Modernism. Against the New Conformists, London: Verso, S. I5I-I6I.

Williams, Raymond (I998): »Drama in einer dramatisierten Gesellschaft«. In: Udo Göttlich/Jörg-Uwe Nieland/Heribert Schatz (Hg.), Kommunikation im Wandel. Zur Theatralität der Medien, Köln: Herbert von Halem Verlag, S. 238-252. 


\section{Ethnographie, Interpretation und Kritik:}

\section{Aspekte der Methodologie der Cultural Studies'}

RAINER WINTER

\section{Einleitung: Das Dilemma von Hermes}

Der amerikanische Anthropologe Vincent Crapanzano vergleicht den Anthropologen mit einem modernen Hermes, einem Kurier,

॥der, ausgestattet mit Methoden, das Verschleierte, Verborgene, Unbewusste zu enthüllen, sich seine Kunde auch durch List verschaffen könnte. Er stellt Sprachen, Kulturen und Gesellschaften zunächst in ihrer ganzen Dunkelheit, Fremdheit, Bedeutungsleere dar, um sodann - wie ein Zauberer, ein Hermeneutiker, wie Hermes selbst - das Dunkle aufzuklären, das Fremde vertraut zu machen, dem Bedeutungsleeren Bedeutung zu verleihen. Er entschlüsselt die Botschaft. Er interpretiert» (Crapanzano 1996: 16I, Hervorhebung R.W.).

Ethnographie und Interpretation sind in dieser Lesart unauflöslich miteinander verbunden. Die folgenden Überlegungen zur Methodologie der Cultural Studies lassen sich davon leiten. Während die ethnographische Sozialforschung in der Bundesrepublik im Wesentlichen einem post-positivistischen Paradigma folgt und davon ausgeht, dass ethnographische Texte eine von ihnen unabhängige Wirklichkeit möglichst realistisch repräsentieren sollen, wird bei den Cultural Studies 
die Rolle des Forschers, seine Position als Beobachter und als Schreibender, radikal problematisiert und die zentrale Bedeutung der Interpretation hervorgehoben. Auf die »Krise der Repräsentation «, die im writing culture-Ansatz innerhalb der Anthropologie herausgestellt und behandelt wird (vgl. Clifford/Marcus I986), wird mit unterschiedlichen Strategien reagiert. Zentral ist hierbei die Vorstellung, dass die Erfahrungen, die qualitative Forscher in ihren Texten beschreiben und analysieren, durch diesen Prozess selbst (mit-)konstituiert werden. Die Praktiken der Forscher stellen situierte Praktiken dar, die kontextspezifische und partikulare »Wahrheiten« produzieren. Der vorliegende Beitrag möchte am Beispiel der Ethnographie einen Einblick in diese Debatten zur qualitativen Sozialforschung geben, die vor allem in den USA geführt werden (vgl. Clough 1992; Denzin 1997; Denzin/Lincoln 2000). Unser Interesse gilt vor allem Verknüpfungen zwischen Cultural Studies und kritischen, interpretativen Methodologien (vgl. Denzin 200I). Nach einer Bestimmung zentraler Merkmale des Projekts der Cultural Studies, das in Großbritannien entstand und heute weltweit betrieben wird, werden wir in Auseinandersetzung mit der aktuellen Diskussion in der bundesrepublikanischen Soziologie die Konzeption einer interpretativen Ethnographie innerhalb der Cultural Studies näher bestimmen und diskutieren.

\section{Das Projekt der Cultural Studies}

Seit ihren Anfängen in Großbritannien Ende der I950er Jahre haben die Cultural Studies eine große Affinität zur Ethnographie. Dies zeigt sich bereits in Richard Hoggarts Studie The Uses of Literacy (1957), in der er den Einfluss des sozialen Wandels nach dem Zweiten Weltkrieg, insbesondere den negativen der kommerziellen Massenkultur, auf die Kulturen der Arbeiterklasse analysiert. Hierzu arbeitet er, in gewisser Weise die Ethnographie von Clifford Geertz vorwegnehmend, mittels dichter Beschreibungen die Eigenart ihrer alltäglichen Praktiken und kulturellen Formen heraus (vgl. Geertz I983). Raymond Williams hat in seinem im darauf folgenden Jahr erschienenen Buch Culture and Society 1780-1950 (1958) in einer differenzierten Auseinandersetzung mit der englischen Literatur und Literaturkritik seit dem I8. Jahrhundert die verschiedenen Bedeutungen von culture entschlüsselt, miteinander verglichen und systematisiert. Einerseits verdichte- 
ten sich in diesem Begriff die Reaktionen auf und die Kritik an der Modernität, andererseits wurde im englischen Kontext eine holistische Konzeption von Kultur entwickelt, die diese als a whole way of life begreift. Kultur wird nicht als ein vom Alltag abgegrenzter Bereich bestimmt, sondern culture is ordinary. Damit waren die Grundlagen für die Arbeit des I964 gegründeten Centre for Contemporary Cultural Studies (CCCS) in Birmingham gelegt. Dessen ursprüngliches Ziel war es, mittels einer Synthese literaturkritischer und soziologischer Ansätze die populäre Kultur zu analysieren. Berühmt geworden sind die Studien zu jugendlichen Subkulturen und zur Medienanalyse und -rezeption.

Hierbei war die Ethnographie die bevorzugte Forschungsstrategie. Zum einen half sie, die schillernden, zum Teil spektakulären und bizarren Jugendwelten der ig6oer und I970er Jahre lebendig und differenziert in ihren alltäglichen Nöten, Kämpfen und kreativen Momenten zu erforschen. Zum anderen zeigen die ethnographischen Studien, dass es neben den dominanten Vorstellungen der durch die Kulturindustrien geprägten Mainstreamkultur auch »abweichende«, residuale und emergente Praktiken, Bedeutungen sowie Werte gibt (vgl. Williams I977). Gegen monolithische und essentialistische Konzeptionen von Kultur heben Cultural Studies deren Vielfalt hervor, welche vor allem die sich im Wandel befindlichen Gesellschaften der Gegenwart bestimmt. So gibt es Kulturen der Klasse, der Geschlechter, der Ethnien, sexuelle und politische Subkulturen, Randkulturen, medial vermittelte Spezialkulturen etc. Vor dem Hintergrund der Enttraditionalisierung und Auflösung stabiler Identitäten legen Cultural Studies dar, dass Kultur ein Kampf um Bedeutungen ist, ein nie zum Stillstand kommender Konflikt über Sinn und Wert von kulturellen Traditionen, Praktiken und Erfahrungen.

Eine der zentralen Einsichten der Cultural Studies ist, dass sich von einer auch noch so gelehrten und raffinierten Interpretation eines kulturellen Textes, einer Ideologie oder eines Diskurses nicht ableiten lässt, wie diese kulturellen Formen tatsächlich im Alltag von verschiedenen Personen und sozialen Gruppen interpretiert, verwendet oder angeeignet werden. Cultural Studies interessieren sich nicht für eine »objektive Sinnstruktur« kultureller Texte, deren Existenz sie generell in Frage stellen, sondern für die oft rasch vorübergehenden, aber keineswegs unbedeutenden Momente der Rezeption kultureller Texte in einem dynamischen, konfliktreichen und widersprüchlichen Alltag. 
In den Prozessen der Interpretation und Aneignung von medialen Ressourcen und kulturellen Symbolen (vgl. Winter I995), im Basteln von Stilen der Selbstdarstellung aus vorgegebenen Materialien oder in den Bemühungen, eine widerständige Identität in Institutionen $\mathrm{zu}$ schaffen und aufrechtzuerhalten, zeigen Cultural Studies die Kreativität und Produktivität kultureller Prozesse auf. Diese Kunst des Eigensinns, die sich in alltäglichen Kontexten entfaltet, kann als eine Kritik der Macht verstanden werden (vgl. Winter 200I).

So ist das primäre Ziel ihres Projekts, kulturelle Prozesse in ihrer kontextuellen Einbindung in Machtverhältnisse zu erforschen. Deren bestimmender und prägender Einfluss auf kulturelle Praktiken soll herausgestellt werden. Deshalb sind z.B. eine semiotische Analyse eines Hollywoodfilms oder die ethnographische Erforschung kultureller Welten ohne Bezug zum Verhältnis von Kultur und Macht keine Cultural Studies. Außerdem wird, Williams folgend, ein umfassender Kulturbegriff verwendet, der sowohl kulturelle Texte als auch Erfahrungen und Praktiken umfasst (vgl. Williams I961). Die herkömmliche Unterscheidung zwischen »Hoch- und Populärkultur« wird als Ausdruck gesellschaftlicher Machtverhältnisse verstanden. Das eigentliche Objekt der Cultural Studies sind so nicht diskrete kulturelle Formen, die losgelöst von ihrem sozialen oder politischen Kontext betrachtet werden. Vielmehr werden, ausgehend von konkreten Fragestellungen, kulturelle Prozesse in ihren verschiedenen Formen in räumlich und zeitlich spezifischen Kontexten analysiert.

Wie Lawrence Grossberg schreibt, werden die Cultural Studies von einem radikalen Kontextualismus geprägt (Grossberg 1999: 58 ff.). Dabei ist der Kontext nicht einfach ein Rahmen, der soziale Praktiken, die sich innerhalb seiner Grenzen ereignen, beeinflusst und bestimmt. Vielmehr konstituieren die Praktiken und Identitäten erst den Kontext, in dem sie Praktiken und Identitäten sind. Für die Analyse bedeutet dies: »Understanding a practice involves theoretically and historically (re-)constructing its context« (Grossberg 1992: 55). So bedingen sich Theorie und Kontext im Rahmen einer Cultural Studies-Analyse gegenseitig, das gewonnene Wissen ist in der Regel kontextspezifisch, wobei Kontexte nie vollständig repräsentiert, sondern nur unter verschiedenen Perspektiven (re-)konstruiert werden können. Ziel der Cultural Studies ist es, mit den jeweils verfügbaren theoretischen Ressourcen und empirischen Forschungen konjunktio- 
nale Prozesse besser zu verstehen und in einem zweiten Schritt auch zu einer Veränderung ihrer Kontexte beizutragen. Dies bedeutet, symbolische Auseinandersetzungen, den Kampf um Bedeutungen und Formen des »Widerstands « zu bestimmen und »Wissen« bereitzustellen, damit die Beteiligten diese Prozesse besser verstehen können.

Die »Erfindung« der Cultural Studies beruhte also auf der Erkenntnis, dass Kultur eine zentrale Bedeutung in der Gegenwart hat und nur im Kontext von Macht und Politik angemessen analysiert werden kann (vgl. Kellner i995). So sind Anlässe für Forschungen oft soziale und politische Probleme bzw. Auseinandersetzungen. Das methodologische Vorgehen der Cultural Studies lässt sich am besten als Bricolage bezeichnen (vgl. Nelson/Treichler/Grossberg I992). Für ein spezifisches Forschungsprojekt werden aus verschiedenen wissenschaftlichen Feldern Theorien und Methoden nach pragmatischen und strategischen Gesichtspunkten ausgewählt, kombiniert und angewendet. Wenn es die Forschungsfrage erfordert, werden auch, aufbauend auf dem Verfügbaren, »neue« Theorien und Methoden »gebastelt« oder entwickelt. So knüpfen Cultural Studies an unterschiedliche Paradigmen und Theorien an, z. B. an kulturalistische oder (post-) strukturalistische Ansätze (vgl. Hall I999). Auch bei den Methoden herrscht eine große Vielfalt, die von der semiotischen Textanalyse über die teilnehmende Beobachtung bis zum narrativen Interview und der Gruppendiskussion reicht. Dabei verfolgen Cultural Studies, wie Stuart Hall, der langjährige Direktor des CCCS feststellt, das emanzipatorische Ziel »to enable people to understand what [was] going on, and especially to provide ways of thinking, strategies for survival, and resources for resistance« (Hall I990: 22). Vor allem unterdrückten und marginalisierten Gruppen soll geholfen werden zu verstehen, wie ihre Lebensbedingungen durch Machtverhältnisse geprägt werden und persönliche Probleme mit sozialen Problemen zusammenhängen (vgl. Mills I973). Im Sinne von Habermas (I968) geht es hier um eine Steigerung der Fähigkeit zur Selbstreflexion.

In neuerer Zeit wurden innerhalb der Cultural Studies-Bewegung verschiedene Standpunkt-Epistemologien ${ }^{2}$ entwickelt, welche die Perspektiven, die Interessen und das Leiden konkreter Subjekte artikulieren, indem sie deren Lebenserfahrungen ins Zentrum rücken. Dabei werden die kulturellen Praktiken thematisiert und analysiert, die Strukturen der Unterdrückung hervorbringen. Auf der Basis die- 
ses »disqualifizierten« Wissens, das lokal, partiell und parteiisch ist, erfolgt dann eine Kritik von Machtverhältnissen. Die Nähe zu Foucaults Projekt der Genealogie ist hier offensichtlich.

„Als IGenealogier bezeichnen wir die Verbindung von gelehrten Kenntnissen und lokalen Erinnerungen, eine Verbindung, die es ermöglicht, ein historisches Wissen der Kämpfe zu erstellen und dieses Wissen in aktuelle Taktiken einzubringen« (Foucault 1999: 17).

Foucaults Einfluss macht sich bei vielen Arbeiten der Cultural Studies bemerkbar. Daneben gibt es aber noch andere Perspektiven, Anschlusspunkte und Vorgehensweisen.

In der empirischen Forschung lassen sich bisher im Wesentlichen drei Ausrichtungen unterscheiden, in deren Spannungsverhältnis und Kombination aber erst gelungene und fruchtbare Cultural Studies-Arbeiten entstehen (vgl. Winter 200I): I. Die Analyse kultureller Texte (wie z.B. von Medienprodukten oder Interviewtranskripten), in der Regel mit strukturalistischen oder poststrukturalistischen Methoden; 2. Die ethnographische Erforschung von Sozialwelten; 3. Die kontextuelle Analyse, die die Verbindungen herstellt zwischen gelebten Erfahrungen, kulturellen Texten und den größeren sozialen, politischen und ökonomischen Verhältnissen in der Gesellschaft. In diesem Rahmen werden die empirischen Studien der Cultural Studies durchgeführt, die grundsätzlich offen und neugierig auf neue Themen, Auswertungsstrategien und Methoden sind. ${ }^{3}$

Bevor ich mich nun den neueren methodologischen Überlegungen der Cultural Studies zur Ethnographie zuwende und insbesondere die in Auseinandersetzung mit dem Poststrukturalismus zentral gewordene Analyse des Forschers und seiner Interpretationen näher betrachte, möchte ich auf die aktuelle Debatte in der Bundesrepublik eingehen, um Verbindungslinien und Differenzen aufzeigen zu können. Insbesondere geht es mir darum, die Bedeutung der Impulse und Anregungen, die Cultural Studies in die qualitative Forschung der englischsprachigen Welt ${ }^{4}$ einbringen, auch hierzulande deutlich zu machen. Vor dem Hintergrund der bisherigen Betrachtungen werde ich exemplarisch das von Stefan Hirschauer und Klaus Amann (I997) vorgelegte Programm »Die Befremdung der eigenen Kultur. Zur ethnographischen Herausforderung soziologischer Empirie« diskutieren. An dieser Stelle kann ich allerdings nur auf einige Aspekte dieses spannenden und interessanten Bielefelder »Reformprogramms« ein- 
gehen. So werde ich mich im Wesentlichen auf wichtige Kritikpunkte beschränken, die sich aus der Perspektive der Cultural Studies ergeben. Anschließend werde ich eine Reform der Reform skizzieren.

\section{Zur Kritik der Ethnographie-Konzeption von Hirschauer/Amann}

Hirschauer und Amann begreifen die Ethnographie als eine »opportunistische und feldspezifische Erkenntnisstrategie« (Hirschauer/ Amann 1997: 20), die sich um ein Verständnis der »Gelebtheit kultureller Ordnungen « (ebd.: 2I) bemüht. In Abgrenzung zur klassischen Ethnographie von Bronislaw Malinowski, die auf das Verstehen einer Kultur als Ganzes ausgerichtet war, bestimmen sie Ethnographie als eine »synchrone Beobachtung lokaler Praxis« (ebd.: 23), die auf der »anhaltenden Kopräsenz von Beobachter und Geschehen « (ebd.: 2I) beruht. Während Malinowski die Maxime ausgab, die Perspektive des Eingeborenen zu übernehmen, und die Repräsentation der Homogenität einer Kultur anstrebte, knüpfen Hirschauer und Amann an die so genannte »Krise der Repräsentation « in der ethnographischen Diskussion an, ohne sich aber - so meine These - angemessen und differenziert auf diese einzulassen.

Ein Auslöser dieser Krise war, dass es in der heutigen globalen Ökonomie von Zeichen und Räumen (vgl. Lash/Urry I994) keine klar abgrenzbaren, unberührten Kulturen mehr gibt, die für sich selbst betrachtet als abgeschlossenes Ganzes umfassend und komplett porträtiert werden können (vgl. Clifford I988). Die Vernetzung der Kulturen, die Mobilität von Zeichen, Kulturen und Menschen - ich erinnere z. B. an Arjun Appadurais diesbezügliche Theorie der »Scapes« (I996) oder an James Cliffords Konzept der »Travelling Cultures« (I999) findet in einem multidimensionalen, transnationalen Raum statt, in dem soziale und kulturelle Praktiken miteinander verknüpft und voneinander abhängig sind. Wie es Clifford Geertz formuliert hat: »The world has its compartments still, but the passages between them are much more numerous and much less well secured « (Geertz I988: I32). Dies bedeute für die ethnographische Forschung, so Hirschauer und Amann, dass sie:

"wes stets mit perspektivisch gebrochenen Feldern zu tun [hat], in denen parteiliche Versionen miteinander konkurrieren ('partial truthsı, Clifford 1986) und sowohl multiple sPer- 
spektivenübernahmenı als auch dezidiert eigenständige Versionen verlangen« (Hirschauer/ Amann 1997: 23).

Hirschauer und Amann gehen davon aus, dass es Ziel der im Feld anwesenden Ethnographen ist, lokales, für die Teilnehmer selbstverständliches Wissen, das diesen aber diskursiv nicht verfügbar ist, zu explizieren. Im Gegensatz zu Interviews haben ethnographische Studien weniger die Absicht, die Welt der Anderen mit deren Augen zu sehen, als deren »Weltsichten als ihre gelebte Praxis zu erkennen« (Hirschauer/Amann I997: 24). Hirschauer und Amann heben also die Differenz zwischen Teilnehmer- und Beobachterverstehen hervor, die im Laufe des Forschungsprozesses wohl temporär und punktuell übereinstimmen können, am Ende dieses Prozesses aber nicht mehr kongruent sind. Der distanzierte und kalt analysierende Blick von Erving Goffman, dessen Interesse nicht Menschen und ihren Situationen, sondern vor allem Situationen und ihren Menschen galt (vgl. Goffman I97I: 9), ist hier explizit Vorbild und Ideal. Er deckt die den Teilnehmenden verborgenen Strukturen sozialer Interaktion auf.

"Das heißt, dass die Ethnographie [...] eine Betrachtungsweise praktiziert, die den Menschen nicht als Sinnzentrum, sondern als Appendix sozialer Situationen betrachtet. Ethnographie ist Teilhabe an der Introspektion sozialer Situationen« (Hirschauer/Amann 1997: 24).

Mit dieser objektivistischen Konzeption von Ethnographie wird, so meine These, jedoch die Pointe der ethnographischen Selbstkritik innerhalb der neueren Anthropologie, z. B. bei Clifford, und neueren Ansätzen der qualitativen Soziologie, z. B. bei Norman Denzin (I995, I997), verschenkt. Wenn bei diesen von parteilichen bzw. partiellen Versionen von »Wahrheit« die Rede ist, ist damit mehr gemeint als Goffmans durch Kenneth Burke angeleitetes Spiel mit Perspektiven, das letztendlich immer auf den Jonglierkünsten eines allwissenden, durch und durch modernen Erzählers beruht. Die neuere amerikanische Diskussion, in der die einengenden Perspektiven von Positivismus und Post-Positivismus überwunden werden, hebt hervor, dass in der ethnographischen Forschung vor allem singuläre und perspektivische »Wahrheiten« konstruiert werden. Ien Ang hat dies den radikalen Kontextualismus der Ethnographie genannt: 
1)edwede kulturelle Beschreibung ist nicht nur konstruktiv [...], sondern auch von provisorischer Natur, schafft eine diskursive 1Objektivierungı und Sedimentation von IKulturs durch das Aussondern und Hervorheben einer Reihe diskontinuierlicher Ereignisse aus einem fortlaufenden, niemals endenden Flussi (Ang 1997: 95).

Gefordert wird im Rahmen von Cultural Studies, einerseits Kultur als Prozess zu begreifen, der viele Stimmen und viele Perspektiven beinhaltet, andererseits den interpretativen Charakter der Ethnographie angemessen zu berücksichtigen. Damit werden die Fragen von Gender, Klasse, ethnischer Zugehörigkeit (race) sowie andere Formen sozialer und kultureller Ungleichheit für die qualitative Forschung wichtig. Denzin stellt hierzu fest:

"We are in a moment of discovery and rediscovery as new ways of looking, interpreting, arguing, and writing are debated and discussed. The qualitative research act can no longer be viewed from within a neutral or objective positivist perspective. Class, race, gender, and ethnicity shape the process of inquiry, thereby making research a multicultural process॥ (Denzin 1997: 19).

Vor diesem Hintergrund stellen ethnographische Forschungen diskursive Konstruktionen dar, die vom objektivierenden und keineswegs neutralen Blick des Forschers geprägt sind, auch wenn er dies selbst nicht wahrhaben möchte. Die Beobachtungen, die er macht und niederschreibt, sind sowohl in seiner Welt als auch in der des Beobachteten sozial verankert. Deshalb muss seine ethnographische Autorität, die »Last der Autorschaft«, dekonstruiert werden, wie Clifford schreibt:

I)Kulturenı halten für ihre Porträts nicht still. Versuche, sie dazu zu bringen, beinhalten immer Vereinfachung und Ausschluss, die Auswahl eines Zeitpunkts, die Konstruktion einer Beziehung zwischen Eigenem und Fremden und das Auferlegen oder die Aushandlung einer Machtbeziehung॥ (Clifford 1986: 10).

Die »Krise der Repräsentation« hat also tief gehendere Implikationen, als Hirschauer und Amann annehmen. Wenn man den post-positivistischen Rahmen in Frage stellt, weitet sie sich zu einer Krise der Legitimation aus, welche die Validität von Ergebnissen problematisiert (vgl. Denzin ı997: 6 ff.). In ihrem Programm wird die ethnographische Empirie abschließend wohl als »eine empirische Variante des 
Dekonstruktivismus« gepriesen. Was Dekonstruktion im poststrukturalistischen Sinne aber bedeutet, bleibt unterbelichtet. Dies zeigt sich auch im Kapitel über das ethnographische Schreiben, in dem das Problem der Repräsentation auf bloße Darstellungsprobleme reduziert wird. Die Dekonstruktion ethnographischer Wirklichkeiten und Darstellungen unterbleibt und wird auch nicht als Aufgabe qualitativer Forschung betrachtet. Während es an der Zeit wäre, z. B. die goffmanschen Texte wie die anderer Klassiker kritisch zu betrachten ${ }^{5}$, wird er wieder einmal als »Virtuose soziologischer Begriffsbildung « gepriesen, der die Analyse empirischer Phänomene fruchtbar mit der Generierung theoretischer Konzepte, Modelle und Metaphern koppelt. Dies mag stimmen, unbeachtet bleibt jedoch der ironische Schluss der Rahmen-Analyse (I977), in dem Goffman selbst sein eigenes Ende als Autor zelebriert und damit auf die Reflexivität der Ethnographie hinweist. Als letzter großer Autor der klassischen Ethnographie in der Soziologie war er sich - anders als Hirschauer/Amann - der Herausforderung der Dekonstruktion und ihrer Bedeutung für die qualitative Sozialforschung durchaus bewusst, was auch seine späte Beschäftigung mit Derridas Kritik an Searle unterstreicht (vgl. Goffman i983).

Zusammenfassend lässt sich feststellen, dass die Ethnographie, wie sie die Gruppe um Hirschauer und Amann betreibt, immer noch der modernistischen Agenda folgt, dass es möglich sei, die Welt gelebter Situationen, verstanden als eine relativ stabile externe soziale Realität, adäquat in der Beobachterperspektive zu erfassen. Auch wenn es vielfältige Interpretationen dieser Realität gebe, strebe der Beobachter doch danach, einen Zugang zur eigentlichen Interaktion zu finden und relativ verlässliche, überzeugende Interpretationen von Strukturen zu machen, die den Untersuchten selbst verborgen bleiben. ${ }^{6}$ Die aktuellen, methodologischen Diskussionen innerhalb der amerikanischen qualitativen Sozialforschung haben diese post-positivistische Ausrichtung qualitativer Forschung überwunden. Dabei ist die zentra-

le Frage, was es bedeutet, sich der »Krise der Repräsentation« und auch der »Krise der Legitimation« ernsthaft zu stellen.

\section{Merkmale einer interpretativen Ethographie}

Wie ich bereits gezeigt habe, werden die Diskurse von qualitativer Sozialforschung, Ethnographie und Cultural Studies in den USA eng 
miteinander verbunden. Es überrascht deshalb nicht, dass seit Ende der I980er Jahre ein geschärftes Bewusstsein für die Rolle der Ethnographie zu finden ist. Während z.B. die frühen Jugendstudien von Paul Willis (1979, I98I), in denen er Howard Beckers (I973) interaktionistischen Ansatz mit einem strukturalistischen Rahmen vermittelt, durchaus realistisch orientiert sind, verschiebt sich das Interesse in den neueren Studien von einer Deskription von Subkulturen oder Milieus hin zu Problemen der Dekonstruktion von Erfahrung und ihrer Interpretation im gesellschaftlichen Kontext. Ich möchte dies an den poststrukturalistischen Arbeiten von John Fiske zur Analyse der Populärkultur erläutern. Anschließend werde ich weitere wichtige Merkmale einer interpretativen Ethnographie diskutieren.

\section{Die Singularität kultureller Praktiken}

Fiske untersucht kulturelle Texte nicht in ihrer Eigenschaft als kulturelle Waren oder ästhetische Objekte (vgl. Fiske 2000; Winter/Mikos 200I). Stattdessen begreift er ihre Formen von Textualität als Orte oder Bereiche, an und in denen sich soziale Auseinandersetzungen um Sinn, Subjektivität und Ideologie ereignen. Auf scharfsinnige und originelle Weise dekonstruiert Fiske in seinen Analysen die unterschiedlichsten populären Texte von Madonna über Stirb langsam bis zu Eine schrecklich nette Familie mit dem Ziel, ihr Potenzial an Bedeutungen aufzuzeigen, das je nach sozialer und historischer Situation der Zuschauer von diesen unterschiedlich realisiert wird. Er zeigt die Inkonsistenzen, die Unabgeschlossenheit, die widersprüchliche Struktur oder die Polyphonie medialer Texte auf, arbeitet heraus, wie eng populäre Texte auf die gesellschaftliche Wirklichkeit bezogen sind und soziale Differenzen artikulieren. Die Rezeption und die Aneignung von Texten wird zu einer kontextuell verankerten gesellschaftlichen Praxis, in der die Texte als Objekte nicht vorgegeben sind, sondern erst auf der Basis sozialer Erfahrungen produziert werden.

Nach seiner Vorstellung hat die Ethnographie der Medienrezeption die Aufgabe, das Publikum bzw. den Prozess der Konstitution eines Publikums in partikularen, kontingenten Raum-Zeit-Momenten ${ }^{7}$ $\mathrm{zu}$ erfassen, zu beschreiben und zu interpretieren. Dabei erforscht Fiske, ob und wie unterschiedliche, miteinander in Konflikt stehende Bedeutungen kontextuell artikuliert werden. Nachdem er deren Organisation in einem spezifischen, lokalen Kontext untersucht hat, stellt 
er Bezüge zu abstrakteren Bedeutungsrahmen, so zur Kultur als einer »ganzen Lebensweise« her. Im Mittelpunkt stehen die sozialen Kämpfe um die Bedeutung kultureller Praktiken. Hierzu knüpft er eng an Gramscis Vorstellung vom kulturellen Kampf und an Foucaults Unterscheidung zwischen Macht und Widerstand an (vgl. Foucault I976). »Widerstand« kann in spezifischen historischen Situationen im Verhältnis von diskursiven Strukturen, kultureller Praxis und subjektiven Erfahrungen entstehen. Fiske begreift den Alltag als kontinuierliche Auseinandersetzung zwischen den Strategien der »Starken« und den Guerillataktiken der »Schwachen« (vgl. Fiske I989: 32-47; Winter/ Mikos 200I). Im Gebrauch der »Ressourcen«, die das System in Form von medialen Texten und anderen Konsumobjekten zur Verfügung stellt, versuchen die alltäglichen Akteure ihre Lebensbedingungen selbst zu definieren und ihre Interessen auszudrücken. Fiske interessiert sich nicht für die Aneignungsprozesse, die zur sozialen Reproduktion beitragen, sondern für den oft heimlichen und verborgenen Konsum, der im Sinne von Michel de Certeau (I988) eine Fabrikation, eine Produktion von Bedeutungen und Vergnügen ist, in der den Konsumenten ihre eigenen Angelegenheiten deutlicher werden und die zur allmählichen kulturellen und sozialen Transformation beitragen kann. Deshalb ersetzt er den Begriff des Publikums durch den der Publikumsformierung:

„Publikumsformierung (audiencing) ist ein Begriff, der nur in einer kritischen Theorie vorkommen kann, die vornehmlich auf das Aufdecken des strukturellen Wirkens des Kapitalismus zielt. Publikumsformierung betrachtet Konsum [...] als einen Akt der heimlichen Produktion auf der Mikro-Ebene, nicht als Reproduktion. Diese heimliche Produktion ist eine Praxis: Sie produziert Bedeutungen, nicht Objekte (ob eine Ware oder ein Text); sie existiert eher in Form eines Prozesses denn in Form eines Produktes und kann so unserer Aufmerksamkeit entgehen. Ihre geringe Sichtbarkeit sollte aber nicht mit geringer Bedeutung gleichgesetzt werden « (Fiske 1999: 261 f.).

Fiske fokussiert sein Interesse auf das Singuläre, das »Nichtrepräsentative« in kulturellen Praktiken, das in Studien, deren Interesse der »normalen Population « gilt und die auf Generalisierung angelegt sind, verloren geht.

Die Ethnographie spezifischer Raum-Zeit-Momente gibt einen Einblick in die Zirkulation von Bedeutungen und affektiven Energien. Nach dem Verständnis von Fiske (I999) entnimmt der Ethnograph 
»Proben«, die er auf kulturelle Praktiken hin untersucht. Diese setzt er in Beziehung zu Proben, die andere, aber damit verbundene Einblicke geben. Bei der Rezeption von Fernsehsendungen sind das z. B. die Produktions- und Marketingstrategien der Fernsehsender. Er stellt dann systemische Beziehungen zwischen den Momenten her, um zu verstehen, wie die Kultur als eine ganze Lebensweise strukturiert wird und wie Subjekte in ihr Lebensweisen entwerfen. Die Texte, die Fiske produziert, zeigen, wie vor allem Subjekte, die zu subordinierten sozialen Formationen gehören, versuchen, einen Sinn für sich zu schaffen sowie eine Identität auszubilden trotz materieller, sozialer und politischer Einschränkungen. Fiske geht also von der Erfahrungsebene von Subjekten in spezifischen Raum-Zeit-Momenten aus, ist sich aber bewusst, dass diese - allein durch sich selbst - nicht verstehbar ist.

\section{Kontextualisierung}

Nicht nur Fiske, auch andere Vertreter der Cultural Studies sind der Auffassung, dass eine Verknüpfung ethnographischer Studien mit den sozialen, kulturellen und ökonomischen Wirklichkeiten, in die sie eingebunden sind, erforderlich sei. So fordert Arjun Appadurai, dass in kontextuellen Analysen die verschiedenen »Scapes « und ihre vielfältigen Interaktionen berücksichtigt werden (vgl. Appadurai I996). Es geht nicht nur um die Bewegungen von Personen und Ideen, sondern auch um die von Geld und Technologien. Darüber hinaus sind die ethnographischen Felder keine abgeschlossenen und diskreten Entitäten, vielmehr sind sie global mit Ereignissen, Praktiken und Orten verknüpft. So hat vor allem James Clifford die Tendenz der traditionellen Ethnographie kritisiert, Orte wie eine Straßenecke, ein Klassenzimmer oder ein Dorf isoliert zu erforschen. In einer globalen Ökonomie von Zeichen und Räumen muss die Ethnographie die Verbindungen zwischen Ereignissen, Praktiken und Diskursen berücksichtigen. Da die Kulturen »auf der Reise« sind, plädiert Clifford für eine Ethnographie, die dies berücksichtigt und den Forscher selbst als Reisenden begreift (vgl. Clifford I999).

Ergänzend hat Grossberg (I988) für die Medienforschung gefordert, dass sie sich nicht alleine auf Texte und ihre bisweilen widerständigen Lesarten konzentrieren, sondern die Rezeptions- und Aneignungspraktiken im umfassenderen Kontext des Alltagslebens verorten solle. Damit ließe sich zeigen, wie z. B. die Rezeptionspraktiken 
im Wohnzimmer mit den übrigen Praktiken in der Familie, mit den Praktiken in der Schule und am Arbeitsplatz zusammenhängen. Da die Untersuchten zwischen Institutionen und Diskursen hin und her pendeln, wird der Ethnograph zu einem Mitreisenden, zu einem nomadischen Kritiker. Seine Aufgabe ist es zu zeigen, wie die Praktiken der Untersuchten in die Machtdispositive einer Gesellschaft eingebunden sind. Denzin (1995) hat darauf hingewiesen, dass eine Ethnographie der postmodernen visuellen Kultur, welche die herkömmlichen Vorstellungen von Realismus dekonstruiert hat, berücksichtigen muss, dass der mediale Apparat zwischen der materiellen Welt und der Welt der alltäglichen, gelebten Erfahrung vermittelt. So schaffen Kino- oder Fernsehtexte Repräsentationen von Erfahrungen, die im Alltag interpretiert, gebraucht und angeeignet werden. Wenn Kultur sowohl eine Zirkulation von Bedeutungen als auch eine ganze Lebensweise umfasst, lässt sich die alltägliche Erfahrung nicht mehr scharf von der medialen Repräsentation abgrenzen.

"The realist logic of the ethnographic text must be deconstructed. At the same time, a new form of ethnography, an ethnography of the video-cinematic text, must be constructed (see Krug 1992). Such an ethnography will attempt to unravel and undo the cinematic texts that themselves map the lived experiences that ethnographers seek, in some final analysis to interpret and critique» (Denzin 1995: $200 \mathrm{f}$.).

\section{Dialog}

Ausgehend von der Kritik an der Autorität des Ethnographen geht es in den neueren Studien der Cultural Studies darum, Untersuchungsund Schreibweisen zu finden, die die »Wahrheit« des Feldes angemessener zum Ausdruck bringen. Wenn Wahrheiten partiell und standpunktbezogen sind, dürfen die Perspektive des Ethnographen und sein objektivierender Blick auf den »Anderen« nicht mehr privilegiert werden. Es geht vielmehr darum, den Dialog sowohl in der Forschungspraxis als auch im Schreibprozess, in den die Untersuchten einbezogen werden sollen, zu einem konstitutiven Prinzip zu machen. Dies bedeutet nicht nur eine kommunikative Validierung der Forschungsergebnisse, sondern ein Einbringen der »Stimmen« der Beobachteten in die Texte der Ethnographen. Dies ist z. B. über autobiographische Texte, über Dialoge, aber auch über literarische Texte und 
verschiedene Formen von Performance möglich. Diese Ergänzungen erlauben es, Erfahrungen, Emotionen und im Alltag gelebte ethische Prinzipien einzubringen, die in auf Kohärenz angelegten realistischen Texten leicht verloren gehen. Die Steigerung der dialogischen Sensibilität geht einher mit einer Dekonstruktion der institutionellen, paradigmatischen, kulturellen und persönlichen Rahmen, die die jeweilige Untersuchung bestimmen. Dieser Prozess bedeutet keine Außerkraftsetzung, sondern ein Bewusstmachen der oft impliziten Rahmenvorstellungen des Forschungsprozesses. Hierbei entstehen selbstreflexive Texte, in denen unterschiedliche Formen der Repräsentation genutzt werden, um verschiedene »Stimmen « in einen Dialog zu bringen. Besonders prägnant wird dies bei performativen Texten.

Daher plädiert Denzin für eine performative Wende der qualitativen Sozialforschung (Denzin 1999: I38 ff.). Dadurch könne sie zum einen der Auffassung der Cultural Studies gerecht werden, dass Kultur ein Prozess ist, zum anderen könne sie Konsequenzen aus den populären Standpunkt-Epistemologien ziehen. Aufführungen ethnographischer Texte machen Räume, Bedeutungen, Ambivalenzen und Widersprüche von Kulturen für das Publikum erfahrbar. Durch die Schaffung eines gemeinsamen Erfahrungsraums von Aufführenden und Publikum können zwangsläufige Objektivierungen des voyeuristischen, ethnographischen Blicks korrigiert werden. Zudem destabilisieren sie die Position des schreibenden Ethnographen.

"Die Aufführung wendet sich an die Erfahrung zurück, die zuvor im ethnographischen Text zur Darstellung gelangte. Sie präsentiert dann wiederum diese Erfahrung dem Publikum in der Aufführung. Sie privilegiert auf diese Weise Erfahrung, den sinnstiftenden Moment, wenn eine Fremderfahrung für das Selbst lebendig wirdı (Denzin 1999: 139).

Wenn die performative Perspektive sich durchsetzen wird, wird sie die Ethnographie und auch die Cultural Studies grundlegend verändern.

\section{$\mathbf{S}$ CHLUSS}

Es ist deutlich geworden, dass es in den neueren ethnographischen Arbeiten der Cultural Studies zum einen darum geht, durch die Berücksichtigung der Vielheit von Stimmen in einem Feld verschiedene Perspektiven und Wirklichkeiten zu repräsentieren. Zum anderen wer- 
den die Untersuchungsfelder in ihren vielfältigen Verbindungen zu diskursiven, ökonomischen und politischen Wirklichkeiten analysiert, um zu neuen Einsichten zu gelangen. Zudem zeigt die Dekonstruktion der Autorität des Forschers, dass ein Ethnograph der Gegenwartsgesellschaft nicht nur mit der »Befremdung der eigenen Kultur « rechnen muss, sondern ebenso mit einer Befremdung des eigenen Forscher-Selbst, dessen Vertrautheit durch ein Aufdecken und eine Berücksichtigung seiner diskursiven und kontextuellen Konstitution erschüttert wird.

Ethnographie stellt für die Cultural Studies ein interpretatives Projekt dar, das sie im Rahmen umfassender Kultur- und Gesellschaftsanalysen betreiben. Jede Stufe im ethnographischen Prozess von der Auswahl des Untersuchungsobjekts bis zum Schreiben des Forschungsberichts - ist wesentlich durch interpretative Praktiken bestimmt. Es scheint an der Zeit zu sein, die entscheidende Bedeutung der »Kunst der Interpretation « in der qualitativen Forschung anzuerkennen und Konsequenzen daraus zu ziehen. Dabei sollte jedoch der Dialog zwischen Hermes und Zeus nicht vergessen werden, an den Crapanzano erinnert: Hermes versprach, keine Lügen zu erzählen, aber auch nicht die ganze Wahrheit (Crapanzano I996: I88).

\section{Ammerkungen}

I Erste Überlegungen zu diesem Thema habe ich 1999 bei der gemeinsam mit Klaus Neumann-Braun veranstalteten Jahrestagung der Sektion Medien- und Kommunikationssoziologie an der Technischen Hochschule Aachen, im Jahr 2000 auf Einladung von Claudia Honegger am Institut für Soziologie der Universität Bern und bei der Third International Crossroads in Cultural Studies Conference an der Universität Birmingham vorgetragen.

2 Vgl. exemplarisch die Arbeiten von Patricia Hill Collins (I99I), die eine afrozentrische, feministische Epistemologie entwickelt, und von Donna Haraway (1995).

3 So versuchen Toby Miller und Alec McHoul (1998) die Ethnomethodologie in die Cultural Studies einzubringen. Pertti Alasuutari (I995) zeigt, dass auch Fragebögen und statistische Auswertungen ihren Platz innerhalb dieser Forschungstradition finden können.

4 Vgl. die Beiträge in Denzin/Lincoln (I994) und (2000). Seit 200I 
gibt Denzin auch die Zeitschrift Cultural Studies $\leftrightarrow$ Critical Methodologies heraus.

5 Vgl. hierzu die instruktive Analyse von Patricia T. Clough (I992).

6 Einen post-positivistischen Ansatz verfolgen auch Neumann-Braun und Deppermann (I998), die der Auffassung sind, dass erst die Analyse transkribierter Gespräche einen Zugang zur eigentlichen Alltagsrealität jugendkultureller Praktiken ermögliche.

7 Bezogen auf unseren Kontext könnten Fragestellungen, seinem Ansatz folgend, folgendermaßen aussehen: Wie interpretieren türkische Jugendliche in Berlin-Kreuzberg Gangsta-Rap, den sie auf MTV an einem Samstagabend im Zimmer eines der Jugendlichen sehen, das mit Postern von Rappern geschmückt ist? Was gefällt den erwachsenen Mitgliedern eines Fanclubs an The Night of the Living Dead bei einem Treffen in einer nebligen Novembernacht in der Nähe einer alten Burgruine am Rhein?

\section{Literatur}

Alasuutari, Pertti (I995): Researching Culture: Qualitative Method and Cultural Studies, Thousand Oaks/London/New Delhi: Sage.

Ang, Ien (I997): »Radikaler Kontextualismus und Ethnographie in der Rezeptionsforschung«. In: Andreas Hepp/Rainer Winter (Hg.), Kultur - Medien - Macht. Cultural Studies und Medienanalyse, Opladen: Westdeutscher Verlag, S. 85-102.

Appadurai, Arjun (I996): Modernity at Large. Cultural Dimensions of Globalization, Minneapolis: University of Minnesota Press.

Becker, Howard S. (I973): Außenseiter, Frankfurt a.M.: S. Fischer.

Clifford, James (ı986). »Introduction: Partial Truths«. In: James Clifford/George E. Marcus (Hg.), Writing Culture, Berkeley: University of California Press, S. I-26.

Clifford, James (I988): The Predicament of Culture, Cambridge: Harvard University Press.

Clifford, James (ı999): »Kulturen auf der Reise«. In: Karl H. Hörning/Rainer Winter (Hg.), Widerspenstige Kulturen. Cultural Studies als Herausforderung, Frankfurt a.M.: Suhrkamp, S. 476-513.

Clifford, James/Marcus, George E. (Hg.) (I986): Writing Culture, Berkeley: University of California Press. 
Clough, Patricia T. (I992): The End(s) of Ethnography. From Realism to Social Criticism, Thousand Oaks/London/New Delhi: Sage.

Collins, Patricia H. (I99I): Black Feminist Thought, New York: Routledge.

Crapanzano, Vincent (I996): »Das Dilemma des Hermes: Die verschleierte Unterwanderung der ethnographischen Beschreibung«. In: Doris Bachmann-Medick (Hg.), Kultur als Text, Frankfurt a.M.: Fischer, S. 98-I2I.

de Certeau, Michel (I988): Kunst des Handelns, Berlin: Merve.

Denzin, Norman K. (I995): The Cinematic Society. The Voyeur's Gaze, Thousand Oaks/London/New Delhi: Sage.

Denzin, Norman K. (I997): Interpretive Ethnography, Thousand Oaks/ London/New Delhi: Sage.

Denzin, Norman K. (I999): »Ein Schritt voran mit den Cultural Studies«. In: Karl H. Hörning/Rainer Winter (Hg.), Widerspenstige Kulturen. Cultural Studies als Herausforderung, Frankfurt a.M.: Suhrkamp, S. II6-I45.

Denzin, Norman K. (200I): »Editor's Introduction«. Cultural Studies $\leftrightarrow$ Critical Methodologies I/I, S. 3-4.

Denzin, Norman K./Lincoln, Yvonna S. (Hg.) (I994): Handbook of Qualitative Research, Thousand Oaks/London/New Delhi: Sage.

Denzin, Norman K./Lincoln, Yvonna S. (Hg.) (2000): Handbook of Qualitative Research. Second Edition, Thousand Oaks/London/ New Delhi: Sage.

Fiske, John (I989): Understanding Popular Culture, Boston: Unwin Hyman.

Fiske, John (I999): »Wie ein Publikum entsteht. Kulturelle Praxis und Cultural Studies«. In: Karl H. Hörning/Rainer Winter (Hg.), Widerspenstige Kulturen. Cultural Studies als Herausforderung, Frankfurt a.M.: Suhrkamp, S. 238-263.

Fiske, John (2000): Lesarten des Populären, Wien: Turia + Kant.

Foucault, Michel (1976): Überwachen und Strafen. Die Geburt des Gefängnisses, Frankfurt a.M.: Suhrkamp.

Foucault, Michel (I999): In Verteidigung der Gesellschaft. Vorlesungen am Collège de France (1975-1976), Frankfurt a.M.: Suhrkamp.

Geertz, Clifford (1983): Dichte Beschreibung, Frankfurt a.M.: Suhrkamp.

Geertz, Clifford (1988): Works and Lives. The Anthropologist as Author, Cambridge: Polity Press. 
Goffman, Erving (I97I): Interaktionsrituale, Frankfurt a.M.: Suhrkamp.

Goffman, Erving (I977): Rahmen-Analyse, Frankfurt a.M.: Suhrkamp.

Goffman, Erving (I983): »Felicity's Condition«. American Journal of Sociology 89/1, S. I-53.

Grossberg, Lawrence (1988): »Wandering Audiences, Nomadic Critics«. Cultural Studies 3, S. 377-391.

Grossberg, Lawrence (I992): We Gotta Get Out of This Place, New York: Routledge.

Grossberg, Lawrence (I999). »Was sind Cultural Studies?«. In: Karl H. Hörning/Rainer Winter (Hg.), Widerspenstige Kulturen. Cultural Studies als Herausforderung, Frankfurt a.M.: Suhrkamp, S. $43-83$.

Habermas, Jürgen (I968): Erkenntnis und Interesse, Frankfurt a.M.: Suhrkamp.

Hall, Stuart (I990): »The Emergence of Cultural Studies and the Crisis of the Humanities«. October 53, S. II-23.

Hall, Stuart (I999): »Die zwei Paradigmen der Cultural Studies«. In: Karl H. Hörning/Rainer Winter (Hg.), Widerspenstige Kulturen. Cultural Studies als Herausforderung, Frankfurt a.M.: Suhrkamp, S. $13-42$.

Haraway, Donna (1995): Die Neuerfindung der Natur. Primaten, Cyborgs und Frauen, Frankfurt a.M./New York: Campus.

Hirschauer, Stefan/Amann, Klaus (I997): »Die Befremdung der eigenen Kultur. Ein Programm«. In: Stefan Hirschauer/Klaus Amann (Hg.), Die Befremdung der eigenen Kultur. Zur ethnographischen Herausforderung soziologischer Empirie, Frankfurt a.M.: Suhrkamp, S. 7-52.

Hoggart, Richard (I957): The Uses of Literacy, London: Chatto \& Windus.

Hörning, Karl H./Winter, Rainer (Hg.) (I999): Widerspenstige Kulturen. Cultural Studies als Herausforderung, Frankfurt a.M.: Suhrkamp.

Kellner, Douglas (I995): Media Culture, New York: Routledge.

Krug, Gary (I992): »Technologies of the Real, and Realist Ethnographies of Video Texts«. Studies in Symbolic Interaction, Jg. I3, S. 59-75.

Lash, Scott/Urry, John (I994): Economies of Signs and Space, London/ Thousand Oaks/New Delhi: Sage. 
Miller, Toby/McHoul, Alec (I998): Popular Culture and Everyday Life, Thousand Oaks/London/New Delhi: Sage.

Mills, Charles W. (I973): Kritik der soziologischen Denkweise, Darmstadt/Neuwied: Luchterhand.

Nelson, Cary/Treichler, Paula/Grossberg, Lawrence (I992): »Cultural Studies: An Introduction«. In: Lawrence Grossberg/Cary Nelson/Paula Treichler (Hg.), Cultural Studies, New York: Routledge, S. I7-22.

Neumann-Braun, Klaus/Deppermann, Arnulf (I998). »Ethnographie der Kommunikationskulturen Jugendlicher«. Zeitschrift für Soziologie 27/4, S. 239-255.

Williams, Raymond (I958): Culture and Society 1780-1950, London: Chatto \& Windus.

Williams, Raymond (I961): The Long Revolution, London: Chatto \& Windus.

Williams, Raymond (I977): Innovationen. Über den Prozeßcharakter von Literatur und Kultur, Frankfurt a.M.: Syndikat.

Willis, Paul (I979): Spaß am Widerstand. Gegenkultur in der Arbeiterschule, Frankfurt a.M.: Syndikat.

Willis, Paul (1981): »Profane Culture«. Rocker, Hippies. Subversive Stile der Jugendkultur, Frankfurt a.M.: Syndikat.

Winter, Rainer (I995): Der produktive Zuschauer. Medienaneignung als kultureller und ästhetischer Prozeß, München: Quintessenz.

Winter, Rainer (2001): Die Kunst des Eigensinns. Cultural Studies als Kritik der Macht, Weilerswist: Velbrück Wissenschaft.

Winter, Rainer/Mikos, Lothar (Hg.) (200I): Die Fabrikation des Populären. Der John Fiske-Reader, Bielefeld: transcript. 


\section{Baustellen in Wien:}

\section{Ein kulturwissenschaftlicher Werkstattbericht}

CHRISTINA LUTTER

Im Juni 2000 fand in Birmingham, dem »Gründungsort« der Cultural Studies, die dritte Crossroads in Cultural Studies Conference statt. Es handelt sich um die weltweit größte Tagung zu Theorien, Methoden und Praxis der unterschiedlichsten Personen und Formationen, die der Umbrella-Begriff Cultural Studies umfasst: In über hundert Arbeitsgruppen wurden nahezu 900 Referate gehalten. Die Bandbreite der Themen reichte von Phänomenen wirtschaftlicher und medialer Globalisierung über Identitäts- und Differenzkonzepte und ihrer politischen Effektivität bis hin zu den unterschiedlichen Entwicklungen der Cultural Studies in verschiedenen geographischen Kontexten, so etwa in Lateinamerika und Südostasien - um nur einige wenige zu nennen ${ }^{\mathrm{I}}$.

Zwei Sessions der Veranstaltung setzten sich mit Unterschieden und Gemeinsamkeiten zwischen »den Cultural Studies« und »den Kulturwissenschaften « im deutschsprachigen Raum auseinander sowie mit bereits erfolgten und möglichen Rezeptionen der Cultural Studies in den deutschsprachigen Ländern. Auch die vorliegende Publikation, deren Konzept wohl nicht ganz zufällig bald nach Birmingham 2000 entstanden ist, beschäftigt sich mit diesen Fragen. Der kurze Text, den die Herausgeber den Autorinnen und Autoren als Leitfaden für ihre Beiträge zur Verfügung gestellt haben, verweist für mich aus zwei Gründen auf ein attraktives Vorhaben: Zum einen 
besteht er zu mehr als der Hälfte aus Fragen, was im hiesigen akademischen Feld leider immer noch unüblich ist, wo lieber Antworten präsentiert werden, bevor die Fragen noch gestellt worden sind. Der Leitfaden hingegen lässt zusammen mit dem Titel »Werkzeugkiste« hoffen, dass es hier darum gehen könnte, etwas Neues zu entwickeln und auszuprobieren und sich darüber zunächst einmal auszutauschen. Außerdem artikuliert der Text (zumindest auch) ein Interesse nicht nur an akademischen Fragestellungen und Debatten, sondern an den »(forschungs-)politischen Implikationen der Cultural Studies« und den »Chancen für ihre Umsetzung «.

Eine der ersten Fragen des Leitfadens gilt den »Vermittlungschancen derjenigen Aspekte [...], die das Spezifische der Cultural Studies ausmachen«, gefolgt von jener, »worin diese Spezifik liegt«. Ich halte diese Fragen insofern für zentral, als ich denke, dass sie weder grundsätzlich noch ein für allemal zu beantworten sind, sondern immer nur mit Bezug auf konkrete (historische) Kontexte und Problemstellungen. Nicht zuletzt darin besteht der »radikale Kontextualismus«, der für zahlreiche Vertreterinnen und Vertreter der Cultural Studies einer der zentralen Aspekte ihrer theoretischen und praktischen Arbeit ist (vgl. Grossberg 2000a). Andererseits lässt sich die Notwendigkeit kaum bestreiten, sich durch Begriffe, die ihrerseits Paradigmen beschreiben, darüber $\mathrm{zu}$ verständigen, worüber man spricht und wie man sein Tun definiert und legitimiert. Derartige Ordnungskategorien laufen allerdings Gefahr, absolut gesetzt zu werden und damit zu erstarren. Die Herausforderung für Vermittlung und Umsetzung von Zugängen der Cultural Studies liegt daher nicht zuletzt darin, Wege zu finden, die eigenen Werkzeuge - und nichts anderes sind Begriffe, Kategorien und Theorien - nicht abstrakt, sondern kontextuell und relational zu definieren und sie in ihrem Gebrauch laufend auf ihre Wirksamkeit zu überprüfen.

Dieser Beitrag ist ein Versuch, das zu tun. Ich möchte daher meine Überlegungen an einem konkreten historischen Ort, in Birmingham, beginnen - wobei es sich im Grunde genommen aus meiner Perspektive und für die Fragestellungen dieses Textes um zwei Orte, um zwei Kontexte handelt: Das wissenschaftliche und politische Paradigma, auf das ich mich in der Folge beziehen werde, ist jenes der »British Cultural Studies«, das im Großbritannien der ig6oer und 7oer Jahre geprägt wurde und sich in der Zwischenzeit in sehr unterschiedliche Richtungen weiterentwickelt hat. Darüber hinaus dient 
mir »Birmingham 2000«, die dort geführten Debatten und ihre Konsequenzen für mögliche kulturwissenschaftliche Praxisfelder im deutschsprachigen Raum als Ausgangspunkt meiner Überlegungen. Diese beiden »Orte« möchte ich zueinander in Beziehung setzen. Meine Verwendung des Begriffs »Cultural Studies« benennt diese Beziehung.

Die Geschichte der britischen Cultural Studies ist mittlerweile oft erzählt und zusammengefasst worden (vgl. Literaturverzeichnis). Ich werde mich daher darauf beschränken, zunächst einige Bausteine dieses Paradigmas zu erläutern, die für mein eigenes Verständnis von Cultural Studies wichtig sind. $\mathrm{Zu}$ diesen Bausteinen gehören unter anderem Konzepte von Raum und Zeit, Geschichtlichkeit und Kontextualität. Bausteine erhalten ihre Bedeutung an den Orten, an denen man sie verwendet, und durch die Gebäude, die man mit ihnen errichten will. Diese Baustellen können Universitäten oder andere Institutionen, (wissenschaftliche) Veranstaltungen oder Forschungsprojekte sein, sie finden sich im Lehrbetrieb genauso wie in künstlerischen und politischen Aktionen, und sie können im Aufbau von Netzwerken bestehen. Eine solche Baustelle werde ich anhand eines konkreten Beispiels vorstellen. Dies wird mich abschließend zur Frage nach den Gebäuden, die wir bauen wollen, - also den Zielen kulturwissenschaftlicher Arbeit - führen. Von ihnen hängt es ab, welche Bausteine wir auf welchen Baustellen verwenden, davon wiederum, wie stabil und gleichzeitig veränderbar und ausbaufähig die errichteten Gebäude sein werden.

\section{Kontext und Praxis}

Eine Annäherung an das Paradigma der britischen Cultural Studies ist am einfachsten über den historischen Kontext, der sie bedingt und ermöglicht hat. Da sich ihre frühen Vertreter (und später auch Vertreterinnen) von Beginn ihrer Tätigkeiten an über ihre projektorientierten, explizit antidisziplinären Interessen und Arbeitsweisen definierten, lassen sich diese kaum in Begriffen wissenschaftlicher Disziplinen beschreiben. Was Cultural Studies in der Birminghamer Tradition ist, lässt sich schwerer sagen, als was die Vertreterinnen und Vertreter des Projekts taten und tun. Zu Beginn stammten viele von ihnen aus dem Arbeitermilieu Nordenglands bzw. Wales' und reagier- 
ten auf einen Bildungsbegriff und eine wissenschaftliche Praxis, die »hohe Literatur « in Form eines literarischen Kanons zur Leitmetapher von »Kultur« überhaupt erhoben. Diese »Hoch-Kultur« war ein zentrales Mittel zur Erziehung der Eliten in England und in den britischen Kolonien. Nach dem Zweiten Weltkrieg hatte die LabourRegierung eine Reihe von Bildungsprogrammen eingerichtet, in denen auch Angehörige der Arbeiterklasse mit dieser Tradition konfrontiert wurden. Sie stießen zunächst als Lernende, später selbst als Lehrende auf Vorstellungen von Kultur, Bildung und Moral, die ihre eigene kulturelle Herkunft ebenso vernachlässigten oder ausgrenzten wie die gleichzeitig zunehmend präsente amerikanische Massenmedienkultur.

In dieser Konstellation wurzelte ihr intellektuell, politisch und pädagogisch motiviertes Interesse, die Zusammenhänge und Wechselwirkungen von sozialer Herkunft, kulturellen Formen und ihrer Bewertung in Forschung und Lehre zugänglich zu machen. Sie räumten der persönlichen Erfahrung (lived experience) der lehrenden und forschenden Personen wie derjenigen, die sie unterrichteten, nicht nur in der pädagogischen Praxis einen zentralen Stellenwert ein, sondern bezogen ihre lebensweltlichen Positionen auch in die wissenschaftliche Darstellung und Reflexion mit ein. Damit verbunden war die Ausweitung des Kulturbegriffes im Sinne von culture as a whole way of life (R. Williams). Dieser Begriff artikuliert ein wissenschaftliches und politisches Interesse an konkreten Menschen, an den ökonomischen und sozialen Strukturen, die ihr Leben bestimmen, aber genauso daran, wie sie im Rahmen dieser vorgegebenen Bedingungen Möglichkeiten für individuelles Handeln (agency) finden: Welche kulturellen Repertoires stehen ihnen dafür zur Verfügung? Unter welchen Umständen und auf welche Weisen werden sie genutzt? Wie gestalten kulturelle Praktiken konkrete Lebenswelten und geben ihnen Sinn? Wie wirkt Praxis ihrerseits auf Bedingungen und Repertoires zurück, und kann sie diese verändern? Im Zentrum der Fragestellungen des sozialen und politischen Projekts Cultural Studies standen also immer konkrete Machtverhältnisse, ihre Effekte auf lebensweltliche Realitäten und ihre meist widersprüchlichen Wechselwirkungen mit kulturellen Praktiken.

Die Sensibilität dafür, wie komplex und widersprüchlich Machtrelationen sind, bedingte gleichzeitig die Kritik an einer angeblich wertfreien, scheinbar »objektiven« Wissenschaft, wie sie im klassischen 
akademischen Betrieb postuliert wird: Zurückgewiesen wurde die Behauptung, Wissenschafterinnen und Wissenschaftler stünden außerhalb dessen, was untersucht wird, und könnten dadurch objektive Aussagen darüber treffen, wie die Welt wirklich ist. Tatsächlich werden so subjektive Interessen verschleiert, die letztlich darauf ausgerichtet sind, die soziale Ordnung und in ihr bestehende Machtverhältnisse aufrechtzuerhalten - eine Kritik, der auch das eigene wissenschaftliche und politische Tun beständig unterzogen wird (bzw. werden sollte).

Für die Konsolidierung der britischen Cultural Studies in den I96oer und 7oer Jahren, die einen sehr offenen, disziplinenübergreifenden und netzwerkartigen Charakter hatte, stehen Institutionen wie das Centre for Contemporary Cultural Studies (CCCS) in Birmingham und die Erwachsenenbildungsprogramme der Open University. Besonders unter der Leitung des CCCS durch Stuart Hall (I968-I979), der seit 1977 auch an der Open University unterrichtete, entstanden jene richtungsweisenden Arbeiten, die wissenschaftliche Forschung mit politischem Engagement verbanden. Texte wie Resistance Through Rituals (vgl. Hall/Jefferson I976) oder Policing the Crisis (vgl. Hall et al. I978) zeigen, wie Hall eine »populärere« Pädagogik mit einem experimentellen Zugang in Forschung und Lehre verband (vgl. McRobbie 2000). Im Zentrum seiner vielfältigen Aktivitäten steht jedoch bis heute die Frage, wie man Forschungs- und akademische Tätigkeiten mit politischer Arbeit vereinbaren, wie man Theorie politisieren und Politik theoretisieren und so auf aktuelle politische Herausforderungen bessere Antworten finden kann (Grossberg 2000a: 262 f.).

Das Interesse gilt daher nicht primär kulturellen Artefakten und den Bedeutungen, die ihnen angeblich inhärent sind. Bedeutung und Sinn spielen zwar eine wichtige Rolle für das Verständnis der Cultural Studies, doch sie liegen nicht in den Dingen an sich, sondern werden in ihrem Gebrauch und daher immer wieder neu hergestellt. Diese Bedeutungen, besonders aber ihre unmittelbaren Wirkungen auf die betroffenen Menschen, entstehen also vor allem in der Praxis. Ein derartiger »praktischer« Kulturbegriff ist ein taugliches Instrument, jene Identitäts- und Gemeinschaftsbegriffe zu dekonstruieren, die von einer normativ festgelegten Identität ausgehen, die wiederum als Basis für Ansprüche auf kulturelle Autorität dient. Wenn hingegen kulturelle Identitäten in und durch kulturelle Praktiken entstehen, können sie niemals »fertig« und abgeschlossen sein, sondern sind ständigen 
Veränderungen unterworfen. »Kultur« äußert sich dann immer im komplexen Wechselspiel von Artikulationen, Prozessen und Praktiken in spezifischen ökonomischen, sozialen und politischen Kontexten.

\section{Raum Und ZeIt}

Identitäts- und Differenztheorien gehören seit den I980er Jahren zu den besonders intensiv diskutierten Feldern der Cultural Studies. Vor allem in den letzten Jahren mehren sich allerdings die kritischen Stimmen zu den theoretischen Prämissen des Nachdenkens über Fragen individueller und kollektiver Identitäten. Für zentral halte ich vor allem die konsequente Kritik an binären Oppositionen, besonders an jener zwischen Identität und Differenz (vgl. Hall/Du Gay i996; Gilroy 2000; Grossberg I995, 2000b). Sie geht davon aus, dass Differenz selbst eine historische Konstruktion ist, ein Produkt moderner Machtstrukturen: Differenz und Identität sind Effekte von Macht. Das moderne Denken funktioniert daher nicht nur über binäre Gegensätze, sondern erzeugt sie als konstitutive Differenzen, in denen der/das Andere immer durch seine »Negativität« gegenüber dem Eigenen definiert wird.

Eine Kritik an der binären Opposition zwischen Identität und Differenz erfordert, so etwa Lawrence Grossberg, eine umfassende Auseinandersetzung mit jener von Zeit und Raum, besonders mit Vorstellungen von Kontinuität, Linearität und Kausalität, durch die Zeit als Geschichte konstruiert wird (Grossberg 200ob: 152 ff.). Grossberg formuliert seine Kritik an der »modernen Konstruktion von Zeit als Geschichte« (ebd.: 154) mit Bezug auf Walter Benjamin und Antonio Gramsci sowie auf Michel Foucault, Gilles Deleuze und Felix Guattari (etwa Grossberg 2000a: 36-38). Foucault, um nur ein Beispiel zu nennen, knüpft in seiner Kritik an Geschichte und Geschichtswissenschaft und zur Entwicklung seines Konzepts der Genealogie an Friedrich Nietzsches Auseinandersetzung mit dem Nutzen und Nachteil der Historie für das Leben (I873) an. ${ }^{2}$ Foucault definiert Genealogie als »Gegensatz zur metahistorischen Entfaltung der idealen Bedeutungen und unbegrenzten Teleologien« (Foucault I987 [I97I]: 69). Er bezieht sich auf die Begriffe Ursprung, Entstehung und Herkunft bei Nietzsche: Die genealogische Geschichtsbetrachtung suche nicht nach dem Ursprung als dem einen Ort der Wahrheit, die 
aller Wissenschaft und Erkenntnis voraus liegt, sondern nach den vielfältigen Episoden und Ereignissen der Geschichte, die nebeneinander und oft gegeneinander existieren (ebd.: 72). Ebenso gehe es bei der Frage nach der Herkunft nicht um die Suche nach (verlorenen) Identitäten, sondern um das Aufdecken von Diskontinuitäten, der »subtilen individuellen und subindividuellen Spuren [...], die sich in einem Individuum kreuzen können und ein schwer entwirrbares Netz bilden« (ebd.: 73). Schließlich übt Foucault mit Nietzsche Kritik an jenen Geschichtsbetrachtungen, die Entstehung ausgehend von der Gegenwart, also vom Endpunkt her definieren, wohingegen ihm zufolge Entstehung immer ein spezifisches historisches Kräfteverhältnis benötigt (ebd.: 76).

Nietzsches Kritik an »jene[r] Historie, die einen überhistorischen Gesichtspunkt einführt«, führt bei Foucault zur Genealogie als »wirkliche[r] Historie« (ebd.: 78). Er stellt damit zwei unterschiedliche Geschichtskonzepte einander gegenüber: Die »traditionelle Historie« sucht Geschlossenheit und Einheit anstelle einer Vielfalt der Ereignisse und Zeiten. Sie harmonisiert Widersprüchlichkeiten und Brüche und konstruiert Kontinuitäten. Sie sucht die Wahrheit und Totalität, nimmt dafür eine »apokalyptische Objektivität« (ebd.: 79) in Anspruch und verschleiert ihren eigenen Standort. Die »wirkliche Historie« hingegen nimmt die Offenheit, Vielfalt und Widersprüchlichkeit der Ereignisse wahr, die genealogische Methode macht Diskontinuitäten und Perspektivität sichtbar (ebd.: 80).

Wie verhält sich diese Vielzahl historischer Zeiten in ihren Überschneidungen zu unterschiedlichen Räumen? Doreen Massey formuliert diese Beziehung so: »Movement and the making of relations also take/make time « (Massey 2000: 227-229). Das Problem besteht ihr zufolge jedoch darin, dass Zeit meist abstrakt, Raum hingegen materiell gedacht wird. Erstens wird Raum häufig im Sinne einer Landschaft ${ }^{3}$ (landscape) als »Ausdehnung « (extension) wahrgenommen und dadurch mit dem Materiellen gleichgesetzt. Zweitens führt die Vorstellung von Raum als Landschaft dazu, dass Raum als Oberfläche (surface) und damit gegeben und kontinuierlich erscheint. Diese Vorstellung wird vor allem durch die Repräsentation von Raum auf Landkarten verstärkt. Tatsächlich jedoch sei die Gegenwärtigkeit der räumlichen Horizontalität das Produkt einer Vielzahl von Geschichten, deren Resonanzen noch immer vorhanden sind. Sie besteht aus der Summe von Zeitpunkten in ihren Geschichten. Während die grundsätzliche 
Komplexität des Räumlichen meist anerkannt wird, geht man laut Massey umgekehrt meist davon aus, dass es möglich sei, das Zeitliche in einer einzelnen Erzählung (in a single narrative) zu repräsentieren. Das Zeitliche werde in der Einzahl gedacht, das Räumliche in einer unendlichen Vielzahl. Denkt man hingegen Zeit und Raum zusammen und als Produkt ihrer Beziehungen, löst sich das Problem, und gleichzeitig erübrigt sich die binäre Unterscheidung zwischen ihnen: Raum ist dann keine Oberfläche, keine durchgängige materielle Landschaft, sondern die zu einem bestimmten Zeitpunkt bestehende Konfiguration einer Vielzahl von Geschichten, die alle im Werden sind.

"Das ist kein „Problemı, außer natürlich man sucht nach der Ordnung der singulären Geschichte und der Lesbarkeit der Glätte einer Oberfläche॥ (ebd.: 229).

Was heißt das für eine »alternative kritische Praxis«? Anstelle Zeit und Raum getrennt zu denken, fragt Grossberg, was es bedeutet, in einem bestimmten Zeit-Raum verankert zu sein. Belonging - d.h. die Orte und Arten der Zugehörigkeit von Menschen - sei weniger eine Frage von Identität als von Identifikation: Identifikationen sind vielfältige Positionen, die wir einnehmen, von denen aus wir die Welt wahrnehmen, uns in Relation zu anderen definieren und Verbindungen zwischen verschiedenen Ereignissen herstellen. Zugehörigkeit (belonging) oder Identifikation ist ein »Set von Praktiken und Ereignissen, ein Milieu« (Grossberg 200ob: 154), eine »gelebte Geographie« (lived geography):

"Gelebte Geographie beschreibt - konstruiert - eine Ökonomie der Zugehörigkeiten (belongings). Sie beschreibt das alltägliche Leben im Sinn der Weisen, wie Menschen und Praktiken sich bewegen und [gleichzeitig] verankert sind (bzw. ruhen). Sie konstruiert eine Karte von Mobilitäten und Stabilitäten, von Räumen und Orten, Vektoren und Kräften. Sie beschreibt - konstruiert - die Transformationen und Überschneidungen, welche die ambivalenten und offenen Möglichkeiten sich verändernder Richtungen, Geschwindigkeiten und Heimaten (homes) definieren.

Aber bei dieser gelebten Geographie, diese Ökonomie, geht es ebenso um Zeitlichkeit und zeitliche Zugehörigkeiten (belongings); sie ist auch eine Geographie des Werdens (becomings)« (ebd.: 156). 


\section{Gegen eine globale Theoriekultur!}

Was bedeuten diese Vorstellungen eines »Zeit-Raums«, einer »gelebten Geographie« für die Anwendung der methodischen Instrumente der Cultural Studies? Mögliche Anknüpfungspunkte sollen in den folgenden Abschnitten skizziert werden: In der Einleitung zu ihrem Buch Too Soon Too Late. History in Popular Culture (1998) wendet sich Meaghan Morris gegen »blockbuster«-Theorien, in denen »riesige, monolithische Subjekte« (»der Westen«, »die Moderne« etc.) über enorme zeitliche und geographische Räume hinweg konstruiert, vereinheitlicht und verallgemeinert werden (ebd.: 2). Es gebe, so Morris in einem Interview mit Christian Höller im September I999, keine »alles nivellierende Globalkultur« (Morris I999: I82), wohl aber eine internationale Theoriekultur. Die Kritik an dieser Theoriekultur ist gleichzeitig eine an der hegemonialen Position der anglo-amerikanischen Cultural Studies. Sie wird in den letzten Jahren immer deutlicher artikuliert, so auch auf der Crossroads in Cultural Studies Conference in Birmingham. Besonders Vertreterinnen und Vertreter jener Formationen, deren wissenschaftliche und politische Arbeit mit Prozessen der Demokratieentwicklung verbunden ist (etwa in Lateinamerika, Südafrika oder Südostasien), beziehen eine äußerst kritische Position gegenüber der gängigen anglo-amerikanischen akademischen Praxis: Die jeweils verschiedenen politischen und akademischen Kontexte und Traditionen würden vernachlässigt oder zugunsten der hegemonialen Cultural Studies-Formationen und ihrer Terminologie homogenisiert ${ }^{4}$; lokale Erfahrungen und Arbeiten würden nach einem euro-amerikanischen Theoriekanon analysiert und so in einen falschen Referenzrahmen gestellt.

Diese Kritik eröffnet Möglichkeiten, darüber nachzudenken, wie und unter welchen Bedingungen zwischen heterogenen, zeit-räumlich verschiedenen und oft konfligierenden Wissensformen Übersetzungen im wörtlichen und im übertragenen Sinne stattfinden können. Gleichzeitig lässt sich damit die Frage reformulieren, welche Relevanz eine je unterschiedliche Praxis von Cultural Studies an verschiedenen Orten hat. Meaghan Morris gibt eine optimistische Antwort und beantwortet damit gleichzeitig die Frage, wozu Cultural Studies Geschichte brauchen: »I tend to want history as a source of a liberating certainty that anything could happen« (Morris I998: 26). Dabei geht es keineswegs um ein liberalistisch verstandenes »anything goes«, das 
Machtverhältnisse ausblendet und gleichzeitig Beliebigkeit wie letztlich Sinnlosigkeit zu handeln nahe legt, sondern im Gegenteil um ein Wahrnehmen der eigenen Verantwortung. In einer theoretisch generalisierenden Form wäre »anything could happen « bedrohlich beliebig, in spezifischen - geographischen und historischen - Kontexten steht jedoch zur Debatte, was konkret geschehen kann, was möglich ist. Diese Möglichkeiten sind zwar immer abhängig und damit auch begrenzt von ihren jeweiligen politischen und sozio-ökonomischen Bedingungen, aber gleichzeitig immer auch offen und damit unbegrenzt in ihrer Vielfalt in Hinblick darauf, wie Menschen innerhalb dieser Rahmenbedingungen handeln und sie damit auch verändern können.

\section{Kategorien und Institutionen}

Diese Sensibilität für Kontexte sollte gerade bei den Überlegungen, welche Elemente »der anglo-amerikanischen Cultural Studies« im deutschsprachigen Raum nützlich sein könnten, vorsichtig machen. Die Methode des »radikalen Kontextualismus« ist auch auf die Ordnungskategorien und Organisationsformen, die Paradigmen und Institutionen der jeweiligen wissenschaftlichen (und politischen) Praxis anzuwenden. Kategorien sind Instrumente, die dazu dienen, die Welten zu beschreiben, in denen wir leben und die wir untersuchen. Sie helfen, Ordnung in ihnen zu schaffen. Als solche sind sie nützliche Werkzeuge - ob es darum geht, mit mittlerweile »klassischen« Kategorien wie »race«, »class« und »gender« die soziale Welt zu erfassen, oder darum, mit Konzepten wie »Kultur« oder »Gesellschaft« die Bedingungen und Optionen zu beschreiben, die Handlungsspielräume eröffnen oder verschließen.

Diese Kategorien und Konzepte müssen allerdings als erkenntnistheoretische Instrumente offen bleiben für Kritik, Modifikationen und gelegentlich auch für die Entscheidung, sie in bestimmten $\mathrm{Zu}-$ sammenhängen nicht mehr zu verwenden. Nach den Kosten und Nutzen von Begriffen zu fragen, bedeutet zu überlegen, inwieweit sie (noch) dafür tauglich sind, das zu beschreiben, was wir ausdrücken wollen, oder ob sie mehr Missverständnisse erzeugen als sie aufklären. Benennungen von Paradigmen und Disziplinen sind ebenfalls solche Kategorien. In einer erhellenden netzwerktheoretischen ver- 
gleichenden Analyse des Birmingham-Paradigmas der Cultural Studies und der »Neuen Kulturwissenschaften« in Deutschland formuliert das Ulf Wuggenig für die universitären Disziplinen so:

"Ein Paradigma (vgl. Kuhn 1977) macht aus einer Gruppe von Wissenschafter/innen eine wissenschaftliche Gemeinschaft, deren Mitglieder gemeinsame Ziele verfolgen oder zumindest untereinander stark kommunizieren, sich teilweise auf die gleiche Literatur beziehen, sich in speziellen Zeitschriften verständigen und sich auch einer spezifischen Sprache bedienen. Die verhältnismäßig unproblematische Kommunikation und die relativ einhelligen Urteile sind möglich, weil es als gemeinsamen Besitz dieser Gemeinschaft eine disziplinäre Matrix mit begrifflichen und theoretischen Elementen gibt» (Wuggenig 1998: 43, Hervorhebung Ch.L.).

Die Cultural Studies der Birminghamer Tradition verfügen, so Wuggenig, trotz der antidisziplinären Haltung vieler ihrer Vertreterinnen und Vertreter über eine derartige disziplinäre Matrix. Das hat Vorund Nachteile: Die Definition von Kategorien und Paradigmen ist Teil von Institutionalisierungsprozessen. Sie sind einerseits notwendig, um Projekte wie das der Cultural Studies auf Dauer sichtbar zu machen, um ihren Bestand zu gewährleisten und denjenigen, die sie betreiben, Handlungsspielräume und Möglichkeiten der Weiterentwicklung zu geben. Dies erfordert eine Praxis der Benennung, um Standpunkte, Ziele, Abgrenzungen im Sinne eines Willens zur Veränderung und Orte der Auseinandersetzung überhaupt erst sichtbar zu machen. Andererseits sind solche Abgrenzungen aber immer auch Ausgrenzungen, Disziplin bedeutet immer auch Disziplinierung. Dazu kommt, dass gerade die Cultural Studies in ihrem Anspruch, keine Disziplin zu sein, dementsprechend auch keinen unveränderbaren Kanon und keine Institutionen begründen wollen, die Autoritäten ein für alle Mal festschreiben. Einen eindeutigen und allgemein gültigen Ausweg aus diesem Dilemma wird es wohl nicht geben. Umso mehr erfordert aber eine Annäherung an mögliche Lösungen eine Kritik an der eigenen Verwendung von generalisierenden Konzepten und Etiketten und die beständige Frage, wie und ob sie noch mit Inhalten zu füllen sind.

Im deutschsprachigen Raum sind es die »Neuen Kulturwissenschaften «, die im Rahmen der beginnenden akademischen Institutionalisierung vielfach über die Definitionsmacht verfügen, was "gute« oder »richtige« Kulturwissenschaft sei, und deren Vertreterinnen und 
Vertreter innerhalb der bestehenden Institutionen (mit)entscheiden, wessen Modell im akademischen Feld sichtbar und relevant wird oder bleibt und wer keinen Zugang zu diesen ökonomischen wie kulturellen Ressourcen hat. Dem wird man allerdings kaum begegnen können, indem man - wie es in letzter Zeit häufig geschieht - diesem quasi-hegemonialen Generalkonzept (ob es über eine disziplinäre Matrix im Sinne Wuggenigs verfügt, sei dahingestellt) unkritisch ein anderes, nämlich das »der Cultural Studies« gegenüberstellt. Dabei handelt es sich einmal mehr um die Konstruktion einer generalisierenden Opposition. Das gilt selbstverständlich auch für andere Wissenschaftsdiskurse, wie etwa die vorwiegend in den USA seit Jahrzehnten geführten Grundsatzdiskussionen zwischen Cultural Studies-Vertreterinnen und -Vertretern des »Kulturalismus« und »Textualismus« und jenen der »Politischen Ökonomie« (vgl. dazu den bezeichnenden Untertitel eines Aufsatzes von Grossberg aus dem Jahr I995 »Is anybody else bored with the debate?«). Dass derlei Debatten nicht nur langweilig sind, sondern für eine disziplinenübergreifende Praxis auch wenig bringen, darüber besteht grundsätzlich große Einigkeit. Gleichzeitig spielen im alltäglichen Verhandeln innerakademischer Machtpositionen (wo offenbar wenig sonst zu gewinnen ist?!) genau solche Gegensätze eine wichtige strategische Rolle: Der »gute«, in einem und für einen bestimmten Zusammenhang definierte und dementsprechend differenzierte eigene Zugang wird dem »schlechten«, generalisierten anderen gegenübergestellt. Aus dem Zusammenhang gerissene Beispiele »guter « und »schlechter« Praxis, welche absolut gesetzt die festgelegten Unterschiede belegen, finden sich allerdings immer auf der einen wie auf der anderen Seite und bestätigen nur die an sich banale Tatsache, dass es in jeder wissenschaftlichen und nichtwissenschaftlichen Praxis bessere und mittelmäßigere (wohl mehr von letzterer) Arbeit gibt.

Dazu kommt, dass die jeweils verwendeten Bausteine nie einem einzigen Paradigma zuzuordnen sind. Georg Simmel und Walter Benjamin, Antonio Gramsci und Louis Althusser, Roland Barthes und Michel Foucault, Gilles Deleuze und Félix Guattari und viele andere, ohne deren theoretische und methodische Instrumente die Werkzeugkisten der »Cultural Studies« wie der »Kulturwissenschaften« schlecht ausgestattet wären, repräsentieren unterschiedliche Traditionen, Denkstile, historische und räumliche Kontexte. Gleichzeitig werden ihre Arbeiten immer wieder neu gelesen, verwendet und angeeig- 
net. Auch ihre Bedeutungen stehen nicht fest, sondern entstehen in ihrem Gebrauch. Eine Archäologie verschiedener kulturwissenschaftlicher Formationen (und auch solcher, die nicht so bezeichnet wurden) macht hingegen deutlich, wie problematisch binäre Oppositionen im Sinne von »deutsche Kulturwissenschaften« vs. »anglo-amerikanische Cultural Studies« sind. Eine zeit-räumlich sensible kulturwissenschaftliche Praxis wird durch solche Konstruktionen mehr behindert als gefördert, da sie Grenzen ziehen, wo die Übergänge fließend sind, und damit das Denken in Netzwerken erschweren. ${ }^{5}$

$\mathrm{Zu}$ fragen ist vielmehr, wie eine Praxis der Benennung kulturwissenschaftlicher Projekte in ihren unterschiedlichen inhaltlichen Ausrichtungen und Organisationsformen aussehen könnte. Eine Benennungspraxis, die gleichzeitig ein kritisches Bewusstsein dafür erhält, dass jede Fest-Schreibung die latente Gefahr der Erstarrung und der Schwerpunktverlagerung von den Prozessen und Inhalten $\mathrm{zu}$ den Etiketten beinhaltet. Kontext, Prozess und Praxis als Schlüsselkonzepte der Cultural Studies könnten Ansatzpunkte bieten, eine Disziplinen und Institutionen überschreitende Verständigung über die Bausteine und Orte kulturwissenschaftlicher Praxis und ihre Benennung und organisatorische Institutionalisierung nicht als Gegensatz zu verstehen: Anstatt nur die identitätsstiftenden Kategorien unserer (akademischen) Beschäftigung zu benennen (Cultural Studies vs. Kulturwissenschaften ${ }^{6}$ ), könnte es vielversprechend sein, auch die Praxis selbst zu beschreiben: Das, was wir tun, wenn wir ein Problem haben und es lösen wollen, wenn wir dafür Fragestellungen entwickeln und nach geeigneten Instrumenten für ihre Beantwortung suchen - mittels und durch kulturelle und soziale Praktiken, die an historisch bestimmten Orten und unter spezifischen historischen Rahmenbedingungen wirksam werden.

\section{Kulturwissenschaft in der Praxis oder für eine PRAKTISChE KULturWissenschaft}

Diese Gleichzeitigkeit der Praxis und ihrer Beschreibung bzw. Benennung zeichnet ein kulturwissenschaftliches Netzwerkprojekt aus, das ich abschließend vorstellen möchte. Es handelt sich dabei um den Forschungsschwerpunkt Kulturwissenschaften/Cultural Studies, der seit I998 vom österreichischen Wissenschaftsministerium finanziert 
wird. In den letzten drei Jahren wurden über 50 Forschungsprojekte, an denen rund I5o Wissenschafterinnen und Wissenschaftler beteiligt sind, in Auftrag gegeben, auf deren Basis es gelungen ist, erstmals ein kulturwissenschaftliches Forschungsnetzwerk in Österreich zu schaffen, welches nun inhaltlich und strategisch ausgebaut werden soll.

Der Titel des Forschungsschwerpunktes - so bewusst sperrig er ist - soll eine prinzipielle Offenheit für die verschiedenen Ansätze kulturwissenschaftlicher Arbeit signalisieren. Es geht also um ein Verständnis von »Kulturwissenschaften« bzw. »Cultural Studies« nicht als abgegrenzte Forschungsbereiche oder -traditionen, sondern als Vielzahl von Disziplinen und Themenfeldern, die durch ihre innovativen methodischen Ansätze gleichzeitig Forschungsstrategien darstellen können. Die Fragestellungen betreffen dementsprechend wissenschaftstheoretische und methodische Herausforderungen ebenso wie gesellschaftliche und soziale Problemstellungen und die Möglichkeiten ihrer Lösung mit Hilfe der Erkenntnisse wissenschaftlicher Arbeit. Dabei stehen Fragen nach der Verteilung von Macht und nach der Begründung von Systemen der Ungleichheit immer in direktem Zusammenhang mit der Analyse von Kultur. Im Unterschied zu traditionellen Kulturbegriffen, die »Kultur« meist auf eine folkloristische oder elitäre Dimension reduzieren, zumindest aber klar von Wissenschaft, Forschung und Technologie trennen, ist es explizites Ziel des Forschungsschwerpunktes, einen wissenschaftlichen und politischen Kulturbegriff für eine konstruktive Diskussion um Stellenwert und Positionierung von Wissenschaft und ihrem Verhältnis zu Gesellschaft und Politik nutzbar zu machen.

Um diese grundsätzlichen Ziele umzusetzen, werden seit Ende der 1999 abgeschlossenen zweijährigen Projekteinreichphase gemeinsam mit einem internationalen Kreis von Expertinnen und Experten $^{7}$ kontinuierlich verschiedene forschungspolitische Instrumente entwickelt und evaluiert: Projekt- und disziplinenübergreifende Workshops und Methodenseminare sollen einen verstärkten Dialog über die theoretischen Grundlagen und die Praxis kulturwissenschaftlicher Arbeit ermöglichen. Sie dienen der Förderung problemorientierter Arbeit und der Vernetzung thematisch und methodisch verwandter Projekte. Diese Veranstaltungen können im Rahmen einzelner Projektgruppen oder in Kooperation mit anderen Projekten stattfinden und sollen gleichzeitig Orte zur Entwicklung neuer, experimentellerer Formen wissenschaftlicher Auseinandersetzung sein. Nach- 
wuchswissenschafterinnen und -wissenschaftler erhalten die Möglichkeit, bei internationalen Veranstaltungen ihre Projekte und Forschungsarbeiten an verschiedenen Orten und in verschiedenen institutionellen Zusammenhängen zu präsentieren. Internationale Fellowships sollen eine nachhaltig wirksame Förderung von jungen Wissenschafterinnen und Wissenschaftlern gewährleisten. Auf diese Weise soll wissenschaftliches Arbeiten im internationalen Zusammenhang mit dem Kennenlernen unterschiedlicher Kontexte des Lehrens und Forschens und einer längerfristigen Einbindung in Forschungsnetzwerke verbunden werden. Zentrales Anliegen des Forschungsschwerpunktes ist außerdem die Entwicklung von strategischen Modellen und die Förderung von Pilotprojekten zur Schaffung von institutionellen Rahmenbedingungen für eine problemorientierte, transdisziplinäre Forschungs- und Förderungspraxis.

Dieses work in progress erfolgt in ständigem Dialog mit den am Forschungsschwerpunkt beteiligten Forscherinnen und Forschern. Anlässlich der jährlichen Treffen des Kreises der Expertinnen und Experten findet heuer bereits zum zweiten Mal ein »Workshop-Tag« statt, zu dem alle Projektbeteiligten eingeladen sind. Sie erhalten dadurch die Möglichkeit, die anderen Projekte kennen zu lernen und in mehreren Arbeitsgruppen miteinander und mit den Expertinnen und Experten über inhaltliche und forschungsstrategische Aspekte ihrer Arbeit zu diskutieren: Im vergangenen Jahr fanden etwa Workshops zu inhaltlichen Themenfeldern:

- Zugänge zum Konzept/Begriff »Kultur«,

- Kultur, Identität und Politik,

- Popularkultur und Medien

sowie Arbeitsgruppen zu forschungstheoretischen und -strategischen Themen statt:

- Theorie und Praxis transdisziplinärer Forschung,

- Nachwuchsförderung durch Einbindung in internationale Forschungsnetzwerke,

- Chancen und Probleme der Institutionalisierung kulturwissenschaftlicher Forschung.

Eine der wichtigsten Zielsetzungen dieser Form des Dialogs, der 
Diskussion und der prozessorientierten Zusammenarbeit war und ist es, den Forschungsschwerpunkt als gemeinsames Projekt zu entwickeln, dessen thematisch-methodische Vernetzung selbst Teil des Programms ist.

Wenn die inhaltliche und strategische Konzeption des Schwerpunktes sich auch grundsätzlich explizit am Birmingham-Paradigma orientiert (vgl. Lutter I999), sollen gleichzeitig jedoch die Vorgaben, was Cultural Studies bzw. Kulturwissenschaften sein und wie sie betrieben werden können, möglichst gering gehalten werden. Im Gegenteil, ein wichtiger Bestandteil des inhaltlichen Konzepts und des beschriebenen Dialogprozesses ist es, anhand der einzelnen Projekte, die sich thematisch mit sehr unterschiedlichen Problemstellungen auseinander setzen, gemeinsam die verschiedenen Traditionen und Formationen kulturwissenschaftlicher Arbeit und ihre personellen wie ideellen Zusammenhänge »archäologisch« zu untersuchen.

Über diese wissenschaftsgeschichtlichen Aspekte hinaus geht es allerdings besonders um ein Nachdenken über das jeweilige Selbstverständnis, um Fragen nach den erkenntnistheoretischen Kategorien der Forschungsarbeiten und ihrer methodischen Umsetzung. Gleichzeitig bedingt diese Offenheit eine große thematische Heterogenität, die es schwierig macht - und das scheint mir symptomatisch zu sein -, die einzelnen Projekte zu »ordnen«. Ein Beispiel: Aktuell entwickeln wir gerade eine Website für den Forschungsschwerpunkt, die nicht nur Informationen über das Programm und die Inhalte der einzelnen Projekte präsentieren, sondern das Medium selbst für den Auf- und Ausbau eines kulturwissenschaftlichen Netzwerkes nutzen soll. ${ }^{8}$ Dabei stellte sich zu Beginn die Frage, nach welchen Kriterien ein Organigramm erstellt werden könnte: So gibt es Projekte, die sich über ihre Beschäftigung mit Jugend-, Popular- und Medienkulturen definieren lassen. Andere untersuchen verschiedene Wissenschaftskulturen. Wieder andere setzen sich mit Fragen zu Diskriminierungen aufgrund von Herkunft, sozialer Situation oder Religion und Möglichkeiten des Zusammenlebens in urbanen Problemgebieten auseinander. Diese Fragestellungen ließen sich noch vergleichsweise leicht kategorisieren.

Gleichzeitig gibt es aber Projekte, deren kulturwissenschaftliche Aspekte primär in der Art der Zusammenarbeit liegen, die etwa als Projektgruppen oder -verbünde im Sinne der frühen Birminghamer Praxis organisiert sind - mit allen Möglichkeiten und Chancen, aber 
auch Schwierigkeiten und Unsicherheiten, die für solche Kooperationen charakteristisch sind. Andere Projekte kann man anhand ihrer Reflexionen über den Kulturbegriff in verschiedenen historischen Zusammenhängen beschreiben, wieder andere über ihren Zugang, Wissenschaft als Praxis zu verstehen und experimentell zu betreiben und zu verändern. Die Liste ließe sich fortsetzen, und alle diese möglichen Ordnungskriterien überschneiden sich in den einzelnen Projekten auf unterschiedliche Weise. Bei der Suche nach Kriterien für die Beschreibung eines kulturwissenschaftlichen Netzwerkes stößt man also schnell an Grenzen. Für ein Projekt, das sich den virtuellen Raum zunutze macht, bietet sich damit aber gleichzeitig die Chance, solche Einteilungen und Klassifikationen nicht alternativ, einander ausschließend zu denken oder verschiedene Kategorien einer zentralen unterzuordnen (etwa Gegenstand, Methode, theoretischer Zugang, wissenschaftsgeschichtliche Tradition etc.), sondern sie gleichzeitig zu denken.

Eine weitere wichtige Kategorie für zahlreiche Projekte sind Fragen nach der Relevanz (kultur-)wissenschaftlicher Arbeit: Wie kann wissenschaftliches Wissen gesellschaftlich umgesetzt werden? An welchen Orten wird welches Wissen überhaupt relevant? Wie können Ansprüche, gesellschaftlich relevantes Wissen hervorzubringen, mit den Strukturen und Spielregeln des akademischen Betriebes vereinbart werden? Und gleichzeitig: Was verstehen wir überhaupt in konkreten Projekten unter einer an gesellschaftlichen Problemen orientierten Praxis? Wie etabliert man einen Dialog zwischen den betroffenen Personen - in den einzelnen Projekten und darüber hinaus? Wo und wie können Orte gefunden oder geschaffen werden, die Spielräume für derartige »Experimente« eröffnen? Angela McRobbie betont in einem Aufsatz über die Besonderheit der Arbeit Stuart Halls den »experimentellen Charakter « seiner pädagogischen und akademischen Praxis (McRobbie 2000: 215) und setzt sein Wirken an der Open University in Milton Keynes zu den Problemen universitärer Strukturen in den I99oer Jahren in Beziehung:

"Wenn Universitäten auch unter widrigen Umständen das bleiben sollen, was Edward Said rutopische Räumer (utopian spaces) genannt hat, wenn sie fähig bleiben sollen, Plätze des Andersdenkens und des Widerspruches (places of dissent) zu sein« (ebd.: 219),

dann könnte die Aufgabe kulturwissenschaftlichen Denkens und 
Handelns nicht zuletzt darin bestehen, »utopische Räume« zu finden bzw. zu gestalten, Laboratorien, an denen eine experimentellere, reflexivere Praxis möglich ist - einfacher gesagt: Orte, an denen es möglich ist, »freier« zu sprechen, Unsicherheiten zu artikulieren, Fehler machen zu können, Fragen überhaupt stellen zu können. Rahmenbedingungen, um derartige »utopische Räume« für »andere«, »riskantere« Formen wissenschaftlicher Praxis gemeinsam zu entwickeln, versucht der Forschungsschwerpunkt zu bieten: Das können kleine Projektarbeitsgruppen sein, Diskussionsgruppen im Internet, aber auch Publikationen, die nicht nur ein akademisches Publikum ansprechen. Gerade hier kann man von der popular pedagogy in der Cultural Studies-Tradition Birminghams viel lernen, einer Lehre, die Studierende unterschiedlichster sozialer Hintergründe in ihren Lebenswelten ernst nimmt und erreichen will.

Denn eine der zentralen Herausforderungen für wissenschaftlich und politisch relevante kulturwissenschaftliche Debatten besteht nicht zuletzt darin, die Praxis unserer Sprache und unserer Auseinandersetzungen zu überdenken. Sie ist bislang und bei weitem nicht nur in den universitären Strukturen von der Trägheit der akademischen Konventionen geprägt. Veranstaltungen etwa werden langfristig geplant und durchgeführt, die vorgetragenen Statements aufgrund der kanonisierten Spielregeln (Fußnoten!), der viel zitierten Arbeitsüberlastung von Akademikerinnen und Akademikern, organisatorischen und finanziellen Hürden nach frühestens einem Jahr veröffentlicht. Zu dem Zeitpunkt also, an dem man die Chance erhält, sich mit den entsprechenden Überlegungen mündlich oder schriftlich auseinander zu setzen, sind sie meist bereits zwei Jahre alt. Gerade wenn es aber darum gehen soll, das Rad nicht ständig neu zu erfinden, scheint es mir eminent wichtig zu sein, zunehmend darüber nachzudenken, mit welchen Instrumenten und Strategien wir Inhalte und Ziele unseres Tuns besser und schneller vermitteln können und damit nicht zuletzt über die »Technik « der Kommunikation auch zu einem wissenschaftstheoretisch und -politisch effektiveren Handeln - dem zentralen Anliegen der Cultural Studies in der Birmingham-Tradition - kommen könnten. 


\section{Anmerkungen}

I http://www.crossroads-conference.org/

2 Die Diskussion selbst ist wesentlich älter als es die diversen »postmodernen« Paradigmenwechsel gelegentlich vermuten lassen, wenn sie auch erst in den vergangenen Jahrzehnten weitere wissenschaftliche Kreise - und dabei längst nicht alle - erfasst hat (vgl. dazu Lutter/Reisenleitner 1999).

3 Die folgenden Übersetzungen und Paraphrasen stammen von mir: Einzelne Begriffe werden zur besseren Nachvollziehbarkeit in Klammern im Original angegeben, wörtliche Übersetzungen werden durch Anführungszeichen ausgewiesen, Paraphrasen mit genauer Angabe der entsprechenden Stelle im Original versehen.

4 Vgl. etwa eine der Keynote Lectures auf der Crossroads Conference, die Daniel Mato (Venezuela) hielt: »Towards a Transnational Dialogue and Context Specific Forms of Transnational Collaboration: Recent Studies on Culture and Power in Latin America, and What our English Speaking Colleagues call Cultural Studies«.

5 Für die bereits bestehenden Versuche von Archäologien der Kulturwissenschaften vgl. etwa Böhme/Matussek/Müller (2000); Musner (1999); Musner/Wunberg/Lutter (200I) sowie den Forschungsschwerpunkt des Internationalen Forschungszentrums Kulturwissenschaften zum Thema »Geschichte der Kulturwissenschaften« vgl. http:// www.ifk.ac.at.

6 Dasselbe gilt für weitere absolut gefasste Gegensätze der akademischen Kategorisierung wie etwa Geistes- und Sozial- vs. Natur- und Technikwissenschaften; Grundlagen- vs. Angewandte Forschung; Cultural Studies vs. einzelne Disziplinen etc.

7 Mitglieder des Kreises der Expertinnen und Experten sind MarieLuise Angerer (Köln); Jochen Fried (Wien); Lawrence Grossberg (NC, Chapel Hill); Friederike Hassauer (Wien); Henry Krips (Pittsburgh); Rolf Lindner (Berlin); Thomas Macho (Berlin).

8 Das Konzept dieses »Projekts im Projekt« wurde von Science Communications (Alexander Martos und Bertram Schütz) gemeinsam mit der Projektkoordination im Wissenschaftsministerium (Christina Lutter) entwickelt und wird im Zusammenwirken mit allen Beteiligten am Forschungsprogramm prozesshaft weiterentwickelt und umgesetzt. Die Website ist seit Juni 200I online zugänglich (http://www. culturalstudies.at). 


\section{Literatur}

Böhme, Hartmut/Matussek, Peter/Müller, Lothar (2000): Orientierung Kulturwissenschaft. Was sie kann, was sie will, Reinbek bei Hamburg: Rowohlt.

Bromley, Roger/Göttlich Udo/Winter Carsten (Hg.) (I999): Cultural Studies. Grundlagentexte zur Einführung, Lüneburg: Zu Klampen.

Foucault, Michel (I987 [I97I]): »Nietzsche, die Genealogie, die Historie«. In: Ders., Von der Subversion des Wissens, hg. und übersetzt von Walter Seitter, Frankfurt a.M.: Fischer.

Gilroy, Paul (2000): Against Race: Imagining Political Culture Beyond the Color Line, Harvard: University Press.

Gilroy, Paul/Grossberg, Lawrence/McRobbie Angela (Hg.) (2000): Without Guarantees. In Honour of Stuart Hall, London/New York: Verso.

Grossberg, Lawrence (I995): »Cultural Studies and Political Economy: Is Anybody Else Bored With This Debate?«. Critical Studies in Mass Communication I22, S. 72-8I.

Grossberg, Lawrence (2000a): What's Going On? Cultural Studies und Popularkultur, Cultural Studies Bd. 3, Wien: Turia + Kant.

Grossberg, Lawrence (200\%b): »History, Imagination and the Politics of Belonging: Between the Death and the Fear of History«. In: Paul Gilroy/Lawrence Grossberg/Angela McRobbie, Without Guarantees. In Honour of Stuart Hall, London/New York: Verso, S. I48-I64.

Hall, Stuart et al. (1978): Policing the Crisis: »Mugging«, the State, and Law and Order, Basingstoke: Macmillan.

Hall, Stuart/Du Gay, Paul (Hg.) (I996): Questions of Cultural Identity, London u.a.: Sage.

Hall, Stuart/Jefferson, Tony (Hg.) (I976): Resistance Through Rituals: Youth Subcultures in Post-war Britain., London: Hutchinson.

Lutter, Christina (I999): »Zur Einrichtung eines Forschungsschwerpunktes Kulturwissenschaften/Cultural Studies«. In: The Contemporary Study of Culture, Kulturwissenschaft Bd. I, Hg. BMWV/IFK, Wien: Turia + Kant, S. I7-20.

Lutter, Christina/Reisenleitner, Markus (I999): »Introducing History (in)to Cultural Studies«. Beiträge zur historischen Sozialkunde, Sondernummer I999, S. 47-57. 
Lutter, Christina/Reisenleitner, Markus (3200I): Cultural Studies. Eine Einführung, Cultural Studies Bd. o, Wien: Turia + Kant.

Massey, Doreen (2000): »Travelling Thoughts«. In: Paul Gilroy/ Lawrence Grossberg/Angela McRobbie, Without Guarantees. In Honour of Stuart Hall, London/New York: Verso, S. 225-232.

McRobbie, Angela (2000): »Stuart Hall: The Universities and the $>$ Hurly Burly«. In: Paul Gilroy/Lawrence Grossberg/Angela McRobbie, Without Guarantees. In Honour of Stuart Hall, London/New York: Verso, S. 212-224.

Morris, Meaghan (1998): Too Soon Too Late: History in Popular Culture, Indianapolis: Indiana University Press.

Morris, Meaghan (I999): »Es gibt keine Globalkultur«. Interview mit Christian Höller. In: Widerstände. Kunst - Cultural Studies - Neue Medien. Interviews und Aufsätze der Zeitschrift springerin 19951999, Wien/Bozen 2000: Folio, S. I82-I95.

Musner, Lutz (I999): »Locating Culture in the US and Central Europe - A Transatlantic Perspective on Cultural Studies«. Cultural Studies I3/4, S. 577-590.

Musner, Lutz/Wunberg, Gotthart/Lutter, Christina (Hg.) (200I): Cultural Turn. Zur Geschichte der Kulturwissenschaften, Kulturwissenschaft Bd. 2, Wien: Turia + Kant.

Turner, Graeme ('²96): British Cultural Studies. An Introduction, London/New York: Routledge.

Wuggenig, Ulf (I998): »Cultural Studies und Kulturwissenschaften«. Merz Akademie 2, Stuttgart I998, S. 43-57. 



\section{Wozu Cultural Studies?}

\section{Lektüreversuch aus einer "widerspenstigen॥}

\section{Perspektive'}

Manuela Ribeiro Sanches

Cultural Studies scheinen ein umstrittenes Thema zu bleiben, auch wenn sie in manchen Gebieten als überholt angesehen werden. Tatsache ist, dass sie weiterhin ein wichtiger Bezugspunkt für bestimmte Forschungsrichtungen sind. Dabei muss betont werden, dass Kritik an und Verteidigung von Cultural Studies sich nicht leicht verallgemeinern lassen. Im englischsprachigen Raum geht es unter anderem darum, entweder Literaturwissenschaft endgültig von den Folgen der cultural wars abzulösen und die Rückkehr zum Literarischen vorzuschlagen (vgl. Culler 2000) oder von den Vorteilen des Ansatzes bei aller Gefahr von Institutionalisierung zu überzeugen (vgl. McRobbie 2000; Grossberg 1997, I999; Giroux 2000). Dabei wird ein stärkerer Rückgriff auf Ethnographie empfohlen (vgl. McRobbie 2000) oder die Möglichkeit, den Überschuss, der jeglicher Artikulation im doppeltem Sinne des Wortes innewohnt (vgl. Hall I996b), als Voraussetzung für ein utopisches Denken und die entsprechenden Praktiken, egal wie unfertig sie sein mögen, anzusehen (vgl. Butler 2000). Es mag zutreffen, dass Kulturanthropologie sich gegen eine zu starke Textualisierung verteidigen möchte bzw. aufgrund eines gefährlichen Verwischens von Grenzen an Identitätsverlust leidet (vgl. Lindner 2000) oder daraus eine anregende Zusammenarbeit zu entwickeln versucht 
(vgl. Clifford I997; Handler 1998). Interessant ist festzustellen, inwieweit Cultural Studies weiter differenziert rezipiert werden, mit unterschiedlichen Tempi und mit widersprüchlichen Folgen. Fest steht, dass innerhalb jeglicher lokalen Rezeption eben danach zu fragen ist, wie bestimmte Ansätze, Methoden, Vorgehensweisen, Fragestellungen verstanden und übersetzt werden sollten (vgl. Hall I996b; Clifford 2000 und Clifford/Sanches 2000).

Wenn im vorliegenden Band der Frage nach der Rezeption der Cultural Studies im deutschsprachigen Raum nachgegangen werden soll, wird allerdings diese Rezeption hier in einer besonderen Perspektive gelesen, und zwar von dem Standpunkt der Auslandsgermanistik aus, in einem südeuropäischen Land, wo überhaupt Germanistik und Cultural Studies wenig Tradition haben bzw. einen geringen Einfluss ausüben konnten. Dabei wird gehofft, dass ein solcher Ansatz thematisch nicht zu sehr an lokale Verhältnisse gebunden bleibt, sondern dass daraus mögliche Fragestellungen entstehen können, die von Relevanz für andere Fächer und Forschungslinien im Allgemeinen sein können.

Dass dieser Standpunkt zu peripher und zu exzentrisch erscheinen mag, wird nicht als nachteilig angesehen. Und es scheint umso einleuchtender zu sein, als diese Perspektive noch besser dem Ansatz der Cultural Studies gerecht werden kann. Cultural Studies stellten sich als eine entscheidende Aufgabe, nach den Selbstverständlichkeiten zu fragen, die aus einem zu eng gefassten Begriff von Kultur resultierten, um daraus radikale Schlussfolgerungen zu ziehen. Minderheitskulturen wurden Gegenstand einer Reflexion, die bereits als festgelegt geltende Fächer und Grenzen umso mehr in Frage stellte, je mehr jene Kulturen von einer zunehmenden Globalisierung gefährdet schienen. Aus einem national oder ethnozentrisch verstandenen Fach (vgl. Gilroy I992; Hall I997) wurden Cultural Studies zunehmend ein Ort des Hinterfragens solcher für selbstverständlich gehaltenen Voraussetzungen, die Nation und Kultur als ein abgeschlossenes, integres Ganzes begriffen, um Platz für ungenaue, hybride, ex-zentrische Identitäten zu machen. Derartige Begriffe von Nation und Kultur hatten eine entscheidende Wirkung im englischsprachigen Raum, vor allem in der Art und Weise, in der Ethnizität, Identität oder Rasse gedacht wurden. Mag sein, dass mittlerweile - und in manchen Zusammenhängen mit Recht (vgl. Almeida 2000) - Hybridität fragwürdig geworden ist, genauso wie jede Art von lokalen Reaktionen auf Globali- 
sierung, nicht zuletzt in Form von virulenten, sich abgrenzenden Nationalismen sich zunehmend, besonders in Europa, bemerkbar macht. Fest steht, dass zur Interpretation solcher lokalen Identitäten der Bezug auf transnationale Elemente und die widersprüchlichen Folgen von Globalisierung umso erforderlicher ist.

\section{Germanistik in Portugal}

Was für einen Einfluss können solche Fragen auf das Selbstverständnis von Fächern wie die Auslandsgermanistik ausüben, die sich hauptsächlich dadurch kennzeichnet, dass sie als Gegenstand ihrer Forschung und Lehre eine nationale Kultur, von einer äußerlichen Perspektive aus gelesen, benutzt? Die folgenden Überlegungen versuchen dieser Frage nachzugehen, indem aus einer ganz konkreten Erfahrung Reflexionen und methodische Ansätze entwickelt werden.

Diese Darstellung beansprucht nicht, die kohärente Wiedergabe einer objektiven Entwicklung zu liefern. Sie besteht vielmehr aus dem Aneinanderreihen von Reflexionen, entstanden aus eigenen, in einigen Jahren gesammelten Erfahrungen innerhalb des Faches »Deutsche Kultur«, das zwar keine Tradition in der Inlandsgermanistik besitzt, aber im Rahmen der portugiesischen Germanistik keine geringe Rolle spielt.

Nach einer chaotischen und innovativen Phase während der I970er Jahre als Folge der »Nelkenrevolution«, die Anlass für ein ständiges Experimentieren mit Fächern und Themen bot, wurde Anfang der 8oer Jahre der Versuch unternommen, neuere systematischere Ansätze einzuführen. ${ }^{2}$ Es sollten der wirtschaftliche, soziale und kulturelle Kontext der Epochen behandelt werden, die im Rahmen von »Deutsche Literatur « - hauptsächlich konzipiert als eine Einführung in die Geschichte der deutschen Literatur mit einem sozialgeschichtlichen Ansatz - berücksichtigt werden.

Allmählich entwickelte sich die Disziplin zu etwas mehr als einer Art »Kulisse« zur Literaturgeschichte, indem eine eigenständige Orientierung erarbeitet und zunehmend bemerkbar wurde, wobei sich trotz der Versuche, disziplinäre Autonomie herzustellen, einige Tendenzen, die den sozialgeschichtlichen Ansatz in der Literatur kennzeichneten, letztendlich zu wiederholen drohten. Das Programm von »Deutsche Kultur« orientierte sich an einem teleologisch verstande- 
nen Begriff von Ideen- bzw. Kulturgeschichte, der parallel zu den Themen, die im Rahmen von »Deutsche Literaturgeschichte« behandelt wurden, einem neomarxistischen Ansatz folgte und einen latenten Hegelianismus verriet, wobei das Bürgertum und der entsprechende Emanzipationsprozess ein Leitmotiv bildeten, das es erlaubte, Reformation, Aufklärung, Naturrecht, Romantik, Vormärz, Gründerjahre und Jahrhundertwende in einen systematisierenden Ansatz mit einem historisch-fortschreitenden Deutungsmuster einzubetten. Natürlich bot ein derartig komplexes Verständnis von Kultur und Aufklärung bzw. Geschichte - von Kritischer Theorie und Rezeptionstheorie beeinflusst - eine Möglichkeit, sich einseitigen Deutungsmustern zu entziehen.

Gleichzeitig zeigte sich aber, dass eine lediglich nationale Perspektive nicht imstande war, die erörterten Fragen adäquat zu erfassen und zu thematisieren. Der Bezug auf einen europäischen Kontext und der Rückgriff auf ein komparatistisches Verfahren erlaubte es, Themen und Fragen auf eine differenzierte Art und Weise zu behandeln, indem sie stets in einen lokalen Kontext übertragen wurden: Man zog beispielsweise Vergleiche zwischen Protestantismus und Katholizismus anhand von Texten wie Max Webers »Die protestantische Ethik und der Geist des Kapitalismus « und konfrontierte das mit den lebensweltlichen Verhältnissen der Studierenden. Der »deutsche Sonderweg« oder die »verspätete Nation« wurden so im Rahmen des europäischen Kontexts besprochen, und man versuchte Parallelen zur spezifisch portugiesischen Situation herzustellen.

Dabei war ein großes Telos zu spüren, das Telos einer dialektischen Aufklärung oder besonderer Tendenzen oder Richtlinien in der deutschen Geschichte, die bestimmte Ereignisse entweder leichter zugänglich machen sollten oder sie mehr oder weniger bewusst einer gewissen Mnemonik zu unterziehen versuchten, wobei die zugrunde liegenden rhetorischen Strategien selten benannt oder als Problem erörtert wurden. Die deutsche Entwicklung konnte so gemäß einer besonderen Tradition deutlich nachgezeichnet werden: Deutsche »Rückständigkeit« war, was »Zivilisationserrungenschaften« betraf, umso belegbarer, da der »Fortschritt« des »objektiven Geistes« oder der »Kultur « gemäß dem deutschen »Sonderweg « nachvollzogen werden konnte und eine »doppelte (deutsche) Seele« sich umso prägnanter in der Kluft zwischen deutscher »Bildung « und nationalsozialistischer »Barbarei« oder zwischen einer deutschen »hohen Kultur« und 
medialer »Trivialität« situieren ließ. Dazu kam, dass ein solches Verständnis es verbot, die Normalisierung oder Infragestellung einer festen nationalen Identität zu denken, was ziemlich beruhigend auf ein postkoloniales Portugal und seine Abneigung wirken musste, sich mit seiner eigenen historischen Vergangenheit auseinander zu setzen. Folglich blieb wenig Platz für brüchige, hybride Identitäten. Minderheiten, wie Juden, wurden behandelt, doch als parallel bestehende Tendenzen, die sich mehr oder weniger peripher zu den allgemeineren Entwicklungslinien herausgebildet hätten. Dasselbe galt für Migranten, die nur nebensächlich erwähnt wurden.

Dabei wurde offensichtlich, dass anhand solcher Deutungsmuster bei den Studierenden, egal wie fasziniert von den »Sonderwegen«, bestimmte Stereotypen sehr leicht auftauchen konnten, da der Ansatz nur schwer bestimmte tradierte Vorstellungen von Deutschland vermeiden konnte. Um es kurz und eventuell vereinfachend zu erklären: Das Bild, das Studierende von der »deutschen Kultur« gewannen und dies trotz allem neo-marxistischen Bemühen, bestimmte geistige Entwicklungen wirtschaftlich und gesellschaftlich $\mathrm{zu}$ erklären, und dem Einbeziehen von aktuellen Texten und Themen -, lief Gefahr, sich letztendlich nicht von einem Land von »Dichtern und Denkern« zu unterscheiden, das im Laufe einer zu schnellen Industrialisierung die Keime der nationalsozialistischen Katastrophe in sich trug.

Dazu kam, dass die chronologische Darstellung und das Bemühen, ein umfassendes, ganzheitliches, kohärentes Bild zu liefern, dazu führten, zeitgenössische Fragen nur sehr unvollständig zu behandeln. Wenn sie erwähnt wurden, geschah das meistens im Rahmen eines begrenzten, elitären Begriffs von Kultur, der nicht umhin konnte, Identitätsfragen wie Frauen, Jugend oder Minderheitskulturen nur relativ sekundär zu behandeln. Dies bedeutete aber nicht, dass solche Themen nicht angesprochen wurden. Von dem mentalitätsgeschichtlichen Ansatz profitierend, wurde Alltagskultur ebenfalls zunehmend wichtiger, wobei methodische Unzulänglichkeiten und Inkohärenzen nicht zu vermeiden waren. Wie waren allägliche Fragen, diskontinuierliche Auftritte, die belanglos für das Fortschreiten der großen Tendenzen waren, in einen kohärenten Zusammenhang zu bringen und mit jenem Telos zu vereinbaren? Fragen wie diejenigen, die eine historische Anthropologie einführen, namentlich die Art und Weise, in der dichotomische Begriffe und vereinfachende Verallgemeinerung durch eine mikrologisch-ethnographische Analyse in Frage gestellt 
werden (vgl. van Dülmen 2000), konnten nicht leicht anhand der vorhandenen Theorien behandelt werden; denn stets traf das integrative Moment (Hörning/Winter I999: 8) auf einen Kultur- und Gesellschaftsbegriff, der jegliche Dissonanz und Widerspenstigkeit als störenden, aber - auf gut hegelianische Art und Weise - aufhebbaren Faktor zu verharmlosen wusste.

Eine zögernde Kenntnisnahme von Norbert Elias' Zivilisationstheorie führte allmählich zu neuen Deutungsmustern, die mit den früheren Ansätzen nicht kollidierten - und dies begründete die zögernde Annahme -, da die teleologische, integrative, eurozentrische Entwicklung, jetzt in »Zivilisationsprozess « »umbenannt«, bewahrt blieb. Obwohl in diesem Ansatz Alltagskultur eine zunehmend wichtige Rolle spielte und eine neue theoretische Legitimation erfuhr, blieb sie Nebensache und ein guter Vorwand, das Telos, das der sozialgeschichtliche Ansatz verlangte, letztendlich nicht in Frage zu stellen. Dichotomien wie Kultur/Zivilisation, höfische/bürgerliche Gesellschaft, Tradition/Moderne, Kontinuität/Bruch, Konservatismus/Revolution, Aufklärung/Romantik, Moderne/Postmoderne, Europa/NichtEuropa, Ost/West, Zentrum/Peripherie bildeten feste Kategorien, die sich umso besser benutzen ließen, als daraus ein kohärentes Deutungsmuster herzustellen war. Keine fließenden Grenzen, keine ambivalenten, »hybriden « Identitäten, keine »mixed feelings« waren dabei erwünscht.

Aber immer wieder machten sich Brüche in dieser kohärenten Erzählung aus der allwissenden, allumfassenden Perspektive eines selbstbewussten Subjekts bemerkbar. Allmählich drangen Feminismus, Diskursanalyse und das entsprechende Hinterfragen von Humanismus und Subjektivität in den souveränen Gang und die Teleologie der Emanzipation ein, indem poststrukturalistische Ansätze, in Portugal früh rezipiert und inzwischen auch von neuen Richtungen in der Germanistik gerechtfertigt, sich als ein wichtiges Gegenstück zu allzu eindeutigen Deutungsmustern erwiesen.

Dabei konnte man sich aber nicht einer gewissen Skepsis entziehen, und zwar besonders dann, wenn solche dekonstruktivistischen Momente der Selbstaufklärung der Aufklärung Schaden zu bringen schienen. Nietzsche und Freud wurden weiter stark rezipiert und gelesen, indem versucht wurde, Grenzen zwischen Subjekt und Objekt, dem Eigenen und dem Fremden nicht nur als fest verankert vorzustellen, sondern sogar angesichts eines deutschen »Sonderwe- 
ges« in ihrer Dichotomie zu verteidigen. Fortschritt konnte in Frage gestellt werden, aber das Projekt der Emanzipation und die unvollendete Moderne durfte letztendlich nicht radikal ins Schwanken geraten.

Solche methodischen Inkonsequenzen konnten aber produktiv bearbeitet werden und dies anhand nicht nur einer gründlichen methodischen Reflexion, sondern auch mittels konkreter Erfahrungen im Rahmen von Forschung und vor allem von Lehre.

\section{Impulse durch die Cultural Studies}

Die Möglichkeit, den Ansatz neu zu gestalten und aus den erwähnten Widersprüchen einen neuen und fruchtbareren Blick zu gewinnen, ergab sich - da deutsche Literatur weiter chronologisch-geschichtsmäßig behandelt wurde - aus der Möglichkeit, »Deutsche Kultur« um zwei Semester als Wahlfach zu verlängern (»Deutsche Kultur II«). Tatsache ist, dass Themen wie Simmels Begriff von Moderne und Kultur, Benjamins kompliziertes Verständnis von Geschichte und Fortschritt, Kracauers Film- und Zeittheorie, Adornos Jazzkritik wichtige Elemente lieferten, um Gespräche über Medien, unvollendete oder überholte Moderne einzuführen, wobei Werbung, Film, Fernsehen, Jazz, Pop- und Jugendkultur, Anlass zu intensiven Diskussionen gaben. Dennoch verharrte man zu eindeutig auf der Ebene einer gewissen Skepsis gegenüber den Medien und der Geschwindigkeit der Bilder: Hermeneutik bildete eine wichtige Voraussetzung, jenen schönen Schein zu durchdringen und gemäß dem adornoschen Ansatz die Manipulation der Kulturindustrie umso deutlicher entlarven zu können. Konsum wurde vereinfachend mit Konsumismus identifiziert und verpönt und dies zu einem Zeitpunkt, in dem Konsumieren zum ersten Mal eine alltägliche Erfahrung der portugiesischen Studenten geworden war. Trotz aller distanzierten Kritik wurde offensichtlich, dass es im Rahmen von »Deutsche Kultur « möglich war, über Alltag und Lebenswelt auf eine konkretere Art und Weise zu sprechen, ohne auf Theorie verzichten zu müssen. Im Gegenteil: Theorie erwies sich umso entscheidender und gerechtfertigter, da sich die alltäglichen Erfahrungen der Seminarteilnehmer als viel komplexer und widersprüchlicher erwiesen als andere zeitlich entlegene Fragen. Dabei wurde deutlich, dass hier schon mit einem erweiterten Begriff von Kultur gearbeitet wurde. Folglich erwies sich der Rückgriff 
auf Sekundärliteratur innerhalb der Cultural Studies als sehr wichtig, da dadurch ein differenzierterer Zugang zu den Themen ermöglicht wurde, indem Begriffe wie »Kulturindustrie« problematisiert, »agency« als Widerstandsfähigkeit eingeführt und dem »Kulturmandarinentum « Grenzen gesetzt wurden. Raymond Williams' Culture is Ordinary und sein problematischer Begriff von Kultur mit den Gemeinsamkeiten zwischen der »deutschen Tradition « - Coleridge, Mathew Arnold - und der nordamerikanischen Kulturanthropologie (vgl. Stocking I982; Handler 1998) wurde ein wichtiges theoretisches Mittel, Kritische Theorie zu relativieren und zu erweitern, indem auf lokale Entwicklungen aufmerksam gemacht wurde.

Auf der anderen Seite stellte sich heraus, dass, obwohl hauptsächlich deutsche Autoren besprochen wurden und auf die besonderen Verhältnisse hingewiesen wurde, die ihre Ansätze beeinflusst hatten wie z. B. Industrialisierung und Moderne im Wilhelminischen Deutschland, in der Weimarer Republik oder im Dritten Reich -, die behandelten Themen und Fragen sich nicht lediglich im nationalen Rahmen erschöpften, sondern stets dazu anregten, sie in einem internationalen und transnationalen Rahmen zu diskutieren. Dabei bildeten die eigene Lebenswelt und Alltagserfahrungen einen wichtigen Bezugspunkt für diese Übertragungsarbeit. Genau solche Erfahrungen, die die Entwicklungen in der portugiesischen Gesellschaft auch stets berücksichtigen mussten, erforderten Mitte der I990er Jahre, das ganze Konzept des Faches »Deutsche Kultur « zu ändern. ${ }^{3}$ Die Tatsache, dass der kulturwissenschaftliche Ansatz sich besser legitimieren ließ, hing nicht zuletzt auch von neueren Trends in der Germanistik ab, wo die Debatte um Kulturwissenschaften immer zentraler wurde.

Was sich ebenfalls immer klarer herausstellte, war die Tatsache, dass es eine Kluft zwischen einem zu engen Begriff von Kultur gab, der in der Wahl der durchgenommenen Themen in Seminaren des Faches »Deutsche Kultur I hervortrat, und dem alltäglichen Umgang mit tradierten Bildern und Stereotypen, die letztendlich auch aus den Unsicherheiten eines großen Migrationen ausgesetzten Portugals entstammten. Nationale Identität und Rassismus bildeten zunehmend wichtige Themen, wobei die eigene oder die durch die Medien vermittelte Erfahrung nicht unberücksichtigt gelassen werden konnte. Dazu kam die hybride Identität portugiesischer Remigrantenkinder, die in Deutschland, in der Schweiz oder in Luxemburg geboren oder aufgewachsen, ihre Zerrissenheit zwischen Sprachen, Alltagskulturen, 
Vorstellungen $\mathrm{zu}$ verbalisieren versuchten, da sie sich von misstrauenden Kollegen angesichts ihrer befremdenden Hybridität eingeengt fühlten. Dies bildete einen wichtigen Anlass, nach Identität und Kultur in einem erweiterten Sinn zu fragen und stereotypisierende Bilder von Deutschland als ein einheitliches Land zu dekonstruieren, indem nach den Zusammenhängen zwischen Sprache, Nation und Kultur und ihrer Tradition im deutschsprachigen Raum gefragt wurde und die portugiesischen Verhältnisse stets thematisiert wurden. Dazu konnte erläutert werden, inwieweit auch Begriffe von portugiesischer Identität auf tradierten Vorstellungen basierten, in denen das »Verwurzelte « und »Volkstümliche« sich noch stark als das »authentisch Portugiesische« darstellen ließen. Dabei war es notwendig, Gegensätze aufzuzeigen, die sich als besonders aufschlussreich erwiesen, namentlich diejenigen zwischen bestimmten Begriffen von nationaler Identität, nicht zuletzt auch durch eine von der deutschen Volkskunde stark geprägte portugiesische Ethnologie (vgl. Leal 2000) beeinflusst, und dem kolonialen Mythos der Entdecker, die noch Kontinuitäten zwischen den vom portugiesischen Faschismus gepflegten Selbstbildern und aktuellen Selbstverständlichkeiten verraten. ${ }^{4}$

Das »postkoloniale« Portugal belegt aber, dass Fragen der Migration sich nicht leicht in ein Nord-Süd- bzw. Ost-West-Schema einbetten lassen, eine Dekonstruktion, die eine noch tabuisierte portugiesische koloniale Vergangenheit umso erforderlicher macht. Ethnizität und Modernisierung von Portugal bilden andere wichtigen Themen, die einen fortschrittlichen Zeitbegriff und teleologische Deutungsmuster in ihrer Unzulänglichkeit erscheinen lassen (vgl. Chambers I996), wobei nicht nur die »Orientalisierung« der Kolonisierten (vgl. Said I995), sondern ebenfalls die »Okzidentalisierung« (vgl. Carrier I995) der kolonisierten Kolonisatoren und ihre Beziehung zu Nordeuropa bzw. Deutschland als Mischung von Neid und Bewunderung umso deutlicher hervortritt.

Zusammengefasst: Aus einem historisch konzipierten Fach wurde »Deutsche Kultur I« zunehmend $\mathrm{zu}$ deutschen Cultural Studies, indem neben Adorno und Benjamin, Horkheimer und Günther Anders, Raymond Williams und Stuart Hall - inzwischen auch in der Anglistik stark rezipiert - James Clifford und Edward W. Said, Renato Rosaldo, Paul Gilroy und bell hooks Anlass geben, entscheidende Themen einzuführen und nicht nur ein national gedachtes Fach wie Germanistik zu hinterfragen, sondern ebenfalls über zentrale Themen 
unserer Gegenwart, wie Globalisierung, Eurozentrismus, Identität, Minderheiten, zu diskutieren. Entscheidend dafür war, die britischen Cultural Studies mit einer renovierten amerikanischen Kulturanthropologie in Zusammenhang zu bringen, indem ebenfalls Ansätze der deutschen Volkskunde und portugiesischen Ethnologie thematisiert und hinterfragt wurden. Das Erweitern der Begriffe und Ansätze vermeidet es demzufolge nicht, auf das Lokale, Spezifische und auf die Notwendigkeit einer vorsichtigen Übertragung von Methoden und Ansätzen bzw. auf einen widersprüchlichen Umgang mit Hybridität angesichts der jeweiligen Traditionen aufmerksam zu machen, indem z.B. auf unterschiedliche Identifikationsdiskurse oder Begriffe von Nation hingewiesen wird. Im Gegensatz $\mathrm{zu}$ einer unbeweglichen deutschen Identität werden hybridere Identifikationsmuster in Portugal dekonstruiert, indem gleichzeitig auf den »toleranten«, vom Faschismus stark rezipierten und instrumentalisierten Lusotropikalismus (vgl. Almeida 2000) hingewiesen wird, der darauf hinausläuft, dass Portugiesen sich durch eine außerordentliche Toleranz und Anpassungsfähigkeit auszeichnen würden, was im Gegensatz zu Deutschland verbietet, Rassismus zu thematisieren.

\section{Transkulturelle und transdisziplinäre Perspertiven}

Bei der zunehmenden Diskussion alltäglicher Fragen wird allerdings auf Geschichte nicht verzichtet. ${ }^{5}$ Brüche und Fragestellungen werden aufgezeigt, die einen eurozentrischen Fortschrittsglauben umso fragwürdiger erscheinen lassen. Grenzen und Gemeinsamkeiten sowohl des Hegelianismus als auch des portugiesischen »Universalismus« (eine andere Darstellung der vermeintlichen portugiesischen Toleranz oder Anpassungsfähigkeit) werden aufgezeigt, wobei der postkoloniale Ansatz (vgl. Chambers/Curti I996; Prakash I995) einen zunehmend wichtigen Platz einnimmt.

Und wenn es darum geht, auf utopische Potenziale des deutschen Idealismus oder einer »unvollendeten Moderne« aufmerksam zu machen, wird es notwendig, auf die unaufhebbaren Momente jener Tradition, namentlich auf das doppelte Bewusstsein eines »Schwarzen Atlantiks« (vgl. Gilroy I993) hinzuweisen, dessen utopische Momente sich nur anhand des Bewusstwerdens der ambivalenten Tendenzen in der Gegenmodernität darstellen und überwinden lassen (vgl. Gilroy 
I993); und dies anhand einer sorgfältigen, stets neu gedachten Übertragungsarbeit, die die lokalen Verhältnisse nicht verleugnet, sondern sie in Betracht zieht und relativiert.

Dies mag als ein sehr anspruchsvolles und nicht leicht durchführbares Programm angesehen werden, wie es übrigens bei jeglichem lokal übertragenen Ansatz von Cultural Studies der Fall ist; denn eine genaue Kenntnis von Daten, Theorien und kanonischen Autoren wird umso mehr verlangt, wenn es um Dekonstruktion von vorhandenen und unbefragten, selbstverständlichen Voraussetzungen geht. Um es ganz konkret zu sagen: Wenn bestimmte Themen nicht als bekannt vorausgesetzt werden können und dazu (zu) viel Information geliefert werden muss, ist es nicht weniger erforderlich, tradierte Vorstellungen wie Geschichtlichkeit, Tradition, Fortschritt, Nation, und Dichotomien wie Kultur und Zivilisation, das Eigene und das Fremde sowohl genau zu erörtern als auch in Frage zu stellen.

Das kann als ein Nachteil des Ansatzes verstanden werden. Dabei ist auch $\mathrm{zu}$ betonen, dass transkulturell gedachte Cultural Studies eben jene doppelte Perspektive erforderlich machen, die, wenn auch schwer praktizierbar, umso differenziertere Ansichten verlangt. Es ist hier nicht beabsichtigt, eine Kulturwissenschaft vorzuschlagen, die als systematisches Ganzes die vorhandenen unterschiedlichen disziplinären Ansätze unter einen allumfassenden Begriff von Kultur zu subsumieren beansprucht oder sich historisch zu legitimieren versucht, indem behauptet wird, es ginge lediglich darum, an einer vergessenen Tradition in der deutschen Germanistik anzuknüpfen (vgl. Böhme/ Matussek/Müller 2000). Hier soll eher auf Vorteile von inter- bzw. transdisziplinären Ansätzen aufmerksam gemacht werden, die anhand von Unterschieden und Gemeinsamkeiten zwischen der literaturwissenschaftlichen und kultursoziologischen bzw. -anthropologischen Methodik umso notwendiger sind. Es geht um die entscheidende Rolle von Kontexten (vgl. Hörning/Winter I999), indem man an der Grenze von Fächern arbeitet, diese deplatziert/verlagert und multiperspektivisch unterschiedliche Ansätze und Methoden miteinander verglichen und konfrontiert und demzufolge neue Fragestellungen und entsprechend differenziertere Schlussfolgerungen gewonnen werden. Close reading von jeglicher Art von Texten, allumfassende Theorien eingeschlossen, soll durch ethnographische Daten korrigiert bzw. relativiert werden, wobei subjektive Konstruktionen und rhetorische Mittel auch zu beachten sind. Und dabei wird nicht vergessen, 
dass die »ursprünglichen « britischen Cultural Studies sich eher durch dieses Gegeneinanderhalten von Perspektiven als durch eine »hybride Mischung aus Literaturkritik und Soziologie« (Lepenies, zitiert in Hörning/Winter I999: 8) ausgezeichnet haben.

Dies ist auch der Grund, warum noch von deutschen Cultural Studies die Rede sein soll, denn es geht hauptsächlich darum, globale Probleme lokal erkennen und übertragen zu können, indem das Nationale am Fach in Betracht gezogen und zugleich relativiert wird, Identitäten ernst genommen und dekonstruiert, Geschichte thematisiert und Teleologien in Frage gestellt, Text und Kontext gegeneinander gehalten werden. Um ein solches hybrides »Fach« zu üben und ein allzu diffuses Verständnis der entsprechenden Forschungslinien und Lehrstrategien zu vermeiden, fehlen einige Grundvoraussetzungen. Zunächst müsste »Deutsche Kultur« sich nicht auf ein einziges Jahr als Pflichtjahr beschränken lassen; dann ist eine Umstrukturierung der Kurse erforderlich, die weniger nationale als thematische Begebenheiten berücksichtigen sollte, indem auf unterschiedliche lokale Ansätze aufmerksam gemacht wird und dennoch vergleichende Verfahrensweisen geübt werden, die sowohl auf Inter- wie Transdisziplinarität zielen. Gerade das Gespräch mit anderen Fächern, national und international betrieben, kann umso besser den lokalen und globalen Ansprüchen gerecht werden, die sich transnational entfalten.

Was verbindet trotz aller spezifischen Fragen diesen Ansatz mit den Cultural Studies? Einmal der Verzicht auf absolute Trennungen, sei es zwischen Text und Kontext, Fremdem und Eigenem, Lokalem und Globalem, sei es zwischen festen Grenzen und sich ausschließenden Methoden. Das Entgegenhalten von anscheinend sich widersprechenden Paradigmen wurde als ein wesentlicher Bestandteil der Cultural Studies von Stuart Hall (vgl. Hall I999) ausgearbeitet, und ein solcher Ansatz liefert einen möglichen Hintergrund, an den sich dieses Vorgehen anknüpfen ließe. Die Unzulänglichkeiten des Kulturalismus kann man bei den zögernden Versuchen nachvollziehen, über einen egal wie erweiterten, aber dennoch zu holistisch gedachten Begriff von Kultur hinauszugehen. Die Herausforderungen von Halls differenziertem Umgang mit dem (post-)strukturalistischen Ansatz kann man nur nachhaltig befürworten, wobei andere Erfahrungen und theoretische Voraussetzungen die Grundlage für eine ähnliche Fragestellungen bilden. Dabei bieten kritische Theorie (vgl. Kögler I999), einfühlsame Auseinandersetzungen mit dem anti-metaphy- 
sischen Poststrukturalismus (vgl. Frank 1997) bzw. poststrukturalistische Versuche, mit und über Hegel hinaus über Subjektivität und Identität zu denken (vgl. Butler 2000), weitere wichtige Anhaltspunkte. Solche Verfahren bieten mögliche methodische und praxisgebundene Modelle, die nicht so sehr neue epistemologische Ansätze einzuführen beanspruchen, sondern anhand der Entgegensetzung und Artikulation (vgl. Hall 1996b, Grossberg 1999, Clifford/Sanches 2000) von (un)versöhnbaren Paradigmen »widerspenstige Kulturen« (Hörning/Winter 1999) in ihrer Eigenständigkeit zu thematisieren versuchen, wobei gemäß Gramscis Begriff von Hegemonie Widerstand und Versöhnung als zwei (un)trennbare Momente erscheinen und artikuliert werden können.

Eine andere Herausforderung an die portugiesische Germanistik ist der Versuch, solche Ansätze mit der hiesigen Rezeption von Cultural Studies, sei es in der Anglistik, Komparatistik, Ethnologie oder Soziologie in Verbindung zu bringen und weitere Berührungspunkte zu entwickeln. Dazu kommt den Postcolonial Studies in Portugal eine zunehmend wichtige und anregende Rolle zu, namentlich - und wie zögernd auch immer - in der Literaturwissenschaft (vgl. Seixo et al. 2000) und in der Ethnologie rezipiert (vgl. Almeida 2000), wobei stärker die Wechselwirkungen zwischen den Postcolonial Studies und den Cultural Studies zu berücksichtigen wären. Der postkoloniale Ansatz in den Cultural Studies scheint umso mehr gerechtfertigt, da er im deutschsprachigen Kontext zu sehr vernachlässigt wurde und in einem zunehmend multikulturell "postkolonialen« Portugal umso entscheidender ist. Fragen nach der nationalen Identität - sei es der »hybrideren « Portugiesen, sei es der »integeren« Deutschen - müssen im Zusammenhang mit »widerspenstigen Kulturen« zusammen gedacht werden, und es müssen Fragen aufgeworfen werden wie: Was für eine Rolle spielen Klasse, Rasse und Geschlecht in einem Begriff von Nation? Wie werden Identifikationsprozesse innerhalb bestimmter hegemonialer Modelle praktiziert, wie wird »Authentizität« bei Minderheiten in Portugal dargestellt und inszeniert, um soziale Abgrenzung zu demonstrieren? Welche Übertragung und Kritik an globalen Modellen sind dort abzulesen? Solche Themen, von der portugiesischen Soziologie und Ethnologie analysiert, sollten dazu beitragen, klassische Begriffe von Kultur zu hinterfragen und daraus die entsprechenden Schlussfolgerungen zu ziehen. Wie soll ein Bild von Europa und deutscher Kulturen vermittelt werden angesichts der zu- 
nehmenden Migrantenströme in Portugal? Die entsprechenden Ansätze findet man eher in den Cultural Studies und ihrem Begriff von Kultur »als Prozess sozialer Ungleichheit« (Hörning/Winter 1999: 9) als in den deutschen Kulturwissenschaften, die dazu tendieren können, lediglich »kulturelle Texte zu analysieren oder Kultur als bloßes Objekt von Management oder Pädagogik engzuführen« (Hörning/ Winter I999: II), als Resultat einer strategischen Bildungspolitik, die sich zu sehr auf einen Begriff des »Ganzen« stützt (Böhme/Matussek/Müller 2000: 23$)^{6}$, einem Verdacht, dem man sich bei der neu gegründeten »Kulturwissenschaft« nicht entziehen kann. Denn was an dem Ansatz zu vermissen ist, so hilfreich und herausfordernd er für die portugiesische Germanistik auch sein mag7, ist eben »das Kreative, gegen die Normalisierung gerichtete Potenzial im Alltag der Gegenwart« (Hörning/Winter 1999: 12). Und dasselbe gilt für ein Überbetonen der deutschen Tradition innerhalb der Kulturwissenschaft(en), indem dabei eine große Erzählung vorgeschlagen wird, die mehr von einem (nationalen) Legitimationsbedürfnis ${ }^{8}$ als von einem innovativen Umgang mit Kultur(en) (Hörning/ Winter I999: 8) zeugt; und dies zu einem Zeitpunkt, in dem sich Identitäten nur global-lokal behaupten können.

Um es so kurz wie möglich zu sagen: Die ex-zentrische Perspektive, die hier vertreten wird, beabsichtigt nicht, verallgemeinernde methodische Ansätze vorzuschlagen, sondern lediglich eine unvollendete Übertragungsarbeit darzustellen, die von einer besonderen lokalen Erfahrung nicht zu trennen ist, aber die nationale und fachliche Abgrenzungen vermeiden will.

Als Auslandsgermanistin ist man eben stets auf das Fremde angewiesen, eine Perspektive, die eher mit derjenigen des Ethnologen als mit dem Cultural Studies-Spezialisten zu vergleichen ist, der sich eben durch sein Insidertum kennzeichnet (vgl. Lindner 2000). Wenn diese letzte Vorgehensweise deutliche Vorteile aufgezeigt hat, müsste sie noch von einer anderen Entwicklung ergänzt werden und zwar derjenigen, die ethnographisch (vgl. Clifford I988) innerkulturelle Perspektiven in Frage zu stellen weiß und die Grenzen zwischen dem Außen und Innen problematisch erscheinen lässt. Eben dieses Schwanken, diese häufig praktizierte, aber selten anerkannte Hybridität einer »widerspenstigen « Auslandsgermanistik wurde hier als Voraussetzung benutzt, um nach Gemeinsamkeiten und Unversöhnbarkeiten 
zwischen einem kulturwissenschaftlichen Ansatz deutscher Prägung und den Cultural Studies zu fragen.

\section{Anmerkungen}

I Für die sorgfältige Korrektur meines Manuskripts bin ich Alfred Opitz besonders dankbar.

2 Ich beziehe mich hier ganz konkret auf die Erfahrung, die ich an der Klassischen Universität in Lissabon gemacht habe, und beanspruche nicht, andere Erfahrungen innerhalb meiner Fakultät oder in anderen Universitäten in Betracht zu ziehen.

3 Eine wichtige Rolle spielte ebenfalls die Zusammenarbeit mit Alfred Opitz und Fernando Clara anlässlich der gemeinsamen Herausgabe eines Bandes zu »Deutsche Gesellschaft und Kultur«im Rahmen der Publikationsreihe der portugiesischen Fernuniversität.

4 Man denke an die Lissabonner Weltausstellung I998 und an die Reihe der Themen, die Portugal als Land von Seeleuten und Kulturvermittlern vorstellten. Dass Portugiesen bereit sind, sich damit $\mathrm{zu}$ identifizieren, belegt die Tatsache, dass solche Themen selten in Frage gestellt wurden trotz aller Proteste angesichts des Unternehmens, das letztendlich einen Grund lieferte, nationalen Stolz offen zu zeigen, etwas was m.E. in einem ähnlichen deutschen Kontext unmöglich wäre. Die Expo Hannover 2000 hatte als Schwerpunkte Zukunft und Technologie, was ebenfalls im Kontext der deutschen nationalen Identität zu hinterfragen wäre, wobei Kultur - im klassischen Sinne des deutschen Wortes - ebenfalls eine entscheidende identifikatorische Rolle spielte, wie man anhand der unternommenen Faust-Inszenierung nachvollziehen kann.

5 Zum Verhältnis zwischen Geschichte und Cultural Studies vgl. Steedman I992 und Catherine Hall I992.

6 Dies kann anhand des vorgeschlagenen Begriffs von Kultur »als Ganzes«, sowohl »Objekt als [...] Rahmen für ihre eigenen Operationen« (Böhme u.a. 2000: IO4) nachvollzogen werden, dessen systematisch-geschlossenes Muster, umso deutlicher herausragt, wenn man es mit dem Ansatz der Cultural Studies vergleicht, der mittels einer Befragung von Gemeinsamkeiten und Übereinstimmungen, die »Vorstellung einer Kultur als Trugbild entlarv[t]« (Hörning/Winter ı999: 
8). Folglich scheint es umso fragwürdiger, wie man aus einem einheitlichen, geschlossenen Begriff von Kultur auf multi- bzw. interkulturelle Prozesse schließen kann, es sei denn, diese werden in jenem Ganzen »aufgehoben«.

7 Vgl. den vor kurzem erschienenen, von Alfred Opitz herausgegebenen Band Erfahrung und Form. Zur kulturwissenschaftichen Perspektivierung eines Problemkomplexes (Opitz 200I).

8 Vgl. den Teil »Zur Geschichte kulturwissenschaftlicher Ansätze in Deutschland « - übrigens einer der umfangreichsten - in Böhme/Matussek/Müller 2000, S. 34-I03.

\section{Literatur}

Almeida, Miguel Vale de (2000): »Tristes luso-trópicos. Raízes e ramificaçoes dos discursos luso-tropicalistas«. In: Ders., Um mar da cor da terra. Raça, Cultura e Política da Identidade, Lisboa: Celta, S. I6I-I84.

Böhme, Hartmut/Matussek, Peter/Müller, Lothar (2000): Orientierung Kulturwissenschaft. Was sie kann, was sie will, Reinbek bei Hamburg: Rowohlt.

Butler, Judith (2000): »Agencies of Style for a Liminal Subject«. In: Paul Gilroy/Lawrence Grossberg/Angela McRobbie (Hg.) (2000), Without Guarantees. In Honour of Stuart Hall, London/ New York: Verso, S. 30-37.

Carrier, James G. (Hg.) (I995): Occidentalism, Oxford: Clarendon Press.

Chambers, Iain (1996): »Signs of Silence, Lines of Listening«. In: Iain Chambers/Lidia Curti (Hg.), The Post-Colonial Question: Common Skies, Divided Horizons, London/New York: Routledge, S. 47-62. Chambers, Iain/Curti, Lidia (Hg.) (1996): The Post-colonial Question: Common Skies, Divided Horizons, London/New York: Routledge.

Clifford, James (1988): The Predicament of Culture. Twentieth-Century Ethnography, Literature, and Art, Cambridge, Mass./London: Harvard University Press.

Clifford, James (1997): Routes. Travel and Translation in the Late Twentieth Century, Cambridge, Mass./London: Harvard University Press.

Clifford, James (2000): »Taking Identity Politics Seriously. The Con- 
tradictory, Stony Ground ...«. In: Paul Gilroy/Lawrence Grossberg/Angela McRobbie (Hg.), Without Guarantees. In Honour of Stuart Hall, London/New York: Verso, S. 94-II2.

Clifford, James/Sanches, Manuela Ribeiro (2000): »The Art of Tacking. An Interview-Dialog«. Etnográfica. Revista do Centro de Estudos de Antropologia Social 4/2, S. 37I-388.

Culler, Jonathan (2000): »The Literary in Theory«. In: Judith Butler/ John Guillory/Thomas Kendall (Hg.), What's Left of Theory? New Work on the Politics of Literary Theory, London/New York, S. 273-292.

Frank, Manfred (1997): The Subject and the Text. Essays on Literary Theory and Philosophy, Cambridge/New York/Melbourne: Cambridge University Press.

Gilroy, Paul (I992): »Cultural Studies and Ethnic Absolutism«. In: Lawrence Grossberg/Cary Nelson/Paula Treichler (Hg.), Cultural Studies, New York/London: Routledge, S. 187-198.

Gilroy, Paul (1993): The Black Atlantic: Modernity and Double Consciousness, Cambridge, Mass.: Harvard University Press.

Gilroy, Paul (2000): Against Race. Imagining Political Culture beyond the Color Line, Cambridge, Mass.: Harvard University Press.

Gilroy, Paul/Grossberg, Lawrence/McRobbie, Angela (Hg.) (2000): Without Guarantees. In Honour of Stuart Hall, London/New York: Verso.

Giroux, Paul (2000): »Public Pedagogy as Cultural Politics. Stuart Hall and the >Crisis< of Culture«. In: Paul Gilroy/Lawrence Grossberg/Angela McRobbie (Hg.), Without Guarantees. In Honour of Stuart Hall, London/New York: Verso, S. I34-I47.

Grossberg, Lawrence/Nelson, Cary/Treichler, Paula (Hg.) (I992): Cultural Studies, New York/London: Routledge.

Grossberg, Lawrence (1997): »Identity and Cultural Studies: Is That All There Is?«. In: Stuart Hall (Hg.), Questions of Cultural Identity, London/Thousand Oakes/New Delhi: Sage, S. 87-I07.

Grossberg, Lawrence (I999): »Was sind Cultural Studies«. In: Karl H. Hörning/Rainer Winter (Hg.), Widerspenstige Kulturen. Cultural Studies als Herausforderung, Frankfurt a.M.: Suhrkamp, S. 43-83.

Hall, Catherine (1992): »Missionary Stories: Gender and Ethnicity in England in the I830s and the I840s«. In: Lawrence Grossberg/ Cary Nelson/Paula Treichler (Hg.), Cultural Studies, New York/ London: Routledge, S. 240-276. 
Hall, Stuart (1996a): »Cultural Studies and its Theoretical Legacies«. In: David Morley/Kuan-Hsing Chen, Stuart Hall. Critical Dialogues in Cultural Studies, London/New York: Routledge, S. 262275 .

Hall, Stuart (I996b): »On Postmodernism and Articulation«. In: David Morley/Kuan-Hsing Chen, Stuart Hall. Critical Dialogues in Cultural Studies, London/New York: Routledge S. I3I-I73.

Hall, Stuart (I996c): »New Ethnicities«. In: David Morley/Kuan-Hsing Chen, Stuart Hall. Critical Dialogues in Cultural Studies, London/ New York: Routledge, S. 44I-449.

Hall, Stuart (I996d): »When Was the >Post-Colonial<? Thinking at the Limit«. In: Iain Chambers/Lidia Curti (Hg.), The Post-Colonial Question: Common Skies, Divided Horizons, London/New York: Routledge, S. 242-260.

Hall, Stuart, (1997): »Old and New Identities, Old and New Ethnicities«. In: Anthony D. King (Hg.), Culture, Globalization and the World-System. Contemporary Conditions for the Representation of Identity, Minneapolis: University of Minneapolis Press, S. 4I-68.

Hall, Stuart (1999): »Die zwei Paradigmen der Cultural Studies«. In: Karl H Hörning/Rainer Winter (Hg.), Widerspenstige Kulturen. Cultural Studies als Herausforderung, Frankfurt a.M.: Suhrkamp, S. $13-42$.

Hall, Stuart/du Gay, Paul (Hg.) (I996): Questions of Cultural Identity, London/Thousand Oakes/New Delhi: Sage.

Handler, Richard (1998): »Raymond Williams, George Stocking, and Fin-de-Siecle U.S. Anthropology«. Cultural Anthropology I3, S. $447-463$.

Hörning, Karl H./Winter, Rainer (I999): »Einleitung«. In: Karl H. Hörning/Rainer Winter (Hg.), Widerspenstige Kulturen. Cultural Studies als Herausforderung, Frankfurt a.M.: Suhrkamp, S. 7-I2.

Kögler, Hans-Herbert (1999): »Kritische Hermeneutik des Subjekts. Cultural Studies als Erbe der Kritischen Theorie«. In: Karl H. Hörning/Rainer Winter (Hg.), Widerspenstige Kulturen. Cultural Studies als Herausforderung, Frankfurt a.M.: Suhrkamp, S. I96237.

Leal, Joao (2000): »A procura do povo português«. In: Ders., Etnografias portuguesas (1870-1970). Cultura popular e identidade nacional, Lisboa: Publicaçoes D. Quixote, S. 27-104. 
Lindner, Rolf (2000): Die Stunde der Cultural Studies, Wien: Wiener Universitätsverlag.

McRobbie, Angela (2000): »The Universities and the >Hurly Burly« In: Paul Gilroy/Lawrence Grossberg/Angela McRobbie (Hg.), Without Guarantees. In Honour of Stuart Hall, London/New York: Verso, S. 212-224.

Nelson, Cary/Grossberg, Lawrence/Treichler, Paula (Hg.) (I992): »Cultural Studies: An Introduction«. In: Dies., Cultural Studies, New York/London, Routledge, S. I-I6.

Opitz, Alfred (Hg.) (200I): Erfahrung und Form. Zur kulturwissenschaftlichen Perspektivierung eines Problemkomplexes, Trier: Wissenschaftlicher Verlag.

Said, Edward (I995): Orientalism. Western Conceptions of the Orient, Harmondsworth: Penguin.

Seixo, Maria-Alzira/Abreu, Graça/Noyes, John/Moutinho, Isabel (2000): The Paths of Multiculturalism. Travel Writings and Postcolonialism, Lissabon: Cosmos.

Steedman, Carolyn (I992): »Culture, Cultural Studies, and the Historians«. In: Lawrence Grossberg/Cary Nelson/Paula Treichler (Hg.), Cultural Studies, New York/London: Routledge, S. 6I3622.

Stocking Jr., George W. (I982): Race, Culture and Evolution. Essays in the History of Anthropology, Chicago/London: Chicago University Press.

van Dülmen, Richard (2000): Historische Anthropologie. Entwicklung, Probleme, Aufgaben, Köln/Weimar/Wien: Böhlau Verlag. 



\section{Sport und Cultural Studies:}

\section{Zur ungleichzeitigen Formierung eines Forschungsfeldes}

Roman Horak und Otto Penz

\section{Neue Perspertiven}

Ende der I980er Jahre begann sich der enge Horizont der österreichischen gesellschaftsbezogenen Sportforschung zu erweitern, wobei außeruniversitäre Studien wesentlich zur Öffnung bzw. Bereicherung des Forschungsfeldes beitrugen. Vor allem zum Fußballspiel - neben dem Skilauf die Hauptsportart in Österreich - oder besser gesagt zur österreichischen Fußballkultur setzte eine systematische (außeruniversitäre) Forschungstätigkeit ein, die anfänglich insbesondere Erkenntnisse der englischen Fußballgewaltforschung aufgriff und für Österreich fruchtbar machte, womit nicht zuletzt Anbindungen der österreichischen Sportforschung an zeitgenössische gesamteuropäische Sportdiskurse hergestellt wurden.

Die Diversifizierung der Forschungsansätze und -methoden ist hierzulande eng mit der einsetzenden Rezeption der Cultural Studies verbunden. Aus einer Forschergruppe junger Postgraduierter (Roman Horak, Wolfgang Reiter und Kurt Stocker), die I984 daranging, das Phänomen des soccer hooliganism zu untersuchen, erwuchs wenig später das Institut für Kulturstudien (IKUS), immerhin der erste Versuch, Cultural Studies im deutschsprachigen Raum institutionell $\mathrm{zu}$ betreiben (vgl. Horak 2002). Den Beweggrund für dieses Forschungsinteresse bildete der Umstand, dass an der Wende von den 
I970er zu den 8oer Jahren die gewaltförmigen Ausschreitungen jugendlicher Fußballfans - zuerst in der Bundesrepublik und dann auch in Österreich - mehr und mehr als ein ernstes soziales Problem öffentlich wahrgenommen wurden. Als Freunde des Fußballspiels nutzte das Forscherteam die Gunst der Stunde und erkor das so genannte »Fußballrowdytum « zu seinem Forschungsgegenstand. Nach Durchsicht der vorliegenden sportwissenschaftlichen Untersuchungen, die entweder auf die Psyche der Jugendlichen (ihre persönlichsubjektiven Defekte) abstellten oder aber kulturpessimistisch gefärbt alte Devianztheorien bemühten, wurde klar, dass dergestalt keine wesentlichen Erkenntnisse zu gewinnen waren. Auf der Suche nach Zugangsweisen, die auch das Fußballspiel selber in ein Erklärungsmodell mit einbezogen, boten sich einerseits die zivilisationstheoretischen Überlegungen von Norbert Elias und Eric Dunning und andererseits die Arbeiten aus dem Umfeld des Birminghamer Centre for Contemporary Cultural Studies (CCCS) an (vgl. Taylor 1971, I976; Clarke I973; Ingham et al. I978). ${ }^{\mathrm{I}}$ Während Erstere allzu linear zu argumentieren schienen, lag der Reiz der CCCS-Arbeiten und damit auch ihre Herausforderung für Untersuchungen in Österreich (vgl. Horak et al. 1987, 1988) zum einen in der doppelten Fokussierung auf die Themenfelder »Fußball« und »Jugend«, die vor dem Hintergrund einer Verschiebung des traditionellen Kulturbegriffs in Richtung eines ethnologischen Bedeutungsgehalts stattfand, und zum anderen darin, dass sie in ihrer konkreten Analyse nicht bei der offensichtlichen Problematik (der Gewalt) stehen blieben, sondern diese in einem komplexen Feld wirksamer Kräfte verorteten. Die Formierung gewaltbereiter jugendlicher Fansubkulturen im Zusammenhang mit der Kommerzialisierung, Medialisierung und Professionalisierung des Fußballsports - sei es nun als symbolischer Protest gegen diese Entwicklung oder als Form der Aneignung eines sozialen Raumes - zu verstehen bedeutete, Raymond Williams Rede von Kultur als a whole way of life ernst zu nehmen.

Ausgehend von der doch recht engen Fragestellung der Zuschauergewalt weitete sich die österreichische Fußballforschung, gespeist aus den Cultural Studies, alsbald rasch aus - und zwar in zweierlei Hauptrichtungen: einerseits in Richtung einer Betrachtung des Fußballspiels als popularkulturelles Phänomen, um das sich Mythen und massenmediale Diskurse ranken, das von hegemonialen Machtverhältnissen durchdrungen ist und subkulturelle Prägungen aufweist 
(vgl. etwa Horak/Reiter 199I; Horak/Marschik 1997), und andererseits in Richtung historischer Fußballforschung im Kontext der Geschichte von Arbeiter- und Stadtkulturen (vgl. Marschik 1994; Horak/Marschik 1995; Horak/Maderthaner 1997). Mit diesen beiden Strömungen, die eng miteinander verschränkt sind, entstand erstmals in Österreich ein kohärentes sportwissenschaftliches Diskursfeld, das sich dadurch auszeichnete, dass der (Fußball-)Sport als konstitutiver Teil eines massenkulturellen Interesses und Vergnügens (der Arbeiterkultur, der Jugendkultur) bzw. als sinn- und identitätsstiftender Bestandteil der low culture begriffen wurde. Typische theoretischmethodische Ansätze der Cultural Studies, etwa die Forderung nach einer breiten Kontextualisierung des empirischen Materials, und wichtige Cultural Studies-Fragestellungen, etwa die Frage nach Elementen des empowerment bei der Verfolgung popularkultureller Interessen, wurden damit erstmals im Hinblick auf die österreichische Sportlandschaft behandelt und in die österreichische Sportforschung eingebracht.

Etwa gleichzeitig mit dieser Entwicklung setzte die Aufmerksamkeit für eine Reihe weiterer sportlicher Themen ein, wenngleich unter anderen theoretischen Prämissen und methodischen Gesichtspunkten. So verstärkte sich generell das historische Interesse am Sport: Ernst G. Eder publizierte kulturhistorische Arbeiten über den österreichischen Wassersport (vgl. Eder I990), Rudolf Müllner beschäftigte sich mit der NS-Vergangenheit der »Leibeserziehung « (vgl. Müllner I992), und Matthias Marschik untersuchte ein paar Jahre später die Entwicklung der Sportverbände in der Zweiten Republik (vgl. Marschik 1999). Das Thema »Körper und Sport« wurde in Österreich aufgegriffen, etwa aus kulturanthropologischer (vgl. Treude 1992) oder diskursanalytischer (vgl. Miller/Penz I993) Sicht (vgl. zudem Peyker 1992); der Geschlechterfrage aus feministischer Perspektive (und z.T. als Import aus Deutschland) wurde größere Aufmerksamkeit zuteil (vgl. den zusammenfassenden Aufsatz von HartmannTews/Rulofs I998). Die Aufbruchstimmung der frühen I990er Jahre, so könnte man die erwähnten Entwicklungen zusammenfassen, manifestierte sich in zwei beachtenswerten Ereignissen: Die Österreichische Zeitschrift für Soziologie (ÖZS) veröffentlichte 1992 unter der redaktionellen Leitung von Gerald Mozetic (zum ersten und einzigen Mal) ein Schwerpunktheft »Sportsoziologie«, das sowohl universitäre als auch außeruniversitäre - und nicht unbedingt streng soziologische - For- 
schungsergebnisse zur Diskussion stellte (vgl. ÖZS 4/1992). Und im Jahr 1993 fand die II. Konferenz des International Committee for Sport Sociology (ICSS) zum Thema »Sport in Space and Time« in Wien statt, wo die namhaftesten Vertreter der europäischen sozialund kulturwissenschaftlichen Sportforschung vertreten waren (vgl. Weiss/Schulz I995).

Aus heutiger Sicht lässt sich konstatieren, dass die österreichische Sportforschung seit damals wohl einen nennenswerten Aufschwung vollzogen hat, der Elan der frühen I99oer Jahre jedoch wieder verflogen ist. Es gibt beispielsweise keinen Sportaufsatz jüngeren Datums in der Österreichischen Zeitschrift für Soziologie und die sozialwissenschaftlichen bzw. kultursoziologischen und -historischen Beiträge im Spectrum der Sportwissenschaften sind dünn gesät. Die Vielfalt der Themen hat zugenommen, und die theoretische Ausrichtung der Forschung ist vielfältiger geworden, aber nach wie vor stellt sich die österreichische Forschungslandschaft als äußerst fragmentiert dar, und große Forschungslücken sind unübersehbar, insbesondere hinsichtlich der Selbstreflexion von historischen und zeitgenössischen Geschehnissen im eigenen Land, aber auch im Hinblick auf internationale Themenstellungen wie massenmediale Sportdiskurse und ethnische Probleme. Der einzige systematisch erforschte Bereich des letzten Jahrzehnts - durchaus mit internationaler Vorbildwirkung ist die österreichische Fußballkultur, wo auch die theoretisch-methodischen Stränge sowie Erkenntnisse der Cultural Studies aufgegriffen wurden und weiterentwickelt werden.

\section{Zur Rezeption von Cultural Studies durch die Sportforschung IN Deutschland ${ }^{2}$}

Keineswegs erfreulicher stellt sich die Situation in Deutschland dar. Eine Durchsicht der letzten zehn Jahrgänge der Zeitschrift Sportwissenschaft bestätigt nicht bloß die von uns weiter unten genauer ausgeführte Dominanz des Funktionalismus in der Sportsoziologie, sie bringt auch die wenig überraschende Einsicht, dass es kaum Elemente der Cultural Studies im Mainstream der deutschen Sportforschung gibt, was diese, wie wir im Schlusskapitel dieses Aufsatzes zu veranschaulichen versuchen werden, von der angloamerikanischen einigermaßen unterscheidet. 
Dabei hatte es in der Bundesrepublik schon sehr frühzeitig eine Bezugnahme auf die Behandlung sportlicher Themen durch die Cultural Studies gegeben. Ein eingehenderes Aufgreifen sportspezifischer Thematiken fand zwar auch in den British Cultural Studies erst mit Beginn der I980er Jahre statt, allerdings trug bereits eines der ersten sieben Projekte des CCCS in Birmingham unter der Leitung von Richard Hoggart den Titel »The Meaning of Sport and its Presentations« (McGuigan I992: 5I), dergestalt die »alltagskulturelle Wende in der literaturwissenschaftlichen Perspektive« (Lindner 2000: 40) mitillustrierend.

Werfen wir zudem noch einen kurzen Blick auf die Liste der ersten 39 bis zum Jahresbeginn 1976 erschienenen CCCS-Papers. Neben den mittlerweile als Klassiker gehandelten »Encoding and Decoding in TV Discourse« (Hall I973), »Working Class Youth Cultures« (Jefferson/Clarke I973) oder »The Style of the Mods« (Hebdige I97I) findet sich auch der Aufsatz »Football Since the War: Study in Social Change and Popular Culture« von Chas Critcher (I974).

Zwei der oben genannten Texte wurden im mittlerweile auch schon historischen Heft 24 von Ästhetik \& Kommunikation (1976) in deutscher Übersetzung vorgestellt: Tony Jeffersons und John Clarkes Aufsatz über die »Jugendlichen Subkulturen in der Arbeiterklasse« sowie ein Auszug aus Critchers Papier. Nimmt man dieses Ä\&K-Heft, dessen Themenschwerpunkt der »Freizeit im Arbeiterviertel« gewidmet war, als einen wichtigen Eckpfeiler der Rezeption von Cultural Studies in Deutschland (vgl. Lindner I998; Horak I998), so muss zugleich hinzugefügt werden, dass dieser Eckpfeiler doppelt - zum einen via Sport/Fußball, zum anderen via Jugendkultur - verankert war. Während allerdings in der Folge die Rede von der Jugendkultur zu einer dominanten diskursiven Formation anwuchs, die Disziplinen wie die Erziehungswissenschaft oder die Sozialpädagogik modernisieren half und schließlich auch in die Marktforschung Eingang fand (vgl. Lindner 1994), blieben die Effekte auf die Sportforschung deutlich geringer.

Die regionalhistorischen und klassenkulturellen Überlegungen Critchers zum Fußball im Kontext der working class communities eröffneten zwar einen neuen Zugang zum Sportspiel Fußball jenseits seiner ideologiekritischen Entlarvung (vgl. Lindner I983), und sie standen auch Pate bei der Hinwendung zu konkreten - meist historischen - Detailstudien, wie sie u.a. von Lindner und Breuer (I979) oder 
Gehrmann (1988) vorgelegt wurden. In die sportsoziologische bzw. -wissenschaftliche Forschung im engeren und eigentlichen Sinne fanden sie jedoch de facto keinen Eingang.

Damit begann eine Geschichte der Nichtrezeption, die bis in die Gegenwart anhält. Weder die an Antonio Gramsci orientierten hegemonietheoretischen Ansätze, die vor allem in Kanada und Großbritannien die traditionellen sportsoziologischen Paradigmen gehörig ins Wanken brachten und dort zu heftigen Debatten innerhalb der Sportforschung führten, noch Momente der Körper-, Gender-, Rassismusoder Ethnizitätsdiskurse der neueren Cultural Studies, die das Gesicht der US-amerikanischen Sportsoziologie einigermaßen verändert haben, konnten in Deutschland eine sichtbare Wirkung entfalten. Die deutsche Sportforschung bleibt sich treu, sie bleibt, was sie immer schon war - nicht allzu innovativ und sportwissenschaftlich hermetisch.

\section{Sportsoliologie in Deutschland}

Gesellschaftstheoretisches Herangehen an Phänomene des Bewegungsverhaltens hat ursächlich mit der Ablösung der europäischen Leibesübungen durch den modernen Sport zu schaffen und ist, wie Bero Rigauer rechtens festhält, im »historischen Kontext der gesellschaftlichen Entwicklung >moderner< (>bürgerlicher<, >zivilisierter<, >industrieller $<$ ) Gesellschaft zu verstehen« (Rigauer I982: I2).

Vom - durch die imperialistische Konkurrenz der beiden Großmächte Großbritannien und Deutschland politisch überformten Widerstreit zwischen englischem Sport und deutschem Turnen um die Wende des I9. zum 20. Jahrhundert bis zur gesellschaftswissenschaftlichen theoretisch-methodisch ausgewiesenen Befassung mit dem, was nun Sport geworden war, sollte allerdings geraume Zeit vergehen.

Es ist hier nicht der Platz für ein Nachzeichnen der diversen Linien und Stränge, die schließlich zu jenem Feld führten, das am ehesten durch die beiden Bezeichnungen »Soziologie des Sports« bzw. »Sportsoziologie« umschrieben werden kann. Festgehalten soll allerdings werden, dass die (internationalen) Prozesse der disziplinären Formierung und Institutionalisierung im Verlauf der ig6oer Jahre wesentlich in Gang kamen: 1965 wurde das erwähnte ICSS gegründet, 
und im selben Jahr erschien auch die erste Nummer des von ihm herausgegebenen Periodikums The International Review of Sport Socio$\log y$ (IRSS).

Eine zentrale Figur bei diesen Bemühungen war Günther Lüschen, der nicht nur erster Generalsekretär des ICSS wurde, sondern I966 auch das erste ICSS-Symposium in Köln organisierte. Ohne hier den Eindruck erwecken zu wollen, dass sich die Geschichte und Entwicklung der gesellschaftswissenschaftlich motivierten Sportforschung in Deutschland auf die Aktivitäten einzelner Personen zurückführen lässt, muss doch jene - unseres Erachtens immer noch dominante - Traditionslinie festgehalten werden, für die Lüschen als einer der Ersten steht. Es ist jene des vor allem von Talcott Parsons herkommenden amerikanischen Funktionalismus, der, wie John Loy und Douglas Booth jüngst zugestanden haben, die Anfänge der Soziologie des Sports in Nordamerika und Europa wesentlich geprägt hat (vgl. Loy/Booth 2000: 23).

In der Bundesrepublik Deutschland trug wohl vor allem auch die enge Anbindung sozialwissenschaftlicher sportbezogener Arbeit an die dominante Sportwissenschaft zur Stärkung und Verbreitung funktionalistischer Ansätze bei. Klaus Heinemann hat jüngst auf die enge Verbindung der Formulierung sportsoziologischer Fragestellungen mit der wachsenden Bedeutung der sportwissenschaftlichen Forschung und der damit zusammenhängenden Modernisierung der Sportlehrerausbildung seit den I970er Jahren hingewiesen (vgl. Heinemann 2000: 536). Die theoretisierende Rede vom gesellschaftlichen Subsystem Sport, von dessen Eigenschaft als »System sui generis« (Lüschen/Weis 1976: I3) passte so recht zur sportimmanenten Praxis der angestrebten Verbesserung der Leistungsfähigkeit im Spitzensport wie zum gesellschaftlichen Ziel der körperlichen Ertüchtigung auf breiter Ebene (»Trimm dich!«). Eine funktionalistische Soziologie des Sports diskutierte und diskutiert ihn vor allem als »soziales System « und »soziale Situation« (vgl. Heinemann I980: 40 ff.). Fragen von politischer, ökonomischer und kultureller Macht wurden nicht als konstitutiv verstanden, sondern bestenfalls anderen Teilsystemen zugeschrieben (der Politik, der Wirtschaft, der Kultur), mit denen das Teilsystem Sport in bestimmte Beziehungen gesetzt wurde (mit je unterschiedlich zugeschriebener Wirksamkeit und Bedeutung).

Neben dem schon erwähnten Reader von Lüschen und Weis (1976) prägten vor allem der Sammelband Texte zur Soziologie des 
Sports (Hammerich/Heinemann I975) sowie Heinemanns Einführung in die Soziologie des Sports (1980) den Mainstream der deutschen sportsoziologischen Forschung. Der Umstand, dass Heinemanns Einführung 1998 in der vierten Auflage erschienen ist, illustriert deutlich die anhaltende Dominanz des systemtheoretisch-funktionalistischen Paradigmas auch in der Gegenwart, ein Eindruck übrigens, der durch das eben erschienene voluminöse Handbook of Sport Studies (Coakley/Dunning 2000), das trotz seiner figurationssoziologischen (an Norbert Elias orientierten) Grundausrichtung im Übrigen bestrebt scheint, eine möglichst bunte Palette gesellschaftstheoretischer Zugänge zum Sport zu präsentieren, bestärkt wird: Von den drei deutschen Autoren sind zwei (Lüschen und Heinemann) als Vertreter des Funktionalismus zu verorten, einzig Bero Rigauer passt nicht so recht in dieses Bild. Und das mit gutem Grund.

I969, mitten in der Phase der ersten Formierung einer internationalen Sportsoziologie im Geiste des strukturellen Funktionalismus, hatte Rigauer mit Sport und Arbeit eine Publikation vorgelegt, der es um die Erhellung »soziologischer Zusammenhänge und ideologischer Implikationen« der beiden im Titel geführten Begriffe ging. Ausgehend von Theodor W. Adornos (vgl. zsf. 1969) und Jürgen Habermas' Überlegungen zum Verhältnis von Arbeit und Freizeit, der Einsicht, dass »die eine nur mit dem Blick auf die andere verstanden werden kann « (Habermas 1958/1967: 29), erarbeitete er eine in der Tradition der Kritischen Theorie stehende Sichtweise von Sportsoziologie, die er zwei Jahre später in einem Aufsatz pointiert folgendermaßen zusammenfasste: Sportsoziologie sei schließlich auch

wals eine Form der Gesellschaftskritik zu verstehen, die nicht nur eine sbloße Verdoppelung der Realitätı beabsichtigt, das meint, nicht deskriptiv bleibt. Gesellschaftliche Phänomene werden auf das hin untersucht (Normen, Werte, Funktionen), was man an ihnen zu verschleiern sucht. In diesem Sinne ist soziologische Kritik zugleich Ideologiekritiku (Rigauer 1971: 13).

»Sportsoziologie als Ideologiekritik«, unter diesem Titel könnte man jenen Strang zusammenfassen, der in den (vor allem frühen) I970er Jahren, wiewohl er im Rahmen des sportwissenschaftlichen Diskurses im engeren Sinne minoritär blieb, im Kontext kritischer gesellschaftstheoretischer Diskussionen durchaus präsent war. Dies lag wohl nicht zuletzt daran, dass nicht nur der gewählte Zugang, sondern auch die 
behandelten Gegenstände und Themenfelder den Rahmen herkömmlicher Sportwissenschaft sprengten und ein durchaus weiteres Interesse ansprachen. Arbeiten wie Ulrike Prokops Soziologie der Olympischen Spiele (1971), erschienen ein Jahr vor den Sommerspielen in München, oder Gerhard Vinnais Fußballsport als Ideologie (1970), publiziert im Weltmeisterschaftsjahr 1970, und der von ihm edierte Band Sport in der Klassengesellschaft (1972) seien hier beispielhaft erwähnt. Wenn Gunter A. Pilz behauptet, dass die neomarxistische Kritik am bürgerlichen Sport »wesentlich dazu beitrug, dass die Sportsoziologie in den Startlöchern hängen zu bleiben drohte« (Pilz I994: 446), dann scheint uns diese Bemerkung zwar reichlich unzutreffend, doch erinnert sie daran, dass der Stachel tief gesessen haben muss. Rückblickend betrachtet hatten die frühen Funktionalisten und die Ideologiekritiker wiewohl auf paradoxe Weise - mehr gemein, als sie zu denken gewagt hätten. Während nämlich die einen zu nahe an ihrem Gegenstand (dem Sport) waren, vielleicht gar zu sehr in ihm steckten (nicht zufällig waren und sind zahlreiche Sportsoziologen heftig bekennende Sportler), trachteten die anderen meist, ihm lebensweltlich nicht $\mathrm{zu}$ nahe zu kommen. Letzteres war auf die Dauer nicht wirklich durchzuhalten, der rein ideologiekritische Zugang hatte sich alsbald erschöpft. Eine Arbeit wie Gerd Hortleders aus dieser Tradition kommende, aber durchaus differenzierter argumentierende Studie über Die Faszination des Fußballspiels (1974) lässt schon in ihrem Titel die unterschiedliche Betrachtung anklingen: Die Faszination des Fußballspiels wird untersucht, nicht sein ideologischer Charakter abgeleitet.

Während also der sportwissenschaftlich geprägte Mainstream die Beiträge, die aus der Tradition der Kritischen Theorie oder, weiter gefasst, aus der des Neomarxismus kamen, als störend zurückwies (vielleicht mit Ausnahme der Arbeiten Rigauers), tat er sich mit bestimmten anderen paradigmatischen Zugängen nicht so schwer. Henning Eichbergs (1973, I978) sozialhistorisch argumentierende, aber durchaus theoretisch gedachte Überlegungen zum Sport als bürgerliche Einrichtung, festgemacht an den Kategorien des Rekords (Messen, Quantifizieren), wurden zwar für die Entwicklung der Disziplin »Sportsoziologie« nicht konstitutiv, aber von ihr wenigstens zur Kenntnis genommen. Einflussreicher allerdings sollten die zahlreichen Arbeiten von Gunter A. Pilz zum Themenfeld »Sport und Gewalt« werden (vgl. Pilz i982; Pilz et al. I982). Über Pilz fand nicht nur besagte Problematik Eingang in die deutsche Sportsoziologie, entlang 
dieser Schiene (genauer: der Linie Hooliganismus, Fußballrowdytum) formierte sich auch der figurationssoziologische Ansatz als ein wichtiges Element der deutschen Sportsoziologie. Dies passierte wohl nicht zuletzt deshalb so erfolgreich, weil auf diese Art mit Norbert Elias ein großer deutscher Soziologe gleichsam symbolisch »heimgeholt« werden konnte und, was nicht vergessen werden sollte, innerhalb der internationalen Sportsoziologie (insbesondere der englischen, vgl. Dunning 197I, 1992, I999; Dunning/Sheard 1979; Elias/Dunning 1986) das von Elias sich ableitende Paradigma eines der wichtigen vorstellt. ${ }^{3}$ Im letzten Jahrzehnt war zudem ein deutlicher Aufschwung von systemtheoretischen Ansätzen, orientiert am Werk Niklas Luhmanns, einem der einflussreichsten Theoretiker der deutschen Soziologie seit den Ig6oer Jahren, in der deutschen Sportforschung zu verzeichnen, dem vor allem Karl-Heinrich Bette den Weg bahnte (dessen Arbeiten allerdings bislang kaum eine nennenswerte Resonanz in der internationalen sportsoziologischen Forschungsgemeinde fanden) (vgl. Bette I992).

Ein sportwissenschaftlicher Außenseiter sei hier noch genannt. Es handelt sich um den frankophilen Sportphilosophen Gunter Gebauer, dessen gemeinsam mit Gerd Hortleder 1986 herausgegebener Band Sport - Eros - Tod (Hortleder/Gebauer I986) der deutschen gesellschaftswissenschaftlich orientierten Sportforschung die Chance geboten hätte, sich theoretisch in mehrfacher Weise zu öffnen. Der Bogen der darin versammelten Aufsätze spannte sich von an Pierre Bourdieu orientierten Gedanken zum Sport im Kontext »sozialer Klassen« bis hin zu einer strukturalistischen Lesart der sportlichen Inszenierungen zwischen den Topoi Eros und Tod. Allein schon der Umstand, dass das Buch erstmalig einen der wichtigsten Texte aus Roland Barthes Mythologies ${ }^{4}$, nämlich »Die Tour de France als Epos«, in deutscher Sprache präsentierte, deutet die Richtung an, in die eine innovationswillige Sportsoziologie bzw. -wissenschaft bei einer eingehenderen Rezeption des Bandes hätte gehen können. ${ }^{5}$

\section{Die lage in Österreich}

Erkenntnistheoretisch habe die österreichische Sozialwissenschaft generell eine große Affinität zu realistisch-positivistischen Ansätzen und kaum eine Tradition in (diskursiver) Gesellschaftstheorie, merk- 
ten Gilbert Norden und Otmar Weiss I992 an, um auf daraus resultierende Unterschiede in der Sportsoziologie Deutschlands und Österreichs hinzuweisen. Während in der ehemaligen BRD in den beginnenden I970er Jahren eine von der Frankfurter Schule bzw. neomarxistisch orientierten Sozialwissenschaftlern losgetretene Diskussion um die Rolle des Sports in kapitalistischen Gesellschaften geführt worden sei, habe es in Österreich keine namhaften Vertreter der Kritischen Sporttheorie bzw. Linken Sportkritik und somit auch keine entsprechenden Kontroversen gegeben (vgl. Norden/Weiss I992: II).

Diese Diagnose scheint stimmig, trifft aber den Kern der Sache nicht genau, nämlich insofern, als in Österreich um I970 schlicht von keinerlei systematischen Sportsoziologie die Rede sein konnte, weder von einer ausgeprägten funktionalistischen Ausrichtung noch von gesellschaftskritischen Ansätzen und somit auch nicht von einem wissenschaftlichen Diskursfeld. Vielmehr befand sich die österreichische Sportsoziologie in dieser Zeit in einer ersten Konstituierungsphase, was sich vielleicht am besten daran ablesen lässt, dass es erst I968 mit der Gründung des »Arbeitskreises für Soziologie des Sports und der Leibeserziehung « zu einer rudimentären Institutionalisierung, einem außeruniversitären Diskussionsforum, der sportsoziologischen Forschung kam. Allein der institutionelle Aufschwung der Sportsoziologie im Anschluss an diesen späten Beginn gestaltete sich keineswegs als Aufholprozess angloamerikanischer oder bundesdeutscher Entwicklungen, sondern verlief äußerst träge und bruchstückhaft. Lediglich drei Wissenschaftler wurden seither für den Bereich Sportsoziologie habilitiert, es gibt in ganz Österreich einen einzigen Lehrstuhl für dieses Fach und kaum soziologische Dissertationen, die der Sportsoziologie zuzurechnen sind (vgl. ebd.: I0). Erst im Jahr I99I wurde eine Sektion »Sportsoziologie« in der Österreichischen Gesellschaft für Soziologie eingerichtet (die allmählich die Funktionen des oben genannten Arbeitskreises übernahm), und seit I989 erscheint zweimal jährlich das Spectrum der Sportwissenschaften, das sich als einzige namhafte wissenschaftliche Zeitschrift in Österreich zumindest sporadisch sportsoziologischen Themen widmet.

Diese Fakten verweisen auf eine bis heute bestehende marginale Position der Sportsoziologie auf dem Gebiet der Sozial- und Kulturwissenschaften, insbesondere auf die mangelnde Verankerung der Disziplin auf universitärer Ebene. Diese defizitäre Situation hat in den letzten Jahrzehnten zumindest drei erhebliche Konsequenzen hin- 
sichtlich der Theoriebildung und inhaltlicher Forschungsschwerpunkte nach sich gezogen: Erstens ist nach wie vor kein klares Profil einer österreichischen Sportsoziologie erkennbar, d.h., ebenso fragmentarisch wie die Institutionalisierung stellt sich die theoretische und inhaltliche Ausrichtung des Forschungsbereichs dar. Zweitens sind nur wenige theoretische Ansätze der internationalen Sportforschung in Österreich rezipiert worden, z.B. hat kaum eine nachträgliche Auseinandersetzung mit der angesprochenen Kritischen Theorie stattgefunden, eher ist ein Aufschwung theorieloser Empirie und (positivistischer) handlungstheoretischer Positionen zu beobachten. Drittens bewirkte das universitäre Forschungsvakuum auf dem Gebiet des Sports, dass der außeruniversitären Beschäftigung mit diesem Thema, auch im Hinblick auf eine theoretische Erneuerung, eine besondere Bedeutung in Österreich zukam.

Von der Konstituierungsphase bis in die späten I980er Jahre bildeten die Bereiche sportbezogene Einstellungen sowie Umfang und Richtung sportlicher Aktivitäten der Bevölkerung die Schwerpunkte der sportsoziologischen Forschung in Österreich. Dazu gehörten (theorielose) für Österreich repräsentative Erhebungen großer Meinungsforschungsinstitute (vgl. etwa IFES I969 oder IMAS I978), an Lebensstilkonzepte angelehnte soziodemographische Analysen zur Sportausübung (vgl. Bässler I989, I990), empirische Einstellungsuntersuchungen wie Reinhard Bachleitners Arbeit aus dem Jahr ${ }_{19} 83$ ebenso wie Studien zum Image des Sports (vgl. Weiss/Russo 1987) oder rollentheoretische, an George $\mathrm{H}$. Mead anknüpfende Arbeiten zum Verhältnis von Sport und Gesellschaft bzw. zur sportlichen Identität der Österreicher (vgl. Weiss I989, I990). In thematischer Hinsicht blieben in diesem Zeitraum alle übrigen möglichen Forschungsbereiche stark unterbelichtet, lediglich vereinzelt findet man etwa kulturvergleichende Analysen, Studien zur sportlichen Sozialisation oder zu sportlichen Kleingruppen. Besonders vernachlässigt wurden die Bereiche Sport und Politik, Sport und Ökonomie sowie Sport und Massenmedien, darüber hinaus existierte kaum eine historische Sportforschung, ganz zu schweigen davon, dass zentrale Themen neuer gesellschaftswissenschaftlicher Ansätze, etwa die Geschlechterund Globalisierungsproblematik und damit die Frage nach hegemonialen Strukturen und Machtverhältnissen, keinerlei Eingang in die österreichische Sportsoziologie der I970er und 8oer Jahre gefunden haben. 
Mit einem Wort: Die österreichische Sportsoziologie präsentierte sich in diesen zwei Jahrzehnten als heterogenes Stückwerk, und die Forschungsergebnisse entsprangen keineswegs einer systematischen sportsoziologischen Debatte, sondern resultierten aus voneinander abgeschotteten Einzelinitiativen. Diese Mängel der Forschungsdisziplin scheinen auch hauptverantwortlich dafür zu sein, dass die österreichische Sportsoziologie im besonderen Maße »Probleme der Identität und der internationalen Akzeptanz« hat, wie Norden und Weiss Anfang der I99oer Jahre schrieben, wobei sie argumentierten, »dass deutschsprachige Soziologien, die außerhalb Deutschlands beheimatet sind (Norden/Weiss I992: I7), generell diese Schwierigkeiten hätten. In diesem Kontext erwies und erweist es sich auch nicht als zielführend, wenn »sich österreichische Sportsoziologen vornehmlich an bundesdeutschen Kollegen orientieren« (ebd.: I6), d.h., sich die österreichische Forschung als rudimentäres Anhängsel der deutschen Sportwissenschaft geriert, anstatt eigene, österreichspezifische Wege zu gehen - noch dazu, da wichtige theoretische Entwicklungen in der Bundesrepublik Deutschland wie der Einfluss der Systemtheorie auf die Sportforschung (vgl. Bette I992) nicht oder kaum wahrgenommen werden.

Die prinzipielle Ausrichtung der österreichischen Forschung an deutschen Sportdiskursen führte in den I970er und 8oer Jahren vor allem dazu, dass bedeutende neuere Theorieansätze britischer oder französischer Provenienz nicht rezipiert wurden (wiewohl sie in Deutschland, wie wir zu zeigen versucht haben, zumindest am Rande des Mainstreams eine gewisse Rolle spielten). So kommt der englischen Figurationssoziologie, die Eric Dunning seit den I970er Jahren im Sportbereich propagiert (vgl. Dunning I97I; Dunning/Sheard I979), bis heute keine Bedeutung in Österreich zu, ebenso wenig hat Pierre Bourdieus Habitus-Konzept (vgl. Bourdieu I982) - dessen Nutzen für Sportanalysen wie gesagt vor allem Gebauer in Deutschland dargelegt hat (vgl. etwa Hortleder/Gebauer I986) - Eingang in die österreichische Sportsoziologie der beiden Jahrzehnte gefunden. Das gleiche gilt für poststrukturalistische und diskursanalytische Ansätze, die erst spät in österreichischen Sportstudien zum ersten Mal erwähnt wurden (vgl. Penz I990; Miller/Penz I992), und ein ähnliches Defizit lässt sich auch bis gegen Ende der ig8oer Jahre im Hinblick auf Cultural Studies-Perspektiven in der Sportforschung feststellen. 


\section{Sport und Cultural Studies international}

Zum gegenwärtigen Stand sportsoziologischer Forschungsansätze im britischen und nordamerikanischen Raum schreiben Jennifer Hargreaves und Ian McDonald resümierend: »The field has become highly contested. Nevertheless, it is widely recognized that cultural studies is one of the key players « (Hargreaves/ McDonald 2000: 48). Diese Bedeutung von Cultural Studies-Analysen erscheint umso erstaunlicher, als der Bereich des Sports bis Anfang der ig8oer Jahre mit Sicherheit nicht $\mathrm{zu}$ den bevorzugten Forschungsgebieten kulturalistisch-strukturalistischer Arbeiten gehörte. Datiert man den Beginn einer regen Cultural Studies-Forschungstätigkeit mit der Gründung des CCCS an der Universität Birmingham im Jahr 1964, wo die theoretischen und methodischen Ansätze von Richard Hoggart (I957), Raymond Williams (1958) und Edward P. Thompson (1963) systematisch weiterverfolgt wurden, so verstreichen beinahe zwei Jahrzehnte, bis sich der Einfluss der neuen Forschungsperspektive auch auf sportlichem Gebiet bemerkbar machte.

Mehrere Gründe lassen sich für diese verzögerte Rezeption anführen: zum einen die Eigentümlichkeit des sportlichen Feldes selbst, d.h. die Vielzahl an Formen und Bedeutungen, die für den Sport charakteristisch sind und eine Analyse des Gesamtgebildes unmöglich machen. Im Unterschied zu anderen, relativ homogenen popularkulturellen Genres wie etwa die »Trivialliteratur« differieren die Bedeutungen und Probleme beispielsweise von aktivem Freizeitsport und Konsum des Schausports beträchtlich - und die Kontextualisierung des Untersuchungsgegenstandes hat damit jeweils auf höchst spezifische Weise zu erfolgen, mit geringen Möglichkeiten, auf allgemeine Erkenntnisse über den Sport zurückgreifen zu können. Daneben erschwert die Unmittelbarkeit und Flüchtigkeit vieler sportlicher Erlebnisse die Analyse, d.h., nur Teilbereiche des Sports lassen sich wiederum im Gegensatz zu anderen Feldern der Popularkultur aufgrund von Artefakten untersuchen, und vor allem die Analyse körperlicher Erfahrungen und Empfindungen wirft neue methodische Probleme auf. Zum anderen und wahrscheinlich der Hauptgrund für die zögerliche Entdeckung des Sports als Forschungsgebiet der Cultural Studies dürfte die fehlende Tradition einer kritischen Sport-Betrachtungsweise gewesen sein. Sowohl in wissenschaftlicher Hinsicht, vor allem durch die Dominanz funktionalistischer Ansätze, als auch 
im Alltagsverständnis galt der Sport in den Ig6oer und 7oer Jahren durchwegs als unproblematisches, unpolitisches, weitgehend konfliktfreies, mit einem Wort rundweg positiv zu bewertendes Betätigungsfeld. Ein Zugang, der darauf abstellt, soziale Ungleichheiten im Sport aufzuzeigen, etwa zwischen den Geschlechtern oder weißen und schwarzen Athleten, oder danach trachtet, hegemoniale Strukturen und Machtverhältnisse offen zu legen, hatte in diesem Kontext erhebliche diskursive Barrieren zu überwinden, um sich als Forschungsrichtung zu etablieren (vgl. Hargreaves I982: I6).

Im Zuge einer generellen Formierung der Sportkritik Anfang der I970er Jahre, die von neomarxistischen Arbeiten bundesdeutscher und angloamerikanischer Provenienz ebenso wie von figurationssoziologischen Studien vorangetrieben wurde (vgl. Hoch 1972; Vinnai I973; Dunning I97I), begannen auch Cultural Studies-Vertreter sich dem Sport zuzuwenden, d.h. dieses kulturelle Massenphänomen im Sinne einer everyday world of lived reality (Valda Blundell) ernsthaft zu hinterfragen. Allerdings erschienen im gesamten Verlauf der I970er Jahre nur sporadisch Veröffentlichungen des CCCS zum Thema »Sport«, und diese konzentrieren sich auf drei Bereiche: Zuschauergewalt im Fußballsport, Sport und Massenmedien sowie die Geschlechterfrage (vgl. etwa Critcher 197I, I974; Clarke I973; Willis 1974). Bahnbrechend und damit konstitutiv für eine umfänglichere Cultural Studies-Sportforschung wirkten erst zwei Publikationen der frühen I980er Jahre, der von Jennifer Hargreaves I9 82 editierte Sammelband Sport, Culture and Ideology und Richard Gruneaus Class, Sports, and Social Development aus dem Jahr I983. »It was very much a reaction to the orthodox Marxist tendency in sport sociology to reduce sport to a mirror of capitalist society«, schreibt Hargreaves im Rückblick auf die Entstehungsgeschichte des damaligen Werkes: »Many of the authors adopted a cultural studies perspective and collectively produced the first sport sociology book with this orientation « (Hargreaves/McDonald 2000: 52; vgl. Hargreaves 1982). Gruneaus oben genanntes Buch wiederum zeichnete sich in erster Linie dadurch aus, Antonio Gramscis Hegemonie-Konzept für den Sport fruchtbar gemacht zu haben, indem er den Machtverhältnissen in der Entwicklungsgeschichte des kanadischen Sports nachspürte und dabei das klassisch-soziologische agency-structure-Dilemma aufgriff und analysierte (vgl. Gruneau I983).

Diesen beiden Publikationen folgten in den I980er und 9oer Jahren im englischen Sprachraum eine Vielzahl an Cultural Studies- 
Sportveröffentlichungen, die sich an den generellen Fragen der gesamten Forschungsrichtung orientierten, so sie etwa Klassen- und Rassenunterschiede im Sport aufgriffen - wobei den massenmedialen Repräsentationen des Sports besondere Aufmerksamkeit geschenkt wurde (vgl. etwa Cantelon/Gruneau I988; Whannel I992) - oder theoretische Ansätze wie etwa Williams dominant-residual-emergentThema weiterentwickelten (vgl. Ingham/Loy I993). Die Geschlechterproblematik und damit zusammenhängend die kritische Analyse der Repräsentationen des Körpers, von Maskulinität und Femininität, spielten in den I99oer Jahren eine zunehmend wichtige Rolle in der Cultural Studies-Sportforschung (vgl. Messner/Szabo I990; Hall I996; zsf. zum Thema »Körper und Sport« vgl. Cole 2000), und neuerdings rücken auch auf dem Gebiet des Sports Identitäts- sowie Globalisierungs- und Neokolonialismusfragen stärker in den Mittelpunkt des Forschungsinteresses (vgl. z.B. Maguire 2000). Zusammenfassend beschreiben Grant Jarvie und Joseph Maguire die Ziele und Ansprüche dieser relativ jungen Forschungsrichtung im Sport folgendermaßen:

"1(i) to consider the relationship between power and culture; (ii) to demonstrate how a particular form of sport or leisure has been consolidated, contested, maintained or reproduced within the context of society as a whole; and (iii) to highlight the role of sport and leisure as a site of popular struggle» (Jarvie/Maguire 1994: 124).

Gegenüber anderen kritischen Ansätzen in der Sportforschung mit einer ähnlichen Programmatik zeichnet sich dieses Projekt vor allem durch seine transdisziplinäre Ausrichtung und eine Engführung von Theorie und Empirie aus, durch die (wie von Jarvie und Maguire angesprochen) breite Kontextualisierung des Untersuchungsgegenstandes, also die Einbettung sportlicher Prozesse in gesamtgesellschaftliche soziale, politische und ökonomische Zusammenhänge - erinnert sei hier noch einmal an Williams Definition von Kultur als whole way of life -, sowie durch den interventionistischen Charakter der Cultural Studies-Forschung. Hargreaves und McDonald zufolge weise "gute« kulturalistisch orientierte Sportforschung vor allem drei grundlegende Charakteristika auf:

„First, it is receptive to, and engages with, different theoretical traditions. Secondly, unlike much of contemporary social theory within the academy, its starting point has 
been the real world, linking theory to empirical investigations and producing, theoretically grounded research. And, thirdly, it has taken sides politically - by developing a form of intellectual engagement that is interventionist. Cultural studies exposes power relationships where none have been assumed, and respects the contribution and creative potential of marginalized, oppressed and exploited groups « (Hargreaves/McDonald 2000: 57 f.).

Diese Forschungsperspektive hat sich seit den frühen I980er Jahren vor allem im angloamerikanischen Raum als ergiebig erwiesen, was sich nicht zuletzt daran ablesen lässt, dass die wichtigsten Cultural Studies-Publikationen Eingang in sportsoziologische Einführungstexte und Lehrbücher gefunden haben (vgl. etwa Hall et al. I99ı, insb. Kap. 2 »Sport and Culture «) bzw. dass diese Art Forschung heutzutage als eigenständige Richtung - u.a. neben der Konflikttheorie, der Frankfurter Schule, der Figurationssoziologie oder poststrukturalistischen Studien - einer kritischen Sportwissenschaft gehandelt wird (vgl. Coakley/Dunning 2000). Ausständig ist nach wie vor eine breite Rezeption der Cultural Studies im deutschsprachigen Raum, wo Ansätze zu einer derartigen Betrachtungsweise des Sports wie skizziert bislang auf wenig Resonanz im etablierten (Sport-)Wissenschaftsbetrieb gestoßen sind.

\section{Schlussbemerkungen}

Der Stellenwert der Cultural Studies in der Sportforschung erweist sich, wie wir zu zeigen versucht haben, als höchst unterschiedlich, je nachdem ob man den angloamerikanischen Raum (mit einer insgesamt stark ausgeprägten Sportsoziologie) oder die deutschsprachigen Verhältnisse betrachtet. Während in Großbritannien und Nordamerika die Cultural Studies-Forschung den vorherrschenden sozial- und kulturwissenschaftlichen Sportdiskurs mit prägt, kann ihr Einfluss in Deutschland und Österreich nur als marginal bezeichnet werden, wobei die Situation in den beiden letztgenannten Ländern doch ein wenig differiert. Hat sich in Österreich zumindest ein kleines kohärentes Forschungsfeld rund um das Fußballspiel herausgebildet, auf dem die interdisziplinäre, kontextuelle und interventionistische Arbeitsweise der Cultural Studies gepflegt und (mit internationaler Beteiligung) vorangetrieben wird, so lassen sich in der gegenwärtigen bun- 
desdeutschen Sportforschung kaum Spuren derartiger Analyseansätze entdecken. Ein gewichtiger Grund, wenn nicht hauptverantwortlich für die mangelnde Offenheit der Sportforschung gegenüber innovativen Sichtweisen, die den Sport im Rahmen übergreifender kultureller Zusammenhänge $\mathrm{zu}$ hinterfragen trachten, dürfte wohl die unter strukturfunktionalistischen Gesichtspunkten propagierte und teilweise realisierte Formierung eines Subsystems »Sportwissenschaft« sein, wodurch u.a. die »Sportsoziologie zur speziellen Sportwissenschaft wird [...] und sich damit allmählich von dem rein fachbezogenen soziologischen Forschungsinteresse löst« (Heinemann I980: 27). Diese Reduktion bzw. Indienstnahme mehrerer Wissenschaftsdisziplinen, etwa auch der Pädagogik oder Sozialpsychologie, scheint sich heutzutage mehr als Manko denn als Segen für eine gesellschaftstheoretische Betrachtungsweise des Sports zu entpuppen, denn bewirkt wird damit letzten Endes eine Verengung des Blickwinkels auf sportinhärente Funktionsweisen und Probleme und eine hermetische Abschließung der Sportforschung gegenüber transdisziplinären und kulturell integrativen Forschungsperspektiven.

\section{Anmerkungen}

I Ian Taylor kommt eigentlich aus der »New Criminology«, die Argumentation und Referenztexte seiner Fußballaufsätze und nicht zuletzt der Umstand, dass er in Jennifer Hargreaves richtungsweisendem Sammelband Sport, Culture and Ideology (1982) mit einem Aufsatz vertreten war, lassen es berechtigt scheinen, ihn hier zu erwähnen.

$2 \mathrm{Zu}$ verschiedenen Aspekten der Rezeption von Cultural Studies im deutschen Sprachraum sind vor allem in den letzten Jahren zahlreiche Publikationen erschienen (vgl. u.a. Lindner 1994; Mikos 1997; Göttlich 1999; Horak 1999, 2002), von denen die meisten, bei allen Unterschieden in der jeweiligen Schwerpunktsetzung und Sichtweise, darin übereinstimmen, dass neben den singulären Anstrengungen von H. Gustav Klaus (vgl. 1983, I993, I994) vor allem der Zeitschrift Ästhetik und Kommunikation (Ä\&K) eine Vorreiterrolle beim Aufgreifen von Theoremen und Debatten aus dem Umfeld der so genannten British Cultural Studies zukommt. Neben diesem Diskursfeld sind, vor allem im Verlauf der späten I98oer und der goer Jahre, mehrere 
andere Rezeptionslinien entstanden, die zum einen, wenngleich mehrfach gebrochen, Veränderungen innerhalb des Projekts Cultural Studies widerspiegeln, zum anderen aber die Schwierigkeiten der traditionellen deutschen akademischen Welt mit jener transdisziplinären Forschungsrichtung sichtbar machen. Fassen wir hier die Aufbereitung der Cultural Studies in Deutschland kurz zusammen: Da gab es zuallererst die sich mehr und mehr pädagogisierende Jugendkulturdebatte, weiterhin wäre jene Rezeption $\mathrm{zu}$ verorten, die, im Umkreis der Zeitschrift Argument, im Rahmen der »Ideologiedebatte« betrieben wurde und an die sich jene der Rassismus- und Ethnizitätsforschung anschloss (vor allem im Rahmen der Aktivitäten des Hamburger Instituts für Migrations- und Rassismusforschung). Die so genannte deutsche Poplinke (Spex) beschließt einmal den Reigen der außeruniversitären Auseinandersetzung mit den Cultural Studies. Mit den (späten) I99oer Jahren beginnen die heftigen Aneignungsversuche seitens einer traditionellen universitären Medien- und Kommunikationswissenschaft, und jüngst scheinen sich auch die klassischen Kulturwissenschaften, deren innere Verfasstheit und Geschichte eine ernsthafte Auseinandersetzung mit den Traditionen der Cultural Studies geradezu ausschließt (vgl. Horak I999), an die - nicht mehr ganz so junge Braut - heranzumachen.

3 Aus Platzgründen ist es hier nicht möglich, auf alle weiteren (eher minoritären) theoretischen und methodischen Strömungen in der frühen Sportsoziologie einzugehen. Zumindest hingewiesen sei aber auf einflussreiche, unterschiedliche Ansätze vertretende Autoren wie Hans Lenk, Christian Graf von Krockow, Volker Rittner oder Wilhelm Hopf.

4 Bei der Herausgabe der deutschen Übersetzung wurde der sportliche Text scheinbar als »unwichtig« erachtet (vgl. Barthes i964). In der englischen Übertragung aus dem Jahre I972 war man da nicht so ignorant.

5 Pierre Bourdieus Überlegungen zum Sport wurden - im Zuge der allgemeinen Bourdieu-Hausse der deutschen Sozialwissenschaften seit den ausgehenden I980er Jahren - wenigstens ansatzweise zur Kenntnis genommen. 


\section{Literatur}

Adorno, Theodor W. (I969): »Freizeit«. In: Ders., Stichworte. Kritische Modelle 2, Frankfurt a.M.: Suhrkamp, S. 57-67.

Bachleitner, Reinhard (1983): Werteinstellungen zum Sport. Empirische Untersuchung zu Einstellungen, Funktionsbewertungen und Stellenwertbestimmungen des Sports, Bad Homburg: Limpert.

Bässler, Roland (1989): Freizeit und Sport in Österreich. Trends im Freizeitverhalten der Bevölkerung unter besonderer Berücksichtigung des Sports, Wien: Wiener Universitätsverlag.

Bässler, Roland (I990): »Sportaktivität und Sportabstinenz«. Spectrum der Sportwissenschaften 2, S. 78-103.

Barthes, Roland (1964): Die Mythen des Alltags, Frankfurt a.M.: Suhrkamp.

Bette, Karl-Heinrich (1992): Theorie als Herausforderung. Beiträge zur systemtheoretischen Reflexion der Sportwissenschaft, Aachen: Meyer \& Meyer.

Bourdieu, Pierre (1982): Die feinen Unterschiede. Kritik der gesellschaftlichen Urteilskraft, Frankfurt a.M.: Suhrkamp.

Cantelon, Hart/Gruneau, Richard (I988): »The Production of Sport for Television«. In: J. Harvey/Hart Cantelon (Hg.), Not Just a Game, Ottawa: University of Ottawa Press, S. I77-I94.

Clarke, John (I973): »Football Hooliganism and the Skinheads«. CCCS Stencilled Occasional Paper Series No. 42.

Coakley, Jay/Dunning, Eric (Hg.) (2000): Handbook of Sport Studies, London: Sage.

Cole, Cheryl L. (2000): »Body Studies in the Sociology of Sport«. In: Jay Coakley/Eric Dunning (Hg.), Handbook of Sport Studies, London: Sage, S. 439-460.

Critcher, Chas (I97I): »Football and Cultural Values«. Working Papers in Cultural Studies, 1 (Spring), S. I03-II9.

Critcher, Chas (I974): »Football since the War: Study in Social Change and Popular Culture«. CCCS Stencilled Occasional Paper Series No. 29.

Dunning, Eric (I97I) (Hg.): The Sociology of Sport, London: Cass.

Dunning, Eric (I992): »Über die Dynamik des Sportkonsums: Eine figurative Analyse«. In: Roman Horak/Otto Penz (Hg.), Sport. Kult \& Kommerz, Wien: Verlag für Gesellschaftskritik, S. 203-222. 
Dunning, Eric (I999): Sport Matters: Sociological Studies of Sport, Violence and Civilization, London/New York: Routledge.

Dunning, Eric/Sheard, Kenneth (I979): Barbarians, Gentlemen and Players. A Sociological Study of the Development of Rugby Football, Oxford: Martin Robertson.

Eder, Ernst Gerhard (I990): »Schwimmdisziplin. Zur Vergesellschaftung körperlicher Fähigkeiten, Fertigkeiten und Möglichkeiten«. Österreichische Zeitschrift für Soziologie 2, S. 52-67.

Eichberg, Henning (I973): Der Weg des Sports in die industrielle Zivilisation, Baden-Baden: Nomos.

Eichberg, Henning (1978): Leistung, Spannung, Geschwindigkeit. Sport und Tanz im gesellschaftlichen Wandel des 18./19. Jahrhunderts, Stuttgart: Klett-Cotta.

Elias, Norbert/Dunning, Eric (1986): The Quest for Excitement: Sport and Leisure in the Civilizing Process, Oxford: Blackwell.

Gehrmann, Siegfried (1988): Fußball - Vereine - Politik. Zur Sportgeschichte des Reviers 1900-1940, Essen: Reimar Hobbing Verlag.

Göttlich, Udo (I999): »Die Wahrnehmung der Cultural Studies«. Sociologia Internationalis 37/2, S. 189-220.

Gruneau, Richard (1983): Class, Sports, and Social Development, Amherst: The University of Massachusetts Press.

Habermas, Jürgen (1958/67): »Soziologische Notizen zum Verhältnis von Arbeit und Freizeit«. In: H. Plessner/E. Bock/O. Gruppe (Hg.), Sport und Leibeserziehung, München: Piper, S. 28-45.

Hall, Ann/Slack, Trevor/Smith, Garry/Whitson, David (I99I): Sport in Canadian Society, Toronto: McClelland \& Stewart.

Hall, Ann (I996): »The Potential of Feminist Cultural Studies«. In: Dies., Feminism and Sporting Bodies, Champaign: Human Kinetics, S. 29-48.

Hall, Stuart (I973): »Encoding and Decoding in the TV Discourse CCCS Stencilled Occasional Paper Series No. 7.

Hammerich, Kurt/Heinemann, Klaus (Hg.) (I975): Texte zur Soziologie des Sports, Schorndorf: Hofmann-Verlag.

Hargreaves, Jennifer (Hg.) (I982): Sport, Culture and Ideology, London: Routledge.

Hargreaves, Jennifer/McDonald, Ian (2000): »Cultural Studies and the Sociology of Sport«. In: Jay Coakley/Eric Dunning (Hg.), Handbook of Sport Studies, London: Sage, S. 49-6o.

Hartmann-Tews, Ilse/Rulofs, Bettina (I998): »Entwicklungen und 
Perspektiven der Frauen- und Geschlechterforschung im Sport«. Spectrum der Sportwissenschaften 2, S. 7I-85.

Hebdige, Dick (I97I): »The Style of the Mods«. CCCS Stencilled Occasional Paper Series No. 20.

Heinemann, Klaus (I980): Einführung in die Soziologie des Sports, Schorndorf: Hofmann-Verlag.

Heinemann, Klaus (2000): »Germany«. In: Jay Coakley/Eric Dunning (Hg.), Handbook of Sport Studies, London: Sage, S. 536-538.

Hoch, Paul (I972): Rip Off the Big Game, New York: Doubleday.

Hoggart, Richard (I957): The Uses of Literacy, Harmondsworth: Penguin.

Horak, Roman (I998): »Ä \& K 24 wiedergelesen. Cultural Studies in Wien«. Ästhetik \& Kommunikation 29/100, S. 99-104.

Horak, Roman (I999): »Cultural Studies in Germany (and Austria) and Why There Is No Such Thing«. European Journal of Cultural Studies 2/I, S. IO9-II5.

Horak, Roman (2002): »Cultural Studies in Deutschland und Österreich. Eine Intervention in Gestalt einer Rezeptionsgeschichte«. In: Ders., Die Praxis der Cultural Studies, Wien: Turia + Kant [im Druck].

Horak, Roman/Maderthaner, Wolfgang (I997): Mehr als ein Spiel. Fußball und populare Kulturen im Wien der Moderne, Wien: Löcker.

Horak, Roman/Marschik, Matthias (I995): Vom Erlebnis zur Wahrnehmung. Der Wiener Fußball und seine Zuschauer 1945-1990, Wien: Turia + Kant.

Horak, Roman/Marschik, Matthias (I997): Das Stadion - Facetten des Fußballkonsums in Österreich, Wien: Wiener Universitätsverlag.

Horak, Roman/Reiter, Wolfgang (Hg.) (I99I): Die Kanten des runden Leders. Beiträge zur europäischen Fußballkultur, Wien: Promedia.

Horak, Roman/Reiter, Wolfgang/Stocker, Kurt (1987): »Soccer Hooliganism. Bericht über eine Studie zur Fan-Subkultur und Fußballgewalt in Österreich«. Journal für Sozialforschung 27/2, S. 233-24I.

Horak, Roman/Reiter, Wolfgang/Stocker, Kurt (Hg.) (1988): »Ein Spiel dauert länger als go Minuten«. Fußball und Gewalt in Europa, Hamburg: Junius.

Hortleder, Gerd (I974): Die Faszination des Fußballspiels, Frankfurt a.M.: Suhrkamp. 
Hortleder, Gerd/Gebauer, Gunter (I986): Sport - Eros - Tod, Frankfurt a.M.: Suhrkamp.

IFES (Institut für empirische Sozialforschung) (I969): Sport und Öffentlichkeit. Ergebnis einer im Auftrag des ASKÖ durchgeführten Untersuchung, Wien.

IMAS (Institut für Markt- und Sozialanalysen) (I978): Umfrage zur Fußball-WM 1978, Linz.

Ingham, Allan/Loy, John (Hg.) (I993): Sport in Social Development: Traditions, Transitions and Transformations, Champaign: $\mathrm{Hu}-$ man Kinetics.

Ingham, Roger et al. (I978): Football Hooliganism: the Wider Context, London: Inter-Action Imprint.

Jarvie, Grant/Maguire, Joseph (I994): Sport and Leisure in Social Thought, London: Routledge.

Jefferson, Tony/Clarke, John (I973): »Working Class Youth Cultures«. CCCS Stencilled Occasional Paper Series No. 18.

Klaus, H. Gustav (I983): »Kultureller Materialismus«. Das Argument I39, S. 372-378.

Klaus, H. Gustav (I993): »Grundprinzipien des kulturellen Materialismus«. In: Ursula Apitzsch (Hg), Neurath - Gramsci - Williams, Hamburg: Argument, S. 8I-98.

Klaus, H. Gustav (I994): »Hoggart - Williams - Thompson. Bemerkung zur zögerlichen Aufnahme der englischen Kulturdebatte in Deutschland«. IKUS-Lectures I7/I8 (Cultural Studies. Eine Intervention), Wien, S. 4I-49.

Lindner, Rolf (Hg.) (1983): Der Satz »Der Ball ist rund « hat eine gewisse philosophische Tiefe, Berlin: Transit.

Lindner, Rolf (I994): »Cultural Studies in der Bundesrepublik. Eine Rezeptionsgeschichte $\ll$. IKUS-Lectures I7/I8 (Cultural Studies. Eine Intervention), Wien, S. 50-58.

Lindner, Rolf (I998): »Kulturanalyse, Kulturwissenschaft, Cultural Studies«. Ästhetik Q Kommunikation 29/100, S. 105-I09.

Lindner, Rolf (2000): Die Stunde der Cultural Studies, Wien: Wiener Universitätsverlag.

Lindner, Rolf/Breuer, Heinrich Th. (I979): »Sind doch nicht alles Beckenbauers«. Zur Sozialgeschichte des Fußballs im Ruhrgebiet, Frankfurt: Syndikat.

Loy, John W./Booth, Douglas (2000): »Functionalism, Sport and So- 
ciety«. In: Jay Coakley/Eric Dunning (Hg.), Handbook of Sport Studies, London: Sage, S. 8-27.

Lüschen, Günther/Weis, Kurt (Hg.) (I976): Die Soziologie des Sports, Darmstadt/Neuwied: Luchterhand.

Maguire, Joseph (2000): »Sport and Globalization«. In: Jay Coakley/ Eric Dunning (Hg.), Handbook of Sport Studies, London: Sage, S. 356-369.

Marschik, Matthias (I994): »Wir spielen nicht zum Vergnügen«. Arbeiterfußball in der Ersten Republik, Wien: Verlag für Gesellschaftskritik.

Marschik, Matthias (1999): Vom Idealismus zur Identität. Der Beitrag des Sports zum Nationalbewußtsein in Österreich (1945-1950), Wien: Turia + Kant.

McGuigan, Jim (1992): Cultural Populism, London/New York: Routledge.

Messner, Michael/Szabo, Don (Hg.) (I990): Sport, Men and the Gender Order: Critical Feminist Perspectives, Champaign: Human Kinetics.

Mikos, Lothar (1997) »Die Rezeption des Cultural Studies Approach im deutschsprachigen Raum«. In: Andreas Hepp/Rainer Winter (Hg.), Kultur - Medien - Macht, Opladen: Westdeutscher Verlag, S. I59-168.

Miller, Leslie/Penz, Otto (1992): »Men are not really thinking about bodybuilding at all. Körperkompetenz und diskursive Strategien von Bodybuilderinnen«. Österreichische Zeitschrift für Soziologie 4, S. 60-74.

Miller, Leslie/Penz, Otto (I993): »Bodybuilderinnen«. In: Hubert Ch. Ehalt/Otmar Weiss (Hg.), Sport zwischen Disziplinierung und neuen sozialen Bewegungen, Wien/Köln: Böhlau, S. 136-45.

Müllner, Rudolf (1992): »Mobile Körper - Zur Faschisierung des Körpers im Sport des Nationalsozialismus«. Spectrum der Sportwissenschaften 2, S. 54-76.

Norden, Gilbert/Weiss, Otmar (1992): »Geschichte der österreichischen Sportsoziologie«. Österreichische Zeitschrift für Soziologie 4 , S. 5-23.

ÖZS (Österreichische Zeitschrift für Soziologie) (1992), I7/4.

Penz, Otto (I990): »Sport and Speed«. International Review for the Sociology of Sport 25/2, S. 157-167.

Peyker, Ingo (1992): »Was weiß mein Körper, das ich nicht weiß?«. Österreichische Zeitschrift für Soziologie 4, S. 75-9I. 
Pilz, Gunter A. (Hg.) (I982): Sport und körperliche Gewalt, Reinbek bei Hamburg: Rowohlt.

Pilz, Gunter A. (I994): »Sportsoziologie«. In: Harald Kerber/Arnold Schmieder (Hg), Spezielle Soziologien. Problemfelder, Forschungsbereiche, Anwendungsorientierungen, Reinbek bei Hamburg: Rowohlt, S. 443-455.

Pilz, Gunter A. et al. (Hg.) (I982): Sport und Gewalt, Schorndorf: Hofmann-Verlag.

Prokop, Ulrike (I97I): Soziologie der Olympischen Spiele. Sport und Kapitalismus, München: Hanser.

Rigauer, Bero (1969): Sport und Arbeit, Frankfurt a.M.: Suhrkamp.

Rigauer, Bero (I97I): »Inhaltliche und methodologische Bestimmungen einer kritischen Soziologie des Sports im Rahmen der Sportwissenschaften«. Die Leibeserziehung I, S. 9-I4.

Rigauer, Bero (1982): Sportsoziologie. Grundlagen, Methoden, Analysen, Reinbek bei Hamburg: Rowohlt.

Taylor, Ian (I97I): »Soccer Consciousness and Soccer Hooliganism«. In: S. Cohen (Hg.), Images of Deviance, London: Penguin, S. I34-I64.

Taylor, Ian (1976): »Spectator Violence Around Football: the Rise and Fall of the >Working Class Weekend «. Research Papers in Physical Education 4/I, S. 4-9.

Thompson, Edward P. (1963): The Making of the English Working Class, Harmondsworth: Penguin.

Treude, Andrea (I992): »Ich bin nichts Halbes und nichts Ganzes. Ich bin dazwischen - Einige Überlegungen zum weiblichen Körper im Sport«. Spectrum der Sportwissenschaften 2, S. 37-53.

Vinnai, Gerhard (I970): Fußballsport als Ideologie, Frankfurt a.M.: Europäische Verlagsanstalt.

Vinnai, Gerhard (Hg.) (I972): Sport in der Klassengesellschaft, Frankfurt a.M.: Fischer.

Vinnai, Gerhard (I973): Football Mania, London: Ocean Books.

Weiss, Otmar (1989): »Image des Skilaufs. Zur Identität des modernen >homo austriacus««. In: K. Kleiner (Red.), Wiener Beiträge zur Sportwissenschaft I989, Wien, S. 230-239.

Weiss, Otmar (I990): Sport und Gesellschaft. Eine sozialpsychologische Perspektive, Wien: Österreichischer Bundesverlag.

Weiss, Otmar/Russo, Manfred (1987): Image des Sports, Wien: Österreichischer Bundesverlag. 
Weiss, Otmar/Schulz, Wolfgang (Hg.) (1995): Sport in Space and Time. Contributions to the $11^{\text {th }}$ International Seminar for Sociology of Sport, Wien: Wiener Universitätsverlag.

Whannel, Garry (1992): Fields in Vision: Television Sport and Cultural Formation, London: Routledge.

Williams, Raymond (1958): Culture and Society, 1780-1950, London: Chatto and Windus.

Willis, Paul (I974): »Women in Sport«. Working Papers in Cultural Studies 5 (Spring), S. 2I-36. 


\section{Cultural Studies und Ethnologie:}

\section{Zu einem schwierigen Verhältnis}

Sibylle NieKisCh

॥)edes Bemühen um Verständnis zerstört den Gegenstand, mit dem wir uns befassen, zugunsten eines anderen Bemühens, das ihn wiederum vernichtet zugunsten eines dritten und so weiter, bis wir Zugang finden zu der einzigen dauerhaften Gegenwart, derjenigen, bei der sich der Unterschied zwischen dem Sinn und dem Fehlen von Sinn verflüchtigt, jener, von der wir ausgegangen waren« (Lévi-Strauss 1981: 408).

Ich beginne in klassisch ethnologischem Stil mit einem Auszug aus »Feldnotizen«, die bei einer Tagung mit dem Thema »Interdisziplinarität: Die Ethnologie und ihre Nachbardisziplinen« im Sommer I999 in Heidelberg entstanden:

„Die Cultural Studies sind was für Leute, die nicht bereit sind, die harte Schule der Ethnologie zu durchlaufen, die sich nicht die Mühe machen wollen, schwierige ethnologische Terminologien zu lernen, die keine Lust haben, eine fremde und komplizierte Sprache einzustudieren und die sich nicht trauen, sich in eine unbekannte und möglicherweise beunruhigende Umgebung zu begeben. Die Cultural Studies sind grundsätzlich ein theoretisch unterentwickelter Versuch, IKulturı in einer bekannten und daher wenig bedrohlichen Umgebung zu untersuchen - am besten in der eigenen Stammkneipe - und zwar mit unseren ethnologischen Methoden, jedoch in völliger Missachtung unserer theoretischen Grundlagen.॥ 
Dieses Zitat stammt von einem der vielen renommierten Ethnologen, die sich dort versammelt hatten, um über den Status der Ethnologie im Verhältnis zu ihren Nachbardisziplinen zu diskutieren. Der zitierte Ethnologe reagierte mit seinem Kommentar auf einen Vortrag von Signe Howell, in dem sie darlegte, weshalb die Cultural Studies der Ethnologie methodisch und theoretisch unterlegen seien. ${ }^{2}$ Dieser Vortrag rief viele spontane Reaktionen aus dem Publikum hervor, das mit meist aggressiven und polemischen Bemerkungen für Howells Argumentation eintrat. Es schien, als hätte Howell der versammelten Zunft aus dem Herzen gesprochen.

Warum diese Vorbehalte den Cultural Studies gegenüber? Um die Vorwürfe, die an die Cultural Studies von Seiten der Ethnologie häufig herangetragen werden, noch einmal kurz zusammenzufassen: Man wirft ihnen Oberflächlichkeit und mangelnde Seriosität vor, aus ethnologischer Sicht vermögen die Cultural Studies nicht, bedeutungsvolle und tief gehende Analysen von sozialem Leben durchzuführen. Sie würden keine Fremderfahrung im existentiellen Sinne des stationären Langzeitfeldaufenthaltes machen ${ }^{3}$, dennoch aber die Methode der teilnehmenden Beobachtung entwenden, allerdings für die Anwendung im eigenen kulturellen Bereich, ohne dabei exakt und systematisch vorzugehen. Damit ignorierten sie den theoretischen Hintergrund der Ethnologie, sodass schlecht fundierte und oberflächliche Untersuchungen daraus resultierten.

Diesen Vorwürfen liegt eine grundsätzliche Implikation zugrunde: dass die Überschreitung einer Grenze, zumeist territorialer Art, und das Verweilen in der Fremdheit den Blick öffnet für kulturelle Phänomene und ein Fremdverstehen ermöglicht. Das erinnert uns an die erkenntnisphilosophische Einsicht, dass sich das Vertraute nur verstehen lässt, wenn man es verloren hat. Der Verlust hebt es aus der Unsichtbarkeit heraus, die Befremdung ermöglicht den Blick mit anderen Augen (vgl. Plessner I982). Nun drängen sich an dieser Stelle sogleich einige Fragen auf: Öffnet die Fremderfahrung, also der Verlust des Eigenen, auch den Blick für das Fremde? Und macht diese Trennung in Eigenes und Fremdes überhaupt noch Sinn in Zeiten der Enträumlichung? Wenn wir aber alle Insider sind, welche Grenzen gilt es dann noch zu verteidigen?

In diesem Aufsatz sollen folgende Thesen aufgestellt und diskutiert werden: Die Gründe für die Verhinderung von Anschlussstellen auf Seiten der Ethnologie liegen erstens in einer unterschiedlichen 
Einstellung zur Analyse des »Eigenen« und des »Fremden«. In der Ethnologie neigt man zu einer Emphatisierung der Feldforschung als Grenzerfahrung, welcher ein ganz eigenes Verständnis von kultureller Fremdheit zugrunde liegt. Das »Fremde« muss aber aus Sicht der Cultural Studies nicht zwangsläufig außerhalb des eigenen Territoriums liegen. Die Position des außenstehenden Beobachters, der mit seinem panoptischen Blick das Fremde bannen kann, gehört ins diskursive Feld der Moderne. Vieles deutet darauf hin, dass wir es seit einiger Zeit mit einem epistemologischen Bruch zu tun haben.

Der zweite Punkt, auf den ich näher eingehen möchte, ist eng mit dem ersten verknüpft: Die von den Cultural Studies praktizierte lived experience wird von der Ethnologie als unwissenschaftlich verbannt, weil es sich dabei um eine subjektive Erfahrung handelt. Den Cultural Studies ist es aber gerade daran gelegen, unterrepräsentierte Erfahrungen in den akademischen Diskurs einzuführen, wenn jemand über Insider-Wissen verfügt, umso besser. Hier kommt eine grundsätzlich andere Einstellung zu »Wissenschaftlichkeit«zum Tragen. Eng damit verknüpft ist eine differierende Auffassung von »Kultur«, auch darauf werde ich zu sprechen kommen.

An letzter Stelle ist für die schleppende Rezeption auf Seiten der Ethnologie die Angst vor »Kolonisation« verantwortlich. Man fürchtet, die Grenzen der akademischen Identität verschwimmen zu sehen, nicht zuletzt aufgrund der Attraktivität, die die Cultural Studies vor allem auf nachwachsende Generationen ausüben. Denn zwei Eigenschaften der Cultural Studies mögen bedrohlich wirken: ihre intellektuelle Praxis, Cultural Studies als ein Projekt zu verstehen, das Theorien aus verschiedenen Disziplinen als Werkzeugkasten (und nicht als »Lehnstuhl«) nutzt, und ihr eng damit zusammenhängendes fehlendes Bestreben, sich zu institutionalisieren. Gerade ihre Disziplinungebundenheit ist notwendige Voraussetzung der Erforschung kultureller Praxen im Sinne der Cultural Studies (vgl. Göttlich/Winter I999: $27 \mathrm{ff}$.).

Doch zunächst soll das Feld abgesteckt werden, in dem sich das Fach Ethnologie in Deutschland bewegt. Dazu lassen wir zu Beginn am besten einige Vertreter des Faches selbst zu Wort kommen. 


\section{Welche Ethnologie? - Fremdheit und Grenzerfahrung}

Wenn wir im Folgenden über das Verhältnis von »Deutscher Ethnologie $\aleph^{4}$ und Cultural Studies diskutieren wollen, sollte an erster Stelle zunächst einmal geklärt werden, welche Position von ethnologischer Seite aus bezogen wird. Die Ethnologie sieht sich in ihrer Selbstdefinition als eine »Wissenschaft vom kulturell Fremden« (Kohl I993: 25). Dabei wird der Ethnologe gern als kultureller Übersetzer und »Vermittler zwischen den Kulturen « (Roth I996: 24$)^{5}$ betrachtet, als kultureller Fremdheitsexperte. Da sich eine Aufteilung der Forschungsobjekte in Europa für die Volkskunde und außereuropäische Gebiete für die Ethnologie in Zeiten der global cultural flows und der ethnoscapes ${ }^{6}$ nicht aufrechterhalten lässt, möchte sich letztgenannte Disziplin nun, in Ermangelung eines klar definierbaren Untersuchungsgegenstandes, wenigstens »vom Ansatz her [...], weil sich die Ethnologie mit dem Anderen, Fremden befasst « (Hauser-Schäublin I999: 139), von anderen Wissenschaften abgrenzen.

Erwählt sich die Ethnologie das Fremde zum Gegenstand ihrer wissenschaftlichen Erforschung, so muss zunächst geklärt werden, was denn unter Fremdheit zu verstehen ist. Oft wird hier von der Prämisse ausgegangen, dass es das zu erforschende, homogene Fremde gibt. Im Folgenden wird davon ausgegangen, dass das untersuchte Fremde nur in Abgrenzung zum Eigenen existiert, also nur innerhalb des eigenen (westlich-wissenschaftlichen) Gedankengebäudes und nicht an sich. »Es war das Ziel, das Eigene und Typische einer bestimmten Kultur und Gesellschaft, vor allem der eigenen westlichen Gesellschaft gegenüber, herauszuarbeiten « (Berg/Fuchs I999: 33).

Bei der Vermittlung von Fremderfahrung muss also der Eindruck erweckt werden, der Gegenstand der Betrachtung sei selbst so, um den Anschein von Wissenschaftlichkeit herzustellen. Verschleiert wird dabei die Tatsache, dass er erst durch die Betrachtung in dieser Form erschaffen wird. Die Repräsentation der Forschungsarbeit vollzieht nämlich

weine mehrfache Transformation auf dem Weg von der direkten persönlichen Interaktion zur ausgefeilten Textualisierung als einer verfestigten Repräsentation, die eigenen Kriterien gehorcht. Es ist dieser hermeneutische Ablösungsprozess, der die Formierung relativ beständiger Diskurse über Andere erlaubt, welche die Fremdheit erst herstellen« (Fuchs 1998: 116). 
Ethnologie zu betreiben bedeutet »die Repräsentation der einen Art von Leben in den Kategorien einer anderen« (Geertz I993: I39). Und Repräsentation bedeutet, »zu zeigen, was man sehen muss« (Said I98I: 278, Hervorhebung im Original).

In der traditionellen ethnologischen Forschung sah man das Problem des richtigen Fremdverstehens höchstens in der Frage danach, wie eine in einer anderen Sprache getroffene Aussage in die eigene Sprache übertragen werden konnte, an der prinzipiellen Möglichkeit der Repräsentation des Anderen zweifelte man nicht.

"Während die europäischen Humanwissenschaften Übersetzung als Aneignung der fremden Wirklichkeit verstehen, wird in den außereuropäischen Kulturen die Übersetzung als ein grundlegender Veränderungsprozess des Eigenen aufgefasstıl ${ }^{7}$ (Shimada 1998: 148).

Bei der Übersetzung von Fremdem in Eigenes ist eine Überbesetzung mit Eigenem nicht zu umgehen, da dabei assoziativ verfahren werden muss. In der ethnographischen Schrift mischen sich Autorrede und fremde Rede, das geschriebene Wort bewegt sich an der Grenze zwischen Eigenem und Fremdem. Diese Schwierigkeit ist nicht aufzulösen.

"Es gehört mit zum Selbstverständnis der westlichen Sozial- und Kulturwissenschaften, zwischen dem Forscher und dem, was erforscht wird, zunächst, methodologisch begründet, eine Distanz herzustellen, und das heißt: ein IFremdheitsı-Verhältnis zwischen ihnen zu stiften« (Matthes 1994: 14).

Forschungsgegenstand der Ethnologie ist, wie der Begriff bereits andeutet, die Ethnie. »Ethnizität« hängt eng zusammen mit »Grenze« und »Fremdheit«.

"Unter IEthnosı oder IEthnies - ein Begriff, in dem sprachgeschichtlich die Fremdheit des Untersuchungsgegenstands mithin bereits anklingt - wird in der Ethnologie heute eine Menschengruppe mit gleicher Kultur, gleicher Sprache, Glauben an eine gleiche Abstammung und ausgeprägtem WWir-Bewusstseinı verstanden. [...] Oft ist es sogar erst dieses WWir-Bewusstsein selbst, das den Glauben an eine gemeinsame Abstammung erzeugt» (Kohl 1998: $270 \mathrm{f}$.$) .$

Ein »Wir-Bewusstsein« kann sich aber nur in Unterscheidung zu anderen herausbilden. Diese Abgrenzung von anderen ist geprägt von einem komplementär-oppositionellen Charakter, denn »über Zugehö- 
rigkeit und Solidarität entscheidet das jeweilige Gegenüber « (Streck I992: IOI). Die Grenzziehungen der abendländischen modernen Gesellschaft in »Fremdes « und »Eigenes « stellen insofern eine kollektive Konstruktion dar, als sie ein kulturelles Produkt sind. Daraus resultiert, dass sie künstlich gezogen sind, um hinterher natürlich zu erscheinen. Lässt man gezogene Grenzen, nachdem die Tatsache ihrer Errichtung in Vergessenheit geraten ist, als natürlich erscheinen, als unverrückbar und objektiv, so entzieht man sie damit der prinzipiellen Veränderbarkeit. In diesem Sinne erschuf die Methode der teilnehmenden Beobachtung, das zentrale Forschungsparadigma der Ethnologie, »das Andere als Objekt intimer und systematischer wissenschaftlicher Betrachtung überhaupt erst richtig: >othering< durch Distanzierung, Kontextualisierung, Eingrenzung (Berg/Fuchs 1999: 35). Im Prozess der Ethnographie ist der Forscher damit beschäftigt, sich das zunächst »Fremde« vertraut zu machen, um es zu verstehen. Bei diesem Aneignungsprozess verändert es sich, das untersuchte Objekt geht - zumeist unwissend oder unfreiwillig, deshalb sein Objektcharakter $^{8}$ - eine Symbiose ein mit dem Forscher. Dem Forscher schien die privilegierte Position des außenstehenden Dritten und Mittler zuzukommen, der die fremde Kultur gleichsam objektiv beschreiben und in einen vergleichenden Kontext stellen konnte. Als Beispiel für dieses Selbstverständnis sei an Louis Dumont erinnert, der

ınicht müde wird zu betonen, dass der Anthropologe sich vor inormalenı Gesellschaftsangehörigen dadurch auszeichne, dass er von der Innen- zur Außenperspektive wechseln könne, was ihm nicht nur erlaube, eher komparativ zu arbeiten, sondern [...] das Denken der Gesellschaftsangehörigen besser zu verstehen, als diese selbst es vermöchten« (Fuchs 1998: 106).

Die Fremderfahrung des stationären Feldaufenthaltes im Sinne »malinowskischer Selbstkasteiung « wird gewöhnlich als persönlicher Ausnahmezustand erlebt - ohne dieses »Initiationsritual« wird man nie wissen, was es heißt, Ethnologie zu betreiben. Bronislaw Malinowski (I884-I942) gilt als »Urvater« des »stationären Feldaufenthaltes«, welcher zu einem Verständnis des Fremden »von innen heraus« führen sollte, sozusagen from the native point of view. Malinowski war freilich nicht der erste langzeitpraktizierende Feldforscher, so hatte Franz Boas (1858-I942), der Begründer des amerikanischen Kulturrelativismus, bereits lange Forschungsaufenthalte bei den Innuit durch- 
laufen. Nichtsdestotrotz etablierte Malinowski die Methode der teilnehmenden Beobachtung für die akademische Wissenschaft Ethnologie. Dabei ist man sich einig, wissenschaftliche Objektivität nur durch ein überzeugendes Dort-gewesen-Sein präsentieren zu können. Anthropologen überzeugen, indem sie vermitteln, dass das, was sie sagen, ein Resultat der Tatsache ist, dass sie »eine andere Lebensform wirklich durchdrungen haben [...], wahrhaft >dort gewesen « sind « (Geertz I993: I4). Das Überzeugen »ist die Basis, auf der alles andere, was die Ethnographie zu tun bestrebt ist - $\mathrm{zu}$ analysieren, erklären, amüsieren, verwirren, preisen, erbauen, entschuldigen, erstaunen, umstürzen -, letztlich beruht« (Geertz I993: I39). Die Konstruktion eines »solchen Bodens ist heute [...] ein Unternehmen, das nicht annähernd so unkompliziert aussieht wie zu der Zeit, als die Hierarchie an ihrem Ort war und die Sprache kein Gewicht hatte (ebd.). ${ }^{9}$ Die Überzeugung, dass »objektive Fakten von subjektiven Ansichten entstellt werden« (Geertz I993: I8), dominierte lange Zeit das Fach. Die Schwierigkeiten beim Entwurf ethnographischer Beschreibungen wurden eher auf »die Problematik der Feldforschung zurückgeführt als auf die von Diskurs« (ebd.). Bei der ethnographischen Schreibarbeit musste folglich der Kunstgriff unternommen werden, den Text sauber zu halten von subjektiven Einflüssen. Die Erfahrungen jedoch, die gemacht werden, sind biografisch ${ }^{\mathrm{I}}$. Als wissenschaftlich kann hier nur gelten, was einen objektiven Charakter trägt.

„Wenn es sich um anders geartete Gesellschaften handelt, ändert sich alles: die Objektivität, die im ersten Fall [bei der Betrachtung der eigenen Gesellschaft, e.E.] unmöglich ist, wird uns ohne weiteres zugestanden« (Lévi-Strauss 1981: 379).

Dass die Fakten bei weitem nicht so klar präsent sind und nur durch teilnehmende Beobachtung "gesammelt« werden brauchen, zeigen die zahlreichen Beispiele der so genannten Restudies in der Ethnologie, die Nachuntersuchungen ${ }^{\text {II }}$. Geertz sagt dazu: Das, »was wir als unsere Daten bezeichnen, [sind] in Wirklichkeit unsere Auslegungen [...]. Schon auf der Ebene der Fakten [...] erklären wir, schlimmer noch: erklären wir Erklärungen«(Geertz I999: I4).

„[Die] Grenzüberschreitung [...] steht im Kern des ethnologischen Berufsethos. In der Feldforschung verlässt der Ethnograph sein vertrautes Ambiente und setzt sich existentiell und territorial der Fremdheit aus॥ (Streck 1997: 15). 
Die Fremdheit der anderen Kultur gegenüber und die Entfremdung von der eigenen Kultur sind zugleich Voraussetzung und Begleiterscheinung des ethnologischen Forschens. Die »chronische Heimatlosigkeit $\ll^{\text {I2 }}$ (Lévi-Strauss I98I: 48) hat zur Folge, dass sich der Ethnologe nie wieder

mirgendwo zu Hause fühlen [wird], er bleibt psychologisch verstümmelt. Wie die Mathematik oder die Musik ist die Ethnographie eine der seltenen wirklichen Berufungen. Man kann sie in sich entdecken, auch ohne studiert zu habenu (ebd., Hervorhebung S. N.).

Im Feld muss er aber feststellen:

"IStatt mir eine neue Welt zu eröffnen, gab mir mein abenteuerliches Leben - ein seltsames Paradox - eher die alte zurück, während mir jene andere, der ich nachgestrebt hatte, zwischen den Fingern zerrannu (Lévi-Strauss 1981: 370).

Die Fremderfahrung wird zwar gemacht, und sie mag auch je nach Kontrast zur bekannten Welt als persönliche Erfahrung sehr herausragend sein, aber der Grenzübertritt stellt keinen Initiationsritus in die Welt der Fremdverstehenden dar. Das wird häufig verwechselt.

॥Das Abgeschiedensein von der eigenen Kultur, die IEinsamkeitı des Feldforschers wird zum Hauptmittel der Erkenntnis. [...] Als ranthropologischer Held [...] bringt er die Erkenntnis des Fremden heim» (Berg/Fuchs 1999: 31 f.).

Indem geographische Grenzen überschritten werden, darf sich nicht der Illusion hingegeben werden, dass nun die räumliche Anwesenheit und die Investition von ausreichend Zeit genügen, um wirklich in das Fremde einzudringen und es anschließend objektiv präsentieren zu können. In diesem Sinne

"Imuss jenes emphatische Element aus dieser IFremdheits-Forschung( herausgenommen werden, das die gesellschaftliche IFremdheits-Erfahrungı, von der sie ausgeht, als einen herausragenden, singulären Tatbestand von zugleich weltumspannender Bedeutung (und Dramatik) erscheinen lässt, - so als ob man es mit einer zentralen Dimension eines weltgeschichtlichen Höhe- oder Wendepunktes zu tun habe, und als ob deren Bewältigung so etwas wie eine iwhite man's burdenı wärell (Matthes 1994: 12). 
Aufgrund dieser fachspezifischen Grenzerfahrungen sieht mancher den Ethnologen innerhalb der benachbarten Universitätsfächer in der Rolle eines

ıinneruniversitären Aufklärer[s] oder antiaufklärerischen Rebell[en], [da] die Mehrzahl der Universitätskollegen jene evolutionistische Kopfhaltung fremden Kulturen gegenüber einnimmt, [...] [und man in] dieser bekennenden Unangepasstheit die bleibende Aufgabe der Ethnologie [sehen kann, diesem] wahrhaft antiimperialistischen, weil antirationalistischen Auftrag treu zu bleiben« (Streck 1997: 35).

Die Schwierigkeit, mit der sich die Ethnologie konfrontiert sieht, besteht in dem Paradox »der Spannung zwischen dem (nicht nur) kognitiven Aneignungsanspruch und dem Anspruch, die Anderen in ihrer Andersheit zu respektieren - was einzuschließen hätte, dass sie sich selbst zur Geltung bringen« (Berg/Fuchs I999: 93).

Der Ethnologie ist anzurechnen, der eigenen Gesellschaft andere Blickwinkel auf bekannte Phänomene zu ermöglichen, jedoch liegt in dieser Möglichkeit auch die Gefahr einer intentionalen Darstellung, einer gezielten Beobachtung, um eigene Interessen durchzusetzen. Der Mittlerrolle wohnt auch immer ein Machtpotenzial inne. In der traditionellen Feldforschungssituation herrschte eine klare Machtasymmetrie vor. Der Informant war nur Objekt einer Information, niemals Subjekt in einer Kommunikation. In dieser monologischen Machtasymmetrie glich die Fragestrategie des Forschers eher einer Untersuchung als einem Dialog. So galten die »Aussagen der Informanten [...] als Aussagen der jeweiligen Kultur und nicht etwa als Ausdruck der Auseinandersetzung der Betroffenen mit ihrer kulturellen Wirklichkeit« (Fuchs I998: I06). Diese Ebene der Forschungsbeziehung verfestigte die Differenzen und reduzierte Andersartigkeit auf homogene Wirklichkeiten. Es kann natürlich nie zu dem Idealfall eines Gesprächs im herrschaftsfreien Raum kommen, da jegliche zwischenmenschliche Beziehung von Machtstrukturen durchsetzt ist. Aber es ist notwendig, dem Beteiligten an der Forschungssituation eine gleichwertige Position »in Augenhöhe« einzuräumen, von der aus er sprechen kann, vorausgesetzt er hat ein Interesse daran.

Der Tatsache, dass kommunikativen Situationen auch immer Machtstrukturen innewohnen, wurde lange Zeit nicht genügend Aufmerksamkeit geschenkt. Man kann ihr nicht genug Bedeutung 
beimessen. Bourdieu illustriert das Verhältnis der symbolischen Gewalt am Beispiel eines Gesprächs zwischen einem Kolonialherren und einem »Einheimischen«:

„Wird der Herrschende als Beweis für sein Bemühen um Gleichheit die Sprache des Beherrschten sprechen? [...] Die symbolische Verneinung [...], die fiktive Ausklammerung des Machtverhältnisses, beutet dieses Machtverhältnis insofern aus, als sie eben die Anerkennung des Machtverhältnisses produzieren soll, auf die sich dieser Verzicht beruft"I (Bourdieu/Wacquant 1996: 177 f.)..$^{13}$

Tritt der weitaus häufigere Fall ein, dass der Beherrschte den Sprachstil des Herrschenden übernehmen muss, so spricht er eine "gebrochene Sprache [...] und sein sprachliches Kapital ist mehr oder weniger vollständig entwertet «4 (Bourdieu/Wacquant I996: I78).

Ebenso wie der Person des Sprechers eine »spezifische soziale Autorität« (Bourdieu/Wacquant 1996: I77) innewohnt, so wohnt sie dem Verfasser eines Textes inne. Diese Asymmetrie wahrzunehmen ist wichtig, um das Fließen und die Relevanz von entstandener Information einschätzen zu können. »Die entscheidenden Besonderheiten des ethnographischen Schreibens liegen wie der entwendete Brief bei $\mathrm{E}$. A. Poe so offen zutage, dass sie sich der Wahrnehmung entziehen « (Geertz 1993: 14) - zumindest der Wahrnehmung eines manchen Ethnologen. ${ }^{\text {I5 }}$ Das, was sich lange der Wahrnehmung entzogen hatte, ist nun, nach der Krise der ethnographischen Repräsentation, hinlänglich bekannt. Geertz vermutet, dass die Gründe für die Furcht der Ethnologie, die dafür verantwortlich ist, dass sie gar nicht wissen will, wie ein anthropologischer Text gemacht ist, in der Ahnung liegt, »dass einige Mythen der Zunft darüber, wie es ihr gelingt zu überzeugen, nicht mehr aufrechtzuerhalten wären « (Geertz I993: I2). Ein Mythos ist, dass sich die Überzeugung aus dem Gewicht der Fakten (Menge, Schlagkraft) speist. Das Problem, das sich hier ergibt, lautet, dass Fakten »von Natur aus « nicht unschuldig sind. Wohl kein Ethnologe geht ohne eine bereits im Kopf bestehende These ins Feld. Die gesammelten Fakten sprechen nicht neutral für sich, sondern werden kontextuell wahrgenommen. Diese Art, Daten zu sammeln, rührt noch aus »alten« Zeiten her, als man mit der Ethnologie eine »richtige« Wissenschaft, orientiert am Vorbild der Naturwissenschaften, betreiben wollte.

Ohne sich in einem dogmatischen Relativismus verstricken zu 
müssen, sollte man sich von der Vorstellung verabschieden, die Anderen wären in ihrem So-Sein präsent, denn sie sind es nur kontrastiv. Ein westlich-wissenschaftlicher Wirklichkeitsbauplan ist nicht universalisierbar, er hat seine Gültigkeit nur innerhalb des abendländischen Kontextes. Aus der Retrospektive lässt sich sagen, dass, wenn diese Position der Differenz nicht mitreflektiert wird, Ethnographie nicht mehr ist als eine literarische Schreibarbeit, nicht die angestrebte Abbildung von Wirklichkeit, sondern die Erfindung von Welten.

Wäre es möglich, die bekannten Dichotomien des wissenschaftlich-westlichen Denkens aufzubrechen, könnte vielleicht ein neues Spiel angefangen werden ${ }^{\mathrm{T} 6}$, auch wenn sich dadurch an der Tatsache, dass es im Machtfeld immer Asymmetrien geben muss, nichts ändern würde. Lediglich die hegemoniale Führung der westlichen Definitionsmacht müsste dann abgegeben werden; denn wer die Definitionsmacht hat, der zieht die Grenzen.

\section{Welches fremde in wessen Kultur?}

»Was geschieht mit der Wirklichkeit, wenn sie in die Fremde verschifft wird? « (Geertz I993: I28). Diese Frage stellt sich freilich erst, seit Imperialismus und Szientismus etwa zur gleichen Zeit fielen. In der kolonialen Epoche gab es keinen Raum für diese Frage, weil niemand sie stellte. Migration, Tourismus, globale Vermischungen aller Art führten dazu, dass eine »der Hauptannahmen, auf denen das Schreiben anthropologischer Texte noch bis gestern ruhte - dass nämlich seine Untersuchungsobjekte nicht nur trennbar wären, sondern auch moralisch nicht zusammenhingen, [...] sich ziemlich vollständig aufgelöst [hat]« (Geertz I993: I29).

»Wen soll man jetzt überzeugen? Afrikanisten oder Afrikaner? [...] Und wovon?« (Geertz 1993: 130). Appadurai versucht dieser Erscheinung einen Namen zu geben, wenn er von »ethnoscapes« (Appadurai I998: II) spricht. Mobilität ist immer häufiger anzutreffen, »Enträumlichung ist demnach eine der zentralen Kräfte der Moderne« (ebd.: I3), handelt es sich nun um Personen, Informationsflüsse oder eine beschleunigte Geld- und Warenzirkulation. Kulturelle Reproduktion und die Herausbildung von Gruppenidentitäten sind nicht mehr räumlich fixierbar. Die globalen Ströme gehen je nach kontextueller Einbettung sehr variierende Verbindungen ein. Die neue Vielfalt der vorgestellten 
Gemeinschaften setzt die zentrale Denkkategorie der räumlichen Einbettung von Identitäten außer Kraft. Für den Ethnographen bedeutet das vor allen Dingen, dass er, wenn er sich einen Ort als Forschungsobjekt wählt, nicht mehr davon ausgehen kann, »auf das, im Vergleich zu einer größeren Perspektive, Grundlegendere, Zufälligere und daher >Realere< zu stoßen « (ebd.: 23). Wir sehen uns hier mit einer kulturellen Wirklichkeit konfrontiert, in der die traditionelle Unterscheidung in »Forschungsobjekt« und Kulturanalytiker keine geeignete Beschreibungskategorie mehr abgibt. ${ }^{\text {I7 }}$

Es gibt ein epistemologisches Bedingungsfeld für die Entstehung dessen, was im spezifisch okzidentalen Sinne unter Kultur verstanden wurde. Die Kategorie Kultur ist demnach selbst ein Mittel der Differenzierung, das Hierarchien impliziert und eine Einheit evoziert.

"Kultur als Kategorie des Denkens ist ein ganz und gar europäisches Phänomen und Teil eines historisch gewachsenen Weltbildes - eine Art siamesischer Zwilling des Eurozentrismus. Nach Niklas Luhmann taucht die Kategorie Kultur, die man als eine Ebene des Beobachtens und Beschreibens und vor allem des Vergleichens zwischen sich selbst und den anderen definieren könnte, in der zweiten Hälfte des 18. Jahrhunderts im Denken Europas aufu (Krasberg 1998: 32).

Nach der Dekonstruktion des hegemonialen Wissenschaftsdiskurses des Westens fand sich die »Wissenschaft vom kulturell Fremden « in einer Repräsentationskrise wieder, welche erdbebenartig die Fundamente in den Bauten der Kultur- und Sozialwissenschaften erschütterte. Der cultural turn grassierte bald auch nach Deutschland, seit Mitte der I980er Jahre lässt sich innerhalb der Sozial- und Kulturwissenschaften von einer »kulturalistische Wende« sprechen (vgl. Reckwitz 2000). Es stellte sich die Frage, ob kulturelle Phänomene angesichts der neuen Vielfalt und des rasenden Wandels überhaupt noch schnell genug und angemessen beobachtet und beschrieben werden können. Die Gegensätze zwischen »Hochkultur« und »Alltagskultur«, zwischen »repräsentativer Kultur « und »Volkskultur « werden heute von niemand mehr ernsthaft in dieser Dualität kontrastiert. Die Kulturwissenschaften sind sich darüber im Klaren, dass sich der Gegenstand ihrer Betrachtung schneller verändert als sie zusehen können.

Auch wenn einige Ethnologen der Ansicht sind, 
1)dass so ziemlich alle Anthropologie, darunter, in einer Art von Kreter-Paradoxon, [...] [die] eigene, sunehrlich, schädlich und eigennützigı ist; dass sie eine Erweiterung des Iwestlichen Gesellschaftsprojektsı [...] ist, das darin besteht, ıalle Fragen zu stellen «1 ${ }^{18}$ (Geertz 1993: 95),

so kann es nicht die Lösung sein, keine Ethnographie mehr zu betreiben und nur noch darüber zu schreiben. Die traditionelle anthropologische Haltung konfrontierte das Fach zwar mit angesprochenen Problemen, der Ausweg kann jedoch nicht darin bestehen, in eine »paralysierende [...] Trance epistemologischer Nabelschau [...] zu verfallen« (Clifford Geertz in Morley I999: 305). Die Reflexion über die Forschungsbedingungen führt nicht dazu, »dass man unmöglich etwas Sicheres über andere Menschen wissen könne« (Morley I999: $\left.305^{\mathrm{I}}\right)$. Es hilft auch nicht, die Augen zu verschließen und zu leugnen, man muss damit leben. Denn weder die Beteuerung des unwissentlichen Handelns noch das Ziehen der Konsequenzen ändern etwas daran, dass das Objekt der Wissenschaft tot ist. Die Möglichkeit der Repräsentation als Sozialtechnik ist an sich fraglich geworden; denn an sich

nist das Dort-Sein eine Postkartenerfahrung (Ilch war in Kathmandu - du auch?). Es ist das Hier-Sein, als Gelehrter unter Gelehrten, was dazu führt, dass eine Anthropologie gelesen wird [...] publiziert, besprochen, erwähnt, gelehrt» (Geertz 1993: 127).

Diese gespaltene Existenz - sich »einige Jahre [...] mit Viehhirten oder Yamsgärtnern herumzuschlagen, [um dann] ein ganzes Leben lang Vorlesungen $\mathrm{zu}$ halten und mit Kollegen $\mathrm{zu}$ diskutieren« (Geertz I993: I28) - führt zu einer

॥weitverbreitete[n] Nervosität im Hinblick auf das ganze Unternehmen, das den Anspruch erhebt, rätselhafte Andere mit der Begründung zu erklären, dass man an ihrem angestammten Wohnsitz mit ihnen verkehrt hat. [Die] Aufrufe zu Reflexivität, Dialog, Heteroglossie, Sprachspiel, rhetorischer Zurückhaltung, performativer Übersetzung, wörtlicher Aufzeichnung und Erzählung in der ersten Person« (ebd.)

wirken weniger als einlösbare Angebote auf die offen bleibenden Fragen, denn als verzweifelte Hilferufe eines vom Untergang bedrohten Fachs. Man will den Anspruch, zu repräsentieren, nicht verlieren 
und retten, was zu retten ist vom sinkenden Schiff. An einigen Planken lässt es sich vielleicht noch eine Zeit lang festklammern, aber ob sich daraus ein neues Schiff erbauen lässt, bleibt fraglich.

\section{Disziplinäre Streifzüge}

Die Überlegung liegt nahe, dass die Gründe für eine Abwehrhaltung in Zeiten akademischer Verunsicherung in der Angst vor »Kolonisation« liegen. Grenzverletzungen wirken hier wie eine Bedrohung der fachlichen Identität. Dieses Verständnis von Identität gründet auf dem Besitz einer eigene Methode (der teilnehmenden Beobachtung), eines unverwechselbaren Gegenstandes bzw. Territoriums (dem Fremden) und der Berufung auf eine eigene Tradition.

„Aus dieser Perspektive entpuppt sich die IAngst des Anthropologens als eine Projektion von Vertretern einer Disziplin, die ihre Identität in erster Linie über Prozesse der Differenzierung $[\ldots]$ und nicht, wie die Cultural Studies, über Prozesse der Synthetisierung [...] herstellt» (Lindner 2000a: 85).

Mit ihrem fehlenden Bestreben, sich zu institutionalisieren, kann man hierzulande wenig anfangen. Aber der »Vorwurf einer Wilderei in >fremden $<$ Wissensbeständen macht allein aus disziplinärer Perspektive Sinn« (Göttlich/Winter I999: 27). Wer mit dem »Plünderungsvorwurf « kommt, vergisst allzu leicht, dass geistiges Wissen kein Eigentum darstellt, niemand hat ein Monopol auf Wahrheit. Die Cultural Studies wollen nicht den angestammten Platz der Ethnologie als Disziplin usurpieren, denn sie sind transdisziplinär orientiert, d.h. sie verfahren nach der »Strategie der Streifzüge in andere disziplinäre Terrains« (Stuart Hall in Linder 2000: 84), ohne irgendwo dauerhaft zu verweilen. Das macht möglicherweise auch ihren Reiz aus und lässt sie in einem zweiten Punkt als gefährlich erscheinen: als Verführerin der jungen Nachfolgegenerationen. Während herkömmliche Ethnologie »ein bisschen langweilig « oder antiquiert wirken mag, sind Cultural Studies jung, attraktiv und faszinierend, oder man könnte sagen: it sells. ${ }^{20}$

Bei der Analyse kultureller Praktiken fragen die Cultural Studies nach den Machtverhältnissen, von denen diese durchdrungen sind. Sie widmen ihr Augenmerk gesellschaftlich unterrepräsentierten, 
marginalisierten und stigmatisierten Gruppen, also kulturellen Details, die nach herkömmlicher Kulturbegriffsdichotomie in »Hochkultur/Massenkultur « Letzterem zugeordnet werden würden, ein weiterer Grund, weshalb sie zuweilen nicht ernst genommen werden. ${ }^{2 \mathrm{I}}$ Diese dichotomisierende Auffassung von Kultur hat bisher eine intensivere Diskussion über Populärkultur in nicht abwertenden Kategorien verhindert. ${ }^{22}$ Während die Perspektive der Cultural Studies einen kreativen Impuls der Massen und das emanzipatorische Potenzial von Populärkultur betont, wird eine solche Sichtweise auf Kultur in deutschen Diskussionen über Populärkultur mit großem Misstrauen betrachtet. Man wirft den Cultural Studies gerne vor, sie seien die Letzten, die sich innerhalb des fachlichen Konkurrenzkampfes der Schulen noch genötigt fühlten, die Waffe des Essenzialismusvorwurfs gegen ihre »Gegner« ziehen zu müssen. Essenzialistische oder substanzialistische Kulturkonzepte werden aber nach wie vor innerhalb der deutschen Kulturwissenschaften - so auch der Ethnologie - verwendet, und der Hinweis darauf lässt sich nicht mit der Vermutung eines möglichen Profilierungswillens auf Seiten der Cultural Studies abschmettern. Es sei noch einmal darauf hingewiesen, dass es aus Sicht der Cultural Studies gar keine Gegner gibt, da sie kein disziplinäres Feld kolonialisieren wollen.

Die analytische Beschäftigung mit dem Eigenen, so eine weitere Prämisse der Ethnologie, sei aufgrund der subjektiven Befangenheit nicht möglich, der Blick für die Besonderheiten werde durch Vertrautheit verstellt. Die Fremderfahrung mag eine notwendige Bedingung für Erkenntnis sein, vergessen wird beim Anbringen dieses Vorwurfs aber, dass man auch innerhalb der eigenen Gesellschaft auf vielfältigste Weise fremd sein kann, die Rede ist vom marginal man $\mathrm{R}$. E. Parks ${ }^{23}$ und den scholarship boys R. Hoggarts und R. Williams ${ }^{24}$ Menschen, die aufgrund ihrer biografischen Erfahrungen »zu Hause« in zwei Welten leben, und zwar für immer. Man könnte von stationärem Feldaufenthalt auf Lebenszeit sprechen. Für sie ist die Heimat nur ein Ort des Übergangs, und für immer mehr Menschen auf der Welt wird das zur gelebten Alltagserfahrung. ${ }^{25}$ Die Cultural Studies geben mit ihrem Interesse für die lived experience die

„Lizenz zur Selbstthematisierung, [...] [das] wirkt befreiend, weil das persönliche Interesse an und die persönliche Erfahrung mit dem Thema nicht länger als Tabu behandelt und hinter dem Paravent der Objektivität verborgen werden muss» (Lindner 2000a: 87). 
Hier werden persönliche Erfahrungen zu kulturellem Kapital, weil sie einzigartiges Erfahrungswissen ins akademische Feld einführen. Die Ethnologie hat sich zwar schon immer mit gelebter Alltagserfahrung beschäftigt, aber die Privilegierung des Exotischen adelte das Fremde auf eigene Art zu etwas Besonderem, es ließe sich sagen, zu einer sublimierten Form von »Hochkultur«.

Den marginal man unterscheidet vom Feldforscher die Art seiner Fremderfahrung: Er hat sich sein Schicksal nicht gewählt, sondern seine existentielle Grenzerfahrung findet aufgrund eines »GeworfenSeins«, eines »Sich-konfrontiert-Sehens-mit« statt, er ist nicht mehr das eine und noch nicht das andere, er ist dazwischen. Der Feldforscher hingegen sucht freiwillig - kraft seiner Entscheidung - die Fremderfahrung, die zumeist mit einem habituellen Prestigegewinn verbunden ist (da die Feldforschung fachintern als »Initiationsritus« gehandelt wird), und er weiß von Anbeginn an, dass er etwa nach ungefähr einem Jahr zurückkehren wird in das Eigene, Vertraute. Dieses Bewusstsein erschafft einen völlig anderen Fremdzugang, als es in ersterem Fall geschieht, in dem der Verlust grundsätzlicher und endgültiger Natur ist. Da der Ethnologe nur scheinbar »einer von ihnen « wird - und going native aufgrund besprochener Subjektivitätsverstrickungen verpönt ist -, verfügt er nicht über die lived experience eines Insiders der fremden Kultur. Sicherlich hat er sich aber andere Augen für das eigene Vertraute erworben. Das wäre der eine Einwand gegen besagte Unfähigkeit zur Analyse des Eigenen.

Der zweite hängt eng mit Ersterem zusammen: In einer Welt, die durch Prozesse der Globalisierung und eine entsprechend hohe Mobilitätsrate gekennzeichnet ist, tauchen Fremderfahrung und Heimatverlust zunehmend in jeder Biografie als gelebte Alltagserfahrung auf. Damit werden wir alle nach und nach zu Insidern, denn der Beobachter ist selbst Teil des beobachteten Feldes, »dieses Begreifen, dass man selber in die Sache verstrickt ist, ist die Voraussetzung jeden Begreifens der Sache selbst« (Lindner 200oa: II3 f.). Während man früher aus methodisch-analytischen Gründen in Subjekt- und Objektperspektive trennte, ist nun ein flexibles Gleiten zwischen den Perspektiven möglich, nun sind »zum ersten Mal [...] persönliche Erfahrungen nicht nur legitim, sondern [sie] haben sogar Gewicht im akademischen Feld « (Lindner 2000a: 87). Indem die Cultural Studies die Lizenz zur Selbstdarstellung geben, umgehen sie das Problem der symbolischen Gewalt durch Fremdrepräsentation, mit dem sich die Ethnologie kon- 
frontiert sieht. Denn sie spricht für andere, die Cultural Studies sprechen für sich selbst. Der marginal man vereint in seiner Person den Einblick des Eingeweihten mit der kritischen Perspektive des Außenstehenden. Sowohl für die Massenkulturuntersuchungen der »Frankfurter « wie auch für Feldforschungen im »klassischen Sinne« war eine Betrachtungsperspektive von oben nach unten üblich, während die Cultural Studies den Blick von unten nach oben geben. Man traut den Rezipienten aktive Entscheidungen und »eigene« Reflexionsfähigkeit $\mathrm{zu}$, wo man sie vormals für unmündig erklärte und als passive Opfer eines Verblendungszusammenhangs sah. Sie werden nicht massenkulturell manipuliert, sondern kodieren und kommunizieren absichtlich durch kreative Stilwahl. Hier wird keine klare Trennung in Objekt und Analytiker angestrebt. Die Angst vor dem Verlust des Eigenen und damit des kritischen Beobachtungswerkzeuges führte zur Verbannung des going native durch die Ethnologie als vorbehaltloser Annahme exotisierender Kulturflucht. Diese Trennung in Eigenes und Fremdes macht aber nur so lange Sinn, wie man der Ansicht ist, durch das Verharren in einer außenstehenden Beobachterposition objektivierbare Fakten sammeln zu können. Nun darf der Anthropologe selbst betroffen sein. »Mehr Mut zu Subjektivismus!«, das könnte die Ethnologie von den Cultural Studies lernen.

Denn die Frage, die sich stellt, ist folgende: Kommt man wirklich $\mathrm{zu}$ »echteren«, weil »objektiveren « Ergebnissen durch »harte Feldforschung «? Oder ist es nicht eher Diskursstrategie derjenigen, die sich selbst noch alle Geißelungen »echter« Feldforschung à la Malinowski auferlegten, da sonst keine akademische Autorität zu erwerben war, und die nun diesen »Initiationsritus « auch dem Nachwuchs abverlangen, sollte er bestrebt sein, die höheren Weihen erteilt zu bekommen? Und muss nicht in erster Linie die institutionalisierte Feldforschung als problematisch erachtet werden, da nur sie es ist, deren Ergebnisse im Nachhinein instrumentalisiert werden durch Einbindung in den akademischen Kontext bzw. deren Fakten bereits im Hinblick auf die Erzeugung eines objektiven Charakters entstanden? Vieles deutet darauf hin. 


\section{Kultur als Text?}

Die Bemerkungen deutscher Ethnologen zum Thema Cultural Studies sind oft verallgemeinernd und tendenziös, wie das Zitat zu Beginn des Aufsatzes beispielhaft verdeutlicht. Sie beruhen meist auf mangelnder Kenntnis und vagen Vorstellungen über das Feld der Cultural Studies. Häufig beziehen sie sich auf irgendeine Form postmoderner oder poststrukturalistischer Theorie, welche willkürlich mit den Cultural Studies in Verbindung gebracht wird, oder sie assoziieren die Cultural Studies mit Popkulturforschung. Wie bereits oft bemerkt worden ist, hat die Rezeption der Cultural Studies in Deutschland bislang fast ausschließlich in den Institutionen der Medienforschung, der Literaturwissenschaft und zum Teil in der Soziologie und der Volkskunde stattgefunden (vgl. Lindner I994; Göttlich/Winter ı999). Wegen dieser disziplinären Einschränkungen der Rezeption der Cultural Studies ist die Bandbreite der Themen, die diskutiert werden, stark limitiert. Es hat auf der einen Seite eine starke Konzentration auf die Themen Arbeiterkultur, Jugendkultur und Populärkultur gegeben, sodass die Cultural Studies für viele deutsche Wissenschaftler gleichbedeutend mit »Subkulturstudien« (Lindner I998: I08) geworden sind, wie Rolf Lindner es formulierte. Auf der anderen Seite hat die amerikanische Variante der Cultural Studies mit ihrem Schwerpunkt auf Textanalyse, Semiotik und Dekonstruktivismus große Aufmerksamkeit in Deutschland erregt, insbesondere innerhalb der Literaturwissenschaften. Das erklärt, warum die Cultural Studies von deutschen Wissenschaftlern oft mit der so genannten postmodernen oder poststrukturalistischen Theorie gleichgesetzt werden.

Aus dieser verengten Wahrnehmung resultiert ein weiterer Vorwurf, der gegen die Cultural Studies ins Feld geführt wird: ihre angebliche Textzentriertheit. Für viele Ethnologen stellen die Cultural Studies lediglich eine Form von Literaturkritik dar, die sich, losgelöst von der »Realität« des sozialen Lebens, nur auf kulturelle Produkte und Repräsentationen konzentriere. Wegen der starken Betonung der Cultural Studies auf diskursive Aspekte, so der Eindruck der Ethnologie, werden soziale, politische und ökonomische Aspekte ignoriert, was ahistorische und dekontextualisierte Untersuchungen hervorbringen würde. Hier wiederholt die Ethnologie den üblichen Vorwurf gegen den Poststrukturalismus: Diskursanalyse reduziere soziales 
Leben zu Text oder, wie David Harvey sagt, »knowledge and meaning to a rubble of signifiers« (Knauft I994: I4I).

So resümiert Signe Howell, dass die Ethnologie keine Angst vor den Cultural Studies zu haben brauche, weil es ihnen nicht darum gehe, »menschliche Wesen in ihrem soziokulturellen Kontext zu untersuchen « (Lindner 2000a: 78). Zugegeben, dieses Argument wirkt irritierend, bedenkt man den zentralen Stellenwert, den Fragen von Macht und Politik in den Cultural Studies einnehmen, und die Tatsache, dass viele Forscher der Cultural Studies sich als sozial engagierte, politisch handelnde Akteure verstehen, die darum bemüht sind, die Position des Erfahrungswissens im akademischen Diskurs zu stärken. Die genannte Anschuldigung basiert auf einer zweifelhaften Unterscheidung zwischen »Diskurs« oder »Repräsentation« auf der einen Seite und »sozialem Leben« auf der anderen. Ein Anliegen der Cultural Studies ist es ja gerade, aufzuzeigen, wie diskursiv vermittelt »Realität« (oder das »soziale Leben«) ist und dass diese »Realität« nur durch Formen der Repräsentation begriffen und vermittelt werden kann. Indem die Ethnologie der Methode der teilnehmenden Beobachtung einen höheren Stellenwert einräumt als der Diskursanalyse, stützt sie sich auf eine Auffassung von »Erfahrung«, welche eng mit dem aufklärerischen Ideal eines rationalen, selbst-determinierten Subjektes verbunden ist, einem Subjekt, das seine Umgebung durch sinnliche Wahrnehmung und transparente Sprache direkt erfassen kann. Dass dieses Ideal aber theoretisch naiv und höchst problematisch ist, wurde versucht $\mathrm{zu}$ verdeutlichen. Mit der Anwendung von diskursanalytischen Methoden als Ergänzung der Methode der teilnehmenden Beobachtung könnte die Ethnologie Einblicke in Dimensionen von »Kultur « gewinnen, die dem konventionellen Feldforscher verborgen bleiben, wie etwa der Aspekt der Konstruktion und Verhandlung von Bedeutung und der Konstituierung von Subjektpositionen durch Sprache. Aspekte wie Macht, Hegemonie, Widerstand und Ideologie, in denen die Cultural Studies, wie Stuart Hall es formuliert, »an astonishing theoretical fluency« (Handler I993: 992) erworben haben, könnten produktiv in die ethnologische Forschung eingebracht werden. 


\section{Ausblick}

Die Ethnologie erforscht das »kulturell Fremde«. Die Gründe dafür sind historisch gewachsen, aber das Erkenntnisinteresse, das den Forscher in damaligen Zeiten antrieb, ist entweder verschwunden oder es schickt sich nicht mehr. Beim Nachdenken darüber, warum sich das Fach neuen Einflüssen und Strömungen verschließt, haben wir die Vermutung aufgestellt und begründet, dass es hier um die Daseinsberechtigung des Faches als akademische Disziplin geht, darum, dass eine disziplinäre Identität und Abgrenzung zu anderen Nachbardisziplinen zwar historisch gewachsen, aber heutzutage nicht mehr vertretbar ist. Berührungsangst geht mit der Angst, selbst in den eigenen Konturen zu zerfließen, einher. Aber war es denn nicht schon immer eine der Stärken der Ethnologie, sich unweigerlich ins Neue und Unbekannte, sei es noch so exotisch und fremd, zu stürzen, um den eigenen Horizont $\mathrm{zu}$ erweitern und $\mathrm{zu}$ erfahren, wie außerhalb der eigenen gewohnten Kategorien noch gedacht und gelebt werden kann? In Zeiten globalen Wissenstransfers kommt derjenige, der sich neuen Denkanstößen verschließt, aufs Abstellgleis. Indem man um jeden Preis die Definitionsmacht dessen, was ein Fach ausmacht, behalten und die Zügel der Autorität nicht in andere Hände abgeben möchte, beschließt man ein Schicksal, welches ein Fach, das sich traditionellerweise mit der Horizonterweiterung beschäftigt hat, nicht verdient. Die Grenze des Denkens, diese Grenze darf nicht verschlossen werden, möchte man sich weiterentwickeln, d.h. möchte man am Leben bleiben. Oder, wie Paul Willis, Ethnologe und Cultural Studies-Forscher, es ausdrückte: »What I really believe is that cultural studies and anthropology need each other« (Willis I997: 33). Eine Strategie der Abwehr ist hier fehl am Platz, denn sie bewirkt genau das, was verhindert werden soll - ein Absterben des Faches.

\section{Anmeruungen}

I Mein ganz besonderer Dank gilt an dieser Stelle Vanessa Ohlraun, deren Vortrag »Watching the Border: The Difficult Relationship between Cultural Studies and German Anthropology«, im Sommer 2000 bei der Tagung »Crossroads in Cultural Studies« in Birmingham gehalten, mir wertvolle Anregungen gab. Für ihre kritische Lektüre 
danke ich Martin Seeh, Boike Rehbein, Martin Hofmann, Kristian Donko und Karsten Kumoll.

2 Signe Howell führt dieses Thema in ihrem Aufsatz »Cultural Studies and Social Anthropology: Contesting or Complementary Discourses?« (Nugent/Shore I997: I03-125) näher aus.

3 Dieser Vorwurf wurde auch schon von amerikanischen Anthropologen erhoben: »With respect to methodology, ethnography is the anthropologist's trump card. Cultural Studies practioners claim they would like to learn to play the card, even though they seem (in this collection at least) mostly to avoid touching it« (Handler I993: 993). Die angelsächsische Debatte über Cultural Studies und Ethnologie dokumentieren Stephen Nugent und Chris Shore (I997) sowie Peter Wade (I997). Vgl. außerdem Rosaldo I994, Knauft I994 und Lindner 20oob.

4 Es kann und muss nicht Aufgabe dieses Aufsatzes sein, einzelne »Schulen« deutscher Ethnologie in Tradition und Gegenwart herauszustellen. Es versteht sich von selbst, dass Differenzen vorhanden sind, aus der Sicht der »Betroffenen« vielleicht sogar erhebliche. In Kontrastierung zu den Cultural Studies kommt jedoch ein deutlicher Grundtenor zum Tragen, welcher vereinheitlichende Züge aufweist. Siehe dazu auch die definitionsbegrifflichen Schwierigkeiten, wie das Fach zu bezeichnen sei. In der DGV (Deutsche Gesellschaft für Völkerkunde) diskutiert man seit einiger Zeit, ob die Begrifflichkeit »Völkerkunde «̈berhaupt noch ethisch tragbar sei und man die Gesellschaft nicht in »Deutsche Gesellschaft für Ethnologie« umbenennen müsse. An dieser Stelle bedarf es einer Bemerkung zur Terminologie: Mit »deutscher Ethnologie« ist das Feld der so genannten »Außereuropäischen Ethnologie« bzw. der »Völkerkunde« in Deutschland gemeint. Das Feld der »Europäischen Ethnologie« (oder »Volkskunde«) fokussiert zumeist andere Themen und hat eine andere Wissenschaftsgeschichte. Wer von der »deutschen Ethnologie« spricht, begeht selbstverständlich einen eigentlich unzulässigen diskursiven Reduktionismus, der dem Fach sicherlich nicht gerecht werden kann. Aus analytischer Perspektive kann hier aber auf diese reduktive Terminologie nicht verzichtet werden. Stuart Hall weist darauf hin, dass diskursive Werkzeuge dieser Art weniger der Abbildung der Realität dienen als ihrer Klassifikation (Hall 2000: 138). Ebenso muss es an dieser Stelle genügen, darauf hinzuweisen, dass bekanntermaßen erhebliche Differenzen bestehen zwischen der amerikanischen Cultural Anthropology, der 
englischen Social Anthropology und der deutschen Ethnologie. Die jeweiligen Abgrenzungen zur Anthropologie, Soziologie, Volkskunde usw. sind im wissenschaftsgeschichtlichen Kontext gewachsen und wurden jeweils durch ganz eigene Erkenntnisinteressen hervorgebracht.

5 Roth sieht den Ethnologen als marginal man (in Anspielung auf Robert E. Park) »ohnehin prädestiniert für die Rolle des Interpreten, Schlichters und Vermittlers" (Roth I996: 24). Auf die Differenz zwischen marginal man und Feldforscher komme ich später zu sprechen. 6 Siehe zum Modell der global cultural flows und seinen Neologismen ethnoscapes und mediascapes bei Arjun Appadurai die Darstellung in Berking I998: »Das Suffix >scape< ist schwer zu übersetzen, weist aber wie in >landscape< vor allem darauf hin, dass es sich um perspektivische Konstruktionen handelt, die je nach der historischen, linguistischen und politischen Situiertheit der Akteure ganz unterschiedliche Blickwinkel implizieren« (Berking I998: 386).

7 Die modernen Humanwissenschaften machten es sich zum Anspruch, der menschlichen Natur alle Geheimnisse zu entreißen, um sie beherrschbar zu machen. Das Dunkle, Geheimnisvolle und Unbewusste sollte erklärbar werden. Der Fremde sollte uns etwas über uns selbst verraten. Die historischen Entstehungsbedingungen der Ethnologie als akademische Wissenschaft als eine »Zwillingsgeburt« von Aufklärung und Kolonialismus sind in anderen Arbeiten ausführlich behandelt worden (vgl. Fink-Eitel I994).

8 Objekt ist der Betrachtete nur aus der Perspektive des Forschers, aber diese Zuweisung zieht keine Objektivitätsgarantie nach sich. Der Blickwinkel der Forschungssituation bringt es so mit sich.

9 Die Veröffentlichung der malinowskischen Tagebücher führte innerhalb der Ethnologie eine »Objektivitätskrise« herbei. Malinowski schrieb während seiner Feldaufenthalte in Neuguinea (I9I4-I5) und auf den Trobriand-Inseln (I9I7-I8) an einer offiziellen Version, der objektiven Fassung der Feldforschung, und an einer inoffiziellen Version, seinem Tagebuch, das nie zur Veröffentlichung bestimmt war und in das er seine gesamten subjektiven Wahrnehmungen »ablassen« konnte. Diese seine strikte Zweiteilung in »erlaubt/verboten«, »öffentlich/privat«, »objektiv/subjektiv« führt uns das Paradigma, mit dem sich das Fach über Jahrzehnte lang in dem Glauben beließ, »harte« Fakten zu sammeln, exemplarisch vor Augen.

10 Die Weigerung, Feldforschungen in Romanform zu respektie- 
ren, hängt eng zusammen mit einem elitären Kulturbegriff, der sich aus der Gleichsetzung »objektiv = wissenschaftlich = ernst $\mathrm{zu}$ nehmen « und »romanesk = unwissenschaftlich = Popkultur « speist und der dem historischen Erbe aus humanistischen Zeiten gehört. An späterer Stelle wird auf die Konsequenzen, die sich aus dieser kulturellen Dichotomisierung ergeben, zurückgekommen.

I I Für Restudies gibt es zahlreiche Beispiele, und auch sie erschütterten den Objektivitätsglauben innerhalb des Faches nachhaltig, so Derek Freeman/Margaret Mead (Samoanerinnen), Li An-Che/Ruth Bendict (Pueblo), Oskar Lewis/Robert Redfield (Teotzlán). Die Nachuntersucher (jeweils die Erstgenannten) kamen zu völlig anderen, oftmals gegenteiligen Ergebnissen. Noch einmal andere Ergebnisse kämen sicherlich zum Vorschein, würde der Nachuntersucher die existente Feldforschung nicht kennen. Denn er wäre dann nicht versucht, kontrastiv zu ermitteln.

12 Was damals zu Zeiten relativer räumlicher Fixierung noch als Besonderheit wahrgenommen werden musste, stellt heute in Zeiten hoher räumlicher Mobilität ein normales, veralltäglichtes Phänomen dar. Fast jeder kennt heutzutage das Gefühl, entwurzelt zu sein, keine einzige Heimat zu haben.

I 3 Diese Aussage stammt von Pierre Bourdieu. Ein anderes Beispiel für diese Form von symbolischer Gewalt, die »Agoraphobie« der Frauen, nennt er in Bourdieu I999: 368.

14 In diesem Zusammenhang weist auch Martin Fuchs darauf hin, dass ein »machtvoller Diskurs andere Diskurse zwingt, sich in dem dominanten Idiom auszudrücken. [...] Übersetzung wird so zur Verdrängung, und im Kampf um die Anerkennung der eigenen Botschaft bringt Übersetzung bestimmte Seiten des Eigenen zum Verschwinden« (Fuchs I998: I32).

I 5 Siehe zur »Writing-Culture-Debatte« vor allen Dingen Clifford/ Marcus i986. Eine ausführliche und gut sortierte Literaturliste findet sich im Anhang des Aufsatzes von Berg/Fuchs I999: 96-ı08.

16 In diesem Sinne möchte Foucault durch seine Herangehensweise neue Perspektiven eröffnen, $\mathrm{zu}$ »neuen Strategien« kommen. Man muss versuchen, »sich von den Mechanismen freizumachen, die stets zwei Seiten erscheinen lassen, um die falsche Einheit [...] aufzulösen« (Foucault i978: 192).

17 Auf etwaige Parallelen der Denkmodelle Forscher/Objekt, Sender/Empfänger, denen ein Kulturverständnis, basierend auf der Tren- 
nung in aktiv/passiv, Hochkultur/Massenkultur zugrunde liegt, komme ich später zu sprechen.

18 Angespielt wird hier von Geertz auf Kevin Dwyer und seine Veröffentlichung Maroccan Dialogues (1982).

19 Von Morley zitiert wird hier James Clifford, aus: Clifford/ Marcus I986: 7 .

20 Siehe dazu Lindner 2000a: 8I f. Natürlich kann auch das den Cultural Studies wieder negativ zu Buche geführt werden: Sie bieten zwar oberflächlich betrachtet fesselnde Themen (gender, Medientheorie, Machtanalytik etc.), in der Tiefe aber stecke nichts dahinter, so die Vorwürfe. Lindner weist darauf hin, dass dieses Argument gerade in Deutschland, mit seiner geistesgeschichtlich gewachsenen Trennung in »Innerlichkeit« und »Äußerlichkeit«, »Tiefe« und »Oberfläche«, gerne angeführt wird. Letztendlich haben die Cultural Studies wohl vor allem eines nicht: eine alte gewachsene Tradition innerhalb Deutschlands und den damit verbundenen »Ahnenkult«. Warum sie diese Autoritätsquelle weder brauchen noch wollen, wurde bereits dargelegt. 2 I Hier kann nicht ausführlicher auf die medientheoretischen Differenzen zwischen der »Massenkulturkritik« der Frankfurter Schule und dem »Populärkulturansatz « einiger Vertreter der Cultural Studies eingegangen werden. Es sei an dieser Stelle nur darauf hingewiesen, dass dem »Frankfurter Konzept« eine Unterscheidung in Hochkultur, zu deren Konsum eine aktive Entschlüsselung erforderlich sei, und in Massenkultur, die leicht zugänglich sei und quasi passiv aufgenommen werden könne, zugrunde liegt, während der Blick auf das Populäre bei den Cultural Studies in eine Richtung geht, die sich nicht auf ein dialektisch fixiertes Sender/Empfänger-Modell festschreiben lässt. Ersterem Ansatz liegt das Ideal einer »vollkommen transparenten Kommunikation « zugrunde, Letzterer geht hingegen von der Möglichkeit einer »systematisch zerstörten Kommunikation« aus. Der Rezipient ist kein »pawlowscher Hund«, für den das Fernsehprogramm eine »Verhaltensprogrammierung [...], etwa dem leichten Schlag auf die Kniescheibe vergleichbar (Hall I999: 98), darstellt. Zu den Kommunikationsmodellen vgl. ebd.: I05 ff.

22 Roman Horak hat diesen Punkt in seinem Aufsatz »Cultural Studies in Germany (and Austria) and why there is no such thing « näher erläutert (Horak I999). Vgl. auch Mikos 1997 sowie Hörning/Winter I999.

23 Siehe zum marginal man: Makropoulus 1988. 
24 Die scholarship boys gehörten im England der I950er Jahre der ersten Arbeiterklassengeneration an, denen eine höhere Schulbildung zuteil wurde. Das entfremdete sie zugleich ihrer Herkunft, sie wurden zu kulturell heimatlosen Persönlichkeiten, die sich mit keiner »Welt« völlig identifizieren konnten.

25 Dafür gibt es inzwischen vielfältigste terminologische Bezeichnungen, erinnert sei hier nur an Homi Bhabas »Dritte Räume«.

\section{Literatur}

Appadurai, Arjun (1998): »Globale ethnische Räume. Bemerkungen und Fragen zur Entwicklung einer transnationalen Anthropologie«. In: Ulrich Beck (Hg.), Perspektiven der Weltgesellschaft, Frankfurt a.M.: Suhrkamp, S. II-40.

Berg, Eberhard/Fuchs, Martin (I999): »Phänomenologie der Differenz. Reflexionsstufen ethnographischer Repräsentation«. In: Dies. (Hg.), Kultur, soziale Praxis, Text. Die Krise der ethnographischen Repräsentation, 2. Aufl., Frankfurt a.M.: Suhrkamp, S. IIIo8.

Berking, Helmuth (1998): »)Global Flows and Local Cultures«. Über die Rekonfiguration sozialer Räume im Globalisierungsprozeß«. Berliner Journal für Soziologie 3, S. 38I-392.

Bourdieu, Pierre (1999): »Narzißtische Reflexivität und wissenschaftliche Reflexivität«. In: Eberhard Berg/Martin Fuchs (Hg.), Kultur, soziale Praxis, Text, Frankfurt a.M.: Suhrkamp, S. 365-374.

Bourdieu, Pierre/Wacquant, Loïc J. D. (1996): Reflexive Anthropologie, Frankfurt a.M.: Suhrkamp.

Clifford, James/Marcus, George E. (Hg.) (I986): Writing Culture. The Poetics and Politics of Ethnography, Berkeley: University of California Press.

Dwyer, Kevin (1982): Maroccan Dialogues, Baltimore: Johns Hopkins Univ. Press.

Fink-Eitel, Hinrich (1994): Die Philosophie und die Wilden. Über die Bedeutung des Fremden für die europäische Geistesgeschichte, Hamburg: Junius.

Foucault, Michel (1978): Dispositive der Macht, Berlin: Merve.

Fuchs, Martin (1998): »Erkenntnispraxis und die Repräsentation von Differenz«. In: Aleida Assmann/Heidrun Friese (Hg.), Identitä- 
ten (Erinnerung, Geschichte, Identität 3), Frankfurt a.M.: Suhrkamp, S. 105-I37.

Geertz, Clifford (1993): Die künstlichen Wilden. Der Anthropologe als Schriftsteller, Frankfurt a.M.: Fischer-Taschenbuch.

Geertz, Clifford (1999): Dichte Beschreibung. Beiträge zum Verstehen kultureller Systeme, 2. Aufl., Frankfurt a.M.: Suhrkamp.

Göttlich, Udo/Winter, Carsten (I999): »Wessen Cultural Studies? Zur Rezeption der Cultural Studies im deutschsprachigen Raum«. In: Roger Bromley/Udo Göttlich/Carsten Winter (Hg.), Cultural Studies. Grundlagentexte zur Einführung, Lüneburg: zu Klampen, S. 25-39.

Hall, Stuart (I999): »Kodieren/Dekodieren«. In: Roger Bromley/Udo Göttlich/Carsten Winter (Hg.), Cultural Studies. Grundlagentexte zur Einführung, Lüneburg: zu Klampen, S. 92-IIо.

Hall, Stuart (2000): »Der Westen und der Rest: Diskurs und Macht«. In: Ders., Rassismus und kulturelle Identität, Ausgewählte Schriften 2, 2. Aufl., Hamburg: Argument, S. I37-I79.

Handler, Richard (1993): »Anthropology is Dead! Long Live Anthropology!«. American Anthropologist 95/4, S. 99I-995.

Hauser-Schäublin, Brigitta (1999): »Vom Reisen und vom Verstehen. Die Ethnologie und das Andere«. Forschung \& Lehre 3, S. I39-I42.

Horak, Roman (I999): »Cultural Studies in Germany (and Austria) and Why There Is No Such Thing «. European Journal of Cultural Studies 2/I, S. IO9-II5.

Hörning, Karl H./Winter, Rainer (1999): »Einleitung«. In: Dies. (Hg.), Widerspenstige Kulturen. Cultural Studies als Herausforderung, Frankfurt a.M.: Suhrkamp, S. 7-I2.

Howell, Signe (1997): »Cultural Studies and Social Anthropology: Contesting or Complementary Discourses?«. In: Stephen Nugent/Chris Shore (Hg.), Anthropology and Cultural Studies, London/Chicago: Pluto Press, S. I03-I25.

Knauft, Bruce (1994): »Pushing Anthropology Past the Posts: Critical Notes on Cultural Anthropology and Cultural Studies as Influenced by Postmodernism and Existentialism «. Critique of Anthropo$\log \gamma \mathrm{I} 4 / 2$, S. II7-I52.

Kohl, Karl-Heinz (1993): Ethnologie - Die Wissenschaft vom kulturell Fremden. Eine Einführung, München: Beck.

Kohl, Karl-Heinz (1998): »Ethnizität und Tradition aus ethnologischer 
Sicht«. In: Aleida Assmann/Heidrun Friese (Hg.), Identitäten (Erinnerung, Geschichte, Identität 3), Frankfurt a.M.: Suhrkamp, S. $269-287$.

Krasberg, Ulrike (1998): »Kultur als Ethnographie der Grenzüberschreitung«. In: Christian Giordano/Roberta Colombo Dougoud/Elke-Nicole Kappus (Hg.), Interkulturelle Kommunikation im Nationalstaat, Münster/München/Berlin: Waxmann.

Lévi-Strauss, Claude (I98I): Traurige Tropen, Frankfurt a.M.: Suhrkamp.

Lindner, Rolf (1994): »Cultural Studies in der Bundesrepublik

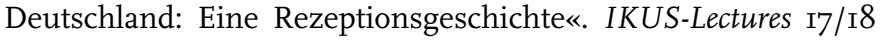
(Cultural Studies: Eine Intervention), Wien, S. 50-82.

Lindner, Rolf (1998): »Cultural Studies«. Ästhetik und Kommunikation 29/100, S. 105-109.

Lindner, Rolf (2000a): Die Stunde der Cultural Studies, Wien: WUV, Ed. Parabasen.

Lindner, Rolf (2000b): »Das Eigene und das Fremde. Cultural Studies versus Cultural Anthropology«. In: Ders., Die Stunde der Cultural Studies, Wien: WUV, Ed. Parabasen, S. 70-96.

Makropoulus, Michael (1988): »Der Mann auf der Grenze. Robert Ezra Park und die Chancen einer heterogenen Gesellschaft«. Freibeuter. Vierteljahreszeitschrift für Kultur und Politik 35, S. 8-22.

Matthes, Joachim (1994): »Vorwort: Ein schwieriger Diskurs. Überlegungen zur zeitgenössischen Fremdheitsforschung«. In: Shingo Shimada (1994), Grenzgänge - Fremdgänge. Japan und Europa im Kulturvergleich, Frankfurt a.M./New York: Campus, S. 7-22.

Mikos, Lothar (1997): »Die Rezeption des Cultural Studies Approach im deutschsprachigen Raum«. In: Andreas Hepp/Rainer Winter (Hg.), Kultur - Medien - Macht: Cultural Studies und Medienanalyse, Opladen: Westdeutscher Verlag, S. 159-170.

Morley, David (1999): »Bemerkungen zur Ethnografie des Fernsehpublikums«. In: Roger Bromley/Udo Göttlich/Carsten Winter (Hg.), Cultural Studies. Grundlagentexte zur Einführung, Lüneburg: zu Klampen, S. 28I-3í.

Nugent, Stephen/Shore, Chris (Hg.) (1997): Anthropology and Cultural Studies, London/Chicago: Pluto Press.

Plessner, Helmuth (1982): Mit anderen Augen. Aspekte einer philosophischen Anthropologie, Stuttgart: Reclam. 
Reckwitz, Andreas (2000): Die Transformation der Kulturtheorien. Zur Entwicklung eines Theorieprogramms, Weilerswist: Velbrück Wissenschaft.

Rosaldo, Renato (I994): »Whose Cultural Studies?«. American Anthropologist 96, S. 524-529.

Roth, Klaus (I996): »Europäische Ethnologie und interkulturelle Kommunikation«. In: Ders., Mit der Differenz leben. Europäische Ethnologie und interkulturelle Kommunikation, Münster/ München/New York:Waxmann, S. II-27.

Said, Edward (I98I): Orientalismus, Frankfurt a.M./Berlin/Wien: Ullstein.

Shimada, Shingo (1998): Grenzgänge - Fremdgänge. Japan und Europa im Kulturvergleich, Frankfurt a.M./New York: Campus.

Streck, Bernhard (I992): »Die Stiftung von Gruppenidentität als ethnologisches Problem«. Sociologus 42/2.

Streck, Bernhard (I997): Fröhliche Wissenschaft Ethnologie. Eine Führung, Wuppertal: Peter-Hammer-Verlag (Edition Trickster).

Wade, Peter (Hg.) (I997): Cultural Studies Will Be the Death of Anthropology, Manchester: GDAT.

Willis, Paul (I997): »For the Motion (2)«. In: Peter Wade (Hg.), Cultural Studies Will Be the Death of Anthropology, Manchester: GDAT, S. 33-4I. 


\section{Das Neue im Zusammenspiel bildungs- und club-kultureller Prozesse}

OLAF SANDERS

Zwischen Kultur und Bildung besteht ein enger Zusammenhang. Die Art des Zusammenhangs wird kontrovers diskutiert. Dessen ungeachtet müsste auch zwischen Bildung und Club-Kultur ein Zusammenhang bestehen. Ich nehme eine Strukturähnlichkeit zwischen den beiden Prozessarten an. Besonders originell ist diese These nicht. Originalität gewinnt sie, wenn das Augenmerk vom Dass auf die Art des Zusammenspiels verschoben wird.

Dieser Text funktioniert ansatzweise ähnlich wie club-kulturelles Material: durch Sampling. Mein Zugriff auf das Thema bleibt in erster Linie bildungsphilosophisch; d.h. ich versuche vom Begriff aus ein Modell zu entwickeln, das es in einem zweiten - hier nicht mehr unternommenen - Schritt empirisch, z. B. anhand von erzählten Bildungsgängen zu prüfen und zu modifizieren gälte. Sein vorrangiges Ziel besteht in der Weitung des Blicks.

Ausgehend von einleitenden Bemerkungen zum Verhältnis der Erziehungswissenschaft zu den Cultural Studies (I) und zur Bildung (II) frage ich mit Cornelius Castoriadis nach den Möglichkeitsbedingungen der Entstehung von Neuem (III). Anhand eines Club-Tracks von Carl Craig will ich dann zu erklären versuchen, wie das Neue in der Clubkultur entsteht (IV). Als zentrale Bezugstheorie dienen dabei Überlegungen des französischen Philosophen Gilles Deleuze, der den »Grundriss einer neuen und zeitgemäßen Ontologie« (Welsch I996: 
356) skizziert, die noch methodologisch und methodisch ausbuchstabiert werden müsste, um zeigen zu können, wie sich Neues in Bildungsprozessen zeigt. Ich beschränke mich - aufgrund des Standes meiner Untersuchungen und der für diesen Beitrag gewählten Perspektive - darauf, kurz nur den Bogen zurück zu Bildungsprozessen zu schlagen, ohne dabei empirische Ansprüche zu erheben (V).

\section{I}

Die Rezeption der Cultural Studies vollzieht sich in der Erziehungswissenschaft - zumindest außerhalb der Medienpädagogik - schleppend. Das verwundert aus zwei Gründen: Erstens ist eine der Wurzeln der Cultural Studies die nachkriegsenglische Erwachsenenbildung, und zweitens zielen sie als kritische Theorie - wie Bildung und Erziehung wenigsten dem Anspruch nach auch - immer auch auf die Verbesserung der Lebensumstände derer, deren Kultur und kulturelle Praxen sie untersuchen. Aus der Perspektive der Allgemeinen Erziehungswissenschaft könnte man die Cultural Studies als pragmatische Bildungstheorie ohne idealistischen Ballast beschreiben. Nun liegt auf der Hand, dass gerade die disziplinäre Befangenheit in der idealistischen Tradition, aus der der klassische Bildungsbegriff stammt, die Rezeption erschwert. Weil diese Tradition durch die historischen Entwicklungen und Ereignisse im letzten Jahrhundert entwertet worden ist und die normative Kraft der großen Ideen seither in Frage steht, könnte es nahe liegen, sie zu ignorieren. Dadurch verspielte man allerdings die Möglichkeit, die Cultural Studies mit bildungstheoretischen Versatzstücken anzureichern, die mögliche, m.E. ernst zu nehmende Einwände seitens kritischer Bildungstheorie entkräften könnten, z. B. den Folgenden: Bei den Cultural Studies handele es sich um eine Bildungstheorie light, der die Option fehle, über den Gedanken der Verbesserung der eigenen Situation hinaus noch einen Maßstab für eine wünschenswerte Entwicklung anzugeben, sodass die Verschlankung einen Niveau-Verlust bedeute, der Tiefe oder Komplexität koste. Im Einklang mit einer Rap-Zeile von Public Enemy gelte es zur Skepsis aufzurufen: »Don't believe the hype«. Dabei schadete es sicher nicht, wenn ein bisschen auf die Bildungstheorie abstrahlte. Sie kann von den Cultural Studies profitieren wie diese von jener. 


\section{II}

Wenn Erziehungswissenschaftler von Bildung reden, war auf dem Kongress der Deutschen Gesellschaft für Erziehungswissenschaft 2000 in Göttingen zu hören, dann werde es ernst. Bildung ist, nachdem sie im Zuge der sozialwissenschaftlichen Wende der Pädagogik gänzlich aus der Mode gekommen war, wieder zur gewichtigen Leitkategorie der erziehungswissenschaftlichen Diskussion aufgestiegen (vgl. z. B. Koller I997: II).

Der moderne deutsche Bildungsbegriff steht schon in seinen klassischen Formulierungen bei Herder, Humboldt und Hegel in engem Zusammenhang zum Neuen. Bildung zeige sich in seiner Realisation oder Emergenz.

Schon im Reisejournal fordert Johann Gottfried Herder ${ }^{7} 69$ als »Prediger der Tugend [s]eines Zeitalters« in jugendlichem Elan einen Menschen, wie er der Kultur-Stufe entspreche. Das wäre »der aufgeklärte, unterrichtete, feine, vernünftige, gebildete, Tugendhafte, genießende Mensch« (Herder 1997: 30). Der Kulturbegriff versieht den Bildungsbegriff mit normativem Gehalt und weist die Richtung. Obwohl Herder - als Theologe liegt das nahe - seine Forderung noch im Namen Gottes formuliert, geht es um die Bildung der Menschheit im und durch den Menschen, die wiederum einhergeht mit der Entwicklung der Menschheit insgesamt und sich mit dieser in Wechselwirkung befindet. Die dritte Komponente des herderschen Bildungsdenkens bildet die individuelle wie historische Sprachentwicklung. Wilhelm von Humboldt verfolgt die Herder-Themen weiter und stellt seine bildungstheoretischen Überlegungen in einen sprach- und sogar kulturphilosophischem Kontext. Der »wahre Zweck« des Menschen, formuliert er I792, »ist die höchste und proportionirlichste Bildung seiner Kräfte zu einem Ganzen« (Humboldt I995: 64). Das Resultat der Bildung ist individuell, der Bildungsgang kontingent, abhängig von der »Mannigfaltigkeit der Situationen «. Als Garantin der Wahrheit fungiert schon bei Humboldt nur noch die Vernunft. Georg Wilhelm Friedrich Hegel schließlich steigert das moderne Selbstschöpfungsmotiv, bläht die Rationalität zu absolutem Geist auf und eliminiert die Kontingenz geschichtsphilosophisch (vgl. Habermas I988: 34 ff.). Interessant an der hegelschen Konstruktion ist die Rolle der Kunst, sie tritt in den Vorlesungen über Ästhetik aus den I82oer Jahren als »erste Lehrerin der Völker« auf (Hegel i986a: 76). Die Kunst als 
Kultur im engeren Sinne vermittelt geistige Gehalte sinnlich an Menschen, deren geistiges Vermögen für direktes Begreifen nicht ausreicht.

Noch Adorno versteht Bildung in der Theorie der Halbbildung (I959) als »Kultur nach Seite ihrer subjektiven Zueignung« (Adorno I998: 94). Die Lebensweise des Einzelnen soll geprägt werden durch den Umgang mit Kulturgütern, die - noch immer in idealistischer Tradition - den Vorschein einer besseren Gesellschaft vermitteln. Genau dieses Potenzial wird den Kulturwaren seitens der älteren Kritischen Theorie und ihren Erben abgesprochen, für frühere Phasen der Pop-Geschichte, die I96oer und I970er Jahre des letzten Jahrhunderts - als Pop noch als Gegenkultur galt -, sicher zu Unrecht. Inzwischen ist Pop trotz seiner fortschreitenden Zersplitterung - Tom Holert und Mark Terkessidis haben dafür die griffige Rede vom »Mainstream der Minderheiten« vorgeschlagen - hegemoniale Kultur (vgl. Holert/Terkessidis I996). Der Bochumer Kunsthistoriker Beat Wyss spricht vom »bedingungslosen Sieg des Pop« (Wyss I997: I23); Diedrich Diederichsen zeigt, dass Pop in den I99oer Jahren allgemein wurde (vgl. Diederichsen I999a: 275). Mit dieser Umwertung sind Vorstellungen einer außerhalb der Ökonomie situierten Kultur obsolet geworden und der Bildung ihre hergebrachten normativen Fundamente weggebrochen. Herders Rede von den »Marktplätzen zur Bildung der Menschheit« (Herder I992 [I774]: 64) gelangt hingegen von Neuem zu Aktualität, auch wenn es inzwischen nicht mehr vorrangig um die Agora, den Markt als öffentlichen Treffpunkt geht, sondern um Handelsplätze. Auf diesen Märkten ist - wie der Karlsruher Kunst-Theoretiker Boris Groys in Über das Neue anmerkt - die »Produktion des Neuen [...] die Forderung, der sich jeder unterwerfen muss, um in der Kultur die Anerkennung zu finden, die er anstrebt« (Groys I999: II).

\section{III}

So wie der Pop in die Kunst eingebrochen ist, scheint das Neue aus ihr ausgewandert und allgemein geworden zu sein, allerdings auf andere Art, als es künstlerische Avantgarden im Sinn hatten. Die Avantgarde-Bewegungen des 20. Jahrhunderts trachteten noch in hegelschem Geist danach, Kunst in Leben zu überführen. Das Neue sollte im radi- 
kalen Traditionsbruch entstehen (vgl. Bürger 1974: 82). Stattdessen mutierte es zur Ring-Richterin im alten Kampf um Anerkennung, wie Hegel ihn schon in der Phänomenologie des Geistes (1807) in aller Deutlichkeit nachzeichnet. Um ihn und die Hegemonie-Verschiebung zugunsten der noch immer Unterdrückten, die - anders als im Falle von Hegels Knecht - nicht mehr von selbst geschieht, geht es auch den Cultural Studies. Die seit den I97oer Jahren verfeinerte Antwort auf die Frage, wie das Neue entstehe, lautet in ihrem Kontext oft: durch bricolage. Bricolage bedeutet nach Claude Lévi-Strauss, der das Wort zum Begriff machte, immer auch »Hindernisse umgehen« (vgl. Lévi-Strauss I973: 29 ff.). Das Haupthindernis für den Bastler liegt in der Ressourcen-Knappheit. Er muss auskommen mit dem, was zur Hand ist, und bisweilen abwegige Mittel und Materialien einsetzen, um seine Ziele zu verwirklichen. Bei den Stilbildungen, wie sie z.B. John Clarke und Dick Hebdige untersucht haben (vgl. Hebdige 1979), entsteht das Neue in einem dreischrittigen Prozess der Dekontextualisierung, Neukombination und Rekontextualisierung. Die Neukombination erfordert »bastelnde Kreativität «, der Michel de Certeau eine »Kunst im Ausnützen« beiseite stellt (de Certeau I988: I2, I8), die der Ressourcen-Knappheit entgegenwirkt.

Die Ressourcen-Knappheit scheint ohnehin eine immer geringere Rolle zu spielen, weil kulturindustrielle Waren im weiteren Sinn in immer größerem Maß verfügbar sind. »Kulturindustrielle Waren im weiteren Sinn« meint in Anlehnung an John Fiske, dass alle industriellen Waren, die kulturelle Bedeutung tragen können, kulturindustrielle Waren sind, die sich außerdem zusehends vom Material lösen oder zumindest einen weiteren Materialbegriff erfordern (vgl. Fiske 2000). Obwohl die Kombinationsmöglichkeiten gegen unendlich tendieren, reicht bricolage nicht hin, um das Entstehen alles Neuen zu erklären.

Um dies zu verstehen, lohnt es sich, sich zu verdeutlichen, dass man mindestens drei Arten des Neuen unterscheiden kann: I. Neues, das nur für einen selbst neu ist, die Aneignung eines vorhandenen Stils etwa; 2. die Bildung oder Teilhabe an der Bildung eines neuen Stils durch Neukombination und Umkodierung, kurz: durch bricolage; und 3. die Emergenz des absolut Neuen, die sich nicht aus Vorhandenem erklären lässt.

Letzteres bildet das zentrale Thema des Hauptwerks von Cornelius Castoriadis' Gesellschaft als imaginäre Institution (I975, dt. 1984). 
Ähnlich den Cultural Studies bricht Castoriadis mit den orthodoxen Spielarten des Marxismus - und zwar mit Hilfe einer zentralen Einsicht Marx', nämlich jener aus der Eingangspassage in Der achtzehnte Brummaire ... -, dass die Menschen ihre Geschichte selbst machen, allerdings unter Bedingungen, die sie nicht selbst geschaffen haben, sondern vorfinden. Castoriadis schlägt dem historischen Materialismus gleichsam Hegel als einer nach Gebrauch fortzuwerfenden Leiter aus dem Kopf und ordnet das Imaginäre dem Realen vor. Castoriadis bestimmt das Imaginäre als Basis jeglicher gesellschaftlicher Realität, genauer als Möglichkeitsbedingung ihrer Emergenz und von Emergenz überhaupt. Schon im Vorwort heißt es:

„Das Imaginäre, von dem ich spreche, ist kein Bild von. Es ist die unaufhörliche und (gesellschaftlich-geschichtlich und psychisch) wesentlich indeterminierte Schöpfung von Gestalten/Formen/Bildern, die jeder Rede von retwası zugrunde liegen. Was wir IRealitätı und IRationalitätı nennen, verdankt sich überhaupt erst ihnen« (Castoriadis 1997: 12).

Die psychische Grundbedingung $\mathrm{zu}$ indeterminierter Schöpfung nennt Castoriadis »radikal Imaginäres«, das ist die Fähigkeit etwas absolut Neues zu denken. Die klassische deutsche Bezeichnung für dieses seit Kant an die Leine genommene Vermögen lautet: Einbildungskraft. Die Leine sollte die »Gefahr der Schwärmerei« abwehren, die Kant in der Kritik der Urteilskraft als »Wahn« begriff, »über alle Grenze der Sinnlichkeit etwas sehen, d.i. nach Grundsätzen träumen (mit Vernunft rasen) zu wollen« (Kant I974 [I790]: A I24). Die gesellschaftlich-geschichtliche Grundbedingung für die Emergenz des Neuen heißt bei Castoriadis »Magma«. »Magma« meint alles »potenziell Gegebene«, eine »Mannigfaltigkeit«, die sich mengenlogisch nicht abbilden lässt, und - folglich - einen überkomplexen Verweisungsraum.

Später schließe ich an Castoriadis' Überlegungen an. Jetzt kehre ich zunächst einmal zur bricolage zurück.

Neben der Untauglichkeit zur Erklärung des absolut Neuen und der zuvor schon benannten Inflation kulturellen Materials gibt es einen weiteren wichtigen Gesichtspunkt im Hinblick auf die Bastelei. Er besteht darin, dass die Bastlerin bzw. der Bastler im allgemeinen Pop nicht mehr in Opposition zur hegemonialen Kultur steht, denn Bastelei ist ein verbreitetes Phänomen des hegemonialen Pop, auch wenn Arten und Weisen durch die Zersplitterung pluralisieren. So 
erklärt auch Gabriele Klein das Fehlen von Widerstand in der Techno-Kultur (vgl. Klein I999: 74). Die letzte Form des Widerstands sei womöglich der Widerstand gegen den Widerstand. Trotzdem oder gerade deshalb beschreibt Klein sie als sehr innovativ. Schließlich bringt sie neue Tanz- und Kleidungsstile, Körperverhältnisse, Urbanitätsverständnisse, Ausdrucksweisen, Gender- wie andere soziale Praxen und weiteres mehr hervor. Innerhalb der Club-Kultur wird gebastelt, es geschieht aber mehr als das.

Kleins Feststellung, dass Dancefloor-Musik textfrei sei, trifft zwar weitgehend zu, sie scheint aber im Wesentlichen dazu zu dienen, die einleuchtende These zu untermauern, dass sie unmittelbar auf den Körper ziele (vgl. Klein I999: I80). Das ist aufgrund ihrer Rhythmus-Orientierung fraglos richtig. Dass sie hingegen keine Geschichte erzähle, stimmt bestimmt nicht - schon weil zeitgenössische Tanzmusik nicht immer textfrei ist, aber auch ohne Text Geschichten erzählt. Die Tracks folgen zwar keiner Songstruktur wie ein klassischer Pop-Song, der sich - Simon Frith hat das in Perfoming Rites (I998) ausführlich vorgeführt - sprachlich und musikalisch interpretieren ließe, sie bedeuten aber trotzdem etwas.

Vielleicht hat das Feuilleton Madonna zur letzten Einigerin des Pop gekürt, weil ihr auf Music, ihrer im September 2000 erschienenen Platte, z. B. mit »Nobody's Perfect« das Wunder gelingt, mit Vocoder-verzerrter Stimme wie vom outer space über einen von Mirvais Ahmadzai produzierten Breakbeat Traurigkeit bekundet, sich entschuldigt, Besserung gelobt und zugleich für mehr Toleranz gegenüber menschlicher Fehlbarkeit plädiert. Sie klingt wie die Predigerin unseres Zeitalters. Der Sound wirkt so neu wie Roxy Music I972.

\section{IV}

Ein weniger weit hergeholtes Beispiel gibt Carl Craig. Craig gehört zur zweiten Generation des Detroit-Techno. Er steht den Urvätern Juan Atkins oder Derrick May nicht nach. Die New Yorker Village Voice definierte ihn als »Goldstandard im 4/4« - 4/4 steht für den technotypischen 4/4-Takt - Das Londoner Trend-Magazin i-D entdeckte schlicht »Gott«. Mit Bug in the Bass Bin hat Craig außerdem ein Schlüsselstück für die Entwicklung des Drum' n' Bass vorgelegt. Platten hat er nicht nur unter seinem eigenen Namen produziert, sein 
Musiker-Ich streute ein Alias ums andere. Er veröffentlichte auch als Psyche, BFC, Rhythm is Rhythm (mit May), Paperclip People, Gg und mit dem Innerzone Orchestra, das I999 beim Montreux Jazz-Festival ein Konzert gab, über das Tom Holert in Spex 9/99 berichtet. Dass das Innerzone Orchestra in Montreux spielte, legt schon die Vermutung nahe, dass es sich bei dieser Spielart des Techno um etwas anderes handeln muss als das, was einem aus den Golfs hierzulande an vielen Straßenkreuzungen in monotonstem 4/4 oft entgegenwummert. Schon für den frühen Detroit Techno rät Ulf Poschardt hinter die fetten Basslinien zu hören, denn »wer gewillt war, genauer hinzuhören, wurde mit den Feinheiten der Musik belohnt. Zart schwebende, kaum hörbare Melodien, perfekt proportionierte Breaks und Rhythmuskonstruktionen ließen musikalische Meisterwerke ahnen« (Poschardt I995: 3I6). Bei Craigs 99er Album mit dem Innerzone Orchestra, Programmed, muss niemand genau hinhören. Holerts Eloge auf Craig soll hier nicht unterschlagen werden:

„Als Musiker und Produzent ist Carl Craig (in meiner moderigen Schreibersprache) ein erfindungsreicher Sounddesigner, ein Apostel des Üppig-Eleganten, ein Philosoph urbaner Mobilität, ein Verfechter edler String-Texturen und intensiver Percussion-Patterns. Aber auch ein apokalyptischer Endzeit-Mystiker, der cyber-naturalistische Gewitter-Atmosphären aufschichtet bis ein Überdruck der Sensualität entsteht. Eine ganze Menge also. Die Vielzahl der musikalischen Qualitäten und die Delikatesse, mit der sie verwendet werden, macht den Begriff «omplexitätı plausibel, eine wichtige Voraussetzung, um von einer besonderen künstlerischen Leistung sprechen zu können« (Holert 1999: 44).

Bekanntermaßen ist es sehr schwer, über Musik zu schreiben. Insofern soll die zitierte Passage keiner Kritik unterzogen werden. Interessant an Holerts Hymne ist auf jeden Fall, dass Carl Craig ein Mensch zu sein scheint oder viele, wie von der Kultur auf ihrer gegenwärtigen Stufe oder Brechung gefordert.

Dass ein »erfindungsreicher Sounddesigner« musikalische Komplexitäten schaffen kann, scheint auf der Hand zu liegen. Er tut dies unter anderem - heißt es - durch »edle Stringtexturen und intensive Percussion Pattern«. Dass Streicher - Streicher-Sets finden sich auf Programmed z. B. in Blakula, auch ein weiteres Alias Craigs (als Keyborder wie C2 als Programmer) und Titel eines Blaxploitation-Films im Techno-Kontext edel wirken, scheint ebenfalls ein Selbstgänger. Das Adjektiv »edel« sagt folglich nicht viel über die Texturen. Für die 
Percussion zeichnet im Innerzone Orchestra Francisco Mora verantwortlich, der schon zum Sun Ra Akestra gehörte, das in den I97oer Jahren auch als Astro Intergalactic Infinity Akestra auftrat. Akestra und Orchestra markieren eine Verwandtschaft, die Verweise auf den outer space bilden einen starken Kontrast zur Innerzone. Stehen sich outer space und Innerzone nicht gegenüber wie Transzendenz und Immanenz, wie künstlerischer Entwurf und kalkuliertes Design. Auch der Londoner Musik-Journalist Kodwo Eshun (I999: oI[OoI]) sieht einen Zusammenhang zwischen Jazz-Fission (Ra) - Fission steht im Gegensatz zu Fusion, Spaltung statt Verdrängung - und Techno (Craig). Die verdrängten Stile ähneln sich durch Derealisierung des Sounds, Verstörtheit und ahnungsschwangeres Zutreiben auf unbestimmte Ursprünge - nicht etwa Zukünfte. Beide - Ra und Craig sind auch Forscher. Sie untersuchen Wucherungen, Craig als Designer womöglich eher im Hinblick auf das »Üppig-Elegante« (Holert), Ra hingegen wirkt durch seine spirituellen Bezüge wie ein »Apostel«, abgesandt von einer anderen Welt. Als »Philosoph der urbanen Mobilität« qualifiziert sich Craig offenbar durch seinen Bezug auf den Film Blade Runner und die Bindung an Detroit, die Autostadt, ein ähnlich »desolater Ort« (Atkins) wie die Filmkulisse, Orte für »apokalyptische Endzeit-Mystiker«. Ra hingegen thematisiert die unendliche Mobilität andernorts oder utopisch: Space is the place. Als prinzipiell endlose Form - gehört auch der Funk in die Ahnen-Reihe des Detroit-Techno. Ra und Craig stehen beide in der Tradition westafrikanischer Musik, die - so Diederichsen - auf einer grundsätzlichen Endlosigkeit basiere und das Ziel verfolge, bei permanenter Einstiegsmöglichkeit die Performance möglichst lange bei hoher Intensität in Gang zu halten (vgl. Diederichsen I999b: 3). Ra sucht die Zeitschleife. Craig erinnert, er fabriziert Erinnerungen. Auf Programmed findet sich ein Stück namens »Manufactured Memories«. Bei diesem Stück handelt es sich um einen clubfähigen Dance-Track, polyrhythmisch und komplex strukturiert.

Oben fiel schon das Stichwort »Breakbeat«. Unter Break versteht man für gewöhnlich eine aus einer Platte »herausgebrochene« Rhythmus-Passage als Element eines Mixes. Kodwo Eshun definiert Breakbeat als aus »molekularen Rhythmuskomponenten zusammengesetzt«; er versetze »kulturelle Trägheit in Bewegung« und sorge für »kulturelle Geschwindigkeit« (Eshun I999: or[OI3]). In Anlehnung an die Arbeiten von Deleuze und Guattari spricht Eshun von »Polyrhy- 
thmaschinen«, die »emergentes Bewusstsein erschaffen« (ebd.: or [0o6]).

Emergentes Bewusstsein ist mehr als bastelnde Kreativität, es basiert auf dem »radikal Imaginären « (vgl. Castoriadis I997). Um dies im Detail zu verstehen, dafür bietet Deleuze in Differenz und Wiederholung ein Modell an, das drei koexistente Zeitreihen ins Verhältnis setzt (vgl. Deleuze 1997 [1968]: 99 ff.). Als konstitutiv beschreibt Deleuze die "gelebte Gegenwart«, die durch die passive Synthese unabhängiger Augenblicke, also durch rhythmische, innerzyklische Wiederholung entsteht. In dieser »lebendigen Gegenwart« entfaltet sich die Zeit. Diese Vorstellung ähnelt den Überlegungen zur inner time, die der Musiksoziologe Simon Frith im Rückgriff auf Alfred Schütz entfaltet und zum Begriff der Jetztzeit (concept of »moment time«) in Relation setzt (vgl. Frith 1998). Demnach beziehe Musik ihre Bedeutung aus dem Jetzt und ermögliche eine - paradoxe - Erfahrung fortgesetzter Präsenz,

"to hold consumption at the moment of desire, before it is regretted. [...] for a moment-for moments-that experience involves ideal time, an ideal defined by the integration of what is routinely kept separate-the individual and the social, the mind and the body, change and stillness, the different and the same, the already past and the still to come, desire and fullfillment॥ (Frith 1998: 157).

Popmusik führt uns durch die Jetztzeit, die schon von Walter Benjamin mit einer wundersamen diachronen Bindekraft ausgestattet wurde, an den Nullpunkt aller Ermächtigung - die ideale Zeit - und von dort aus - mit Glück, manchmal - zur Bildung als Realisation besserer Zeit (vgl. Benjamin I99I [1942]). Benjamins Überlegungen zur Jetztzeit, die die vorbeihuschenden Bilder der Vergangenheit mit der messianischen Zukunft kurzschließen, führen zurück zu Deleuze: Die Gewohnheit, die sich aus vielen Teilgewohnheiten zusammensetzt, erzeugt Kontinuität. Deleuze unterscheidet aber die Gründung der Zeit durch die Sinnlichkeit von ihrem Grund, dem Gedächtnis, das die zweite Zeitreihe konstituiert, die Vergangenheit, wie sie auch in den Arbeiten Craigs hörbar werden. Von dieser Reihe aus betrachtet erscheint die Gegenwart als zyklische Wiederholung. In der Gegenwart entsteht Neues durch Metamorphose, die Setzung von Differenzen, deren Grundbedingung das Imaginäre bzw. die Einbildungskraft ist. Das Gedächtnis ermöglicht die »Schachtelung der Gegenwarten« 
(Deleuze I997: II2), indem sie die lebendige Gegenwart mit einer zusätzlichen Dimension versieht, in der das Vergangene repräsentiert werden und als Ressourcen-Pool dienen kann. So können Vergangenheit und Gegenwart interferieren. So weit vertiefen die Überlegungen Deleuzes nur jene zur bricolage. Das »absolut Neue« einsteht durch die dritte Reihe der Zeit, die Zukunft. Die Zukunft denkt Deleuze nietzscheanisch als überschießende ewige Wiederkunft des Formlosen, des Differenten oder des Ungleichartigen. Die Zukunft taucht die Gegenwart in das »Chaosmos« (Deleuze 1997: 37I), den zugleich »unbestimmten Ursprung« (vgl. Eshun I999).

Und genau dies geschieht durch Dancefloor-Musik, durch den komplexen Bau und durch den Sound. Sound ist ein weiteres wesentliches Moment zeitgenössischer Tanzmusik: offener als Ton, eher Geräusch, das hier wie bei den Experimenten John Cages dem Ton gegenüber emanzipiert wird. Trotz seiner Offenheit trägt Sound Bedeutung, weil er in eigene und in die eigenen Erinnerungssysteme eingebunden ist. Eshun beschäftigt sich mit der sonic fiction und ihren ganz eigenen Erzählstrukturen. Interessant ist dabei erstens, dass Eschun durch den Sound die unterdrückte blackness in den Diskurs zurückkehren sieht, samt aller ihrer Implikationen. Clubber stehen noch immer in der Mod-Linie des »white negro« und artikulieren Widerstand, womöglich ohne es $\mathrm{zu}$ wollen. Und zweitens, dass man durch Eshun im Sound das Molekulare am Werk sehen kann. In Tausend Plateaus erheben Gilles Deleuze und Félix Guattari das Rhizom zur zentralen Analysekategorie von Gesellschaft und Individuum. Rhizome meinen Allianzgefüge, Zusammenhänge, Wurzelgeflechte ohne Anfang und Ende, die zugleich von zweierlei Struktursorten durchzogen seien, welche sie molar und molekular nennen (vgl. Deleuze/Guattari 1997). Die qualitative Differenz zwischen dem Molaren und dem Molekularen sehen Deleuze/Guattari in den Wirkungsweisen (ebd.: 305): Das Molare wirkt definitorisch und produziert Dichotomien (Paare in verschiedenen Segmenten wie Geschlechter, Klassen etc.), während das Molekulare dekodiert und deterritorialisiert, also flieht, flüchtet. Der Sound entzieht sich der Bestimmbarkeit oder Notation und stellt klassifizierende Dichotomien gänzlich in Frage. Der Grund steigt auf und wird der Figur gleichwertig im Nebel der Mikroperzeptionen - ganz ähnlich wie in Barnett Newmanns Who's Afraid of Red, Yellow and Blue-Farbfeldmalereien. Die vier monochromen Riesenbilder gehören ja wegen ihrer verstörenden Wirkung zu 
den meistzerstörten modernen Kunstwerken. Ihre Erhabenheit verleitet dazu, auf sie einzustechen, was ja fraglos eine - wenn auch, zumindest aus Sammler-Perspektive ungewollte - Form der Überwindung kultureller Trägheit darstellt.

Die Soundcollagen und Polyrhythmien besserer DJ-Musik vermitteln diesseits der Reflexion ein Gefühl für die Formlosigkeit, der sich Form geben lässt. Diese zweischrittige Figur erinnert an Kants Definition des Erhabenen und kehrt sie um. Bei Kant überfordert das Erhabene die Einbildungskraft, die es aufgrund seiner Größe oder Dynamik nicht synthetisieren - sprich: repräsentieren - kann. Der überforderten Einbildungskraft springt dann die Vernunft beiseite, die das Überfordernde an sich selbst misst und so indirekt die eigene kategoriale Größe nachweist, auf die sich erhabenes Gefühl ebenso bezieht wie auf den Gegenstand. Nach dem Sturz der Metaphysik lädt die Formlosigkeit, das Magma, die Einbildungskraft oder das radikal Imaginäre ein, neue Formen zu erfinden. Der Weg führt vom Gefühl zur Artikulation (vgl. Lyotard i989).

Im Falle von Craigs Manufactured Memories handelt es sich um bessere Dancefloor-Musik. Der Track erzählt viele Geschichten, schon ohne zu erzählen. Das Stück ist allerdings nicht textfrei, sondern tendenziell babylonisch. Er beginnt Holländisch. Man schnappt auf, dass jemand vom anderen Planeten gekommen sei. Danach setzt ein später wiederkehrender ritueller Gesang ein, dessen Sprache mir unbekannt ist. Ich tippte auf eine afrikanische Sprache. Holert präzisiert: Es handelt sich um einen »Yoruba-Gesang«. Die Joruba sind eine in Benin und Nigeria verbreitete Ethnie mit einer bis ins II. Jahrhundert zurückreichenden Geschichte. Nach 52 Sekunden folgt die die Überlegungen Deleuzes perfekt illustrierende Erklärung auf Englisch:

„From the minds in the universe comes a new future, The future that brings the spirits together to move 'em over in music The inspiering beats from the I The melodies from the bottom' Which give a new modell Alike gided by Sun Ra, Miles, Clayton, Coltrain Alike the gides of brothers to a new level A level that vagrants can touch»

Sun Ra steht für besagtes »afrodelisches Weltraumprogramm« (Eshun I999: оI[O०I]) und die vom outer space importierten »Futurythmaschinen«, die den vagrant, den Nomaden, Vagabunden oder outcast auf eine neue Ebene heben. Durch die vier Namen - von Ra bis Alice Coltrain - wird auf ein Erinnerungssystem schwarzer Musik ange- 
spielt, die noch immer unterpräsent ist oder nach wie vor verdrängt wird. Das Stück thematisiert somit - zumindest implizit - wesentliche Fragen der Cultural Studies und kann zugleich, um einen Begriff aus ihrer Gründerzeit $\mathrm{zu}$ aktualisieren, als »guiding fiction« fungieren (vgl. Hall/Whannel I964). Die »orientierende Erzählung« wirkt zeitgemäß, sie wirkt sicher nicht bewusst. Zumindest hinsichtlich ihres Sounds ist sie das Resultat von »Maschinenfehlern« (Eshun). Die Zukunft stellt sich als Fehler ein, als Unfall, ähnlich wie bei vielen biographischen Innovationen. Weil Tracks wie Manufactured Memories dem Individuum - Deleuze spricht von »larvenhaften Subjekten« - als eine Art sonic unconcious voraus sind, sind sie bildungsrelevant. Aus dem gleichen Grund wirken sie auf das Affektive (vgl. Grossberg I999), das - wie schon Williams' (I96I) »unbewusste Gefühlsstruktur« (structure offeeling) - ähnlich rhizomatisch strukturiert scheint wie das musikalische Material, das hier zum Modell wird.

Die An- und Abschlussfrage lautet, ob und wie sich derartige Erzählstrukturen in biographischen Erzählungen niederschlagen oder sogar wiederfinden lassen, denn so ließen sich konkrete Bildungsprozesse empirisch erforschen. Eine Antwort kann ich hier nicht mehr geben. Nur so viel: Die Artikulation von Konflikten bzw. Schweigen oder Fehlleistungen können auf Bildungsprozesse hindeuten (vgl. Koller I997). Desgleichen gilt auch für Idiosynkrasien, die sich zu Idiolekten oder Idiomen entwickeln können, wodurch das Neue diskursiv und mithin gesellschaftlich wirksam würde und wird (vgl. Lyotard I989; Castoriadis I997). Drittens schließlich könnten Ähnlichkeiten zwischen rhetorischen und ästhetischen Figuren in kulturellen und biographischen Texten Hinweise auf mögliche Übergänge und ihre Art geben.

Einen Lösungsschlüssel verspricht das Unbestimmte. Schon Hegel positioniert die Musik zwischen bildender Kunst und Poesie (Hegel I986b: I3I ff.). Von der Malerei unterscheide sie, dass sie die Trennung zwischen objektiver Erscheinung und Subjekt aufhebe, sodass musikalische Erfahrung unmittelbare Selbsterfahrung sei. Sie ermöglicht also besser, sich in der Fragmentierung zu erfahren. Verglichen mit der Poesie sei ihr Inhalt aber unbestimmt. Und auch das 
halte ich - nach den bisherigen Ausführungen nicht überraschend für einen Vorteil: Denn die artikulierte Unbestimmtheit schafft Räume, in denen Neues entstehen kann. In gewisser Weise bildet sie eine Unbestimmtheitssemantik, die der hegelschen »Leidenschaft des Bestimmens (Gamm I994: 60) resistent bleibt und der Möglichkeit so dauerhaft ihren Platz im Wirklichen sichert. Das wäre wirklich nicht nichts.

\section{Anmerkung}

I Ich danke den Teilnehmern der Ad hoc-Gruppe Cultural Studies auf dem Kongress der Deutschen Gesellschaft für in Köln 2000 und dem Hamburger Forschungsschwerpunkt »Bildung und gesellschaftliche Transformationsprozesse « für die kritischen und anregenden Diskussionen. Gewidmet ist dieser Text Christina Müller.

\section{Alben}

Innerzone Orchestra: Programmed, Talkin' Loud/Universal Jazz I999 Madonna: Music, Maveric/Warner Bros. 2000

Sun Ra: Space is the Place, Impulse/MCA 1998 (zuerst I973 Blue Thumb)

\section{Literatur}

Adorno, Theodor W. (1998 [I959]): »Theorie der Halbbildung«. In: Ders., Soziologische Schriften I, Gesammelte Schriften, Bd. 8, Darmstadt: Wissenschaftliche Buchgesellschaft, S. 93-I2I.

Benjamin, Walter (I99I [1942]): »Über den Begriff der Geschichte«. In: Ders., Abhandlungen, Gesammelte Schriften, Bd. I/2, Frankfurt a.M.: Suhrkamp, S. 69I-704.

Bürger, Peter (1974): Theorie der Avantgarde, Frankfurt a.M.: Suhrkamp.

Castoriadis, Cornelius (1997 [1984]): Gesellschaft als imaginäre Institution. Entwurf einer politischen Philosophie, Frankfurt a.M.: Suhrkamp (Taschenbuchausg.). 
Certeau, Michel de (I988): Kunst des Handelns, Berlin: Merve.

Clarke, John/Cohen, Phil/Corrigan, Paul et al. (Hg.) (I979): Jugendkultur als Widerstand. Milieus, Rituale, Provokationen, Frankfurt a.M.: Syndikat.

Deleuze, Gilles (I997 [I968]): Differenz und Wiederholung, München: Fink.

Deleuze, Gilles/Guattari, Félix (I997 [1980]): Tausend Plateaus. Kapitalismus und Schizophrenie, Berlin: Merve.

Diederichsen, Diederich (I999a): Der lange Weg nach Mitte. Der Sound und die Stadt, Köln: Kiepenheuer \& Witsch.

Diederichsen, Diederich (I999b): »Endlos und afrikanisch«. Du, November i999/70I, S. I ff.

Eshun, Kodwo (I999): Heller als die Sonne. Abenteuer in der Sonic Fiction, Berlin: ID-Verlag.

Fiske, John (2000): Lesarten des Populären, Wien: Turia + Kant.

Frith, Simon (1998): Performing Rites. On the Value of Popular Music, Cambridge (Mass.): Havard University Press (Paperback Ed.).

Gamm, Gerhard (I994): Flucht aus der Kategorie. Die Positivierung des Unbestimmten als Ausgang aus der Moderne, Frankfurt a.M.: Suhrkamp.

Grossberg, Lawrence (I999): »Zur Verortung der Populärkultur«. In: Roger Bromley/Udo Göttlich/Carsten Winter (Hg.), Cultural Studies. Grundlagentexte zur Einführung, Lüneburg: zu Klampen.

Groys, Boris (I999): Über das Neue. Versuch einer Kulturökonomie, Frankfurt a.M.: Fischer.

Habermas, Jürgen (I988): Der philosophische Diskurs der Moderne, Frankfurt a.M.: Suhrkamp (Taschenbuchausg.).

Hall, Stuart/Whannel, Paddy (I964): The Popular Arts, London: Hutchinson.

Hebdige, Dick (I979): »Die Bedeutung des Mod-Phänomens«. In: John Clarke/Phil Cohen/Paul Corrigan et al. (Hg.), Jugendkultur als Widerstand. Milieus, Rituale, Provokationen, Frankfurt a.M.: Syndikat.

Hegel, Georg Wilhelm Friedrich (1986a): Vorlesungen über die Ästhetik I, Werke i3, Frankfurt a.M.: Suhrkamp.

Hegel, Georg Wilhelm Friedrich (I986b): Vorlesungen über die Ästhetik III, Werke I5, Frankfurt a.M.: Suhrkamp.

Herder, Johann Gottfried (I992 [I774]): Auch eine Philosophie der Geschichte zur Bildung der Menschheit, Stuttgart: Reclam. 
Herder, Johann Gottfried (1997): Journal meiner Reise im Jahr 1769. Pädagogische Schriften, Werke in zehn Bänden, Band 9/2, Frankfurt a.M.: Deutscher Klassiker Verlag.

Holert, Tom (I999): »Mit Leuten: eine Welt«. Spex 9/99, S. 42 ff.

Holert, Tom/Terkessidis, Mark (Hg.) (I996): Mainstream der Minderheiten, Berlin/Amsterdam: Edition ID-Archiv.

Humboldt, Wilhem von (I995 [I792]): »Ideen zu einem Versuch, die Gränzen der Wirksamkeit des Staats zu bestimmen«. In: Ders., Werke in fünf Bänden, Bd. 1, Schriften zur Anthropologie und Geschichte, Stuttgart: Cotta, S. 56-233.

Kant, Immanuel (I974 [I790]): Kritik der Urteilskraft, Werkausgabe, Bd. X, Frankfurt a.M. Suhrkamp.

Klein, Gabriele (1999): Electronic Vibration. Pop Kultur Theorie. Hamburg: Rogner \& Bernhard.

Koller, Hans-Christoph (1997): Bildung im Widerstreit, München: Fink. Lévi-Strauss, Claude (1973): Das wilde Denken, Frankfurt a.M.: Suhrkamp.

Lyotard, Jean-François (1989): Der Widerstreit, München: Fink.

Poschardt, Ulf (1995): DJ Culture, Hamburg: Rogner \& Bernhard.

Welsch, Wolfgang (1996): Vernunft, Die zeitgenössische Vernunftkritik und das Konzept der transversalen Vernunft, Frankfurt a.M.: Suhrkamp.

Williams, Raymond (1961): The Long Revolution, London: Chatto \& Windus.

Wyss, Beat (1997): Die Welt als T-Shirt. Zur Ästhetik und Geschichte der Medien. Köln: DuMont. 


\section{Zur Rezeption der Cultural Studies in ॥SPEX - Magazin für Pop-Kultur»}

Richard GEBHARdT

\section{Einleitung}

Die Theorietradition der Cultural Studies, die die Produktion und Aneignung von Populärkultur als Feld von Konflikten, sozialen Auseinandersetzungen, abweichenden Rezeptionen, Widersprüchen und Selbstermächtigungen begreift, findet spätestens seit Mitte der I99oer Jahre in der Bundesrepublik Deutschland eine über den akademischen Bereich hinausreichende Aufmerksamkeit, die maßgeblichen Einfluss auf den zeitgenössischen Diskurs über Pop-Kultur hat.

Ziel dieses Beitrags ist die Untersuchung der publizistischen Praxis des auch am Cultural Studies-Diskurs orientierten Printmediums SPEX - Magazin für Pop-Kultur. Einleitend wird von der Geschichte der I980 in Köln gegründeten Zeitschrift, ihren einflussreichsten Autorinnen und Autoren, Themen und Diskursen die Rede sein. Aufgezeigt wird, in welcher Form hier der Cultural Studies-Approach in den Pop-Journalismus eingeführt wurde. Anhand eines ausgewählten Textbeispiels über die US-Rockband Hole und deren Sängerin Courtney Love wird unter Bezugnahme auf die Berichterstattung über die Riot Grrrls-Bewegung die journalistische Arbeit dieser Zeitschrift dargestellt. Das Beispiel der Beiträge über die Riot Grrrls steht hier paradigmatisch für eine vom Cultural Studies-Diskurs beeinflusste Schreibweise des Pop-Journalismus, die in SPEX ebenfalls in Bezug 
auf andere, hier nur am Rande erwähnte Stile, Jugendbewegungen und popkulturelle Phänomene (HipHop, Techno, Rechts-Rock) angewendet wurde bzw. wird.

Aufgezeigt werden soll, dass die Cultural Studies in der Bundesrepublik Deutschland, vermittelt durch das Medium SPEX, einen wichtigen Einfluss auf die öffentliche Rede über Jugend- und PopKultur ausüben und dass zudem mit der Bezugnahme auf die Schriften der Cultural Studies neuartige Perspektiven auf die in diesem Feld zu verhandelnden Themen freigelegt werden - Perspektiven nämlich, die die Beschränkungen des etablierten, meist nur auf Konsumberatung zielenden Pop-Journalismus überwinden und auch das Feld der Pop-Kultur als Feld von sozialen und kulturellen Konflikten betrachten.

\section{„SPEX॥ - Magazin für Pop-Kultur}

Die I980 von Gerald Hündgen, Peter Bömmels, Wilfried Rütten und anderen in Köln gegründete Musikzeitschrift SPEX - Musik zur Zeit (heute: Magazin für Pop-Kultur) hat als außerakademische Zeitschrift mit ihrer Pop-Berichterstattung sowie nicht zuletzt mit zwei Schwerpunktausgaben im Sommer 1995 (Ausgabe 7: 48-55 und Ausgabe 8: 46-51; eine Aktualisierung folgte in der Ausgabe 6/i997: 58-62) zur Bekanntmachung der Cultural Studies bei einem breiteren Publikum beigetragen. ${ }^{I}$ SPEX, das »Zentralorgan der Popdissidenz « (die tageszeitung) zählt zwar nicht $\mathrm{zu}$ den auflagenstärksten, jedoch $\mathrm{zu}$ den einflussreichsten Musikzeitschriften in deutscher Sprache. Bands und Künstler mit großer Popularität wie Guns 'n' Roses oder REM waren in SPEX schon lange vor ihrem Massenerfolg ein Thema; über Madonna wurde in SPEX als eine der ersten Zeitschriften überhaupt bereits Anfang der I980er Jahre berichtet. Innovative Stile der Populärkultur wie HipHop, Techno, House und Jungle/Drum \& Bass waren längst vor ihrer Entdeckung durch ein großes Publikum Gegenstand der Berichterstattung. Da in SPEX häufig Themen verhandelt wurden, die von anderen Medien erst mit einiger Verspätung übernommen oder entdeckt wurden (Madonna, HipHop, Independent-Rock), entwickelte sich die Zeitschrift im Laufe der Jahre zum Forum für subkulturelle Strömungen und »alternative« Musikstile jenseits von Radio-Airplay und Charts-Platzierung. Die politischen Implikationen von HipHop 
vor dem Hintergrund der sozialen Situation der afroamerikanischen Community in den USA beispielsweise waren bereits in den I980er Jahren Thema der frühen Beiträge von Günther Jacob und Lothar Gorris, also lange bevor die Feuilletons der großen Zeitungen oder die Mehrheit der anderen Musikblätter das Thema entdeckten. Durch die guten Kontakte zur US-amerikanischen Musikszene und die genaue Lektüre zahlreicher internationaler Musikzeitschriften und Fanzines (d.h. nichtkommerzielle Magazine, die von Fans bestimmter Musikrichtungen herausgegeben werden) konnte ebenfalls rasch auf den Boom amerikanischer Rockbands Anfang der I99oer Jahre reagiert werden. Einige Autorinnen und Autoren - neben Mark Terkessidis, Tom Holert, Günther Jacob, Jörg Heiser, Jutta Koether und Lothar Gorris ist hier vor allem Diedrich Diederichsen zu nennen - wiederum konnten in den Medien (Spiegel, die tageszeitung, WDR, HR, Die Woche, jungle world, Die Zeit, Frankfurter Rundschau, Berliner Seiten der Frankfurter Allgemeinen Zeitung etc.) zu gefragten Spezialisten und Sinndeutern von Pop-Phänomenen oder auch Themen wie Multikulturalismus, moderne Kunst und Literatur werden. SPEX-Mitarbeiter, die sich mit den frühen Jugendstudien der Birmingham School (vgl. exemplarisch P. Willis I979 und I981; Hall/Jefferson I976) oder den Beiträgen zur Pop- und Jugendkultur (vgl. exemplarisch Hebdige I979; Grossberg 1992) befasst haben, konnten über die spezifische Berichterstattung in SPEX hinaus in diesen und anderen Publikationen auch zur Popularisierung von Vertretern der Cultural Studies beitragen. Die Macher des Neuen Funkkollegs des Hessischen Rundfunks zur Jugendkultur (Titel: »Zwischen Protest und Party«) beauftragten beispielsweise SPEX-Autorinnen und -Autoren mit Radiofeatures, in denen u.a. Dick Hebdige ausführlich vorgestellt wurde (für die hervorragenden Einzelbeiträge des Funkkollegs vgl. Kemper et al. I999). Hebdige kann neben dem britischen Soziologen Simon Frith und dem US-Amerikaner Lawrence Grossberg als einer der für den Pop-Diskurs wohl wichtigsten Vertreter der Cultural Studies gelten, ähneln seine Vorträge mit DJ-Pult und visuellen Medien in den letzten Jahren zumal eher einer Performance denn einer klassischen Vorlesung. Die hier genannten Standardwerke werden in den Artikeln jener Autorinnen und Autoren, die sich auf die Cultural Studies beziehen, jedoch nur selten explizit aufgeführt. Im Unterschied zur akademischen Rezeption der Arbeiten des Centre for Contemporary Cultural Studies in Birmingham (CCCS) und ihrer US-amerikanischen Ver- 
bündeten verläuft die Rezeption der Cultural Studies in SPEX eher sporadisch, ohne Anspruch auf klassische Belegführung oder Weiterführung der Werkdiskussion.

\section{Diedrich Diederichsen - SOUNDS, SPEX und die Weiterentwicklung der Sprechweisen über Pop}

Diedrich Diederichsen - oftmals auch mit einem leichten Anflug von Ironie als »deutscher Pop-Papst« bezeichnet - hat seit Ende der I970er Jahre mit unzähligen Artikeln über Pop, Kunst und Politik ein neues Idiom in den deutschen Pop-Journalismus eingeführt. ${ }^{2}$ Diederichsen setzte ab I979 als sehr junger Chefredakteur den Schwerpunkt der Berichterstattung der zuvor recht stilkonservativen Rockzeitschrift SOUNDS auf die Musik aus dem britischen Punk-/New Wave-/Independent-Bereich sowie US-amerikanischen Underground und förderte bzw. schrieb die Rezensionen und Artikel über die zahllosen deutschen Bands Ende der I970er/Anfang der I980er Jahre (Fehlfarben, Mittagspause, Syph etc.). Diederichsen bediente sich dabei einer neuartigen Schreibweise, die aus ausladenden, mit Neologismen, Fachtermini und Anglizismen durchsetzten Schachtelsätzen mit zum Teil hohem Abstraktionsgrad bestand und die sich bereits in ihrem frühen Stadium zum Teil auch explizit auf postmoderne bzw. poststrukturalistische Autoren und Positionen bezog (vgl. dazu auch Diederichsen I985: 52 f.). Diederichsen bediente sich dabei auf unkonventionelle und zum Teil eklektizistische Art und Weise bei so unterschiedlichen Richtungen wie der Semiotik, dem (Post-)Strukturalismus, der Kritischen Theorie und dem westlichen Marxismus. Er war es auch, der zusammen mit dem 1993 verstorbenen Journalisten Olaf Dante Marx bereits in den I980er Jahren ein grundlegendes Standardwerk der Cultural Studies für die Analyse der Pop- und Jugendkultur herausgab und zugleich für die deutsche Ausgabe einen eigenen Beitrag verfasste - Subculture - The Meaning of Style von Dick Hebdige erschien unter dem Titel Schocker. Stile und Moden der Subkultur als Sammelband bei Rowohlt (vgl. Diederichsen/Hebdige/Marx 1983). Als die Zeitschrift SOUNDS schließlich 1983 ihr Erscheinen einstellte, wechselte Diederichsen nach einem kurzen Intermezzo in einer Düsseldorfer Werbeagentur zur Zeitschrift SPEX, die bereits seit I980 vor allem in einem inhaltlichen Konkurrenzverhältnis zu SOUNDS existierte und bei den 
Hamburger Kollegen mangelnde Radikalität in politischen und ästhetischen Positionen beklagte. Diederichsens Ansatz fiel hier in den Folgejahren auf einen breiten Resonanzboden. Neben einer Vielzahl von Schreibweisen, die in SPEX ihr Forum haben bzw. hatten (neben zahlreichen informativen Musikartikeln sind dies etwa der von großer Sachkenntnis getragene Rock-Journalismus des Musikers und Kritikers Michael Ruff, die literarischen Beiträge eines Rainald Goetz, die Artikel zu Dub-Reggae und Pop des Schriftstellers Marcel Beyer, die Kunstkritiken von Jutta Koether etc.), konnte Diederichsen hier zusammen mit anderen endgültig eine Form des Journalismus entwickeln, in der Urteile über Pop-Kultur in einen größeren theoretischen Rahmen eingebunden waren, der sich zur Analyse von Pop-Kultur auch in eigenwilliger Form der oben genannten akademischen Richtungen zwischen Cultural Studies, Strukturalismus und Postmoderne bediente. ${ }^{3}$

\section{Cultural Studies in nSPEX॥}

Zahlreiche der in SPEX diskutierten Themen - von der Mitarbeitern bevorzugt »Issues « genannt - gehören zu den zentralen Gegenständen der Cultural Studies. In den frühen r9goer Jahren erschien beispielsweise kaum ein Artikel über HipHop, der nicht den Zusammenhang zwischen kultureller bzw. postkolonialer Identität und Rassismus, zwischen »schwarzer« Musik und »weißer« Aneignung diskutiert hätte. Inwieweit in der subversiven Praxis von Jugend- und Popkultur auch ein widerständiges Potenzial zu finden sei, zählt von Beginn an zu den zentralen Fragestellungen vieler Beiträge. Die Gründe dafür sind vielfältig: Die frühe Generation der SPEX-Autorinnen und -Autoren hat ihre journalistische Tätigkeit begonnen, als in der Bundesrepublik Deutschland die Debatte über Poststrukturalismus und vor allem Postmoderne ihren ersten Höhepunkt erreicht hatte. Die schmalen Bändchen des Berliner Merve-Verlages ${ }^{4}$ beispielsweise, in denen kleinere Arbeiten u.a. von Foucault, Deleuze und Guattari sowie Lyotard erschienen, waren in jenen Jahren Ausweis für ein besonderes (»cooles«) Wissen, ein Distinktionszeichen gegenüber dem bürgerlichen Universitätsbetrieb, aber auch gegenüber den verbliebenen traditionalistischen linken Studentenverbänden. Die Rede vom »Patchwork der Minderheiten« (Lyotard) wurde in 
den Subkulturen zum gängigen Begriff, die zeitdiagnostischen Postulate der Postmoderne und des Poststrukturalismus - Disziplinierung des Körpers, Ästhetisierung der Lebenswelt, Verlust der Wirklichkeit fanden im Zuge der Rezeption neben Begriffen wie Mikropolitik, Rhizome, Netzwerke, Dezentralisierung, hybride Identitäten, Dezentrierung des Subjekts, De- und Reterritorialisierung, Diskurse, Vielheiten, anything goes ihren Niederschlag in subkulturellen Publikationen und wurden teilweise recht beliebig verwendet. ${ }^{5}$ Von besonderer Relevanz für die SPEX der I990er (die Zeitschrift hat in den letzten Jahren eine Phase der Umgestaltung durchlaufen, die mit dem Rückzug des alten Herausgeberkreises im Jahr 2000 seinen vorläufigen und viel beachteten Höhepunkt fand ${ }^{6}$ ) war zu Beginn des Jahrzehnts die Herausbildung eines »Diskurszusammenhangs« zwischen den Autorinnen und Autoren von SPEX, neu gegründeten Instituten wie der Stuttgarter Merz-Akademie, der etablierten Presse und den in Kleinauflagen verbreiteten Kunst- und Theoriezeitschriften, die Anfang der I990er mit Titeln wie Texte zur Kunst, Heaven Sent, Die Beute, 17 Grad Celsius, Symptome - Zeitschrift für epistemologische Baustellen zahlreich gegründet und teilweise inzwischen wieder eingestellt worden sind. Diese Zeitschriften, in denen ebenfalls für die Cultural Studies wichtige Autoren wie Edward Said und Stuart Hall ausführlich behandelt wurden bzw. mit nachgedruckten Artikeln erschienen, waren zum Teil auch eine Reaktion auf die nach dem Ende des Staatssozialismus offenkundig gewordene Niederlage der Linken. In Abgrenzung zu den traditionellen Varianten des Marxismus, vom Realsozialismus bis zur Kritischen Theorie, knüpften diese Projekte nicht zuletzt an die Tradition des Poststrukturalismus und der Diskursanalyse an. Zeitgleich mit dem Erscheinen der deutschen Ausgabe der Mille Plateaux (Tausend Plateaus) von Deleuze und Guattari ${ }^{7}$ begann hier eine Analyse der deutschen Gegenwart - Pogrome in Rostock, Mölln und Hoyerswerda, Neue Rechte, Rechtsrock -, die sich teilweise in Terminologie und Verfahren des poststrukturalistischen Denkens sowie der Diskursanalyse bediente - Denktraditionen und Verfahrensweisen, die für das Projekt der Cultural Studies von zentraler Bedeutung sind. In den bereits genannten Schwerpunktausgaben zum Thema »Cultural Studies « im Sommer 1995, aber auch in den in loser Folge erschienenen Artikeln, Interviews und Rezensionen hat SPEX zur Rezeption der Cultural Studies in der Bundesrepublik Deutschland beigetragen. Einige Beispiele: Es wurden Bücher von 
Simon Frith und Andrew Goodwin kurz aufgeführt (SPEX 9/1990: 7I f. $)^{8}$, später folgten Beiträge zur Theorie des Fernsehens (SPEX Iо/ 1993: 6 und 5/1994: 30-37), im Jahresrückblick auf das Jahr I994 wurde das Stichwort »Cultural Studies « gesondert behandelt (SPEX I/1995: 34). ${ }^{9}$ Im September 1999 erschien eine Sammelbesprechung neuerer Cultural Studies-Bände, die im Sommer 1999 gleich mehrfach veröffentlicht worden waren (SPEX 9/1999: 24). ${ }^{\text {IO }}$ In der Einleitung $\mathrm{zu}$ den beiden Schwerpunktausgaben, dem damaligen Höhepunkt in der Rezeption, heißt es zu den Parallelen zwischen der journalistischen Arbeit von SPEX und dem Ansatz der Cultural Studies:

„Populärkultur ernst nehmen - für diese Forderung muss in SPEX wahrscheinlich nicht noch heroisch eine Lanze gebrochen werden. Die campy-lustvolle Freistil-Analyse von Fernsehshows und Soap Operas ist sowieso schon eins der liebsten Themen an Tresen und Telefon ('Hast Du gestern Boulevard Bio gesehen?). Die eh schon essentialismenfeindliche und hochkulturkritische essayistische Schreibpraxis, die dafür Vorlage und Munition ist, nimmt das IErnstnehmen allerdings auch noch mal ernst: kein folgenfreies Rumdozieren, pleasell (Redaktion SPEX 1995: 48).

Der Ansatz der Cultural Studies wird hier wie folgt beschrieben:

»n den Fünfzigern und Sechzigern muss es erst einmal ein ziemlich befreiender Gedanke gewesen sein, die Produktions-, Distributions- und Konsumtionsformen der Populärkultur nicht einfach nur als bloßes Realitätwerden des kapitalistischen Verwertungsprozesses zu sehen, sondern auch als Realitätwerden der sozialen Relationen und sogar Widerstände, die er auslöst. Zunehmend werden die Potenziale beschrieben, die aus den populären (Sub-)Kulturen selbst erwuchsen, um der Warenlogik ihre Widersprüche entgegenzuschleudern.

An der Universität Birmingham etablierte sich seinerzeit dafür das Centre For Contemporary Cultural Studiesı, das zunächst Stil und Habitus des jungen Working-ClassMannes im Blick hatte und erst mit dem Wirken von Stuart Hall seine Perspektive für das Feld öffnete [...], in dem sich spezifische marginalisierte Positionen im Dreieck von IRaces, IClassı und IGenderı ausmachen ließen. Mit dieser Öffnung erweiterte sich auch das Theorie-Werkzeug von Marxismus/Pragmatischer Soziologie - zum Beispiel um (post-) strukturalistische Kritiken des klassischen Subjektbegriffs. In den USA hinterließen die Krise des Siebziger-Jahre-Feminismus und das Ende des zivilen bzw. die Zerschlagung des militanten schwarzen Widerstandes ein Vakuum, das eine neue Analyse der Prozesse von Dominanz und Unterdrückung vordringlich machte. Um die Politik von People of Color, 
Frauen, Lesben, Schwulen, von Leuten aus der postkolonialistischen so genannten IDritten Welt zu formulieren, mussten erstmal wieder die Mechanismen ihrer diskriminierenden Repräsentation oder Nicht-Repräsentation in den dominierenden Diskursen/Märkten beschrieben werden. Zugleich musste die nie ganz abbrechende Praxis des Widerstandes darüber konkretisiert und zur Allianzfähigkeit aufgebaut werden« (ebd.: 58).

In der Anwendung einer an dem oben skizzierten theoretischen Bezugsrahmen orientierten Berichterstattung über Künstler, Bands, Labels, Stile und kulturelle Phänomene, die bei aller Heterogenität und unterschiedlichen Artikulationsformen den Schreibstil zahlreicher Autorinnen und Autoren prägt, hat SPEX eine eigene Sprechweise über Kultur eingeführt, die nicht unumstritten ist: Der Nürnberger Krisentheoretiker Robert Kurz beispielsweise ordnete I999 in seiner Streitschrift Die Welt als Wille und Design. Postmoderne, Lifestyle-Linke und die Ästhetisierung der Krise die Kölner Zeitschrift gar in die Reihe jener postmodernen Denker und Denkschulen, welche nach marxistischer Auffassung mit rasender Geschäftigkeit die Verhältnisse affirmieren und bereits bloßem »anders gearteten« Konsum ein widerständiges Potenzial zuschreiben würden (vgl. Kurz I999). ${ }^{\text {II }}$

\section{Pop-Diskurse der 1990er}

Die Pop-Kultur der I99oer Jahre war durch einige signifikante Besonderheiten bestimmt, die eine Erweiterung des Vokabulars und eine Neufassung des theoretischen Bezugsrahmens von Pop-Journalismus notwendig machten. Während die rassistischen Angriffe und Pogrome nach Hoyerswerda eine hedonistisch orientierte und depolitisierte Subkultur wieder politisch aktivierten, ${ }^{\text {I2 }}$ wurden durch HipHop die Journalisten und das Publikum mit den aktuellen sozialen Auseinandersetzungen des »schwarzen« Amerika konfrontiert, was angesichts des mangelnden Hintergrundwissen über die Geschichte des Kampfes der »schwarzen« US-Amerikaner für einige Irritationen und Missverständnisse sorgte ${ }^{\mathrm{I3}}$. Der Erfolg rechter Subkulturen ${ }^{\mathrm{I} 4}$ (Böhse Onkelz et al.) ließ die Hoffnung auf ein emanzipatorisches Element des Rock 'n' Roll und der Jugendkultur unzeitgemäß, wenn nicht sogar verdächtig erscheinen, der Massenerfolg von Bands wie Nirvana und anderen aus der Sparte »Alternativer Rock« die Grenzen zwischen »Underground « (im Idealbild eine »authentische«, unabhängige und 
dissidente Form musikalischer Produktion und Distribution) und »Mainstream« (d.h. die chartskompatible, kulturindustrielle Variante des Pop) verschwimmen. Zudem waren zahlreiche Vertreter der zeitgenössischen elektronischen Musik bemüht, die Techno-Rezeption in einen »poststrukturalistischen « Rahmen einzuordnen. ${ }^{\text {I5 }}$ In der BRD wurden, angestoßen von dem Musiker Heinz-Rudolf Kunze, Debatten über Quoten für deutschsprachige Musik im Radio geführt, zeitgleich hatten Vertreter der »türkischen« Pop-Musik in Deutschland bislang unbekannte Erfolge. Die Presse reagierte auf diesen Prozess der Repolitisierung von Pop-Kultur mit einer verstärkten Berichterstattung über »Pop und Politik « ${ }^{16}$, international wurden Symposien und Kongresse zum Thema abgehalten (beispielsweise 1993 im österreichischen Graz unter dem Titel Musik Macht Politik). Hintergrund dieses Interesses an Pop war die Prämisse, dass mit dem sich verändernden sozialen und kulturellen Gefüge der Pop-Kultur eine neue Perspektive notwendig wird, welche sich nicht auf musikalische Kategorien beschränkt (»guter Groove«, »tolles Gitarren-Riff« etc.), sondern, wie im Fall der im Folgenden behandelten Subkultur der Riot Grrrls, sich auch über soziale und kulturelle Hintergründe verständigt und die subversiv-widerständigen Elemente in der künstlerischen Praxis herausarbeitet.

\section{Die Riot Grrrls - eine «problematische Subkultur»?}

Courtney Love, die Ehefrau des verstorbenen Nirvana-Sängers und Gitarristen Kurt Cobain, zählt zu den Schlüsselpersonen im Diskurs um die Riot Grrrls, wenn auch die Zuordnung der Band zum Riot Grrrls-Umfeld umstritten ist, da zentrale Musikerinnen der Szene wie Kathleen Hannah (Bikini Kill, Le Tigre etc.) mit Love heftige Auseinandersetzungen hatten. Riot Grrrls - diese meist von jungen amerikanischen Frauen getragene musikalische und politische Bewegung wird in Heft 3/1998 der Zeitschrift Politik und Unterricht (Themenschwerpunkt »Kein Ich ohne Wir«) unter der Überschrift »Krawallmädchen« als »Bildungsbaustein« »problematische Subkultur« behandelt. Die Autorin des zitierten Textes verweist dabei auf eine in den USA und England existierende Bewegung, die den Begriff »Grrrk »aggressiv« von girl abgewandelt habe. Riot Grrrls seien »Mädchen, die wilde Knurrgeräusche von sich geben« (Heiliger I998: 8I; zur Zitierweise vgl. die Buchausgabe). Weiter schreibt die Autorin: 
"Sie haben kurzgeschorene Haare, tätowieren sich und treten in Springerstiefeln auf. Sie setzen dem gängigen weiblichen Schönheitsideal à la Marilyn Monroe gezielt Hässlichkeit und ein Schminktabu entgegen. Sie repräsentieren ein neues, aggressives Mädchenbewusstsein und wollen Raum schaffen für eine neue Mädchenidentität, die sich abwendet von zugeschriebener weiblicher Schwäche und Machtlosigkeit. Ein Netzwerk solcher Mädchengruppen will weibliche Freiräume schaffen, ohne sich mit der männlichen Kultur auseinander setzen zu müssen. Sie definieren sich nicht über ihre Probleme, sondern wollen ihre Bedürfnisse ausleben und dazu brauchen wir zu allererst einmal viel Zeit für uns selber.l Ihr Verhältnis zur Gewalt ist eindeutig: Ilch bin gegen Gewalt, sagt eine der jungen Frauen, laber ich habe keine Angst sie anzuwenden [...]« (ebd.).

\section{Riot Grrrls - Praxis einer subkulturellen Jugendbewegung}

Die Riot Grrrls, vom hier angeführten Unterrichtsmaterial als »problematische Subkultur« begriffen, haben seit Anfang der I99oer Jahre - ihre erste internationale Aufmerksamkeit erlangte die Bewegung um I99I - auf spezifische Weise die Musikszene mit der zeitgenössischen Feminismus- und Gender-Diskussion konfrontiert. Der Begriff »Riot Grrrls« steht hier als Bezeichnung für Bands wie Hole, Bikini Kill, Babes in Toyland, Le Tigre et al., die zu feministisch inspirierten Texten lautstarken Rock spielen und in einer dem Independent-Sektor vergleichbaren Struktur von Netzwerken (zu dem nicht nur musikalische Vernetzungen zählen, sondern auch Fanzines, Gedichte, Treffen, Internet-Workshops, Selbstverteidigungskurse etc.) organisiert sind. In dem I99० veröffentlichten Manifest »Revolution Girl Style Now « ${ }^{\text {I7 }}$ wenden sich die Bands Bikini Kill und Bratmobile gegen den »Seelentod« junger Mädchen und fordern dazu auf, durch aggressive Aneignung die Muster des US-amerikanischen Hardcore zu nutzen, um mit martialischer Geste gegen die »männliche Hegemonie« anzutreten. Neben dem oben erwähnten Manifest war die »Feminist Mayority Foundation«, ein Verein für Frauenrechte, ebenfalls entscheidend für die Entwicklung der neuen weiblichen Musikszene. Dieser Verein organisierte I99I in Hollywood das erste große »Rock for Choice-« Konzert mit Bands wie Nirvana, Hole, L7 und Sister Double Happiness, dem sich zahlreiche weitere Konzertreihen anschlossen, die einen explizit politischen Hintergrund hatten und sich stark mit »Frauenthemen« (Abtreibung, sexuelle Gewalt) beschäftigten. 


\section{„Express Yourself» - Riot Grrrls und die feministische Theorie}

Wurden Frauen in der männerdominierten Pop-Kultur zunächst meist als schreiende Fans, Groupies oder Star-Diven wahrgenommen und ihre künstlerische Arbeit zugleich lediglich zum Objekt männlicher Zuschreibungen unterschiedlichster Art (vgl. hierzu auch Baldauf/Weingartner 1998 und McDonnal/Powers I995), änderte sich dies spätestens mit dem Erfolg von Madonna (»Express Yourself«): Frauen wurden im Pop-Diskurs anders wahrgenommen und Rollenverhalten, Strategien der Selbstermächtigung sowie die Artikulation von Themen junger Frauen (mediale Männermacht, Liebeskummer, Ess-Störungen, Schönheitsideale, Männergewalt, Vergewaltigungen) thematisiert. Im Anschluss an die Diskussion um den feministischen Dekonstruktivismus und der Differenztheorie hielt der feministische Diskurs $^{\mathrm{I} 8}$ auch in die Riot Grrrls-Netzwerke Einzug, die eine eher »segregationistische« Praxis durchführten, in der männlichen Journalisten zuweilen Interviews verweigert wurden, frauenspezifische Kommunikationskanäle organisiert und »Womens-Only-Konzerte« veranstaltet wurden. Politisch geprägt war diese Zeit von der Debatte über den so genannten Backlash (Susan Faludi), d.h. der Diagnose des Rückschritts in wesentlichen Zielen und Forderungen der zeitgenössischen Frauenpolitik. In Anschluss an die Arbeiten u.a. von Judith Butler wurden Begriffe wie »Performativität « auch in den Pop-Diskurs eingeführt (vgl. Butler I995). Durch die Zitierung und Verfremdung von sozial normierten Mustern wie dem der »Mädchenrolle« werden ganz im Sinne der von Butler postulierten »performativen Praxis« die vermeintlich stabilen Idealbilder symbolisch überladen und somit Gegenstand der Kritik. Bands aus der Riot Grrrls-Bewegung karikieren in diesem Sinne die gängigen Muster von Frauen, Models und Idealtypen des konventionellen Rollenbildes und grenzen sich somit von normierten und affirmativen Weiblichkeitsmustern ab. Courtney Love von Hole tritt beispielsweise gerne in puppenhaften MädchenKleidern auf und »dekonstruiert« somit die Geschlechterrolle des »Girlies«. Love, die Ex-Stripperin in Mädchenkleidern, überlädt in ihrer künstlerischen Praxis das Verhaltensmuster »Girlie« und grenzt sich somit von einer festgelegten Rollenzuweisung ab. Das für die Riot Grrrls-Bewegung typische Muster der verfremdeten und symbolischen »Aneignung « dominanter Geschlechterrollen wird somit zur Strategie der Selbstermächtigung. In der Verwendung des männlichen Vokabu- 
lars und der »positiven Umdeutung « negativer Zuschreibungen bezeichnen sich Musikerinnen von Bands wie Hole oder Babes in Toyland beispielsweise als sluts (Schlampen) und verwenden somit die Fremdzuschreibung zur Selbstdarstellung, wobei slut ähnlich als Ausdruck des »Selbstbewusstseins « zu bewerten ist wie die Selbstdarstellung von schwarzen Rapperinnen wie Missy Elliot, die sich selbst als bitches bezeichnen. Die bewusst inszenierte »Hässlichkeit« der Bands, die das herrschende Bild vom »schönen Geschlecht« auflöst, »spielt« mit dem voyeuristischen Blick des Zuschauers und entzieht sich zugleich seiner Kontrolle. In diesem Sinne wäre die Bühnenpräsenz der Riot Grrrls-Bands mit ihrer extrem überzeichneten Zurschaustellung von engen, verschmutzten T-Shirts, löcherigen MiniRöcken, verfilzten Zöpfen und Zahnspangen eine politische Praxis, ein Akt des »symbolischen Widerstands « gegen die Identifikation weiblicher Künstlerinnen mit ihren Rollenklischees und den dazugehörigen Sexualisierungen. Passend zur Tendenz der I990er, dem Jahrzehnt der kulturindustriellen Vereinnahmung subkultureller Tendenzen, wurden den führenden Musikerinnen aus der Riot GrrrlsBewegung von der Großindustrie Verträge angeboten, die Bands wie Hole oder Babes in Toyland zum einen größere Verbreitung und aufwendigere Produktionen ermöglichten, zum anderen aber ein Muster kulturindustrieller Dominanz bestätigten, das schon 1979 (Erscheinungsdatum der englischsprachigen Originalausgabe von Subculture The Meaning of Style) von Dick Hebdige herausgearbeitet wurde. Dieser beschrieb die warenförmige Vereinnahmung von Subkultur durch den Mainstream als einen Vorgang, durch den die Gefahren (ideologische, kulturelle, symbolische, politische) der Subkulturen »handhabbar und kontrolliert« werden (vgl. Hebdige I979). Einst ein Forum für »Deformation, Transformation und Verweigerung « (Hebdige 1979 über die Subkultur des Punk), wird das zuvor rebellische Zeichenreservoir in die gefahrlose Warenform z. B. des Laufstegs der Mode-Industrie übersetzt. Die Bewegung der Riot Grrrls ist damit aber nicht vollständig von der Pop-Kulturindustrie absorbiert worden. Weiterhin existieren die unabhängigen Netzwerke der Aktivistinnen und ermöglichen so ein weitgehend unzensiertes Sprechen, das nicht notwendigerweise nach den Maßstäben von Platten-Management, Radio-Stationen etc. umformuliert werden muss. Darüber hinaus sind - wie das folgende Textbeispiel zeigt - die aus der Bewegung kommenden Künstlerinnen zudem Gegenstand von publizistischen An- 
griffen und Zuschreibungen, deren ideologischer Gehalt hier von Interesse ist.

\section{Textbeispiel: Courtney Love - „Die Frau, die alle sterben SEHEN WOLLEN II}

Vor dem Hintergrund der an die Riot Grrrls-Bewegung angekoppelten feministischen Diskurse analysiert Sandra Grether in ihrem Beitrag über Courtney Love in SPEX die Presseberichterstattung über die Künstlerin und deren Band Hole (vgl. Grether 1995a). Der Artikel selbst ist eine Form des Pop-Journalismus, der sich auch an den Paradigmen der Cultural Studies (hier verläuft diese Diskussion zumeist unter den Schlagworten »Women and Gender«) und des feministischen Dekonstruktivismus orientiert.

Nach dem Tod von Kurt Cobain wurden Grether zufolge die vermeintlichen familiären Hintergründe des Selbstmords in den amerikanischen Medien breit ausgeschlachtet, die Witwe Courtney Love war Gegenstand eines breiten Interesses in den Medien, die alle - z.T. lediglich auf Grundlage von Gerüchten - den Drogenkonsum und die Vernachlässigung des Kindes thematisierten. Das zerfahrene Äußere der Künstlerin und ihre symbolisch aufgeladenen obsessiv-exhibitionistischen Texte (»I Was a Teenage Whore«) haben maßgeblich zum Negativimage von Love beigetragen und bestimmen das von PopJournalen und Boulevard-Magazinen verbreitete öffentliche Bild der Künstlerin. Sandra Grether kennzeichnet in ihrem unter der Überschrift »Hole: Die Band, die alle sterben sehen wollen« erschienenen Beitrag die persönliche Faszination zum Gegenstand wie folgt:

"Da ging es plötzlich wieder um das alte Punk-Ding: rauskommen, aber wissen, wohin. Teil-einer-Jugendbewegung-sein-Wollen als politischer Akt, aber diesmal unter feministischen Vorzeichen« (Grether 1995a: 20).

Grether erzählt die Bandgeschichte seit 1992, als Hole die Riot Grrrls-Bewegung mit initiierte und die Debüt-Platte von der New Yorker Stadtzeitschrift Village Voice zur »Platte des Jahres« gewählt wurde. Courtney Love fragte daraufhin in einem Interview mit dem Melody Maker nach den Hintergründen für diese künstlerische Anerkennung und spekulierte, ob sie in drei Monaten nicht die neue Nancy 
Spungen $^{\text {I9 }}$ sein könnte. Grether verweist in diesem Zusammenhang darauf, das Love in der Presse später tatsächlich in erster Linie als die Frau an der Seite von Cobain wahrgenommen wurde, also keine eigenständige Position besaß.

Später, Monate nach der Veröffentlichung der Platte »Live Through This« Anfang 1993 (der Titel bezieht sich auf die Drogenexzesse und die Partnerschaft mit Cobain), bezog sich die Wahrnehmung der Künstlerin vor allem auf ihre Rolle als Witwe ihres noch prominenteren Mannes. Love, die ehemalige Stripperin und Kind von zwei in den USA relativ bekannten Alt-Hippies, fand die Aufmerksamkeit der Medien, nicht nur der US-Pop-Journale, als sie in einem Artikel der Zeitschrift Vanity Fair - laut Grether »ein klassisches, sexistisches Stück Dreck von Journalismus « (Grether I995a: 22) - über ihre Partnerschaft sprach. Zum gleichen Zeitpunkt, Anfang der I990er Jahre, als in den USA die »Family Values«-Kampagne von George Bush lief, wurde in dem Artikel vor allem unter Berufung auf Gerüchte und namentlich nicht genannte Personen ein Bild von Love gezeichnet, das die Künstlerin als selbstbezogen und hysterisch beschrieb und die Nachricht verbreitete, Love habe während der Schwangerschaft Heroin genommen. Grether berichtet in ihrem Beitrag, dass diese Meldung eine öffentliche Kampagne gegen das Ehepaar Cobain losgetreten habe, in deren Verlauf sogar mit der sofortigen Heimeinweisung des Neugeborenen durch die amerikanischen Sozialbehörden zu rechnen gewesen sei. Grether über den weiteren Verlauf der Ereignisse:

»Als es schließlich auf die Welt kommt (es war tatsächlich jemand von der Sozialbehörde da, der es mitnehmen wollte), präsentieren sich die beiden als die stolze, gesunde Familie, geben dem SubPop-Gründer Jonathan Poneman für ISpin ein vertrauliches Interview, in dem die Kleinfamilie gepriesen wird. Überall wird das Kind hergezeigt. Seht her! Es ist gesund! Das ist nebenbei auch noch alles ziemlich behindertenfeindlichu (Grether 1995a: 22).

Im weiteren Verlauf des Textes referiert Grether »zwei übliche Herangehensweisen an Bands mit Frauen « (ebd.), in denen die Künstlerinnen entweder auf ihre »Weiblichkeit« reduziert oder geschlechtsspezifische Kontexte nivelliert werden. Grether betont in diesem Zusammenhang, dass sich die Qualität von Musik auch aus ihrem gesellschaftlichen Entstehungszusammenhang ableitet (ebd.). Auch die 
Künstler selbst hatten den Kontext ihrer eigenen Arbeit immer mitbedacht. So antwortete Kurt Cobain auf die Frage des Melody Maker, ob Nirvana überhaupt subversiv sei (für die Journalistin Everett True war Hole tatsächlich ein subversives feministisches Bandkonzept) mit den Worten:

„Nein. Es ist überhaupt nicht möglich für uns, in der kommerziellen Welt subversiv zu sein, weil völlig übersehen wird, wofür wir wirklich einstehen« (Cobain, zitiert nach Grether 1995a: 23).

Cobain begründete diese Auffassung damit, dass der Massenerfolg seiner Gruppe längst schon nicht mehr durch eigene Intentionen zu steuern sei und er keinen Einfluss mehr auf das Publikum habe, unter dem sich längst schon auch Personen befinden würden - Cobain nannte an dieser Stelle häufig Rassisten oder homophobe Männer -, die der Musiker nie habe erreichen wollen. Dies sei der schlimmste Aspekt des kommerziellen Erfolgs der Band. Grether schließt ihren Text, der hier als Beispiel zur Verdeutlichung einer auch an den Cultural Studies geprägten Arbeitsweise vorgestellt wurde (Kennzeichnung der Strategien der Subkulturen als Form der Selbstermächtigung, Herausarbeitung der symbolischen Aneignung von scheinbar festgelegten Zeichenpotenzialen, »widerständige« Arbeit im Pop-Kontext, Stil als absichtliche Kommunikation), mit einem Hinweis auf die weiteren Projekte der Band und die Zeit nach dem »medial« verbreiteten physischen und psychischen Zusammenbruch der Künstlerin. Dieser Artikel macht das Arbeitsprinzip zahlreicher SPEX-Autoren deutlich, für die häufig auch die Arbeiten von Cultural Studies-Theoretikern wie Dick Hebdige, Lawrence Grossberg, Stuart Hall, bell hooks u.a. Bezugsrahmen waren. Band und Künstlerin werden vor dem Hintergrund der US-amerikanischen Gesellschaft und ihrer Subkulturen betrachtet, kontextbildend sind vor allem die Medienberichte in Großbritannien und den USA. Diskursanalytische Verfahrensweisen werden hier mit dem Fan-Standpunkt der Journalistin verbunden, der Text selbst ist voller Bezüge, Analogien und Querverweise und ermöglicht somit einen Blick auf musikalische, biografische und gesellschaftliche Implikationen der Band Hole. 


\section{Resümee zu Cultural Studies, Spex und Pop-Kultur}

Im Frühjahr 2000, mitten in der Debatte um die Auflösung der alten SPEX-Verlagsgesellschaft und die Übernahme des Blattes durch die Münchner Piranha-Mediengruppe, charakterisierte der ehemalige SPEX-Redakteur Mark Terkessidis in einem mit der Überschrift »Langer Abschied vom Anderssein« versehenen Beitrag für die Berliner tageszeitung die bislang erschienenen Artikel der Zeitschrift als »Mischung aus popkulturellem Fachwissen, Musikfeuilleton und Cultural Studies« (Terkessidis 2000: I7). Diese Reihenfolge umfasst treffend die verschiedenen Sprechweisen über populäre Kultur, die in SPEX ihr Forum hatten und haben. Spezifisch für die Rezeption der Cultural Studies in SPEX war freilich die kursorische, über mehrere Hefte verstreute Berichterstattung in Form von einzelnen Beiträgen, Rezensionen, Interviews und Themenschwerpunkten. In den Artikeln, die deutlich vom Cultural Studies-Approach beeinflusst wurden, besteht die Verbindung vor allem im gemeinsamen Erkenntnisinteresse bezüglich der Praxis jugendlicher Subkulturen, die nicht lediglich in das Erklärungsmuster kulturindustrieller Manipulation eingefügt werden, und in der Analyse populärkultureller Phänomene vor dem Hintergrund der Diskussion zu Gender, Race, Ethnizität und Identitätspolitiken. Die Rezeption der Cultural Studies in SPEX bedeutet jedoch nicht, dass einzelne Werke des CCCS in den Artikeln über Pop-Musik explizit aufgeführt werden - dies ist eher selten der Fall -, vielmehr bilden diese die Folie, anhand derer die Autorinnen und Autoren sich dem Gegenstand nähern. In diesem Sinne vollzog sich die Rezeption der Cultural Studies in SPEX mit einer gewissen Unschärfe parallel und unabhängig vom akademischen Diskurs, in dessen Einzeldisziplinen die Schriften aus Großbritannien und den USA erst in den letzten Jahren zur Kenntnis genommen wurden.

Terkessidis' oben erwähnter Artikel über »die beste Band Deutschlands« (so die liebevolle Bezeichnung des Hamburger Blumfeld-Musikers Jochen Distelmeyer für die SPEX) durchzieht ein elegischer Tonfall, der auch für einen bestimmten Leserinnen- und Leser-Typus der »alten« SPEX signifikant ist: Viele von denen, die einstmals bei manchen Artikeln derart fasziniert mitgingen, dass sie die Kernpassagen während der Lektüre dick mit Rotstift unterstrichen, kennen die »neue« SPEX bestenfalls noch vom Durchblättern und registrieren kopfschüttelnd die neuen Mode-Fotostrecken auf Hochglanz-Niveau, 
das ambitionierte Layout und die großformatigen Anzeigen des Musik-TV-Senders VIVA 2 mit dem Slogan »Radikalisiert das Leben!«.

Mark Terkessidis hatte in seinem Artikel vorgeschlagen, das Ende der alten »Arbeitsweise « und Produktionsbedingungen als »Symptom für gesellschaftliche Veränderungen« (Terkessidis 2000: I7) zu lesen. Zusammen mit Tom Holert hatte Terkessidis I996 den deleuzianisch inspirierten Sammelband Mainstream der Minderheiten. Pop in der Kontrollgesellschaft herausgegeben, der sich unter Bezugnahme u.a. auf Lawrence Grossberg vom eigentümlichen Modell der kritischen Theorie der »manipulierten« Konsumenten abgrenzte, den Zerfall des »Mainstreams« in zahlreiche Submilieus verzeichnete und somit die Auflösung des alten Independent-Mainstream-Antagonismus konstatierte. In der Tat hatte der Erfolg der Grunge-Rocker Nirvana aus Seattle die Underground-Szene und sukzessive den Bereich nichtkommerzieller Independent-Netzwerke völlig durcheinandergewirbelt und einen gesamten Zweig verändert. »Radikalisiert das Leben!« wäre vor I992 vielleicht der Titel eines Punk-Samplers oder eines Artikels über die französischen Situationisten um Gy Debord gewesen, heute füllt die »progressive« Abteilung der Musikindustrie damit breitesten Anzeigenraum. Hatten zuvor schon Techno, HipHop, House, Drum $\&$ Bass/Jungle die Pop-Kultur zu einem für ein »Zentralorgan« kaum überblick- und kontrollierbaren Feld tausendfacher Segmente, Querverbindungen, Allianzen und Subströmungen erweitert und damit eine Zeitschrift wie SPEX zusätzlich in Legitimationsprobleme bezüglich ihrer Avantgarde-Position in Bezug auf die richtungsweisende Berichterstattung gebracht, stehen Beobachter der Jugend- und Populärkultur spätestens seit dem keinesfalls neuen, in dieser Form aber noch nicht da gewesenen Erfolg rechter Bands und Subkulturen vor einem Karneval der Zeichen. Kann auf einem Techno-Rave noch eine bunte, vielgestaltige, nicht eindeutig decodierbare Bricolage aus hippesken Emblemen, Plastik und Esoterik-Accessoires »gelesen« werden, stellt die rechte Subkultur die alten popkulturellen Zuordnungen vollends in Frage. So berichtet der Berliner Journalist Burkhard Schröder in Nazis sind Pop von einer jungen Interviewpartnerin, die sich als »nationale Sozialistin« definierte und Rasta-Zöpfe trug (Schröder 2000: I57); Rammstein, eine nach Selbstauskunft vorgeblich apolitische deutsche Rock-Band, begeistert ein jugendliches Publikum mit Video-Clips, die die protofaschistische Leni-Riefenstahl-Ästhetik imitieren und auf ein ähnlich diffuses Milieu einwirken. Entscheidend 
ist in diesen Fällen vor allem, wie die Codes der Musiker vom Publikum rezipiert, verändert und symbolisch angeeignet werden. Zum Beispiel: Die wegen ihrer Neonazi-Vergangenheit einschlägig bekannte Gruppe Böhse Onkelz konnte nach ihrer Distanzierung vom rechten Spektrum auf Konzerten Gäste mit Transparenten, die die Aufschrift »Hoyerswerda grüßt die Onkelz« trugen, begrüßen - die Botschaft wird verstanden! Keine andere Epoche der Pop-Geschichte stellte bislang derart die dringliche Aufgabe, die journalistische und akademische Berichterstattung über Pop der affirmativen Verbraucherberatung und nutzlosen Pädagogisierung zu entreißen, keine andere bot weiter so viel Anlass, dem »Resistance through Rituals« als möglicherweise emanzipatorischer Form des Protestes zu misstrauen. $\mathrm{Zu}$ den besten, inzwischen ein wenig zurückliegenden Zeiten, hat SPEX trotz aller theoretischen Überladungen diese Aufgabe erfüllt, für die jüngeren Ausgaben gilt dies nicht mehr ohne Einschränkung, obwohl auch heute noch gelegentlich hervorragende Artikel im Blatt veröffentlicht werden.

Ob Pop nun aber in seinen besten Momenten die ästhetische Vorwegnahme der Emanzipation oder doch nur kulturindustriell vereinnahmte Scheindissidenz ist, soll an dieser Stelle nicht entschieden werden. In Bezug auf die Geschlechterrepräsentation im Pop kann zumindest festgehalten werden, dass die Entwicklung der letzten Jahre zwar nicht zur Auflösung tradierter Hierarchien geführt hat, wohl aber - wie das Riot Grrrls-Netzwerk zeigt - zur Durchsetzung divergierender Entwürfe: vom transsexuellen Image der New York Dolls in den frühen I970ern über die offensive Strategie von Madonna bis hin zum

Erfolg hervorragender jüngerer Bands wie Sleater Kinne. Auch wenn aus dem Erfolg dieser »dissidenten« Modelle wenig in Bezug auf die Änderung der Warenförmigkeit kultureller Produkte folgt, so sind, da weibliche Pop-Stars lange schon nicht mehr lediglich auf die Rolle der »Girlies« oder der fragilen Künstlerinnen reduziert werden können, die emanzipatorischen Konsequenzen doch deutlich sichtbar.

\section{Anmerkungen}

I In Heft 7/1995 wurde neben einer redaktionellen Einleitung ein Beitrag von Kerstin Grether über die feministischen Rock-Kritikerinnen Ellen Willis (Professorin für Journalismus an der New York Uni- 
versity und Autorin von No More Nice Girls. Countercultural Essays) und Ann Powers (Musikredakteurin der traditionsreichen New Yorker Stadtzeitung Village Voice) veröffentlicht (vgl. K. Grether I995). Die Journalisten Ralf Niemczyk und Christian Höller publizierten unter dem Titel »Dick Hebdige. Ein Ringkampf mit Engeln« ein Interview mit Dick Hebdige (vgl. Niemczyk/Höller I995), während Tom Holert in dem Beitrag »College Rock. Die Ästhetik von Cultural Studies« über den Zusammenhang von Cultural Studies und Rock-Kritik berichtete (vgl. Holert I995a). Die Fortsetzung in Heft 8/1995 enthielt einen Beitrag von Christian Storms über Andrew Goodwin, dessen Buch Dancing in the Distraction Factory (1992) und die Problematik einer Medienkritik zwischen Postmoderne, Kritischer Theorie und MTV (vgl. Storms I995). Manfred Hermes verfasste mit »Suspekte Subjekte« einen Bücherbericht über neuere Publikationen zum Bereich der Gender Studies (vgl. Hermes I995). Abgeschlossen wurde dieses Special mit einer Kolumne für grundlegende Buchempfehlungen (hier wurden u.a. Arbeiten von Ellen Willis, John Fiske, Raymond Williams und Paul Gilroy aufgeführt) sowie mit Artikeln von Tom Holert (vgl. Holert I995b) und Sandra Grether (vgl. Grether I995b) zur Situation der Cultural Studies an deutschen Universitäten und Kunstakademien. Für das redaktionelle Update im Heft 6/I997 führte Jan Engelmann ein Interview mit Simon Frith (vgl. Engelmann I997), Mark Terkessidis berichtete unter dem Titel »Nicht ohne revolutionäre Perspektive« über die »Geburt der britischen Cultural Studies aus dem Geist der Neuen Linken« (vgl. Terkessidis I997). Letzter Beitrag dieses Themenhefts war ein Lektürebericht von Tom Holert über Neuerscheinungen zum Thema »Jugendkultur« und deren Verbindungen zu den Jugendforschungen der Cultural Studies (vgl. Holert I997).

2 Ralf Hinz beschreibt in seiner detailgenauen und höchst ausführlichen Dissertation Cultural Studies und Pop. Zur Kritik der Urteilskraft wissenschaftlicher und journalistischer Rede über populäre Kultur mit anderer Akzentsetzung ebenfalls Diederichsens Werdegang und Einfluss auf den deutschen Pop-Journalismus (Hinz I998, S. I97-209 und S. 259-267). Seine vor allem an die Kategorien von Marx, Adorno und Pierre Bourdieu anknüpfende Arbeit ist zugleich eine übersichtliche Darstellung der Diskussion über die Möglichkeiten und Grenzen einer zeitgemäßen Kritik der politischen Ökonomie der Pop-Kulturindustrie. Hinzes Arbeit steht in dieser Hinsicht beispielhaft für die im letzten Jahrzehnt vollends entfaltete akademisch-theoretische Analyse 
pop-kultureller Stile, Bewegungen und Kulturformen und hat nicht zufällig vor allem SPEX als Gegenstand der wissenschaftlichen Untersuchung ausgewählt.

3 Diederichsens Vorwort zur I989 erschienenen Kritikensammlung 1500 Schallplatten 1979-1989 trägt den bezeichnenden Titel $\gg$ Musik und Dissidenz in den 8oer Jahren - Inhaltsverzeichnis einer Theorie«. Vgl. Diederichsen I989, S. II-I9; zur neueren Diskussion vgl. Diederichsen I999.

4 »Das neue Merve-Bändchen war für eine gewisse Zeit für gewisse Kreise so etwas wie die neue Indie-Platte (Merve war und ist da sowas wie ein Major-Indie, das Rough-Trade unter den Theorie-Verlagen)« (Diederichsen I993a: I63).

5 Der Journalist und testcard-Herausgeber Martin Büsser kritisiert im Vorwort seines Buches Anti-Pop den leichtfertigen Umgang mit den Geistesgrößen des akademisch orientierten Pop-Diskurses: »Antipop meint strenge Absage. Bitte wieder mit Marx, Adorno und Marcuse beschäftigen, mit Foucault und Deleuze. Aber was heißt schon >wieder $<$ - hat das je jemand getan? Als wir >testcard \#3< in ein Fake-Cover verhüllten, das die Merve-Ausgabe von >Mille Plateaux< nachstellte, ist dies fast niemandem aufgefallen. Der Philosoph, vom dem die hippe Popisten reden, als sei er der Schlüssel, über den sich Belanglosigkeiten wie die FUGEES als rhizomatisch minoritär verkaufen lassen, ist in den entsprechenden Kreisen nur über Schlagwörter präsent« (Büsser 1998: 9). Die in ambitionierter Buchform erscheinende Pop-Theorie-Reihe testcard stellt einen außerhalb der Zeitschrift SPEX agierenden Arbeitszusammenhang dar, welcher eine ästhetische Untersuchung der Pop-Kultur betreibt und jenseits popkultureller Mythenbildung nach ihrer gesellschaftlichen Relevanz fragen will.

6 Obwohl für die Weiterentwicklung des Blattes entscheidend, ist die Frage nach der »neuen « SPEX für diesen Überblick weniger wichtig, da die entscheidende Phase der Rezeption der Cultural Studies in den Jahren vor 1998 stattfand. Zum jetzigen Zeitpunkt kann eine deutliche, sich bereits länger abzeichnende Umgestaltung festgestellt werden, die sich vor allem im höchst ambitionierten und kunstvollen Layout des Blattes niederschlägt, während die Bezugnahme auf die Cultural Studies inzwischen einen weniger breiten und expliziten Raum einnimmt. Über diese Debatte berichtet ausführlich Klaus Walther unter dem Titel »I just wasn't made for these times.< Leben mit >Spex« in der Zeitschrift Texte zur Kunst (vgl. Walther 2000). 
7 Die Rezension von Diedrich Diederichsen enthält zugleich einen Abriss über das geistige Klima, in der die Rezeption dieser Denkrichtungen stattfinden konnte. Das Erscheinen der deutschen Übersetzung der Mille Plateaux wurde in der SPEX zudem euphorisch begrüßt (vgl. dazu auch Diederichsen i993a, S. I59-I8I).

8 On Record, herausgegeben von Simon Frith und Andrew Goodwin, in dem von Theodor W. Adorno bis Greil Marcus unterschiedlichste Beiträge zur (Rock-)Kulturtheorie veröffentlicht wurden (vgl. Frith/ Goodwin I990).

9 Festgestellt wurde hier die damals noch weitgehend fehlende Aneignung von Cultural Studies im klassischen akademischen Bereich und die »halblegitime Stellung « der mit Cultural Studies befassten Theoretiker. Der Leserschaft empfohlen wurde der »Theorie-Hammer« The Location of Culture (I993) von Homi K. Bhabha. Als Resümee zum damaligen Zeitpunkt bilanziert der Autor Birger Hübel die Rezeption der Cultural Studies mit den Worten: »Allerdings wird sich die Wirksamkeit vieler Theoretiker aus den Bereichen der Cultural Studies erst dann konkretisieren, wenn ihre Dialoge und Vorschläge auch, und das bleibt zu hoffen, im hiesigen kulturellen und politischen Mainstream-Diskurs eingreifen können, und andererseits eine Menge von Leuten sich ihren >Nebenwiderspruch $<$ sparen. Zunächst hoffen wir erst einmal, dass >Yo Hermeneutics<, >black looks< und >Körper von Gewicht< endlich mehr deutsche Übersetzungen anstoßen« (Hübel I995: 34). Der letzte Satz bezieht sich auf damals aktuelle Veröffentlichungen von bell hooks, Judith Butler und Diedrich Diederichsen.

10 Unter dem Titel »Phobien, Synopsen, forcierte Wissensstände!? Jetzt: Boom? Deutschsprachige Cultural Studies-Publikationen versuchen den Diskurs zu ordnen« erschien eine Sammelbesprechung des Autors Christian Höller von folgenden Bänden: The contemporary Study of Culture, herausgegeben vom Internationalen Forschungszentrum Kulturwissenschaften des Bundesministeriums für Wissenschaft und Verkehr in Wien; Cultural Studies - Eine Einführung von Christina Lutter und Markus Reisenleitner; Cultural Studies - Grundlagentexte zur Einführung, herausgegeben von Roger Bromley, Udo Göttlich und Carsten Winter, sowie Die kleinen Unterschiede - Der Cultural StudiesReader, herausgegeben von Jan Engelmann.

I I Florian Schneider weist in seiner Rezension des Buches Empire von Antonio Negri und Michael Hardt (Cambridge/London 2000) auf 
eine aktuelle Version des Vorwurfs hin, die Cultural Studies würden letztlich nur der Konsumforschung und hierarchisierenden Marktsegmentierung dienen: »Postmoderne Theorie hat die Welt nur dekonstruiert, es kommt aber darauf an, sie zu verändern - so ließe sich das Credo von Michael Hardt und Toni Negri vielleicht am treffendsten umschreiben. Hart gehen die beiden Autoren mit der Scheinradikalität der Postcolonial und Cultural Studies ins Gericht. Diese hätten zwar dazu beigetragen, dass es mittlerweile auch >für schwule i8- bis 22-jährige Latino-Amerikaner< eine spezielle Marketingstrategie gebe. Die Theoretisierung aller möglichen Verschiedenheiten und deren Realisierung im Rahmen des Weltmarktes führten aber keineswegs zu Gleichheit und zum freien Spiel der Kräfte, sondern zu neuen Hierarchien oder eher einem Prozess andauernder Hierarchisierung <. Postmoderne Tugenden wie Differenz, Hybridität und Freizügigkeit blieben Privilegien von Eliten, solange eine wirklich revolutionäre Praxis sich nicht auf die Ebene der Produktion beziehe« (Schneider 2000: 86 ff.).

12 Nach der Wiedervereinigung Deutschlands konnte in subkulturellen Zusammenhängen eine verstärkte Repolitisierung verzeichnet werden. Zahlreiche Autoren aus dem SPEX-Umfeld (zu nennen sind hier Diedrich Diederichsen und Günther Jacob) sowie bekannte Bands wie die Hamburger Gruppen Die Goldenen Zitronen oder Blumfeld bereisten Anfang der I99oer die neuen Bundesländer, um in aktionistischer Form gegen die rassistischen Pogrome in Hoyerswerda und anderen Städten zu demonstrieren. Personen und Zusammenhänge, die sich in den I980ern vor allem auf publizistische und künstlerische Praxis beschränkt hatten, nahmen in diesem Zeitraum an Kongressen (z.B. konkret-Kongress in Hamburg, Juni I993; Kongress der Wohlfahrtsausschüsse, Köln, Juli I993) teil oder intervenierten in Form von Demonstrationen und situativen Happenings. Beispielhaft ist hier die zum selben Zeitraum in Köln stattfindende Verhinderung der Aufführung von Filmen des Regisseurs Hans-Jürgen Syberberg, dem u.a. aufgrund seiner kulturpolitischen Schriften Antisemitismus vorgeworfen wurde. Diese Interventionen waren eigenständige Artikulationen eines Milieus, welches sich über Jahre von der traditionellen (arbeiterbewegten oder reformistischen) deutschen Linken abgegrenzt hatte und nun, nach ihrem Rückzug aus dem subkulturellen Mikrokosmos, mit autonomen linken Gruppen gemeinsam agierte (vgl. dazu ausführlich Wohlfahrtsausschüsse 1993). 
13 Zur Debatte um HipHop, Rassismus und die Problematiken der Identitätspolitik vgl. das kenntnisreiche Standardwerk Agit Pop. Schwarze Musik und weiße Hörer (I993) von Günther Jacob.

14 Vgl. hierzu Annas/Christoph I993, für die neueren Entwicklungen Searchlight et al. (Hg.) 2000. In SPEX wurde die Debatte unter dem Titel »Das Ende der Jugendkultur wie wir sie kennen« eröffnet (vgl. Diederichsen I992).

I 5 Ein bekanntes Frankfurter Label aus dem Techno-Bereich gab sich den bezeichnenden Namen Mille Plateaux, in Zeitschriften wie de:bug - Zeitschrift für elektronische Lebensaspekte findet dieser Ansatz auch publizistischen Niederschlag.

16 So der Titel eines Spiegel-Spezial-Hefts, in dem die führenden internationalen und deutschen Pop-Journalisten zu Wort kamen und die unterschiedlichen Positionen der damaligen Debatte referiert wurden (vgl. Spiegel Spezial i994).

I Vgl. zur Geschichte der Riot Grrrls den Beitrag von Gottlieb und Wald in Eichhorn/Grimm I994.

18 Annette Weber beschreibt in jungle world prägnant die Praxis der Riot Grrrls »zwischen subversiver Praxis und koketter Frechdachs-Attitüde« und liefert mit ihrem journalistischen Artikel an anderer Stelle ebenfalls einen Beitrag, der bestens zu der im Folgenden untersuchten Textsorte einer am Cultural Studies-Diskurs orientierten Annäherung an popkulturelle Phänomene passt (vgl. Weber I998).

19 Nancy Spungen war die Freundin des Sex Pistols-Bassisten Sid Vicious und gilt in der Geschichtsschreibung des Punk als geisteskranke Heroinsüchtige, die von ihrem Freund später durch Messerstiche ermordet wurde. In dem Film Sid Q Nancy von Alex Cox (USA I988) spielt Courtney Love in einer Nebenrolle die Freundin von Nancy Spungen.

\section{Literatur}

Annas, Max/Christoph, Ralf (Hg.) (I993): Neue Soundtracks für den Volksempfänger. Nazirock, Jugendkultur und rechter Mainstream, Berlin/Amsterdam: Edition ID-Verlag.

Baldauf, Anette/Weingartner, Katharina (Hg.) (I998): Lips, Tits, Hits, Power? Popkultur und Feminismus, Wien: Folio.

Bhabha, Homi K. (I993): The Location of Culture, London: Routledge. 
Bromley, Roger/Göttlich, Udo/Winter, Carsten (Hg.) (I999): Cultural Studies - Grundlagentexte zur Einführung, Lüneburg: zu Klampen.

Büsser, Martin (I998): Antipop, Mainz: Ventil.

Butler, Judith (I995): Körper von Gewicht, Frankfurt a.M.: Suhrkamp.

Deleuze, Gilles/Guattari, Felix (1992): Tausend Plateaus, Berlin: Merve.

Diederichsen, Diedrich (I985): Sexbeat. 1972 bis heute, Köln: Kiepenheuer \& Witsch.

Diederichsen, Diedrich (1989): 1500 Schallplatten. 1979-1989, Köln: Kiepenheuer \& Witsch.

Diederichsen, Diedrich (1992): »The Kids Are Not Alright «. SPEX II/ I992, S. 28-34.

Diederichsen, Diedrich (I993a): Freiheit macht arm. Das Leben nach Rock 'n' Roll 1990-93, Köln: Kiepenheuer \& Witsch.

Diederichsen, Diedrich (Hg.) (1993b): Yo! Hermeneutics! Schwarze Kulturkritik, Berlin/Amsterdam: Edition ID-Archiv.

Diederichsen, Diedrich (1999): Der lange Weg nach Mitte. Der Sound und die Stadt, Köln: Kiepenheuer \& Witsch.

Diederichsen, Diedrich/Hebdige, Dick/Marx, Olaf D. (1983): Schocker. Stile und Moden der Subkultur, Reinbek: Rowohlt.

Eichhorn, Cornelia/Grimm, Sabine (Hg.) (I994): Gender Killer. Texte zu Feminismus und Politik, Berlin: ID-Verlag.

Engelmann, Jan (I997): »Roter Faden Performanz. Interview mit Simon Frith«. SPEX 6/1997, S. 58.

Engelmann, Jan (Hg.) (1999): Die kleinen Unterschiede - Der CulturalStudies-Reader, Frankfurt a.M.: Campus.

Frith, Simon/Goodwin, Andrew (Hg.) (I990): On Record: Rock, Pop and the Written Word, London: Routledge.

Goodwin, Andrew (1992): Dancing in the Distraction Factory: Music Television and Popular Culture, Minneapolis: University of Minnesota Press.

Gottlieb, Joanne/Wald, Gaby (I994): »Smells Like Teen Spirit«. In: Cornelia Eichhorn/Sabine Grimm, Gender Killer. Texte zu Feminismus und Politik, Berlin: ID-Verlag.

Grether, Kerstin (I995): »Ellen Willis \& Ann Powers. Rock She Wrote«. SPEX 7/1995, S. 50-51.

Grether, Sandra (I995a): »Hole. Die Band, die alle sterben sehen wollen«. SPEX I/1995, S. 20-23. 
Grether, Sandra (I995b): »Ich zieh' mein Studium durch. Kunstakademien im Test «. SPEX 8/1995, S. 51.

Grossberg, Lawrence (I992): We Gotta Get Out Of This Place. Popular Conservatism and Postmodern Culture, New York/London: Routledge.

Hafeneger, Benno/Japsen, Mechthild M./Klose, Christiana (Hg.) (I998): »Mit fünfzehn hat man noch Träume ...« Lebensgefühl und Lebenswelten in der Adoleszenz, Opladen: Leske + Budrich.

Hall, Stuart/Jefferson, Tony (Hg.) (I976): Resistance Through Rituals. Youth Subcultures in Post-War Britain, London: Hutchinson.

Hebdige, Dick (I979): Subculture. The Meaning of Style, London/New York: Methuen.

Heiliger, Anita (I998): »Weibliche Lebensentwürfe: Vielfalt und Blockierungen. Starke Vorbilder, enge Grenzen«. In: Benno Hafeneger/Mechthild M. Japsen/Christiana Klose (Hg.), »Mit fünfzehn hat man noch Träume ...« Lebensgefühl und Lebenswelten in der Adoleszenz, Opladen: Leske + Budrich.

Hermes, Manfred (I995): »Suspekte Subjekte«. SPEX 8/ı995, S. 48-49.

Hinz, Ralf (I998): Cultural Studies und Pop. Zur Kritik der Urteilskraft wissenschaftlicher und journalistischer Rede über populäre Kultur, Opladen: Westdeutscher Verlag.

Höller, Christian (I999): »Phobien, Synopsen, forcierte Wissensstände!? Jetzt: Boom? Deutschsprachige Cultural-Studies-Publikationen versuchen den Diskurs zu ordnen«. SPEX 9/ı999, S. 24 .

Holert, Tom (I995): »College Rock. Die Ästhetik von Cultural Studies«. SPEX 7/1995, S. 54-55.

Holert, Tom (I995): »Kulturwissenschaften zu Cultural Studies. Wo geht es hier zum Institut?«. SPEX 8/1995, S. 50-51.

Holert, Tom (I997): »Gegen-»Kultur««. SPEX 6/1997, S. 60-62.

Holert, Tom/Terkessidis, Mark (Hg.) (I996): Mainstream der Minderheiten. Pop in der Kontrollgesellschaft, Berlin: ID-Verlag.

Hübel, Birger (ı995): »Cultural Studies«. SPEX I/ı995, S. 34.

Jacob, Günther (1993): Agit-Pop. Schwarze Musik und weiße Hörer. Texte zu Rassismus und Nationalismus, HipHop und Raggamuffin, Berlin/Amsterdam: Edition ID-Verlag.

Kemper, Peter/Langhoff, Thomas/Sonnenschein, Ulrich (Hg.) (I999): 
Alles so schön bunt hier. Die Geschichte der Popkultur von den Fünfzigern bis heute, Stuttgart: Reclam.

Kurz, Robert (1999): Die Welt als Wille und Design. Postmoderne, Lifestyle-Linke und die Ästhetisierung der Krise, Berlin: Edition Tiamat.

Lutter, Christina/Reisenleitner, Markus (I998): Cultural Studies - Eine Einführung, Wien: Turia + Kant.

McDonnall, Evelyn/Powers, Ann (Hg.) (I995): Rock She Wrote. Woman Write About Rock, Pop and Rap, New York: Plexus.

Negri, Antonio/Hardt, Michael (2000): Empire, Cambridge/London: Harvard University Press.

Niemczyk, Ralf/Höller, Christian (I995): »Dick Hebdige. Ein Ringkampf mit Engeln«. SPEX 7/1995, S. 52-53.

Redaktion SPEX (I995): Special - Cultural Studies. Teil Eins. SPEX 7/ I995.

Schneider, Florian (2000): »Empire. Rezension zu Negri/Hardt«. SPEX Iо/2000, S. 86-89.

Schröder, Burkhard (2000): Nazis sind Pop, Berlin: Espresso.

Spiegel Spezial (I994): Pop und Politik, Ausgabe 2/I994, Hamburg.

Storms, Christian (I995): »Die Fabrik tanzt«. SPEX 8/1995, S. 47.

Terkessidis, Mark (I997): »Nicht ohne revolutionäre Perspektive!«. SPEX 6/1997, S. 59 .

Terkessidis, Mark (2000): »Langer Abschied vom Anderssein«. die tageszeitung vom I7.2.2000, S. I7.

Walther, Klaus (2000): »>I Just Wasn't Made for these Times.< Leben mit >Spex««. Texte zur Kunst, Heft 38 Juni 2000, S. I75-180.

Weber, Annette (I998): »Was in der Kleidchennummer steckt. Riot Grrrls zwischen subversiver Praxis und koketter Frechdachs-Attitüde«. jungle world I8/ı998.

Westbam/Goetz, Rainald (I997): Mix, Cuts \& Scratches, Berlin: Merve.

Willis, Ellen (I994): No More Nice Girls. Countercultural Essays, New England: Wesleyan University Press.

Willis, Paul (I979): Spaß am Widerstand. Gegenkultur in der Arbeiterschule, Frankfurt a.M.: Syndikat.

Willis, Paul (I98I): »Profane Culture«. Rocker, Hippies: Subversive Stile der Jugendkultur, Frankfurt a.M.: Syndikat.

Wohlfahrtsausschüsse (Hg.) (I993): Etwas besseres als die Nation, Berlin/Amsterdam: Edition ID-Verlag. 


\section{Die Politik des Kulturellen:}

\section{Cultural Studies in Wien und in Birmingham}

GÜNTHER SANDNER

\section{Die britischen Cultural Studies und die Politik des Kulturellen}

Basiert die deutschsprachige Tradition der Kulturwissenschaften eher auf einem ästhetischen Kulturbegriff, so stützt sich die angelsächsische stärker auf einen anthropologischen, der Kultur als Lebensweise definiert (Burns I995: I). Als repräsentativ für den angelsächsischen Kulturbegriff gilt die I958 von Raymond Williams geprägte Formulierung, der Kultur als »the whole way of life of a group of people« (Williams I958: xvi) definierte. Die damit gleichermaßen korrespondierende und weiterführende Formel prägte Williams in einem gleichnamigen Essay: Culture is ordinary. E.P. Thompson führte mit seinem Ansatz einer »Geschichte von unten« eine konflikttheoretische Komponente ein, indem er kulturelle Ausdrucksformen der Arbeiterklasse als Bestandteil gesellschaftlicher Hegemoniekonflikte interpretierte. Culture as a whole way of struggle definierte Kultur als politisch umkämpften Ort. Damit positionierte Thompson die Cultural Studies in einer Theorietradition des britischen Marxismus und grenzte sich gegenüber einer traditionellen Lesart des Marxismus ab, der in einem allzu schematischen Basis-Überbau-Modell Kultur im Wesentlichen aus den Produktionsverhältnissen ableitete. Bei Thompson erschien das kulturelle Feld als eines, das die Herrschaftsverhältnisse einer Klassengesellschaft nicht nur spiegelt, sondern das Macht gleichermaßen 
produziert. In nahezu allen relevanten Analysen der Geschichte der Cultural Studies werden ihre Vorläufer im Arbeiter- und Erwachsenenbildungsmilieu der britischen Gesellschaft der I930er, 4oer und 5oer Jahre verortet. Dort waren auch die späteren Protagonisten der Cultural Studies, also Raymond Williams, Richard Hoggart und E.P. Thompson aktiv (vgl. Steele 1997). Wie Raymond Williams festgestellt hat, sind die Cultural Studies und ihre Entstehung deswegen nicht mit einem Kanon grundlegender Texte gleichzusetzen, sondern diese sind letztlich als Produkt eines kulturpolitischen Prozesses aufzufassen:

"Cultural Studies was extremely active in adult education. It only got into print and gained some kind of general intellectual recognition with these later books (Williams 1989: 154).

Sie entstanden also nicht im universitären Milieu, ihre Adressaten waren nicht so sehr Studierende aus dem bürgerlichen Milieu, sondern Angehörige der Arbeiterklasse, deren Lebenswelt auf zweifache Art präsent war. Zum einen durch ihre Resonanz im Bildungsprozess, zum anderen weil insbesondere Raymond Williams oder Richard Hoggart selbst aus der Arbeiterklasse kamen und als cultural hybrids, als Grenzgänger zwischen den Kulturen, agierten (vgl. Lindner 2000), gewissermaßen beide Welten kannten und diese Erfahrung in ihrer wissenschaftlichen und pädagogischen Arbeit einfließen lassen konnten. Damit ist auch eines der zentralen Anfangs- und Ausgangsthemen der Cultural Studies bestimmt, nämlich die Arbeiterkultur, die in verschiedene Richtungen hin analysiert wurde: historisch-genetisch, literaturkritisch und gegenwartsbezogen-politisch im Sinne ihrer gesellschaftlichen Funktion. Diese Aktivitäten in der Erwachsenenbildung entsprangen aber durchaus nicht nur der Not der outsider der akademischen Welt, sondern sie spiegeln vielmehr den politischen Anspruch, Bildung und Kultur zu demokratisieren. Deswegen verstanden sich die Cultural Studies als politisches Projekt. Ihr sozialer Ort war weniger das institutionalisierte akademische Leben, sondern die Schnittpunkte zwischen der Gelehrtenwelt und dem, was Stuart Hall als die »dirty world outside« bezeichnet hat, bestimmten ihre Themen: Die niedergehende Arbeiterkultur, die Kultur der Massen und die Popularkultur wurden auf der Basis eines Kulturbegriffs erforscht, der das Kulturelle, Kulturproduktion und Kulturkonsumtion, in seiner Wechselwirkung und in seinen Verbindungslinien zu 
Ökonomie und Politik interpretierte; der Gegenstandsbereich der Cultural Studies konnte somit mit der Gramsci-Formel der »Politik des Kulturellen« umrissen werden. Könnte die Vergegenwärtigung dieser Frühgeschichte der britischen Cultural Studies den Blick dafür schärfen, das, was im Folgenden als austromarxistisches cluster bezeichnet wird, als Bestandteil einer Geschichte der Kulturwissenschaften wahrzunehmen? Kann ein komparativer Ansatz ein scheinbar dem Vergessen überantwortetes Kapitel der österreichischen Kultur- und Politikgeschichte gewissermaßen neu kontextualisieren? Der Gründungsmythos der Cultural Studies in Großbritannien erschien lange Zeit unangefochten, ein stabiler Abschnitt der Wissenschaftsgeschichte. Doch jede hegemoniale Geschichtsschreibung ist der Kritik, dem Versuch, Gegengeschichte zu konstruieren, ausgesetzt. Dies führte dazu, aus unterschiedlichen Blickwinkeln die Ursprungsgeschichte der Transdisziplin, die im Centre for Contemporary Cultural Studies an der Universität Birmingham im Jahr I964 institutionalisiert wurde, zu hinterfragen, alternative Wissenschaftstraditionen anzuführen. »If cultural studies started with progressive adult literacy«, fragt Handel K. Wright (I998: 42), »doesn't that mean it started in Denmark?« Und wie war das in Österreich?

\section{Das austromarxistische "Clusteril in den Kulturwissenschaften}

Der Begriff Austromarxismus bezeichnet insbesondere die politische Theorie der österreichischen Sozialdemokratie von der Jahrhundertwende bis 1933/34. Als Austromarxisten im engeren Sinn gilt eine Kerngruppe politischer Theoretiker und Philosophen (Glaser 198I: I8). Genannt werden in der Regel Otto Bauer, Karl Renner, Max Adler und Friedrich Adler. Als theoretische Strömung manifestierte sich der Austromarxismus in bestimmten Schriftreihen und Publikationen. Hier sind insbesondere die von Max Adler herausgegebenen MarxStudien und das unter anderem von Otto Bauer editierte Theorieorgan Der Kampf zu nennen. Schon die Aufzählung dieser Namen verweist auf die Heterogenität des Phänomens. Liest man die teils erbitterten und polemischen Auseinandersetzungen zwischen Max Adler und Karl Renner nach, so kann man vermuten, dass beide wenig Freude an der Vorstellung hatten, zu einer Gruppe oder gar Schule zusammengefasst zu werden. Trotzdem gibt es freilich Gemeinsamkeiten: 
So unterschied den Austromarxismus etwa von der deutschen Sozialdemokratie die stärkere Betonung marxistischer Systemtranszendenz, darüber hinaus die Betonung des subjektiven Faktors. Damit verbunden war eine relativ hohe Relevanz von Phänomenen wie Ethik oder Kultur für die austromarxistische Theorie und die Emphase für eine politische Bewegung als Erziehungsinstanz. Dies sollte grundsätzlich vereinbar mit der marxistischen Theorie sein, der die geistige und ethische Dimension quasi eingeschrieben sei, wie sich Max Adler überzeugt gab (vgl. Adler I928). Vom Bolschewismus trennte ihn ganz klar das Bekenntnis zu einer parlamentarischen Strategie, zu einem friedlichen Weg der Machtergreifung, auch wenn die Rute der defensiven Gewalt und der Diktatur des Proletariats folgenschwer dem politischen Gegner im Linzer Programm von I926 ins Fenster gestellt wurde. Für die österreichische Sozialdemokratie als Kulturbewegung engagierten sich zahlreiche Wissenschaftler und Intellektuelle, mit starkem Überlappen in den liberalen und bürgerlichen Bereich, wie jene berühmte »Kundgebung des geistigen Wien«, ein Wahlaufruf zahlreicher prominenter Persönlichkeiten des so genannten geistigen Lebens für die Wiener Sozialdemokratie aus dem Jahr I927, demonstriert (Pfoser I980: I79). Nicht selten waren unter diesen Wissenschaftlern aber auch jene, die Randfiguren im herrschenden Wissenschaftsbetrieb waren, sich nicht an der Universität verankern konnten, die politisches Engagement und wissenschaftliche Aktivitäten verbinden wollten. Gemeinsam mit den kulturtheoretischen Reflexionen der genannten Kerngruppe des Austromarxismus sollen diese im Bildungswesen der österreichischen Sozialdemokratie wirkenden Persönlichkeiten als austromarxistisches cluster bezeichnet werden. Dass sich im Rahmen dieses clusters in politischer Hinsicht höchst unterschiedliche Persönlichkeiten finden, hängt auch mit dem Stellenwert und dem Begriff von Wissenschaft im Austromarxismus zusammen. Zwar wären wohl nur wenige dem geradezu fortschrittseuphorischen Verdikt von Karl Kautsky gefolgt, wonach

„kein Triumph [...] stolzer, erhabener und reiner sein [könne] als der der wissenschaftlichen Eroberer, keine Tätigkeit beglückender als die, in ihrem Gefolge zu kämpfen« (Karl Kautsky, zitiert nach: Küenzlen 1997: 84),

doch ein gewisser Abglanz dieser Fortschritts- und Wissenschaftsemphase beseelte den Austromarxismus. Denn auch im Austromarxis- 
mus hatte die Wissenschaft generell den Charakter einer objektiven Schlichtungsinstanz gesellschaftlicher Konflikte und eines Garanten von politischem und sozialem Fortschritt. Vielfach wurde der Marxismus als wissenschaftliche Methode, nicht als Weltanschauung interpretiert, wie insbesondere Karl Renners feinsinnige Unterscheidung zwischen einem ideologischen (d.h. abzulehnenden) und einem wissenschaftlichen Marxismus unterstrich (vgl. Renner 1928a). Diese Sichtweise schloss keineswegs aus, dass die herrschende Wissenschaft als bürgerliche, d.h. als an Besitzinteressen gebundene, wahrgenommen wurde. Gerade Käthe Leichters Reflexionen über ihren Lehrer Max Weber zeugen von intensiver Auseinandersetzung mit der Rolle des Wissenschaftlers in der bürgerlichen Gesellschaft (vgl. Leichter I926). Gleichzeitig wurde versucht, den progressiven Gehalt bürgerlicher Wissenschaft herauszuarbeiten - und an dem Anspruch einer wertfreien Wissenschaft zumindest als Idealbild festzuhalten. Für den für die Sozialdemokratie engagierten Philosophen Otto Neurath waren wissenschaftliche Weltauffassung und Sozialismus quasi gleichbedeutend (vgl. Neurath i98ia). Die Rekonstruktion eines austromarxistischen kulturwissenschaftlichen clusters bedeutet nun nicht unbedingt, dass die Wissenschaftler als Gruppe oder Forscherteam zusammengearbeitet haben - auch Hoggart, Williams und Thompson haben das nicht oder nur bedingt. Vielmehr geht es darum, gemeinsame Strukturmerkmale und kulturtheoretische Grundannahmen zu analysieren.

\section{Der Austromarxismus und sein Gegenteil}

Die Cultural Studies werden unter anderem durch das Prinzip der Kontextualisierung näher bestimmt (vgl. Grossberg 1997). Diesen Anspruch auf ihre Geschichte selbst anzuwenden, bedeutet zumindest, das Kräftefeld zu veranschaulichen, in dem sich die austromarxistischen Kulturdiskurse bewegten, die Widerstände und Gegenbewegungen zu umreißen, kurz: den Austromarxismus mit seinem Gegenteil zu konfrontieren. Die Erste Österreichische Republik war geprägt von der Existenz unterschiedlicher politischer Lager, die bereits in der Historiographie der I95oer Jahre definiert wurden und in der aktuellen zeitgeschichtlichen Politikforschung mit dem Begriff der Politisch-kulturelle(n) Integrationsmilieus und Orientierungslager in einer polarisierten Massengesellschaft bezeichnet worden sind (vgl. Lehnert 
I995). Dabei handelte es sich um eine Polarisierung, die in latenter wie in manifester politischer Gewalt ihren Ausdruck fand und gerade an den Schnittpunkten von Kultur und Politik virulent wurde. Diese Lager-Dichotomie bedingte, dass es zwischen den stark abgeschotteten Milieus nur zu relativ wenig Austausch - etwa im Sinne von Wählerfluktuation - gekommen ist. Damit war auch eine gewisse Abtrennung der Lebenswelten, der Sphären des Alltags und der Freizeit, verbunden. So konnte es zur parallelen Existenz verschiedener Kulturen im Sinne von Lebensweisen, zur Ausbildung von dominanten Kulturen und Gegenkulturen kommen. Diese schroffe Gegenüberstellung wurde durch die Dichotomie eines ab 1920 bürgerlich regierten Staates und der sozialdemokratisch dominierten Hauptstadt verstärkt. Sie führte $z u$ unverhüllter Aggression des deutschnationalen und katholischkonservativen Lagers gegen das »rote Wien«. Kulturpolitische Konflikte prägten das Klima dieser Ersten Republik, doch während die konservative Hegemonie in den Bundesländern wenig Dissonanz zuließ, entzündeten sich in der Hauptstadt Wien zahllose Kontroversen. Dies zeigte sich etwa in der Auseinandersetzung um die Aufführung von Arthur Schnitzlers Stück Der Reigen in Wien I92I. Der sozialdemokratische Bürgermeister genehmigte die Aufführung, der christlichsoziale Innenminister setzte jedoch ein Aufführungsverbot durch. Zuvor hatte die katholische Kirche in Hirtenbriefen gegen das Stück Stimmung gemacht. Bei einer Veranstaltung vor Wiener Katholiken fand Bundeskanzler Seipel klare Worte:

IDas sittliche Empfinden unseres bodenständigen christlichen Volkes wird fortgesetzt aufs schwerste verletzt durch die Aufführung eines Schmutzstückes aus der Feder eines jüdischen Autorsu (zit. n. Pfoser 1980: 193).

Ein Akt der »Selbsthilfe junger christlicher Männer«, wie die katholische Reichspost es formulierte, erzeugte schließlich jenes öffentliche Ärgernis, das zur Absetzung des Stückes vom Spielplan führte (ebd.: I94). Dabei dürfte das wissenschaftliche, intellektuelle und künstlerische Klima der Zeit eher durch betonte (Partei-)Politikferne bei gleichzeitiger Virulenz einer konservativ traditionalistischen Statusquo-Orientierung der geistigen Eliten bestimmt gewesen sein. Das weitgehende Fehlen manifester gesellschaftsverändernder Impulse aus dem Kulturbereich wurde jedenfalls häufig diagnostiziert (vgl. Pfoser I980: I75-I90). Doch für das klerikale und konservative wie für 
das nationale Lager verkörperte der in der furchterregenden Gestalt der österreichischen Sozialdemokratie auftretende Marxismus - austauschbar in den zeitgenössischen Kontroversen auch als Bolschewismus bezeichnet - gerade im kulturellen Bereich eine ernst $\mathrm{zu}$ nehmende Bedrohung der herrschenden Ordnung und Wertestruktur. Die proletarische Körperkultur signalisierte Sittenverderbnis, die Kulturvereine der Sozialdemokratie, ob Freidenker, ob Naturfreunde, ob Bildungsvereine, standen stellvertretend für Materialismus und Atheismus, die Massenveranstaltungen der Arbeiterkulturbewegung erschienen als anmaßende Gegeninszenierung zu den Manifestationen des politischen Katholizismus. Diese kulturpolitische Konfliktkonstellation entlud sich in aufsehenerregender Gewalt, aber auch in einem Kulturkampf in Permanenz. Aufsehenerregend waren etwa die Ermordung des unter Pornografieverdacht stehenden Schriftstellers Hugo Bettauer im Jahr I925 oder die gewalttätigen Auseinandersetzungen um den Remarque-Film Im Westen nichts Neues im Jahr I93I, die $\mathrm{zu}$ wahren Straßenschlachten führten. Die Sozialdemokratie distanzierte sich sowohl von Arthur Schnitzler als auch von Hugo Bettauer, die als mehr oder minder dekadente Repräsentanten eines bürgerlichen Kulturbegriffes gesehen wurden; gegen Zensurbestrebungen, die von den Christlichsozialen und Deutschnationalen mit ungeheurer Vehemenz artikuliert wurden, trat sie aber auf.

\section{Austromarxistische Kulturtheoreme: Oppositionen und Brüche}

Nach E.P. Thompson muss jede Kulturtheorie die dialektische Spannung zwischen Kultur und dem, was nicht Kultur ist, berücksichtigen. Diese Kontrastierung des Kulturellen mit dem Nichtkulturellen, die Verbindungen und Überlappungen von Kultur mit Politik und Ökonomie, die Verhandlungen darüber, was Kultur und was nicht Kultur ist, bestimmten die austromarxistischen Kulturdiskurse. An drei Beispielen soll dies veranschaulicht werden.

\section{Kultur und Macht}

Das Linzer Programm der österreichischen Sozialdemokratie von I926 analysierte, dass die politische Herrschaft der Bourgeoisie nicht mehr auf politischen Privilegien beruhe, sondern darauf, 
"Idass sie mittels ihrer wirtschaftlichen Macht, mittels der Macht der Tradition, mittels der Presse, der Schule und der Kirche die Mehrheit des Volkes unter ihrem geistigen Einfluss zu erhalten vermag॥ (Linzer Programm 1926, zit. n. Frei 1991: 15).

Die politische Relevanz kultureller Macht hatte Otto Bauer schon zwei Jahre davor in seiner I924 veröffentlichten Schrift Der Kampf um die Macht formuliert, in der er einerseits die Befreiung der Arbeiterklasse »von der Hegemonie des Bürgertums« (Bauer I924: I9) postulierte und andererseits die Herstellung klassenübergreifender Bündnisse zum Kampf gegen die Großbourgeoisie forderte. Verzahnt mit der Frage der politischen Macht tauchen zwei Dimensionen des Kulturbegriffes auf. Kultur als Bildungskultur und Erziehung erscheint als politisches Instrument der geistigen Befreiung und somit als Voraussetzung der (politischen) Machtübernahme, als emanzipatorisches wie als antizipatorisches Moment gleichermaßen. Politische Macht wird dabei nicht nur in ihrer institutionellen Dimension gesehen, sondern auch als kulturelle Hegemonie. Besonderen Ausdruck fand dieses Verständnis von kultureller Macht im Bildungswesen der Sozialdemokratie, insbesondere im Wien der Zwischenkriegszeit. Vor allem die Verbindung aus Wissenschaftsvermittlung und -popularisierung in der Arbeiter- und Erwachsenenbildung mit politischem Engagement ist hier von Bedeutung. Bei den Wissenschaftlern im Umfeld des Austromarxismus spielte auch ein gewisses akademisches outsider-Dasein eine Rolle. Nicht selten hatten diese durch ihr außeruniversitäres politisches Engagement wie auch durch ihren wissenschaftlichen Nonkonformismus mit Widerständen und erbitterter Gegnerschaft an der Universität zu rechnen. Dies fiel mit dem Anspruch zusammen, das Erwachsenenbildungswesen zu einem Ort der Demokratisierung von Wissenschaft zu machen. Aufgrund der ungleichen Verteilung von Bildungs- und Lebenschancen in der österreichischen Gesellschaft der Zwischenkriegszeit barg dieser Anspruch eine gewisse soziale Sprengkraft. Bemerkenswert dabei war sowohl das Vorhaben, Bildungs- und Kulturfragen mit Fragen der Lebenswelt zu verknüpfen als auch die Interpretation der Wissensproduktion als kollektiver und sozialer Prozess. Über diese Dimension des Kulturbegriffs hinaus wurde Kultur als Lebensweise - nämlich als Lebensweise der Arbeiterschaft - konzeptualisiert. Zu Recht wird dabei zwischen der organisierten Arbeiterkulturbewegung und der Arbeiterkultur an sich unterschieden. Die Sozialdemokratische Partei anerkannte die Funk- 
tion der Arbeiterkulturbewegung und versuchte, sie als politisches Instrument zu nutzen. Sie hatte aber letztlich ein ambivalentes Verhältnis zu ihr. Insbesondere in der Gründungsphase vieler Vereine und Verbände waren die Beurteilungen der Parteiführung mitunter skeptisch bis ablehnend, ein Wildwuchs an Organisationen rief parteioffiziellen Vereinheitlichungswillen hervor. Die Kräfte sollten gebündelt, Multifunktionen verhindert werden, zentralistische Ausschüsse die Kulturarbeit steuern. Doch mit diesen Bestrebungen hatte die Sozialdemokratie wenig Erfolg - die Frage, ob die Kulturbewegung der Machtgewinnung dienlich war oder die Zersplitterung der Kräfte beschleunigte, blieb letztlich kontrovers.

\section{Kultur und Masse}

Über die Frage, was die Intellektuellen zum Sozialismus treibe, machte sich der politisch äußerst wendige Soziologe Robert Michels schon in den I930er Jahren seine Gedanken. Es könne durchaus die unbewusste oder auch bewusste »Spekulation auf das glänzende Rohmaterial sein, das die proletarischen Massen jedem politischen Ehrgeizling« böten (Michels I987: I95 f.). Mit dieser Masse und ihrem Verhältnis zur Kultur hatten sich die sozialdemokratischen Kulturtheoretiker auseinander zu setzen. Sie tauchte letztlich in zweierlei Gestalt in ihren Diskursen auf. Zum einen tatsächlich als jene rohe Masse im Sinne von Michels, die es zu bearbeiten oder besser zu erziehen gelte - deren tatsächliches Kulturverhalten freilich einer strengen Kritik und entschiedenem Tadel unterzogen wurde; zum anderen als disziplinierte, politisch erzogene Masse, die nun selbst wieder zum kulturellen und zum politischen Faktor wurde. Das Verhältnis von Kultur und Masse im Austromarxismus ist also ebenfalls gebrochen: Einerseits ist es gerade das Massenhafte, das kulturelle Macht symbolisieren soll; andererseits wird die »reale« Kultur der Massen zum Angelpunkt der Kritik - zumal das Proletariat ja ursprünglich, wie Max Adler ausführt, »eine rohe Masse darstellte, undiszipliniert und vertiert durch die Ausbeutung des Kapitalismus, sodass man mit dieser Masse damals keinen Freiheitskampf hätte anfangen können « (Adler I928a: 26). Es war also erst die dirigierte, paternalistisch präformierte Kultur der Massen, die für den Austromarxismus die beiden Begriffe miteinander versöhnte. 
Kultur der Masse. Die Bereiche, in denen die Kulturvorstellungen führender Sozialdemokraten und das Verhalten des kulturpädagogisch anvisierten Proletariats einander widersprachen, waren zahlreich. An den Entlehnstatistiken der Arbeiterbibliotheken wurde der Konsum von Trivialliteratur abgelesen und getadelt, das Unproletarische bei Arbeiterfesten kritisiert, der Alltag der Kulturorganisationen als »Verspießerung« wahrgenommen. Bemerkenswert daran ist zum einen eine gewisse grundsätzliche Feindschaft gegenüber dem Populären, das zum quasi naturgemäßen Agenten des Kapitalismus erklärt wurde; zum anderen aber, dass die Grenzen zwischen Kitsch und Schund auf der einen Seite und dem echten Kunstwerk auf der anderen meist implizit vorausgesetzt wurden, also auf einem stillschweigend akzeptierten Kanon basierten, der den Gedanken an die Fragwürdigkeit oder zumindest an die Durchlässigkeit dieser als stabil gedachten Grenzen erst gar nicht aufkommen ließ. Wenn ein sozialdemokratischer Kulturtheoretiker mit dem schillernden Namen Richard Wagner über die vermeintlich verbürgte Tatsache räsonierte, dass Kitsch und Schund auf die überwiegende Mehrzahl der Menschen schlichtweg stark wirkten, während dies Kunstwerke nicht vermöchten, so waren die diesem Urteil zugrunde liegenden Kategorien gewissermaßen prädiskursiv und wurden auch kaum herausgefordert (Wagner I927: I29). Dies mag zum Teil erklären, dass die Sozialdemokratie mit großer Skepsis und bisweilen Ablehnung auf die beiden großen Massenmedien der damaligen Zeit, auf Kino und Radio, reagierte und es bei Projekten bewenden ließ, deren Konsum durch Arbeiter zu steuern.

Die Masse als Kulturbewegung. Die kulturell geformte, pädagogisch disziplinierte Masse hingegen war eine Zielperspektive sozialdemokratischer Politik; die Masse sollte zur Kulturbewegung werden. Symbolische Manifestationen von kultureller Macht, eine Fahnen- und Massenromantik, eine gewaltige ästhetische Formierung prägten eine mitunter pompöse Fest- und Feierkultur, von Sängerfestivals bis zur legendären Arbeiter-Olympiade I93I. Eine Massenästhetik entwickelte sich auf der Basis von Massenpsychologie, die strategisch bewusst eingesetzt wurde. Um dem politischen Gegner Macht zu demonstrieren, um Zusammenhalt zu vermitteln, aber eben auch, wie der Sozialdemokrat Jacques Hannak meinte, um »gefährliche(r) Massentriebe« einzudämmen. Das Arbeiter-Turn- und Sportfest wurde für Hannak zur eindrucksvollen Manifestation der Beherrschung und Disziplinie- 
rung gefährlicher Körperinstinkte und Leidenschaften, die durch proletarische Körperkultur sublimiert und in eine Gemeinschaftskultur übergeführt werden sollten: »Wenn ein einzelner Mensch die Last seiner Kleider abwirft, so sperrt man ihn in ein Irrenhaus«, formulierte Hannak, »wenn es die Massen tun, so wird eine Kulturbewegung daraus« (Hannak i926: 273 f.).

\section{Kultur und Natur}

Als in der Tradition aufgeklärten Denkens stehende Kulturbewegung reflektierten die austromarxistische Theorie und die Diskurse in der Sozialdemokratie und ihren Verbänden eine Dichotomie des Naturbegriffs, die in eben jenem aufklärerischen Denken selbst angelegt ist. Die Rede ist von den Naturkonzepten von Voltaire und Rousseau. Denn die Diagnose der Korrumpierung der menschlichen Natur durch Gesellschaft und Zivilisation widersprach dem Deutungsmuster der Veredelung von Mensch und Gesellschaft durch die Lösung der durch die Natur auferlegten Fesseln. Natur wurde in den austromarxistischen Diskursen zur Projektionsfläche höchst divergierender politischer Vorstellungen und Konzeptionen, in deren Differenz sich jeweils das Kulturelle konstituierte. In evasionistischen Diskursen erschien sie als ein Bereich der Auflösung von Widersprüchen, als Kontrastbild zur herrschenden Kultur, die das Versprechen der Auflösung repressiver gesellschaftlicher Verhältnisse in sich barg, die ein Stück Zukunft vorwegzunehmen schien; Natur war also eine Gegenkultur, die, als Norm konzeptualisiert, der Klassengesellschaft den Spiegel ihrer notwendigen Auflösung entgegenhielt. Gleichzeitig war es aber auch die Aneignung von Natur, die Gestaltung von Naturräumen, das Entziffern und Entschlüsseln ihrer Geheimnisse, die als Faktor jenes kulturellen Aufstiegs interpretiert wurden, der das Selbstverständnis und die Identität der Arbeiterkulturbewegung so wesentlich prägte. Kultur war folglich mit Fortschritt verknüpft, dessen Preis in der Unterwerfung und Disziplinierung von Natur - der menschlichen wie der äußeren Natur - bestand. Kultur wurde somit zur Gegennatur. Es waren die utopischen Konzepte, die hier die Auflösung der Widersprüche, die Zurückgewinnung der Einheit von Mensch und Natur, versprachen. Man denke an August Bebels Sozialismus-Utopie (Die Frau und der Sozialismus), in der eine humanisierte Technik die friedliche Koexistenz von Mensch und Natur zu gewähr- 
leisten schien, jene marxsche Vorstellung der umfassenden Entfaltung menschlicher Fähigkeiten und Bedürfnisse also nicht mehr an Schranken des Natürlichen stieß; oder im Falle des Austromarxismus an jenen neuen Menschen - eine Denkfigur, die, wie Gottfried Küenzlen (I994: 94 ff.) nachgewiesen hat, originär mit utopischem Denken verknüpft ist -, der im dichten Netz sozialistischer Gegenkultur präformiert wird, um in einer neuen Gesellschaft, deren Konturen freilich sehr blass verblieben, zur vollen Entfaltung zu gelangen. Der neue Mensch lebte politisch bewusst, war durch Partei- und Gewerkschaftsarbeit erzogen, umfassend geistig gebildet, befand sich auf der Höhe der Wissenschaften seiner Zeit und schöpfte seine Kraft aus der Naturbegegnung: Er vermochte jene »durchdachte und rational nachvollziehbare Alternative« (Saage I99I: 3), die als Merkmal politischer Utopien gilt, bereits ein Stück weit im Hier und Jetzt zu verwirklichen.

\section{Austromarxismus und Kulturwissenschaften exemplarisch: Otto Neurath und Edgar Zilsel}

Mit Otto Neurath und Edgar Zilsel sollen nun zwei Persönlichkeiten ins Blickfeld geraten, die - beide dem linken Flügel des so genannten Wiener Kreises zugehörend - jene Intellektuellen im Umfeld des Austromarxismus geradezu idealtypisch verkörpern. Wissenschaftstheoretisch vertrat der Wiener Kreis das Programm einer »wissenschaftlichen Weltauffassung und den Ansatz des Logischen Empirismus.

Politisch bestand die Absicht, die Alltagskultur der unteren Schichten, der Arbeiterklasse zu analysieren und zugleich zu reformieren, mit dem Ziel einer Demokratisierung der Gesellschaft. Neurath und Zilsel waren politisch in der Sozialdemokratie und pädagogisch in der Arbeiterbildung engagiert, publizierten regelmäßig im austromarxistischen Theorieorgan Der Kampf; beide waren, obwohl gerade von der Nachwelt wissenschaftlich anerkannt, im Österreich der Zwischenkriegszeit eher outsider der akademischen Welt - und beide standen in einer gewissen Opposition zu den kulturpolitischen Vorstellungen wie sie Otto Bauer und insbesondere Max Adler vertraten. 


\section{Edgar Zilsel - Genieverehrung als Mengenverachtung}

Edgar Zilsel wurde I89I als Sohn eines Rechtsanwalts in Wien geboren. Er studierte an der Wiener Universität Philosophie, Physik und Mathematik. Als Lehrender fand er sein Betätigungsfeld jedoch vorrangig im Rahmen des Wiener Volkshochschulwesens. Sein I923 unternommener Versuch, sich mit einer Arbeit zur Geschichte des Geniebegriffs an der Wiener Universität für Philosophie zu habilitieren, scheiterte am Widerstand der Fakultät. Als Sozialdemokrat wie als Jude waren die Voraussetzungen für eine solche Karriere auch alles andere als günstig gewesen, denn soziale Exklusion und Antisemitismus waren im universitären Bereich wesentliche Phänomene. Nach dem Februar I934, der schließlich gewaltsamen Beendigung der Demokratie durch den Austrofaschismus, musste er seine Tätigkeit an den Volkshochschulen einstellen. I938, nach dem »Anschluss« Österreichs an das nationalsozialistische Deutschland, emigrierte Zilsel mit seiner Familie zunächst nach London, dann in die USA. In der Emigration konnte er weder wissenschaftlich noch emotional und persönlich ein neues Zuhause finden; im März I944 beging er Selbstmord. Edgar Zilsel setzte sich in seinen Schriften insbesondere mit zwei kulturgeschichtlichen Themen auseinander. Als Wissenschaftshistoriker thematisierte er die sozialen Ursprünge der neuzeitlichen Wissenschaften. In mehreren Abhandlungen beschäftigte er sich darüber hinaus mit dem Geniebegriff. Die Grundannahme der von ihm so bezeichneten Geniereligion, dass die Menschheit kulturell betrachtet in zwei Kategorien zerfalle, nämlich in wenige Genies einerseits und die Menge andererseits, wird für ihn zum Ausgangspunkt einer Kritik am Persönlichkeitskult und an einem elitären Kulturbegriff. Zwischen die beiden zitierten Gruppen tritt nach Zilsel noch die kleine Gruppe jener, die das Genie erkennen und auf die deswegen ein gewisser Abglanz desselben fällt - die Priester der Geniereligion gewissermaßen. Für den Rationalisten und Materialisten Zilsel erscheint die Geniereligion als bloße Metaphysik, deren Grundannahmen der Prüfung durch die Vernunft nicht standhalten. Ihre Gefährlichkeit gewinnt sie durch die Kehrseite, die sie unablässig produziert: die Entmündigung der Menge und somit die Verhinderung der Selbstentfaltung und Autonomie der vom Kulturelitismus verächtlich so genannten »Dutzendmenschen«. Bemerkenswert an dieser Schrift ist, dass Zilsel in seiner Auseinandersetzung eine der impliziten Vorausset- 
zungen der austromarxistischen Kulturtheorie, nämlich das Vorhandensein eines gleichsam seichten Massengeschmackes, der sowohl mit dem bürgerlich-klassischen Erbe als auch mit den Manifestationen einer sozialistischen Gegenkultur kontrastiert und deswegen durch Erziehungsarbeit korrigiert werden müsse, konterkariert. »Der Schwärmer vermeint zuerst, die Welt sei aus den Angeln, wenn es den Operettenkomponisten besser ergeht als den Philosophen«, führt er dazu aus, »dann hält er es für moralischer, dass man Handwerker, die die >Lustige Witwe< genießen wollen, ihr gutes Geld dafür ausgeben lässt, dass sie die Neunte Symphonie anhören müssen; und schließlich entrüstet er sich darüber, dass man ein Volk für Krämerinteressen in den Krieg ziehen lässt, statt für Goethe, Beethoven und Kant« (Zilsel I990: I85). »Einer reinlichen Gesinnung«, fährt Zilsel fort,

"will es dagegen scheinen, dass sich die Handwerker für ihr Geld zunächst kaufen sollen, was ihnen Freude bereitet, dass Krämerinteressen die einzigen sind, für die man Krämer ihr Leben wagen lassen darf, und dass es keine gefährlichere und unmenschlichere Verblendung gibt, als seinen Mitmenschen Physisches zu nehmen um sie mit Metaphysischem zu bezahlen« (ebd.).

Besonders provokant musste den austromarxistischen Erziehungstheoretikern dann die Schlussfolgerung erscheinen:

"Deshalb erscheint heute weit wichtiger als Mengenverachtung die Aufgabe, jenen ungezählten Mitmenschen, die von einem unklaren Enthusiasmus als Dutzendmenschen verhöhnt werden, Mut zu machen, auf dass wir Menschen wieder unseren (sic!) harmlosen und heiteren Vergnügungen leben können - und wären sie auch spießbürgerlich -, auf dass vor allem die sachlichen Menschen sich wieder hervorwagen, ohne den Vorwurf der Seichtheit fürchten zu müssen« (ebd.: 190).

Ohne Zweifel kündigt sich hier eine gewisse Opposition zur kulturpädagogischen Haltung Max Adlers an, die dieser insbesondere in seinem Werk Neue Menschen entwickelte. Sie entlud sich letztlich auch in einer Kontroverse zwischen den beiden in der Zeitschrift Der Kampf, in der Max Adler Zilsels Forderungen nach einer populären Lesart des Materialismus als unzulässige »Akkomodation an das Massenunverständnis« (Adler I93I: I30) und als Zugeständnis an eine »zur Denkfaulheit geneigte(n) Menschennatur« (ebd.: I26) bewertete. Auch wenn Zilsels kühl-rationalistische Argumentation bisweilen auf ihre 
Art wieder anachronistisch anmutet, so ist das eigentlich Interessante daran der Bruch mit der im austromarxistischen Mainstream dominanten Vorstellung der wertlosen Massenvergnügungen. Verwiesen sei darauf, dass viele dieser Argumentationslinien Zilsels sich auch bei Raymond Williams in Culture and Society finden, in seinem Eintreten eben gegen jede dirigistische Kulturpolitik, der Linken wie der Rechten, der Einsicht, dass Kultur (im Wesentlichen) nicht planbar ist und der gegen diese Planungs- und Normierungstendenzen leicht resignativ formuliert:

"Wir handeln so als Konservative und versuchen, alte Formen zu verlängern; wir handeln so als Sozialisten und versuchen, den neuen Menschen vorzuschreiben« (Williams 1972: 403).

\section{Otto Neurath - Kultur als (proletarische) Lebensweise}

Otto Neurath wurde I882 geboren, begann I90 in Wien sein Studium und setzte es in Berlin fort. Er engagierte sich politisch für die Bayrische Räterepublik, beschäftigte sich nicht zuletzt in diesem Zusammenhang mit Fragen der Sozialisierung und mit der Kriegswirtschaft und ihren Applikationsmöglichkeiten in Friedenszeiten. Als die Bayrische Räterepublik Anfang Mai I9I9 blutig zerschlagen wurde, kam Neurath in Haft und wurde wegen Hochverrats verurteilt, nach einer Intervention österreichischer Sozialdemokraten allerdings nach Österreich abgeschoben. Im Wien der Zwischenkriegszeit wirkte er in der Volks- und Arbeiterbildung. I924 wurde in Wien das Gesellschaftsund Wirtschaftsmuseum gegründet, dessen Direktor Neurath bis zu dessen Auflösung im Jahr I934 blieb. I934 musste Neurath in die Niederlande flüchten, um einer Verhaftung in Österreich zu entgehen. I940 flüchtete er vor den deutschen Truppen nach England. I945 verstarb er dort im Exil. Wissenschaftstheoretisch ist er dem Logischen Empirismus zuordenbar, als dessen »big locomotive« er von Rudolf Carnap bezeichnet worden ist (Hegselmann I993: I5). In Otto Neuraths Schriften wird Kultur einerseits als Ausdruck der sozialen und politischen Lebensverhältnisse begriffen, andererseits auch als Instrument, diese Lebensverhältnisse neu zu gestalten. Am wahrscheinlich deutlichsten hat er dies in einer I928 veröffentlichten Schrift mit dem Titel Lebensgestaltung und Klassenkampf zum Ausdruck gebracht. In ihr wird die sozialistische Gemeinschaftskultur mit der individua- 
listischen Kultur der bürgerlichen Gesellschaft kontrastiert. Die Entdeckung neuer Lebens-, Ausdrucks- und Kulturformen und die Überwindung der Tradition - auch in geistiger Hinsicht - wird zur Voraussetzung gesellschaftlicher Veränderung. Von den Formen des Wohnens, der Architektur oder Fragen der Lebensreform ausgehend - hier wird etwa der in der Sozialdemokratie populäre Abstinenzgedanke entschieden ventiliert (vgl. Neurath I98Ib) -, diskutiert Neurath die Perspektiven einer neuen Gemeinschaftskultur, die immer in Relation zur sozialen und politischen Ordnung gesehen wird:

„Wer die Wohnung, die Kleidung, die Feste, die Lektüre, das Liebesleben, die Geselligkeit, den Alltag wie den Feiertag, kurzum, das persönliche Leben ernsthaft ändern will, muss die Machtverhältnisse, die gesamte Gesellschaftsordnung ändern wollen« (Neurath 198Ib: 236).

Der Grundgedanke von Otto Neurath, dass Lebensgestaltung und Weltanschauung, dass Kultur als Lebensweise wie auch Kultur als »Überbau«, eng mit der Gesellschafts- und Wirtschaftsordnung verknüpft sind, innerhalb derer sie entstehen - jedoch nicht daraus abgeleitet werden kann - könnte mit einem wesentlich später geprägten Terminus als kultureller Materialismus bezeichnet werden. Neurath schrieb dem Kulturellen gesellschaftsveränderndes Potenzial, ein durchaus antizipierendes Moment und ein konfliktives movens zu. Doch gleichzeitig distanzierte er sich von Bestrebungen der Überreglementierung des Alltags durch die Vorgaben einer proletarischen Lebensreform. In diesem Kontext sind auch seine Bemühungen um eine Popularisierung von Wissenschaft zu sehen. Während Zilsel gegen das Elitäre der Geniereligion anschreibt - und damit gegen die Massenverachtung - rückt Neurath die Lebenswelten dieser Massen ins Zentrum wissenschaftlicher Betrachtung, verbunden mit wissenschaftlicher Aufklärung, für welche die von ihm entwickelte Methode der Bildstatistik beispielhaft stand. Statistische Zusammenhänge sollten visualisiert und in politisch-aufklärerischer Absicht eingesetzt werden - also Zusammenhänge zwischen sozialer Lage und Lebensqualität, zwischen Wohnverhältnissen und Säuglingssterblichkeit etwa. Entgegen einer rezenten Kritik vermittelte die Statistik für Neurath keineswegs ein reduktionistisches Menschenbild, sondern wies, ganz im Gegenteil, einen Weg zum Begreifen, zum Mitfühlen und 
zum Verändern. »Die statistische Denkweise entfernt nicht vom lebendigen Menschen«, führt Neurath aus,

»sie führt zum lebendigen Menschen hin. Sie zeigt, wo der einzelne mitleiden kann, wo er sich mit zu freuen vermag. Sich mit den anderen als eine Gemeinschaft zu fühlen, kann man nur, wenn man lebhaft vor Augen sieht, wie die Gemeinschaft leidet und sich freut» (ebd.: 280).

Das mit Unterstützung der Stadt Wien und der regierenden Sozialdemokratie errichtete Museum für Gesellschaft und Wirtschaft sollte ganz im Dienste dieser Idee stehen (vgl. Mulley I984).

\section{Resümee}

Zusammenfassend lässt sich sagen, dass die für das deutschsprachige Einflussgebiet charakteristische Deutung des Kulturbegriffes, die Georg Bollenbeck als »semantischen Sonderweg « bezeichnet hat, in den Kulturdiskursen des Austromarxismus gebrochen wird. Die Trennung zwischen Ästhetik und Alltag, zwischen den Ebenen des Geistigen, des Emotionalen und Idealistischen einerseits, der materiellen Welt andererseits, wird zwar zum Teil aufgegriffen, gleichzeitig aber in Frage gestellt. Sie wird aufgegriffen in den Vorstellungen einer anzueignenden Bildungskultur und Wissenschaft, in deren bloßer Akkumulation schon politischer und kultureller Fortschritt gesehen wurde. Implizit in Frage gestellt wird sie durch die Verknüpfung dieser Konstruktion von Kultur mit dem Konzept einer Kultur als Lebensweise, in dem die materielle Existenz mit Kulturproduktion und Kulturkonsumtion verbunden wird. Damit war auch für die mit dem Austromarxismus verknüpfte Wissenschaft ein Feld abgesteckt, das äußerst innovative Impulse auf den kulturwissenschaftlichen Diskurs ausüben konnte: Phänomene wie Freizeit und Lebenswelt konnten unter kulturtheoretischen Gesichtspunkten analysiert werden, das Soziale und das Politische waren nicht mehr das Gegenteil des Kulturellen, sondern konnten zusammen gedacht werden. Relationen wurden hergestellt zwischen Kultur und Klasse, zwischen Kultur und politischer Macht, zwischen Kultur und der Spannung zu dem, was nicht Kultur ist. Gerade diese Verzahnungen und damit letztlich die Herausbil- 
dung einer differenzierten Theorie politischer Macht und kultureller Hegemonie lassen erkennen, dass auch die politische Theorie des Austromarxismus nicht nur ideengeschichtlich relevant ist. Kulturtheoretische Fragen standen jedenfalls nicht getrennt von kultureller Praxis, wie insbesondere die Tätigkeiten der Kulturorganisationen und des sozialdemokratischen Bildungswesens zeigten. Hier sind auch die wesentlichen Überschneidungen mit der politisch-kulturellen Formation der birmingham group festzustellen: Kultur wird als (proletarische) Lebensweise, die im Kampf und in der Auseinandersetzung mit konträren Lebensweisen steht, begriffen. Damit geht eine beträchtliche Entgrenzung kulturwissenschaftlicher Thematiken einher, die nun gesellschaftliche Bereiche wie Freizeit und Konsum oder Popularkultur und Massenkultur umfassen. Auch die kulturellen Praktiken von Kulturorganisationen werden somit ins Blickfeld gerückt. Kulturelle Theorie und Praxis werden zu einer »Politik des Kulturellen« konzeptualisiert. Diese Verbindung von Kultur mit Politik und Ökonomie verbindet die Theorie und Praxis der birmingham group und des austromarxistischen clusters. Beide entstehen an jenen sozialen Orten, an denen die akademische Welt mit einem nicht-wissenschaftlichen Publikum zusammentrifft, wo sich alltägliche und wissenschaftliche Praktiken treffen. Die beiden Formationen differieren freilich erheblich in der Reflexion der eigenen Position, in der Bestimmung des Verhältnisses von Wissensproduzenten einerseits und »Wissenskonsumenten « andererseits. Durch die Heterogenität des Austromarxismus als politische Theorie, seine Offenheit gegenüber verschiedenen wissenschaftstheoretischen Positionen, konnte zwar ein weites diskursives Feld betreten werden, das zahlreiche Schnittpunkte mit einer Geschichte der Kulturwissenschaften aufweist. Gleichzeitig aber hatte die austromarxistische Theorie mit einem Widerspruch zu ringen, der nicht ohne Folgen für die Praxis bleiben konnte. Denn weil das aus der Sicht der austromarxistischen Kulturtheoretiker Nicht-Normierbare, nicht eindeutig politisch Instrumentalisierbare, sozusagen Nicht-Steuerbare unter den pauschalen Manipulationsverdacht einer bürgerlichen Unterhaltungsindustrie gestellt wurde, das Populäre oft per se abgelehnt wurde, ohne die für eine solche Ablehnung grundlegenden Kategorien überhaupt nur in Frage zu stellen, hinkten die kulturtheoretischen Vorstellungen oft den Praktiken der arbeitenden Bevölkerung und der sozialdemokratischen Kulturorganisationen hinterher. Dies führte, um in der Terminologie des Austromarxismus 
zu bleiben, zu einer höchst bürgerlichen Kulturbetrachtung, die Mengenverachtung mit einschloss, die Vorstellung einer zu formenden rohen Masse, die nach den Vorstellungen der führenden austromarxistischen Theoretiker zur Kultur erzogen werden musste. Vorstellungen also, die - wie wir gesehen haben - von Persönlichkeiten wie Otto Neurath oder Edgar Zilsel zumindest partiell in Frage gestellt wurden. Dazu mag beigetragen haben, dass - übrigens im Gegensatz zu den Vertretern der birmingham group - die führenden Austromarxisten weniger die von Rolf Lindner analysierte Grenzerfahrung zwischen den Kulturen, der herrschenden und der Arbeiterkultur, gemacht hatten, sondern eine sozialisationsbedingte Kulturerfahrung in ihre politische Arbeit einfließen ließen, in der die Kultur der Massen letztlich etwas zutiefst Fremdes blieb, das nur unter dem Gesichtspunkt seiner notwendigen Umformung wahrgenommen wurde. Die starke Konzentration des Austromarxismus auf Kulturfragen, die ihn als politische Theorie in eine Tradition des so genannten kulturellen oder komplexen Marxismus einordnenbar erscheinen lässt, wurde in zahlreichen Analysen der I970er und 8oer Jahre zur Erklärung seiner politischen Schwäche und seines moralischen Versagens herangezogen. Die Behaglichkeit einer sozialistischen Gegenkultur habe, so heißt es, in der Sozialdemokratie Illusionen über die politischen Machtverhältnisse erzeugt und die Gefahren des aufkommenden Faschismus verdeckt. Die Politisierung der Kultur könnte funktional betrachtet vielmehr eine Kulturalisierung und Pädagogisierung von Politik innerhalb des eigenen Lagers ohne gesamtgesellschaftlichen Veränderungsimpuls gewesen sein (vgl. Negt/Kluge I972), die letztlich zu jener realpolitischen Blindheit beigetragen hätten, die in der Februarniederlage von 1934 münden musste. Gleichzeitig war es aber genau jenes gegenkulturelle Milieu, das die Rahmenbedingungen eines austromarxistischen kulturwissenschaftlichen clusters definierte. So betrachtet könnte es paradoxerweise gerade seine politische Schwäche gewesen sein, die den Austromarxismus als Bestandteil einer Geschichte der Kulturwissenschaften wieder als aktuell erscheinen lässt. 


\section{Literatur}

Adler, Max (1920): Der Kampf der geistigen Arbeiter, Wien: F. Kainz.

Adler, Max (I972 [1923]): Neue Menschen. Gedanken über sozialistische Erziehung, Wien/München: Verlag Jungbrunnen.

Adler, Max (I928a): Über psychologische und ethische »Läuterung« des Marxismus, Berlin: E. Laub.

Adler, Max (I928b): »Praktischer und unpraktischer Klassenkampf«. Der Kampf 21/5, S. I97-206.

Adler, Max (I93I): »Wozu schreibt man Bücher? Melancholische Betrachtung zu einer Buchbesprechung«. Der Kampf 25/3, S. I25I3I.

Albers, Detlev (1983): Versuch über Otto Bauer und Antonio Gramsci. Zur politischen Theorie des Marxismus, Berlin: Argument-Verlag.

Bauer, Otto (1924): Der Kampf um die Macht, Wien.

Bauer, Otto (I928): »Klassenkampf und Ideologie«. Der Kampf 2I/7, S. $28 \mathrm{I}-288$.

Bollenbeck, Georg (I994): Bildung und Kultur. Glanz und Elend eines deutschen Deutungsmusters, Frankfurt a.M.: Insel-Verlag.

Burns, Rob (Hg.) (I995): German Cultural Studies, Oxford: Oxford University-Press.

Frei, Alfred Georg (I99I): Die Arbeiterbewegung und die »Graswurzeln« am Beispiel der Wiener Wohnungspolitik 1919-1934, Wien: Braumüller.

Glaser, Ernst (198I): Im Umfeld des Austromarxismus. Ein Beitrag zur Geistesgeschichte des österreichischen Sozialismus, Wien/München/ Zürich: Europa-Verlag.

Grossberg, Lawrence (I997): »Cultural Studies: What's in a Name? (One More Time)« (I995). In: Ders., Bringing it All Back Home. Essays on Cultural Studies, Durham/London: Duke University Press, S. 245-27I.

Hannak, Jacques (1926): »Die neue Großmacht: Sport. Zum Arbeiter-Turn- und Sportfest in Wien«. Der Kampf I9/7, S. 273-280.

Hegselmann, Rainer (I993): »Otto Neurath, der Wiener Kreis und das Projekt einer empiristischen Aufklärung«. In: Ursula Apitzsch (Hg.), Neurath - Gramsci - Williams, Hamburg: ArgumentVerlag, S. 13-36.

Küenzlen, Gottfried (I997): Der Neue Mensch. Eine Untersuchung zur 
säkularen Religionsgeschichte der Moderne, Frankfurt a.M.: Suhrkamp.

Lehnert, Detlef (I995): »Politisch-kulturelle Integrationsmilieus und Orientierungslager in einer polarisierten Massengesellschaft $\ll$. In: Tálos, Emmerich et al. (Hg.), Handbuch des politischen Systems Österreichs. Erste Republik 1918-1933, Wien: Manz, S. 43I-443.

Leichter, Käthe (1926): »Max Weber als Lehrer und Politiker«. Der Kampf I9/9, S. 374-385.

Lindner, Rolf (2000): Die Stunde der Cultural Studies, Wien: Wiener Universitätsverlag.

Michels, Robert (I987 [I933]): »Historisch-kritische Untersuchungen zum politischen Verhalten der Intellektuellen«. In: Robert Michels, Masse, Führer, Intellektuelle. Politisch-soziologische Aufsätze 1906-1933, Frankfurt a.M./New York: Campus, S. I89-213.

Müller, Karl H. (I996): »Sozialwissenschaftliche Kreativität in der Ersten und in der Zweiten Republik«. Österreichische Zeitschrift für Geschichtswissenschaften 7/I, S. 9-43.

Mulley, Klaus-Dieter (I984): »Demokratisierung durch Visualisierung - Zur Geschichte des Gesellschafts- und Wirtschaftsmuseums in Wien«. In: Helmut Konrad/Wolfgang Maderthaner (Hg.), Neuere Studien zur Arbeitergeschichte, Band III: Beiträge zur Kultur- und Geistesgeschichte, Wien: Europa-Verlag, S. 747-763.

Negt, Oskar/Alexander Kluge (I972): Öffentlichkeit und Erfahrung. Zur Organisationsanalyse von bürgerlicher und proletarischer Öffentlichkeit, Frankfurt a.M.: Suhrkamp.

Neurath, Otto (I981a [1929]): »Wissenschaftliche Weltauffassung«. In: Otto Neurath. Gesammelte philosophische und methodologische Schriften, Band I, hg. von Rudolf Haller und Heiner Rutte, Wien: Hölder-Pichler-Tempsky, S. 345-347.

Neurath, Otto (I98Ib [I929]): »Lebensgestaltung und Klassenkampf«. In: Otto Neurath. Gesammelte philosophische und methodologische Schriften, Band I, hg. von Rudolf Haller und Heiner Rutte, Wien: Hölder-Pichler-Tempsky, S. 228-289.

Pfoser, Alfred (1980): Literatur und Austromarxismus, Wien: Löcker.

Renner, Karl (I928a): »Einige Erfahrungen praktischen Klassenkampfes«. Der Kampf 2I/4, S. I42-I53.

Renner, Karl (1928b): »Ist der Marxismus Ideologie oder Wissenschaft!«. Der Kampf 2I/6, S. 245-256. 
Saage, Richard (1991): Politische Utopien der Neuzeit, Darmstadt: Wissenschaftliche Buchgesellschaft.

Steele, Tom (1997): The Emergence of Cultural Studies. Adult Education, Cultural Politics and the »English « Question, London: Lawrence \& Wishart.

Wagner, Richard (1927): »Welterleben und Kunsterleben«. Der Kampf $20 / 3$, S. $129-138$.

Williams, Raymond (1958): Culture and Society 1780-1950, London: Lawrence \& Wishart.

Williams, Raymond (I972): Gesellschaftstheorie als Begriffsgeschichte. Studien zur historischen Semantik von »Kultur«, München: Rogner \& Bernhard.

Williams, Raymond (I989): »The Future of Cultural Studies«. In: Ders., The Politics of Modernism. Against the New Conformists, London: Verso, S. $\mathrm{I}^{\mathrm{I}-\mathrm{I} 62 .}$

Wright, Handel (1998): »Dare We De-Centre Birmingham? Troubling the >Origin Journal of Cultural Studies I/I, S. 33-56.

Zilsel, Edgar (1990 [1918]): Die Geniereligion. Ein kritischer Versuch über das moderne Persönlichkeitsideal, mit einer historischen Begründung, herausgegeben und eingeleitet von Johann Dvorak, Frankfurt a.M.: Suhrkamp. 


\section{Denken (und Schreiben) in Netzwerken:}

\section{Antonio Gramsci, Walter Benjamin und Antonio Machado}

BIRGIT WAGNER

Transdisziplinäre Fragestellungen sind nicht nur ein Schlagwort, sondern durchaus auch eine Forschungsrealität: und zwar - wenn auch nicht auf gleiche Weise - sowohl in den anglophonen Cultural Studies als auch in den deutschsprachigen Kulturwissenschaften. Sie machen einen wichtigen Teil des innovativen Potenzials dieser Forschungsprojekte aus. Transdisziplinäres Arbeiten erlaubt die Emergenz von neuen Denkobjekten, erlaubt Gegenstandskonstruktionen, für die es bisher keinen Raum im hierarchischen Gebäude der Wissenschaften gab, erlaubt eine Annäherung an komplexe soziale und kulturelle Realitäten, die sich bekanntlich hartnäckig den Klassifikationsschemata der klassischen Disziplinen und Subdisziplinen entziehen. Wer transdisziplinär arbeitet, hat allerdings unvermeidlich schwerwiegende forschungspraktische Probleme: Probleme mit dem Amateurstatus, den man notwendigerweise in den »anderen« Disziplinen einnimmt, denn Professionalität ist eben nur durch Spezialisierung zu haben, Probleme mit der Notwendigkeit, den persönlichen Kontakt zu den Vertretern und Vertreterinnen anderer Disziplinen zu suchen und sich dem manchmal mühsamen und immer zeitaufwendigen Geschäft des interdisziplinären Dialogs auszusetzen. Doch nur, wenn das in den Kulturwissenschaften traditionelle solitäre Arbeiten aufgegeben wird, kann Kreativität als kollektiver Prozess für disziplinenübergreifende Fragestellungen überhaupt freigesetzt werden. In 
der Praxis stehen dem häufig der Narzissmus der Forschenden und der Disziplinierungseffekt von Institutionen und Forschungsförderungsrichtlinien entgegen.

Dies alles ist bekannt. Ich will hier ein anders gelagertes und häufig unterschätztes Problem des transdisziplinären Arbeitens thematisieren: nämlich die Darstellungsproblematik, die Fragen, in welchen Formen, Schreibweisen (écritures) und Medien sich die Arbeitsprozesse abspielen und wie sich formale und mediale Bedingtheiten in die Ergebnisse transdisziplinären Arbeitens einschreiben. In dieser Fragestellung ist gewiss meine eigene disziplinäre Herkunft aus der Literaturwissenschaft erkennbar. Die Fragestellung ist aber, wie ich argumentieren möchte, insofern zentral, als sie sich in der Praxis des transdisziplinären Arbeitens unvermeidlich stellt. Die Lösungen, die für sie gefunden wurden und werden, lassen kulturraumspezifisch verschiedene Muster erkennen. Ein Grund für die zögerliche Rezeption der Cultural Studies im deutschsprachigen Raum mag auch darin zu suchen sein, dass manche Schreibweisen und Darstellungskonventionen, wie sie sich innerhalb der Cultural Studies ausgebildet haben, auf dem europäischen Kontinent als unüblich und »unwissenschaftlich« gelten.

Kulturraumübergreifend bringen die neuen Medien einen technikinduzierten Innovationsschub für die Forschungspraxis. Die kommunikationstechnischen Grundlagen der Gegenwartskulturen haben, in der Sichtweise mancher neuerer Medientheorien, bestimmte Denkstrukturen nicht nur gefördert, sondern in vieler Hinsicht erst produziert. Dies gilt sowohl für die Kommunikationstechnologien im engeren Sinne, wie Internet, E-Mail und CD-ROMs, als auch für die neuen Darstellungsformen, die die Digitalisierung ermöglicht haben: hypertext, chatrooms, workspaces, online-Zeitschriften, homepages und digitale Präsentationstechniken. Diese Kommunikationstechniken und Darstellungsformen haben die gegenwärtige Forschungspraxis der Kulturwissenschaften entscheidend verändert, haben dazu beigetragen, Vernetzungsprozesse zwischen Wissenschaftlern und Wissenschaftlerinnen bedeutend $\mathrm{zu}$ erleichtern, qualitativ $\mathrm{zu}$ verbessern und für Ergebnisse wissenschaftlichen Arbeitens neue Darstellungsmöglichkeiten zu bieten. So haben quantitative Errungenschaften - schnellere Kommunikation, leichterer Zugriff auf internationale Forschungsergebnisse, größere und billigere Speicherplätze - nicht zuletzt eine qualitative Auswirkung auf das transdisziplinäre Arbeiten gehabt. 
Naturgemäß besitzen aber auch diese »neuen« Qualitäten zeitgenössischer Forschungspraxis ihre Geschichte. Und es ist nützlich, diese Geschichte präsent zu machen, präsent zu halten, will man nicht einem deterministisch-reduktiven Verständnis der Beziehung zwischen technischer und kultureller Innovation Vorschub leisten. Einen kleinen archäologischen Beitrag zu dieser Geschichte möchte ich mit den folgenden Bemerkungen zu drei europäischen Denkern, Philosophen und Schriftstellern leisten: Antonio Gramsci, Walter Benjamin und Antonio Machado. Diese drei haben, jeder auf seine Weise, neue Formen des Denkens und Schreibens gesucht und gefunden, weil das traditionelle Instrumentarium wissenschaftlichen und literarischen Schreibens für ihre Schreibprojekte in ihrer vordigitalen Zeit sich als zu eng erwies. Die Lösungen, für die sie sich entschieden haben, sind sowohl forschungspraktisch als auch theoretisch relevant, und dies gerade im Rahmen der Cultural Studies und ihrer produktiven Rezeption und Weiterentwicklung.

\section{Gramsci, Benjamin, Machado: im Kontext der Zwischenkriegszeit und in der Perspektive der Kulturwissenschaften/Cultural Studies}

Gewiss trennt die drei als archäologische Bezugspunkte genannten Autoren auch Vieles: die Verschiedenheit ihrer Herkunftsländer Italien, Deutschland und Spanien, die verschiedenen Sprachen, in denen sie schreiben, ihre sehr unterschiedliche regionale, nationale, politische und religiöse Sozialisation. Trotz des Trennenden lässt sich aber zeigen, in welcher Hinsicht diese drei Denker zum gemeinsamen Erbe sowohl der Cultural Studies als auch der Kulturwissenschaft gehören (oder gehören sollten).

Antonio Gramsci, einer der Gründer der nunmehr bereits historischen italienischen KPI und wichtigster italienischer Philosoph des 20. Jahrhunderts, ist ja in der Tat eine der zentralen nichtanglophonen Bezugsfiguren der Cultural Studies. Die breite Rezeption Gramscis seit den I970er und I980er Jahren wurde durch eine Vielfalt von Gramsci-Readern in Großbritannien und den USA ermöglicht (siehe Bibliografie). Das in den USA von Joseph Buttigieg begonnene Projekt der Gesamtübersetzung der italienischen kritischen Ausgabe der Prison Notebooks ist allerdings unabgeschlossen.

Gesteuert wurde die Rezeption des italienischen Philosophen 
durch ganz bestimmte politische und thematische Affinitäten. So interessierte an Gramsci die »kulturalistische« Wendung des Hegemoniebegriffs, die Möglichkeit, unter dem Stichwort »Hegemonie« kulturelle Praktiken im Rahmen einer Machttheorie zu denken; weiterhin interessierte die Theorie der Zivilgesellschaft als institutioneller Ort, wo der politische Spielraum kulturellen Handelns zu finden ist; schließlich und vor allem die Schriften zur Populärkultur, deren produktives Potenzial Gramsci als einer der ersten thematisiert hat. Stuart Hall ist in diesem Bereich der vielleicht bekannteste Gramsci-Leser. Sein Projekt einer Open University, verbunden mit der Schriftenreihe, die zum Selbststudium gedacht ist, ist von der Konzeption her ein genuin gramscianischer Versuch, den Alltagsverstand (bei Gramsci: senso comune) der Vielen und nicht nur der akademischen Elite zu verändern. ${ }^{\mathrm{I}}$ Das Denken Gramscis hat auch bei vielen anderen Autoren Spuren hinterlassen; stellvertretend seien Ernesto Laclau, Chantal Mouffe, Edward Said, Cornel West, Homi K. Bhabha und Lawrence Grossberg genannt (vgl. Forgacs I993; Wagner 1999).

Im deutschen Sprachraum, wo die Gefängnishefte durch die vorbildliche Edition von Bochmann und Haug neuerdings komplett verfügbar sind, ist Gramsci zwar sowohl von der orthodoxen als auch der heterodoxen Linken gelesen worden, kaum aber von den Vertretern der Kulturwissenschaften. Die neuesten Handbücher zum Thema nennen den Namen Gramsci an keiner einzigen Stelle (vgl. Böhme/ Scherpe I996; Böhme/Matussek/Müller 2000), während ein deutschsprachiges Handbuch zu den Cultural Studies, wie das von Lutter und Reisenleitner (1998), selbstverständlich mehrere Einträge zu Gramsci verzeichnet. Dies hängt wesentlich mit der im Vergleich zu den Cultural Studies geringeren Politisierung der deutschen Kulturwissenschaften zusammen. Nur sporadisch und vergleichsweise viel unverbindlicher haben deutsche Kulturwissenschaftler eine politische Funktion für ihre Schriften reklamiert; der für Gramsci (und seine »fernen« Schüler wie Said oder Hall) selbstverständliche Zusammenhang von Denken und politischer Praxis kann im deutschen Sprachraum immer noch zu dem Vorwurf mangelnder »Wissenschaftlichkeit« führen. Aleida Assmann hat diesen Unterschied zwischen Wissenschaftskulturen kürzlich erst aus ihrer Sicht kommentiert. ${ }^{2}$

Walter Benjamin, dissidentes Mitglied der Frankfurter Schule und einer der frühen Medientheoretiker des 20. Jahrhunderts, ist in der deutschsprachigen Tradition der Kulturwissenschaft zwar nicht omni- 
präsent, aber doch sehr gut vertreten: und zwar hauptsächlich mit seiner Geschichtsphilosophie sowie mit seinen medientheoretischen Texten und seinen Anregungen für eine Kulturgeschichte der Technik. So spielt Benjamin zum Beispiel für die Studien zum historischen Gedächtnis eine wichtige Rolle; daneben existiert natürlich die breite Rezeption der germanistisch-literaturwissenschaftlichen Seite Benjamins (vgl. Garber I987). Was es meines Wissens nicht gibt, ist eine kulturwissenschaftliche Lektüre des Passagen-Werks. Eine solche Lektüre könnte herausarbeiten, auf welche Weise Benjamin die Auffassung von culture as a whole way of life (Raymond Williams) antizipiert hat. Das anspruchsvolle Projekt, eine umfassende Darstellung einer Stadt, der Stadt Paris, und ihrer vielfältigen sozialen, technischen und kulturellen Äußerungsformen im I9. Jahrhundert zu geben, war, wie Benjamin selbst formuliert hat, als »Kommentar zu einer Wirklichkeit« (Benjamin I983: 574), zu einer disziplinär nicht zurechtgeschnittenen und nicht parzellierten Wirklichkeit konzipiert; in ihm kommt ein umfassender Kulturbegriff zum Tragen, der Populärkultur, Lebensstile und technische Veränderungen der Lebenswelt mit einschließt. ${ }^{3}$ Generell lässt sich feststellen, dass der »französische« Benjamin in der deutschsprachigen Rezeption unterrepräsentiert ist.

Auch in den Cultural Studies ist Benjamin ein häufig zitierter Autor: zum Beispiel bei Iain Chambers, Homi K. Bhabha, Trinh T. Minh-ha und Benedict Anderson, aber auch bei vielen anderen. Eine sehr selektive Lektüre und eine gelegentlich etwas willkürliche Integration benjaminscher Gedanken lassen sich allerdings beobachten. Eine integrale kulturwissenschaftliche Lektüre des Passagen-Werks scheint mir auch hier zu fehlen; im Übrigen wurde dieses Textkonvolut erst 1999 durch die Übersetzung von Howard Eiland und Kevin McLaughlin zur Gänze auf Englisch verfügbar.

Antonio Machado schließlich, ein spanischer Lyriker, Essayist und eigenwilliger Denker, ist nur im Kanon der spanischen Literatur vertreten, einem Kanon, der außerhalb der spanischsprachigen Welt nie die geringste Verbindlichkeit erreicht hat. Das äußert sich u.a. in der überaus unvollständigen Übersetzung dieses Autors ins Deutsche und ins Englische. Machado ist meines Wissens bisher auch nie im Zusammenhang mit Cultural Studies und Kulturwissenschaft genannt worden. Warum es trotzdem Sinn macht, ihn in eine Reihe mit Gramsci und Benjamin zu stellen, will ich im Folgenden argumentieren. 
Machado, im Spanien seiner Zeit ein berühmter und geliebter Dichter, ist ein Kind der schmalen linksliberalen Elite seines Landes und hatte in der Zweiten Republik (193I-36) und auch in den Jahren des Spanischen Bürgerkriegs die Funktion einer moralischen Autorität inne. Die Sprechposition einer kritischen öffentlichen Stimme konnte er vorwiegend aufgrund seines philosophisch-essayistischen work in progress unter dem Titel Juan de Mairena einnehmen. Es handelt sich dabei um eine Sammlung von Texten, die aus meiner Sicht ein kulturwissenschaftlicher Gründertext $\mathrm{zu}$ werden verdiente. Entstanden als fortlaufender Zeitkommentar, angesiedelt zwischen Literatur, Journalistik und Philosophie, begleiten diese fragmentarischen Prosatexte die spanische Geschichte von I934 bis I939 und berühren Themen wie die Populärkultur, Religion und populäre Formen des Religiösen, Pädagogik und Didaktik, eine literarische Dekonstruktion des Subjektbegriffs, Politik, Literatur und Literaturkritik, Amerikanisierung des Alltagslebens und schließlich, in den Jahren des Bürgerkriegs, auch Krieg und Ideologie. Wer je Gramscis Gefängnishefte in der Hand gehabt hat, wird sofort das bei aller Verschiedenheit Vergleichbare sehen: den weiten, umfassenden Kulturbegriff, in dem populäre Denk- und Ausdrucksformen ihren Platz finden, und die politische Relevanz des Kulturellen.

\section{Drei Lebensgeschichten, Drei Schreib-Geschichten}

An dieser Stelle müssen nun in aller Kürze einige biografische Markierungen hinzugefügt bzw. in Erinnerung gerufen werden; denn ich will argumentieren, dass der historisch-biografische Kontext der drei Philosophen mutatis mutandis strukturell vergleichbar ist und dass sie aus ihrer vergleichbaren Lebenserfahrung den Anstoß zu einer ganz bestimmten Weise des Schreibens und Denkens erhalten haben. Diese Schreib- und Denkweise nenne ich »Denken (und Schreiben) in Netzwerken«: ein Denken und Schreiben, das sich der Komplexität der sozialen und kulturellen Realität stellt, ein Denken und Schreiben jenseits der Trennlinie, die zwischen literarischem und wissenschaftlichem Schreiben verläuft. Denn angesichts dessen, was sie sagen wollten, angesichts der politischen Situation, in der sie schrieben, und im Rahmen der materialen und medialen Produktionsbedingungen ihres Tuns mussten diese drei Autoren neue Darstellungsformen 
finden. Diese kreative Leistung steht zwar unleugbar unter dem Einfluss des Technologieschubs der zoer und 3oer Jahre des 20. Jahrhunderts, findet aber noch völlig innerhalb der Schriftlichkeit statt und muss mit diesem Medium ihr Auslangen finden.

Es handelt sich um drei intellektuelle Lebensläufe, die in den zoer und zoer Jahren auf unterschiedliche Weise vom Marxismus geprägt worden sind; drei Lebenskurven, die alle durch die reale Macht der europäischen faschistischen Bewegungen eine leidvolle Wendung nehmen und in einem bitteren Tod enden. Nicht nur das jeweilige Schicksal, sondern auch das jeweilige Lebenswerk werden so unter gewaltigem und gewaltsamen äußeren Druck krisenhaft radikalisiert: zu jenen Formen und Texten, für die die drei Autoren für die Nachwelt berühmt wurden.

Walter Benjamin muss I933 aus dem nationalsozialistischen Deutschland emigrieren, zunächst nach Paris, in eine Stadt, die aber selbst kein dauerhafter Zufluchtsort sein konnte. I940 geht die Flucht weiter in den französischen Süden. Sein letzter Weg führt Benjamin bekanntlich über die Pyrenäengrenze von Frankreich nach Port Bou in Spanien, wo er sich im September 1940 im Alter von 48 Jahren in bedrohter Situation das Leben nimmt. In Port Bou, auf spanisch-katalanischem Boden, an der Mittelmeerküste ganz nahe an der Grenze zu Frankreich, wurde er auch begraben.

Wenige Kilometer entfernt, auf der anderen Seite der Grenze, im französischen Teil Kataloniens, liegt das Städtchen Collioure, wo Antonio Machado gestorben ist und auch zu Grabe getragen wurde: und zwar im Februar 1939, drei Wochen nach der mit äußerster Erschöpfung bezahlten Flucht aus dem siegreichen Franco-Spanien. Machado ist 63 Jahre alt geworden. Die Wege Machados und Benjamins, zweier Menschen, die sich in der Realität niemals begegnet sind, kreuzen sich so auf höchst berührende Weise in der symbolischen Geographie der Opfer.

Antonio Gramsci schließlich verbrachte die Jahre von 1926 bis I937 als politischer Häftling in verschiedenen Gefängnisanstalten des faschistischen Italien und hat sein philosophisches Lebenswerk, die Quaderni del carcere (Gefängnishefte), im Gefängnis geschrieben. Gestorben ist er im Alter von 46 Jahren: im April 1937 an den Folgen seiner Haft und der mangelnden medizinischen Betreuung, auch er also ein direktes Opfer politischer Gewalt.

Die Gefängnishefte, das Passagen-Werk und die zwei Teile des Juan 
de Mairena sind jeweils unabgeschlossene Werke, gewaltige TextSteinbrüche, aus denen die Autoren, hätten sie überlebt, für die Publikation erst Texte ausgewählt, sie überarbeitet, in eine Reihenfolge gebracht und weitergeschrieben hätten. So sind sie unfertig geblieben, fragmentarische Monumente der großen europäischen Krise. Sie haben daher ihre späteren Herausgeber vor nicht geringe editorische Probleme gestellt.

Es lässt sich allerdings auch argumentieren, dass das Unfertige Teil dieser Schreibprojekte war: Die Darstellung und reflexive Durchdringung von culture as a whole way of life seien notwendig prozesshaft und könnten nur willkürlich zu einem Abschluss gebracht werden. Unabhängig von dem fremdbestimmten Schlusspunkt, den der Tod jeweils unter diese Werke gesetzt hat, spiegelt sich das Prozesshafte des Denkens in ihrer Darstellungsform, für die ein »Ende« allenfalls eine Konvention des Buchmarktes hätte bedeuten können.

Im Übrigen handelt es sich um Textsammlungen, die programmatisch außerhalb der universitären Welt angesiedelt sind. Ihre Autoren hatten jeweils mit den akademischen Institutionen ihrer Herkunftsländer ambivalente Erfahrungen gemacht: Gramsci hat sein Studium der Sprachwissenschaft in Turin nie abgeschlossen, weil ihm, solange er nicht daran gehindert wurde, seine politische Praxis wichtiger war; Benjamins marginale Position in der deutschen akademischen Welt und das Scheitern seines Habilitationsprojekts an der Universität Frankfurt sind sprichwörtlich geworden; Antonio Machado hat in seinem glanzlosen Lehrer-Dasein in der spanischen Provinz mühsam und jahrelang akademische Prüfungen nachholen müssen, um seine berufliche Existenz zu verbessern. Alle drei hatten aufgrund ihrer Lebenserfahrung keinen Anlass, der universitären Wissensproduktion einen irgendwie privilegierten Status zuzuschreiben. Sie schrieben und argumentierten in dem Bewusstsein, aus der Marginalität zu schreiben und zu argumentieren: Philosophie aus der Gefängniszelle, aus der Emigration, und, vergleichsweise glückhaft, Philosophie, die auf einem Dichterruhm ${ }^{4}$, einem als Dichter erworbenen symbolischen Kapital aufbaut. Die Marginalität ihrer Wissensproduktion hat sich jeweils mit der politischen Krise zugespitzt: Nur Antonio Machado hat durch diese Krise vorübergehend eine neue Öffentlichkeit gewonnen, die Öffentlichkeit des republikanischen Spanien des Bürgerkriegs (eine Öffentlichkeit, die ihn freilich als Symbolfigur der Verliererpartei auch äußerst exponiert hat). Gramsci hatte zunächst 
überhaupt keine Öffentlichkeit und schrieb als Ersatz für politische Tätigkeit in der aufgezwungenen Muße der Gefängniszelle. Benjamin schließlich schrieb unter dem Damokles-Schwert: in einem Exil, das niemals Hafen war, in der vagen, der allervagesten Aussicht auf spätere Publikationsmöglichkeiten.

Es handelt sich also um Schreibprojekte, die die paradoxe Freiheit des Ungeschütztseins ausgenutzt haben, um sich den üblichen Normen zu verweigern: dem akademischen Stil, dem Zwang zur Gattungskohärenz und der Verpflichtung, sich innerhalb der Grenzen einer institutionalisierten Disziplin zu bewegen. Das doppelt Krisenhafte der Lebenssituationen und der mangelnde institutionelle Rahmen sind die Produktionsbedingungen, die den innovativen Schreibprojekten der drei Autoren zugrunde liegen, die sie gewiss nicht »erzeugt« haben, die aber doch ihren Rahmen bilden.

\section{Transdisziplinäres Arbeiten, experimentelle Darstellungsformen}

In einem solchen Rahmen, der von Krise, Mangel und Absenz gebildet wird, entstehen also Werke, die einerseits fragmentarisch und unabgeschlossen, andererseits kulturwissenschaftlich umfassend und transdisziplinär orientiert sind. Es kann nicht verwundern, dass die Autoren dieser Werke mit der Frage der Darstellungsform und den Leistungsmöglichkeiten ihres Mediums, der Schriftlichkeit des Buchs (des Manuskripts), gerungen haben. Sie haben Lösungsmöglichkeiten gefunden, die ich formal zum Teil als handwerkliche Antizipationen digitaler Textverarbeitung bezeichnen möchte, und die zum Teil genuine Erweiterungen literarisch-essayistischer Ausdrucksmöglichkeiten darstellen (und als solche uns auch vor Augen führen können, was Kommunikationstechnologie allein nicht leisten kann).

Beginnen wir mit einem sehr alläglichen Problem der kulturwissenschaftlichen Forschung, nämlich der Notwendigkeit, große Datenmengen zu sammeln, die sich zunächst einmal durch die Heterogeneität ihrer Herkunft und ihrer Inhalte auszeichnen. Es handelt sich bei diesen Daten um Fragmente der Realität, insofern sie nämlich in die Kategorie tatsächlich gemachter Äußerungen (positivités im Sinne der foucaultschen Diskurstheorie) fallen. Solche Daten haben Gramsci und Benjamin vorwiegend in Form von Texten, Textfragmenten, Exzerpten, Zitaten und bibliografischen Angaben gesammelt, Benjamin 
zusätzlich auch als Bildmaterialien. Die Dokumentation ihrer Sammlertätigkeit ist Teil ihres Werks geworden: was nicht nur mit dessen jeweiliger »Unfertigkeit« zusammenhängt, sondern mit der Funktion, die dem einzelnen Fragment zukommt. Denn es gilt, in Benjamins Worten,

„1das Prinzip der Montage in die Geschichte zu übernehmen. Also die großen Konstruktionen aus kleinsten, scharf und schneidend konfektionierten Baugliedern zu errichten. Ja in der Analyse des kleinen Einzelmoments den Kristall des Totalgeschehens zu entdecken« (Benjamin 1983: 575).

Oder an einer anderen Stelle:

"Geschichte schreiben heißt also Geschichte zitieren. Im Begriff des Zitierens liegt aber, dass der jeweilige historische Gegenstand aus seinem Zusammenhang gerissen wird» (Benjamin 1983: 595).

Das ist nun exakt die Vorgangsweise der Montage. Eine derartige Form der Darstellung ist auf eine aktive und kreative Rezeption angewiesen und fördert eine solche.

Eben diese Rezeptionshaltung erfordert auch die Lektüre der Gefängnishefte. Gewiss sind diese weiter ausgearbeitet als das Passagen-Werk, sie enthalten mehr durchlaufende Texte ihres Autors; doch auch sie bestehen $\mathrm{zu}$ einem Gutteil aus bibliografischen Angaben, Zitaten und Zitatkommentierungen. Und auch Gramsci begreift die gewaltige Fülle an Textfragmenten, die er ausgewählt und notiert hat, nicht als Datenschutt, sondern als Sammlung, in der jedem einzelnen Stück sein Wert zukommt. Davon spricht ein oft zitiertes Wort aus dem elften Heft (Introduzione alla filosofia/Einführung in das Studium der Philosophie), wo es darum geht, wie die irreduzibel vielfältige Realität zum Gegenstand des Denkens gemacht werden kann:

„Die Erfahrung, auf der die Philosophie der Praxis beruht, kann nicht schematisiert werden; sie ist die Geschichte selbst in ihrer unendlichen Mannigfaltigkeit und Vielfalt, deren Studium zur Geburt der IPhilologies als wissenschaftlicher Methode für das konkrete Erfassen singulärer Fakten und zur Geburt der Philosophie als allgemeiner Methodik der Geschichte führen kann« (Gramsci 1975: 1428 f.).

Eine solche »Methodik der Geschichte« ist also immer darauf ange- 
wiesen, die singuläre Erfahrung, das einzelne Dokument, die Spur im Gewebe der Realität ernst zu nehmen: Das wäre die - im übertragenen Sinne - »philologische« Qualität dieser Vorgangsweise (vgl. Wagner 1993). Die Autoren des jüngsten Orientierungsbandes zu den deutschsprachigen Kulturwissenschaften haben unlängst erst eine Glosse zu diesem Kerntheorem der Quaderni formuliert und es zu einem Kerntheorem des Projekts Kulturwissenschaft erklärt (wie gesagt, ohne Gramsci zu kennen oder zumindest ohne ihn zu nennen):

1)ede Theorie muss aus dem Material erarbeitet werden. Das ist eine für die Theorie der Kulturwissenschaft gültige Einsichtı (Böhme/Matussek/Müller 2000: 73).

Nimmt man die Forderung, »Theorie« aus dem »Material« zu erarbeiten, ernst, geht es um jene »Passage«, jenen Zwischen-Ort des Denkens und Schreibens, wo die singulären Daten in einen Kontext gestellt und ein Text erstellt wird. Dem singulären Realen steht die textuelle Konstruktion von Bedeutung gegenüber. Letztere entsteht schon, wie Benjamin gezeigt hat, durch die schiere Montage von Fragmenten, explizit wird sie durch den Metatext, der letztlich durchaus auch Benjamins Ziel war. Seine Zielvorstellung beschreibt er einmal als »die ganze Gewalt des [...] sich bietenden Panoramas« (Benjamin 1983: 575), als die Panorama-Perspektive, die sich am Ende des Schreibprozesses herstellen soll. Doch wie kommen wir jeweils vom einen zum anderen? Wie kommen wir von der Präsentation unserer Daten zu unserem Text? Kulturwissenschaftliches Datenmaterial präsentierte sich den beiden Autoren und präsentiert sich uns heute ausnahmslos fragmentarisch, in der Form von Bruchstücken eines phantasmierten Ganzen, das wir beim Schreiben im Kopf zu haben vermeinen. Gäbe es nicht die Dialektik von phantasmiertem Ganzen und Fragment, würde kein Mensch je auch nur ein Zitat auswählen können. Diese Dialektik kann im Übrigen durchaus »in geordneten Bahnen« verlaufen. Wissenschaftsdisziplinen, wie beispielsweise die Geschichtswissenschaften oder die Literaturwissenschaft, haben Methodologien und auch mehr oder minder automatisierte Denkschemata ausgebildet, wie die einzelnen Daten zu Reihen organisiert werden können, die zumindest eine entfernte Ähnlichkeit mit dem vorgestellten Ganzen im Kopf besitzen. Dies sind die Wege des disziplinären, des disziplinierten Denkens: das sich verpflichtet, in den Bahnen der 
freiwilligen Selbstbeschränkung zu verlaufen, auf Um- und Abwege und vor allem: auf gekreuzte und verschlungene Wege zu verzichten.

Was geschieht aber, wenn die Fragmente sich diesen schematischen Ordnungen verweigern, weil sie, nach den Standards der Einzeldisziplinen, irreduzibel heterogen sind? Wenn zum Beispiel Daten aus den Bereichen Glücksspiel, Feuilleton-Roman, Populärmentalität, Theologie und Marxismus in einem Text zu organisieren sind, wie das Gramsci in einem berühmten Fragment getan hat (vgl. Heft I6, \ I: La religione, il lotto e l'oppio della miseria/Die Religion, das Lotto und das Opium der Armen). Oder Daten $z \mathfrak{u} »$ Traumstadt und Traumhaus, Zukunftsträume, anthropologischer Nihilismus, Jung«, wie der Titel einer Abteilung der benjaminschen Aufzeichnungen und Materialien durchaus rätselhaft ankündigt. In solchen Fällen gibt es keine methodologischen Auto-Bahnen zum Phantasma des Ganzen. Die Wege, die man beschreiten kann, sind bedroht durch die Beliebigkeit der Anordnung einerseits und die Unangemessenheit von Ordnungsprinzipien andererseits.

Benjamin glaubte, diese gefahrvolle Passage zwischen Szylla und Charybdis durch die Technik (die Metapher) der Montage bewältigen zu können. Als Technik kannte er sie aus den formalen Experimenten der avantgardistischen Malerei und Literatur sowie aus der jungen Filmtechnik. Was er reflektiert, kann man heute in Begriffen der Intermedialität denken: Er überträgt ein Konstruktionsprinzip von literarischen Texten und Bildern bzw. die durch die Bedingungen der Technik geprägte Verknüpfung von Elementen im Film auf die Praxis des wissenschaftlichen Schreibens. Insofern ist »Montage « auch eine Metapher. Er hat diesen Vorgang bekanntlich mit überaus erhellenden Kommentaren und Metareflexionen begleitet, die man in der Abteilung »Erkenntnistheoretisches, Theorie des Fortschritts « des Passagen-Werks nachlesen kann. Zum Beispiel folgendes, freilich übertreibend-zuspitzendes Fragment: »Methode dieser Arbeit: literarische Montage. Ich habe nichts zu sagen. Nur zu zeigen« (Benjamin I983: 574).

Gramsci nun, der den Montagebegriff an keiner einzigen Stelle erwähnt, der im Vergleich zu Benjamin eine weniger technikorientierte Aufmerksamkeit für Medien entwickelt hat, Gramsci tut dasselbe, ohne es explizit zu machen. Das Verhältnis der Zitate zu den ausformulierten Teilen seiner Texte entspricht durchweg dem benjaminschen Begriff der Montage, und sein produktiver Umgang mit Meta- 
phern macht aus ihnen Konstellation, Bilder, »worin das Gewesene mit dem Jetzt blitzhaft zu einer Konstellation zusammentritt« (Benjamin $1983: 578$ ).

Es lohnt, die Metapher der Konstellation ihrerseits wörtlich zu nehmen. Konstellationen von Gestirnen ziehen über den Nachthimmel und verändern sich für das Auge der menschlichen Beobachter durch ihre Position relativ zu den Himmelskoordinaten und durch das Dazwischentreten der Planeten. Ebenso müssen die Denkkonstellationen der Kulturwissenschaft beschaffen sein: metaphorische und terminologische Gitterpunkte in Netzwerken, die elastisch und entwicklungsfähig sind und deren Bedeutung von dem jeweiligen Kontext, den das Netz durchzieht, mitgeformt wird. Verknüpfungspunkte von Netzwerken, die nach Bedarf sich auflösen und neue Verbindungen eingehen können. Eben diese »radikale Kontextualität« hat Lawrence Grossberg als ein zentrales Merkmal der Cultural Studies bezeichnet: »practicing cultural studies involves constantly redefining it in response [...] to the changing context in which it works « (Grossberg I999: 24) und »cultural studies tends to be strategic with its theoretical commitments so a particular formation is often characterized not only by its theoretical hybridity but by its theoretical instability and fluidity« (ebd.: 30): Konstellationen in Kontexten.

\section{Textuelle Wanderschaften und gedankliche Konstellationen}

Es ist im Übrigen wohl kein Zufall, dass Metaphern der Wanderschaft und der Verknüpfung für die Prozesse des Denkens und Schreibens gerade im Werk von Theoretikerinnen und Theoretikern der Postcolonial Studies wiederkehren, die von einem Migrantenleben geprägt worden sind. Trinh Minh-ha, eine Benjamin-Leserin, verwendet solche Metaphern in ihrem Kontext, dem der allegorischen Erzählung, mit der sie Woman, Native, Other einleitet: »In a remote village« (d.i. irgendwo außerhalb des »Westens«) wird eine Dorfversammlung abgehalten.

"Never does one open the discussion by coming right to the heart of the matter. For the heart of the matter is always somewhere else than it is supposed to be. To allow it to emerge, people approach it indirectly by postponing it until it maturesu (Minh-ha 1989: I). 
In einem Interview, das sie einige Jahre später gegeben hat, übersetzt sie diese kleine Erzählung in eine methodologische Reflexion:

nif one goes directly to an object, if one tries to seize it, one would always somehow lose it. [...] a creative event does not grasp, it does not take possession, it is an excursion« (Minh-ha 1996: 3).

Konstellationen, Wanderschaften, Flanerien: Die digitalen Medien erlauben und ermöglichen uns heute textuelle Streifzüge, sie erlauben ein kreatives Umgehen mit einem langfristigen work in progress. Das Denken und Schreiben in Netzwerken ist für uns durch die Neuen Medien ungeheuer erleichtert worden; denn wie Daten gespeichert und abrufbar gemacht werden und welche Verweissysteme man installieren kann, ist eine zentrale mediale Vorentscheidung für das Schreiben. Im vordigitalen Zeitalter mussten Gramsci, Benjamin und auch Machado gewissermaßen handwerkliche Lösungen für die Probleme finden, die sich mit dem Aufzeichnen und Ordnen von heterogenen Datenmengen stellen. Gramsci wählte die Methode, seine Exzerpte und die Texte, die er dazu schrieb, in verschiedene Hefte zu schreiben. Er führte im Übrigen »thematische« Hefte mit einschlägigen Titeln und »wilde« Hefte, in denen die Aufzeichnungen ohne thematische Fixierung einander folgen. Diese Vorgangsweise ermöglichte ihm die gleichzeitige Arbeit an Fragestellungen, die sich an der Oberfläche höchst unterschiedlich ausnehmen, zum Beispiel an der Geschichte des italienischen Risorgimento einerseits und der politischen Theorie Machiavellis andererseits. Gerade durch dieses gleichzeitige Arbeiten am Disparaten aber entstehen »Konstellationen«: so wie auch die Sternbilder am Nachthimmel keineswegs »Nachbarn« sind, sondern unterschiedlichen Himmelsregionen angehören.

Diese Konstellationen manifestieren sich unter anderem in einem verzweigten und wildwüchsigen System von Verweisen und Rückverweisen. Es lässt sich behaupten, dass die Quaderni so etwas wie einen vordigitalen Hypertext darstellen. Bis zu einem gewissen Grad kann das auch für das Passagen-Werk gelten, obwohl es, wie gesagt, doch eine vorläufigere Arbeitsphase dokumentiert als Gramscis Gefängnishefte. Ein Blick auf Machado kann darüber belehren, dass es hier aber nicht um die im Grunde zufällige Abgeschlossenheit oder Unfertigkeit von Texten geht, sondern um eine innovative Darstellungsform. Der erste Teil des Juan de Mairena ist ja zu des Autors Lebzeiten im Jahr 
I936 erschienen und stellt daher eine autorisierte und abgeschlossene Ausgabe dar. Die Texte des zweiten, von Machado unabgeschlossenen Teils waren zum Großteil als journalistische Einzelpublikationen also doch jeweils als abgeschlossene Texteinheiten - gedruckt worden. Trotzdem präsentiert sich der Juan de Mairena insgesamt als eine Sammlung von Fragmenten, die verschiedenen literarischen Textsorten angehören und scheinbar disparaten Themen gewidmet sind. Die Hauptfigur, der fiktive Rhetorik-Professor Juan de Mairena, hat eine escuela de sabiduría popular (eine Schule für populäre Weisheit) ${ }^{5}$ gegründet, führt Dialoge mit seinen Schülern, äußert sich in Form von Vorträgen, Interviews, Gesprächen und Kommentaren, ja, er äußert sich sogar noch nach seinem fiktiven Tod (unter dem rekurrenten Zwischentitel »Was Juan de Mairena gesagt hätte«). Das genuin Literarische dieser Darstellungsform liegt in der Einführung apokrypher Autoren (Juan de Mairena und sein Lehrer, Abel Martín) sowie im extensiven Gebrauch des sokratischen Dialogs: Beides macht den Juan de Mairena zu einem Höhepunkt essayistischer Prosa. Die SternBilder, zu denen sich die Gedanken und Themen verdichten, bilden auch in diesem Werk Konstellationen, Konstellationen auf der Wanderschaft, wie man es füglich von einem Dichter erwarten darf, der in einem seiner bekanntesten Gedichte geschrieben hat:

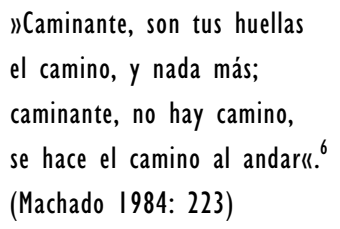

Was im Juan de Mairena zur literarischen Meisterschaft gebracht wird, ist das Prinzip der erweiternden Wiederholung und der wiederholenden Variation: Man kann das ein Verweissystem literarischer Art nennen; es ist eine Schreibweise, die kontextuelles Denken und aktivproduktive Rezeptionshaltungen in hohem Maße fördert. Als Schreibweise ist sie gerade auch für Kulturwissenschaften zu brauchen. Dass das so ist, haben Gramscis und Benjamins nachgelassene Werke bewiesen. Alle drei Denker, die ich als Gründerfiguren und gemeinsame Bezugspunkte für die Kulturwissenschaften und die Cultural Studies betrachte, haben ein Gutteil ihrer Aufmerksamkeit und ihrer Kreativität der Frage der Darstellungsform und den Möglichkeiten 
und Grenzen von Medien gewidmet. Sie haben nicht von Formen und Medien ihr Heil erwartet, doch sie waren sich bewusst, dass Denken und Schreiben nicht immaterielle Prozesse sind, sondern im Austausch mit der Materialität des Medialen vor sich gehen. Sie haben ihr Denken und Schreiben in Netzwerken mit einer anspruchsvollen metatheoretischen Reflexion begleitet. Ihre Ansprüche mögen dabei größer als manche der Resultate gewesen sein, die sie zu ihren Lebenszeiten erzielen konnten: Aber sie haben von ihren Ansprüchen nicht abgelassen. Insofern stellen sie eine schwierige Erbschaft dar für eine Epoche, in der der Eklektizismus den Essayismus und die Beliebigkeit der Zitatauswahl die konstruktive Montage zu verdrängen drohen. ${ }^{7}$

\section{Anmeriungen}

I Als Beispiel möge der von Stuart Hall edierte Band Representation. Cultural Representations and Signifying Practices (1997) gelten. Gramsci spielt darin thematisch eine große Rolle (wie im Übrigen auch Benjamin). Ich sehe seine Präsenz aber vor allem in der Darstellungsform vermittelt: in der großen Sorgfalt, mit der komplexe Theorien und Zusammenhänge in eine allgemein verständliche Sprache gebracht werden, in dem Fortschreiten von Denkmotiven, die eingeführt, später wieder aufgenommen, erweitert und in neue Kontexte gestellt werden, im Verfahren der Montage von Textausschnitten.

2 »While American and British cultural studies redefine culture in such a way as, to provide ways of thinking, strategies for survival, and resources for resistance for the marginalized, German Kulturwissenschaften seem to do the very opposite; they cool rather than ignite, they ward off rather than encourage political action « (Assmann I999: 9I): Ob dies wirklich zureichend mit einer historischen »Impfung « gegen »fatal politicization « zu erklären ist, wie die Autorin kurz andeutet, ist eine Frage, die ich hier offen lassen will.

3 Das Inhaltsverzeichnis der Aufzeichnungen und Materialien listet Phänomene aus folgenden Bereichen auf: Architektur und Innenarchitektur (Passagen, Museum, Straßen, Interieur, altes Paris), Technik (Eisenkonstruktionen, Beleuchtungsarten, Eisenbahnen, Automat) Stadtplanung (Haussmannisierung), Ökonomie (Ausstellungen, Reklame, Börse), Gesellschaft (Flaneur, Langeweile, Prostitution, Spiel, Müßiggang, Konspirationen, soziale Bewegung, die Kommune), Phi- 
losophie (Theorie des Fortschritts, Fourier, Marx), Medien (Panorama, Fotografie, Lithografie), Kunst (Malerei, Literatur) auf. Alle diese Themenbereiche stehen jedoch nicht unverbunden nebeneinander, sondern durchdringen einander selbst in dem fragmentarisch-provisorischen Zustand, den die Aufzeichnungen erreicht haben.

4 Zum besseren Verständnis muss hinzugefügt werden, dass in Spanien die Kunstform Lyrik und mit ihr die Dichter und Dichterinnen traditionell und bis heute ein ungleich höheres Ansehen genießen als im deutschen Sprachraum.

5 Diese escuela de sabiduría popular trägt im Spanischen zwei Bedeutungen: eine Schule für Lernende aus dem Volk und eine Schule für das Wissen, das in den populären Wissensbeständen angesammelt ist. Ein ähnlicher als folklore bezeichneter Komplex findet sich unter demselben Stichwort (!) auch bei Gramsci und eröffnet eine weitere Parallele zwischen den beiden.

6 »Wanderer, deine Spuren sind der Weg, sonst nichts; Wanderer, es gibt keinen Weg: Das Gehen erst bahnt ihn«(Übersetzung B.W.).

7 Dieser Text ist die überarbeitete Version eines Vortrags, den ich im Rahmen einer von Wolfgang Müller-Funk und dem German Department der Universität Birmingham organisierten Sektion der Crossroads Conference on Cultural Studies (Juni 2000) in Birmingham gehalten habe.

\section{Literatur}

Assmann, Aleida (I999): »Cultural Studies and Historical Memories«. In: Bundesministerium für Wissenschaft und Verkehr/Internationales Forschungszentrum Kulturwissenschaften (Hg.), The Contemporary Study of Culture, Wien: Turia + Kant, S. 85-1oo.

Benjamin, Walter (I990): »Über den Begriff der Geschichte«. In: Rolf Tiedemann/Herrmann Schweppenhäuser (Hg.), Gesammelte Schriften Vol. I.2, Frankfurt a.M.: Suhrkamp, S. 69I-706.

Benjamin, Walter (1983): Das Passagen-Werk, 2 Bde., herausg. von Rolf Tiedemann, Frankfurt a.M.: Suhrkamp. Englische Ausgabe: The Arcades Project (I999), (übersetzt von Howard Eiland und Kevin McLaughlin), Cambridge, Mass./London: Belknap Press of Harvard University Press.

Böhme, Hartmut/Scherpe, Klaus R. (I996): Literatur- und Kulturwis- 
senschaften. Positionen, Theorien, Modelle, Reinbek bei Hamburg: Rowohlt.

Böhme, Hartmut/Matussek, Peter/Müller, Lothar (2000): Orientierung Kulturwissenschaft, Was sie kann, was sie will, Reinbek bei Hamburg: Rowohlt.

Forgacs, David (I993): »National-popular: genealogy of a concept«. In: Simon During (Hg.), The Cultural Studies Reader, London/New York: Routledge, S. I77-I92.

Garber, Klaus (I987): »Stationen der Benjamin-Rezeption I940-I985«. In: Ders., Rezeption und Rettung. Drei Studien zu Walter Benjamin, Tübingen: Niemeyer, S. I2I-I94.

Gramsci, Antonio (I975): Quaderni del carcere, 4 Bde., herausg. von Valentino Gerratana, Turin: Einaudi. Deutsche Ausgabe: Gefängnishefte, herausg. von Klaus Bochmann und Wolfgang Fritz Haug, Hamburg: Argument (I99I ff., zehnter und letzter Bd. erscheint 200I). - Englische Ausgabe: Prison Notebooks, herausg. von Joseph Buttigieg, New York: Columbia University Press (I992 ff., bis jetzt 2 Bde. erschienen: Heft I-5). Siehe auch: Quintin Hoare und Geoffrey Nowell-Smith (Hg.) (I97I), Selections from the Prison Notebooks of Antonio Gramsci, London/New York: Lawrence \& Wishart sowie Derek Boothman (Hg.) (I995), Further Selections from the Prison Notebooks, London/New York: Lawrence \& Wishart.

Grossberg, Lawrence (I999): »Globalization and the >Economization< of Cultural Studies«. In: Bundesministerium für Wissenschaft und Verkehr/Internationales Forschungszentrum Kulturwissenschaften (Hg.), The Contemporary Study of Culture, Wien: Turia \& Kant, S. 23-46.

Hall, Stuart (Hg.) (I997): Representation. Cultural Representations and Signifying Practices, London etc.: Sage.

Lutter, Christina/Reisenleitner, Markus (I998): Cultural Studies. Eine Einführung, Wien: Turia \& Kant.

Machado, Antonio (1986): Juan de Mairena, 2 Bde., herausg. v. Antonio Fernández Ferrer, Madrid: Cátedra. Deutsche Ausgabe (ts.): Juan de Mairena. Sentenzen, Späße, Aufzeichnungen und Erinnerungen eines apokryphen Lehrers, Frankfurt a.M: Suhrkamp (I956). Englische Ausgabe (ts.): Juan de Mairena. Epigrams, Maxims, Memoranda, and Memoirs of an Apocryphal Professor, with an appen- 
dix of poems from the Apocryphyl songbooks, Berkeley: University of California Press (1963).

Machado, Antonio (1984): Poesías completas. Prólogo de Manuel Alvar, Madrid: Austral/Espasa Calpe.

Minh-ha, Trinh T. (1989): Woman, Native, Other. Writing Postcoloniality and Feminism. Bloomington/Indianapolis: Indiana University Press.

Minh-ha, Trinh T. (1996): »The undone interval. Trinh T. Minh-ha in conversation with Annamaria Morelli«. In: Iain Chambers/Lidia Curti (Hg.), The Post-colonial Question. Common Skies Divided Horizons, London/New York: Routledge, S. 3-16.

Wagner, Birgit (I993): »Die Methode ist ein Politikum. Thesen zu Gramscis >lebendiger Philologie«. In: Johanna Borek/Birge Krondorfer/Julius Mende (Hg.), Kulturen des Widerstands. Texte zu Antonio Gramsci, Wien: Verlag für Gesellschaftskritik, S. I73I8o.

Wagner, Birgit (I999): »Argomenti di cultura. I >Quaderni< alla luce delle scienze culturali«. In: Rita Medici (Hg.), Gramsci. Il linguaggio della politica, Bologna: Clueb, S. 89-99. 



\title{
Medienkultur als „Nationalkultur» im Wandel:
}

\section{Cultural Studies und die Perspektive einer}

\author{
transkulturellen Medienforschung im
}

\section{deutschsprachigen Raum}

Andreas Hepp

\section{Die Globalisierung von Medienkommunikation und eine transkulturelle Perspertive}

Schaltet man gegenwärtig an einem beliebigen Tag in Deutschland den Fernsehapparat ein und zappt durch die Kanäle, so hat man recht schnell einen Blick dafür, was die Globalisierung von Medienkommunikation lokal bedeutet: Neben scheinbar »eindeutig deutschen« Fernsehsendungen wie Mundart-Theater oder einer Tatort-Sendung mit Lokalkolorit, kann man unter einer Vielzahl von amerikanischen, britischen, französischen, australischen und teilweise auch Sendungen aus anderen kulturellen Kontexten wählen. Die Sportübertragungen, die zu sehen sind, werden bei großen Ereignissen nicht selten aus sehr verschiedenen Ländern der Welt gesendet, und vereinzelt ist zumindest auf ARTE oder 3sat auch ein Beitrag aus einem Land der so genannten Dritten Welt zu sehen. Ein zweiter, etwas detaillierterer Blick - und dies bestätigen wissenschaftliche Untersuchungen (vgl. Hallenberger 200I) - würde aber gleichzeitig zeigen, dass zur »Primetime« zumindest im Bereich der fiktionalen Fernsehsendun- 
gen nach wie vor eine Tendenz zu nationalkulturellen Produkten besteht.

Ein solches fiktives Zapping durch die Kanäle macht deutlich, was mit dem Begriff der Globalisierung von Medienkommunikation gemeint ist: Globalisierung der Medienkommunikation heißt, dass, vermittelt durch die unterschiedlichen elektronischen Medien, die weltweiten kommunikativen Beziehungen zwischen verschiedenen Kulturen und Lokalitäten zugenommen haben. ${ }^{{ }^{2}}$ Dabei findet auf Produktebene in einem bestimmten Rahmen ein wenn auch nicht immer ausgeglichener Transfer statt, ein Transfer, der, wie das Beispiel der Daily Soaps zeigt, teilweise auch nur ein Transfer von Produktideen ist, die im jeweiligen kulturellen Kontext adaptiert und zu »eigenen« Produkten weiterentwickelt werden. Insgesamt hat aber und auch dies macht das fiktive Zapping deutlich - die Globalisierung von Medienkommunikation nicht zu einer gänzlichen Auflösung von Nationalkulturen und lokalen Sinnwelten geführt. Allerdings hat durch sie das Geflecht von Kommunikationsbeziehungen, in denen beide stehen, umfassend zugenommen. Damit sind sowohl Nationalkulturen als auch lokale Sinnwelten einem Wandlungsprozess unterzogen.

Grundlegend könnte man - und dies tut implizit die internationale und interkulturelle Kommunikationsforschung - bezogen auf einzelne Staaten im Hinblick auf die Globalisierung von Medienkommunikation von einer Außen- und einer Binnenorientierung sprechen: Durch die Globalisierung von Medienkommunikation haben sich die Kommunikationsbeziehungen »zwischen Staaten« (z.B. durch Produkttransfers) ebenso verändert, wie die Globalisierung von Medienkommunikation »in den einzelnen Staaten« selbst stattfindet (z. B. durch die Zugänglichkeit von Medienprodukten aus anderen kulturellen Kontexten). Ein differenzierter Blick zeigt aber, dass diese Unterscheidung von Binnen- und Außenorientierung, die die medien- und kommunikationswissenschaftliche Auseinandersetzung mit dem Themenfeld im deutschsprachigen Raum strukturiert hat, mehr und mehr als heuristisches Artefakt erscheint. Auch im nationalen Kontext produzierte Sendungen stehen zunehmend in einem Diskurs des Globalen, indem beispielsweise bei einzelnen Medienprodukten Narrationen und thematisierte Lebensstile als Teil einer (fiktiven) kosmopolitischen (Konsum-)Welt inszeniert werden. ${ }^{2}$ Selbst wenn solche Narrationen und Stilentwürfe wiederum ausgehend von lokalen kultu- 
rellen Kontexten angeeignet werden, so machen solche Überlegungen doch deutlich, dass der Gegensatz von Binnen- und Außenperspektive an Trennschärfe verloren hat. ${ }^{3}$ In den Cultural Studies jedenfalls wird in diesem Kontext in einer Reihe von Arbeiten auf die zunehmende »Deterritorialisierung « von Kulturen und lokalen Sinnwelten hingewiesen. Die gegenwärtigen, mediatisierten Kulturen werden immer schwerer in traditionellen, territorial bezogenen Konzepten der Nationalkultur greifbar.

In meinem Beitrag möchte ich die These aufstellen, dass sich mit der fortschreitenden Globalisierung von Medienkommunikation innerhalb des deutschsprachigen Raums auch die Auseinandersetzung mit Medien geändert hat. In Abgrenzung zur interkulturellen und internationalen Kommunikationsforschung hat sich eine Perspektive entwickelt, die versucht, den Wandlungsprozessen, denen Kulturen ausgesetzt sind, gerecht $\mathrm{zu}$ werden. Diese Perspektive lässt sich als transkulturelle Medienforschung bezeichnen. Auffallend sind dabei insbesondere drei Bezüge, die zwischen transkultureller Medienforschung und Cultural Studies bestehen:

I. In der transkulturellen Medienforschung wird ein Kulturbegriff verwendet, der sich u.a. an die Cultural Studies anlehnt.

2. Ähnlich wie die Vertreterinnen und Vertreter der Cultural Studies ihren Ansatz als transdisziplinäres Projekt verstehen, wird davon ausgegangen, dass eine Auseinandersetzung mit transkultureller Kommunikation auch »offene Grenzen« in der Medien- und Kommunikationswissenschaft erfordert.

3. Diese Auseinandersetzung geschieht innerhalb der transkulturellen Medienforschung in dem Sinne selbstreflexiv, dass thematischer Fokus der Beschäftigung mit der Globalisierung von Medienkommunikation auch der eigene kulturelle Kontext ist.

Für diese Beziehungen gibt es zumindest zwei Gründe, wobei der erstere eher auf »phänomenologischer Ebene« zu sehen ist, der zweite auf der Ebene direkter Bezugnahme. Erstens befinden sich im Kontext der bisher skizzierten Prozesse der Globalisierung von Medienkommunikation auch die Nationalkulturen im deutschsprachigen Raum im Wandel. Dieser Sachverhalt fordert geradezu eine selbstreflexive Auseinandersetzung ein. Ein zweiter Erklärungsansatz ist, dass innerhalb der transkulturellen Medienforschung - und hier auch vermittelt 
durch die dort verhandelten Themen - selbst auf Autorinnen und Autoren der Cultural Studies referiert wird. Dies hat letztlich ein vielfältiges Beziehungsgeflecht zwischen transkultureller Medienforschung und Cultural Studies entstehen lassen.

Im Weiteren möchte ich dieses Beziehungsgeflecht näher betrachten. Dazu erscheint es mir zuerst notwendig, die Tradition der interkulturellen und internationalen Kommunikationsforschung aus Perspektive der Cultural Studies kritisch zu skizzieren, um das spezifisch Neue der transkulturellen Medienforschung im deutschsprachigen Raum deutlich zu machen. Ausgehend hiervon soll die Perspektive der transkulturellen Medienforschung im Hinblick auf zwei der dort primär behandelten Themen - kulturelle Identität und Minderheiten - näher betrachtet und dabei Bezüge zu den Cultural Studies herausgearbeitet werden. Abschließend möchte ich dann aus Sicht der Cultural Studies Differenzen zur transkulturellen Medienforschung deutlich machen - um zu argumentieren, dass sich mit der transkulturellen Medienforschung neue Möglichkeiten für Cultural Studies im deutschsprachigen Raum ergeben. Vor diesem Hintergrund geht es mir auch um die Frage, was es bedeutet, Cultural Studies zu betreiben, wenn »Kultur « in den verschiedensten Bereichen der Medienforschung zunehmend »Konjunktur« hat. All dies geschieht letztlich vor dem Hintergrund einer »phänomenologischen Folie« kultureller Wandlungsprozesse, die Vorstellungen von homogenen und territorial bezogenen Nationalkulturen immer mehr als fragwürdig erscheinen lassen. Sicherlich bergen solche umfassenden Darstellungen die Gefahr, dass man eine dominante und nicht unproblematische »Geschichte« konstruiert, eine Gefahr, vor der auch meine weitere Argumentation nicht gefeit ist. Entsprechend gehe ich auch nicht davon aus, alle Entwicklungslinien der deutschsprachigen medien- und kommunikationswissenschaftlichen Beschäftigung mit der Thematik der »Medien im globalen Kontext« behandelt zu haben. Insgesamt aber werden im Weiteren diejenigen diskutiert, die notwendig sind, um das Spannungsverhältnis zwischen Cultural Studies und transkultureller Medienforschung zu fassen. 


\section{Interkulturelle Und internationale Kommunikation als Tradition: Von Entwicklungsländern zur Weltgesellschaft}

Die in der deutschsprachigen Tradition der wissenschaftlichen Auseinandersetzung mit der Globalisierung von Medienkommunikation dominierenden Theorien sind sicherlich die der interkulturellen und internationalen Kommunikation. Eine aus Sicht der Cultural Studies grundsätzlich zu kritisierende Tendenz dieser Tradition wird schon an der Unterscheidung der beiden Begriffe interkulturelle und internationale Kommunikation durch Gerhard Maletzke - einem der Begründer der deutschsprachigen Forschung in diesem Bereich - deutlich. Maletzke weist in seinem Aufsatz »Interkulturelle Kommunikation und Publizistikwissenschaft« bereits I966 darauf hin, dass Fragen der Interkulturalität zu einem zunehmend für die Medien- und Kommunikationsforschung relevanten Bereich zählen, den es entsprechend wahrzunehmen und zu theoretisieren gilt. Er grenzt dabei interkulturelle und internationale Kommunikation wie folgt voneinander ab: Internationale Kommunikation ist für ihn im Anschluss an die amerikanische Diskussion die Kommunikation zwischen Ländern, also über Staatsgrenzen hinweg, interkulturelle Kommunikation dagegen ganz allgemein der Prozess der Gedanken- und Bedeutungsvermittlung zwischen einzelnen Menschen oder Gruppen, die verschiedenen Kulturen angehören (vgl. Maletzke I966: 319). ${ }^{4}$

In der von mir in der Einleitung eingeführten Unterscheidung kann man davon sprechen, dass internationale Kommunikation bezogen auf einzelne (National-)Staaten interkulturelle Kommunikation ausschließlich in einer Außenorientierung fasst, interkulturelle Kommunikation den entsprechenden Gegenstandsbereich sowohl außen- als auch binnenorientiert. Das Problem, das Maletzkes Ansatz dabei von Anbeginn hat, ist, dass er durch ein von westlichen Vorstellungen dominiertes Denken geprägt ist: Zwar gesteht Maletzke die prinzipielle Möglichkeit des Bestehens von Subkulturen ein bzw. nimmt die Existenz von Minderheiten innerhalb von einzelnen Staaten zur Kenntnis, implizite Richtschnur ist für ihn jedoch die in sich homogene Nationalkultur, die sich territorial mit den jeweiligen Grenzen eines Staates deckt - nur so macht die von ihm vollzogene Unterscheidung von internationaler und interkultureller Kommunikation überhaupt Sinn. Letztlich handelt es sich hierbei aber um eine zuerst einmal europäische Konstruktion, die historisch durch politisch bzw. 
kulturell dominierende Machtblöcke in Gesellschaften erreicht wurde.

Auch wenn ein solches Konzept von Nationalkultur jenseits seiner Ideologie historisch gesehen auch für kein europäisches Land vollkommen stichhaltig gewesen ist, so sind es solche westlich geprägten Vorstellungen, die die medien- und kommunikationswissenschaftliche Auseinandersetzung mit Medien im globalen Kontext in ihrer Anfangszeit prägen, trotz aller Bekenntnisse zu einem Kulturrelativismus. Dies zeigt die sich an den Aufruf Maletzkes zu einer »interkulturellen Publizistik « anschließende, aber nicht unbedingt auf ihn berufende Tradition der interkulturellen und internationalen Medienforschung. Dabei sind vor allem zwei Entwicklungsphasen auszumachen, in denen interkulturelle bzw. internationale Kommunikation jeweils unterschiedlich theoretisiert werden. Dies ist erstens eine insbesondere auf Fragen der Modernisierung und Dependenz von Entwicklungsländern fokussierte Phase, die bis Ende der i98oer Jahre andauert, und zweitens eine durch Systemtheorie und Funktionalismus dominierte Phase, in der der Fokus der Betrachtung die Kommunikation in der »Weltgesellschaft $\ll$ ist.

Modernisierungs- und Dependenztheorie bezeichnen zwei international einflussreiche sozialwissenschaftliche Konzepte der Betrachtung internationaler Beziehungen im Allgemeinen bzw. internationaler (Medien-)Kommunikation im Speziellen. ${ }^{5}$ Ausgehend von einer Zurkenntnisnahme der internationalen Forschung war die Beschäftigung mit interkultureller bzw. internationaler Kommunikation in den I970er Jahren im deutschsprachigen Raum anfangs eher vereinzelt, nahm allerdings mit Ende der 7oer und Anfang der 8oer Jahre zu. Der Grund hierfür ist insbesondere darin zu sehen, dass mit Verbreitung der Satellitenübertragung und den Anfängen der computervermittelten Kommunikation Fragen der Globalisierung von Medienkommunikation drängender wurden, was sich auch darin manifestierte, dass die UNESCO ihren Augenmerk auf eine Weltkommunikationsordnung legte.

Im Kontext dieser Diskussion ist das Sonderheft der Zeitschrift Publizistik zu sehen, das sich 1984 intensiv mit dieser Thematik auseinander setzt und als charakteristisch für die deutschsprachige Diskussion in diesem Zeitraum gelten kann. Im Gegensatz zu der dezidiert auf Fragen von Kultur fokussierten Herangehensweise Maletzkes spielen in den meisten Artikeln - wie auch in einem Überblick zur 
Lage der internationalen Medienforschung in den USA (vgl. Hardt I984) - kulturtheoretische Überlegungen eine nur untergeordnete Rolle. Ausnahmen stellen hier allenfalls die Artikel von Holger Rust und Gernot Wersig dar, in denen eine kulturtheoretische Reflexion der Thematik von Medien im globalen Kontext eingefordert wird. Rust wünscht sich eine »ethnologische Fundierung der Kommunikationswissenschaft« (Rust I984: 384) als Bezugspunkt für eine interkulturelle Beschäftigung mit Medienkommunikation - sowohl in der Außenorientierung des internationalen Rahmens als auch in der Binnenorientierung von multikulturellen Gesellschaften. Dabei bleibt er allerdings modernisierungstheoretischen Vorstellungen treu. ${ }^{6}$ Gernot Wersig fordert im Hinblick auf eine zunehmende Ausbreitung der »Informationsgesellschaft« als notwendiges Komplement eine »Informationskultur «, mit der er im Rahmen einer normativen Betrachtung die Stabilisierung von Lebenswelt sowie den Schutz vor umfassender Informatisierung und technischer Rationalität verbindet (vgl. Wersig I984: 392), jedoch ohne den »europäischen Blick« auf das westliche Konzept der Informationsgesellschaft zu überwinden.? Den sicherlich bemerkenswertesten Ansatz für eine kulturtheoretische Fundierung der Betrachtung von internationaler Kommunikation legt in diesem Zeitraum aber Horst Reimann vor, wenn auch in einem gänzlich anderen Publikationskontext. ${ }^{8}$ Ausgehend von kultursoziologischen Ansätzen vertritt Reimann die These, dass die Aneignung kommunikativer Technologien in verschiedenen Kulturen unterschiedlich verläuft und die Annahme einer durch die Globalisierung von Medienkommunikation vermittelten Homogenisierung in Richtung einer globalen »Einheitsgesellschaft « oder »Welt-Öffentlichkeit« (Reimann I983: 275) nicht haltbar ist. Insgesamt sind seine Darlegungen aber auch für ihn eine Skizze, bei der »viele Fragen offen « (Reimann 1983: 277) bleiben - aus Perspektive der Cultural Studies vor allem die Frage, wie er den konfliktären Zusammenhang von kulturellem Wandel und Globalisierung von Medienkommunikation theoretisieren will. ${ }^{9}$

Diese sicherlich nicht vollständige, in ihren Grundlinien aber exemplarische Übersicht lässt zweierlei erkennen: Erstens wird eine umfassende Theoretisierung der Thematik von internationaler bzw. interkultureller Kommunikation aus kulturtheoretischer Perspektive zwar vereinzelt eingefordert, aber nur ansatzweise geleistet. Zweitens bleibt der Fokus in diesem Themenbereich tendenziell auf außen- 
orientierte Fragen der medienvermittelten Kommunikationsbeziehungen zur Dritten Welt bzw. Entwicklungsländern gerichtet, unabhängig davon, ob man eher modernisierungs- oder dependenztheoretischen Überlegungen anhängt bzw., wie Horst Reimann vermittelt, über die Kultursoziologie eine Betrachtungsperspektive »zwischen« beiden Paradigmen wählt. ${ }^{\text {Io }}$

Ein solcher Fokus der internationalen bzw. interkulturellen Kommunikationsforschung auf die Beziehung zwischen erster und dritter Welt ist mit einem Diskurs verbunden, den Stuart Hall als den Diskurs vom »Westen und dem Rest« bezeichnet hat. In dem Aufsatz »Der Westen und der Rest« setzt sich Stuart Hall damit auseinander, welche Rolle die Gesellschaften außerhalb Europas bei der Konstitution dessen haben, was als (westliche) »Modernität« bezeichnet wird. Er arbeitet hier heraus, dass die Formierung der heutigen Vorstellung von »westlicher Modernität« mittels eines diskursiven Musters von »dem Westen und dem Rest« geschehen ist: Grundlegend beruht die diskursive Konstruktion »westlicher Modernität« auf der Abgrenzung gegenüber dem »Anderen«. Durch diskursive Strategien »wird der Rest als etwas definiert, das der Westen nicht ist - sein Spiegelbild. Er wird als das absolute, wesenhafte, verschiedene Andere repräsentiert« (Hall I994: I67, Hervorhebung im Original). Dieses Andere wird wiederum binär gefasst auf der Basis von Dichotomien wie Westen: Rest, zivilisiert: unreif, entwickelt: zurückgeblieben; unedel: edel. Genau dieses Muster vom »Westen und dem Rest« lässt sich auch in den frühen Arbeiten in der Tradition der interkulturellen und internationalen Kommunikation ausmachen: Während modernisierungstheoretische Konzepte offensichtlich mit einer Vorstellung operieren, man müsse (zurückgebliebene) Länder der Dritten Welt nach dem Modell des Westens entwickeln - Denkrahmen ist hier die Dichotomie entwickelt: zurückgeblieben -, wird in dependenztheoretischen Überlegungen eine (kommunikative) Loslösung der dritten von der ersten Welt gefordert - ein Denken, das insofern ebenfalls durch den Diskurs vom »Westen und dem Rest« geprägt ist, als hier das romantisierende Bild einer Kultur evoziert wird, deren Lebenswirklichkeit (fast) paradiesisch war, bis der kolonialisierende Westen in sie eingebrochen ist (vgl. Ang/Stratton I996; Ang 200I). Die Vorstellung des »edlen Wilden« lässt grüßen.

Als erstes Zeichen für eine Öffnung der auf die »restlichen Länder« und ihre Beziehung zum Westen fokussierten Auseinanderset- 
zung kann man das Symposium »Internationalisierung von Medienangelegenheiten « im Juni I990 ${ }^{\text {II }}$ begreifen. Dort zeichnete sich als ein weiterer wichtiger Bezugspunkt der Diskussion die mit der Einführung des dualen Systems in den I980er Jahren zunehmende Kommerzialisierung der Medienlandschaft im europäischen Kontext ab. In verschiedenen Beiträgen wurde eine Internationalisierung der Medienmärkte konstatiert, und hierbei wurden insbesondere die Fragen diskutiert, inwieweit damit eine Entwicklung zur Transnationalität von Medienproduktion einhergehe oder einhergehen solle bzw. ob dies zur Veränderung von europäischen Nationalkulturen selbst beitragen könne (vgl. Kiefer I990; Langenbucher I990; Ronneberger I990). ${ }^{\text {I2 }}$ Was interessierte war nicht mehr nur die Kommunikationsbeziehung zu so genannten Entwicklungsländern, sondern verstärkt auch die interkulturelle Kommunikation innerhalb Europas, motiviert durch die auch normativ aufgeladene Frage, ob (europäische) Medien bei der Konstitution einer geteilten »europäischen Identität der Vielheit « einen Beitrag leisten könnten. ${ }^{\text {I3 }}$

Während der Außenfokus der interkulturellen bzw. internationalen Medienforschung sich so von Entwicklungsländern weg hin zum europäischen Ausland bewegt, gewinnt auch der Binnenfokus in der Diskussion an Stellenwert: Hierfür stehen Ende der ig8oer, Anfang der 9oer Jahre eine Reihe von Inhaltsanalysen, die sich beispielsweise mit der Darstellung von Fremden, Ausländern und Migranten in den deutschsprachigen Medien befassen. ${ }^{\text {I4 }}$ Während solche Ansätze zumindest perspektivisch in die Richtung einer transkulturellen Medienforschung weisen, so wurde Mitte der I99oer Jahre im Rahmen der Systemtheorie ein weiterer Versuch der umfassenden, primär außenorientierten theoretischen Fundierung der internationalen Kommunikationsforschung unternommen. Dieser Fokus dominiert insbesondere in dem von Miriam Meckel und Markus Kriener herausgegebenen Band Internationale Kommunikation. Die Herausgeber verstehen diese Publikation explizit als »eine aktuelle Überblicksdarstellung [...], die wesentliche Themensegmente der Internationalen Kommunikation beleuchtet« und damit eine »thematische Lücke« im deutschen Sprachraum füllt (Meckel/Kriener I996, Vorwort). Es sind aus systemtheoretischer Perspektive vor allem zwei Punkte, in Bezug auf die die vorhergehende internationale Kommunikationsforschung kritisch gesehen wird, nämlich erstens, dass bisherige Ansätze davon ausgingen, Medien würden eindimensional und wirkmächtig Infor- 
mationen transferieren, könnten also direkt das Bewusstsein ihrer Nutzerinnen und Nutzer beeinflussen (Transfer-Annahme). Zweitens wurde von einer (politischen) Steuerbarkeit der Medien als Institutionen ausgegangen, die insbesondere bezogen auf transnational agierende Unternehmen nicht zutreffend ist (Steuerbarkeits-Annahme). Ins Zentrum der systemtheoretischen Überlegungen $\mathrm{zu}$ internationaler Kommunikation wird in Anschluss an Niklas Luhmann der Begriff der »Weltgesellschaft« gerückt (vgl. Görke/Kollbeck I996: 263 und Kriener/Meckel ı996: I4). Der Ausdruck »Weltgesellschaft« verweist - ausgehend von der systemtheoretischen Vorstellung, dass sich soziale Systeme auf Kommunikation gründen - auf die Ausdehnung von Gesellschaft (verstanden als die Gesamtheit der füreinander erreichbaren Kommunikationen) über nationale und regionale Beschränkungen hinaus. Weltgesellschaft setzt entsprechend die globale Verbreitung von Kommunikationen voraus, ergibt sich in der Theorie also durch die Globalisierung von Medienkommunikation. ${ }^{\text {I5 }}$

Wie ist nun internationale Kommunikation in diesem Begriffsrahmen zu fassen? Was leistet er für die Theoretisierung der Auseinandersetzung mit Medien im globalen Kontext? Stellt man sich diese beiden Fragen, so fällt zuallererst einmal die weitgehende Eliminierung des Begriffes »Kultur« auf. ${ }^{\mathrm{I} 6}$ Der Zugang zu diesem Themenfeld geschieht nicht aus der Perspektive einzelner Kulturen, sondern aus der Perspektive der Funktion und Leistung von Medien, die als geschlossenes System begriffen werden (vgl. Görke/Kollbeck I996: 266). Dies heißt, dass man von einer selbstreferentiellen Operationsweise des Mediensystems ausgeht, also von der Vorstellung, dass Medien(institutionen) nach ihren systeminternen Regeln operieren, um ihren eigenen Fortbestand zu sichern. Aus diesem Verständnis von Medien folgern Görke und Kollbeck bezogen auf internationale Kommunikation zweierlei: Erstens sind Medien systemextern nicht steuerbar, weswegen der Funktion, die die Medien für die (Welt-)Gesellschaft haben, enge Grenzen gesetzt sind. Zweitens »bilden« Medien Ereignisse des Weltgeschehens nicht einfach »ab«, sondern sie konstruieren ihre Sicht als Beobachter auf die Welt. Diese Konstruktion ist notwendig selektiv, kann also grundlegend als Konstruktion nicht gewertet werden.

Dieser hier skizzierte, systemtheoretische Zugang zu internationaler Kommunikation stellt sicherlich unter gewissen Gesichtspunkten einen Fortschritt gegenüber frühen Ansätzen in diesem Themenfeld 
dar. So bricht er durch das Konstrukt der zuerst einmal wertneutral gefassten Weltgesellschaft mit einer (eurozentrischen) Gleichsetzung der internationalen Kommunikation mit Kommunikationsbeziehungen gegenüber Entwicklungsländern. Dabei wird Abschied von geradlinigen Modernisierungsvorstellungen genommen, indem - statt von kulturellen Transfervorstellungen auszugehen und dabei die Dichotomie vom »Westen und dem Rest « implizit zu übernehmen - einzelne Nationen als gleichberechtigt konzeptionalisiert werden und die Kommunikation »zwischen ihnen « betrachtet wird. ${ }^{\mathrm{I7}}$ Ebenso wichtig erscheint, dass hier eine theoretische Fundierung der Thematik unternommen wird, die in ihrem Anti-Essentialismus klar über frühere Theoretisierungen hinausgeht: Soziale Systeme werden nicht als gegeben aufgefasst, sondern als in einem Kommunikationsprozess konstruiert.

In diesem Anti-Essentialismus könnte möglicherweise eine Beziehung zu den Cultural Studies gesehen werden, ${ }^{\mathrm{I} 8}$ von denen sich die Systemtheorie aber u.a. durch eine »Blindheit gegenüber sozialen und kulturellen Identitäten « (Stäheli 2000: 324) unterscheidet. ${ }^{\text {I9 }}$ Nicht zuletzt dies trägt dazu bei, dass ein ausschließlich funktionalistisch-systemtheoretischer Zugang zu internationaler Kommunikation aus Perspektive der Cultural Studies einen unbefriedigenden Eindruck hinterlässt. Zwar erkennt er auf einer ersten Ebene Differenz an Differenz, die auch begründet, warum Medien innerhalb von internationaler Kommunikation keine umfassende Integrationsinstanz darstellen können -, jedoch wird Differenz in dieser Perspektive als Systemdifferenz einer Weltgesellschaft konstruiert und damit auf einer zweiten Ebene wiederum funktional integriert: Was zählt ist die Frage, wie einzelne Systeme auch in Bezug auf internationale Kommunikation »strukturell gekoppelt « sind, und dies gilt es analytisch zu beschreiben. Die Blindheit gegenüber differenten kulturellen Identitäten, die »quer« zu einzelnen Funktionssystemenen liegen, durch die Individuen unterschiedlicher kultureller Kontexte aber beispielsweise keinen gleichen Zugang $\mathrm{zu}$ unterschiedlichen Funktionssystemen einer Weltgesellschaft haben, führt zu einer unangemessenen Fokussierung auf Stabilität und Integration, wenn auch auf der Ebene des Horizonts einer Weltgesellschaft.

Genau in diesem »Funktionalismus der Weltgesellschaft« erscheinen die skizzierten systemtheoretischen Überlegungen aus Perspektive der Cultural Studies als unangemessen für eine Betrachtung 
der Globalisierung von Medienkommunikation. Problematisch an einer solchen Fokussierung auf Stabilität und Systemintegration erscheint zweierlei, das ich an anderer Stelle als Konsensfiktion kritisiert habe (vgl. Hepp 2000a): Erstens wird durch eine solche funktionale Betrachtung die Widersprüchlichkeit und Unsicherheit von Medienkommunikation unter den Bedingungen einer kapitalistischen Postmoderne zwar nicht übergangen, indem man die prinzipielle Unwahrscheinlichkeit von Verstehen betont; die sich hieraus ergebenden Probleme von Missverstehen werden aber in Fragen der Beobachterperspektive aufgelöst und nicht in Bezug auf kulturelle Differenzen theoretisiert (vgl. Ang I996: I64). Zweitens geht mit dieser Perspektive eine weitergehende Marginalisierung von Fragen der Macht, des Konflikts und der Auseinandersetzung einher. Der Grund hierfür ist, dass die als widerspruchslos konstruierte Funktion und Leistung von Medien auf einzelstaatlicher Ebene unproblematisiert auf die internationale Ebene übertragen werden. ${ }^{20}$

Eine kritische Auseinandersetzung mit der Globalisierung von Medienkommunikation und dem damit einhergehenden Wandel von (National-)Kulturen muss aber neue Fragen in den Mittelpunkt rücken und kann nicht traditionelle Theoriekonzepte unhinterfragt auf den aktuellen Kontext übertragen. Lassen sich Vorstellungen von geschlossenen und damit »reinen« Systemen vor dem Hintergrund der mit der Globalisierung verbundenen Hybridisierungsprozesse überhaupt aufrechterhalten? Ist die homogen angenommene Nation bzw. Nationalkultur und die damit verbundene politische Größe des Nationalstaates überhaupt noch die primär relevante Bezugsgröße der Beschäftigung mit der Globalisierung von Medienkommunikation? Wie genau sind Prozesse der kulturellen Produktion von Medieninhalten und ihre globale Distribution zu fassen? Wie hängen damit Fragen der Dominanz und Hegemonie zusammen? Und stellt man gegen die Strategien der Medienproduktion die eher taktische Aneignung der Rezipierenden, welche Räume für eigene Bedeutungsproduktion bestehen hier? Welche Stellung haben bei der Medienaneignung häusliche Welt und Lokalität, aber auch Geschlecht, Ethnie und kulturelle Identität?

Es sind genau solche Fragen, die mit der Globalisierung von Medienkommunikation drängend geworden sind und $\mathrm{zu}$ denen die systemtheoretische, internationale Kommunikationsforschung bisher 
keinen befriedigenden Zugang gefunden hat. Vor dem Hintergrund verwundert es nicht, dass sich der systemtheoretische Ansatz nicht zu der von Miriam Meckel und Markus Kriener erhofften Theoriebasis der Beschäftigung mit der Globalisierung von Medienkommunikation entwickelt hat. Dies zeigen auch gegenwärtige, traditionell medienund kommunikationswissenschaftliche Publikationen in diesem Bereich, die sich insbesondere durch eine detaillierte Auseinandersetzung mit Einzelaspekten interkultureller Kommunikation auszeichnen und in denen Binnen- und Außenorientierung interkultureller Kommunikation gleichberechtigt Gegenstand sind. ${ }^{2 \mathrm{I}}$ Der Bereich der Medien- und Kommunikationsforschung, der sich aktuell am umfassendsten den oben genannten Fragen öffnet, ist aber ein anderer, nämlich der der transkulturellen Medienforschung.

\section{Transkulturelle Medienforschung als Perspertive: Kulturelle Identität und Minderheiten}

Mit fortschreitender Globalisierung hat ein soziokultureller Wandel eingesetzt, der innerhalb der Cultural Studies und der britischen Kulturtheorie zunehmend mit dem Konzept der Deterritorialisierung gefasst wird (vgl. García Canclini I995; Tomlinson 1999). Deterritorialisierung kann man in Anlehnung an Nestor García Canclini als »the loss of the snatural relation of culture to geographical and social territories« (García Canclini 1995: 229) definieren. Kultur erscheint also durch die mit der Globalisierung von Medienkommunikation zunehmenden, kommunikativen Beziehungen (kommunikative Deterritorialisierung), aber auch durch Migrationsbewegungen (physische Deterritorialisierung) immer weniger als etwas, das man als eine auf einen Staat fokussierte, homogene Nationalkultur territorial fassen kann. ${ }^{22}$ Dies lässt sich insbesondere auf der Ebene der kommunikativen Deterritorialisierung festmachen, und zwar an der mit der Verbreitung von Kommunikationstechnologien verbundenen Öffnung der häuslichen Welt zum Globalen. So stellt beispielsweise John Tomlinson bezogen auf Fernsehen, Radio und computervermittelte Kommunikation in Anlehnung an die Überlegungen von David Morley fest: 
"These communication technologies are the most striking ways in which modern houses become settings for distanciated relations. Though relations of presence may still dominate here, increasingly homes are open to the world: our sitting rooms places iwhere the global meets the localıu (Tomlinson 1999: $53 \mathrm{f}$.).

Dass physische und kommunikative Deterritorialisierungstendenzen auch im deutschsprachigen Raum auszumachen sind, manifestiert sich u.a. in den Themen, die zunehmend auch die medien- und kommunikationswissenschaftliche Auseinandersetzung prägen: Die Beziehung zwischen Medien und Minderheiten erfährt hier ebenso ein gesteigertes Interesse wie die Beziehung zwischen Medien und kulturellen Identitäten. Die Orientierung, die bei der Beschäftigung mit diesen beiden Themen besonders heraussticht, ist, wie gesagt, die Perspektive, die ich transkulturelle Medienforschung nennen möchte. $^{23}$

Transkulturalität wird hier ausgehend von den Überlegungen von Wolfgang Welsch gefasst. Dieser hat den Begriff des Transkulturellen gezielt gegen den der Interkulturalität gestellt: Interkulturalität - und, wie man in Anschluss an Welschs Überlegungen ebenfalls behaupten kann: Internationalität - von Kommunikation ist ein zweistufiges Konzept, d.h., es geht auf einer Primärebene von wohl abgegrenzten und verschiedenen Kulturen aus und fragt auf einer Sekundärebene dann, wie Mitglieder dieser Kulturen miteinander kommunizieren, sich verstehen können usw. (vgl. Welsch I992: 5 und Welsch i999). Welsch hält bereits die mit dem Ausdruck Interkulturalität verbundene Primärbehauptung, es gäbe territorial definierte (National-)Kulturen mit dem Nationalstaat als Grenze, für die heutige Zeit für nicht mehr zutreffend. Deutlich schwingt hier die Vorstellung von Deterritorialisierung mit: Wie er argumentiert, sind an die Stelle von »Kulturen alten Zuschnitts«, also National- oder Regionalkulturen, mit der Globalisierung von Medienkommunikation diverse »Lebensformen« getreten (vgl. Welsch I992: 5). Diese Lebensformen oder Lebensstile machen nicht an den Grenzen der »alten Kulturen« halt, sondern gehen quer durch diese hindurch, weshalb sie auch mit den herkömmlichen Kulturkategorien nicht mehr zu fassen sind. Vor diesem Hintergrund führt Welsch nun den Begriff der Transkulturalität als ein seiner Meinung nach angemesseneres Konzept ein: 
»Transkulturalitätı will beides anzeigen: dass wir uns heute jenseits der klassischen Kulturverfassung befinden; und dass die neuen Kultur- bzw. Lebensformen durch diese alten Formationen wie selbstverständlich hindurchgehen« (Welsch 1992: 5).

Hier ist es erforderlich, eine Beziehung zu Überlegungen zu sehen, wie sie im Rahmen der Cultural Studies beispielsweise Stuart Hall dargelegt hat. Er hat darauf hingewiesen, dass je stärker global vernetzte Medien das gesellschaftliche Leben durch weltweite Vermarktung von Stilen und Bildern durchdringen, desto mehr Identitäten von bestimmten Zeiten, Orten, Geschichten und Traditionen entbunden werden (vgl. Hall I992: 303). Ganz ähnliche Überlegungen hat Stuart Hall auch in dem bereits I99I von Anthony King herausgegebenen Band Culture, Globalization and the World System formuliert. In dieser Publikation wird in den verschiedenen Beiträgen - weit bevor dies im deutschsprachigen Raum Gegenstand der Diskussion war - die Frage aufgeworfen, welchen Wandlungsprozessen Kulturen mit der Globalisierung ausgesetzt sind und wie diese Prozesse mit Bezug auf das Entstehen eines ökonomischen Weltsystems im Sinne von Wallerstein analysiert werden können. ${ }^{24}$ Dabei bringt Stuart Hall die Widersprüchlichkeit der Globalisierung von Medienkommunikation und der damit verbundenen Wandlungsprozesse auf den Punkt, wenn er schreibt: ${ }^{25}$

"Diese neue Art der Globalisierung [...] geht mit einer neuen Form globaler Massenkultur einher, die sich stark von derjenigen unterscheidet, die mit der englischen Identität oder anderen, in einer früheren Phase mit dem Nationalstaat verknüpften kulturellen Identitäten verbunden war. Die globale Massenkultur wird durch die modernen Mittel der kulturellen Produktion bestimmt, durch das Bild, das die Sprachgrenzen schneller und einfacher überschreitet und über sie hinweg in einer sehr viel unmittelbareren Weise spricht. Sie wird dadurch bestimmt, dass die visuellen und grafischen Künste direkt in die Umgestaltung des Alltagslebens, der Unterhaltung und der Freizeit hineinwirken. Sie wird durch Fernsehen, Film und das Bild, die Metaphorik und die Stile der Massenwerbung bestimmt. Als Inbegriff all dieser Formen der Massenkommunikation könnte das Satellitenfernsehen gelten. Nicht, weil es das einzige Beispiel wäre, sondern weil das Satellitenfernsehen nicht ohne seine Verankerung in einer bestimmten, hoch entwickelten nationalen Ökonomie und Kultur verstanden werden kann, während sein eigentlicher Zweck gleichzeitig darin besteht, dass es nicht länger durch nationale Grenzen eingeschränkt werden kann« (Hall 1994: 52). 
Wolfgang Welschs Argumentation und die Argumentation von Stuart Hall verbindet also die konstruktive und kritische Perspektive, dass durch die Globalisierung von Medienkommunikation bestimmte semiotische Ressourcen an verschiedensten Orten der Welt zugänglich sind und so nationenübergreifende Kristallisationsmaterialien von kulturellen Identitäten sein können. Kultur und kulturelle Identität sind nicht (mehr) territorial fixiert, sondern stehen in einem komplexen, widersprüchlichen und konfliktären Prozess der Globalisierung.

In diesem allgemeinen Diskussionsrahmen, den Stuart Hall hier auf »phänomenologischer Ebene« klar absteckt, muss auch die Entwicklung der transkulturellen Perspektive in der deutschsprachigen Medien- und Kommunikationswissenschaft gesehen werden. Die Publikation, durch die Transkulturalität als Konzept insbesondere in den Blickwinkel der Medien- und Kommunikationswissenschaft gerückt ist, stellt der von Kurt Luger und Rudi Renger herausgegebene Band Dialog der Kulturen dar (vgl. Luger/Renger I994). ${ }^{26}$ Exemplarisch sei hier auf Lugers Überlegungen in dem Aufsatz »Offene Grenzen in der Kommunikationswissenschaft « verwiesen. Im Anschluss an Wolfgang Welsch streicht er heraus, dass eine durch verschiedenste Kommunikationstechnologien vernetzte Welt die Vorstellung von separierten und autonomen Kulturen als irreal ausweist. Entsprechend greifen die eindimensionalen Ansätze der internationalen und interkulturellen Kommunikationsforschung auch zu kurz, indem in ihnen - ob als Dependenztheorie, Kultur- oder Medienimperialismusansatz - Kulturen als geschlossene Zusammenhänge begriffen werden, auf die global übertragene Medieninhalte »wirken«, worauf die jeweiligen Kulturen wiederum »reagieren« (vgl. Luger I994: 46 f.). Eine kulturtheoretisch begründete, transkulturelle Medienforschung

»lenkt ihre Aufmerksamkeit hingegen mehr auf die Transformationsprozesse, versteht die Beziehung zwischen Kultur bzw. Individuum und der Kulturindustrie nicht als Einbahn, sondern als Interaktionsprozess und kulturellen Wandel nicht notwendig als Beeinflussungsprozess» (Luger 1994: 47).

Kulturen werden dabei nicht als »in sich geschlossen « und »von außen durch Medien beeinflusst« begriffen, sondern selbst als mediatisiert, multiethisch und stark differenziert nach Milieus, Lebensformen und ausgeprägten Lebensstilen, die translokal bestehen, wobei man auch 
hier eine Beziehung zu den Cultural Studies sehen kann. Solche Beziehungen der transkulturellen Medienforschung zu den Cultural Studies werden - wie ich eingangs bemerkt habe - detaillierter an drei Punkten deutlich, nämlich erstens anhand der Bezüge beim Kulturbegriff, zweitens anhand der Überlegungen zu disziplinären Orientierungen und drittens anhand der insbesondere in empirischen Studien greifbar werdenden Selbstreflexivität der transkulturellen Medienforschung.

Erstens: In Bezug auf die Cultural Studies - und hier vor allem anlehnend an die Arbeiten des Centre for Contemporary Cultural Studies in Birmingham bzw. die Arbeiten von Raymond Williams wird der Kulturbegriff in der transkulturellen Perspektive entwickelt. Kurt Luger ${ }^{27}$ beispielsweise zitiert hier John Clarke, Stuart Hall, Tony Jefferson und Brian Roberts mit ihrem Aufsatz über Subkulturen, in dem diese Kultur definieren als die

॥distinkte Lebensweise [einer] Gruppe oder Klasse, [als] die Bedeutungen, Werte und Ideen, wie sie in den Institutionen, in den gesellschaftlichen Beziehungen, in Glaubenssystemen, in Sitten und Bräuchen, im Gebrauch der 0bjekte und im materiellen Leben verkörpert sind. Kultur ist die besondere Gestalt, in der dieses Material und diese gesellschaftliche Organisation des Lebens Ausdruck findetu (Clarke/Hall//efferson/Roberts 1979: 41).

Kultur enthält diesem Verständnis nach »die >Landkarten der Bedeutung <, welche Dinge für ihre Mitglieder verstehbar machen« (ebd.). ${ }^{28}$ Zwar schließt sich Kurt Luger diesem Kulturbegriff zuerst einmal an, greift also die Cultural Studies mit ihrem Kulturverständnis auf, dass seine Überlegungen sich dann aber im Theoriediskurs der Medienund Kommunikationswissenschaft bewegen, macht die Perspektive deutlich, unter der er den Kulturbegriff weiter diskutiert. Was ihn als Medien- und Kommunikationswissenschaftler interessiert ist die konstruktivistische Dimension des Kulturbegriffs der Cultural Studies, die diesen für ihn in eine gewisse Nähe zum radikalen Konstruktivismus rückt (vgl. Luger 1994: 35). Entsprechend kann man argumentieren, dass die Perspektive der transkulturellen Medienforschung gegenwärtig eine Öffnung der deutschsprachigen Medien- und Kommunikationswissenschaft zu den Cultural Studies darstellt, indem hier auf die Kulturtheorie der Cultural Studies Bezug genommen wird. ${ }^{29}$ Allerdings erscheint - und dies soll noch gezeigt werden - eine weitergehende Auseinandersetzung mit den Cultural Studies notwendig, 
um deren kulturtheoretischen Zugang zur Globalisierung von Medienkommunikation für eine transkulturelle Medienforschung zugänglich zu machen.

Zweitens: Eine weitere Beziehung zwischen transkultureller Medienforschung und Cultural Studies, die aber nicht zu einem wechselseitigen Ineinanderaufgehen führt, wird auch am zweiten von mir genannten Punkt deutlich, nämlich dem der disziplinären Orientierung. In gewisser Parallelität dazu, dass die Vertreterinnen und Vertreter der Cultural Studies ihren Ansatz selbst als transdisziplinäres Projekt verstehen (vgl. Hepp I999: i5 f.), wird im Kontext der transkulturellen Medienforschung betont, dass eine Auseinandersetzung mit der Globalisierung von Medienkommunikation auch »offene Grenzen « in der Medien- und Kommunikationswissenschaft erfordert (vgl. Luger I994: 40). Dass diese Parallelität aber wiederum auch ihre »Grenzen« hat, machen bereits die unterschiedlichen Formulierungen deutlich: Was in der transkulturellen Medienforschung eingefordert wird, ist inter- bzw. transdisziplinäre Zusammenarbeit. Als klarer Referenzpunkt für eine solche Zusammenarbeit gilt jedoch die Medien- und Kommunikationswissenschaft, wobei sie als Forschungsfeld aber dazu übergehen sollte, den Anforderungen einer Beschäftigung mit der Globalisierung von Medienkommunikation »durch einen integrativen Ansatz gerecht zu werden« (Luger 1994: 40). Die Transdisziplinarität der Cultural Studies ist im Vergleich dazu etwas anderes. Hier wird Transdisziplinarität nicht nur verstanden als Zusammenarbeit über mehr oder weniger offene Disziplinengrenzen hinweg, sondern es geht darum, eine kritische Perspektive auf Kultur und kulturelle Bedeutungsproduktion jenseits von Disziplinengrenzen zu entwickeln. In diesem Sinne wäre es vielleicht sinnvoller, mit Bezug auf die Cultural Studies von einem postdisziplinären Projekt zu sprechen. Es ist diese postdiziplinäre Orientierung der Cultural Studies, die einen Teil ihres Innovationspotenzials begründet.

Drittens: Als dritten Berührungspunkt zwischen transkultureller Medienforschung und den Cultural Studies habe ich genannt, dass die Auseinandersetzung mit der Globalisierung von Medienkommunikation auch innerhalb der transkulturellen Medienforschung selbstreflexiv bezogen auf die Kultur des Analysierenden geschieht. Das heißt, das Phänomen der transkulturellen Kommunikation interessiert nicht nur in der Außenorientierung der Kommunikationsbeziehung zwischen Staaten wie in der internationalen Kommunikationsforschung, 
sondern jeder Staat wird selbst als kulturell gebrochen konzeptionalisiert. Entsprechend ist Transkulturalität auch ein umfassender Gegenstandsbereich für eine binnenorientierte Betrachtung - oder, um genauer zu sein: In einer transkulturellen Perspektive lösen sich außen- und binnenorientierter Fokus auf, da die Globalisierung von Medienkommunikation als ein Prozess begriffen wird, der zunehmend alle Kulturen durchdringt und entsprechend homogenisierende, territorial fixierte Nationalkulturkonzepte hinfällig macht. ${ }^{30}$ Ein solcher selbstreflexiver Fokus wird an den beiden Hauptthemenbereichen deutlich, die bisher im Kontext der transkulturellen Medienforschung fokussiert worden sind, nämlich erstens die Auseinandersetzung mit Medien und kultureller Identität und zweitens die Beschäftigung mit Medien und Minderheiten.

Die Zentralität der Identitätsthematik im Rahmen einer transkulturellen Medienforschung wird bereits an den Beiträgen des Bandes Dialog der Kulturen deutlich. Marie-Luise Angerer beispielsweise setzt sich in ihrem Artikel mit (Multi-)Kulturalität und Identität aus feministischer Perspektive auseinander (vgl. Angerer 1994) ) $^{3 \mathrm{I}}$, andere Beiträge behandeln die Möglichkeiten des Lebens unterschiedlicher kultureller Identitäten innerhalb von multikulturellen Gesellschaften, und es findet eine Auseinandersetzung damit statt, inwieweit »kulturelle Vielfalt« - verstanden als auf unterschiedliche kulturelle Identitäten fokussierte Medienproduktion - im bestehenden, globalisierten medienökonomischen Rahmen zu realisieren ist (vgl. Grisold I994; Larcher 1994; Wolfgruber 1994). Schließlich wird das Verhältnis von transkulturellem Lernen und der Entwicklung von kultureller Identität diskutiert (vgl. beispielsweise Hipfl 1994 und Stotz I994). ${ }^{32}$ Inwieweit das Themenfeld der kulturellen Identität auch für die gegenwärtigen Arbeiten Kurt Lugers prägend ist, macht dessen Studie zu Populärkultur und Identität in Österreich deutlich. Theoretischer Ausgangspunkt ist für ihn die Überlegung, dass Identität und nationale Erzählung in einer Beziehung zueinander stehen: So wird in Nationalkulturen Identität konstruiert, indem einzelne »Bausteine« als Identifikationsangebote angeboten werden (vgl. Luger I998: I22). Die »Bausteine« sind beispielsweise Nationalfeiertage, nationale Veranstaltungen wie Bälle, nationale Architektur oder Mythen. Solche »Bausteine« sind lokalisiert in einer umfassenden Erzählung der Nation und können so Ressourcen für die Konstruktion nationaler Identität sein. Kernpunkt ist dabei, dass solche nationalen Identitätsressourcen 
für den beträchtlichen Teil der Bevölkerung eines Staates nur medienvermittelt zugänglich sind. Hieraus ergibt sich letztlich die Frage, was die relevanten medialen Inszenierungen für die Konstruktion einer nationalen Identität sind.

Kurt Luger untersucht hier bezogen auf Österreich insbesondere drei solcher Inszenierungszusammenhänge, nämlich erstens den Heimatfilm, zweitens die Volksmusik und drittens einen neuen Boulevard-Journalismus (vgl. Luger 1998: 123-130).33 Vor dem Hintergrund von Wolfgang Welschs Begriff der Transkulturalität ist allerdings offensichtlich, dass dies nur ein Moment der Auseinandersetzung mit der Konstruktion von Identität in Österreich sein kann. Indem in Österreich - wie in allen europäischen Staaten auch - durch die Medien zunehmend Identitätsressourcen aus gänzlich anderen kulturellen Kontexten zugänglich sind, ist die Erzählung der österreichischen Nationalidentität gegenwärtig mit transkulturellen Erzählungen konfrontiert, die die Frage nach einer homogenen österreichischen Nationalkultur als zunehmend falsch gestellte Frage erscheinen lässt:

»Österreich gehört zu jenen westlichen Industriestaaten, in denen eine Fülle individueller wie kollektiver Muster der Lebensführung nebeneinander existieren. Ihre kulturellen Manifestationen befruchten, ignorieren und bekämpfen einanderu (Luger 1998: 134).

Nimmt man für die Betrachtung des Themenfeldes »Minderheiten « in einer transkulturellen Perspektive ebenfalls die Beiträge in dem Band Dialog der Kulturen als Ausgangspunkt, so wird die Grundorientierung der Diskussion deutlich: Jerzy J. Smolicz beispielsweise setzt sich hier mit der Problematik von Konzepten wie dem der Nation, des Staats und der Minderheit auseinander (vgl. Smolicz I994) ${ }^{34}$, daneben befasst sich eine Anzahl von Artikeln mit der Stellung von Minderheiten innerhalb von multikulturellen Gesellschaften und der medialen Berichterstattung über sie bzw. beleuchtet in diesem Kontext die bestehende Medienpolitik (vgl. Busch 1994; Çinar 1994; Hummel I994; Trappel 1994). Diese Diskussion wurde jüngst in dem Themenheft 2/1999 Medien und Minderheiten der Zeitschrift Medien Journal aufgegriffen..$^{35}$ In ihrem das Themenheft einleitenden Aufsatz konstatiert Brigitta Busch bei der seit den I990er Jahren zunehmenden Beschäftigung mit Medien und Minderheiten einen Paradigmenwechsel: Während in früheren Arbeiten, die insbesondere durch einen 
menschenrechtlichen und einen sprachpolitischen Ansatz geprägt waren, die Seite der Produktion und medialen Strukturen im Vordergrund stand, »bekommt nun die Rezipientenforschung und ein umfassender Cultural-Studies-Zugang mehr Gewicht« (Busch I999a: 8).

Als ein Beispiel für eine empirische Auseinandersetzung mit Minderheitenmedien im Rahmen der Perspektive einer transkulturellen Medienforschung kann man Brigitte Buschs Studie zu den Medien der slowenischen Minderheit in Kärnten begreifen (vgl. Busch I999b). Busch beschreibt hier u.a. slowenische Minderheitenmedien als einen »virtuellen Dorfplatz«, eine Metapher, mit der sie den Sachverhalt fassen möchte, dass diese auf eine fast intime Weise Bestandteil der Gemeinschaft der slowenischen Minderheit sind: So kennen nicht nur die von Busch befragten Angehörigen der slowenischen Minderheit einen beträchtlichen Teil der thematisierten bzw. auftretenden Personen, auch sehen sie eine zentrale Funktion der Medien darin, sich über das Geschehen in der eigenen Minderheit zu informieren. Medien ersetzen so teilweise den direkten Kontakt mit anderen Mitgliedern der slowenischen Minderheit (vgl. Busch I999b: 208-210). Dabei sind die slowenischen Medien in Kärnten identitätsstiftend für die dortige Minderheit. Busch verweist hier drauf, dass die slowenischen Medien eine »Welt in der Welt« schaffen, in der in einem eigenen Code über bestimmte Themen kommuniziert werden kann, die sonst nirgends vertreten sind (vgl. Busch I999b: 225). So wird erst durch die Minderheitenmedien ein umfassender Kommunikationsraum geschaffen, der drei historisch nicht zusammengehörende Täler, in denen Slowenisch gesprochen wird, zu der »Region « der slowenischen Minderheit in Kärnten verbindet (vgl. Busch I999b: 240). Zwar ist die slowenische Minderheit nur in geringem Maße in der allgemeinen medialen Öffentlichkeit Österreichs präsent, umgekehrt haben ihre Minderheitenmedien aber zentrale Funktionen für ihren kommunikativen Zusammenhalt - auch wenn die Minderheitenmedien sich konstant aufgrund der nur geringen Zahl von möglichen Publika unter ökonomischen Druck befinden. ${ }^{36}$

Fasst man das in diesem Kapitel bisher Dargelegte zusammen, so ergibt dies ein widersprüchliches Bild. Die transkulturelle Medienforschung ist eine Perspektive in der Medien- und Kommunikationswissenschaft, durch die diese Anschluss an die gegenwärtig drängenden Fragen der Globalisierung von Medienkommunikation gewonnen hat. Hier wird versucht, den mit der Globalisierung von Medienkommuni- 
kation einhergehenden Wandel von (National-)Kulturen zu analysieren. Dabei werden Tendenzen der Hybridisierung und des Synkretismus ebenso berücksichtigt, wie der Nationalstaat als alleiniger Referenzpunkt der Betrachtung der Globalisierung der Medienkommunikation aufgegeben wird. Fragen der Dominanz und Hegemonie werden ebenso gestellt, wie Minderheiten und kulturelle Identität als Referenzpunkte der Betrachtung von transkultureller Kommunikation zu wichtigen Theoriekonzepten erhoben werden. Bei der Theoretisierung dieser Konzepte wird über die Fachgrenzen der Medien- und Kommunikationswissenschaft hinausgehend auch auf die Cultural Studies Bezug genommen. Darüber hinaus erfolgt in der transkulturellen Medienforschung die Auseinandersetzung mit der Globalisierung von Medienkommunikation selbstreflexiv, indem die damit verbundenen Wandlungsprozesse bezogen auf den eigenen kulturellen Kontext betrachtet werden. Dies heißt umgekehrt aber nicht, dass eine transkulturelle Medienforschung sich auf eine Auseinandersetzung mit dem eigenen kulturellen Kontext beschränken würde: Die verschiedenen Beiträge des Sammelbandes Transkulturelle Kommunikation verdeutlichen exemplarisch, dass mit der Perspektive der transkulturellen Medienforschung eine kritische Auseinandersetzung mit der Globalisierung von Medienkommunikation im Allgemeinen verbunden ist (vgl. Hepp/Löffelholz 200I). Insgesamt jedenfalls sind deutliche Beziehungen zwischen transkultureller Medienforschung und Cultural Studies zu sehen. Gleichzeitig werden aber auch Differenzen deutlich, die nicht nur zeigen, dass die transkulturelle Medienforschung nicht einfach »ein umfassender Cultural-Studies-Zugang « (Busch I999b: 8) ist, sondern ausgehend von denen auch argumentiert werden kann, dass trotz aller Berührungen einige Aspekte der transkulturellen Medienforschung aus Sicht der Cultural Studies zu kritisieren sind. Eine solche Kritik möchte ich im folgenden Abschnitt leisten.

\section{Möglichieiten: Cultural Studies als transkulturelle Medienforschung?}

Die in den Cultural Studies aktuell wohl interessanteste Arbeit zur Thematik von Lokalität und Globalisierung von Medienkommunikation ist David Morleys Studie Home Territories. Media, Mobility and 
Identity. In Bezug auf die bereits behandelten drei Berührungspunkte zwischen transkultureller Medienforschung und Cultural Studies ergeben sich aus der Studie drei korrespondierende Kritikpunkte an der transkulturellen Medienforschung. Dies ist erstens, dass die Theoretisierung der Widersprüchlichkeit und des konfliktären Charakters von Kultur in der transkulturellen Medienforschung nicht hinreichend erscheint, zweitens, dass die transkulturelle Medienforschung durch ihre disziplinäre Orientierung umfassendere Wechselbeziehungen unzureichend fasst und drittens, dass ihr Ansatz von Kritik in einer zunehmend deterritorialisierten Welt als unbefriedigend erscheint.

Erstens: Eine umfassende Widersprüchlichkeit bzw. einen Konfliktcharakter von Kultur haben verschiedene Vertreterinnen und Vertreter der Cultural Studies herausgestrichen. Betont wird dabei, dass Kultur als die Summe der unterschiedlichen Klassifikationssysteme und diskursiven Formationen, auf die in Prozessen der Bedeutungskonstitution rekurriert wird, in sich nicht homogen und harmonisch ist (vgl. Hall 200I). Die Leistung der Studie von Morley ist darin zu sehen, dass er zeigt, wie sich dies in der Medienkommunikation artikuliert. So bezieht Morley Stellung gegen Arbeiten wie die von Paddy Scannell, der argumentiert, dass die lokal lebenden Menschen durch die Medien in eine öffentliche Sphäre integriert werden, wodurch letztlich das Vorhandensein einer »nationalen Familie« - die Zugehörigkeit zu einer bestimmten Nationalkultur - konstruiert wird (vgl. Scannell I989). Solche Vorstellungen gehen nach Morley letztlich von einer bürgerlichen und durch einen »männlichen« Zugang dominierten Konzeption von Öffentlichkeit aus, die in dieser Form nie Realität gewesen ist (vgl. Morley 2000: II3-II8). Zentral ist für ihn, dass im Gegensatz dazu mit der Globalisierung von Medienkommunikation eine Vielzahl von Öffentlichkeiten bestehen. Beispielsweise erklärt sich die Unzufriedenheit der schwarzen britischen Bevölkerung gegenüber dem terrestrischen Fernsehen in Großbritannien darüber, dass deren Öffentlichkeiten in diesem Medium unzureichend repräsentiert sind. Dies heißt aber nicht, dass die schwarze Bevölkerung generell von Öffentlichkeit ausgeschlossen wäre - sie verfügt über andere Öffentlichkeiten, die zunehmend transnational sind: Ihre »diasporic public spheres« (Morley 2000: I25) zeichnen sich dadurch aus, dass sie durch den Einsatz verschiedener Medientechnologien (Video, Satellitenfernsehen usw.) nicht auf einen Staat bzw. eine Nationalkultur fixiert sind. Jedenfalls kann nicht - wie Paddy 
Scannell dies macht - damit gerechnet werden, dass sich solche Bevölkerungsgruppen mit der Öffentlichkeit einer »nationalen Familie« identifizieren, dass Fernsehen also zwangsläufig ein Medium ist, das eine homogene Nationalkultur (mit) konstituiert. Entsprechend manifestiert sich gegenwärtig die Auseinandersetzung um bestimmte kulturelle Bedeutungsdefinitionen auch in einer Auseinandersetzung in den und um die verschiedenen medial konstituierten Öffentlichkeiten.

An diesem Punkt gehen die Cultural Studies mit ihrer Theoretisierung von kommunikativer Deterritorialisierung wesentlich weiter als die meisten Vertreterinnen und Vertreter der transkulturellen Medienforschung. Will man nämlich wirklich der Widersprüchlichkeit gegenwärtiger Kulturen gerecht werden, so genügt es nicht, sich bei der Diskussion des Kulturbegriffs an die Cultural Studies anzulehnen, sondern es erscheint nötig, darüber hinaus eine Vielzahl häufig unproblematisiert übernommener Konzepte zu hinterfragen und bezogen auf aktuelle Wandlungsprozesse neu zu definieren bzw. zu theoretisieren. Ist nämlich mit der Globalisierung von Medienkommunikation ein Wandel von Nationalkultur verbunden, warum sollte man dann beispielsweise an dem idealisierenden Konzept einer nationalen Öffentlichkeit festhalten? Aber auch eine Vielzahl anderer, tradierter Konzepte gehört in Bezug auf die Globalisierung von Medienkommunikation und die damit verbundenen Wandlungsprozesse in Frage gestellt und neu theoretisiert - angefangen von Konzepten der kulturellen Identität, die vielleicht mehr und mehr zu einer »Medienidentität« (vgl. Hepp/Winter 200I) wird, über Vorstellungen der Lokalität bis hin zur Theoretisierung von transkultureller Medienproduktion und transkulturellem Medienmanagement (vgl. hierzu Negus 200I und Winter 200I). Dabei handelt es sich um eine bisher nicht geleistete Aufgabe, für die die Cultural Studies die dazu notwendige Kritikpraxis zur Verfügung stellen.

Zweitens: Mit einer Theoretisierung der Interdependenz von Betrachtungsebenen hat sich David Morley bereits in früheren Publikationen auseinander gesetzt, insbesondere mit dem Wechselverhältnis von Mikro- und Makroprozessen beim alltäglichen Umgang mit dem Medium Fernsehen (vgl. Morley I992). Solche Überlegungen führt Morley fort, wenn er Stellung zur Diskussion um die Lokalisierung von Medieninhalten in häuslichen Kontexten bezieht. Morley weist hier darauf hin, dass es ein Fehler ist, den Mikrobereich bzw. das 
Lokale mit dem eher Konkreten und Empirisch-Fassbaren gleichzusetzen und den Makrobereich bzw. das Globale dagegen mit dem Abstrakten, Theoretischen. Seiner Argumentation nach sind Makroprozesse jederzeit auch lokal greifbar, und umgekehrt ist das Lokale nur durch eine auch abstrakte Theoretisierung analytisch zu fassen. Folglich besteht mit Bezug auf die Globalisierung von Medienkommunikation die Notwendigkeit,

munser Verständnis von Ort in dem Kontext der Transformationen und Destabilisierungen zu überdenken, die getrieben werden sowohl durch die Kräfte der ökonomischen Globalisierung als auch durch die globalen Medienindustrien« (Morley 2000: 5; Übersetzung A.H.).

Es ist letztlich genau dies, was Morley in seiner Studie leistet, wenn er Lokalität in den Diskursen des Zuhauses und der Heimat verortet und dabei herausarbeitet, dass Medieninhalte nicht einfach auf eine gesicherte Identität einer häuslichen Welt treffen, sondern diese bereits in widersprüchlichen, auch medienvermittelten Diskursen konstituiert ist (vgl. Morley 2000: I05).

Gerade bei ihrer kritischen Theoretisierung von kulturellen Prozessen im Spannungsverhältnis von Mikro und Makro werden innerhalb der Cultural Studies als »postdisziplinärem Projekt« Zusammenhänge diskutiert, zu denen in der Perspektive der transkulturellen Medienforschung gegenwärtig noch kein Zugang besteht. Dies liegt nicht zuletzt daran, dass die transkulturelle Medienforschung - trotz aller Forderungen von »offenen Grenzen« - sich primär im disziplinären Bereich der medien- und kommunikationswissenschaftlichen Auseinandersetzung mit Medien bewegt. Dabei gerät eine umfassende, kritische Kontextualisierung von Medienproduktion, Medienprodukten und Medienaneignung aus dem Blick. Gegen eine solche disziplinäre Engführung haben die Vertreterinnen und Vertreter der Cultural Studies immer argumentiert, und es ist letztlich diese umfassende, empirische und theoretische Auseinandersetzung mit konfliktären kulturellen Praktiken und Wandlungsprozessen, die auch die transkulturelle Medienforschung stimulieren kann.

Drittens: Solche Überlegungen verweisen auf die auch für Morley zentrale Frage der Theoretisierung von Kritik im Kontext der Globalisierung von Medienkommunikation. Der Gedankengang, den David Morley hier entwickelt, ist, dass eine in diesem Zusammenhang sinn- 
volle Kritik nicht einfach bei der Idee eines unberührten Lokalen ansetzen kann. Er weist hier in Anlehnung an Celeste Olalquiaga darauf hin, dass das Zuhause in solchen Denkzusammenhängen zu einer nostalgischen Sehnsucht geworden ist, einem Verlangen nach einem romantisierenden Sinn von Zugehörigkeit (vgl. Morley 2000: 246). Allerdings ist dies auch eine "postmoderne Nostalgie«, die für ein Suchen nach Gemeinschaft und emotionaler Zugehörigkeit kennzeichnend ist, was zuerst einmal nicht reaktionär sein muss, sondern gegen eine umfassende Individualisierung und Rationalisierung von Gesellschaft gerichtet sein kann. Problematisch ist hier aber, dass die politische Rechte in den I980er Jahren genau diese "postmoderne Nostalgie« in ihrem Sinne besetzt hat. ${ }^{37}$ Die Kritik, die Morley dem entgegensetzt, bezieht sich vor allem auf eine begriffliche Trennung zwischen Zuhause (verstanden als Lokalität) und Heimat (verstanden als emotionalen Identifikationszusammenhang). Ein und dieselbe Lokalität - beispielsweise eine Nachbarschaft - kann also von verschiedenen Leuten, und hier beeinflusst auch von medialen Diskursen, ganz unterschiedlich (oder auch nicht) als Heimat definiert werden, ein Gedanke, auf den bereits Doreen Massey hingewiesen hat (vgl. Massey 1992). Die Folgerung, die man aus solchen Überlegungen ziehen kann, ist ein Plädoyer für ein Leben mit Differenz. Kritik wäre dann keine exklusive Kritik anderer Kulturen, sondern eine »multiperspektivische Kritik« von Praktiken, die ein solches Leben in Differenz in Frage stellen..$^{38}$ Gerade an diesem Punkt befindet sich die transkulturelle Medienforschung mit ihrer Position der Selbstreflexivität auf dem Weg zu den Cultural Studies, ist meiner Meinung nach aber noch nicht bei diesen angekommen.

Dies verweist darauf, dass die transkulturelle Medienforschung in der Medien- und Kommunikationswissenschaft einen wichtigen Anschlusspunkt für das aktuelle Betreiben von Cultural Studies bieten kann. So hat Lawrence Grossberg darauf hingewiesen, dass die Cultural Studies gerade als trans- oder postdisziplinäres Projekt mit ihrer Etablierung jeweils ihren Platz in den verschiedenen Disziplinen finden müssen (vgl. Grossberg 1994). Die Berührungspunkte von transkultureller Medienforschung und Cultural Studies zeigen, dass bei aller Differenz die transkulturelle Medienforschung innerhalb der deutschsprachigen Medien- und Kommunikationswissenschaft ein Kontext sein kann, in dem auch die Cultural Studies ihren Platz finden. Ich möchte hier nicht missverstanden werden: Was ich nicht sage 
ist, dass transkulturelle Medienforschung und Cultural Studies gegenwärtig eins wären. Aber die transkulturelle Medienforschung bietet innerhalb der deutschsprachigen Medien- und Kommunikationswissenschaft Raum für die Cultural Studies, mit der Möglichkeit, an die Diskussionen der deutschsprachigen Forschungslandschaft anzuknüpfen. Und genau aus diesem Grund erscheint es meines Erachtens notwendig, Cultural Studies auch als transkulturelle Medienforschung zu betreiben.

\section{Anmerkungen}

I Eine umfassende Diskussion des vielschichtigen Begriffs der Globalisierung kann hier nicht geleistet werden, findet sich aber beispielsweise in den Beiträgen in Beck 1998 oder Robertson-Wensauer \& Winter 2000 (insbesondere Winter 2000). Meine eigene Position habe ich in Hepp I999: 242-253 dargelegt.

2 Mit dem Diskurs des Globalen ist also die Inszenierung einer kosmopolitischen Welt gemeint, in der Figuren unterschiedlicher kultureller Kontexte ebenso auftreten, wie Handlungsorte ihres direkten lokalen Bezugs enthoben werden. Greifbar werden solche Diskurse des Globalen insbesondere in der Werbung, aber beispielsweise auch in den Videos von Musikkanälen wie MTV oder VIVA. Deren Repräsentation als »multikulturelle Musik- und Lebensstilensemble« besteht unabhängig von der Fokussierung von MTV und VIVA auf einzelne, nationale Märkte: Auch in einem deutschsprachigen Musiksender wird die Welt der Musikkultur(en) als in dem Sinne multikulturell konstruiert, dass »schwarzer Rap« und »deutscher Pop« nebeneinander stehen können, dass Videos von scheinbar kosmopolitischen, zu verschiedensten Ethnien zählenden Moderatorinnen und Moderatoren angesagt werden usw. Man muss also klar zwischen der diskursiven Repräsentation durch Medienprodukte, der medienökonomischen Fokussierung von Programmen auf einzelne Märkte und ihrer Aneignung unterscheiden.

3 Als ein Konzept, diesen Bereich der Unschärfe zu theoretisieren, habe ich das der Translokalität vorgeschlagen. Siehe dazu Hepp zooob und Hepp 20orb.

4 Entsprechend können interkulturelle und internationale Kommunikation identisch sein, sind es aber nicht zwangsläufig, da es durch 
zunehmende Mobilität und Migration interkulturelle Kommunikation auch innerhalb von einzelnen Staaten gibt.

5 Modernisierungstheoretische Konzepte gehen davon aus, dass die Einführung von Massenmedien in so genannten Entwicklungsländern einen wichtigen Schritt für ihre Modernisierung von traditionalen Gesellschaften über transitionale hin zu modernen bzw. postmodernen Gesellschaften darstellen. Dependenztheoretischen Annahmen liegt im Gegensatz dazu die Überlegung zugrunde, dass die Globalisierung von Medienkommunikation - wie auch globale Wirtschaftsbeziehungen - zur Dominanz von westlichen Staaten gegenüber Staaten der Dritten Welt beitragen und diese zunehmend zur Peripherie eines westlich dominierten, kapitalistischen Weltsystems machen. Vgl. beispielsweise Kunczik I985: 69-152.

6 Seine Argumentation bleibt klar der Modernisierungstheorie Parsons mit ihrer Unterscheidung von »primitiven Kulturen mit geringem Außenkontakt«, »primitiven Kulturen mit regelmäßigen Außenkontakten«, »archaischen Hochkulturen« und »modernen Gesellschaften« verpflichtet (Rust I984: 375-377).

7 Dabei hat für ihn der Raum im Rahmen einer geforderten Informationskultur eine neue Bedeutung, indem mit räumlicher Virtualität der Aufenthaltsort des bzw. der Einzelnen und die regionale Topik (das Befinden eher im Zentrum oder an der Peripherie) an Stellenwert verlieren, während gleichzeitig Polymorphie (Vervielfachung der Kommunikationsmöglichkeiten innerhalb von unterschiedlichen Gruppierungen) und Globalität (verstanden als prinzipielle kommunikative Erreichbarkeit) von Medienkommunikation zunehmen.

8 Daneben unternimmt auch Gerhard Maletzke einen zweiten Begründungsversuch einer interkulturellen Medienforschung im deutschsprachigen Raum (vgl. Maletzke I98I), der sich in den grundlegenden Annahmen aber nicht von seinem ersten Versuch unterscheidet.

9 Reimann greift Anfang der r99oer Jahre seinen hier formulierten Ansatz unter dem Schlagwort der »transkulturellen Kommunikation « auf, leider ohne den Anschluss an die aktuelle kulturtheoretische Diskussion zu finden. Siehe dazu meine Argumentation weiter unten. 10 Beide Tendenzen werden auch an der Überblicksdarstellung Massenmedien und Entwicklungsländer von Michael Kunczik deutlich (vgl. Kunczik I985). In dieser Monographie stellt Kunczik den I985 aktuellen Forschungsstand zu der Thematik der internationalen Kommunikation dar und betrachtet insbesondere modernisierungstheore- 
tische und dependenztheoretische Ansätze sowie die internationale Kulturimperialismus-Diskussion. Auch wenn Kunczik hier dezidiert für einen kulturellen Relativismus und gegen einen Ethnozentrismus argumentiert (Kunczik 1985: 22-32), so geht er in der Publikation nicht über einen - sicherlich hoch einzuschätzenden - Forschungsblick bezogen auf das Feld internationale Kommunikation und Entwicklungsländer hinaus. Vor allem eine kulturtheoretisch fundierte Theoretisierung des Gegenstandsbereichs sucht man vergeblich.

I I Hierbei handelt es sich um das achte Wissenschaftliche Gespräch der Bundesregierung über Fragen der Kommunikationswissenschaft und -politik, das am 28. und 29. Juni 1990 in Mayschoß (Ahrtal) stattfand.

12 Marie-Luise Kiefer betrachtet beispielsweise das Spannungsverhältnis zwischen transnationaler Produktion und nationalkultureller Identität bei der europäischen Fernsehproduktion, bei dem in der Folge einer Fokussierung auf mehrere nationale Märkte nationalkulturelle Bezüge in transnationalen Medienprodukten zunehmend vermieden werden (Kiefer 1990: 7I). Wolfgang Langenbucher weist auf die bisherigen Leistungen des Rundfunks als »nationales Kulturgut« (Langenbucher 1990: 147) hin, die er mit zunehmender Kommerzialisierung und Internationalisierung von Medienkommunikation gefährdet sieht. Und Franz Ronneberger setzt sich aus einer sicherlich nicht unproblematischen, national-konservativen Perspektive mit der »kulturelle[n] Verträglichkeit« (Ronneberger I990: 159) in der internationalen Medienkommunikation auseinander.

$13 \mathrm{Zu}$ einer Kritik vorschneller Annahmen der Entwicklung einer europäischen Identität vgl. Morley/Robins 200I. Gegenwärtig beschäftigt sich auch das Projekt Eurofiction mit dieser Thematik (vgl. Hallenberger 200I).

14 Zwar gab es schon zuvor Untersuchungen in diesem Themenbereich wie die Studie von Jesus M. Delgado (vgl. Delgado I972), jedoch nahm die Anzahl von Untersuchungen zu diesem Themenbereich im oben genannten Zeitraum erheblich zu (vgl. beispielsweise die Beiträge in Schatz et al. 2000).

15 In den Worten Luhmanns selbst: »Die Bestimmung der Gesellschaft als das umfassende Sozialsystem hat zur Konsequenz, dass es für alle anschlussfähige Kommunikation nur ein einziges Gesellschaftssystem geben kann« (Luhmann I997: I45).

16 Hier übrigens im Gegensatz zu den Überlegungen Luhmanns, 
der beispielsweise bei der Diskussion des Begriffs »Weltgesellschaft« die Frage von Problemen interkultureller Kommunikation aufwirft (Luhmann 1997: 170).

17 Problematisch bleibt hier allerdings das Konstrukt der Nation. Vgl. dazu meine Darlegungen in Hepp 2000b.

18 Vgl. zu dieser Beziehung im Speziellen Stäheli 2000: 322, zum Anti-Essentialismus der Cultural Studies im Allgemeinen Hepp I999: I6 ff.

19 Allerdings muss man Miriam Meckel zugute halten, dass sie gegenwärtig versucht, ihren Ansatz theoretisch auf eine breitere Basis zu stellen und dabei auch Fragen kultureller Identität zu berücksichtigen (vgl. Meckel I998.

20 Dies spiegelt sich übrigens auch in dem Festhalten an dem Begriff der internationalen Kommunikation in diesem Bereich wider und das im Kontext eines Globalisierungsprozesses, der einen Transnationalismus bei der kritischen Betrachtung von Medien geradezu fordert.

21 Vgl. beispielsweise die Beiträge in Scheffer 1997, Brosius 2000 und Quandt/Gast 1998 sowie Kunczik/Zipfel I996.

22 Falls dies jenseits von Ideologie überhaupt jemals möglich gewesen ist. Siehe dazu meine Argumentation im vorherigen Abschnitt.

23 Worum es im Weiteren gehen wird, ist also eher eine Perspektive der deutschsprachigen Beschäftigung mit Medien, die sich von der oben skizzierten Tradition der interkulturellen und internationalen Kommunikation abgrenzt, denn eine in sich geschlossene »Schule«.

24 Zum Stellenwert der Publikation allgemein im Kontext der Auseinandersetzung mit Kulturwandel und Globalisierung vgl. Winter 2000: 35-39.

25 Etwas unglücklich erscheint die deutsche Übersetzung von orig. »popular culture« mit »Massenkultur«.

26 Für 1992 könnte man hier auch auf das von Horst Reimann herausgegebene Buch Transkulturelle Kommunikation und Weltgesellschaft verweisen (vgl. Reimann I992), dessen Konzeption von transkultureller Kommunikation allerdings nicht die Dimension aufweist, die mit der von Kurt Luger herausgegebenen Publikation vorliegt. Dies ist nicht zuletzt in dem engeren und eher an modernen denn postmodernen Überlegungen orientierten Begriff von transkultureller Kommunikation bei Reimann begründet, der sehr stark auf die Vorstellung 
abgeschlossener Kulturen rekurriert. Reimann begreift transkulturelle Kommunikation als grenzüberschreitenden Vorgang von einer »kulturellen Einheit« zur anderen, wobei für ihn »der Akzent nicht auf dem wechselseitigen Austausch kultureller Elemente [liegt], sondern auf der oft durchaus einseitigen Grenzüberschreitung (und Beeinflussung), wie sie prinzipiell bei Massenkommunikation (>Ein-Weg-Kommunikation `) der Fall ist, auch wenn hierbei die selektive Kapazität auf der Empfangsseite (im Sinne der Aus- und Abwahlmöglichkeit, Interpretation und Einpassung in die jeweils eigene Kultur) die Annahme einer bloßen Ein-Weg-Perspektive konterkariert« (Reimann I992: I4).

27 Ebenfalls Bezug auf den Kulturbegriff der Cultural Studies nehmen Renger 1994, Angerer 1994 und Hipfl 1994.

28 Auch aktuell lehnt sich Kurt Luger an diesen Kulturbegriff an, wenn er formuliert: »Eine Kultur enthält die >Landkarten der Bedeutungく, welche Dinge für ihre Mitglieder verstehbar macht« (Luger 1998: II7).

29 Kurt Luger hat hier aber sicherlich einen Schritt zu früh mit der Auseinandersetzung mit den Cultural Studies aufgehört und deren Theoretisierungen zu schnell in den etablierten Theoriekanon der Medien- und Kommunikationswissenschaft eingeordnet. Auf die zusätzliche Perspektive der Cultural Studies, die dadurch in seinen Überlegungen verloren geht, gehe ich im letzten Abschnitt dieses Beitrags ein.

30 Hiermit ist nicht gemeint, dass sich Nationalkulturen als solche gänzlich aufgelöst hätten. Wogegen argumentiert wird ist ein Verständnis von Nationalkultur als in sich abgeschlossen, homogen und territorial fixiert.

3 I Dabei streicht sie insbesondere die durch eine männlich-weiße Perspektive geprägte Konstruktion offizieller, westlicher Kultur und die Ausgrenzung weiblicher Kultur in die private Sphäre heraus.

32 Ich würde hier die Arbeiten von Marie-Luise Angerer und Brigitte Hipfl nicht bruchlos unter die Perspektive der transkulturellen Medienforschung subsumieren, ist die Arbeit von beiden doch der innerhalb des deutschsprachigen Raums bestehenden Tradition der Cultural Studies zuzuordnen (vgl. Angerer 1999; Hipfl I999). Jedoch kann man das Aufnehmen und die Positionierung der beiden Artikel in dem von Kurt Luger und Rudi Renger herausgegebenen Band sicherlich als charakteristisch für den Fokus der transkulturellen Medienforschung auf diese Thematik nehmen - ebenso wie für die Bezie- 
hungen zwischen transkultureller Medienforschung und Cultural Studies. Diese Beziehungen werden auch dadurch verdeutlicht, dass Brigitte Hipfl beispielsweise zusammen mit Brigitte Busch und Kevin Robins gegenwärtig den Band Bewegte Identitäten. Medien in transkulturellen Kontexten herausgibt (vgl. Busch/Hipfl/Robins 200I).

33 Der Heimatfilm mit seiner »alpinen Symbolik« war vor allem nach dem Zweiten Weltkrieg bei der Neudefinition der nationalen Identität Österreichs nicht mehr als Donaumonarchie, sondern als Alpenrepublik zentral. Die filmischen Mythen der Heimatfilme - von denen bis 1965 in Österreich I22 gedreht wurden - enthalten zahlreiche Identitätsbausteine aus dem ländlichen Milieu, die dann zur prototypischen Identitätsressource österreichischer Identität wurden: »herb, patriarchal, aber auch idealisierend als Sommerfrische« (Luger I998: I24). Dieser Diskurs des Heimatfilms wurde später in der österreichischen Fremdenverkehrswerbung aufgegriffen, aber auch im neuen Heimatfilm - hier allerdings als Negativfolie, von der sich ein eher problematisierendes Heimatverständnis abgrenzt. In der Musik war es insbesondere die Volksmusik bzw. die damit eng verbundene volkstümliche Musikshow Musikantenstadl (Erstausstrahlung I98I), die als Kompensationsraum für eine zunehmend als entfremdet empfundene Heimat zu einer wichtigen Ressource der Konstruktion von österreichischer Nationalidentität wurde. Solche eher konservativen Fokusse manifestieren sich auch in der Erfolgsgeschichte der Boulevardzeitung Neue Kronen Zeitung, die sich selbst nicht nur als Ressource für nationale Identität stilisiert, sondern darüber hinaus als nationale Stimme des Volkes: »Die Zeitung erzeugt [...] eine Nähe zwischen sich und den Lesern, simuliert eine Handlungseinheit, die sie letztlich als >Stimme des Volkes<, etwa bei den zahlreichen Kampagnen, laut werden lässt« (Luger I998: г28).

34 Dabei arbeitete er die Spezifik des deutschen Nationkonzepts heraus, dessen Staatsbürgerlichkeit auf der Vorstellung eines auf »Blut-Erbe« rekurriert, sowie die daraus folgenden Problemlagen für unterschiedliche »Minderheiten« innerhalb des gesamtdeutschen Staates (Smolicz I994: I33 f.).

35 Bereits früher setzten sich Beiträge in einem Themenheft des Medien Journals mit Medien und Fremdenhass auseinander, wobei auch hier schon die Überlegungen von Stuart Hall zum Rassismus ein zentraler Referenzpunkt gewesen sind (Liebminger I993: $\mathrm{I}_{3}$ und Stocker 1993: 158). 
36 Im Einzelnen verweist Busch dreimal in der Studie auf Arbeiten, die dem Umfeld der Cultural Studies entstammen (Busch i999b: II6, I65 und 257), ordnet dabei aber beispielsweise auch John Fiske eher als Kommunikationswissenschaftler denn als Vertreter der Cultural Studies ein.

37 In diesem Kontext hat eine neue Alltagstheorie der natürlichen Xenophobie Verbreitung gefunden: Der Ausschluss von Migranten aus dem eigenen Zuhause wird hier territorialisierend begründet, d.h., (National-)Kultur wird in dem Sinne territorialisiert, dass das jeweilige kulturelle Erbe örtlich fixiert und hierüber monosemiert gedacht wird - »jede (National-)Kultur hat ihren Ort« (Morley 2000: 248 f.).

38 Erste Ansätze für eine solche multiperspektivische Kritik finden sich in Hepp 2о0га.

\section{Literatur}

Ang, Ien (I996): Living Room Wars. Rethinking Media Audiences for a Postmodern World, London/New York: Routledge.

Ang, Ien (200I): »Verzweifelt Grenzen bewachen: Mediale Globalisierung, >kultureller Imperialismus und der Aufgang Asiens«. In: Andreas Hepp/Martin Löffelholz (Hg.), Transkulturelle Kommunikation. Ein internationaler Reader, Konstanz: UVK [in Vorbereitung].

Ang, Ien/Stratton, Jon (I996): »Asianing Australia: Notes Toward a Critical Transnationalism in Cultural Studies«. Cultural Studies Iо/I, S. I6-36.

Angerer, Marie-Luise (I994): »Vom Unbehagen der Geschlechter in der Kultur. Über Differenz, Andersheit und Identität. Feministische Perspektiven«. In: Kurt Luger/Rudi Renger (Hg.), Dialog der Kulturen: Die multikulturelle Gesellschaft und die Medien, Wien: Österreichischer Kunst- und Kulturverlag, S. IIO-I28.

Angerer, Marie-Luise (I999): »Medienkörper: Zur Materialität des Medialen und der Medialität der Körper«. In: Andreas Hepp/ Rainer Winter (Hg.), Kultur - Medien - Macht. Cultural Studies und Medienanalyse. Zweite, überarbeitete und erweiterte Auflage, Opladen: Westdeutscher Verlag, S. 307-318.

Beck, Ulrich (Hg.) (I998): Perspektiven der Weltgesellschaft, Frankfurt a.M.: Suhrkamp Verlag. 
Brosius, Hans-Bernd (Hg.) (2000): Kommunikation über Grenzen und Kulturen, Konstanz: UVK.

Busch, Brigitta (I994): »Minderheiten und Massenmedien«. In: Kurt Luger/Rudi Renger (Hg.), Dialog der Kulturen: Die multikulturelle Gesellschaft und die Medien, Wien: Österreichischer Kunst- und Kulturverlag, S. 263-279.

Busch, Brigitta (I999a): »Von Minderheitenmedien zu Medien in multilingualen und multikulturellen Situationen. Versuch eines Überblicks über das Forschungsfeld«. Medien Journal 2, S. 3-I2.

Busch, Brigitta (I999b): Der virtuelle Dorfplatz. Minderheitenmedien, Globalisierung und kulturelle Identität, Klagenfurt: Drava.

Busch, Brigitta/Hipfl, Brigitte/Robins, Kevin (200I): Bewegte Identitäten. Medien in transkulturellen Kontexten, Klagenfurt: Drava.

Çinar, Dilek (I994): »Vom Nutzen des (Multi-)Kulturalismus«. In: Kurt Luger/Rudi Renger (Hg.), Dialog der Kulturen: Die multikulturelle Gesellschaft und die Medien, Wien: Österreichischer Kunstund Kulturverlag, S. I70-I79.

Clarke, John/Hall, Stuart/Jefferson, Tony/Roberts, Brian (I979): »Subkulturen, Kulturen und Klasse«. In: John Clarke/Phil Cohen/ Paul Corrigan et al. (Hg.), Jugendkultur als Widerstand. Milieus, Rituale, Provokationen, Frankfurt a.M.: Syndikat, S. 39-I3I.

Delgado, Jesus M. (I972): Die »Gastarbeiter « in der Presse. Eine inhaltsanalytische Studie, Opladen: Leske + Budrich.

García Canclini, Néstor (I995): Hybrid Cultures. Strategies for Entering and Leaving Modernity, Minneapolis: University of Minnesota Press.

Görke, Alexander/Kollbeck, Johannes (I996): »(Welt-)Gesellschaft und Mediensystem. Zur Funktion und Evolution internationaler Medienkommunikation«. In: Miriam Meckel/Markus Kriener (Hg.), Internationale Kommunikation. Eine Einführung, Opladen: Westdeutscher Verlag, S. 263-28I.

Grisold, Andrea (I994): »Die Medien - Marktplatz der Kulturen. Wie marktfähig ist kulturelle Vielfalt«. In: Kurt Luger/Rudi Renger (Hg.), Dialog der Kulturen: Die multikulturelle Gesellschaft und die Medien, Wien: Österreichischer Kunst- und Kulturverlag, S. 24I$25 \mathrm{I}$.

Grossberg, Lawrence (I994): »Cultural Studies. Was besagt ein Name?«. IKUS Lectures I7/I8 (Cultural Studies. Eine Intervention),Wien, S. II-40. 
Hall, Stuart (1992): »The Question of Cultural Identity«. In: Stuart Hall/D. Held/T. McGrew (Hg.), Modernity and Its Futures, Cambridge: Polity Press, S. 273-326.

Hall, Stuart (I994): Rassismus und kulturelle Identität. Ausgewählte Schriften 2, Hamburg: Argument Verlag.

Hall, Stuart (200I): »Die Zentralität von Kultur: Anmerkungen zu den kulturellen Revolutionen unserer Zeit«. In: Andreas Hepp/ Martin Löffelholz (Hg.), Transkulturelle Kommunikation. Ein internationaler Reader, Konstanz: UVK [in Vorbereitung].

Hallenberger, Gerd (200I): »Fiktionale Fernsehsendungen in Europa. Das Projekt Eurofiction«. In: Andreas Hepp/Martin Löffelholz (Hg.), Transkulturelle Kommunikation. Ein internationaler Reader, Konstanz: UVK [in Vorbereitung].

Hardt, Hanno (I984): »Internationale Medienforschung in den Vereinigten Staaten von Amerika: eine Kritik«. Publizistik 29, S. 553$55^{8}$.

Hepp, Andreas (I999): Cultural Studies und Medienanalyse. Eine Einführung, Opladen: Westdeutscher Verlag.

Hepp, Andreas (2000a): »Internet und Cultural Studies. Konsensfiktion in der Netzkommunikation«. In: GMK (Hg.), Denkräume: Szenarien zum Informationszeitalter, Bielefeld, S. 8I-90.

Hepp, Andreas (2000b): »Transkulturalität, Synkretismus und Lokalität: Auf dem Weg zu einer kritischen Betrachtung von Medien im globalen Kontext«. In: Thomas Düllo/Arno Meteling/André Suhr/Carsten Winter (Hg.), Kursbuch Kulturwissenschaft, Münster: LIT Verlag, S. I87-207.

Hepp, Andreas (200Ia): »Translokale Orientierung und multiperspektivische Kritik: Perspektiven einer kritischen Ethnografie und Diskursanalyse im Kontext der Cultural Studies«. In: Matthias Karmasin/Marco Höhn (Hg.), Die Zukunft der empirischen Sozialforschung, Graz: Nausner \& Nausner [in Vorbereitung].

Hepp, Andreas (200Ib): »Translokalität und Medienkulturen«. In: Andreas Hepp/Martin Löffelholz (Hg.), Transkulturelle Kommunikation. Ein globaler Reader, Konstanz: UVK [in Vorbereitung].

Hepp, Andreas/Löffelholz, Martin (Hg.) (200I): Transkulturelle Kommunikation. Ein internationaler Reader, Konstanz: UVK [in Vorbereitung].

Hepp, Andreas/Winter, Carsten (200I): Medienidentitäten, TU Ilmenau, Typoskript. 
Hipfl, Brigitte (I994): »Zum Umgang mit dem Fremden in der Familie«. In: Kurt Luger/Rudi Renger (Hg.), Dialog der Kulturen: Die multikulturelle Gesellschaft und die Medien, Wien: Österreichischer Kunst- und Kulturverlag, S. 307-324.

Hipfl, Brigitte (I999): »Inszenierungen des Begehrens: Zur Rolle der Phantasien im Umgang mit Medien«. In: Andreas Hepp/Rainer Winter (Hg.), Kultur - Medien - Macht. Cultural Studies und Medienanalyse, Zweite, überarbeitete und erweiterte Auflage, Opladen: Westdeutscher Verlag, S. I45-I57.

Hummel, Romas (I994): »Chancen für Multikulturalität. Ein Versuch, das medienpolitische Terrain abzustecken«. In: Kurt Luger/Rudi Renger (Hg.), Dialog der Kulturen: Die multikulturelle Gesellschaft und die Medien, Wien: Österreichischer Kunst- und Kulturverlag, S. 252-255.

Kiefer, Marie-Luise (I990): »Audiovisuelle Medienprodukte: National, international, transnational«. In: Walter A. Mahle (Hg.), Internationalisierung der Medienangelegenheiten, München: Ölschläger Verlag, S. 67-72.

Kriener, Markus/Meckel, Miriam (I996): »Internationale Kommunikation. Begriffe, Probleme, Referenzen«. In: Miriam Meckel/ Markus Kriener (Hg.), Internationale Kommunikation. Eine Einführung, Opladen: Westdeutscher Verlag, S. II-I8.

Kunczik, Michael (I985): Massenmedien und Entwicklungsländer, Köln: Böhlau Verlag.

Kunczik, Michael/Zipfel, Astrid (I996): »Mediengiganten in Lateinamerika: Globo und Televisa«. Nord-Süd aktuell го/4, S. 712- 726. Langenbucher, Wolfgang R. (I990): »Der Rundfunk als nationales Kulturinstitut - das Ende einer Epoche?«. In: Walter A. Mahle (Hg.), Internationalisierung der Medienangelegenheiten, München: Ölschläger Verlag, S. I47-I52.

Larcher, Dietmar (I994): »Minimudus Periculosus. Die Einübung von Ethnozentrismus, Nationalismus, Eurozentrismus und Rassismus im ganz alltäglichen Leben«. In: Kurt Luger/Rudi Renger (Hg.), Dialog der Kulturen: Die multikulturelle Gesellschaft und die Medien, Wien: Österreichischer Kunst- und Kulturverlag, S. I93209.

Liebminger, Michael (I993): »Skinhead-Fanzines in Österreich«. Medien Journal 3, S. I63-I72.

Luger, Kurt (I994): »Offene Grenzen in der Kommunikationswissen- 
schaft. Über die Notwendigkeit eines interkulturellen Forschungsansatzes«. In: Kurt Luger/Rudi Renger (Hg.), Dialog der Kulturen: Die multikulturelle Gesellschaft und die Medien, Wien: Österreichischer Kunst- und Kulturverlag, S. 23-65.

Luger, Kurt (1998): »Populärkultur und Identität«. In: Ulrich Saxer (Hg.), Medien-Kulturkommunikation. Publizistik Sonderheft 2, Opladen: Westdeutscher Verlag, S. II5-I38.

Luger, Kurt/Renger, Rudi (1994): Dialog der Kulturen: die multikulturelle Gesellschaft und die Medien, Wien: Österreichischer Kunstund Kulturverlag.

Luhmann, Niklas (1997): Die Gesellschaft der Gesellschaft. 2 Bde., Frankfurt a.M.: Suhrkamp.

Maletzke, Gerhard (ig66): »Interkulturelle Kommunikation und Publizistikwissenschaft«. Publizistik II, S. 318-33I.

Maletzke, Gerhard (198I): »Internationale und interkulturelle Kommunikation «. Publizistik 3, S. 345-352.

Maletzke, Gerhard (1996): Interkulturelle Kommunikation. Zur Interaktion zwischen Menschen verschiedener Kulturen, Opladen: Westdeutscher Verlag.

Massey, Doreen (1992): »A Place called Home?«. New Formations I7, S. 3-15.

Meckel, Miriam (1996): »Die vernetzte Welt. Perspektiven der globalen Informationsgesellschaft«. In: Miriam Meckel/Markus Kriener (Hg.), Internationale Kommunikation. Eine Einführung, Opladen: Westdeutscher Verlag, S. 299-321.

Meckel, Miriam (1998): »Kommunikative Identität und Weltöffentlichkeit«. Publizistik 43, S. 362-375.

Meckel, Miriam/Kriener, Markus (Hg.) (1996): Internationale Kommunikation. Eine Einführung, Opladen: Westdeutscher Verlag.

Morley, David (1992): Television Audiences and Cultural Studies, London/New York: Routledge.

Morley, David (2000): Home Territories. Media, Mobility and Identity, London/New York: Routledge.

Morley, David/Robins, Kevin (200I): »Globalisierung als Identitätskrise: Die neue globale Medienlandschaft«. In: Andreas Hepp/ Martin Löffelholz (Hg.), Transkulturelle Kommunikation. Ein internationaler Reader, Konstanz: UVK [in Vorbereitung].

Negus, Keith (200I): »Produktionskulturen und die soziale Vermittlung von symbolischen Formen«. In: Andreas Hepp/Martin 
Löffelholz (Hg.), Transkulturelle Kommunikation. Ein internationaler Reader, Konstanz: UVK [in Vorbereitung].

Quandt, Siegfried/Gast, Wolfgang (Hg.) (I998): Deutschland im Dialog der Kulturen. Medien - Images - Verständigung, Konstanz: UVK.

Reimann, Horst (1983): »Die Bedeutung kultureller Codes für die internationale Kommunikation«. In: Manfred Rühl/Heinz-Werner Stuiber (Hg.), Kommunikationspolitik in Forschung und Anwendung, Düsseldorf: Droste Verlag, S. 269-279.

Reimann, Horst (I992): »Transkulturelle Kommunikation und Weltgesellschaft«. In: Ders. (Hg.), Transkulturelle Kommunikation und Weltgesellschaft. Theorie und Pragmatik globaler Interaktion, Opladen: Westdeutscher Verlag, S. I3-29.

Reimann, Horst (Hg.) (I992): Transkulturelle Kommunikation und Weltgesellschaft. Theorie und Pragmatik globaler Interaktion, Opladen: Westdeutscher Verlag.

Renger, Rudi (I994): »Der Kultur auf der Spur. Materialien zur Interkulturellen Forschung«. In: Kurt Luger/Rudi Renger (Hg.), Dialog der Kulturen: Die multikulturelle Gesellschaft und die Medien, Wien: Österreichischer Kunst- und Kulturverlag, S. 66-83.

Robertson-Wensauer, Caroline/Winter, Carsten (Hg.) (2000): Kulturwandel und Globalisierung, Baden-Baden: Nomos Verlagsgesellschaft.

Ronneberger, Franz (I990): »Kulturelle Verträglichkeit in der internationalen (Medien-)Kommunikation«. In: Walter A. Mahle (Hg.), Internationalisierung der Medienangelegenheiten, München: Ölschläger Verlag, S. I59-165.

Rust, Holger (I984): »Kommunikationsprobleme in multikulturellen Gesellschaften. Eine ethnologische Perspektive zur Begründung einer kommunikationspolitischen Moral«. Publizistik 29, S. 36I386.

Scannell, Paddy (I989): »Public Service Broadcasting and Modern Public Life«. Media, Culture and Society 2/II, S. I35-166.

Schatz, Heribert/Holtz-Bacha, Christina/Nieland, Jörg-Uwe (Hg.) (2000): Migranten und Medien. Neue Herausforderungen an die Integrationsfunktion von Presse und Rundfunk, Opladen: Westdeutscher Verlag.

Scheffer, Bernd (Hg.) (I997): Medien und Fremdenfeindlichkeit. Alltägliche Paradoxien, Dilemmata, Absurditäten und Zynismen, Opladen: Leske + Budrich. 
Smolicz, Jerzy J. (I994): »Nation, State and Ethnic Minorities. From an Euro-Arab Perspetive«. In: Kurt Luger/Rudi Renger (Hg.), Dialog der Kulturen: Die multikulturelle Gesellschaft und die Medien, Wien: Österreichischer Kunst- und Kulturverlag, S. I29-I43.

Stäheli, Urs (2000): »Das Populäre zwischen Systemtheorie und Cultural Studies«. In: Udo Göttlich/Rainer Winter (Hg.), Politik des Vergnügens. Zur Diskussion der Populärkultur in den Culturals Studies, Köln: Halem Verlag, S. 321-336.

Stocker, Günther (I993): »Ein Vorkrieg. Rassismus in der Neuen Kronen Zeitung«. Medien Journal 3, S. I53-162.

Stotz, Günther (I994): »Kultur, Kommunikation, Bildung«. In: Kurt Luger/Rudi Renger (Hg.), Dialog der Kulturen: Die multikulturelle Gesellschaft und die Medien, Wien: Österreichischer Kunst- und Kulturverlag, S. 289-296.

Tomlinson, John (I999): Globalization and Culture, Cambridge, Oxford: Polity Press.

Trappel, Josef (I994): »Vom Knirschen im Gebtriebe der medienpolitischen Konzeption«. In: Kurt Luger/Rudi Renger (Hg.), Dialog der Kulturen: Die multikulturelle Gesellschaft und die Medien, Wien: Österreichischer Kunst- und Kulturverlag, S. 256-262.

Welsch, Wolfgang (I992): »Transkulturalität. Lebensformen nach der Auflösung der Kulturen«. Information Philosophie 2, S. 5-20.

Welsch, Wolfgang (I999): »Transculturality - The Changing Forms of Cultures Today«. In: Bundesministerium für Wissenschaft und Verkehr/Internationales Forschungszentrum Kulturwissenschaften (Hg.), The Contemporary Study of Culture, Wien: Turia + Kant, S. $2 \mathrm{I} 7-244$.

Wersig, Gernot (I984): »Informationsgesellschaft, Informationskultur und Veränderung des Raumkonzeptes als kommunikative Herausforderung«. Publizistik 29, S. 387-400.

Winter, Carsten (2000): »Kulturwandel und Globalisierung. Eine Einführung in die Diskussion«. In: Caroline Robertson/Carsten Winter (Hg.), Kulturwandel und Globalisierung, Baden-Baden: Nomos Verlagsgesellschaft, S. I3-73.

Winter, Carsten (200I): »Transkulturelles Medienmanagement«. In: Andreas Hepp/Martin Löffelholz (Hg.), Transkulturelle Kommunikation. Ein internationaler Reader, Konstanz: UVK [in Vorbereitung].

Wolfgruber, Elisabeth R. (I994): »Minimudus Periculosus. Die Ein- 
übung von Ethnozentrismus, Nationalismus, Eurozentrismus und Rassismus im ganz alltäglichen Leben«. In: Kurt Luger/ Rudi Renger (Hg.), Dialog der Kulturen: Die multikulturelle Gesellschaft und die Medien, Wien: Österreichischer Kunst- und Kulturverlag, S. 216-238. 


\title{
Kulturimperialismus und Kulturindustrie ade?
}

\section{Zur Notwendigkeit einer Neuorientierung der}

\section{Erforschung und Kritik von Medienkultur in den}

\section{Cultural Studies'}

CARSTEN WinTER

\begin{abstract}
"Doch wenn Globalisierung zumindest eine Dimension der Konjunktur definiert, die Cultural Studies anzusprechen haben, dann scheint mit zu folgen, dass die Cultural Studies explizit zu Fragen der Ökonomie zurückkehren müssen, zu Fragen, die aus verschiedenen Momenten ihrer Geschichte aus strategischen Gründen ausgeklammert waren« (Grossberg 1999: 294).
\end{abstract}

\section{VORBEMERKUNG}

Im Beitrag wird die Auffassung vertreten, dass bei der Erforschung von Kulturwandel und Globalisierung die ökonomischen Bedingungen dieses Wandels bislang zu wenig berücksichtigt wurden. Dies gilt auch für die Cultural Studies. Sie haben seit Mitte der I98oer Jahre fast aufgehört, die Momente (Produktion und Allokation) des Kreislaufs von Kommunikation und Kultur zu erforschen, von denen aus die globale Kommerzialisierung von Medienkultur geplant und - im 
Kontext politisch legitimierter Liberalisierung in Form von Deregulierung und Privatisierung - umgesetzt wird. Dieses Problem wurde mir im Anschluss an eine eigene Arbeit, die ich 1997 abgeschlossen habe, bewusst (C. Winter 2000a). In einer an Hall (I999a) orientierten Kritik an konstruktivistischen Versuchen, Kulturwandel zu beschreiben, habe ich darauf hingewiesen (C. Winter I998: 310-3I3). Damals war ich der Auffassung, dass Cultural Studies die theoretische Integration von Produktions- und Rezeptionsanalysen »bereits vollzogen« hatten (Göttlich/C. Winter I999: 37). Heute, nachdem ich mich in den Diskurs der Ökonomie und des Managements in der Medienbranche eingearbeitet habe (vgl. C. Winter 2000a/b/c und 200I) meine ich, dass diese Aussage zu schnell getroffen wurde.

Der Text versucht, die Notwendigkeit einer stärkeren Berücksichtigung ökonomischer Voraussetzungen von Medienkultur im Kontext von Arbeiten aus den Cultural Studies historisch und systematisch zu entfalten. Es kann gezeigt werden, dass die Cultural Studies bislang über keinen entsprechenden theoretischen Bezugsrahmen und kein entsprechend differenziertes Vokabular zur Beschreibung von Kulturwandel und Kommerzialisierung verfügen, das die Rolle von Unternehmen angemessen berücksichtigt. Vor dem Hintergrund der irreversiblen Tatsache, dass diese weltweit fast die gesamte materiale Basis von Medienkulturen produzieren und allozieren, wird ein umfassendes Verständnis von Medienkultur immer stärker von den Möglichkeiten abhängen, den strategischen Diskurs in der »Medien- und Kulturindustrie « zu rekonstruieren und zu verstehen. Nach der Darstellung zentraler Aspekte des Diskurses Medienmanagement (vgl. Karmasin/C. Winter 2000) wird gefragt, wie dieser mit den Cultural Studies vermittelt werden kann.

\section{Ausgangsfragen: Globalisierung und Kulturwandel ohne "Basisu?}

Ende der I980er Jahre wurde in der gerade entstehenden Diskussion insbesondere über die Globalisierung von Strukturen (vgl. Giddens I995; Robertson I991; Robertson I992) u.a. festgestellt, dass Marken, Produkte und Medienangebote global agierender Konzerne zunehmend das Lokale prägen, das immer häufiger als eine komplexe Reaktion auf entfernte Veränderungen erscheint (vgl. Featherstone I99०; King I99ı; darin inbes. Hall ı99ıа; Hall ı99ıb; zur Entstehung dieser 
Diskussion vgl. Winter 2000a: 26-39). Dieser Sachverhalt wird Gegenstand einer spezifisch kulturtheoretisch orientierten Erforschung kultureller Selbstverhältnisse im Kontext von Kulturwandel und Globalisierung - explizit steht dabei nicht der großflächige Wandel von Strukturen oder Systemen, wie des Weltsystems oder der Gesellschaft, im Vordergrund. Es war erwartet worden, dass die empirische Erforschung von Werten, Orientierungen, Gegenständen und Praktiken in konkreten Lebensverhältnissen ein angemesseneres Verständnis der Widersprüche, Materialität und repräsentationalen Rolle von Kultur im Kontext von Kulturwandel und Globalisierung erlauben würde als das im Hinblick auf konkrete Aspekte von Kulturwandel doch sehr abstrakte und trennungsscharfe "global babble« der Globalisierungsoder Weltsystemtheorie (vgl. Abu Lughod I99I).

In diese zunehmend international geführte Diskussion bringen die Cultural Studies ihre Erfahrung in der Erforschung konkreter kultureller Praxen und Kontexte von Identität sehr erfolgreich ein (vgl. zum frühen Kontext Wolff I99I; zusgef. C. Winter 2000a: insbes. 24 f. und 37 f.). Das theoretisch und methodisch offenere Vorgehen, das in den Cultural Studies in Studien zur Arbeiter- und Jugendkultur, zu Rasse, Gender und ethnischen Minoritäten zur Beschreibung dieser spezifischen kulturellen Kontexte und zur Überwindung von Ungleichheitsverhältnissen entwickelt worden war, wird - vor dem Hintergrund der Kritik an den Globalisierungskonzepten insbesondere von Wallerstein, Giddens, Robertson und Hannerz - bei der differenzierten Erforschung des komplexen, synkretischen Umgangs mit glokaler Kultur sogar forschungsorientierend (vgl. Wolff I99I; Bromley 2000). Im Zusammenhang mit diesen Entwicklungen verlagern sich in den Cultural Studies die Schwerpunkte. Die Erforschung des Konsums hybrider und globaler Medienangebote (vgl. R. Winter 2000b) ist zunehmend durch Psychoanalyse, Postmoderne und Poststrukturalismus geprägt und weniger durch Feminismus und Marxismus (vgl. Nelson/Treichler et al. 1992: 2). Insbesondere die Abkehr von den marxistischen Wurzeln der Cultural Studies (vgl. Johnson 1999) wird in der Folge auch kritisch als Entkontextualisierung und Entpolitisierung beschrieben (Bromley I999: 2I) - eine Entwicklung, die auch eine weitgehende Aufgabe der Erforschung von Produktionsund Distributionsvoraussetzungen von Medienkultur zur Folge hatte. Trotzdem wurde aber der politische Anspruch der Cultural Studies, der seit der Kritik von E.P. Thompson an Williams in den I96oer 
Jahren für die Cultural Studies konstitutiv war (vgl. Thompson 1999), nie aus den Augen verloren. Aktuell wird dieser Anspruch wieder stärker eingefordert. Es wird immer deutlicher, dass die Cultural Studies als politisches Projekt vor allem durch das Ziel aufeinander bezogen bleiben, hegemoniale Bestrebungen aufzudecken, zu kritisieren und zu überwinden (vgl. Ang I999; Nelson/Treichler et al. 1992; Hall I992; Grossberg I999; zuletzt Göttlich/C. Winter I999; Bromley 1999).

\section{Die Cultural Studies als Alternative zu Kulturindustrie- und Kulturimperialismustheorie?}

Nur vor dem Hintergrund dieses politischen Anspruchs lässt sich verstehen, wie Cultural Studies im Kontext der Diskussion um Kulturwandel und Globalisierung eine Alternative zu sozial-strukturellen und systemtheoretischen Konzeptualisierungen und den in kultur-kritischen Diskursen vorherrschenden Konzepten »Kulturimperialismus « und »Kulturindustrie « werden konnten. Letztere enthalten ja in Teilen bereits die Vorstellung eines globalen Prozesses und haben damit Aspekte der Diskussion über Globalisierung antizipiert. Kulturimperialismus bezeichnet - vereinfacht ausgedrückt - die Unterdrückung klar unterscheidbarer Kulturen durch andere Kulturen, wie sie etwa aus der Kolonialzeit bekannt ist. Später bezeichnet der Terminus vor allem als zielgerichtet angenommene dominante Prozesse wie Amerikanisierung oder McDonaldisierung (vgl. insbes. Tomlinson I99I, I997). Kulturindustrie ist ein begriffliches Konzept, das von Horkheimer und Adorno im Kulturindustriekapitel ihrer »Dialektik der Aufklärung « erstmalig verwendet wurde (vgl. Horkheimer/Adorno I988: I28-I76). Darin stellen sie Kulturprodukte und Kulturproduktion erstmalig konsequent in den Kontext industrieller Produktion der kapitalistischen Gesellschaft. Sie beschreiben sodann, wie charakteristische Merkmale kapitalistischer Produktion - Warenförmigkeit, Standardisierung und Massenhaftigkeit - immer häufiger auch für Kulturgüter gelten. Diese Einsicht nehmen sie zum Anlass für die Entwicklung der Kulturindustrietheorie, wonach die Warenförmigkeit industriell gefertigter Produkte die systemaffirmative Anpassung individueller Verhaltensdispositionen nach sich zieht. An den Grundzügen dieser These hält Adorno auch zwanzig Jahre später noch fest: 
»Kulturindustrie ist willentliche Integration ihrer Abnehmer von oben« (Adorno I972: 347).

Anders als die Kulturimperialismus- und die Kulturindustrietheorie verfügen die Cultural Studies über keine feststehenden Vorstellungen von spezifischen Konstitutionsmerkmalen kultureller Hegemonie. Die theoretische Klarheit, die zugleich Stärke und Schwäche der Kulturimperialismus- und Kulturindustrietheorie ist, charakterisiert Cultural Studies nicht. Ihre Stärke liegt vor dem Hintergrund eines konflikttheoretischen Verständnisses von Kultur vielmehr in der wechselseitigen Korrektur von abstrakter Konzeptualisierung und der umfassenden empirischen Erforschung kultureller Verhältnisse, die aber und das ist zentral - prinzipiell als konfliktär aufgefasst werden! Es war diese Stärke, welche die Cultural Studies in der Diskussion um Kulturwandel und Globalisierung wichtig werden ließ - sie verfügten über methodische und theoretische Voraussetzungen für einen Umgang mit Komplexität. Das wird von vielen als wichtigste Voraussetzung für die Entdeckung, begriffliche Entfaltung und kritische Reflexion von Kulturwandel im Kontext von Globalisierung gesehen (vgl. etwa Featherstone 2000; Tomlinson 2000). Kulturimperialismus- und Kulturindustrietheorie hingegen verfügen über diese Möglichkeiten zur Entfaltung empirischer und theoretischer Neugier und weiter zur differenzierten Beschreibung von Kulturwandel nicht (vgl. bereits Tomlinson I99I: I73-I79). Dies heißt freilich nicht, dass der Kulturimperialismus der Kolonialzeit, Verwestlichung, Amerikanisierung, McDonaldisierung oder die industrielle Standardisierung nicht weiter zentrale Aspekte von Kulturwandel sind - sie können nur nicht mehr als die Losung zu dessen innerer Logik ausgegeben werden.

\section{Eine «Neuorientierung» der Cultural Studies?}

Ihren eigenen Anspruch, Kultur und Kulturwandel im Kontext von Globalisierung angemessen kritisch und umfassend $\mathrm{zu}$ erforschen, haben aber auch die Cultural Studies immer noch nicht eingelöst. Auch sie scheitern bislang an der Komplexität kultureller Globalisierungsprozesse. Zwischen dem Anspruch, die Bedingungen und Voraussetzungen von Kultur und Kulturkritik umfassend zu erforschen und kritisch auf allgemeine Bedingungen und Voraussetzungen von Medienkultur zu beziehen - das ist der Ausschnitt, der hier interes- 
siert -, und ihren Forschungen klaffte zuletzt eine immer größere Lücke. Diese Lücke entstand Ende der I980er Jahre dort, wo in den Cultural Studies ursprünglich der Kern marxistischer Kulturtheorie, die Frage nach dem Verhältnis des Überbaus Kultur zur materiellen Basis im Kontext von produktionstheoretischen Konzepten diskutiert wurde (vgl. Johnson I999: I53 f.). Die Rolle transnational agierender Konzerne in der Medien- und Kulturindustrie sowie deren strategisches Management werden im Kontext von Kulturwandel und Globalisierung praktisch nicht erforscht (vgl. Morris I990; Ang 1999; Hall I992; zuletzt Grossberg 2000).

Die transnationalen großen Medienunternehmen produzieren und vertreiben einen immer größeren Teil der materialen Basis populärer Kultur. Populäre Kultur kann nicht mehr verstanden werden, wenn es nicht auch gelingt, die Strategien der Unternehmen zu verstehen und hinsichtlich ihrer Bedeutung für kulturelle Kontexte zu beschreiben und zu beurteilen. Ausgehend von einer Erinnerung an die »Basis-Problematik«, die zuletzt in den Cultural Studies in Vergessenheit geraten ist, werden sodann die zentralen Aktivitäten von Unternehmen sowie das Managementhandeln von Medienunternehmen vorgestellt. Freilich hat es auch Arbeiten zur Produktion von Medienkultur gegeben (vgl. Gay/Hall et al. 1997; Gay 1997). Weiter wird auch seit Halls encoding/decoding-Modell in den Cultural Studies gefordert, Medienunternehmen und -organisationen als »diskursproduzierende « Institutionen in die Analyse einzubeziehen (Hall I999a; Hall I997: 44). Eine kritische Erforschung des Managements von Medienunternehmen, dem Motor der globalen Kommerzialisierung, hat bislang trotzdem noch nicht eingesetzt. Solange damit nicht begonnen wird, dürfte ein Verständnis von Medienkultur aber unvollständig bleiben. Die Vorschläge zur Konkretisierung der Erforschung und Kritik des strategischen Umgangs mit Medienangeboten, also desjenigen Umgangs, der über Ressourcen und Produktionsmittel verfügt, verstehen sich als Ergänzung der in den Cultural Studies geleisteten Erforschung des taktischen Umgangs ${ }^{2}$ mit Medien und ihren Angeboten (vgl. R. Winter 1995; Lutter/Reisenleitner 1998; Göttlich/C. Winter I999 sowie die Beiträge in Hepp/R. Winter 1999 und Düllo et al. 2000).

Bei der Entwicklung einer stärkeren Orientierung auf ökonomische und managementrelevante Aspekte von Medienkultur waren u.a. folgende Fragen orientierend: Welche Relevanz haben Diskurse des 
Managements der Kultur- und Medienbranchen für ein Verständnis des Gesamtzusammenhangs von Medienkultur? Wie konstruieren Manager und Managementtheoretiker Kontexte von Medienkultur und wie kann dieser Diskurs aus der Perspektive der Cultural Studies dekonstruiert werden? Wie kann die kritische empirische Erforschung der Vielfalt und Widersprüchlichkeit der Mediennutzung um eine entsprechend angemessene Erforschung der Komplexität kommerzieller Medienproduktion und -allokation ausgedehnt werden? Welche Rolle spielt das an Bedeutung gewinnende Management in der Medien- und Kulturbranche im Kontext von Globalisierung und Kulturwandel?

\section{Zur \#Basisi von Medienkulturforschung und - kritik in den Cultural Studies}

\section{Die „Basis-Problematik» im Umfeld der frühen British Cultural Studies}

Die theoretischen Grundlagen der Kulturforschung und -kritik in den Cultural Studies wurden insbesondere im Anschluss an Edward P. Thompsons Kritik an Raymond Williams »The Long Revolution« entwickelt. Thompson hatte die Fiktion einer gemeinsamen (National-)Kultur - wie sie in Williams' Konzeption von Kultur als whole way of life durchschimmerte - kritisiert und als whole way of conflict re-konzeptualisiert (vgl. Thompson I999). Die Kulturforschung und -kritik der Cultural Studies war seit Thompson explizit an sozialtheoretischen und ökonomischen Prämissen orientiert (vgl. Hall ı999b). Bezüge zu Marx sowie zur Politischen Ökonomie entwickelten und differenzierten den Horizont der Cultural Studies hinsichtlich der Wahrnehmung von Ideologien, Klassengegensätzen und hegemonialen Verhältnissen. Williams selbst hat später die grundlegenden Brücken zu marxistischen und materialistischen Kulturtheorien aus der Perspektive der Cultural Studies sowie deren Kritik in seinem Beitrag »Zur BasisÜberbau-These in der marxistischen Kulturtheorie« formuliert (Williams I983). Er kritisiert die zu einfache Annahme, dass es eine bestimmende Basis und einen bestimmten Überbau (Kultur) gäbe und konzentriert sich auf das Konzept »Basis«, das auch von Marx, der die Kräfte der Basis als »spezifische Tätigkeiten und Beziehungen wirkli- 
cher Menschen aufgefasst und untersucht« hat (ebd.: I86), keinesfalls statisch verwendet wurde. Williams resümiert: Die Kräfte, die als »Basis« bezeichnet werden, nehmen »einen viel aktiveren, komplizierteren und widersprüchlicheren Sinn an, als es die metaphorische Vorstellung von >der Basis « uns zu denken nahe legt« (ebd.). Williams fordert, in der Forschung "nicht nach den Komponenten eines Produkts, sondern nach den Bedingungen der Praxis zu suchen« (ebd.: 200).

Während Williams »Zur Basis-Überbau-These in der marxistischen Kulturtheorie« schrieb, kursierte in der jungen Media Group am Centre for Contemporary Cultural Studies (CCCS) ein Beitrag, der Williams' Forderung, nach den Bedingungen der Praxis zu suchen, für die Medienforschung der Cultural Studies formuliert. Halls ebenfalls zuerst I973 publizierter Beitrag »Enkodieren/Decodieren« (I999a) wurde aber nur selten als marxistisch fundierte Kritik vorherrschender Kommunikationsforschung rezipiert, deren lineare Modelle die Verbindung zwischen den verschiedenen Momenten von Praxis im Prozess der Kommunikation nicht aufzuzeigen vermögen. Dies genau war aber Halls - in der politischen Tradition der Cultural Studies formuliertes - umfassendes und materialistisches Anliegen (vgl. Abb. I):

॥Doch es ist denkbar und auch sinnvoll, diesen Prozess als eine Struktur aufzufassen, die durch die Artikulation miteinander verbundener, aber eigenständiger Momente produziert und aufrechterhalten wird: Produktion, Zirkulation, Distribution/Konsum, Reproduktion der Produktion« (Hall 1999a: 92 f.).

Halls Modell hebt die Verbindung und die Spezifität der Formen hervor, »in denen das Produkt dieses Prozesses zu jedem Zeitpunkt >erscheint«« (ebd.). Für Hall steht die maßgebliche Rolle der Produktion in diesem Prozess außer Frage (ebd. 95).

\section{Vom »encoding/decoding-Modell« zum »Kultur-Zirkulationsmodell«}

Halls Beitrag wurde Bezugspunkt einer nicht mehr überschaubaren Zahl von Studien. Ihr Bezug war meist der Hinweis, dass jedes Moment der Kommunikation seine »eigenen Existenzformen und -bedingungen hat« (ebd.). Die Studien, die dann die Widersprüchlichkeit und Unvorhersehbarkeit des taktischen Umgangs mit Medienangeboten erforschten, halfen, Engführungen von Konzepten zu korrigieren, 
Abbildung 1: Stuart Halls encoding/decoding-Modell

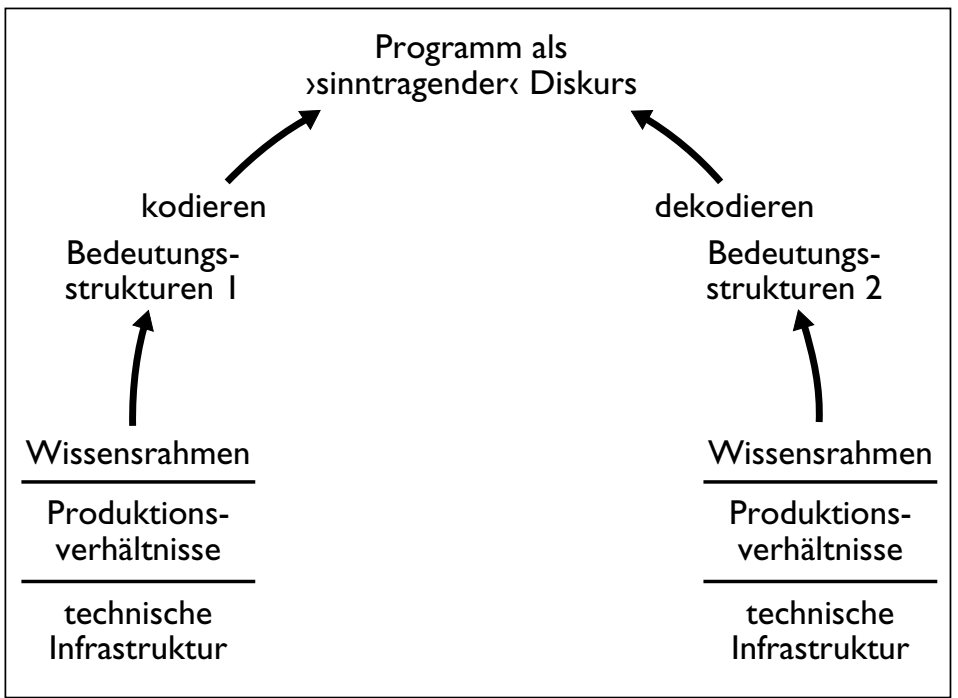

Quelle: Hall ı999a: 97

die - wie Kulturimperialismus- und Kulturindustrietheorie - unterstellten, dass von der Kontrolle der Produktion oder den Produkten auf die Rezeption oder deren Wirkung geschlossen werden könne (vgl. Hepp I999: 164-253; R. Winter 2000a, 200ob). Kritische Beiträge zu Strategien von Unternehmen und ihrem Umgang mit Medien und ihren Angeboten oder zur Produktion und Distribution bzw. Allokation finden sich dagegen kaum. ${ }^{3}$ Eine Ausnahme bildet hier Hall. Er hat in einer Arbeit - die an Williams' Text zur Basis-Überbau-These erinnert - versucht, den ideologischen »Effekt« der Medien auf Kultur zu bestimmen (vgl. Hall I977). Halls Versuch, das Verhältnis von Kultur, Medien und Macht im Kontext von »Ideologie« konkreter auf der Ebene von empirisch ausweisbaren Effekten zu entfalten, gelingt aber - wie er selbst einräumt - nur bedingt. Erst zum Ende des Beitrags geht er, indem er - wie Williams - Engführungen marxistischer Kulturtheorie beklagt, konkret auf Medien und das Problem ein, wie »Domination « verstanden werden müsse, um den ideologischen Effekt der Medien bestimmen zu können. Als die wichtigsten Mechanismen der ideologischen Wirkung der Medien bestimmt Hall schließlich aber nicht etwa intentionale Handlungen von Produzen- 
ten, sondern die »professionellen Codes« im Kontext der Produktion und Allokation von Medienangeboten (ebd.: S. 342). Diese Codes, die - so Hall - zumeist auch für die an der Produktion Beteiligten weitgehend unsichtbar bleiben, werden aber von ihm in seinem Beitrag nicht weiter untersucht. Halls Fazit fällt dann auch eher zurückhaltend aus:

"We can speak, then, only of the tendency of the media - but it is a systematic tendency, not an incidental feature - to reproduce the ideological field of a society in such a way as to reproduce, also, its structure of dominance»l (ebd.: 346 ).

Das von Hall ursprünglich in Anlehnung an Marx entwickelte encoding/decoding-Modell wurde in den 8oer Jahren um ein Zirkulationsmodell von Kultur ergänzt, das Richard Johnson (ebenfalls in Anlehnung an Marx) in dem einflussreichen Beitrag »Was sind eigentlich Cultural Studies?« (Johnson I999) vorstellt. Der Gesamtzusammenhang von Kultur und Kommunikation wird bei Johnson durch die Anordnung der Elemente in einem Kreismodell sichtbar und ist nicht mehr nur - wie bei Hall - über ein »Sinnangebot« verbunden (vgl. Abb. 2):

W)eder Kasten ist ein Element dieses Kreislaufs, das seinerseits von den anderen abhängt und für das Ganze unverzichtbar, zugleich aber auch von den anderen unterschieden ist und für jeweils charakteristische Veränderungen der Form steht. Wenn wir uns also in einem bestimmten Element dieses Kreislaufs befinden, können wir nicht unbedingt erkennen, was bei den anderen Elementen geschieht. Die Formen, die für uns im Hinblick auf ein Element die größte Bedeutung haben, können sich von denen eines anderen stark unterscheiden. Prozesse verschwinden in Resultaten. So werden zum Beispiel alle Kulturprodukte notwendigerweise produziert, aber die Produktionsbedingungen lassen sich aus ihrer Analyse als ,Texter nicht erschließenu (ebd.: 148 f.).

Im Anschluss an die Vorstellung seines Modells präsentiert Johnson die Stärken und Schwächen der drei in den Cultural Studies bestehenden Forschungsrichtungen: »produktionstheoretische und texttheoretische Untersuchungen sowie Forschungen zur Kultur als Lebensweise« (ebd.: I80). Diese Unterscheidung ist für Johnson so nahe liegend, dass er sicher nicht erwartet hat, dass die von ihm so genannten produktionstheoretischen Untersuchungen seit Beginn der I99oer Jahre in den Cultural Studies kaum mehr angetroffen werden können. 
Abbildung 2: Johnsons Zirkulationsmodell von Kultur

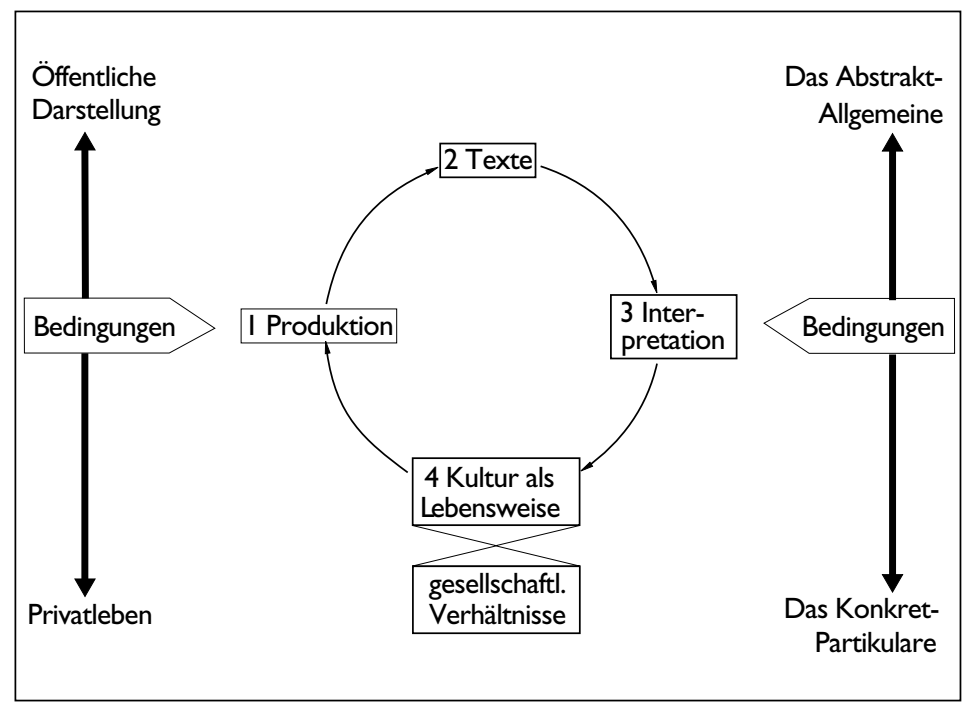

Quelle: Johnson I999: 148

Exemplarisch dokumentiert dies der Band zur Tagung »Cultural Studies jetzt und in der Zukunft« in Illinois (Grossberg/Nelson et al. I992). In diesem, für die Entwicklung der Cultural Studies in den USA wichtigen Band findet sich trotz des Verweises auf die Relevanz der Erforschung von sozialen Transformationsprozessen und von Kulturwandel in der Einleitung (Nelson/Treichler et al. I992: 15) kein Bezug zu den ökonomischen Bedingungen dieser Prozesse - andererseits wird die umfassende und konfliktorientierte Anlage der Kulturforschung und -kritik der Cultural Studies ausführlich herausgestellt (vgl. ebd.: 4 ff.).

\section{Die Forderung nach einem neuen theoretischen Bezugsrahmen}

Im Kontext dieser veränderten Rahmenbedingungen von Cultural Studies, die nicht mehr auf marxistische Produktions- und Kulturtheorien verweisen, fordert Ien Ang Anfang der I99oer Jahre eine umfassende ethnographische Kritik des Medienkonsums im transnationalen Mediensystem: »wenn die Cultural Studies nicht bloß eine raffiniertere Form der empirischen Publikumsforschung sein wollen, 
sondern $\mathrm{zu}$ einem umfassenderen strukturellen und historischen Verständnis unserer gegenwärtigen kulturellen Lebensverhältnisse beitragen « wollen (Ang 1999: 323), dann muss die »ethnographische Perspektive in einen neuen »umfassenden theoretischen Rahmen eingebunden werden« (ebd.). Angs Forderung knüpft dabei nicht mehr an Marx und die Politische Ökonomie an, sondern an eine kritische Ethnographie:

"Zunächst müssen wir ein konkretes Verständnis der hegemonialen Kräfte entwickeln, die die Welt von heute beherrschen. In vielen Beiträgen bleibt das Verständnis von Hegemonie sehr abstrakt und theoretisch und mit den Anspielungen auf grundlegende Konzepte wie IKlassee, IGeschlechtı und IRasses wird sie eher beschworen als analysiert. Wir müssen über dieses paradigmatische Verständnis von Hegemonie hinausgehen und ein spezifischeres, konkreteres und kontextualisierteres, kurz, ein ethnographisches Verständnis des Hegemonialen entwickeln« (Ang 1999: 329 f.).

Die Erforschung des Hegemonialen als konstitutiver Bestandteil des whole way of conflict der Medienkulturen sollte dabei aus ihrer Sicht ansetzen an den "grundlegenden Veränderungen des weltweiten Mediensystems, das zweifelsohne ein Stützpfeiler der hegemonialen Kräfte ist« (ebd.: 330). Begründet wird dies wieder aus der Perspektive der Rezeptionsforschung. Explizit hebt Ang die Rolle der Unternehmen hervor und kritisiert ihre Rolle im Prozess des Wandels von Medienkulturen, weil sie mit ihren Produkten letztlich die Kontexte konstituieren, in denen sich Praktiken von Medienrezeption und -konsum herausbilden.

॥Am Ende des Jahrhunderts befinden sich die Kommunikationsindustrien als Teil des weltweit expandierenden kapitalistischen Systems in einem Prozess der tief greifenden ökonomischen und institutionellen Umstrukturierung, die sich durch beschleunigte Transnationalisierung und Globalisierung auszeichnet. Erkennbar wird dieser Prozess an dem Aufkommen wirklich globaler und dezentrierter Unternehmen, die verschiedenste Medienprodukte (Film und Fernsehen, Presse und Bücher, Musik und Videos) kombinieren und in Kommunikationsimperien wie Bertelsmann, Murdoch, Berlusconi und Time-Warner integrieren. [...] Diese historischen Entwicklungen formen auf spezifische Weise die strukturellen und globalen Konfigurationen der Hegemonie, innerhalb derer sich die gegenwärtigen Praktiken von Medienrezeption und -konsum herausbilden« (ebd.: $330 \mathrm{ff}$.).

Konkrete Beispiele für die Erforschung dieser historischen Entwick- 
lungen in der Medien- und Kulturindustrie, die auf »spezifische Weise die strukturellen und globalen Konfigurationen formen«, innerhalb derer sich die »gegenwärtigen Praktiken von Medienrezeption und -konsum herausbilden«, gibt Ang - wie auch schon Williams und Hall - aber nicht. Dort, wo sie Vereinfachungen marxistischer Kulturtheorie nicht mehr kritisiert, kritisiert sie Antworten, wie sie »die Kulturimperialismustheorie versucht hat« (ebd.: 33I). Anders als Williams und Hall fordert Ang eine partikularistische Forschung, die »das voreilige Abschotten unserer Auffassungsgabe gegen kontextuelle Besonderheiten und Widersprüche vermeiden« hilft (ebd.). Ihre abschließende Forderung (ebd.: 33I ff.) gibt zwar ihren umfassenden Anspruch wieder, aber sie enthält keinen Hinweis auf die von ihr geforderte umfassende Theorie, in die ihrer Meinung nach eine kritische ethnographische Forschung einzubinden wäre.

Aber wie und in welchem Bezugsrahmen lassen sich die ökonomischen Bedingungen der Momente von Medienkommunikation verstehen? Insbesondere dann, wenn diese - wie Williams hervorgehoben hatte - nicht statisch missverstanden werden dürfen (vgl. Williams I983: I86), sondern hinsichtlich sich verändernder Bedingungen von Praxis immer wieder neu erforscht werden müssen. Angs Vorschläge öffnen die Cultural Studies stärker für die Erforschung der Praxis-Momente des Zusammenhangs von Medienkultur, die diese konstituieren. Ihre im Kontext ethnographischer Forschung entwickelte Kritik fordert die Cultural Studies dabei empirisch und theoretisch heraus: Sie sollen einerseits stärker als bisher die Unternehmen und ihre Strategien empirisch erforschen und andererseits diese Forschung theoretisch im Gesamtzusammenhang von Medienkultur neu reflektieren lernen - weil sie ja sonst, wie oben zitiert, »bloß eine raffiniertere Form der empirischen Publikumsforschung« bleiben (Ang 1999: 323). Angs Forderung nach einer empirischen Erforschung und theoretischen Berücksichtigung von Medienunternehmen im Kontext von Kulturwandel und Globalisierung wurde zuletzt von Lawrence Grossberg ähnlich geäußert (vgl. Grossberg I997, 2000).

Ausgehend von der Forderung, dass die Cultural Studies »ihren eigenen Ansatz für die politische Ökonomie finden« sollen (Grossberg I997: I9), konkretisiert er später, die Cultural Studies müssten »explizit zu Fragen der politischen Ökonomie zurückkehren, zu Fragen, die zu verschiedenen Momenten ihrer Geschichte aus strategischen Fragen ausgeklammert waren« (Grossberg 2000: 293). Cultural Studies 
können, so Grossberg weiter, »die von der Globalisierung aufgeworfenen ökonomischen Probleme nicht ansprechen, indem sie einfach jene Formen politischer Ökonomie aufgreifen, die sie bereits für inadäquat befunden hatten« (ebd.). ${ }^{4}$ Konkret fordert er schließlich: »Wir müssen Cultural Studies »ökonomisieren« und Ökonomie >diskursivieren « (ebd.: 294). Bei Grossberg liegt der Schwerpunkt aber auf der theoretischen Herausforderung (vgl. auch Grossberg I997: I6 f.). Die folgende Forderung von Grossberg (2000) kann fast als eine Arbeitsanleitung gesehen werden, weshalb sie hier auch als eine Art Aufgabenstellung vollständig wiedergegeben wird: Cultural Studies

॥müssen von einem anderen Ort beginnen, denn nun müssen sie ihr Gehör der Vielzahl der Stimmen im und zum Kapitalismus schenken, die rings um den Globus laut werden. Cultural Studies müssen anerkennen, dass es keinen einzigen Ort und keine einzige Erfahrung des Kapitalismus gibt (und müssen daher auf ihre eigene Verortung im Kapitalismus wie auch in der globalen Kultur reflektieren). Aber es geht weder darum, dass Cultural Studies-Forscher einfach ihre Lieblingstheorie ausschlachten, noch dass sie sich einem fundamentalistischen Marxismus zuwenden - keines von beidem würde sie mit fertig verpackten Antworten und Zusammenhängen ausstatten, ohne dass irgendetwas von der theoretischen Arbeit, die Marx' kritische politische Ökonomie ausmachte, getan worden wäre. Stattdessen müssen wir genug ökonomische Literatur lesen, um zu wissen, was wir wählen, wenn wir eine bestimmte Theorie wählen, und eine Entscheidung darüber auf uns nehmen, welche Daten sowohl relevant als auch verlässlich sind - und wie sie zu interpretieren sind. Wir müssen - noch radikaler - sogar erkennen, dass Ökonomie selbst ein Diskurs ist, dass die Wirtschaft selbst immer auf komplexe Weise durch kulturelle Praktiken artikuliert ist. Die Herausforderung besteht nicht einfach darin, Marx supzudatenı, sondern die Formen anzuerkennen, auf die Marx' Theorien des Kapitalismus selbst historisch und diskursiv konstruiert waren, und solche kulturellen Determinationen herausfordern. Wir müssen die Frage der diskursiven Konstruktion des Kapitalismus und des Subjekts des Kapitals erneut denken, nicht nur als totalisierendes Ganzes (und wie Gibson-Graham argumentiert, haben wir vielleicht einen über-totalisierenden Kapitalismus), sondern auch als ein System von Differenzen (z.B. den Differenzen zwischen Finanz-, Industrie- und Rentenkapital)« (ebd.: 294).

Wie für Ang scheinen für Grossberg klassische Konzepte wie die traditionelle Politische Ökonomie, »Kulturimperialismus« oder »Kulturindustrie« nur mehr als historische Orientierungen akzeptabel. Für die 
Cultural Studies könnte eine solche Situation eine Möglichkeit werden, ihre Leistungsfähigkeit in der kritischen Aneignung und Entwicklung von Theorien und Konzepten zu Medienkultur vor dem Hintergrund ihrer erfolgreichen Erforschung insbesondere des taktischen Umgangs mit Medien unter Beweis zu stellen. Diese Perspektive wird vielleicht deutlicher, wenn die Vielfalt von Formen der Mediennutzung, die in den Studien der Cultural Studies erforscht und dokumentiert wurden (vgl. R. Winter 200oa sowie zusf. Hepp I999: I64-253), in den Kontext strategischer Erwägungen von Medienunternehmen gestellt werden. Es kann angenommen werden, dass diese Vielfalt nicht ganz zufällig ist, sondern auch in den Strategien des Managements von Medienunternehmen reflektiert wird - nur dass es bislang in den Cultural Studies keinen Bezugsrahmen gibt, der in der Lage wäre, diese Strategien und ihre Bedeutung für Medienkultur zu verstehen und zu beschreiben. Dazu freilich dürfte es für die Cultural Studies erforderlich werden, die Binnenrationalität der Kulturen und Diskurse des Managements von Medienunternehmen zu rekonstruieren und sich ausführlich und kritisch mit deren Denkmodellen, Begriffen, Methoden und Theorien auseinander zu setzen.

\section{Die unternehmensstrategisch forcierte globale Kommerzialisierung der Medienkultur seit Mitte der 1980er Jahre}

Als Bezugspunkt für ein Verständnis der Diskurse, Binnenrationalitäten und Strategien des Managements von Medienunternehmen bieten sich die strukturellen Veränderungen der Medienökonomie seit Mitte des letzten Jahrhunderts an. Der Strukturwandel der Medienbranchen ist seit dieser Zeit von Unternehmen vorbereitet, begleitet und forciert worden. Die Deregulierung, Privatisierung und Kommerzialisierung von zumeist nationalen Mediensystemen, die gegenwärtig in dieser Form aufhören zu existieren und besser als Teilsysteme einer globalen Medienökonomie verstanden werden sollten (vgl. Karmasin/C. Winter 2000: 2I-27), stellt eine Entwicklung dar, die nicht nur in der Politischen Ökonomie der Medien (vgl. insbes. Herman/McChesney I997 oder McChesney I998), den Medien- und Kommunikationswissenschaften (vgl. Picard I997; Jarren/Meier I998 oder Kleinsteuber/Thomaß I999), sondern auch in der Betriebswirtschafts- und Manage- 
mentlehre ausführlich für verschiedene Branchen diskutiert und untersucht wird (vgl. zuletzt Sjurts 2000 sowie zum internationalen Medienmanagement Apfelthaler 2000).

\section{Globalisierung und Kommerzialisierung: Die Richtung des Wandels}

Die globale Kommerzialisierung (vgl. ausf. C. Winter/Karmasin 200I) markiert die offenkundigste Veränderung der Bedingungen und Voraussetzungen von Medienkultur, zu der neben Veränderungen der Produktions- und Allokationsvoraussetzungen insbesondere Entwicklungen in der Werbebranche, der Politik und der Technik beigetragen haben. Die größten Antreiber und Gewinner der globalen Kommerzialisierung waren freilich die großen und transnational agierenden Medienunternehmen (vgl. Herman/McChesney I997; aus der Perspektive von Medienmanagement Karmasin/C. Winter 2000: 22-29). Um den strategischen Diskurs des Medienmanagements einordnen und nachvollziehen zu können, werden diese Entwicklungen hier kurz in Erinnerung gerufen.

Globalisierung ist seit Levitts These von der Globalisierung der Märkte 1983 zentrales Thema im Management jeder größeren Medien-Unternehmung (vgl. Levitt I983). Diese These besagt, dass Unternehmen nur wettbewerbsfähig bleiben, wenn sie global konkurrieren können. Wie sehr sich diese These für die Medienbranche bewahrheitet hat, zeigen Entwicklungen auf regionalen Medienmärkten, die einmal als geschlossene galten - man denke an den Kölner Zeitungskrieg, in dem skandinavische Unternehmen einen regionalen Zeitungsmarkt durcheinander wirbelten, oder an Engagements von CNN bei regionalen Fernsehsendern. Kommerzialisierung steht in diesem Zusammenhang für das Ziel von Unternehmen, die Möglichkeiten marktorientierter Refinanzierung zu verbessern. Dieses Ziel verfolgen Medienunternehmen im Hinblick auf die Steigerung von Verkaufs- und vor allem von Werbeerlösen, deren Zunahme die maßgeblichere Veränderung darstellt. Eine weiterer Entwicklung ist in diesem Zusammenhang weiter die generelle Zunahme der Bedeutung ökonomischer Rationalität zur Beurteilung der Medien und des Mediensystems, die als Ökonomisierung diskutiert wird und die im Kontext von Maßnahmen zur Deregulierung und Privatisierung die Medienökonomie erheblich verändert hat. Diese politischen Entwicklungen bedingen strategische Neuorientierungen vieler Unternehmen, 
die nun verstärkt versuchen, ihre Marktpositionen durch externes Wachstum, also durch Übernahmen, Allianzen und Kooperationen zu sichern und zu entwickeln. Sie ist spätestens seit der Übernahme des Medienmultis Warner-Communication durch das Pressehaus Time Inc. im Jahr I989 für jedermann nachvollziehbar. Nun entwickelten fast alle größeren Medienunternehmen geostrategische Optionen, die über ihre angestammten Märkte und vor allem über die Grenzen von Ländern hinaus zielen. Für die nun einsetzenden strategischen Überlegungen ist der Terminus Konglomeration geprägt worden, der in engem Zusammenhang mit der Strategie der »vertikalen Integration« steht. Vertikale Integration meint die Ausweitung der Aktivitäten vor allem auf vor- und nachgelagerte unternehmerische Aktivitäten - also etwa den Zukauf technologischer Kenntnisse in der Produktion oder etwa den von Know-how im Service oder der Kundenbetreuung. Als letzte grundlegende Veränderung der Bedingungen von Medienkultur muss schließlich die Konvergenz der bislang weitgehend getrennt operierenden Branchen Medien, Telekommunikation und Computer genannt werden. Digitalisierung und Globalisierung einer entsprechenden technischen Infrastruktur verändern derzeit global die Märkte, Dienste und Wertschöpfungsaktivitäten von Medienunternehmen. Selbst mit Blick auf die USA, wo nach Meinung vieler Experten bestimmte Entwicklungen in Europa vorweg genommen werden (vgl. Picard 1997), ist es sicherlich nicht möglich, die Folgen der Integration der Märkte und Geschäftsfelder von Telekommunikations-, Medien- und Computerbranche vorherzusagen. Die kurze Erinnerung dürfte ausreichen, um die Annahme eines erheblichen vor allem unternehmerischen, aber auch politischen, technischen und geographischen Wandels insbesondere der materialen Bedingungen und Voraussetzungen von Medienkultur zu belegen.

\section{Der istrukturelle Gesamtzusammenhang" des strategischen Handelns: "Management» aus der Perspektive auf die Wettbewerbsfähigkeit der Aktivitäten von Unternehmen}

Diese Darstellung der strukturellen Entwicklungen in der Medienökonomie berührt den Diskurs des strategischen Medienmanagements nicht. Sie kennt auch keine Handelnden oder konkrete Strategien einzelner Unternehmen. Die eher an Strukturen orientierte Darstellung bietet - das wissen wir seit der Kritik am Strukturalismus (vgl. 
Hall I999b) und der frühen Diskussion über Globalisierung (vgl. Wolff I99I; Ang 1999) - keine Ansatzpunkte für die Beschreibung von konkreten Veränderungen in der »Produktion « und »Allokation« von Medienkultur, die auch in Halls encoding/decoding-Modell oder Johnsons Zirkulationsmodell nicht erfasst werden können, weil sie nicht für die Differenzierung organisationaler und wettbewerbsspezifischer Unternehmensaktivitäten entwickelt worden sind. Sie und der Wandel auf der Handlungsebene werden sorgfältig und kontrovers in den Führungsetagen von Medienunternehmen und der Managementlehre analysiert und diskutiert - freilich nur im Hinblick auf ihre Bedeutung für Unternehmen, nicht für Kultur und Gesellschaft. Führungsetagen und Business-Schools sind die Orte des komplexen Diskurses »strategisches Management« (vgl. Montgomery/Porter 1996; Mintzberg/Ahlstrand et al. 1999), der die verschiedensten Aktionsund Reaktionsmöglichkeiten von Unternehmen auf unterschiedlichsten Ebenen kennt, die ihrerseits Gegenstand eigener Diskurse und Managementschulen sind (vgl. die Darstellung der »Managementschulen « sowie die Literatur bei Mintzberg/Ahlstrand et al. I999; zum strategischen Medienmanagement vgl. Maier 2000).

Den international erfolgreichsten Bezugsrahmen zur Analyse und Gestaltung strategischer Aktivitäten von Unternehmen hat Michael E. Porter entwickelt (vgl. Porter 1999). Sein Modell der Wertkette (vgl. Abb. 3) stellt alle Aktivitäten der Unternehmung in einen strukturellen Gesamtzusammenhang und kann in seiner Bedeutung für die Managementlehre und -praxis nicht unterschätzt werden (vgl. exempl. für die Medienbranche Zerdick et al. 1999).

Porters Modell etabliert eine Sichtweise auf den strukturellen Gesamtzusammenhang von Produktion, Allokation, Rezeption und Nutzung konsequent von der »anderen Seite«, vom Nutzer, Kunden bzw. Konsumenten her. Dadurch unterscheidet es sich von den klassischen Modellen der Güterproduktion, wie sie etwa für Halls encoding/decoding oder Johnsons Zirkulationsmodell orientierend waren, die von der Produktionsseite ausgehen, sondern auch von herkömmlichen, am Arbeitsablauf orientierten Produktionsmodellen aus der Betriebswirtschaft, die ebenfalls bei der Produktion, an der Entwicklung von Ideen und Produkten ansetzen und die erst Produktion, Preisfestlegung sowie Werbung und Allokation berücksichtigen, bevor in ihnen schlussendlich der Konsument oder Kunde auftaucht.

Im Vordergrund des Modells von Porter stehen primäre Unter- 
Abbildung 3: Porters Modell der Wertkette

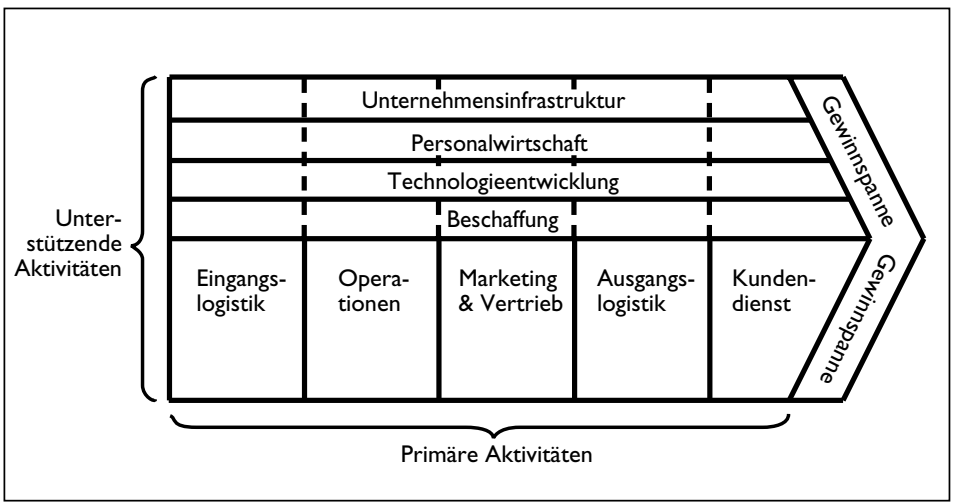

Quelle: Porter 1999: 66

nehmensaktivitäten (Eingangslogistik usf.). Auf diese und den Wandel ihrer Bedingungen und Voraussetzungen auf den jeweiligen Stufen der unternehmerischen Leistungserstellung ist die folgende Darstellung konzentriert. Die sekundären oder unterstützenden Unternehmensaktivitäten (Personalwirtschaft usf.) dienen - wie der Name schon sagt - der Unterstützung der primären Unternehmensaktivitäten und werden auf diese ausgerichtet. Das strategische Handeln des Managements von Medienunternehmen kann nur verstanden werden, wenn es gelingt, die Ausrichtung und die operativen Ziele der primären Unternehmensaktivitäten $\mathrm{zu}$ verstehen. In diesem Zusammenhang ist es - sowohl in der Planung als auch in der Analyse und der Kritik - von Vorteil, eine Vorstellung davon entwickeln zu können, welche unternehmensexternen und unternehmensinternen Veränderungen zu einer Verbesserung der Leistungserstellung im Prozess der unternehmerischen Werterstellung beitragen können. Als Ausgangspunkt für die Darstellung wird hier ein modifiziertes Modell der Wertschöpfung verwendet, das den wichtigen Unterschied kommerzieller Wertschöpfung gegenüber nichtkommerziellen Formen der Produktion von Medienkultur betont. Zentral ist in dieser Darstellung der Zusammenhang von Investition und Konsum: Kommerzielle Medienunternehmen produzieren (überwiegend) für eine bestehende oder erwartete Marktnachfrage. Das modifizierte Modell stellt nach wie vor Porters Leistung heraus: die konsequente Orientierung des strategi- 
schen Handelns von Unternehmen an der Erstellung von Wert für den Kunden. Es hebt hervor, dass Erlöse wieder zu Investitionen werden, wenn sie erneut Erlöse versprechen (vgl. Abb. 4).

Abbildung 4: Grundmodell der Darstellung primärer Unternehmensaktivitäten in einer Wertschöpfungskette

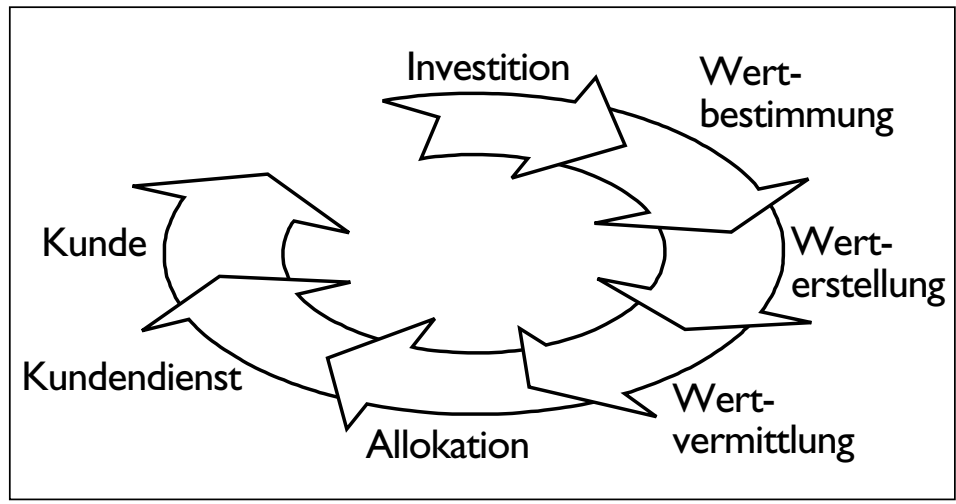

\section{Die globale Transformation der Bedingungen und Voraussetzungen primärer Unternehmensaktivitäten}

Die Wertkette bietet eine sehr konkrete Orientierung, wie die oben genannten Entwicklungen (Globalisierung usf.) in den Kontext des strategischen Handelns von Medienmanagement gestellt werden können. Zunächst macht die Wertkette deutlich, dass natürlich nicht nur Märkte, sondern die Aktivitäten auf jeder Stufe unternehmerischer Leistungserstellung zunehmend in einen globalen Wettbewerb eintreten. Wettbewerbsvorteile auf Medienkulturmärkten entstehen durch die Leistung auf den einzelnen Stufen der Wertketten und die Art und Weise der Abstimmung dieser Leistungen. Durch das strategische Managementhandeln in kommerziellen Medienorganisationen kommt es aber nicht nur zu Wettbewerbsvorteilen oder -nachteilen. Vielmehr bedingt dieses Handeln und die Ausweitung dieses Handelns auf immer mehr ursprünglich nationale Mediensysteme und -branchen einen substanziellen Wandel der vormals zu einem größeren Teil 
nichtkommerziellen und öffentlich-rechtlich produzierten und allozierten materialen Basis von Medienkultur. Bevor der Wandel von einer eher öffentlich-rechtlichen hin zu einer privat-kommerziellen Medienkultur diskutiert und analysiert werden kann, sollte freilich die kommerzielle Medienkultur angemessen, also auch im Hinblick auf ihre internen Widersprüche und ihre faktische Komplexität, verstanden sein. Es ist demnach $z u$ fragen und zu erforschen, wie Unternehmen mit einzelnen Stufen medialer Wertschöpfung auch die Bedingungen und Voraussetzungen des Umgangs mit der materialen Basis von Medienkultur verändern.

Das Modell der Wertkette bietet sich als Folie für eine Beschreibung der Aktivitäten, Praktiken, Orientierungen und Ziele von Akteuren an, die in unterschiedlichen Kontexten an der medialen Wertkette anzutreffen sind. In der Regel handelt es sich dabei um Organisationseinheiten von Unternehmen, etwa um Profit-Center oder Strategische Geschäftseinheiten, die durch ihre Aufgaben und Struktur als eigenständige Einheit identifiziert und abgegrenzt werden können. Sie unterscheiden sich aufgrund unterschiedlicher Aufgaben, vor deren Hintergrund sie ihre eigenen Praktiken und Werte entwickelt haben. Es ist durchaus möglich und in der Betriebswirtschaftslehre fast schon üblich, hier von Unternehmensteilkulturen zu sprechen, um die je spezifischen Systeme »von Wahrnehmungs-, Denk- und Verhaltensweisen « unterscheiden zu können, die in diesen Kontexten »als Voraussetzung für erfolgreiche Arbeit« (Buhr 1989: 44) entstanden sind. In der folgenden Darstellung des Handelns auf den einzelnen Wertschöpfungsstufen werden diese als »Kulturen« dargestellt, wodurch Unterschiede und Widersprüche zwischen den Stufen - im Sinne eines konflikttheoretischen Kulturbegriffs, wonach das Handeln und die Orientierungen von Gruppen immer auch in Konflikt stehen mit denen anderer Gruppen - besser sichtbar werden. Orientiert ist die Darstellung an den in Abb. 4 angezeigten Stufen.

Investition: Die Globalisierung der Finanzmärkte hat insbesondere für börsennotierte Unternehmen der Medien- und Kulturbranche neue Finanzierungs- und Investitionsmöglichkeiten geschaffen. Diesen Möglichkeiten stehen aber die Zwänge einer höheren Renditeerwartung gegenüber: Der Shareholder-Value wird zu einem Unternehmensziel. Im Kontext dieses Ziels gewinnen Übernahmen, Allianzen und Joint-Ventures an Bedeutung. Diese Art von Aktivitäten hat 
häufig größeren Einfluss auf den Wert eines Unternehmens an der Börse als unternehmensinterne Aktivitäten, wie z. B. die Entwicklung neuer Produkte. Korrespondierend gewinnen für das Management Investor-Relationsaktivitäten in international tätigen börsennotierten Medienunternehmen an Bedeutung. Durch diese Entwicklungen haben sich börsennotierte Medienunternehmen den Einflüssen der Kapitalmärkte geöffnet und die Shareholder, die Aktionäre, zur dominierenden Anspruchsgruppe dieser Unternehmen gemacht.

Wertbestimmung: Die Öffnung von Medienmärkten und technische Entwicklungen wie z.B. Satelliten-Fernsehen haben die Differenzierung und Vermischung von Orientierungen und entsprechend auch von Konsumstilen und Produkten verstärkt. Diese Entwicklung macht gesicherte Aussagen über marketingrelevante Orientierungen und Werte von Kunden immer schwieriger. Darüber hinaus erschweren sie die Selektion von Märkten und die Positionierung gegenüber den Wettbewerbern. Die Nähe zum Markt, die häufig ein Vorteil von regionalen und kleineren Medienunternehmen ist, wird auf dieser Stufe zunehmend durch die Möglichkeiten größerer Unternehmen zur Investition in Forschung und Entwicklung wettgemacht. Auch haben größere Unternehmen zunehmend mehr Möglichkeiten zur Entwicklung von Verbundstrategien, die im Zuge der Digitalisierung von Content immer wichtiger werden. Auch auf dieser Stufe nimmt der Aufwand für Forschung und Entwicklung zu. Es entstehen neue Forschungs- und Entwicklungskulturen, in denen andere Anspruchsgruppen als die Mitarbeiter, Konsumenten oder Shareholder in den Blick geraten, die aber nicht über strategische Macht verfügen.

Werterstellung: Die Werterstellung ist zunächst mit weiteren Überlegungen zur Produktentwicklung (medienformübergreifend, medienformintegrierend oder medienformdifferenzierend) konfrontiert. Immer wichtiger sind auf dieser Stufe Entscheidungen über Eigen- oder Fremdproduktion, über Standorte, Teams, Netzwerke usf., die Kostenkalkulation sowie die differenzierte Entwicklung von Leistungsangeboten für verschiedenste Medienmärkte und Zielgruppen. Die Entwicklungen auf dieser Stufe ist vor allem durch die so genannte Flexibilisierung der Produktion gekennzeichnet, die vor allem eine Ausgliederung darstellt. Quer durch alle Medienbranchen ist auf dieser Stufe die Entstehung eines neuen Produktionsunternehmertums zu beobachten, das - seien es Radiobeiträge, journalistische Meldungen, 
Fernsehsendungen usf. - aus relativ kleinen Einheiten besteht. Diese Produzenten und Dienstleister verfügen nur über eingeschränkte Finanzierungs- und Trainingsmöglichkeiten und sind von vertriebsstarken Großunternehmen abhängig.

Kommunikative Wertvermittlung: Unter Bedingungen des Überangebots unterliegt die Medienwirtschaft zunehmend den Regeln einer Ökonomie der Aufmerksamkeit und machen Marketing und Werbung zu einem immer entscheidenderen Erfolgsfaktor - »Wer nicht wirbt, der stirbt« gilt heute mehr denn je, insbesondere deshalb, weil sich die kulturellen Kontexte der Konsumenten immer stärker mit jenen Kulturen überlappen, die durch Werbung/Marketing für diese Zielgruppen konstituiert werden. Abzulesen ist dies an den steigenden Aufwendungen der Medien- und Kommunikationsbranche für Werbung, die noch höher ausfallen würden, wenn Ausgaben für PR, Marketing usf. hinzugerechnet würden.

Allokation: Durch politische und technische Entwicklungen einerseits, die eine Globalisierung von Vertriebsstrukturen ermöglicht haben, und andererseits durch die Fragmentierung und Differenzierung von Medienmärkten im Zuge von E-Commerce und Datenbanken, durch die neuen Möglichkeiten zur Individualisierung des Vertriebs geschaffen werden können, entstehen für Unternehmen eine Vielzahl von neuen Möglichkeiten und Herausforderungen. Auf dieser Stufe hat sich zuletzt gezeigt, dass auf neu entstehenden Märkten Schnelligkeitsvorteile wichtiger sind als Größenvorteile. Die zunehmende Bedeutung der Technik für den Vertrieb verändert klassische Vertriebskulturen. Während diese einmal vor allem vom persönlichen Engagement der Verkäufer - sei es auf Messen, gegenüber Großkunden oder etwa gegenüber Experten - abhingen oder zumindest geprägt waren, spielen nun Neuerungen wie etwa Vertriebsautomatisierungen oder die elektronische Steuerung von Lieferketten (Supply Chain Management) eine immer wichtiger Rolle. Auf dieser Stufe ist Bertelsmann derzeit weltweit das mit Abstand technisch am stärksten integrierte Medien-Vertriebsunternehmen.

Kundendienst: Der Stellenwert der Nähe von Produkten und Dienstleistungen zum Konsumenten führen den Kundendienst im weitesten Sinne in das Zentrum von Unternehmensaktivitäten. Die Betreuung von Endkunden am Produkt gewinnt an Bedeutung, und Kundenbindung wird ein immer kostbareres Gut. Für ausgewählte 
Kunden wird es zunehmend Events sowie andere produkt- und angebotsbezogene, aber nicht klassisch werbliche bzw. PR-Leistungen geben. Dieser Kundendienst ist von Aktivitäten zu unterscheiden, in denen potenzielle Kunden im Mittelpunkt stehen, die hier auf der nächsten Stufe unter »Kunde« berücksichtigt sind. Durch immer ausgefeilteres CRM (Customer-Relationship-Management) wird versucht, Medien und Medienangebote zu einem immer wichtigeren kulturellen Bestandteil des Lebens der Konsumenten zu machen. Hier gibt es ganz verschiedene Wege, wie etwa von Disney (Disney Parks) oder Bertelsmann (vor allem Clubs).

Kunde: Der durch Medienentwicklung und Wettbewerb induzierte Wandel der Medien und ihrer Angebote erfordert es, neue Angebote immer wieder auf Neukunden auszurichten. Um hier dennoch immer wieder anschlussfähig anbieten zu können, wird Motivforschung zur Medienethnographie und betreibt eine an den Cultural Studies orientierte Erforschung der Lebens- und Mediennutzungsformen ihrer Kunden.

Die Darstellung der Anforderungen und Einflüsse auf die Stufen der medialen Leistungserstellung zeigt einen Prozess, bei dem ständig die Grenzen von Unternehmen, Nationen und Sprachen und vor allem von Praktiken und ihren Diskursen überschritten werden. In der Sprache der Managementlehre wird daher - wie ich finde zu Recht - von verschiedensten »Wertschöpfungskulturen« gesprochen, von »Shareholder-Value-Kulturen «, »Forschungs- und Entwicklungskulturen« von in Netzwerke eingebundenen flexiblen »Produktionskulturen«, von »Agentur-Kulturen«, von »Vertriebskulturen«, von »Dienstleistungskulturen « und wieder von »Forschungskulturen«, die aber nicht auf Innovation und Entwicklung, sondern auf Alltagskultur spezialisiert sind. Diese »Kulturen« folgen keinesfalls einer Logik oder einem professionellen Code i. S. von Hall (I977). Vor diesem Hintergrund überrascht es nicht, dass Medienangebote hybride Bedeutungsstrukturen aufweisen können und mit Bezugsrahmen wie der Kulturimperialismus- oder Kulturindustrietheorie nicht angemessen zu verstehen sind. Weiter überrascht es auch nicht, dass Medienunternehmen alles Erdenkliche unternehmen, um aus möglichen Kunden tatsächliche Kunden zu machen, und dass sie dazu die Planung ihrer Leistungserstellung so frühzeitig wie möglich an der Erstellung von möglichen Leistungen für Konsumenten orientieren. Die Darstellung zeigt aber vor allem, dass sich nationale und branchenspezifische 
Formen der Produktion und Allokation von Medienkultur auflösen, wenn sie global unter Kommerzialisierungsdruck geraten.

Unter diesen Bedingungen wird die Art und Weise der Abstimmung der unternehmerischen Leistungserstellungsprozesse zur zentralen und wettbewerbsentscheidenden unternehmerischen Aktivität. Diese Abstimmung der Aktivitäten auf den Stufen ist die Aufgabe des Managements. Das Medien-Management ist der rationale Kontext des strategischen Handelns in Medienunternehmen.

Die zunehmende Komplexität der Aufgaben, des Wettbewerbs sowie der Integration und Vernetzung in der Branche verändert aber im Sinne einer Managementorientierung nicht nur die Anforderungen an Führungskräfte in der Medienbranche (vgl. Stürzebecher I997; C. Winter 2000b). Vielmehr verändern die im Kontext von Management implizit erwarteten und explizit formulierten Anforderungen auch die Anforderungen auf allen anderen Stufen und insbesondere auch an Kreative und Journalisten (vgl. Raulf I997). Deutlich ist dies belegt durch die Transformation klassischer Organisationsstrukturen von Medienunternehmen, bei der ursprünglich auf geographische Orte und entsprechend strukturierte Aufbau- und Ablauforganisationen sowie überschaubare nationale Märkte abgestimmte Organisationen in glokale, lose gekoppelte Projektstrukturen aufgelöst werden. Und dennoch folgt das Handeln von Managern und ihr Management von Leistungserstellungsaktivitäten keinesfalls einem Code oder einer spezifischen professionellen Rationalität i.S. von Hall. Die Aktivitäten des Managements sind so widersprüchlich und vielfältig wie die Mediennutzung der Konsumenten. Vor dem Hintergrund der fortschreitenden Kommerzialisierung stellen sich erneut die bereits angeführten Fragen: Wie formen die im Kontext des Diskurses Management beschriebenen Entwicklungen »auf spezifische Weise die strukturellen und globalen Konfigurationen der Hegemonie, innerhalb derer sich die gegenwärtigen Praktiken von Medienrezeption und -konsum herausbilden«? (Ang I999: 330). Wie können die »diskursproduzierenden « Institutionen in die Analyse einbezogen werden? (Hall I997: 44). Wie kann vor dem Hintergrund von Grossbergs Forderung: »Wir müssen Cultural Studies >ökonomisieren vieren« (Grossberg 2000: 294) der (Management-)Diskurs diskursiviert werden? 


\section{Voraussetzungen umfassender Medienkulturforschung und - kritik UNTER den Bedingungen eINER gLOBALEN KOMMERZIALISIERUng DURCH Medienmanagement}

An dieser Stelle gibt es mehrere Möglichkeiten, sich dem Diskurs Medienmanagement zu nähern, um die Voraussetzungen bestimmen zu können, unter denen unter den Bedingungen einer globalen Kommerzialisierung durch Medienmanagement gegenwärtig eine umfassende, also nicht auf einzelne Momente und Aspekte gerichtete Erforschung und Kritik von Medienkultur geleistet werden könnte. Ein wichtiger Bezugspunkt ist dabei sicherlich die frühe philosophische Kritik am Management als einem Symptom der moralischen Krise der Gegenwart (vgl. MacIntyre I9987). Dieser eher philosophische Diskurs hat den Nachteil, dass er - ebenso wie der Managementdiskurs - noch mit den Cultural Studies vermittelt werden müsste. Es bietet sich an, von dieser Vermittlungsproblematik ausgehend, die angesprochenen Probleme und Perspektiven im Kontext eines in den Cultural Studies eingeführten Diskurses $\mathrm{zu}$ thematisieren. Nahe liegend ist derzeit eine Orientierung an dem Modell von Kultur, das im Kontext des Kurses D 3I8 »Culture, Media and Identities« an der Open University verwendet wird. Im Zusammenhang mit diesem Kurs ist das Modell des Circuit of Culture entstanden, an dem sich sechs (!) Bände orientieren und das als eine Weiterentwicklung von Johnsons Zirkulationsmodell von Kultur bezeichnet wird (vgl. du Gay/ Hall et al. I997: 3; vgl. hier Abb. 5).

Es stellt sich nun die Frage, ob und wie im Rahmen dieses Modells eine Beobachtung, Analyse und Kritik der von mir beschriebenen kommerziellen und managementgetriebenen Durchdringung der Produktion und Allokation von Medienkultur möglich ist, bei dessen Einführung die theoretischen Gründe - anders als bei den referierten Modellen von Hall und Johnson - nicht explizit ausgeführt wurden (vgl. ebd.). Dieses Modell vermittelt zuallererst die Einsicht, dass alles irgendwie und mit allem zusammenhängt und dass Kultur dabei sehr wichtig ist. Diese Einsicht und dieser Hinweis sind wichtig, aber das reicht nicht. Das erinnert zu sehr an die Kulturstudien von Raymond Williams vor der Kritik durch Edward P. Thompson (s.o.). Zweifelsohne - und das soll hier auch nicht vergessen werden - thematisiert das Modell wichtige Perspektiven der Cultural Studies und entwickelt manche sogar weiter. Die Herausgeber haben mit den oben erwähn- 


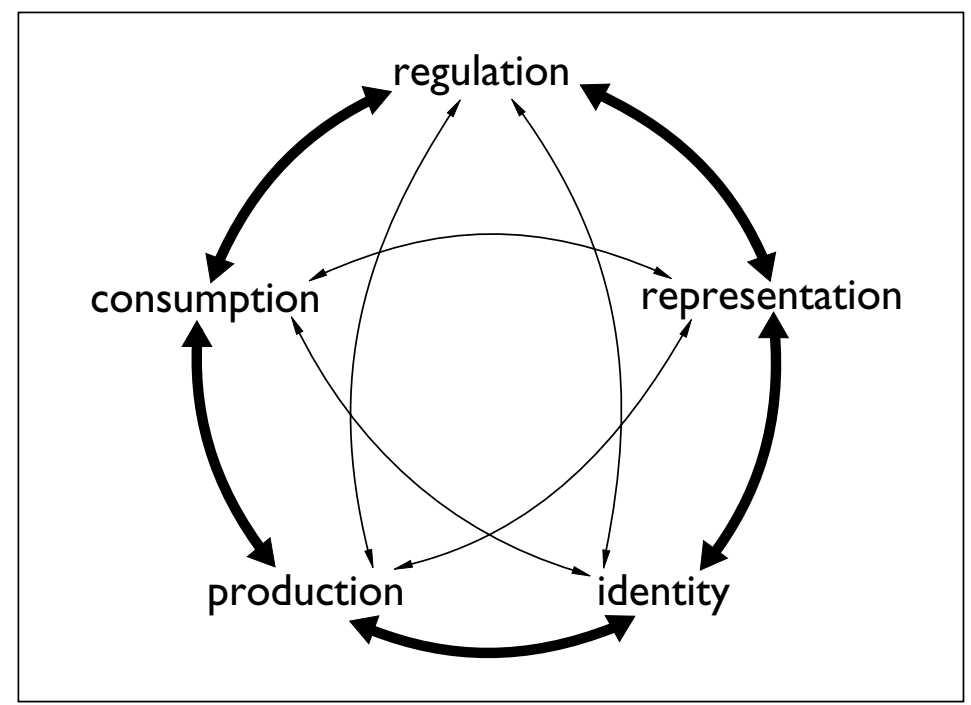

Quelle: du Gay 1997: 3

ten Bänden das Gesichtsfeld der Cultural Studies erweitert und im Zusammenhang mit der Erforschung von Medienkultur die Unternehmung und spezifische Bedingungen und Voraussetzungen des Handelns von und in Unternehmen in die Perspektive gerückt. $\mathrm{Zu}$ nennen sind Aspekte wie »Unternehmenskultur « (vgl. du Gay/Hall et al. I997: 44 ff.; Negus 1997) und die Rolle von Kreativen/Symbolanalysten in der Kulturindustrie (du Gay/Hall et al. 1997: 62 ff.), die im Einzelband auf die Rolle von Managern ausgeweitet wird, die aber nicht beim Namen genannt werden (vgl. insbesondere Robins 1997).

Der von mir aufgezeigte und managementinduzierte Wandel der Bedingungen und Voraussetzungen der materialen Basis von Medienkultur wird sehr deutlich im Fazit des Kapitels »What in the world's going on? « von Kevin Robins (1997). Robins setzt sich fast ausschließlich mit Thesen von Kenich J. Ohmae, Leiter der McKinsey Niederlassung in Tokyo, auseinander und weicht dabei der von ihm im Titel gestellten Frage so gut aus, wie es eben geht. Er setzt sich gerade nicht mit der Handlungslogik der von ihm so genannten "global business perspective« (ebd.: 44) auseinander und umkreist seine eingangs gestellte Frage: »What in the world's going on?« mit Zitaten 
aus Texten von Managern wie Ohmae, von denen ersichtlich wird, dass sie ihm nicht geheuer sind: "You really have to believe, deep down, that people may work sin< different national environments but are not >of $<$ them. What they are >of $<$ is the global corporation« (ebd.: 49). Die globale Unternehmung steht aber im Zentrum seiner Frage »What in the world's going on? « und der Frage nach den Bedingungen und Voraussetzungen von Medienkultur. Robins bleibt jedoch eine Antwort auf die Frage schuldig. Sein im letzten Satz geäußertes Fazit bleibt im Rahmen des Modells Circuit of Culture und hebt noch einmal hervor, dass alles mit allem komplex verbunden ist:

IThe globalization process must be seen in terms of the complex interplay of economic and cultural dynamics, involving confrontation, contestation and negotiation. The global future is therefore sure to have surprises in store for us॥ (ebd.: 45).

Die Allgemeinheit dieser Antwort werte ich als zwingendes Argument für eine Neuausrichtung der Cultural Studies, für die ich hier abschließend argumentieren möchte. Der Circuit of Culture ist in seiner jetzigen Form ungeeignet, um Theorien und Methoden weiter zu entwickeln, eine Arbeit, die bislang zu den wichtigsten Stärken der Cultural Studies zählt. Insbesondere im Hinblick auf die Erforschung der materialen Basis von Medienkultur, der Produktion und der Allokation/Distribution, entsteht der Eindruck, dass die Produktionsanalyse und die Ökonomie »zum fünften Rad am Wagen« der Kulturanalyse werden (Grossberg I997: I9). Ich plädiere dafür, dass die kritische und empirisch fundierte konstruktive Auseinandersetzung mit Gesellschafts- und Kulturwandel und mit neuen Theorien und Konzepten, aber auch mit anderen Positionen und Perspektiven nicht nur bewahrt, sondern weiter ausgebaut werden sollte. Dazu werden hier Forderungen von Hall (I977 u. I999a), Ang (I999) und Grossberg (2000) - »Wir müssen Cultural Studies >ökonomisieren nomie >diskursiveren«« - in den Kontext des Circuit of Culture gestellt, der - um Fragen und empirische Forschungen anzuregen - dazu ergänzt wurde. Es geht um die Frage, ob - und wenn ja: mit welchen Folgen - die Verbindungen zwischen den in der Cultural StudiesForschung relevanten Momenten unter Einfluss vom Medien-Management als dem dominanten hegemonialen »professionellen Code« geraten oder geraten sind. Konkret hieße dies im Hinblick auf den Circuit of Culture, dass jeweils der konkrete Einfluss des Management 
auf die im Modell durch die Doppelpfeile angegebenen Beziehungen zu untersuchen wäre.

Die Umstellung der Koordination der Bedingungen und Voraussetzungen von Medienkultur von eher regionalen, medienbranchenspezifischen und häufig öffentlich-rechtlich organisierten und legitimierten Verfahren auf ein strategisches Management kommerzieller globaler Unternehmen bedeutet hier eine beachtliche Veränderung. Die Folgen dieses Wandels, die theoretisch sicher kaum prognostizierbar sind, können im Hinblick auf widersprüchliche Entwicklungen im Zusammenhang mit dem Circuit of Culture empirisch erforscht werden. Medienmanagement tritt in eine sehr ungleiche Konkurrenz mit nichtkommerziellen kulturellen Orientierungen und Angeboten, deren Erhaltung und Wahrnehmung unter Bedingungen einer globalen Kommerzialisierung immer schwieriger werden. Die Art und Weise des Umgangs mit dem Diskurs und der Praxis von Medienmanagement wird mit darüber entscheiden, ob und wie Cultural Studies auch zukünftig angemessen Kulturforschung und Kulturkritik betreiben können. Abschließend will ich meine These, wonach Medienmanagement zunehmend stärker und grundsätzlicher Einfluss auf die Kultur nimmt, im Zusammenhang mit zwei Neuerscheinungen auf dem Buchmarkt erläutern. Sie belegen nach meinem Dafürhalten die Notwendigkeit und die Chancen einer »Neuorientierung« in den Cultural Studies.

Bei den beiden Publikationen handelt es sich um Douglas Rushkoffs sehr unglücklich mit »Der Anschlag auf die Psyche« übersetzte Arbeit »Coercion. Why We Listen to What >They< Say« (Rushkoff 2000) und Naomi Kleins »No Logo!« (Klein 200I) - hier unterscheidet sich der deutsche Titel von dem kanadischen nur dadurch, dass ein Ausrufezeichen hinzugekommen ist.

Rushkoffs Darstellung strategisch geplanter und erfolgreicher Manipulationen des Handelns von Konsumenten kann als eine Ergänzung zu ethnographischen Kulturstudien gelesen werden, in denen vor allem die taktischen Möglichkeiten von Konsumenten aufgezeigt werden. Er zeigt, dass Strategien des Verkaufens, Überzeugens und Beeinflussens bereits viel differenzierter sind, als es auch noch so kritische Konsumenten erahnen. Deutlich wird, dass viele der im Diskurs-Management als wichtig angesehenen Unternehmensaktivitäten, insbesondere die auf die Aufmerksamkeit und das »Verhalten« von Konsumenten gerichteten, nur dazu dienen, die Kontrolle über 
das Verhalten von Konsumenten zu vergrößern - wobei die Konsumenten selbst ihr Verhalten in diesen Fällen vielleicht als aktives und kritisches Handeln beschreiben würden. Die empirische und diskursanalytische Erforschung der Diskrepanz zwischen der Wahrnehmung strategischer unternehmerischer Aktivitäten als entweder manipulativ oder aber als wertsteigernd kann nicht mehr angemessen von einem Fach allein geleistet werden. Aus diesem Grund halte ich eine kulturwissenschaftliche, also die Grenzen von Fächern überschreitende, rationalitätskritische und teilnehmende Forschung für wichtig. Die seit der Debatte über Rationalität und das Verstehen fremder Kulturen bekannte Einsicht (vgl. C. Winter 2oooa: ig f.), dass Handeln nur aus dem Kontext der Praxisrationalität erschlossen werden kann, gilt auch für die professionellen Kontexte des Managements von Unternehmensaktivitäten, die wohl nur über eine Integration von Praxiserfahrungen und Praxiswissen verstanden werden können. ${ }^{5}$

Rushkoffs Beitrag, der auf die Beschreibung konkreter manipulativer Interaktionsstrategien gerichtet ist, erscheint vor dem Hintergrund von Naomi Kleins »No Logo!« aus kulturtheoretischer Perspektive noch überschaubar. Während Rushkoff konkrete Strategien, die auf das Handeln und Verhalten von Konsumenten gerichtet sind, als Manipulationsstrategien entlarvt, stehen bei Naomi Klein sehr viel umfassender angelegte Strategien im Vordergrund. Sie beschreibt in ihrem Buch, wie global agierende Konzerne den Konsum ihrer Angebote in basale kulturelle Differenzierungscodes integrieren. Klein konzentriert sich nicht auf die Mikroebene der Beeinflussung konkreter Konsumentenentscheidungen. Sie konzentriert sich auf Strategien, die auf eine immer umfassendere und vollständigere Integration von kulturellen Differenzierungsmerkmalen angelegt sind, mit denen Konsumenten sich von anderen Konsumenten als Menschen unterscheiden und die im glokalen Wettbewerb mit nichtkommerziellen kulturellen Differenzierungsmerkmalen zunehmend erfolgreich sind.

Die Bedeutung des Managements von global agierenden Konzernen und ihren Entscheidungen für die ganz konkrete Ausgestaltung des Lebens von immer mehr und immer unterschiedlichen Gruppen von Menschen wird bei Klein noch offenkundiger als bei Rushkoff insbesondere dort, wo sie den Einzug kommerzieller Strategien in vormals öffentliche Bereiche und Räume beschreibt: Das Management von Konzernen verändert durch strategische Bemühungen um Wettbewerbsvorteile und Marktmacht die Bedingungen und Vorausset- 
zungen von Kultur auf der ganzen Welt grundlegend. Kommerzielle Unternehmen produzieren einen zunehmenden Teil der Ressourcen für identitätsbildende Prozesse, ohne dass es einen öffentlichen oder rechtlich legitimierten Kontext jenseits des Marktes gibt, in dem diese Entwicklungen politisch-ethisch angemessene normativ reflektiert werden können.

Genau dazu bedarf es einer besseren Erforschung des Einflusses von Medienmanagement auf den umfassenden Kontext von Medienkultur. Ich bin der Auffassung, dass sich diese Forschung zu einem gewissen Grad auf die Terminologie der Managementwissenschaften einlassen muss, um den dort erreichten Grad wissenschaftlicher Differenzierung nicht zu weit zu unterlaufen. Ein Anfang sollte mit der Vorstellung entsprechender Begrifflichkeiten und Modelle sowie der Rekonstruktion der bereits im Kontext der Cultural Studies geleisteten Erforschung und Kritik, insbesondere der Produktion und Allokation von Medienkultur, geleistet sein. Es wird nun darauf ankommen, ob es gelingt, die Einflüsse des neuen professionellen Codes Medienmanagement genauer zu erforschen und zu beschreiben und diese Erkenntnisse auch in den Diskurs über Strategisches Management und die für Liberalisierung, Deregulierung und Privatisierung zuständigen politischen Kontexte hineinzutragen.

Im Kontext transdisziplinär angelegter und auch die Wirtschaftswissenschaften integrierenden Arbeiten, dürfte diese Entwicklung des Projekts Cultural Studies vor allem in den offenen Kultur-, Kommunikations- und Sozialwissenschaften die Chance haben, die skizzierte doppelte empirische und theoretische Herausforderung einer Erforschung der hegemonialen Managementpraktiken und -diskurse erfolgreich anzunehmen. Im deutschsprachigen Raum scheinen insbesondere die »Neueren Kulturwissenschaften« sowie die Mediensoziologie und die Medienökonomie geeignet, eine kritische Erforschung des Managements von Medienkultur zu leisten (vgl. exemplarisch C. Winter 1996; Münch 1996; Cornely/Macho I996; zuletzt Düllo/C. Winter 2000 und C. Winter 2000 b sowie absehbar die Beiträge in Karma$\sin /$ Knoche/C. Winter 200I). 


\section{Anmeriungen}

I Für hilfreiche Kritik und Kommentare zu einer ersten Fassung des Beitrags danke ich Udo Göttlich, Andreas Hepp und Rainer Winter.

2 Den strategischen Umgang unterscheidet bekanntlich vom taktischen Umgang, dass er über Ressourcen der Produktion und Allokation verfügt, während der taktische Umgang immer ein Umgang mit vorgegebenen Produkten und Angeboten bleibt (vgl. de Certeau I988;

C. Winter I998; Hepp I999: 70 f.).

3 Im Folgenden wird an Stelle der Bezeichnung Distribution von Allokation gesprochen. Dadurch soll bewusst gehalten werden, dass nicht nur interessiert, dass etwas von a nach b gelangt, sondern vor allem auch, wie, an wen und unter welchen Bedingungen Medienangebote vertrieben werden.

4 Diese Abgrenzung ist insbesondere gegenüber der Politischen Ökonomie der Medien erforderlich, weil diese den Cultural Studies vorwerfen, dass sie es weder theoretisch noch politisch kompensieren konnten, die Brücken zu ihrer materialistisch-marxistischen Vergangenheit abgebrochen zu haben. Diese Kritik war häufig wenig konstruktiv und polemisch. Dies zeigt etwa der Beitrag »Politische Ökonomie und die Praxis der Cultural Studies« von Nicolas Garnham (I997). Sie erschweren eine gemeinsame Arbeit an wichtigen Fragen, die bei Garnham durchaus zu finden sind: »Where in the contemporary cultural studies' literature or research program are the studies of the cultural producers and of the organisational sites and practices the inhabit and through which they exercise their power?« (ebd.: 6I).

$5 \mathrm{Zu}$ Vorschlägen, wie dies unter Berücksichtigung der Erfahrungen von Studierenden zu leisten wäre, vgl. C. Winter zooob.

\section{Literatur}

Abu Lughod, J. (I991): »Going Beyond Global Babble«. In: Anthony D. King (Hg.), Culture Globalization and the World-System: Contemporary Conditions for the Representation of Identity, London: Macmillan Press, S. I3I-I37.

Adorno, Theodor W. (I972): »Résumé über Kulturindustrie«. In: Dieter Prokop (Hg.), Massenkommunikationsforschung 1: Produktion. Frankfurt a.M.: Fischer Taschenbuchverlag, S. 347-354. 
Ang, Ien (I999): »Kultur und Kommunikation. Auf dem Weg zu einer ethnographischen Kritik des Medienkonsums im transnationalen Mediensystem«. In: Roger Bromley/Udo Göttlich/Carsten Winter (Hg.), Cultural Studies. Grundlagentexte zur Einführung, Lüneburg: zu Klampen, S. 317-340.

Apfelthaler, G. (2000): »Medienmanagement als Internationales Management«. In: Matthias Karmasin/Carsten Winter (Hg.), Grundlagen des Medienmanagements, München: Fink, S. I97-218.

Bromley, Roger (I999): »Cultural Studies gestern und heute«. In: Roger Bromley/Udo Göttlich/Carsten Winter (Hg.), Cultural Studies: Grundlagentexte zur Einführung, Lüneburg: zu Klampen, S. $9^{-24}$.

Bromley, Roger (2000): »Multiglobalismen - Synkretismus und Vielfalt in der Populärkultur«. In. Caroline Y. Robertson/Carsten Winter (Hg.), Kulturwandel und Globalisierung, Baden-Baden: Nomos, S. 189-206.

Bromley, Roger/Göttlich, Udo/Winter, Carsten (Hg.) (I999): Cultural Studies. Grundlagentexte zur Einführung, Lüneburg: zu Klampen.

Buhr, Regina (1989): Unternehmen als Kulturräume: eigensinnige betriebliche Integrationsprozesse im transnationalen Kontext, Berlin: Ed. Sigma.

Certeau, Michel de (1988): Kunst des Handelns, Berlin: Merve Verlag.

Cornely, Bernd/Macho, Thomas (I996): »Kulturwissenschaft als Projekt. Ein Gespräch zwischen Bernd Cornely und Thomas Macho«. In: Carsten Winter (Hg.), Kulturwissenschaft: Perspektiven, Erfahrungen, Beobachtungen, Bonn: ARGult-Media, S. 5I-65.

Düllo, Thomas/Winter, Carsten (2000): »Die Kunst der Navigation: Kulturwissenschaft für das 2I. Jahrhundert«. In: Thomas Düllo/Arno Meteling/André Suhr/Carsten Winter (Hg.), Kursbuch Kulturwissenschaft, Münster: LIT Verlag, S. I-I3.

Featherstone, Mike (Hg.) (I990): Global Culture: Nationalism, Globalization and Modernity, London: Sage.

Featherstone, Mike (2000): »Postmodernismus und Konsumkultur. Die Globalisierung der Komplexität«. In: Caroline Y. Robertson/Carsten Winter (Hg.), Kulturwandel und Globalisierung, Baden-Baden: Nomos, S. 77-Io6.

Garnham, Nicolas (I997): »Political Economy and the Practice of Cultural Studies«. In: Marjorie Ferguson/Peter Golding (Hg.), Cultural Studies in Question, London: Sage, S. 56-73. 
Gay, Paul du (Hg.) (I997): Production of Culture/Cultures of Production, London: Sage.

Gay, Paul du/Hall, Stuart et al. (1997): Doing Cultural Studies: The Story of the Sony Walkman, London: Sage.

Giddens, Anthony (I995): Konsequenzen der Moderne, Frankfurt a.M.: Suhrkamp.

Göttlich, Udo (2000): »Schmiermittel für den Äther. Daily Soap Operas im Spannungsfeld globaler Formatverbreitung und lokaler Produktion«. In: Thomas Düllo/Arno Meteling/André Suhr/ Carsten Winter (Hg.), Kursbuch Kulturwissenschaft, Münster: LIT Verlag, S. I77-I85.

Göttlich, Udo/Winter, Carsten (I999): »Wessen Cultural Studies? Die Rezeption der Cultural Studies im deutschsprachigen Raum«. In: Roger Bromley/Udo Göttlich/Carsten Winter (Hg.), Cultural Studies: Grundlagentexte zur Einführung, Lüneburg: zu Klampen, S. 25-39.

Göttlich, Udo/Winter, Rainer (Hg.) (2000): Politik des Vergnügens. Zur Diskussion der Populärkultur in den Cultural Studies, Köln: Herbert von Halem Verlag.

Grossberg, Lawrence (I997): »Der Cross Road Blues der Cultural Studies«. In: Andreas Hepp/Rainer Winter (Hg.), Kultur - Medien - Macht: Cultural Studies und Medienanalyse, Opladen: Westdeutscher Verlag, S. 13-29.

Grossberg, Lawrence (I999): »Globalization and the >Economization< of Cultural Studies«. In: Bundesministerium für Wissenschaft und Verkehr/Internationales Forschungszentrum Kulturwissenschaften (Hg.), The Contemporary Study of Culture, Wien: Turia + Kant, S. 23-46.

Grossberg, Lawrence (2000): Globalisierung, Medien und Agency. What's Going on? Cultural Studies und Popularkultur, Wien: Turia + Kant, S. 287-315.

Grossberg, Lawrence/Nelson, Cary/Treichler, Paula (Hg.) (I992): Cultural Studies, New York/London: Routledge.

Hall, Stuart (I977): »Culture, the Media and the >Ideological Effect««. In: J. Curran/M. Gurevitch/J. Woollacott (Hg.), Mass Communication and Society, London: Edward Arnold, S. 315-348.

Hall, Stuart (I991a): »The Local and the Global: Globalization and Ethnicity«. In: Anthony D. King (Hg.), Culture Globalization and 
the World-System: Contemporary Conditions for the Representation of Identity, London: Macmillan Press, S. I9-39.

Hall, Stuart. (I99rb): »Old and New Identities, Old and New Ethnicities«. In: Anthony D. King (Hg.), Culture Globalization and the World-System: Contemporary Conditions for the Representation of Identity, London: Macmillan Press, S. 4I-68.

Hall, Stuart (I992): »Cultural Studies and its Theoretical Legacies«. In: Lawrence Grossberg/Cary Nelson/Paula Treichler (Hg.), Cultural Studies, New York/London: Routledge, S. 277-294.

Hall, Stuart (Hg.) (I997): Representation: Cultural Representations and Signifying Practices, London: Sage.

Hall, Stuart (I997): »The Work of Representation«. In: Ders. (Hg.), Representation: Cultural Representations and Signifying Practices, London: Sage, S. I3-74.

Hall, Stuart (I999a): »Kodieren/Dekodieren«. In: Roger Bromley/Udo Göttlich/Carsten Winter (Hg.), Cultural Studies: Grundlagentexte zur Einführung, Lüneburg: zu Klampen, S. 92-IIo.

Hall, Stuart (I999b): »Cultural Studies. Zwei Paradigmen«. In: Roger Bromley/Udo Göttlich/Carsten Winter (Hg.), Cultural Studies: Grundlagentexte zur Einführung, Lüneburg: zu Klampen, S. II3I38.

Hepp, Andreas (I999): Cultural Studies und Medienanalyse. Eine Einführung, Opladen: Westdeutscher Verlag.

Hepp, Andreas/Winter, Rainer (Hg.) (I999): Kultur - Medien - Macht. Cultural Studies und Medienanalyse. 2., überarbeitete und erweiterte Auflage, Opladen: Westdeutscher Verlag.

Herman, E.S./McChesney, R.W. (I997): The Global Media: The New Missionaries of Corporate Capitalism, London/Washington: Cassell.

Horkheimer, Max/Adorno, Theodor W. (I988): Dialektik der Aufklärung: Philosophische Fragmente, Frankfurt a.M.: Fischer Taschenbuchverlag.

Jarren, Otfried/Meier, Werner A. (I998): »Globalisierung der Medienlandschaft und ihre medienpolitische Bewältigung: Ende der Medienpolitik oder neue Gestaltungsformen auf regionaler und nationaler Ebene? «In: Patrick P. Donges/Otfried Jarren/Heribert Schatz (Hg.), Globalisierung der Medien? Medienpolitik in der Informationsgesellschaft, Opladen: Westdeutscher Verlag, S. 23I-249. 
Johnson, Richard (I999): »Was sind eigentlich Cultural Studies?«. In: Roger Bromley/Udo Göttlich/Carsten Winter (Hg.), Cultural Studies. Grundlagentexte zur Einführung, Lüneburg: zu Klampen, S. I39-188.

Karmasin, Matthias/Winter, Carsten (2000): »Kontexte und Aufgabenfelder von Medienmanagement«. In: Dies. (Hg.), Grundlagen des Medienmanagements, München: Fink (UTB), S. 15-39.

King, Anthony D. (Hg.) (I99I): Culture Globalization and the WorldSystem: Contemporary Conditions for the Representation of Identity, London: Macmillan Press.

Klein, Naomi (200I): No Logo! Der Kampf der Global Players um Marktmacht. Ein Spiel mit vielen Verlierern und wenigen Gewinnern, Gütersloh: Bertelsmann.

Kleinsteuber, Hans-J./Thomaß, Barbara (I999): »Kommunikation, Medien, Wissensgesellschaft: Globale Kommunikation und lokale Sprachlosigkeit - Alte und neue Medien - Wissensgesellschaft und Kommerz - Globale Medien und lokale Kulturen - Meinungsfreiheit, Zensur, Kriegspropaganda - Ordnungspolitik und Markt«. In: Ingomar Hauchler/Dirk Messner/Franz Nuscheler (Hg.), Globale Trends 2000. Fakten, Analysen, Prognosen, Frankfurt a.M.: Fischer Taschenbuchverlag, S. I4I-I65.

Levitt, Theodor (1983): »The Globalization of Markets«. Harvard Business Review 61/3, S. 92-102.

Lutter, Christina/Reisenleitner, Markus (1998): Cultural Studies. Eine Einführung, Wien: Turia + Kant.

MacIntyre, Alasdair (1987): Der Verlust der Tugend. Zur moralischen Krise der Gegenwart, Frankfurt a.M.: Campus.

Maier, Matthias (2000): »Medienmanagement als strategisches Management«. In: Matthias Karmasin/Carsten Winter (Hg.), Grundlagen des Medienmanagements, München: Fink, S. 59-92.

McChesney, Robert W. (1998): »The Political Economy of Global Communication«. In: Robert W. McChesney/Ellen Meiksins Wood/John Bellamy Foster (Hg.), Capitalism and the Information Age: The Political Economy of the Global Communication Revolution, New York: Monthly Review Press, S. I-26.

Mintzberg, Henry et al. (1999): Strategy Safari. Eine Reise durch die Wildnis des strategischen Managements, Wien: Überreuther.

Montgomery, Cynthia A./Porter, Michael E. (Hg.) (1996): Strategie: Die brillanten Beiträge der weltbesten Experten, Wien: Überreuther. 
Morris, Meaghan (1990): »Banality in Cultural Studies«. In: Patricia Mellencamp (Hg.), Logics of Television. Essays in Cultural Criticis, Bloomington: Indiana University Press, S. I4-43.

Münch, Richard (1996): »Mut zum Spagat. Transdisziplinäres Studieren in den Kulturwissenschaften«. In: Carsten Winter (Hg.), Kulturwissenschaft: Perspektiven, Erfahrungen, Beobachtungen, Bonn: ARCult-Media, S. I5-30.

Negus, Keith (1997): »The Production of Culture«. In: Paul du Gay (Hg.), Production of Culture/Cultures of Production, London: Sage, S. 68-II8.

Nelson, Cary/Treichler, Paula/Grossberg, Lawrence (1992): »Cultural Studies: An Introduction«. In: Lawrence Grossberg/Cary Nelson/Paula Treichler (Hg.), Cultural Studies, New York; London: Routledge, S. I-I6.

Picard, Robert G. (1997): »Entwicklung der Kommunikations- und Medienstrukturen in den USA. Auswirkungen auf Entscheidungen in Europa.«In: Gerd G. Kopper (Hg.), Europäische Öffentlichkeit: Entwicklung von Strukturen und Theorie, Berlin: Vistas Verlag, S. I09-I2I.

Porter, Michael E. (1999): Wettbewerbsvorteile. Spitzenleistungen erreichen und behaupten, Frankfurt a.M./New York: Campus Verlag.

Raulf, Holgar (1997): »Anforderungen an Nachwuchskräfte in Verlagen«. In: Jürgen Deters/Carsten Winter (Hg.), Karriere in der Medienbranche: Anforderungen, Schlüsselqualifikationen, Ausbildungssituation, Frankfurt a.M./New York: Campus Verlag, S. 2I-30.

Robertson, Roland (199I): »Sociel Theory, Cultural Relativity and the Problem of Globality«. In: Anthony D. King (Hg.), Culture Globalization and the World-System: Contemporary Conditions for the Representation of Identity, London: Macmillan Press, S. 69-90.

Robertson, R. (1992): Globalization: Social Theory and Global Culture, London: Sage Publications.

Robins, Kevin (1997): »What in the World's going on?«. In: Paul du Gay (Hg.), Production of Culture/Cultures of Production, London: Sage, S. II-66.

Rushkoff, Douglas (200I): Der Anschlag auf die Psyche. Wie wir ständig manipuliert werden, Stuttgart; München: DVA.

Sjurts, Insa (2000): »Chancen und Risiken im globalen Medienmarkt - Die Strategien der größten Medien-, Telekommunikations- 
und Informationstechnologiekonzerne«. In: Hans Bredow Institut (Hg.), Internationales Handbuch für Hörfunk und Fernsehen, Hamburg: Nomos, S. 3I-45.

Stürzebecher, Gerd/Schröter, Thomas (1997): »Multimedia - Anforderungsprofile im Wandel?«. In: Jürgen Deters/Carsten Winter (Hg.), Karriere in der Medienbranche: Anforderungen, Schlüsselqualifikationen, Ausbildungssituation, Frankfurt a.M./New York: Campus Verlag, S. 45-54.

Thompson, Edward P. (I999): »Kritik an Raymond Williams' >The Long Revolution «. In: Roger Bromley/Udo Göttlich/Carsten Winter (Hg.), Cultural Studies: Grundlagentexte zur Einführung, Lüneburg: zu Klampen, S. 75-9I.

Thompson, Kenneth, (Hg.) (1997): Media and Cultural Regulation, London: Sage.

Tomlinson, John (I99I): Cultural Imperialism. A Critical Introduction, London: Pinter Publishers.

Tomlinson, John (I997): »Cultural Globalization and Cultural Imperialism.« In: Ali Mohammadi (Hg.), International Communication and Globalization: A Critical Introduction, London: Sage, S. I70I90.

Tomlinson, John (2000): »Globalisierung, Kultur und komplexe Vernetzungen«. In: Thomas Düllo/Arno Meteling/André Suhr/Carsten Winter (Hg.), Kursbuch Kulturwissenschaft, Münster: LIT Verlag, S. $17-43$.

Williams, Raymond (1983), »Zur Basis-Überbau-These in der marxistischen Literatur.«In: Gustav H. Klaus (Hg.), Raymond Williams. Innovationen. Über den Prozeßcharakter von Literatur, Frankfurt a.M.: Suhrkamp, S. I83-20I.

Winter, Carsten (1996): »Einleitung«. In: Ders. (Hg.), Kulturwissenschaft: Perspektiven, Erfahrungen, Beobachtungen, Bonn: ARGult Media, S. 9-I4.

Winter, Carsten (I998): »Die Rolle der Medien im Kontext von Kulturwandel. Ansätze und Probleme ihrer kulturwissenschaftlichen Bestimmung«. In: Thomas Düllo et al. (Hg.), Einführung in die Kulturwissenschaft, Münster: LIT-Verlag, S. 295-327.

Winter, Carsten (2000a): »Kulturwandel und Globalisierung. Eine Einführung in die Diskussion«. In: Caroline Y. Robertson/ Carsten Winter (Hg.), Kulturwandel und Globalisierung, Baden-Baden: Nomos, S. 13-73. 
Winter, Carsten (2000b): »Synkretismus - Kulturwissenschaft? - Karriere. KULTUR-wissenschaftlich studieren für interessante berufliche Perspektiven in der Medien- und Kommunikationsbranche«. In: Thomas Düllo/Arno Meteling/André Suhr/Carsten Winter (Hg.), Kursbuch Kulturwissenschaft, Münster: LIT Verlag, S. 299-320.

Winter, Carsten (2000c): »Medienmanagement als interkulturelles Medienmanagement«. In: Matthias Karmasin/Carsten Winter (Hg.), Grundlagen des Medienmanagements, München: Fink (UTB), S. $245^{-278 .}$

Winter, Carsten (200га): »Das Management interkultureller Kommunikation. Neue Perspektiven für die Erforschung von Kommunikationskulturen und -netzwerken im Wandel.« In: Ursula Maier-Rabler/Michael Latzer (200I) (Hg.), Kommunikationskulturen zwischen Kontinuität und Wandel. Universelle Netzwerke für die Zivilgesellschaft (Proceedings of the DGPuK Conference 2000 in Vienna), Konstanz: UVK Verlag, S. 373-391.

Winter, Carsten/Karmasin, Matthias (200I): »Ökonomisierung aus unternehmensstrategischer Perspektive: Ursachen, Formen und Folgen der globalen Kommerzialisierung medialer Wertschöpfungsprozesse.«In: Otfried Jarren, O./Werner A. Meier (Hg.), Ökonomisierung der Medienindustrie: Ursachen, Formen und Folgen, Themenheft der Zeitschritt Medien und Kommunikation, Baden-Baden: Nomos, S. 206-2I7.

Winter, Rainer (I995): Der produktive Zuschauer. Medienaneignung als kultureller und ästhetischer Prozeß, München: Quintessenz.

Winter, Rainer (2000a): »Die Kunst des Handelns unter globalen Bedingungen. Zum Verhältnis von Populärkultur und Postmodernismus«. In: Caroline Y. Robertson/Carsten Winter (Hg.), Kulturwandel und Globalisierung, Baden-Baden: Nomos, S. I53-I74.

Winter, Rainer (2000b): »Differenz und Hybridität in der Medienrezeption«. In Thomas Düllo/Arno Meteling/André Suhr/Carsten Winter (Hg.), Kursbuch Kulturwissenschaft, Münster: LIT Verlag, S. 22I-23I.

Wolff, Janet (I99I): »The Global and the Specific: Reconciling Conflicting Theories of Culture«. In: Anthony D. King (Hg.), Culture Globalization and the World-System: Contemporary Conditions for the Representation of Identity, London: Macmillan Press, S. I6II73. 
Zerdick, Axel et al. (1999): European Communication Council Report. Die Internet Ökonomie. Strategien für die digitale Wirtschaft, Berlin et al.: Springer-Verlag. 


\section{Cultural Studies, Medienanalyse und Rezeptionsästhetik}

LOTHAR MiKos

\section{Vorbemerkung}

In den 7oer Jahren des 20. Jahrhunderts wurden in der Bundesrepublik Deutschland erstmals die Arbeiten der britischen Cultural Studies rezipiert. Auf die Rezeptionsgeschichte soll hier nicht weiter eingegangen werden, das ist an anderer Stelle bereits ausführlich geschehen (vgl. Lindner I994; Mikos I997a). Es seien jedoch einige Bemerkungen dazu erlaubt, warum die Rezeption gerade in der Zeit und vor allem nicht in der Literaturwissenschaft, sondern in den Sozialwissenschaften einsetzte. Einer der Gründe liegt im sozialen Wandel der damaligen Zeit. Die allmählich Auflösung starrer Klassengrenzen ging mit einer Unsicherheit über die sozialen Orte einher, an denen die Individuen ihre Identitäten ausbildeten. Das Bildungssystem in der Bundesrepublik Deutschland hatte ähnlich wie in Großbritannien die Universitäten für Kinder aus der Arbeiterklasse und dem Kleinbürgertum durchlässiger werden lassen. Immer mehr Angehörige dieser sozialen Schichten strömten an die Hochschulen. Dort fühlten sie sich jedoch nicht richtig aufgehoben. Trotz aller Idealisierung der Kritischen Theorie und der Politischen Ökonomie herrschten die Gepflogenheiten der bürgerlichen Kultur vor. In den linken Zirkeln konnte man sich mit »echten « Arbeiterkindern ein Stück Authentizität der Arbeiterkultur sichern, wenn auch nur legitimatorisch. Erst all- 
mählich bildete sich auch in Deutschland eine Neue Linke heraus, die sich immer mehr für die Alltagskultur der Menschen, für die Arbeiterkultur und für die Mentalitätsgeschichte der »einfachen« Menschen interessierte.

In dieser Situation fielen die Arbeiten der britischen Cultural Studies auf einen fruchtbaren Boden. Richard Hoggart und Raymond Williams boten mit ihrem Verständnis von Kultur als etwas Alltäglichem einen Ausweg aus einem Kulturverständnis, »das sich ausschließlich auf ästhetische und intellektuelle Werke und Prozesse bezieht« (Lindner 2000: 19). Das Alltagshandeln der Menschen gelangte allmählich als bedeutungsvolle kulturelle Praxis in den Blick, und damit konnte die Rede von der Kultur als ganze Lebensweise erst ihre Bedeutung im sozialwissenschaftlichen und intellektuellen Diskurs der damaligen Zeit entfalten. Rolf Lindner hat in seinem Essay über die Entstehungsgeschichte der Cultural Studies in Großbritannien dazu angemerkt:

"Es geht hier letzten Endes, und diese Dimension ist bei der Analyse der Genese der Cultural Studies bislang sträflich vernachlässigt worden, um eine Politik der Anerkennung, in einem Kontext, in dem Kultur zum Mittel symbolischer Gewalt wird» (Lindner 2000: 21 ff.).

Das trifft auch auf die Situation in der Bundesrepublik Deutschland zu. Denn es ging letztlich auch um die Anerkennung der Lebensweise derjenigen, die aus der Arbeiterklasse und dem Kleinbürgertum an die Hochschulen gekommen waren. Sie hatten im universitären Milieu schnell festgestellt, »dass den Kultivierten jegliche Vorstellung davon fehlt, wie das Leben für die große Mehrheit der Bevölkerung aussieht« (ebd.: 24). Mit den Cultural Studies wurde die gelebte Erfahrung dieser großen Mehrheit der Bevölkerung zum Gegenstand intellektueller Diskurse in den Universitäten. Zugleich fanden die Aufsteigerkinder in diesem Ansatz eine intellektuelle Heimat. Faszinierend war für sie insbesondere, dass die gelebten Erfahrungen, ja die ganze Lebensweise ihrer Herkunftsklassen nicht mehr mit einem abfälligen Blick aus der hohen Warte bürgerlicher Kultur und bürgerlichen Intellekts bedacht wurden, sondern sie wurden mit ihren eigenen Lebenserfahrungen ernst genommen. Was Rolf Lindner für die Vertreter der British Cultural Studies gezeigt hat, galt auch für die deutschen Vertreter, biographische Erfahrungen wurden zum kulturellen Kapital (ebd.: 
40). Diese biographischen Erfahrungen waren seit Ende der I950er Jahre auch wesentlich durch die Popkultur und durch die Medien, insbesondere das Fernsehen bestimmt.

Aus diesem Grund wurden in der Bundesrepublik zunächst vor allem diejenigen Arbeiten des Birminghamer Centre for Contemporary Cultural Studies (CCCS) rezipiert, die sich mit der Arbeiterkultur, jugendlichen Subkulturen und dem Medienumgang im Alltag befassten. Es dominierte zunächst die ethnographische Ausrichtung, Textanalysen wurden kaum betrieben. Das war insofern erstaunlich, als die britischen Cultural Studies versuchten, beide Richtungen in einem Ansatz zu vereinen. Aus literaturwissenschaftlichen Traditionen geboren (vgl. als Überblick Göttlich I997a; Maas I980), betrieb das CCCS in den I970er Jahren in seinen Jugendstudien explizit ethnographische Forschung, indem die Homologien zwischen den Lebensweisen der Jugendlichen und den kulturellen Ausdrucksformen, zu denen auch massenmediale Produkte zählten, untersucht wurden (vgl. als Überblick Lindner 1995; Winter 1997a). Erst mit der Untersuchung der Stile jugendlicher Subkulturen leitete Dick Hebdige 1979 eine Wende zur Textanalyse ein (vgl. Lindner 1995: 35), die in der Bundesrepublik jedoch erst in den I98oer Jahren vor allem in der Jugendsoziologie nachvollzogen wurde. Der ethnographische Ansatz war in den I970er Jahren für die Aufsteigerkinder an den Universitäten nicht nur interessant, weil ihre Herkunftsklasse ernst genommen wurde, sondern weil darin auch eine Parteinahme lag. Denn letztlich ging es darum, über das ethnographische Verstehen der Kultur unterdrückter Klassen und Minderheiten diesen zur Selbstermächtigung zu verhelfen. Das konnte nur dann gelingen, wenn deren Anliegen in den politischen Diskurs, der maßgeblich von Intellektuellen mitbestimmt war, einfließen konnten.

In der Beschäftigung mit den Medien, insbesondere dem Fernsehen, wurden zunächst Rezeptionsstudien besonders wichtig. Die hatten in der bis dahin dominierenden Publizistik- und Kommunikationswissenschaften eher ein Schattendasein geführt, fühlte sich die Disziplin doch nach wie vor mehrheitlich den Paradigmen der Wirkungsforschung verpflichtet. Daher hatten Rezeptionsuntersuchungen vor allem experimentell psychologischen Charakter (vgl. als Überblick Charlton 1997; Schenk 1987). Daneben betrieb man hauptsächlich Kommunikatorstudien und Inhaltsanalysen. Ähnlich wie in den Cultural Studies gab es eine eher medien- bzw. textzentrierte Arbeitsweise 
und eine eher rezeptionsorientierte Arbeitsweise, wobei sich Letztere in den genannten Disziplinen durch ihren methodologischen Zugriff unterschied. Erst Mitte der I980er Jahre setzten sich auch in Deutschland mit einer Zunahme der so genannten qualitativen Medienforschung ethnographische Rezeptionsstudien durch, die teilweise von den britischen Cultural Studies beeinflusst waren.

Forschungen ebenso wie theoretische Entwicklungen in den jeweiligen Richtungen wurden weitgehend unabhängig voneinander betrieben, eine Verbindung nur selten gesucht. Während Rezeptionsstudien die Texte weitgehend ausblendeten, nahmen Kommunikatorstudien und Inhalts- bzw. Textanalysen den konkreten Umgang der Zuschauer mit den Medien nicht in den Blick. Immerhin wurde in den Cultural Studies dieser Zustand reflektiert (vgl. exempl. Grossberg I997; Walters 1995). Es ist m. E. dringend geboten, beide Richtungen miteinander zu verbinden. Sinnvoll scheint die Entwicklung einer Rezeptionsästhetik der audiovisuellen Medien, die in einen weiteren Ansatz der Cultural Studies eingebettet ist. Einerseits kann so die konkrete Interaktion zwischen Text und Zuschauer untersucht werden, andererseits kann diese Interaktion dann in einen weiteren Kontext (einen gesellschaftlichen, sozialen, politischen, ökonomischen, rechtlichen, technologischen, historischen und kulturellen) gestellt werden, zu dem das Projekt Cultural Studies den theoretischen Rahmen liefert. ${ }^{\mathrm{I}}$

\section{Text und Zuschauer als Konstituenten einer Rezeptionsästhetik}

Weder mediale Texte noch Zuschauer existieren als ontologische Gegebenheiten, sondern sie entstehen, indem sie sich in der Zeit an einem Ort realisieren. Ein Film- oder Fernsehtext ist zwar in der Regel produziert worden, hat aber als Ergebnis dieses Produktionsprozesses lediglich materielle Qualitäten. Erst indem er auf der Leinwand oder dem Bildschirm zu sehen ist und vom Zuschauer gesehen wird, realisiert er sich als Text. Gleiches gilt für die Zuschauer: Erst indem sie sich mit einem medialen Text auseinander setzen, realisieren sie sich als Zuschauer. Sowohl Text als auch Zuschauer existieren lediglich in einem latenten Stadium. Erst in der Interaktion miteinander konkretisieren sie sich in ihren jeweiligen Funktionsrollen. Der Zuschauer ist somit als Funktionsrolle in einer Interaktionssituation zu sehen, in 
der er zwar nicht mit einer anderen Person interagiert, sondern mit einem Text, der auch als eine Funktionsrolle zu sehen ist, da er in der Interaktion mit dem Zuschauer symbolisches Material bereitstellt. Birgitta Höijer hat in Bezug auf die Fernsehrezeption festgestellt: »The interpretation of a television programme should thus be regarded as a continuous interplay between the viewer and the programme« (Höijer I992: 292). Dies ist jedoch nicht nur auf die Interpretation von Texten zu beziehen, sondern gilt für alle Aktivitäten des Zuschauers, die in der Rezeption eine Rolle spielen. Das reziproke, dialogische Text-Zuschauer-Verhältnis im Rahmen der kommunikativen Konstellation kann generell als permanentes Wechselspiel zwischen Text und Zuschauer gesehen werden. Die Rezeption von Film- und Fernsehtexten lässt sich ähnlich dem Lesen von schriftlichen Texten als »Prozess einer dynamischen Wechselwirkung von Text und Leser« (Iser I984: I76) bzw. Zuschauer beschreiben. In der Interaktion zwischen Text und Zuschauer entsteht etwas gemeinsames Drittes, der rezipierte Text, der nicht mit dem »Originaltext«, dem Film auf der Leinwand oder der Sendung auf dem Bildschirm, identisch ist.

Im Rahmen einer Rezeptionsästhetik des Fernsehens wird das Text-Zuschauer-Verhältnis weder als eindimensionaler Prozess der Massenkommunikation begriffen, bei dem eine wie auch immer geartete Medienbotschaft auf einen wie auch immer dispositionierten Zuschauer trifft und dort ihre Wirkung entfaltet, noch als eindimensionaler Prozess, bei dem ein in ein soziales Umfeld eingebetteter Zuschauer motiviert ist, bestimmte Medienbotschaften oder Programmtypen zu nutzen. Für die Betrachtung dieses Text-ZuschauerVerhältnisses spielt so weder der Produzent des Textes noch seine Intentionen eine Rolle, sondern lediglich die beiden Teile, die dieses Verhältnis bestimmen, der Text und der Zuschauer, sowie die lebensweltliche und gesellschaftliche Einbindung dieser Interaktionsform.

Das Text-Zuschauer-Verhältnis wird daher als eine kommunikative Konstellation begriffen, im Rahmen derer ein produzierter medialer Text mit einem im Rahmen eines spezifischen kulturellen Kontextes sozialisierten Zuschauer vor dessen lebensweltlichem Hintergrund interagiert. Mit dem Zuschauer ist zunächst kein empirischer $\mathrm{Zu}$ schauer gemeint, sondern der Zuschauer als neben dem Text modellhafter Teil eines Interaktionsverhältnisses, das gemeinhin als Rezeption bezeichnet wird, sowie der Zuschauer als Konstrukt im Text selbst, mit dem Rezeptionsprozesse vorstrukturiert werden. Mit Text 
ist hier nicht der Gesamttext des Fernsehens als Programmfluss gemeint, sondern ein einzelner Film oder eine einzelne Sendung bzw. Teile davon, mit denen ein Zuschauer interagiert. ${ }^{2}$ Diese kommunikative Konstellation, in der ein Text mit einem Zuschauer interagiert, ist insofern reziprok organisiert, als der Text Zuschaueraktivitäten vorstrukturiert, dabei aber auf einen Zuschauer trifft, der seinerseits als vorstrukturierendes Element auf einen Text trifft. Allerdings spielen in diesem Zusammenhang Persönlichkeitsmerkmale der Zuschauer keine Rolle, da sie keinerlei Einfluss auf die grundsätzliche Struktur des Text-Zuschauer-Verhältnisses als kommunikative Konstellation haben, sondern lediglich die Modalitäten der Realisation dieses Verhältnisses regeln, wie z.B. die Intensität der Interaktion oder die Motivation der Zuwendung.

Die Vorstrukturierung der Zuschaueraktivitäten ist nur möglich, weil der Text die Bedingungen enthält, die eine Interaktion mit ihm gelingen lassen. Denn er verortet sich einerseits im Universum bereits vorhandener Texte, andererseits weist er grundsätzlich einen Bezug zum Wissen der Zuschauer auf, ohne den er sich gar nicht realisieren ließe. Ein Text kann einerseits durch seine Ästhetik, seine Narration und seine Rhetorik die Bedingungen der Interaktion mit ihm deutlich machen. Andererseits enthält jeder Text, der sich als Handlungsanweisung an den Zuschauer versteht, ein implizites Gebrauchswertversprechen, das auf die Interaktion selbst zielt, nach dem Motto: »Interagiere mit mir, benutze mich, und du wirst mich verstehen und mir Sinn zuweisen können«. Die impliziten Handlungsanweisungen kann man auch als die Appellstruktur der Texte bezeichnen (vgl. dazu Iser I984). Dabei handelt es sich gewissermaßen um die Strukturierung des Zuschauers im Text, der vom Text als Interaktionspartner angesprochen wird. Es geht also um die Verankerung von Rezeptionsaktivitäten im Text selbst und damit um Strukturierungsmerkmale des Textes. Der Zuschauer ist sozusagen als Struktur im Text vorhanden. Da der Text darauf angelegt ist, mit einem Zuschauer zu interagieren, kommt der Struktur des Zuschauers im Text Aufforderungscharakter zu. Der Text selbst wird zur Handlungsanweisung für den Zuschauer.

"Vollendet sich der Text in der vom Leser zu vollziehenden Sinnkonstitution, dann funktioniert er primär als Anweisung auf das, was es hervorzubringen gilt, und kann daher selbst noch nicht das Hervorgebrachte sein« (Iser 1984: 175). 
Allerdings beziehen sich die Anweisungen des Textes nicht nur, wie Iser es hier beschrieben hat, auf die $\mathrm{zu} »$ vollziehende Sinnkonstitution«, sondern eben auf alle Textoperationen des Zuschauers, z.B. informationsverarbeitende Prozesse, die einer Sinnkonstitution vorgelagert sind. Die Zuschauer sind in der Rezeption physiologisch, kognitiv und emotional aktiv. All diese Rezeptionsaktivitäten werden in der Text-Zuschauer-Interaktion vom Text initiiert, d.h., sie sind als Anweisungen in der Struktur der Texte vorhanden.

Die Beschaffenheit der Texte muss daher in Bezug zu den Rezeptionsaktivitäten stehen. Filme und Fernsehtexte lassen sich zunächst einmal als Bilderzählungen klassifizieren, die sich verschiedener Symbolebenen bedienen, auf denen die Handlungsanweisungen an den Zuschauer angesiedelt sein können. Filme und Fernsehsendungen sind grundsätzlich mediale Bearbeitungen von Realität, unabhängig davon, ob sie fiktionale Geschichten erzählen, die für die Kamera inszeniert wurden, oder ob sie Ereignisse abbilden, die in der gesellschaftlichen Realität passiert sind. Diese mediale Bearbeitung zeigt sich auch in ihrer ästhetischen Gestaltung. Es geht also nicht nur darum, was in den Film- und Fernsehtexten erzählt wird, sondern auch darum, wie es als Erzählung inszeniert ist, um die Wahrnehmung und Aufmerksamkeit der Zuschauer zu erregen. In diesem Sinn sind alle Film- und Fernsehtexte ästhetisch gestaltet, und diese Gestaltung zielt auf Aktivitäten der Zuschauer. Ästhetik ist hier als Strukturmerkmal der Film- und Fernsehtexte gemeint, unabhängig davon, ob ein wie auch immer gearteter Autor bewusst oder unbewusst ästhetische Mittel eingesetzt hat. Für die Text-Zuschauer-Interaktion ist lediglich Ästhetik als strukturelles Textmerkmal relevant. Als solches strukturiert sie Aufmerksamkeit sowie emotionale und kognitive Aktivitäten des Zuschauers vor. In diesem Sinn ist die Ästhetik eines Films oder einer Fernsehsendung immer funktional zur Rezeption.

Die ästhetische Gestaltung und die Inszenierung geschehen sowohl auf einer formalen als auch auf einer inhaltlichen Ebene. Dabei können formale Elemente allerdings eine inhaltliche Funktion haben. Zur formalen Gestaltung von Film- und Fernsehtexten gehören Elemente wie Bildausschnitt, Perspektive, Kamerabewegung, Licht, Töne, Mise-en-Scène, Montage und andere. Zur inhaltlichen Gestaltung gehören Aspekte der Plotgestaltung, Dramaturgie, Erzähltechniken, die Ausgestaltung der Charaktere und andere (vgl. Mikos I996a+b, 
I997c+d+e, I998a+b, I999, 2000). Wenn man davon ausgeht, dass alle diese Elemente auf der formalen und inhaltlichen Ebene einen Aufforderungscharakter für die Zuschauer haben, dann kann man in diesem Zusammenhang nicht nur von der Ästhetik, sondern auch von der rhetorischen Struktur der Film- und Fernsehtexte sprechen. Die narrative Struktur der Texte ist in diesem Sinn immer rhetorisch vermittelt.

Mit dem Wissen, das Zuschauer an einen Film oder eine Fernsehsendung herantragen, tun sie zweierlei: Einerseits interagieren sie mit dem Filmtext und entwickeln so den rezipierten Text als die konkretisierte Bedeutung des »Originaltextes«, andererseits benutzen sie diesen rezipierten Text möglicherweise in ihrem Alltag. In diesem Sinn ist zwischen Rezeption und Aneignung zu unterscheiden (vgl. auch Mikos I994: 4I ff., 200Ia: 72 f., 200Ib). Mit Rezeption ist die konkrete Interaktion zwischen Film- oder Fernsehtext und Zuschauer bezeichnet, in der von beiden gemeinsam der rezipierte Text produziert wird. Sie ist mit der Dauer der Zuwendung identisch. Sie entspricht der Realisation des Textes durch den Zuschauer in der Zeit. »Aneignung ist demgegenüber die Übernahme des rezipierten Textes in den alltags- und lebensweltlichen Diskurs und die soziokulturelle Praxis des Zuschauers « (Mikos 200Ib: 63). Die Texte selbst können sowohl die Rezeption als auch die Aneignung strukturieren, indem sie entsprechende Angebote machen. Ein Film kann beispielsweise nur zu einem Kultfilm werden, wenn es ihm gelingt, sich im sozialen Netz spezifischer Zielgruppen mit bestimmten dort zirkulierenden Bedeutungen zu verankern. Der Text strukturiert hier nicht nur die konkrete Interaktion vor, sondern ebenso die Aneignung, indem er auf soziale Kontexte verweist. Die Unterscheidung zwischen Rezeption und Aneignung bietet den Vorteil, die konkrete Interaktion zwischen Film- oder Fernsehtext und Zuschauer von den Kontexten, in die diese Interaktion eingebettet ist, analytisch zu trennen, auch und gerade weil diese Kontexte in der Interaktion wirksam sind.

\section{Rezeptionsästhetische Medienanalyse}

Für das Verhältnis der Film- und Fernsehtexte zum Zuschauer ist bedeutsam, dass Texte grundsätzlich zum Wissen der Rezipienten hin geöffnet sind (vgl. dazu Wulff I985: 13; Mikos 1996a+b). Ein einfaches 
Beispiel mag das verdeutlichen: Um zu erkennen, dass es sich bei den Figuren auf der Leinwand um Menschen handelt, die in einem Restaurant an einem Tisch sitzen, muss der Zuschauer die ihm dargebotene Bildinformation entsprechend verarbeiten: Er muss wissen, was Menschen sind, was ein Restaurant ist und was ein Tisch ist. Das alles hat zunächst noch nichts mit der Bedeutung dieser Szene zu tun, sondern ist reine Informationsaufnahme. Die Bedeutung dieser Restaurantszene ergibt sich nun daraus, dass sie in einem narrativen Kontext des Films steht - der Zuschauer also aus der bisherigen Erzählung an dieser Stelle schließen kann, dass nicht in erster Linie der Zusammenhang von Hunger und Essen diese Szene kennzeichnet, sondern dass das Gespräch der Personen beim Essen bedeutsam ist. Zugleich ergibt sich die Bedeutung aber auch daraus, dass der $\mathrm{Zu}$ schauer um diese Möglichkeiten weiß, d.h. in diesem Fall, dass es nicht nur um das abgebildete Essen im Restaurant geht, sondern um das Gespräch, das dabei geführt wird. Der Zuschauer hat also, kurz gesagt, ein Wissen um die soziale Bedeutung von Restaurants, das er nun in der Rezeption aktivieren kann, weil der Filmtext zu diesem Wissen hin geöffnet ist. Es ist leicht vorstellbar, wie komplex diese Bezüge werden, wenn es nicht um so einfache Alltagsbegebenheiten wie Restaurantbesuche geht, sondern z.B. um Hierarchien, Geschlechterverhältnisse oder religiöse Praktiken.

Filme und Fernsehsendungen enthalten keine abgeschlossenen Bedeutungen, die Zuschauer oder analysierende Wissenschaftler »objektiv« freilegen könnten, sondern ihre Bedeutung entfaltet sich erst in der Rezeption und der Aneignung durch die Zuschauer.

"Die Wirkung eines Films kann man als ein Angebot an Bedeutungen, Zeichen, Gefühlsanregungen und Identifikationsmöglichkeiten begreifen, aus dem die Zuschauer und Zuschauerinnen ihr Filmerlebnis zusammensetzen und die sie zur Deutung ihrer Lebenswelt nutzen« (Lowry 1992: 123).

Dieses Filmerlebnis und seine Vorstrukturierung durch die Film- und Fernsehtexte muss im Mittelpunkt einer rezeptionsästhetischen Medienanalyse stehen. Sie muss z. B. herausarbeiten, wie die Texte auf das Wissen verschiedener Zuschauergruppen oder Publika hin geöffnet sind, wie die Texte während der Rezeption Wissen des Zuschauers aufbauen, oder anders ausgedrückt, wie Texte und Zuschauer die Interaktion vorstrukturieren und wie der rezipierte Text als Ergebnis der 
Interaktion entsteht und welche Bedeutung die Rezipienten ihm zuweisen. Dazu ist es aber gerade notwendig, nicht nur, wie das meistens in den Text- und Diskursanalysen gemacht wird, auf den Inhalt oder das Erzählte in den medialen Produkten einzugehen, sondern eben gerade auch auf die Darstellungs- und Gestaltungsmittel, mit denen die Narration vorangetrieben und die Rezipienten durch die Rezeption von Filmen und Fernsehtexten geführt werden. Es handelt sich also um eine strukturfunktionale Analyse, die in den Strukturen des Film- oder Fernsehtextes das Interaktionsangebot herauszuarbeiten versucht, durch das sich die Texte dann funktional für die $\mathrm{Zu}-$ schauer über deren kognitive und emotionale Aktivitäten erschließen. Wenn mediale Texte verschiedene Lesarten nahe legen, dann tun sie dies nicht nur auf einer Inhaltsebene, sondern auch auf der Ebene der Gestaltungsmittel, mit der die Wahrnehmung und die Aufmerksamkeit der Zuschauer gelenkt werden.

Diese Lenkung der Aufmerksamkeit ist notwendig, weil Film- und Fernsehtexte zwar die Komplexität der Welt reduzieren, doch sind Film- und Fernsehbilder an sich ausgesprochen komplex, »visueller Reichtum « (Chatman I990: 39) zeichnet sie aus. Aufgrund dieser Komplexität sind die Zuschauer in der Rezeption genötigt, die Bilder auf die wichtigen Informationen abzutasten. Dabei geht es allerdings nicht darum, einzelne Bildinformationen gewissermaßen herauszunehmen und als relevant anzusehen, sondern die Aktivität des $\mathrm{Zu}-$ schauers liegt darin, die verschiedenen Aspekte des Bildes zueinander in Beziehung zu setzen (vgl. Ellis I992: 54). Dabei kommt den einzelnen gestalterischen Mitteln besondere Bedeutung zu. Sie dienen den Zuschauern als Hinweise, die zum Verständnis der Filmerzählung beitragen und Erwartungen auf den Fortgang hervorrufen können. Sie sind für die Geschichte im Kopf der Zuschauer unentbehrlich.

Es sind diese spezifischen filmischen Darstellungsmittel, die die Zuschauer während der Rezeption eines Films oder einer Fernsehsendung an das Geschehen auf der Leinwand oder dem Bildschirm binden. Mit diesen formalen, stilistischen Mitteln werden die $\mathrm{Zu}-$ schauer vor allem emotional durch die Erzählung geführt, sie werden in bestimmte Stimmungen versetzt, ihre Aufmerksamkeit wird auf spezifische Aspekte im Film- oder Fernsehbild gelenkt, ohne dass ihnen dies immer bewusst wäre. Denn gerade die formalen und stilistischen Möglichkeiten bewegter Bilder machen die Erlebnisqualität eines Films oder einer Fernsehsendung aus. Sie beruhen allerdings 
auf Konventionen der Darstellung, aus denen sich auch das Wissen der Zuschauer um die filmischen Darstellungsweisen speist. Da sie auf Konventionen beruhen, können sie einerseits gelernt werden, andererseits wird das Wissen um sie zur Routine, sodass die Prozesse, die bei der Film- und Fernsehrezeption in Bezug auf die Darstellungsweisen ablaufen, vorbewusst und teilweise unbewusst sind. Das gilt auch für Wissenschaftler, die sich text- oder diskursanalytisch mit Filmen auseinander setzen. So beschreibt Jane Caputi eine Szene in Pretty Woman (USA I989, Garry Marshall), in der es offenbar zum ersten sexuellen Kontakt zwischen den beiden Protagonisten Vivian und Edward kommt, mit den Worten: »She crawls over to perform fellatio on him « (Caputi I99I: 5). Dieser Akt ist jedoch im Film gar nicht zu sehen, sondern aus der Montage der Bilder hat die Autorin als Rezipientin geschlossen, dass dem so sei. Sie hat also ihr Wissen benutzt, um die Leerstelle, die der Film gelassen hat, zu verstehen und zu interpretieren. Hier zeigt sich auch, wie ein Text sich mit dem kulturellen Diskurs verknüpft, und zwar über die Bedeutungszuweisung der Zuschauer.

\section{Kontextualisierung und Cultural Studies}

Hier zeigt sich bereits, dass die Bedeutungszuweisung über die konkrete Interaktion des Textes mit dem Zuschauer hinausweist, insofern als der Text im gesellschaftlichen Diskurs verankert wird, sowohl durch den Verweis auf andere Texte und die Produktion als auch durch den Verweis auf die Rezeption durch die Zuschauer. Auch das Verständnis von den Zuschauern weist über die konkrete Interaktion mit dem Text hinaus, insofern als die Rezeptionshandlungen der Zuschauer in lebensweltliche und kulturelle Diskurse und die soziokulturelle Praxis eingebettet sind, auf die sie auch wieder zurückwirken. Im Rahmen der Arbeiten der Cultural Studies, die sich mit der medialen Kommunikation und der Rolle der Medien in spätkapitalistischen Gesellschaften auseinander setzen, wurde denn auch der Begriff des Publikums (audience) zentral. Mit diesem Begriff wurde das Fernsehpublikum gegen die Zuschauerschaft (spectatorship) des Films abgegrenzt. Marie-Luise Angerer hat angemerkt, dass für beide Konzepte die »Frage nach dem Subjekt« zentral ist: 
"Doch das Subjekt der Filmtheorie - um es überspitzt zu formulieren - ist eines der Signifikation, es ist ein Knotenbündel vielfältiger enunziatorischer Verfahren, während das Zusehersubjekt des Fernsehens ein ssoziales Subjektı ist [...]» (Angerer 1994: 4).

In der weiteren Diskussion sollten beide Konzepte zusammengeführt werden, doch blieb das einzelne Zuschauersubjekt des Fernsehens als soziales Subjekt immer Teil des Publikums. Denn die Rezeption des Fernsehens ist stärker als die Filmrezeption in die soziokulturelle Praxis und die alltäglichen, kulturellen Diskurse eingebettet, denen die Zuschauer verhaftet sind. Es wird deutlich, dass die Text-ZuschauerInteraktion nicht unabhängig von diesen Kontexten gesehen werden kann.

In der jüngsten Zeit ist verschiedentlich versucht worden, die Bedeutung der Kontexte für die Text-Zuschauer-Interaktion (vgl. Mikos I994: 4I ff., 200Ia: 74 ff.) bzw. für die Medienrezeption generell (vgl. Göttlich I997b, 200I; Krotz I997, 200I) hervorzuheben. So hat Lawrence Grossberg ausdrücklich darauf hingewiesen, dass die Cultural Studies an der kontextuellen Idee diskursiver Praktiken und Wirkungen festhalten sollten: »Sowohl Texte als auch Publika sollten innerhalb umfassenderer Kontexte, die die Identität und die Wirkungen jeder Praxis artikulieren, betrachtet werden« (Grossberg I997: 22). Damit weist er auf eine der generellen Merkmale der Cultural Studies hin, die »radikale Kontextualisierung « (vgl. dazu auch Ang I996 und I997; Grossberg I994). Denn im Rahmen der Cultural Studies sind die sozialen, kulturellen, politischen, ökonomischen und historischen Kontexte bedeutsam, in denen Texte und ihre Nutzer interagieren (vgl. dazu auch Winter I995: Io8 ff.). Nur so können Macht- und Herrschaftsverhältnisse in den Blick geraten. Denn den Cultural Studies geht es darum, die spezifische Formation von Dominanz und Macht, Ökonomie und Leiden für spezifische Menschen als Agenten des sozialen und kulturellen Wandels zu verstehen (vgl. Grossberg I997). Verweist die Text-Zuschauer-Interaktion auf die konkreten textuellen sowie kognitiven und emotionalen Operationen, die den rezipierten Text hervorbringen, so kann deren Bedeutung erst im Rahmen der radikalen Kontextualisierung erschlossen werden.

„Aus der Sicht des radikalen Kontextualismus jedoch kann die Bedeutung des Fernsehens für die Rezipienten - textuell, technisch, psychologisch und sozial - außerhalb der multidimensionalen, intersubjektiven Netzwerke, in die das Objekt integriert und in konkreten 
kontextuellen Settings mit Bedeutung versehen wird, nicht bestimmt werden« (Ang 1997: 89).

Um herauszufinden, welche konkreten Bedeutungen in der Interaktion zwischen Text und Zuschauer aktualisiert werden und wie der rezipierte Text von den Zuschauern in den Alltag übernommen wird, müssen die Kontexte bestimmt werden, in die die Film- und Fernsehrezeption eingebunden ist.

In der Analyse von Film- und Fernsehtexten muss daher untersucht werden, wie die Texte zum Wissen der Zuschauer hin geöffnet sind, und die ethnographisch orientierte Rezeptionsforschung muss sich mit den situativen Kontexten befassen, in die die Text-Zuschauer-Interaktion eingebunden ist. Eine Rezeptionsästhetik der audiovisuellen Medien verbindet daher die Analyse von Film- und Fernsehtexten mit der Analyse von Rezeptionshandlungen. Sie legt ihren Fokus auf die Text-Zuschauer-Interaktion in der Rezeptionssituation, ohne dabei jedoch deren Einbettung in lebensweltliche, soziale, kulturelle und gesellschaftliche Kontexte außer Acht zu lassen, zumal sowohl Text als auch Zuschauer bereits immer selbst auf ihre Kontexte verweisen. Sie muss ganz im Sinne des Programms des »Projekts Cultural Studies« (Grossberg I994) die Kontexte mit berücksichtigen, wenn sie die Text-Zuschauer-Interaktion als Sinn stiftendes Handeln und als bedeutungsvoll im Rahmen der gesellschaftlichen Kommunikationsverhältnisse begreifen will. Sie ist damit handlungstheoretisch zu begründen. Dabei muss sie »die interkontextuelle Unendlichkeit« (Ang I997: 93) beachten, denn jede Text-Zuschauer-Interaktion als Handlungssituation ist durch »eine unbegrenzte Vielzahl von Kontexten charakterisiert «, wobei »Kontexte sich nicht gegenseitig ausschließen, sondern ineinander greifen und miteinander interagieren, ineinander geschachtelt und letztlich auch in Zeit und Raum unbegrenzt sind« (ebd.: 92). Mediale Texte verweisen nicht nur auf den Kontext der Rezeption und Aneignung, sondern auch auf Kontexte der Produktion, der technologischen Bedingungen, der intertextuellen Bezüge etc., ebenso wie die Zuschauer auf Kontexte des Alltags, der Kultur und der Lebenswelt, des Wissens, der Sozialisation etc. verweisen. Studien, die sich mit Medienphänomenen als kulturelle Praktiken befassen, müssen versuchen, von der konkreten Text-Zuschauer-Interaktion ausgehend, die Kontexte zu erfassen, die für ihr spezifisches Erkenntnisinteresse von Bedeutung sind. Dann ließe sich die inter- 
kontextuelle Unendlichkeit zumindest forschungsökonomisch einschränken.

\section{Zusammenfassung}

Grundlage jeglicher Bedeutung, die audiovisuelle Medien für die $\mathrm{Zu}-$ schauer erlangen können, ist die Text-Zuschauer-Interaktion in der Rezeptionssituation. Hier entfalten die Texte ihre Appellstruktur und erschaffen mit ihren Rezeptionsvorgaben den Möglichkeitsraum, in dem sich das Subjekt handelnd als Zuschauer realisieren und entfalten kann. Da die Texte zum Wissen der Zuschauer hin geöffnet sind, ist es dieses Wissen der Zuschauer, das die Texte in die soziale und kulturelle Zirkulation von Bedeutungen einbindet. Sowohl die Texte als auch die zuschauenden Subjekte sind sozial konstituiert, da sie einerseits den lebensweltlichen Kontexten verhaftet sind und andererseits in einem gegebenen historischen Kontext in gesellschaftlichen Strukturen handeln. Der ästhetische Aspekt liegt darin, dass die Gestaltung der Texte als ästhetische Struktur funktional als Element der Rezeption zu sehen ist. Zugleich gründet der ästhetische Aspekt aber auch darin, dass die Rezeptionserwartungen der Zuschauer, die sie in die Interaktion mit den Texten einbringen, ebenfalls ästhetisch strukturiert sind, nicht nur im Sinne einer »populären Ästhetik«, wie sie Pierre Bourdieu (I984: 64 ff.) gekennzeichnet hat, sondern in einem umfassenden Sinn als Wahrnehmung und Empfindung. Eine Rezeptionsästhetik hat dann die ästhetischen Strukturen der Texte als eine Funktion der Rezeption selbst zu untersuchen, denn in ihrer Appellstruktur ist die Aufforderung an den Zuschauer enthalten, seine Rezeptionserwartungen an ihr zu prüfen sowie sich in der Rezeption von ihr leiten zu lassen, um zu einer Empfindung und Erfahrung der Rezeptionshandlung $\mathrm{zu}$ gelangen. In diesem Sinn mündet die Text-Zuschauer-Interaktion als Rezeptionshandlung in rezipierte Texte als Rezeptionserlebnis. Ästhetik wäre dann das Movens, das die TextZuschauer-Interaktion als dynamischen Prozess initiiert. Zugleich kann sie aber auch darüber hinausweisen, indem sie nicht nur die Rezeption, sondern auch die Aneignung des Textes im Alltag der Zuschauer vorstrukturiert. Erst über die Interaktion mit den Zuschauern können Texte in deren alltägliche und lebensweltliche Kontexte einge- 
hen, indem sie angeeignet werden; denn über das Wissen der $\mathrm{Zu}$ schauer verknüpfen sie sich mit kulturellen Diskursen.

Eine Rezeptionsästhetik der audiovisuellen Medien, deren Grundlinien hier skizziert wurden, wäre dann eine Voraussetzung für die Untersuchung diskursiver Praktiken, wie sie von den Cultural Studies geleistet wird. Ebenso wären die Cultural Studies mit ihrer Forderung nach einer »radikalen Kontextualisierung« ein geeigneter Rahmen für eine Rezeptionsästhetik, da so die Kontextabhängigkeit der Text-Zuschauer-Interaktion in den Blick gerät. Allerdings bedarf es weiterer theoretischer Überlegungen, um eine Rezeptionsästhetik der audiovisuellen Medien auszudifferenzieren und um sie - auch unter forschungsökonomischen Gesichtspunkten - für empirische Untersuchungen fruchtbar zu machen. Möglich wäre dies nur mit einem interdisziplinären Vorgehen (vgl. dazu auch Charlton I997), für das die Cultural Studies als inter- und transdisziplinäres Projekt (Nelson/ Treichler/Grossberg I992: 4) Vorbild sein können.

Zugleich kann damit ein Weg beschritten werden, der die Cultural Studies und die Analyse populärer Medien an die Idee des politischen Projektes zurückbindet. Schließlich geht es darum, den alltäglichen Umgang mit den verschiedenen Medien ernst zu nehmen und ihn als Feld sozialer Auseinandersetzungen zu begreifen. Das scheint insbesondere in Zeiten der so genannten reflexiven Moderne und in Zeiten der lokalen Aneignung globalisierter Medienkultur wichtig zu sein. Der Medienumgang der verschiedenen Bevölkerungsgruppen im Rahmen ihrer sozialen Lebenslagen muss von einer von den Cultural Studies inspirierten Medien- und Kommunikationswissenschaft ernst genommen werden. Dann kann es gelingen, das »magische Dreieck« der Cultural Studies von Kultur - Medien - Macht als Kontextualisierung von Medienanalysen und Rezeptionsstudien zu institutionalisieren und um die Dimensionen des Alltags und der Ökonomie zu erweitern. Zwar scheinen viele Kämpfe in der reflexiven Moderne auf symbolischer Ebene als Kampf um Bedeutungen ausgetragen zu werden, doch dahinter stehen noch immer »echte Menschen«, die in materielle und soziale Lagen verstrickt sind. Es muss daher auch in der Medienanalyse und der Rezeptionsästhetik ganz im Sinne der britischen Cultural Studies um eine Politik der Anerkennung von Lebenspraktiken gehen. 


\section{Anmeriungen}

I Auf die Traditionslinien und Grundlagen einer Rezeptionsästhetik in der Kunst- und Literaturwissenschaft gehe ich hier nicht näher ein (vgl. dazu als Überblick Eagleton I994: 40 ff.; Groeben/Vorderer i988: I48 ff.; Kemp i992).

2 Der hier zugrunde gelegte Textbegriff geht auf die poststrukturalistische Sichtweise zurück, nach der Texte nicht als geschlossene Systeme zu sehen sind, sondern als Produkte des Schreibens und des Rezipierens (vgl. als Überblick Eagleton I994: IIo ff.). Das bedeutet, dass Texten kein abgeschlossener Sinn eigen ist, der entschlüsselt werden kann. Fiske bezeichnet denn auch Fernsehsendungen und andere populäre Texte in Anlehnung an Barthes nicht nur als produzierte, sondern als produzierbare Texte (Fiske I987: 95 ff.), d.h., sie bedürfen der Produktivität der Rezeption, denn erst dort entfalten sie sich. Über diese Produktivität der Rezeption verankern sie sich in der kulturellen Zirkulation von Bedeutungen.

\section{Literatur}

Ang, Ien (I996): »Ethnography and Radical Contextualism in Audience Studies«. In: James Hay/Lawrence Grossberg/Ellen Wartella (Hg.), The Audience and its Landscape, Boulder/Oxford: Westview, S. 247-262.

Ang, Ien (I997): »Radikaler Kontextualismus und Ethnographie in der Rezeptionsforschung«. In: Andreas Hepp/Rainer Winter (Hg.), Kultur - Medien - Macht. Cultural Studies und Medienanalyse, Opladen: Westdeutscher Verlag, S. 85-102.

Angerer, Marie-Luise (I994): »)Was, wenn nur der Hund fernsieht? Anmerkungen zu aktuellen Tendenzen in der TV-Forschung im Rahmen der Cultural Studies«. Medien Journal I, S. 3-9.

Bourdieu, Pierre (1984): Die feinen Unterschiede. Kritik der gesellschaftlichen Urteilskraft, Frankfurt a.M.: Suhrkamp.

Caputi, Jane (I99I): »Sleeping with the Enemy as Pretty Woman, Part II?«. Journal of Popular Film and Television I9/I, S. 2-8.

Charlton, Michael (I997): »Rezeptionsforschung als Aufgabe einer interdisziplinären Medienwissenschaft«. In: Ders./Silvia Schnei- 
der (Hg.), Rezeptionsforschung. Theorien und Untersuchungen zum Umgang mit Massenmedien, Opladen: Westdeutscher Verlag, S. I6-39.

Chatman, Seymour (1990): Coming to Terms. The Rhetoric of Narrative in Fiction and Film, Ithaca/London: Cornell University Press.

Eagleton, Terry (1994): Einführung in die Literaturtheorie, 3. Auflage, Stuttgart/Weimar: Metzler.

Ellis, John (I992): Visible Fiction. Cinema, Television, Video, 2. Auflage, London/New York: Routledge.

Fiske, John (I987): Television Culture, London/New York: Methuen.

Göttlich, Udo (I997a): »Kultureller Materialismus und Cultural Studies: Aspekte der Kultur- und Medientheorie von Raymond Williams«. In: Andreas Hepp/Rainer Winter (Hg.), Kultur - Medien Macht. Cultural Studies und Medienanalyse, Opladen: Westdeutscher Verlag, S. I03-II6.

Göttlich, Udo (I997b): »Kontexte der Mediennutzung. Zur handlungstheoretischen Modellierung der Medienrezeption«. montage/av 6/I, S. I05-II3.

Göttlich, Udo (200I): »Zur Kreativität der Medienrezeption. Eine theoretische Skizze zu Aspekten und Problemen einer handlungstheoretischen Modellierung der Medienkommunikation«. In: Patrick Rössler/Uwe Hasebrink/Michael Jäckel (Hg.), Theoretische Perspektiven der Rezeptionsforschung, München: Reinhard Fischer, S. I2I-I35.

Groeben, Norbert/Vorderer, Peter (I988): Leserpsychologie: Lesemotivation - Lektürewirkung, Münster: Aschendorff.

Grossberg, Lawrence (I994): »Cultural Studies: Was besagt ein Name?«. IKUS Lectures I7/I8 (Cultural Studies. Eine Intervention), S. II-40.

Grossberg, Lawrence (I997): »Der Cross Road Blues der Cultural Studies«. In: Andreas Hepp/Rainer Winter (Hg.), Kultur - Medien Macht. Cultural Studies und Medienanalyse, Opladen: Westdeutscher Verlag, S. 13-29.

Hebdige, Dick (I979): Subculture. The Meaning of Style, London/New York: Methuen.

Höijer, Birgitta (I992): »Reception of Television Narration as a Sociocognitive Process: A Schema-theoretical Outline«. Poetics 2I/4, S. 283-304. 
Iser, Wolfgang (1984): Der Akt des Lesens. Theorie ästhetischer Wirkung, 2. Auflage, München: Fink.

Kemp, Wolfgang (I992): »Kunstwissenschaft und Rezeptionsästhetik«. In: Ders. (Hg.), Der Betrachter ist im Bild. Kunstwissenschaft und Rezeptionsästhetik, Berlin, S. 7-27.

Krotz, Friedrich (I992): »Kommunikation als Teilhabe. Der >Cultural Studies Approach««. Rundfunk und Fernsehen 40/3, S. 4I2-43I.

Krotz, Friedrich (I995): »Fernsehen kultursoziologisch betrachtet. Der Beitrag der Cultural Studies zur Konzeption und Erforschung des Mediengebrauchs«. Soziale Welt 46/3, S. 245-265.

Krotz, Friedrich (I997): »Kontexte des Verstehens audiovisueller Kommunikate. Das sozial positionierte Subjekt der Cultural Studies und die kommunikativ konstruierte Identität des Symbolischen Interaktionismus«. In: Michael Charlton/Silvia Schneider (Hg.), Rezeptionsforschung. Theorien und Untersuchungen zum Umgang mit Massenmedien, Opladen: Westdeutscher Verlag, S. 73-89.

Krotz, Friedrich (200I): »Der Symbolische Interaktionismus und die Kommunikationsforschung. Zum hoffnungsvollen Stand einer schwierigen Beziehung«. In: Patrick Rössler/Uwe Hasebrink/ Michael Jäckel (Hg.), Theoretische Perspektiven der Rezeptionsforschung, München: Reinhard Fischer, S. 73-95.

Lindner, Rolf (I994): »Cultural Studies in der Bundesrepublik Deutschland. Eine Rezeptionsgeschichte«. IKUS Lectures I7/I8 (Cultural Studies. Eine Intervention), S. 50-57.

Lindner, Rolf (I995): »Kulturtransfer. Zum Verhältnis von Alltags-, Medien- und Wissenschaftskultur«. In: Wolfgang Kaschuba (Hg.), Kulturen - Identitäten - Diskurse. Perspektiven Europäischer Ethnologie, Berlin: Akademie, S. 3I-44.

Lindner, Rolf (2000): Die Stunde der Cultural Studies, Wien: Wiener Universitätsverlag.

Lowry, Stephen (I992): »Film - Wahrnehmung - Subjekt. Theorien des Filmzuschauers«. montage/av I/I, S. II3-I28.

Maas, Utz (I980): »Kulturanalyse«. OBST (Osnabrücker Beiträge zur Sprachtheorie) I6, S. I18-162.

Mikos, Lothar (I994): Fernsehen im Erleben der Zuschauer. Vom lustvollen Umgang mit einem populären Medium, Berlin/München: Quintessenz.

Mikos, Lothar (I996a): »Bilderfaszination und Kommunikation. 
Strukturfunktionale Film- und Fernsehanalyse. Teil I «. Medien Praktisch 20/3, S. 52-56.

Mikos, Lothar (I996b): »Die Geschichte im Kopf des Zuschauers. Strukturfunktionale Film- und Fernsehanalyse. Teil 2«. Medien Praktisch 20/4, S. 57-62.

Mikos, Lothar (I997a): »Die Rezeption des Cultural Studies Approach im deutschsprachigen Raum«. In: Andreas Hepp/Rainer Winter (Hg.), Kultur - Medien - Macht. Cultural Studies und Medienanalyse, Opladen: Westdeutscher Verlag, S. 159-169.

Mikos, Lothar (1997b): »Das Publikum und seine soziale Strukturiertheit. Zu Morleys Kategorie des >Haushalts«. montage/av 6/I, S. $89-96$.

Mikos, Lothar (I997c): »Aus weiter Ferne so nah. Strukturfunktionale Film- und Fernsehanalyse. Teil 3«. Medien Praktisch 2I/I, S. 44-49.

Mikos, Lothar (I997d): »Licht und Schatten. Strukturfunktionale Filmund Fernsehanalyse. Teil 4«. Medien Praktisch 21/2, S. 57-62.

Mikos, Lothar (1997e): »Monster und Mutanten in CinemaScope. Strukturfunktionale Film- und Fernsehanalyse. Teil $5 \ll$. Medien Praktisch 2I/3, S. 53-56.

Mikos, Lothar (1998a): »Kontinuität durch Schnitt und Montage. Strukturfunktionale Film- und Fernsehanalyse. Teil 6«. Medien Praktisch 22/I, S. 45-50.

Mikos, Lothar (1998b): »Helden, Versager und andere Typen. Strukturfunktionale Film- und Fernsehanalyse. Teil $7 \ll$. Medien Praktisch 22/4, S. 48-54.

Mikos, Lothar (I999): »Erlebnisse im intertextuellen Universum der Populärkultur. Strukturfunktionale Film- und Fernsehanalyse. Teil 8«. Medien Praktisch 23/3, S. 44-48.

Mikos, Lothar (2000): »Von möglichen und realen Welten. Strukturfunktionale Film- und Fernsehanalyse. Teil 9«. Medien Praktisch 24/I, S. 57-59.

Mikos, Lothar (200Ia): Fern-Sehen. Bausteine zu einer Rezeptionsästhetik des Fernsehens, Berlin: VISTAS.

Mikos, Lothar (200Ib): »Rezeption und Aneignung - eine handlungstheoretische Perspektive«. In: Patrick Rössler/Uwe Hasebrink/ Michael Jäckel (Hg.), Theoretische Perspektiven der Rezeptionsforschung, München: Reinhard Fischer, S. 59-7I.

Nelson, Cary/Treichler, Paula A./Grossberg, Lawrence (I992): »Cul- 
tural Studies: An Introduction«. In: Lawrence Grossberg/Cary Nelson/Paula A. Treichler (Hg.), Cultural Studies, London/New York: Routledge, S. I-I6.

Schenk, Michael (1987): Medienwirkungsforschung, Tübingen: J.C.B. Mohr.

Walters, Suzanna Danuta (1995): Material Girls. Making Sense of Feminist Cultural Theory, Berkeley/Los Angeles/London: University of California Press.

Winter, Rainer (I995): Der produktive Zuschauer. Medienaneignung als kultureller und ästhetischer Prozess, München: Quintessenz.

Winter, Rainer (I997a): »Vom Widerstand zur kulturellen Reflexivität. Die Jugendstudien der British Cultural Studies«. In: Michael Charlton/Silvia Schneider (Hg.), Rezeptionsforschung. Theorien und Untersuchungen zum Umgang mit Massenmedien, Opladen: Westdeutscher Verlag, S. 59-72.

Winter, Rainer (1997b): »Cultural Studies als kritische Medienanalyse: Vom sencoding/decoding «-Modell zur Diskursanalyse«. In: Andreas Hepp/Ders. (Hg.), Kultur - Medien - Macht. Cultural Studies und Medienanalyse, Opladen: Westdeutscher Verlag, S. 47-63.

Wulff, Hans Jürgen (1985): Die Erzählung der Gewalt. Untersuchungen $z u$ den Konventionen der Darstellung gewalttätiger Interaktion, Münster: MAkS. 


\section{Die Autorinnen und Autoren}

Richard Gebhardt (*I970), gelernter Assistent an Bibliotheken. Studium der Politischen Wissenschaft, Soziologie und Philosophie an der RWTH Aachen.

Arbeitsschwerpunkte: Neofaschismus/»Neue« Rechte, Jugend- und Populärkultur, Sozialstrukturanalyse

Udo Göttlich (*I96I), Dr. phil., M.A., Studium der Soziologie, Politischen Wissenschaft und Komparatistik an der RWTH Aachen. I99II996 wiss. Mitarbeiter am Sonderforschungsbereich 240 »Bildschirmmedien « der Universität Siegen; seit I996 wiss. Mitarbeiter am Rhein-Ruhr-Institut für Sozialforschung und Politikberatung (RISP) an der Gerhard-Mercator-Universität Duisburg, stellvertretender Projektleiter im Forschungsprojekt: »Daily Soaps und Kult-Marketing« im DFG-Schwerpunktprogramm »Theatralität«.

Arbeitsschwerpunkte: Medien-, Kommunikations- und Kultursoziologie, Cultural Studies, Rezeptionsforschung und Fernsehnutzung, qualitatitive Methoden der Kommunikationsforschung.

Publikationen (Auswahl): Kritik der Medien. Reflexionsstufen kritisch-materialistischer Medientheorien am Beispiel von Leo Löwenthal und Raymond Williams (Opladen I996); Kommunikation im Wandel. Zur Theatralität der Medien (gem. hg. mit J.-U. Nieland und H. Schatz, Köln I998); Cultural Studies. Grundlagentexte zur Einführung (gem. hg. mit R. Bromley und C. Winter, Lüneburg I999); Politik des Vergnügens. Zur Diskussion der Populärkultur in den Cultural Studies (gem. hg. mit R. Winter, Köln 2000); Daily Soaps und Daily Talks im Alltag von Ju- 
gendlichen (gem. hg. mit F. Krotz und I. Paus-Haase, Schriftenreihe Medienforschung der Landesanstalt für Rundfunk NRW, Bd. 38 , Opladen 200I).

Lawrence Grossberg (*1947), Morris Davis Professor of Communication Studies and Cultural Studies an der University of North Carolina in Chapel Hill.

Publikationen: We Gotta Get Out of This Place. Popular Conservatism and Postmodern Culture (New York/London I992); Dancing in Spite of Myself. Essays on Popular Culture. (Durham/London 1997); Bringing It All Back Home. Essays on Cultural Studies. (Durham/London 1997); What's going on? Cultural Studies und Popularkultur (Wien 2000); z.Zt. Vorbereitung einer Einführung in die Cultural Studies für den Sage-Verlag.

Andreas Hepp (*I970), Dr. phil., M.A., wiss. Assistent im Fachgebiet Medienwissenschaft am Institut für Medien- und Kommunikationswissenschaft der TU Ilmenau. I995-I997 wiss. Mitarbeiter im DFGProjekt »Über Fernsehen sprechen« an der Universität Trier, I997I999 Postdoktorand an der Universität Trier, I999 wiss. Angestellter an der Universität Karlsruhe (TH) am Interfakultativen Institut für Angewandte Kulturwissenschaft (IAK).

Arbeitsschwerpunkte: Medien- und Kulturtheorie, Mediensoziologie, Cultural Studies, Transkulturelle Medienforschung, Medienaneignungsforschung und qualitative Methoden der Medienforschung. Publikationen (Auswahl): Kultur - Medien - Macht. Cultural Studies und Medienanalyse (gem. hg. mit R. Winter, Opladen I997, 2. erw. Auflage 1999); Fernsehaneignung und Alltagsgespräche. Fernsehnutzung aus der Perspektive der Cultural Studies (Opladen I998); Cultural Studies und Medienanalyse. Eine Einführung (Opladen I999); Transkulturelle Kommunikation. Ein internationaler Reader (gem. hg. mit M. Löffelholz, Konstanz 200I).

Roman Horak (“I953), Dr. phil., Universitätsdozent an der Universität für angewandte Kunst in Wien. Mitbegründer und bis 1992 Co-Leiter des 1994 geschlossenen Instituts für Kulturstudien (IKUS) in Wien; Wintersemester $1988 / 89$ Honorary Visiting Fellow am Department of Sociology (University of Leicester, U.K.); 1999 Habilitation »Über Politik und Kultur. Auf dem Weg zu einer österreichischen Formation 
von Cultural Studies«; seit I998 Mitglied des International Board der Zeitschrift Cultural Studies.

Arbeitsschwerpunkte: Urbane Kulturen, Cultural Studies, Popularkultur, Ethnographie.

Publikationen (Auswahl): Metropole Wien. Texturen der Moderne (gem. hg. mit W. Maderthaner, S. Mattl, G. Meißl, L. Musner, A. Pfoser, 2 Bd., Wien 200o); Stadt.Masse.Raum. Wiener Studien zur Archäologie des Popularen (gem. hg. mit M. Maderthaner, S. Mattl, L. Musner, Wien 200I); Die Praxis der Cultural Studies. Über Politik und Kultur (erscheint 2002 in Wien).

Christina Lutter (*I970), Dr. phil., Historikerin; Mitglied des Instituts für Österreichische Geschichtsforschung; Herausgeberin der Reihe Cultural Studies sowie Autorin des gleichnamigen Einführungsbandes bei Turia + Kant (beides gemeinsam mit Markus Reisenleitner); seit I998 Lehraufträge und Gastprofessuren in Wien, Klagenfurt, Linz sowie Berlin und Lüneburg; seit I994 im österr. Wissenschaftsministerium: Entwicklung und Organisation der Forschungsschwerpunkte Cultural Studies/Kulturwissenschaften und Gender Studies.

Vorträge und Publikationen: Verfassungs-, Kultur- und Geschlechtergeschichte des Mittelalters und der Frühen Neuzeit; Cultural Studies und Gender Studies. Zur Zeit Arbeit an einem Habilitationsprojekt zum Thema »Geschlecht und Wissen im I2. Jh.«.

Lothar Mikos (*I954), Dr. phil. habil., Dipl.-Soz., Professor für Fernsehwissenschaft an der Hochschule für Film und Fernsehen »Konrad Wolf $\ll$ in Potsdam-Babelsberg. Studium der Soziologie, Psychologie und Publizistik. Seit I98I Lehrtätigkeiten und Gastprofessuren an Hochschulen in Berlin, Dresden, Florenz, Kassel, Klagenfurt, Leipzig und Potsdam. Mitglied der Redaktion der Zeitschrift tv diskurs, Mitglied des Redaktionsbeirats der Zeitschrift Medien Praktisch und Mitglied des Herausgebergremiums der Online-Zeitschrift Medienpädagogik.

Arbeitsschwerpunkte: Fernsehtheorie, Rezeptionstheorie und -forschung, Cultural Studies, Populärkultur, qualitative Methoden der Medienforschung, Film- und Fernsehanalyse, Mediensport, Gewaltdarstellungen in den Medien.

Publikationen (Auswahl): Sepp Herberger. Ein deutsches Fußballeben (gem. hg. mit H. Nutt, Frankfurt I997); Im Auge der Kamera. Das 
Fernsehereignis Big Brother (gem. hg. mit P. Feise, K. Herzog, E. Prommer, V. Veihl, Berlin 200I); Fern-Sehen. Bausteine zu einer Rezeptionsästhetik des Fernsehens (Berlin 200I); Die Fabrikation des Populären. Der John-Fiske-Reader (gem. hg. mit R. Winter, Bielefeld 200 I.

Sibylle Niekisch (*I973), Studium der Soziologie und Ethnologie in Freiburg/Br. Studienbegleitend: Entwicklungshilfe bei einer NGO in Brasilien/Mato Grosso; Webdesign in einer Werbeagentur; Mitarbeit am Institut für Soziologie der Universität Freiburg.

Otto Penz (*I955), Adjunct Associate Professor für Soziologie an der Universität Calgary, Lehrtätigkeiten an der Wirtschaftsuniversität Wien sowie an der Karl-Franzens-Universität Graz.

Arbeitsschwerpunkte: Neben Themen rund um den Sport, kulturwissenschaftliche Themen wie Körper und Schönheit, Freizeit und Tourismus, Konsum oder Massenkommunikation.

Publikationen (Auswahl): Schönheit des Körpers (gem. hg. mit W. Pauser, Wien I995); Massentourismus und sozialer Wandel (gem. hg. mit R. Bachleitner, München/Wien 200o); Metamorphosen der Schönheit. Eine Kulturgeschichte moderner Körperlichkeit (Wien 200I).

Manuela Ribeiro Sanches (*I95I), Professorin an der Geisteswissenschaftlichen Fakultät der Universität Lissabon, Fächer »Deutsche Kultur « (Fachbereich Germanistik) und »Identität und Minderheiten « (Europäische Studien). Promotion über Georg Forster.

Forschungsinteressen und Veröffentlichungen: Georg Forster, Reiseliteratur, Geschichte der Anthropologie, Cultural Studies, Postcolonial Studies.

Olaf Sanders (*I967), Dr. phil., wiss. Assistent am Institut für Allgemeine Erziehungswissenschaft der Universität Hamburg.

Arbeitsschwerpunkte: Philosophie und Geschichte der Bildung, Populäre Kultur, Jugendforschung, interkulturelle Kommunikation.

Aktuelle Publikation: Romantik, Zerstörung, Pop. Studien zu einer Theorie der Selbstbildung (Opladen 2000).

Günther Sandner (*I967), Lehrbeauftragter für Politikwissenschaft (Politische Theorie) an der Universität Salzburg, wiss. Mitarbeiter am 
Forschungsprojekt »The Making of History« im Rahmen des Wittgensteinpreises an der Österreichischen Akademie der Wissenschaften. Bisherige Tätigkeiten in den Bereichen politische Erwachsenenbildung, Wissenstransfer und als Research Fellow am Internationalen Forschungszentrum für Kulturwissenschaften (IFK) in Wien.

Arbeitsschwerpunkte: Politische Kulturtheorien, Sozialdemokratie, Politische Ökologie, Geschichtspolitik.

Publikationen (Auswahl): Die Natur und ihr Gegenteil. Politische Diskurse der sozialdemokratischen Kulturbewegung bis 1933/34 (Frankfurt a.M. et al. I999); Umkämpfte Erinnerung. Die Wehrmachtsausstellung in Salzburg (gem. hg. mit H. Embacher und A. Lichtblau, Salzburg/Wien I999).

Birgit Wagner, o. Prof. für romanische Literaturwissenschaft an der Universität Wien.

Arbeitsschwerpunkte: Gender Studies und Cultural Studies in Verbindung mit den Literaturen romanischer Länder, Avantgardetheorie und Filmgeschichte der romanischen Länder.

Publikationen (Auswahl): Gramsci, Pasolini. Ein imaginärer Dialog (Wien I987); Technik und Literatur im Zeitalter der Avantgarden. Ein Beitrag zur Geschichte des Imaginären (gem. hg. mit J. Borek und S. Puntscher Riekmann, München I996).

Carsten Winter (*I966), M.A., Universitäts-Assistent am Institut für Medien- und Kommunikationswissenschaft der Universität Klagenfurt. Studium der Angewandten Kulturwissenschaften mit den Schwerpunkten Medien- und Kommunikationswissenschaft und Betriebswirtschaftslehre; I995-I998 Mitarbeiter am Institut für angewandte Medienforschung an der Universität Lüneburg; I998-2000 Mitarbeiter am Institut für Medien- und Kommunikationswissenschaft (Lehrstuhl Medienmanagement) an der TU Ilmenau.

Arbeitsschwerpunkte: Cultural Studies, Mediensoziologie, Globalisierung, Medienmanagement und Medienkulturgeschichte.

Publikationen (Auswahl): Medienwirtschaft und Gesellschaft 1. Medienunternehmen und die Kommerzialisierung von Öffentlichkeit (gem. hg. mit M. Karmasin u. M. Knoche, Münster 200I); Kursbuch Kulturwissenschaft (gem. hg. mit Th. Düllo, A. Meteling u. A. Suhr, Münster 200I); Grundlagen des Medienmanagements (gem. hg. mit M. Karmasin, 
München 200I); Kulturwandel und Globalisierung (gem. hg. mit C. Robertson. Baden-Baden 200I); Cultural Studies. Grundlagentexte zur Einführung (gem. hg. mit R. Bromley u. U. Göttlich, Lüneburg I999).

Rainer Winter (*1960), Dr. phil. habil., Dipl.-Psych., M.A., Studium der Psychologie, Philosophie und Soziologie. Privatdozent am Institut für Soziologie der Technischen Hochschule Aachen, Lehrbeauftragter für Medienwissenschaft an der Universität des Saarlandes und der TU Dresden; 200I Gastprofessuren an der Universität Gießen und der Universität Klagenfurt.

Arbeitsschwerpunkte: Kultur- und Mediensoziologie, Cultural Studies, qualitative Sozialforschung.

Publikationen (Auswahl): Der produktive Zuschauer. Medienaneignung als kultureller und ästhetischer Prozeß (München/Köln I995); Kultur-Medien-Macht. Cultural Studies und Medienanalyse (gem. hg. mit A. Hepp, Opladen I999, 2. Aufl.); Widerspenstige Kulturen. Cultural Studies als Herausforderung (gem. hg. mit K.H. Hörning, Frankfurt a.M. I999); Politik des Vergnügens (gem. hg. mit U. Göttlich, Köln 2000); Die Kunst des Eigensinns. Cultural Studies als Kritik der Macht (Weilerswist 200I). 


\section{transcript Kulturwissenschaften Novitäten Herbst 2001}

Georg Christoph Tholen,

Gerhard Schmitz,

Manfred Riepe (Hg.)

Übertragung - Übersetzung Überlieferung

Episteme und Sprache in der

Psychoanalyse Lacans

Oktober 2001, 452 Seiten,

kart., 49,80 DM

ISBN: 3-933127-74-2

Hans-Joachim Lenger

\section{Vom Abschied}

Ein Essay zur Differenz

Oktober 2001, 242 Seiten,

kart., 49,80 DM

ISBN: 3-933127-75-o

Annette Keck,

Nicolas Pethes (Hg.)

Mediale Anatomien

Menschenbilder als

Medienprojektionen

Oktober 2001, 454 Seiten,

kart., 49,80 DM

ISBN: 3-933127-76-9

Stefan Weber

Medien - Systeme - Netze

Elemente einer Theorie der

Cyber-Netzwerke

Oktober 2001, 128 Seiten,

kart., 24,80 DM

ISBN: 3-933127-77-7
Rainer Winter,

Lothar Mikos (Hg.)

Die Fabrikation

des Populären

Der John Fiske-Reader

(Cultural Studies 1, hrsg. von

Rainer Winter)

Aus dem Englischen

von Thomas Hartl

Oktober 2001, 372 Seiten,

kart., 49,80 DM

ISBN: 3-933127-65-3

Udo Göttlich, Lothar Mikos,

Rainer Winter (Hg.)

Die Werkzeugkiste

der Cultural Studies

Perspektiven, Anschlüsse und

Interventionen

(Cultural Studies 2, hrsg. von

Rainer Winter)

Oktober 2001, 348 Seiten,

kart., 49,80 DM

ISBN: 3-933127-66-1

Leseproben und weitere Informationen finden Sie unter: www.transcript-verlag.de 